


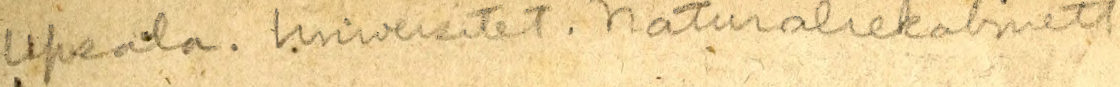

1

D. $D$.

\section{MUSEUM NATURALIUM ACADEMIÆE UPSALIENSIS.}

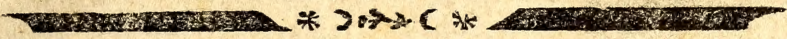

CUJUS

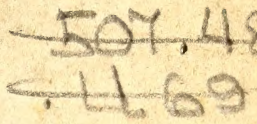

PARTEM PRIMAM

CONSENSU EXP. FAC, MED. UPSAL.

PR ESIDE

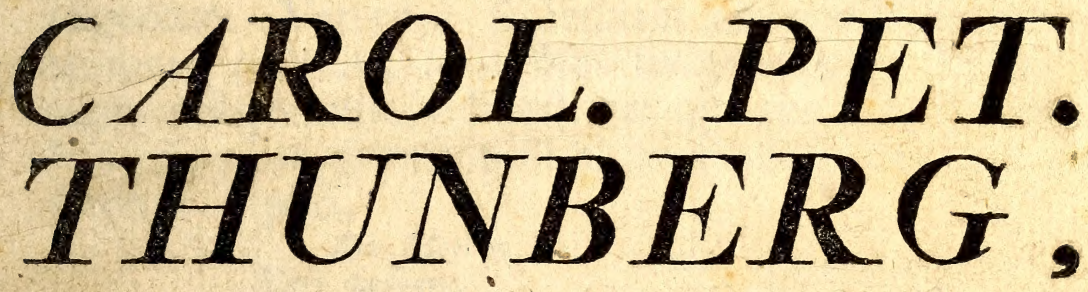

Equits Aurat. Reg. Ord, de Vasa,

Medic. Doct. Profess, Med, et Botan, Reg. egt Ord., Acad. Casar Nat. Curios, Reg. Scient. Holmiens. Societ. Scient. Ursar. Patr Holm. Berol. Nat, Scrut. Lund. Harlem. Amsteld. Zeland. Nidro siens. Medic. Endimburg. et Nat. Studios. Ibid. Membro; nec non Acad. Monsfeliens. Agricult. Paris. et Batavin, Correspond.

Publico examini proponir.

FRIDERICUS WILHELM. RADLOFF,

Stockbolmienfis.

in aUditorio Gust. MAJ. D. Xiv. April. mDCclixịX, H. A. M. S.

\section{U P S A L I A E,}

Typis Edmannianis, 


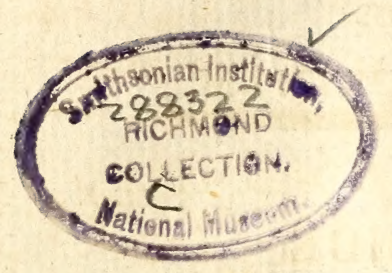




\section{9}

\section{PRAFATIO.}

A dminiculis ftudia non tantum modo adjuvantur, fed ad fummum etiam advehuntur faftigium. A multis retro fæculis, tempore jam olim OsymanDUÆ, Bibliothecas, ubicunque litteræ aliquo fuere pretio, haud fine cura collectas atque fervatas effe, neminem fugit. Noftro etiam tempore, maximæ librorum collectiones, omnibus Mufarum in delubris \& majoribus urbibus, minores vero apud omnes, quibus ftudia curæ cordique funt, fervantur, nec non augentur. Veterum fapientiam ad noviffima usque tempora retinent, \& codem tempore, quo ftudia honores affequuntur, Bibliothecæ quoque amplificantur. His etiam ex promtuariis infruetisfimis posfunt ftudiorum cupidi,

$$
\begin{aligned}
& \text { Apis Matina } \\
& \text { More modoque } \\
& \quad \text { Grata carpentis thyma. Horat. }
\end{aligned}
$$

artes haurire. Nemo umquam utilitatem earum ferio impugnabit, \& vix ullum Athenæum illis caruit, alia aliis licet fcientiis adminicula defiderio fuere.

Mathematicorum inftrumentorum ope, in facili eft cum utilitate cceleftia corpora obfervare \& quam celerrime fine ullo errore tempora atque fpatia ingentia emetiri.

Legum atque operationum naturx cognitionem \& explicationem Phyfica inftrumenta nobis edocuerunt, \& vix fine experimentis vel Eleßtrica vis, vel aquæ aut aëris proprietates, aut alia Phœnomena intelligi posfent.

Columella fua jam olim ætate miratus eft, agri- 
culturam Doetoribus carere; noftro tamen fxculo refervatum fuit, agriculturam, libero homini, Cicerone tefte, digniffimam artem \& opificum artificia publicis in acroateriis Academicis audire, \& varia agricultorum \& opificum inftrumenta, cum ufus explicatione videre.

Priusquam Chemia faftigium illud, quo jam confpicitur, attigit, inftrumentis Chemicis experimenta nftituere neceffe fuit; hac ex ratione etiam illa, in Athenxis tamquam neceffaria fcientix, hodie debito in honore habitx, comparata funt. Lithophylazia inftituta fuerunt, ut nulla fufcepta peregrinatione facile atque fine temporis difpendio poffint Metallicolx, qux montibus inclufa funt, cognofcere.

Hiftoria naturalis, vaftiffma fcientia ratione ob. jectorum, quæ per totum tellurem Iparfa obveniunt, haud facile fine animalium atque herbarum collectione, quæ noftra attentione digna funt, addifcitur. Majoribus in Lyceis Horti quidem Botanici, quibus herbx diffitis ex oris collecta coluntur, tamquam neceffarii habiti fuerunt; animalium vere \& ficcatarum herbarum collectiones diu Magnatibus \& divitibus curiofis fuere relictx, usque dum ufus earum magis perfpicuus factus, \& mufea earum ob utilitatem quibusdam in Academiis exftrueta. Collectiones namque multorum cura atque induftria factx, haud exigux utilitatis funt omnibus in Athenæis; uno enim loco poffumus multa Naturæ producta, omnium partium orbis terraquei intueri, quorum ad cognitionem adquirendam alias longinqua itinera, longo tempore \& magno fumptu fufcipienda forent. Plura quoque 
paucis diebus in Mufeis, quam per plures annos moleftiflimis in itineribus oculis luftrare poffumus, \& in naturalium collectionibus omnia animalia omnesque herbæ, quæ conveniunt aliqua ex parte, fxpiffime autem toto orbe funt divifa, juxta fe invicem pofica intueri, optimeque heic catenam omnia natura regna nectentem, vel potius, fecundum Donatr \& BoNNET, mirabile naturæ rete perfpicere \& admirari valemus.

Non ullus, cui præcordia meliori luto finxit Ti. tan, unquam denegavit fuam collektonibus naturali. um utilitatem; multa enim Omnipotentis miracula dum natura divcrfis removit oris, omniaque fere animalia hominis adfpectum refugiunt, multa quoque vel peregrinatorum ex ignorantia ficta vel ob tempo. ris anguftiam minus adcurate defcripta funt, in $\mathrm{Mu}$. feis inftruetis præfentia oculis pollunt fubjici, invicem comparari, \& accuratius examinari.

Aves atque earum varietates rite cognofcere, vix ac ne vix quidem fine collectione horum, facile oculis noftris fe fubtrahentium Volatilium fperare pofumus; non eft itaque quod miremur, Ornithologiam ad recentiora ufque tempora negleetam a multis fuiffe. Amphibia, hæc tetra animalia, quorum nomen etiam quandam negligentiam Hiftoricorum naturalium argu. it, maxima tamen attentione digna effe, contendimus, ut certo indagentur venenatæ \& noxia f́pecies \& ut conftet, quibus ex hac claffe vefci absque noxa animalibus poffimus.

Pifcium cognitio, valde in re cibaria utilis \& neceffaria, multos etiam veterum errores antiquavic. En- 
tomologia hodie egregie culta, cultorum labores abunde compenfat. Noxas Infectorum evitare, \& multas fpecies horum animalculorum noftrum in ufum convertere nobis edocuit. Stupenda exilitas, \& eximia quorundam pulchritudo præfertim Indicorum, omnium admirationem movent. Vermium Hiftoria, certe non inutilis indicat, cujus iudolis vermes homines infert 7 tes funt, non fine maxima in Practica Medicina utiitate. Omnem facile cognitionem Hiftorix Nituralis ftatim deftruetis Mufeis in antiquum ruere chaos, certo certius perfvafus fum, dum, hifce confervatis, veterum refervantur inventa; in his peregrinatores fpolia reponunt pretiofifima; \& fumma cum voluptate fcientiæ amatores poffunt inter mirabilia Optimi Creatoris verfari, illa cum reverentia quadam contemplari \& facili negotio nobiliffimam illam fcientiam addifcere, quæ opera Omnipotentis Creatoris pertractans, fummam erga infinitum Deum pietatem quoque excitat \& infpirat.

Noftrum vero non eft, vel in laudes Mufeorum rerum naturalium excurrere, vel late patentem eorum utilitatem demonftrare; fed Tux, B. L. cenfuræx mitiori fubjicere Catalogum illarum, ex animali inprimis \& vegetabili Regno, rerum Naturalium, qux in Mufeo Academix Upfalienfis fervantur, ut fciant Scientiæ Amatores, \& quorum liberalitate inftruetum \& ditatum fuit Mufeum, \& quænam heic animalia \& herbx, eorum in utilitatem \& commoda publico fumptu confervantur \& ut alii ad numerum earum adaugendum incitentur, in Creatoris veram gloriam \& Scientiarum incrementum certiffimum. 


\section{- ) $7(12$ \\ x. DONATIO I744.}

\section{CAR O L I G L LENBOR G,}

COMITIS, REGIS REGNIQUE SENATORIS,

REGI瓜 CANCELLARIÆ PR ÆSIDIS, ACAD.

UPSALIENSIS CANCELLARII *).

oce

Teftudo Mydas a1).

Mydas $\beta$ 2).
Teftulo Lutaria 3).

Geometrica 4).

*) Donatio faßta fuit 1744. vid. Acta Confift. Upfal. 3744. d. I Sept. p. 274. Inventarium confectum ut vid. ex act. eodem anno, d. 6 Oct. p. 360 . 387. Spiritus vini, Lagenæ vitreæ \& thecæ procuratæ eodem anno Act. d. 13 Oct. p. 39 I.

Volucrum imitamenta arte facta nonnulla, dono data mucore jam obducta, læfa \& omnino deperdita inveniuntur. Coralliorum \& minerarum pulcerrimæ varietatis mentio fit Am. acd. I p. 522. ex qua tantum Chryftalli nonnulli montani fuperfunt.

Cor humanum, quod inter hæc collectanea inveniebatur, ftatim in Nofocomio fervandum erat. Act. Confift. 1744 d. 13 Ost. p. 392. 


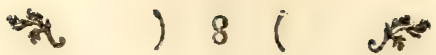

Rana Arborea.

Draco Volans.

Lacerta Crocodylas.

Igvana.

Ameiva.

Teguixin.

Marnorata.

Lemnifcata.

Salamandra.

Cordylus.

Gecko.
Coluber Berus.

Abatulla 163: I50.

Natrix.

Cobella 154: 52.

Domicella 119: 60.

Lemnifcatus 250: 33 .

Angulatus.

Petbola 205: 86.

Annulatus 190: $8 \mathrm{I}$.

Rbinocerotis cornu 5).

\section{DO.}

i) Integra eft, cum corpore ficcata, Lepadibus balanis fparfis ornata, mediocris magnitudinis.

2) Tefta tantum fuperior, priori major.

3) Magnitudo fere volre manus; tefta rotunda, parum convexa. Palmæ \& plantæ $5 \cdot$ dactylæ, membrana tectæ \& palmatæ, digitis 4 ungiculatis, cauda femoribus dimidio brevior.

4) Tefta major hujus tantum in Mufeo fervatur.

5) Dono datum 1746 vid. Act. Confift. 1746. d. 8 Nov. p. 994. 


\section{) 96 (4)}

\section{DONATIO 1745 .}

\section{ADOLPHI FRIDERICI,}

PRINCIPIS EO TEMPORE HÆREDIT. POSTEA

REGIS SVECIÆ GLORIOSISSIMI *).

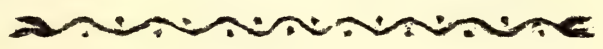

Simia apedia.

Lemur Tardigradus 1).

Didelpbis Marfupialis.

Murina 2).

Dafypus 7 - cinctus 3).

Verjpertilio Vampyrus.

Myrmecopbaga 3-dactyla.

Sciurus Flavus.
Teftudo Mydas ( 4).

Rana Boans.

Gibbofa.

Arborea $\beta$.

Pipa.

Paradoxa 5).

Lacerta Principalis.

Iguana var.

B

*) Vid. Act. Confift. Acad. Upfal. 1745. fem. pofter. p. II \& I 40 ubi individua, Spiritu-vini fervata numero 87 enumerantur, fcilicet: Serpentes 2r. Lacertæ 20. Ra. næ 5. Teftudo I. Quadrupedia 7. Infecta 7. Vermes 4. Pifces 22.

i) Cranium tantum.

2) Cum pullis.

3) Fœtus eft fine dentibus.

4) Defcribitur integra in Amcenit. Acad. fed non exftat, nifi tefta fuperior, fatis magna.

5) Completa \& larvata. 


\section{*) $10($ \%}

Lacerta Calotes.

Marmorata var. Ameiva var.

Cbamaleon. Aurata.

Seps.

Superciliofa.

Monitor.

Bicarinata.

6 - lineata 6).

Orbicularis.

Umbra.

Agilis 7).

gecko var.
Crocodylus 8).
Paluftis 9).
Crotalus Dryinus.

Boc Hortulana.

Canina.

Controtrix.

Coluber carinatus 10) 155:116. Annulatus 103: 95. Cencbor. Plicatilis $130: 46$. Vittatus. Cobella var. 151: 52. Sibilans 158: 103.

Co.

6) Cauda abrupta in hac uti \& in Lacerta principali.

7) Varietas: Supra nigra areis maculisque ocellaribus ex punctis elevatis albis; fubtus alba; latera parum ccerulefcunt. Palmæ \& plantæ fifræ, 5-dactylæ, ungviculatæ. Cauda teres, verticillata, artenuata.

8) Pullus.

9) Lacerta hæc ccerulefcens maculis oblongis fparfis albidis. Sebæ Thefaur. T. 2. p. 15. T. 12. fig. 5. Lacerta cordylus ut duplicata refpicienda.

10) Coluber Dipfas, fibon, cæruleus, Tetraodon Lagocephalus, Silurus Callichthus, labrus linearis, Gymnotus carapo, Argentina in Amcen. Acad. 1. p. 583 . feqq. nominantur, jam vero in Mufeo non invenienda. 


\section{- * ) iI (}

Coluber Petbola 202: 99. Blennius Cornutus. Atrox 193: 60. Superciliofus.

Laticaudatus ui) 216:32. Murana Helena.

Agilis? 184: 50. Echeneis Remora.

Pullatus 216: 109. Neucrates.

Anguis Scytale 240:13,234,II. Exoccetus Volitans.

Ampbisbena Fuliginofa. Cyprinus Auratus.

Creilia Glutinofa. Aranea avicularia.

Raja Torpedo.

Tarantula.

Tetraodon tefudineus.

Diodon Hyfrix 22).

Syngnatus Hippocampus.

Gobius elotris.

Silurus Ajpredo.

Cbetodon faxatilis.

Striatus.

Perca Radula. capiftratus.
Apbrodita aculeata.
Actinia Fudaica.
Apbrodita aculeata.
Actinia Fudaica.

Mantis Religiofa.

Gryllus Pupus.

Scorpio afer.

Scolopendra morfitans.

Fulus Indus 23).

Sepia officinalis.

B 2 Senilis。 Do-

11) Cauda obtufa, parum compreffa, corpus æqualis craffitiei.

12) Siccatus.

13) Fractus \& deperditus. 


\section{* ) $12(\%$ \\ 3. DONATIO 1746. \\ ERICI PETREI,}

CONSIILIARII SUPREM.DICASTER. HOLMENSIS *).

Echinus Efculentus.

Rofaceus.

Lepas Balanus.

Tintinnabulum.

Anatifera.

Mya Truncata.

Arenaria.

Margaritifera.

Solen Vagina.

Siliqusa.

Sirigilatus.

Tcllina Radiata.

Carnaria.

Pifformis.

Cardium Medium.

Ecbinatuma.

Ciliare.

Ilocardia.
Serratum.

Eáule.

Rufticum.

Mactra Stultorum.

Glabrata.

Solida.

Donax Trunculus.

Venus Dione.

Dyfera.

Verrucofa.

Cancellata.

Cbione.

Deflorata.

Reticulata.

Prolirata.

Tigrina.

Edentula.

Rotundata.

*) Act Confift Upr. 1746 fem. prius p. 629.

Conchylia initio in Bibliotheca Upfalienfi fervaban. tur. Act. Confift. 1746 p. 165 , deinde vero ad Hortum Botanicum tranfportata fuerunt \& inventario debito inftructa. Aet. Confift. Acad. Upf.d. 3 febr. \& 3 OA. 


\section{*) 13 (}

Spondylus Gaderopus.

Chama Gigas.

Hippopus.

Antiquata.

Arca Noe.

Antiquata.

Undata.

Glycymeris.

Pilofa.

Oftrea Maxinza.

Facobea.

Pallium.

Nodofa.

Pes felis.

Sangvinea.

Varia.

Puffio.

Edulis.

Lina.

Anomia Cepa.

Mytilus Margaritiferus.

Litbopbagus.

Edulis.

Argonauta Argo.

Nautilus pompilius.

Spirula.

Conus marmotatus.
Conus Litteratus.

Generalis.

Ebraus.

Stercus mufcarum.

Varius.

Magus?

Striatus.

Textile.

Geograpbus.

Tulipa.

Cypraa Exantbema.

Arabica.

Argus.

Stercorarie.

Carneola.

Zebra.

Ametbyfther.

Lurida.

Caput Serpentis.

Mauritiana.

Vitelins.

Mus.

Tigris.

Lynx.

Ijabella.

Clandefina.

Hirundo.

Alellus. 


\section{\# ) $14($}

Cyprea Monetn.

Annulus.

Caurica.

Eroja.

Helvola.

Poraria.

Pediculus.

Nucleus.

Bulla Ovum.

Gilbora.

Naucum.

Ampulla.

Ficus.

Terebellum.

Virginica.

Achatina.

Voluta Oliva.

Monilis.

Perficula.

Glabella.

Ruftica.

Cancellata.

Mufica.

Vepperitio.

Turbinel!us.

Cymbium.

Olla.

Mitra cardinalis.
Buccinum Perdix.

Pomum.

Oleariunt.

Dolium.

Plicatum.

Rufum.

Tefticulus.

Areola.

Glaucum.

Vilex.

Arcularia.

Mutabile.

Harpa.

Spiratum.

Undatum.

Maculatum.

Subolatune.

Strombus Pes Pelecani.

Cbiragra.

Lambis.

Lentiginofus.

Auris Diance.

Pugilis.

Marginatus.

Gibberulus.

Gigas.

Canariun.

Epidromis. 


\section{$\Rightarrow 1) 15($}

Strombus Vittatus. Murex Haufellum.

Tribulus.

Brandaris.

Trunculus.

Ramofus.

Rana.

Femorale.

Anus.

Nodus.

Melongena.

Cutaceus.

Morio.

Colus.

Aruanus?

Despectus.

Antiquus.

Iritonis.

Aluco.

Vertagus.

Evinaceus.

Trocbus Niloticus.

Perspectivus.

Magus.

Varius.

Veftiarius.

Labio.

Conulus.

Turbo Petbolatus.

Cbryfoftomus.

rugofus.

Pica.

Margaritaceus.

Delphisus.

Terebra.

Exoletus.

Imbricatus.

Clatbrus.

Helix Scarabaus.

Arbuftorum.

Pertuja.

Nennoralis.

Lucorum?

Vivipara.

Nerita Canrena.

Glaucina.

Littoralis.

Exuvia.

Haliotis tuberculats.

Patella Saccbarina.

Granatina.

Carulea.

Mamillaris.

Nimblofa.

Dentalium Elepbantinum. Entalis.

Teredo Navalis. 


\section{) I6 ( 此 4. DONATIO 1747 . \\ C L A U D I GR I L L,}

SOCIET. IND. COMMERC. DIRECTOR. EQVIT. DE STELLA POLARI AURAT. ATQUE REG. ACAD. SCIENT. HOLMENS. MEMBRI *)

Bradypus Tridactylus. Coluber Cyaneus i) 165:122.

Crotalus Duvisfus,

Bos Conftrictor.

Cecilia Tentaculata.

Lacerta Cbameleon. var.

Cobella var. 150.55 .

Afculapii 2) 183: 44. 188: 73 .

Abretulla 162: 139 .

Lenunifcatus 242: 36 .

Angvis Scytale 227: 18.

Gymnotus Electricus.

Gryllus Criftatus 3). 388.

\%) Act. Confift. Acad. Upf. 1747. ferm. prior. p. 205.

1) Amphisbæna fuliginofa, Lacerta Teguixin, Aranea avicularia, Scolopendra morfitans ad duplicatas referri debent.

2) Numero fcutarum \& fquamarum valde variare deprehenditur.

3) Mantis Gongylodes fpiritu diffoluta \& lacerata omni. no deperdita fuit. Amphisbænæ fuliginof variet. Am. Acad. I p. 500. non invenitur; neque Fringilla Jamai. cenfis, Coluber pallidus, domicella \& pethola. 
D. D.

\section{MUSEUM NATURALIUM ACADEMIIE UPSALIENSIS. \\ (1) \\ CUJU'S}

\section{PARTEM SECUNDAM}

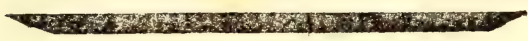

CONSENSU EXP. FAC, MED. UPSAL.

PR ESIDE

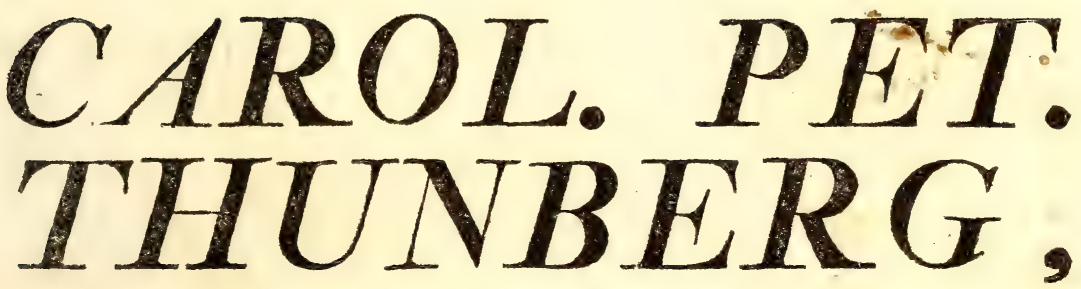

Equite aurat. Reg. Ord, de Vasa,

Medic. Doct. Frofess. Med, et Botan, Reg. et Ord, Acad. Censar, Nat. Curios, Reg. Scient, Holmiens. Societ. Scient. Ufsal. Patr. Horm. Berot. Nat, Scrut. Lund. Harlem. Amsteld. Zeland. Nidro. siens. Medic. Edimeurg. et Nat. Studios. Ibid. Membro; nec nom Agad. Mongzzliens. Agricy maris. ext Batavin. Correspond.

\section{Pullico examini proponic,}

\section{LAUR. MAGN. HOLMER,}

Stipendiarius Regius. Dolia. Wermelandus.

IN AUDIT. GUST. MAJ. D. XIV APRIL. ANNI MDCCLXXXVII. H. P. M. S.

\section{U P S A L I E,}

LitTERIS DiREctor. JOHAN. EDMAN. 
.0

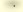

(1) 


\section{$\Rightarrow \quad$ ) $19($ )}

\section{DONATIO. 1748 .}

MAGNI LAGERSTROEM,

REG. COLLEG. COMMERCIOR. CONSILIAR. SOCIET. COMMERC. IND. OR. DIRECT. ACA D. SCIFNT. STO KH. ET UPSAL. MEMBRI.*)。

Tanagra Militaris i $^{\text {. }}$

Emberiza Mixta.

Loxia Dominicana 2).

Sangviniroltris.

Oryzivora.

Flavicans.

Fufca.

Lophius Hiftrio.

Balliftes Vetula 3).

Tetraodon bifpidus.

Chatodon argenteus.

Scomber Tracburus.

Gobius Pectiniroftris
Clupea Triza.

Myjtus.

Cyprinus Auratus. 5).

Cancer Grapfus.

fulus ovatus.

Nereis Carulea.

Holotburia Pbyfalis.

Actinia Equina. Priapus.

Medusa Porpita. Afterias PeEtinata. Pennatula Pbospborea. Mirabilis.

*) Ast. Confift. Acad. Upf. 1748 ferm, pofter. p. 345 \& 1750 d. 13 ORt. Promifit qunque ulterius mittere, sct. $1750 \mathrm{~d}$. 8 Dec.

1) Manis pentadnctyla, ficcara a tineis fucceffu temporis omnino confumta: Pfitracus Galgulus, Buceros bicomis, Pelecanus aquilus \& pifcator, Sterna ftulida non inveniuntur in Mufeo.

2) Loxis Cyanea, pefine occifa, in Spiritu Vini macerata inconfpicua eft.

3) Siccata.

4) Gobius elotris ad diplieatas numerandus.

5) Varietas cauda bifica. D. $\frac{1}{16}$ F. 15 V. 8. A. $\frac{2}{6}$ C. 20. Clupea Triza lrefa uti et Chiton punctarus.

Defiderantur Loxia cordinalis, Labrus opercularis, Cliztodon pinna. tus, Julus fufcus \& craffus, Hirudo muricara, Pennatula fagitta, Hydra conglomerata, Madrepora polygama, Corallinm Chinenfe, Fueus tendo filaque inde facta, quorum fit mentio Am. Acad. T. 4. p. 236. \& feqq. 


\section{$=$ ) $20($ (}

6. ET. 7 DONATIO I749 ET SEQUENTIBUS anNiS

\section{JONA ALSTRÓMER,}

EQUIT. REG. ORD. DE STELLA BOREALI, REG. COLL. COMMERCIOR. CONSILIAR, NEC NON CIV. et MERC. GOTHOBURGENSIS, ATRUE REG. ACAD. SCIENT. HOLM. MEMBRI "). NEC NON

\section{C $\triangle R O L I$ A INNÉ,}

EQUIT. DE STELLA BOREALI, ARCHIAT. REG. ET PROFESS. UPS. PLURIUM ACAD. LITT. MEMBRI **).

Lemur Catta.

Viverra Erminea.

Muftela Lutreola.

Talpa Europea.

Caftor Fiber.
Mus Letimus.

Terrelivis?

Paludojus.

Gerboa jaculus.

Sciurus Sagitta i).

Vulgaris: albus 2).

*) Dono data effe Naruralia, conftat ex Actis Confift. Acad. Upf. 1749 d. 20 Dec. cum vero nullus eorum exftet catalogus, hanc donationem cum Linneana conjungere coadti fuimus.

**) Plura naturalia, Spiritu-vini fervata liberalitati D. Arch. \& Equit. à Linné debere Mufeum Upfalienle, nullum eft dubium, licer certum ejus catalogum confrce. re nos non poffimus, vel illa femper ab aliorum fparfis donis diftingvere.

1) Ex infula Java attulit P. Nordgren.

2) Caprus fuit in Sylva juxta Kongsór \& donatus a 
Sus Cbinenfis 3).

Gracula fetida.

Alcedo Hifpida.

Rallus capenfs. 4).

Parra Facana.

Loxia virens.

Hirundo Apus: alba.

Ovum Teftudinis.

Teftudo roftrata 5).

Scabra.

Imbricata.

Rana Bombina.

Rubeta.

Mavina.

Typbonia.

Marginata.

Efculenta.
Lacerta agilis var.

it. var.

Crocodylus var.

Lacerta Rrevipes 6).

Lateralis a. 7).

Siren Lacertina.

Coluber Calamarius r44: 46 8).

Cbersea 9).

Prefiter 148: 32.

Buccatus.

Trifcalis.

Guttatus.

Mycterizans.

Carule/cens? 215: I13. Exoletus 152: 138. Lineatus 167: 82.

Petolarius 158: 57 .

(152: 50 .

$\mathrm{C}_{3}$ $\mathrm{Ne}-$

Comit. Cronfedt. Totus albus oculis aurantiacis vid. Fifcberftrims befkrifning om Málaren p. 151.

3) Pullus.

4) In promontorio bonæ fpei Sclopeto occifus a D. Præfide \& ad Arch. Linné anno I 774 transmisfus.

5) Teftudo roftrata: pedibus palmatis, tefta integra ca. rinata elevato-ftriata fcabra.

6) Angvis quadrupes LinN, Syft. Naturæ Anim. Holm. 1765. p. 390.

7) Cauda tereti attenuata mediocri, palmis plantisque pentadactylis, linea latersli nigra.

8) Cauda in figura Mufei Adolphi Frideric1 valde ob. tufa, forfan abrupta fuit, in noftro fpecimine acuta, longior \& pluribus fquamis inftructa.

9) Variat fcutis \& fquamis 148: 32, $147: 36,144: 44$. 


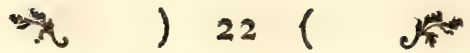

Nebulatus 180: 84. Ova Pifcium. Miliaris.

Virgatus 20).

Strigilis 11).

Ammodytes.1 42:32.12)

Anguis Ventralis. 128: 230 . Fragilis, I19: 129.13).

Petronsyzon Fluviatilis.

Raja Clavata.

Squalus Acantbias.

Squatina.

Catulus.

Carcharias.

Lopbius Vespertilio.

Balliftes Monoceros.

Oftracion Triqueter.

Cornutus.

Tetraodon Ocellatus.

Cyclopterus Lumpius.

Syngnatbus acus.

Accipenfer Sturio.

Pelagicus.
Murana Jerpens.

Miyrus.

Gymnotus .Afiaticus.

Tricbiurus Lepturus.

Annmodytes Tobianus.

Tracbinus Draco.

Gadus Mevlangus.

Merluccius.

Cailareas.

Carbonarius.

Pollacbius.

Lota.

Blennius Gunellus. Viviparus.

Raninus.

Gattorugine.

Capola Tania.

Rubefcens.

Corypheen. Virens. 14).

Gobuus Niger.

$$
\text { Fofo. }
$$

Co.

io) Pallidus fufco nebuloilis. 244: I 76.

12) Tenuis pedalis totus pallidus, ftriis nigris brevibus par paria approximatis, in dorfo, inprimis prope caput fparfis 150: 78 .

12) Dono datus ab Edvardo Carleforn, Reg. Cancellariæ Confiliario. Linn. Amcen. Acad. Tom. 1. p. 517.

13) Angvis fragilis variat numero fquamarum I I9: 129. I37: 69. 1 $31: 82$.

14) D. 20. T. II. V. 5. A. 14. C. 12. 
Goüius Angvillavis. Cottus Catapbractus. Quadricornis. Scorpius.

Zeus Vomer.

Uranofcopus fcaber. Pleuronectes flefus.

Sparus Cbromis.

Honoratus 15).

Labrus melops.

Olfifragus.

Cbinen is.

Lunaris -

Sciena Unimaculata.

Umbra 26).

Ferca Fluviatilis: $\alpha$.

B. Monfrofa.

Polymnia.

Cottoides.

Scriba.

Cernua.

Gafterofteus Aculeatus. Ovatus.

Scomber Scombrus. Trigla Catapbracta.

Gurnardus.

Hirundo.
Cobitis Tania.

Silurus Galeatus.

Loricaria Catapbracta.

Salmo Fario.

Lacuftris.

Eperlanus.

Efox Belone.

Gymnocepbalus.

Atberina bepsetus.

Mugil Albula.

Cbatodon Nigricans. Arcuatus.

Clupea Harengus.

Encraficolus.

Atberinoides.

Cyprinus Apbya.

Griflagine.

Alburnus.

Cepbalus.

Scorpio Americanus. Europaus.

Gordius Aquaticus. Medinenfis?

Fafciola Hepatica. Intefinalis. Apbrodita Syuamata. Pennata 17).

Scyl-

15) Cauda lunata corpore flavo, pinnarum ventralium radio primo fetaceo D. $\frac{7}{15}$ P. 14. V. 5. A. $\frac{2}{12}$ C. 12.

26) D. $\frac{17}{9}$ P. 14. V. $\frac{1}{5}$ A. $\frac{3}{8}$ C. $18 \cdot$

17) E. China mifit D. Staf. A. Oblonga fafciculis fetaceis XXXVI, papillis penniformibus utrinque XXXVI. 


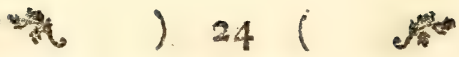

Scyllea Pelagica.

Sepra Octopodia. Media.

Loligo.

Tenia Vulgaris.

Lata.
Aferias Rubens.

Opbiura.

Teredo Nivalis.

Sabella Penicillus.

Lumbricus Marinus.

Myxine Glutinofa.

Animalia, quæ in Horto Batanico viva fuerunt \& mortua jam in Muleo fervantur.

Simia cepbeus: B cetbiops. jaccbus.

Erinaceus europecus.

Mus aguti.

Pfittacus Macro. criftatus.
Cafuarius fetotus.

Strutbio camelus.

Ardea grus.").

8 DO.

*) Omnium ante enumeratorum Naturalium Inven. tarium Confiftorio Academico ab Arch. a Linvé tradi. tum fuit anno 1768 , ut vid. ex Act, d. 12 Mart. p. 262. 


\section{* ) $25($ 莶}

\section{DONATIO. 1775.}

\section{CAROL. PETR. THUNBERG ,}

EQUIT. REG. ORD. VASRI, PROF.| REG. UPS. ET PLURIUM ACAD, SCIENT. MEMBRI.

\section{$M A M M A L I A$.}

Simia Satyrus $x)$.

Silenus. 2).

Sabea.

Lemur tardigradus. tarfic 3).

$V$ espertilio auritus.

murnus.

Myrmecophaga didactyla. Viverra genetta.

martes.
Viverra erminea. nivalis.

Felix lynx. muftela lutra.

Urfus meles. Lepus timidus. cuniculus. Mus Mufculus. porcellus.

1) Vivam e Java attulit Peregrinator illuftris, Dn. Cl. Fr. Hornftedt, cariff. olim Cel. Præfid. Difcipulus, jam Med. Doct. \& ad Gymnafium Lincop. Med. \& Hiftor. Natur. Leet. in Patriam reverfus, \& de Hiftoria Naturali optime meritus, quique hanc fingularem \& rariffimam Simiam, uti \& Muftelam lutrem, Mofcum pygmæum \& multas alias Indiæ orientalis fpeciofas Gazas cumDn.Præfide communicavit.

2) Cauda corpore longior. Animal facile manfvefcit \& familiare evadit.

3) Rariffimum ex Indiæ Orientalis Infulis Animalculum, cauda longa prehenfili vid. Buffon Hiftor. Nat. Qvadrup. Tom. 13. p. 51, 52. Tab, I0. 


\section{- ) $26(x$}

Mus Avellanarius.

Sylvaticus.

Terreftris.

Sciurus vulgaris.

ceilonicus 4).

volans.

Bos gnu 5).

Capra dorcas.

oreas.

pygargus.

fyluatica.

leucophea.

Arepficeros.

Mo cus pygmaus.

Equus Zebra.

Falco apivorus.

palumbarius.

nifus.

rufticolus.

pygargus.

tinnunculus.

aruginofus.

gentilis.

fubbuteo.

albicilla.

Milvus.

Strix bubo.

aluco.

nyctea: nigromaculata.

Jcops.

pafferina.

funerea.

fabirica.

\section{AVES.}

Strix otus.

flammea.

Lanius excubitor.

collurio. 8 of

collaris.

Corvus cornix. monedula. carax. graculus. pica. caryocatactes. glandarius. infouftus.

Coracias garrula. Oriolus galbula. Paradifea apoda.

Cuculus canorus. Fynx torquilla.

4) E Ceilona. Supra ater, fubrus flavefcens, pilis rigidis. vid. Pennant. Quadrup. p. 408. Vocatur a Ceilo. nenfibus: Rukkia. manfvef́cit lepidum animal. Holm.

5) Ex interiori Africa Auftrali. vid. Sparman, Adt. 
औ ) 27 ( )

Picus martius. 1 q major.

medius.

minor. 1 of

tridactylus. 89

viridis.

Sitta Europea.

Gracula religiofa.

Alcedo capenfis.

Merops apiafter.

Certbia familiaris. cbalybea. famofa.

Trocbilus pegafus.

Anas nigra.

fufar.

crecia. 5 운

mallifirma. iq

byenalis: glacialis. 우

clangula. 8

tadorna.

mavila.

clypeata.

penelops.

acuta.

ferina.

fuligula.

Mergus ferrator. merganfer. Ifq albellus.

Alca torda.

Procellaria capenfis.
Pelecanus baffanus. pifcator. carbo.

Colymbus grylle. troile.

Septentrionalis. arEticus. auritus.

Larus marinus. urinator. fuscus. canus. parafiticus.

Sterna birundo. nigra. cappica. Rolida.

Ardea cineven. ftellavis.

Scolopax arquata. capenfis. rufticola. gallinago. phaopus. totanus.

Tring a bypoleuchos. byperborea. pugnax. $\hat{\delta}$ ocbropbus. littorea. interpres. vanellus. 占 alpina? 


\section{*) $28(\%$}

Phrenicopterus raber. Tantalus falcinellus.

Cbaradrius morinellus. pluvialis. biaticula.

Hernatopus oftralegus. Fulica atra.

Rallus crex.

Tetrao tetrix. 1 if urogallus. lagopus. bonafia. bybridus.

Columba Ariata.

bispanica.

dafypus.

gutturofa.

cucullats.

turbita.

laticaudu.

coronata.

turtur.

riforia.

Pav crifatus. ?

Alauda arvenfis. pratenfis.

arborea.

Sturnus valgaris. cinclus.

Turdus capen/s.

pilaris.

vifcivorus.

iliacus.
Turdus muficus. merula.

Ampelis garrulus.

Loxia capenfis. 余 curviroftra. coccotbrauftes. pyrrbula. If cbloris. enucleator. cyanea. orix. I

Emberiza nivalis. citrinella. hortulana. fchaniclos.

Fringilla coelebs. 1 ? cannabina. linaria. 19 domeftica. lulerfis. canarina. it. bylirida. carduelis. it. bybrida. spinus. flaviroftris. lapponica. montifringilla. montana.

Mufcicapa grifeola. atricapilla.

Motacilla alba. arzantbe. 
Motacilla rubetra.

regulus. 令承

fvecica.

bypolais.

falicaria.

phanicurus.

trocbilus.

Sylvia.

troglodytes.

moduralis.

flava.

atricapilla.

Tanagra violacea.
Parus paluftris.

$$
\text { criftatus. }
$$

caruleus.

major.

ater.

caudatus.

Hirundo urbica.

ruftica.

apus: atra.

viparia.

Caprimulgus europaus.

\section{AMPHIBIA。}

Teftudo geometrica: pufilla. Lacerta crocodylus: pullus. orbicularis. japonica 1). areolata 2).

Lacerta agilis: war. palutris: var. listeralis $\beta$ 3). gecko var. 4). abdominalis 5 ).

$D_{3}$

Lacer:

1) T. Japonica: pedibus pinniformibus uni-ungviculatis, tefta carinata crenata poftice quadribola.

2) T. Areolata: pedibus digitatis, tefta gibbofa fcutellis elevatis fubquadrangulis ftriatis medio depreffis f́abris.

3) L. lateralis: cauda tereti attenuata mediocri, palmis plantisque 5 -dactylis, linea laterali nigra. Varietatem $\beta$ communicavit cum Exp. D. Præfide, Demonftrat. Botanices Dn. Mag. Adam. Afselius.

4) L. gecko: cauda tereti longa, dorfo papillofo, vitta longitudinali alba, pedibus dilatato, palmatis.

5) L. abdominalis: pedibus remoriflimis, cauda fubnulla, corpore cinereo. 


\section{* ) $301 \%$}

Lacerta amboinen/is $q 6$ ). japonica 7).

Coluber natrix.

naja.

aeculapii $175: 44$. var. 179: 36 . var. 183: 47. var. 182: 45 .

Regine 140: 67. vittatus. abcetulla $172: 162$. petolavius 2 $12: 100$. Berus: niger 144:39. var. $144: 40$. cherfea var. $150: 33$. guttatus var. $220: 52$. pelias 180: 100.

Coluber minerva 240: 90. domicella $120: 56$. finus 124: 44 . lati caudatus var.224:44. calamarius 140: 42 . Arigilis var. 154:94. Anguis frytale 226: 15. var. 232: 13. fragilis? $13 \mathrm{r}: 33$. Ampbisbeena reticulata 8). Petromyzon brancbialis. Lopbius pifcatorius. Balliftes aculeatus. Tetraodon teftudineus. Oftracion tricornis.

Diodon Atringa. bexagonus 9). PISCES.

Murana conger.

$$
\begin{aligned}
& \text { anguilla. } \\
& \text { myrus. }
\end{aligned}
$$

Stromateus argenteus. cbinenfis.

Callyonymus Indicus.
Callyonymus Dracunculus. Gadus cbinenfis. aglefinus. barbatus. norrbua.

Ga-

6) Hornftedt Act. Holmenf. 1785, p. 130. cum figura.

7) Lacerta Japonica: cauda compreffa longa, linea dorfali alba, plantis muticis.

8) Annulis 130: 21. fupra rubicunda, fubtus alba, pulcherrime reticulata, vitta utrinque alba.

9) Oftracion hexagonus: tetragonus lateribus linea elevata, fpinis dorfalibus ventralibusque binis. 


\section{- ) 311}

Gadus merlangus. virens.

Coryphena ama.

pompilius.

Uranofcopus fealer.

Blennius viviparus.

Cottus volans.

scorpius.

cataphractus.

Gobius patella 10). bipes.

Scorpana porcus.

Pleuronectes cynogloffus.

$$
\begin{aligned}
& \text { papillofus. } \\
& \text { platefJa. } \\
& \text { limanda. }
\end{aligned}
$$

Chatodon macrolepidotus. vofzratus.

Sparus spinus. rbomboides?

$$
\text { argyrops: }
$$

Labrus guazo.
Labrus auritus. Sciena lepifma. cappa.

Perca marina. labrax. nobilis. fcbretfer. ocellata. picta i1). lucioperca. Gafterofteus jpinacbia. pungitius.

Scomber glaucus. orcidentalis.

fcombrus.

Mullus barbatus.

$$
\text { imberbis. }
$$

Trigla volitans. afiatica. rubicunda 12). cuculus.

Silurus catus.

lineatus 13).

20) Gobius parella : pinna pectorali orbiculata concavo-patelliformi, corpore ferrugineo, pinnis dorfalibus nigro-fafciatis.

11) Perca picta: pinna dorfali ramentacea, corpore albo lineis fex nigris.

12) Triga rubicunda : digitis geminis, roftro obtufo, fpiraculis lateralibus folitariis. $\mathrm{E}$ Java Dr. Hornftedt.

23) Silurus lineatus: pinna dorfali poftica \& ani cum caudali unita, cirris 8 , lineis quatuor albis. 


\section{a) $32(\ldots$}

Cobitis barbatula.

Salmo fario.

Clupea tbriza.

eperlanus.

lavaretus.

albula.

tbymallus.

Efox brafilienfis.

lucius.

spbyrana.

Mugit albula.

Fiftularia tabacaria.

my/tus.

Jprattus.

fima.

tropica?

barengus $\beta$.

Cyprinus barbus. americanus. gonorynchus. rutilus. leucifcus.

Obf. r:o Sanguinem habent frigidum \& Ampbibia \& Pijces.

2:0 Corde uniloculari \& uniaurito non nofcuntur Amppibia, fed pulmonibus, cute nuda \& generandi modo.

3:0 Pifces interdum difficile diftinguuntur ab Amspbibiis Nantilus, nifi refpectus habeatur branchiarum, pinnarum \& imprimis pulmonum.

4:0 Singulare nobis obvenit, Siren lacertinam fore larvam, nec amphibium completum, branchiis brachiisque absque pedibus inftructum.

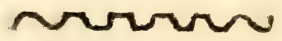




\section{D. \\ MUSEUM NATURALIUM ACADEMIE UYSALIENSIS.

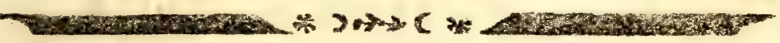 \\ cujus}

PARTEM TERTIAM.

CONSENSU EXP. FAC. MED, UPSAL.

PR IESIDE

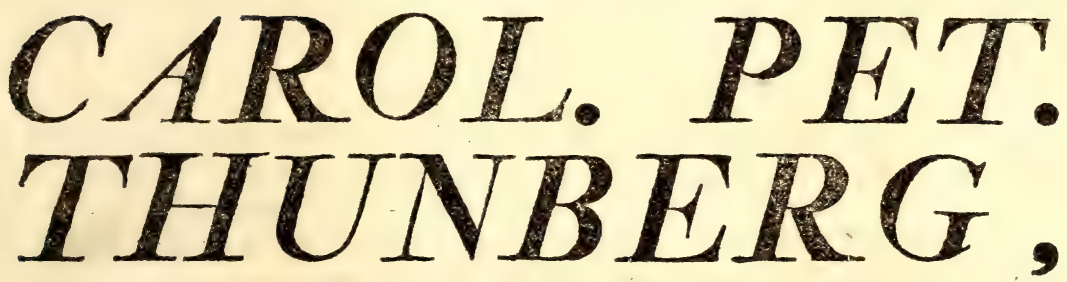

Equite Aurat. Reg. Ord. dr Vasa,

Medic. Doct. Profess, Med, et Botan, Reg. ét Ord, Acad, Chasar. Nat. Curios, Reg. Scient. Holmiens. Societ. Scient. Upsal. Patr. Holm. Berol. Nat. Scrut. lund. Harlem. Amsteld. Zeland. Nidrosiens, Medic. Edimburg. et Nat. Studios. Ibid. Membro; nec non Acad. Monstaliens. Agricult. Paris. et Batavin. Ind, Or. Correspond.

\section{Publicn examini proponit, \\ AND. GUSTAV. EKEBERG. \\ Stipendiarius Starrfeldianus, Uplandus.}

IN AUDIT. GUST. MAJ. D. XXI. JUNIr. ANNI MDCCLXXXVIr. H. A. M. $S$.

U P S A L I E,

EITTERIS DIRECTOR. JOHAN. EDMAN, 


\section{VIRO \\ NOBILISSIMO ET SPECTATISSIMO, \\ DOMINO \\ JOSEPHO ERICO EKEBERG, \\ AD CLASSEM REGIAM \\ ARCHITECTO \\ EXPERIENTISSIMO, \\ PARENTI INDDULGENTISSIMO,}

Accipias, procor bunc, Pater o dilecte! libellum,

1 Quem facrum tibi vult, filioli pietas.

Si debent majora dari pro munere totum

Omnia, qui dederas me tibi reddo Patri.

Ipje Deus vires cum viribus augeat annos,

Cumque tuis annis augeat usque decus. 


\section{DONATION, THUNBERGIANÆ I 1785 CONTINUAT. I.}

\section{INSECTA.}

Paufus clavicornis.

Hifter major.

unicolor.

gigas.

cyaneus.

brunneus.

pygmeus.

bimaculatus.

4.maculatus.

elongatus 1 ).

Coccinella pallida.

fimbriata.

marginata.

impunctata.

minuta.

I punctata.

2-punctata.

3-punctata.

3-notata.

s-punctata.

б.punctata.

6-maculata.

6 -notata.

7-punctata.

8-punctata.
Coccinella 8-maculata.

y.punctata.

Oculata.

3.fajciata.

larva.

bieroglypbica.

japonica.

flexuofa. 2)

flexa. 3)

repanda.

isf.

ocellata.

grandis.

10-punctata.

11-punctata.

13-punctata.

gigantea.

23-notata. 4)

Similis.

borealis.

capenfis.

28.punctata.

Hebraea.

14.punctata.

conglomerata.

I) Lineari - elongatus niger.

2) Coccin. flexuofa. Fabric.

$3)$ Coccin. Hexuola. Thunb. Disfert, nov, inf. Spec. p. I.

4) Coccin. I3. maculata Fabr. 


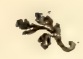

Coccinella conglobata.

triangularis.

I6 punetata.

26. maculata. 5).

19-punctata.

$22 \cdot$ punetata.

lunats.

24-punctata.

25 -punctata.

diftineta.

pufilla.

iridea.

flavicollis.

undulata.

gibba.

Crux.

lineats.

comma.

flavipes.

marginella.

2-puftulata.

colon.

baemorrboidalis.

4.puftulata.

6-puftulata.

70.puftulata.

rivosa.

14-pufulata.

dentatn.

birta.

20-pultulata.
$34 \cdot($

Coccinella tigrina.

cacti.

laevis.

10. guttata.

12.guttata 6).

14-guttata.

16-guttata.

18.guttata.

20-guttata.

oblongo-guttats.

Elopborus aquaticus.

minutus.

Byrrbus fcropbularie.

musfeorum.

pimpinella.

fafciatus.

ater.

Curculio zamine.

nucun.

colon.

cylindrivoftris.

palmarum.

longipes.

betula.

populi.

cupreus.

ruficollis.

violaceus.

cyaneus.

fagi.

campanulae.

5) Coccinella ${ }^{6}$ punctata Fabricii.

6) habitat in Svecia. 


\section{苏 ! 35 搭}

Curculio ceraf.

fexetis a. B. alliavia.

vifcarice? ovalis. ocridulus. nigriroliris.! alni. tortrix. polnorums. drupartim. quercus. purpureus. granarius. oryza.

frumentarius. dorfalis. beccabunga. falicis. pedicularius. fcropbularia. germanus. carbonarius. Lapatbi. equijeti. pericarpius. abietis. pini. rumicis. polygoni. 2-prnctatus. s-punctatus. 4.maculatus. T. album.
Curculio ancborago. pavaplecticus. cylindvicus. grifeus? fulcatus. tesfellatus. murinus. ligufici. incanus. ruficornis. capitatus. lineatus. tereticollis. ecbinatus. oblongus.

pyri.

rotatus. imperialis. latiroftris. albinus. fprenglevi. arugino/us. viridis. argentatus. morio. ovatus. triftis. lineellus. ater. rufipes. trunculus. fcaber. fcabriculus. lividus. 


\section{* ) $36($}

Curculio acuminatus.

Scarabeus aygulus.

cervinus. a. $\beta$.

asperatus.

obtufus.1

crenulatus.

nebulofus.

fulciroftris.

niveus.

apterus.

nodulofus.

Attelabus coryli a. B.

gemmatus.
betulc.

Bulbocerus cepbalotes. I $q$ Lucanus dama

cervus major. 令字 minor.

interruptus. parallelipipedus. caraboides. If $q$ capenfis.

piceus.

carinatus. 1)

Scaraborus bercules $\hat{\delta}$

rbinoceros. 章早

naficornis. 昘?

gideon.

typboeus of \&

barbarosfa.

dispar. 5 우

filenus. cylindricus. of 9

fimetarius. $a, \beta$.

erraticus.

conspurcatus.

luridus

apelles.

Aercorarius.

vernalis.

fosfor.

fubterraneus.

granarius.

feniculus. If $q$

lunaris. of

vacca.

bonafus.

taurus. 1 q

furcatus.

fagittarius.

jaccbus.

fabceus. molosfus. a. $\beta . \gamma_{0}$ bucepbalus. carolinus. bispanus. bifon. 1 오 spbinx.

nucbicornis $\hat{\delta}$ 웅 mimas. 3 车 carnifex. plagiatus.

Melo.

1) Hanc \& plurimas alias lpecies benevole communicavit Celeb. Dn. Doct. Hornftedt. 


\section{$\Rightarrow$ ) 37 ( )}

Scarabaus 4-maculatus. Melolontba cincta. fobreberi. quisquilius. vufipes.

Melolontba fullo of 우 vulyaris. ruficornis? folfitialis. punctata. reflexa. feftiva. brunnea. vitis. notata. 7) friscbii $\beta$. triftis. crinita. urfus.

lynx. hirfuta 8). vulpes. borticola. e. $\beta$. arvicola? argentea. farinofa. fubspinofa. rupicola. iongimana. fafciata 9).

indus. 9) bemiptera of \& 9). cbinentis. aurata. a. $\beta_{.} \gamma_{0} \delta_{0} \varepsilon_{0}$ morio. maculata. nobilis. 8-punctata. fafcicularis.! capenfis. a. B. $\gamma$. fignata. marginella. nitida. olivacea. finuata. lineola. a. B. y. $\delta$. elegans. africana. cbry/is. 4-dactyla. gagates. evemita. a. B. 2 웅. ftictica. pilofa. beemorrboidalis. variegata.

7) Melol. fignata Fabr.

8) Melol. hirta Fabr.

9) Trichius Fabric. 


\section{光) 38 ( )}

Copris Bacsbus 20).

laticollis.

freer.

kanigii.

fobefferi.

bilularius.

cupreus.

Throx fuberofus.

Subuingus.

fulcatus. "i)

filpboides 12).

Hydrous piceus.

olivaceus.

caraboides.

luvidus.

fufcipes.

Spberidium pilula.

varium.

nenคum.

atrum.

beenorrboidale.

fcarabroides.

psyllium.

melanocepbalum.
Spheridium rubrum.

globofuns.

feminulum a. B.

colon 13!.

luteum.

Ips typograpbus.

polizrapbus.

calcograpbus.

foabra.

piniperda. domeftica.

2 punctata.

joolytus.

monacbus.

capucinus.

Dermeftes carnivorus.

marginatus (4).

lardirius.

piceus.

naturinus.

pellivo.

undatus.

interruptus.

10) Genus Geofroiæ, affine Scarabæis, diftinctum cly. peo emarginato, dentato, thorace convexo lato.

iI) T. fulcarus: thorace trifulcato, elytris fulcatis punctato-reticularis hifpidis.

r2) T. filphoides: thorace trifulcato, elytris lulcatis hifpidis.

13) Dermeftes colon Linn.

14) Dermeftes vulpinus Fabric. 


\section{- I 39 (}

Dermeftes fajciatus.

bitafciatus.

maeulatus. a. B. $\gamma$.

feneftratus.

feneftralis.

ferraticornis.

ferrugineus.

fanicus.

fercoreus 15).

aeftivus i6).

pedicularius.

paniceus.

2-puftulatus.
Anobium nigruns.

ceruleum.

violaceum.

viride.

vufipes.

ruficolle.
Anobium netbiopicum.

2-fafciatum.

Tritoma fufca i7).

colon 18).

Aercorea 19).

fulcata 20).

rufipes 22 ).

fuccincta 22).

Nitidula littoralis a. $\beta$.

germanica 23).

indica 23).

vefpillo.

prulicaria.

pubescens.

difcoidea.

colnn.

biguttata.

depresfa.

grijea.

15) Dermeftes fumatus Linn. Syft. p. 564. Dermeft. ftercoreus Linn. Syft. p. 564. Torus rufefcens villofus oculis nigris.

I6) Dermeftes flavefcenti-teftaceus tomentofus oculis nigris, antennis pedibusque rubris Derm. fumatus Fabr. Spec. inf. p. 66 an. Silpha æftiva? Linn. Faun. Svec. p. 152.

17) Silpha fufca Linn.

18) Silpha colon. Linn.

19) $T$. ferruginea tota, glabra elytris ftriatis.

20) T. picea, elytris antice rubris apice fufcis, fulcatis.

21) Silpha rufipes Linn. Syft. nat. p. 573.

22) Silpha fuccincta Linn. Tritoma crenata Fabric.

23) Nicrophorus Fabric. 


\section{* $) 401$.}

Nitidula verbafci.

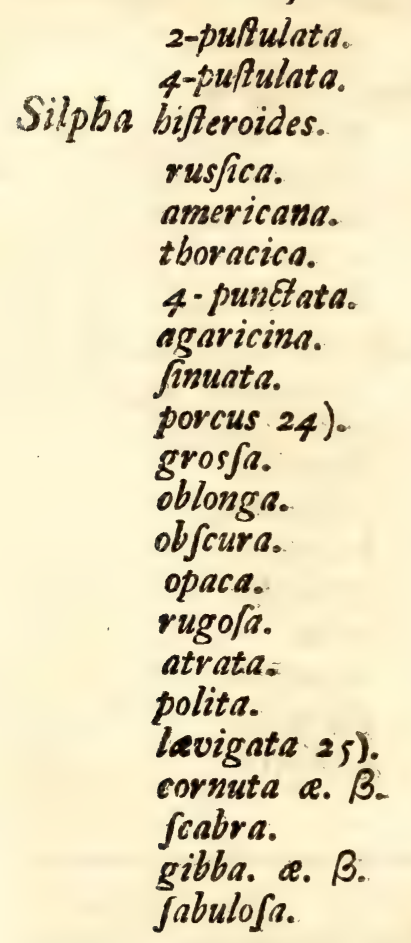

Silpba friata 26.)

minuta 26.)

fulcata

ferruginea.

Hispa mutica.

capen/is.

atra.

Cassida cruciata.

fangvinicollis.

lateralis.

nebulofa. a. B. 1).

affinis.

murraa.

vibex.

nobilis.

maculata.

purpurea 2).

veficularis 3).

reticulata.

viridis.

brunnea. 4)

bicornis.

grosfa.

Casi

24) Tota fetofa, cinerea, lineis elytrorum elevatis. E bonæ fpei promontorio.

25) Hanc uti \& plura alia infecta rariora com: municavit D. Dr. \& Adj. P. Afzelius.

26) Opatrum ftriatum \& minutum. Fabric.

I) Variat colore flavefcente \& virefcente, punctis nigris variegata.

2) Similis valde veficulari, fed differt eo, quod lævis fit, pellucida, macula dorfali rubra.

3) Tota adfperfa veficulis parvis; habitat in Japonia.

4) Tota brunnea; habitat in Svecia. 


\section{औ) 4 I (}

Casfida variegrata.

marginata.

flava.

Clerus apiarius.

formicarius.

mollis.

birtus 5 ).

elongatus. a. B. 6)

villofus. a. $\beta . \gamma .7$ )

Meloe profcarabaus.

majalis.

bimaculatus.

veficatorius.

fyriacus.

cicborei.

citratus 8).

Meloe erytrocepbalus.

marginatus.

algivicus.

nitidulus.

capenfis.

Erodius teftudineus.

minutus. borridus 9):

punctatus. tuberculatus. globofus. ecbinatus. glomeratus.

bifidus. crenatus.

Mordella rafuta.

5) Lagria hirta Fabr. Spec. Infect. p. 160.

6) Lagria elongata Fabr. variat thorace rubro \& cæruleo in Finlandia.

7) Lagria villofa Fabr. variat colore elytrorum.

8) totus ater.

9) Hæc \& fequentes fpecies omnes novæe capite bo: næ fpei Africæ.

Horridus: thorace lateribus fpinofo, elytris lineis tribus elevatis fpinofis.

Punctatus: thorace lævi, elytris lineis tribus elevatis lævibus.

Tuberculutus : thorace lævi, elytris elevato-punctatis, lineis tribus elevatis obfoletis.

Globolus: thorace glabro, elytris aculeis frequentisfimis totis hifpidis.

Echinatus: thorace glabro, elytris lineis tribus eleva. tis fulcisque interjectis muricatis.

Glomeratus: thorace punctato, elytris rugofis: lineis qua. tuor elevatis ferratis. 


\section{$-7) 42(\ldots$}

Mordella aculeata.

frontalis.

Altica fafcicornis.

thoracica.

lens.

atricilla.

flava.

exoleta.

bumeralis.

tenella.

fafciata.

nemorum.

Altica oleracea 10).

byolcyami.

ferruginea.

belxines.

erytrocepbala.

nitidula.

rufipes.

bolfatica. pulicaria.

Modéri.

exclamntionis.

2 -puftulata.

Bifidus: thorace glabro, elytris lineis tribus elevatis ferratis: laterali poftice bifida.

Crenatus: thorace glabro, elytris lineis quatuor elevatis crenatis.

ro) Alticas nos dicimus omnes Chryfomelas Linnæi, femoribus pofticis incrasfatis taltatoriis, quæ fub generi: bus Fabricii Chryfomelæ \& Alticæ comprehenduncur.

Obf, 1:0 Metbodum entomologicam Linneanam, cum matatione beic ufitata, optimam merito cenfemus.

2:0 Ex antennis infectorum defuntos Cbaracteres aque esje certos credimus, ac ab aliis quibusdam eorum partibus.

3:0 Fulto pauciora infectorun genera exbibet fyRenas Linnoanum; naturalna nimis lacerat in plura metbodus Fabriciana.

4:0 Quo magis ab externis infecti partibus generuns dejumi posfunt Cbaracteres, eo facilius evadic Syytema Entomologicum. 


\section{D. \\ MUSEUM NATURALIUM ACADEMIE UPSALIENSIS.

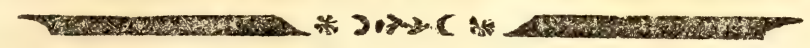 \\ cUJUS \\ PARTEM QVARTAM. \\ CONSENSU EXP. FAC, MED. UPSAL。 \\ PR EESIDE

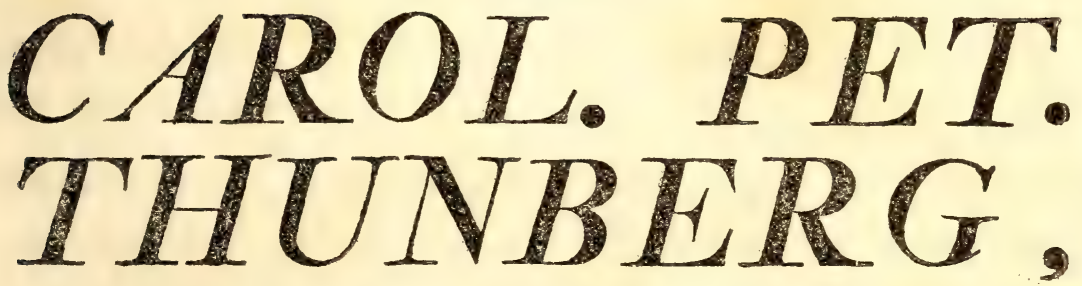

Equite Aurat. Reg. Ord. de Vasa,

Medic. Doct. Profess. Med, et Botan。 Reg. ex Ord., Acad. Cefesar. Nat. Curios, Reg. Scient. Holmens, Societ. Scient. Ursal, Patr. Holm. Berol. Nat. Scrut. Lund. Harlem. Amsterd. Zei and. Nidrosienso Medic. Edimburg. et Nat. Stedios. Ibid. Membro; nec non Acado Monspeliens. Agricult, Paris. Florentin.et Batavin, Ind。

Or. Corraspond.

Publico examinz fubjicit, PETRUS A BJERKEN, Stị, Leijell, Angermannus.

IN AUDIT. GUST. MAJ. D, XIX DEC, MDCCIXXXVII. H. A. M. S

U P S A L I $Æ$, LITTERIS Director. JOH. EDMAN. 


\section{ASSESSORSKAN, \\ VÅLBORNA FRU \\ JOHANNA MARGARETHA AF BIERKÉN,}

\section{MIN HULDASTE MODER}

Min fórfat ocb min Aơrfta lycka ir, at vara fódd af en Mor, fon frän forrfa futnden af min lefnad lanunat mig den

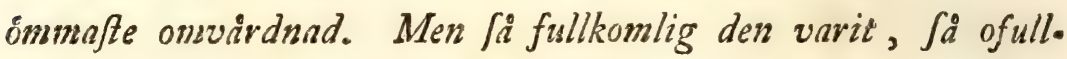
komliga áro de tackfamhets prof jag derfore kan frambaira. Ofvertygad, at min Huldafte Moder med noje anfer, äfven det ringafte bland desfe, tilégnar jag Eder detta Academi$J_{k} a$ arbete, under trognafte ón/kan at Allmagten mà gơra Edra dagar mainga ocb lyckliga.

Frambirdar med oaflatelig vôrdnad MIN HULDASTE MODERS

lydigfte fon PEHR AF BIERKÉN. 


\section{DONATION. THUNBERGIANÆ I785. CONTINUAT. II.}

Chryfomela tenebricofa. goettingen/s. jurinamenfis. afiatica. cyanea. graminis.

enea. atbiopica 2). polita.

ftapbylaa. $\alpha . \beta$. bemoptera. bemorrboidalis. vulgata. vitellina. alni. betule. armoracie. obfcura. 10-punctata 2).
Chryfomela viminalis. lurida. populi. pallida. undata 3). coccinea. 3-maculata. comma 4). brunnea. a. B. 5). Juturalis. litura. a. $\beta$. g-vittata. polygoni. limbata. analis. fangvinolenta. collaris. lutefcerss 6). marginata.

r) Ovata viridis futura margineque cupreis, E capite bonæ Ppei.

2) Quam maxime variat hæc fpecies \& numero pun. ctorum in elytris \& magnitudine, nexu atque fitu eorum.

3) Flavefcens tota elytris fafciis duabus nigris undatis.

4) Ovata nigra, elytris flavis futura vittaque nigra. Ex India Orient. Doet. Hornstedt.

5) Ovata brunnea, elytris margine vittaque fefquialtera flava. Fig. 7 .

6) Oblonga flava, thorace viridi marginibus flavis, elytris cupreis. 


\section{* ) 44(}

Cbryfomela undulata. ${ }^{*}$ ). bannoveriana. 7 .

lapponica. a. $\beta$.

boleti.

cyanicornis.

Favanica, 8).

6-lineata. 9).

faftuofa. a. $\beta$.

cerealis.

fuperba. 10).

coccinelloides. $\alpha, \beta . \gamma \cdot \dagger$ )

aftuans. I2).

2-pufulata. 13).

8-puftulata. 14).

10-puftulata. 15).

20-puftulata. 16).

22-guttata. 37).

longipes. I8).

4-puftulata.

Cbry-

7) Habitat in Svecia. Gyllenhahl。

8) Ovata cyanea, thorace marginibus elytrisque fa. fciis undatis rubris aureisque. Ex Infula Java.

9) Ovata cyanea, thorace violaceo, elytris marginibus lineaque media flavis.

I0) oblonga viridis, thorace lunula elytrisque lineis octo aureis.

II) Ovata atra, elytris margine pofticeque flavis: maculis tribus nigris, antice nigris punctis quatuor flavis.

82 ) Chryfomela æeftuans. Linn. Syft. p. 593. Thunberg Disfert. Nov. Infect. Spec. p. I. Coccinella 8-guttata.

13) Ovata atra, elytris margine punctis duobus rubris.

14) Ovata atra, elytris margine apice punctisque quatuor luteis.

15) Ovata ferruginea, elytris atris margine punctisque decem rubris. Fig. 4.

16) Ovata atra capite thoraceque flavo-maculatis, ely. tris punetis viginti luteis.

17) Ovata ferruginea, elytris 12-guttatis. Ex India Orient. Doet. Hornttedt. Fig. 6.

18) Cryptocephalus, Fabr.

t) Fig. 5. 
Coryfomela longimana. 19!. Brucbus pif.

tridentata. I 8 ).

unipunctata. I9).

colon. 20 ).

4-punctata.

Gyrinus amevicanus.

capenfis. I).

conme. $2 \%$.

natator.

Bruchus fcabrofus.

tbeobronice.

Seminarius.

bactre. granarius.

varius.

cbinen/is.

pectinicornis.

Notoxus monoceros.

cornutus. 4).

coruleus. 5).

ater. 6)

antberinus, 7 \%

foralis. 7).

deu(tus. 8).

flavus. 9).

19) Tota fupra flava humeris unipunctatis. E. Cap. Bon. Spei.

20) Nigra margine thoracis flavo, elytris flavis pun. ctis quatuor nigris. Fig. 9.

r) Virefcens thoracis elytrorumque marginibus luteis, elytris Aavo-ftriatis. E Capite Bonæ Spei aquis.

2) Niger thoracis elytrorumque marginibus luteis, elytris linea nigra nirida. Ex India Orient. Doct. Hornftedt.

3) Chryfomela hemiptera. Thunberg. A\&t. Nov. Upfal. Vol. 4.

4) Thorace cornuto violaceus, elytris 2-puftulatis. E Cap. Bon. Spei.

5) Thorace inermi totus violaceus immaculatus. E Cap. Bon. Spei.

6) Thorace inermi totus ater. Habitat in Svecia.

7) Lagria Fabric.

8) Thorace inermi capiteque nigris, elytris flavis apice fufcis. E Capite Bonæ Spei.

9) Thorace inermi totus flavus, capite antennis pedibusque nigris. E Cap. Bon. Spei. 


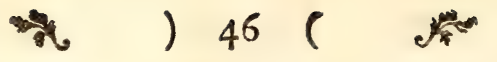

Notoxus melanurus. 10). Spondylis bupreftoides. indicus. 10).

Ptinus perizinax.

mollis.

teftaceus.

pectinicornis.

cinereus.

imperialis.

fur.

faber. II).

latro.

Melyris viridis.

fericeus. 12).

caruleus. I 3).

nizer.

marginatus. 14).

Spondylis ceramboides.

femoratus. 15).

Cryptocephalus nitens.

fericeus.

bypocbseridis.

2-puftulatus. I).

morai.

botbnicus. a. B.

y barbarea.

labiatus.

auritus.

8-puftulatus. 2).

2-lineatus.

vittatus.

octavius. 3).

2-punctatus.a. B. . . ..4).

melanocepbalus. 5).

Cry.

10) Attellabus Linné.

iI) Derm. Tesfellatus Fabr.

12) Totus viridis rugofus, elytris lineis tribus eleva. tis; multoties minor M. Viridi.

13) Totus cæruleus rugofus, elytris lineis tribus elevatis.

14) Niger thorace elytrorumque marginibus flavis.

15) Alurnus femoratus Fabric.

I) Habitat in Svecia. Sam. Odman.

2) Ater capite punctisque quatuor elytrorum rubris.

3) Thorace rufo lunula flava, elytris nigris linea resquitertia obliqua flava. Ex India Orient. Doct. Hornftedt.

4) Variat punctis duobus \& quatuor, lineaque lata loco punctorum nec non magnitudine corporis \& punctorum. Cryptocephalus quadrum hujus eft varietas in Svecia \& Germania occurrens.

5) Ruber abdomine capite punctisque quatuor elytro. rum transverfis nigris. 


\section{倠 ) 47}

Cryptocepbalus 6 punctatus.a. . . Crioceris cyanella. 10-maculatus. $\alpha$. 6 . flavipes. 10-notatus. 6). cordigerus. $\alpha, \beta . \gamma . \delta . \varepsilon .7)$. coryli.

pini.

Crioceris calmarien/s.

cuprea. nymipbar. tenella. gibba. I). merdigera. cerafi. $\alpha_{0} \beta .2$ ). fulpburea. cervina. $\alpha . \beta .3$ ). murina. melanopa. tanaceti. aulacea 4). tetrapuncta. 5). betulina. 6\%. insuba. 4). s-vittata. 4). elongata. pbellandrii. lata. 7). 2-fafciata. fcopolina. bioculata. $\alpha . \beta . \gamma$. asparagi. 12-punctata. 4-maculata.

Crio

6) Flavus abdomine capite punctisque elytrorum decem nigris.

7) Variat multum figura \& numero macularum lutearum in thorace uti \& numero punctorum in elytris. Fig. 10. I) Oblonga fufca elyt:is acuminatis incurvatis. Habibitat in Svecia. Chryfomela gibbofa Thunb. Act. Nov. Upfal. Vol. 4.

2) Variat thorace flavo \& nigro.

3) B. Ciftela cinerea Fabric.

4) Ciftela aulica, innuba, 5-vittata. Fabric.

5) Thorace rufo, capite elytris punctisque thoracis quatuor cyaneis.

6) Oblonga fufca, linea elytrorum obfoleta pedibusque ferrugineis. E Lapponia Dn. Paykull.

7) Thorace flavo, elytris flavis marginibus lineaque media nigris. E Cap. Bon. Spei。 


\section{到) 48 ( )}

Crioceris caijanenfis. 8).

jubpinoja.

flasipeda. 9).

Sepidium oblongun. 1).

caraboides. $\alpha . \beta$.

notatum. 2).

marginatum. 3).

fricium. 4).

reticulatums. $\left.\alpha . \beta,{ }^{*}\right)$.

lacunofum. 5).

vittatum. $\alpha . \beta$.

lineatum. 6).

Terzebrio mortifagus.

violaceus. 7 .
Tenebrio quisquilius.

femoralis. asper. 8). molitor. culinaris.

nigrita. difformis. †). roftratus. longipes. lanipes. pallens. plumnofus. 9). fosfor.

Pimelia Spinosa.

Pime-

8) Thorace nigro, elytris rubris maculis quatuor virefcentibus. Fig. 11. E Cajana.

9) Erotylus flavipes Fabric.

I) Oblongum atrum ftriis elytrorum obfoletis, thorace fubquadrato.

2) Ovatum atrum ftriis elytrorum obfoletis, thorace rotundato.

3) Ovatum atrum elytris oftofulcatis fulcis rugofis.

4) Ovatum atrum elytris octofulcatis fulcis lævibus, Itria media majori.

5) Oblongum cinereo-nigrum thorace elytrisque rugofis. Fig. 14.

6) Ovatum atrum thorace muricato, elytris lineis fex albis. Fig. I6.

7) Oblongus violaceus elytris ftriatis punctatis.

8) Oblongus ater totus punctis elevatis fcaber. Fig. 2 I.

9) Ater elytris ftriatis punctatis tibiis ciliatis. Fig. 17. 10) Oblongus ater, thorace 4-fpinofo depresfo elytris

*) Fig. 15 .

t) Fig. 19. 


\section{- ) $49($ 儒}

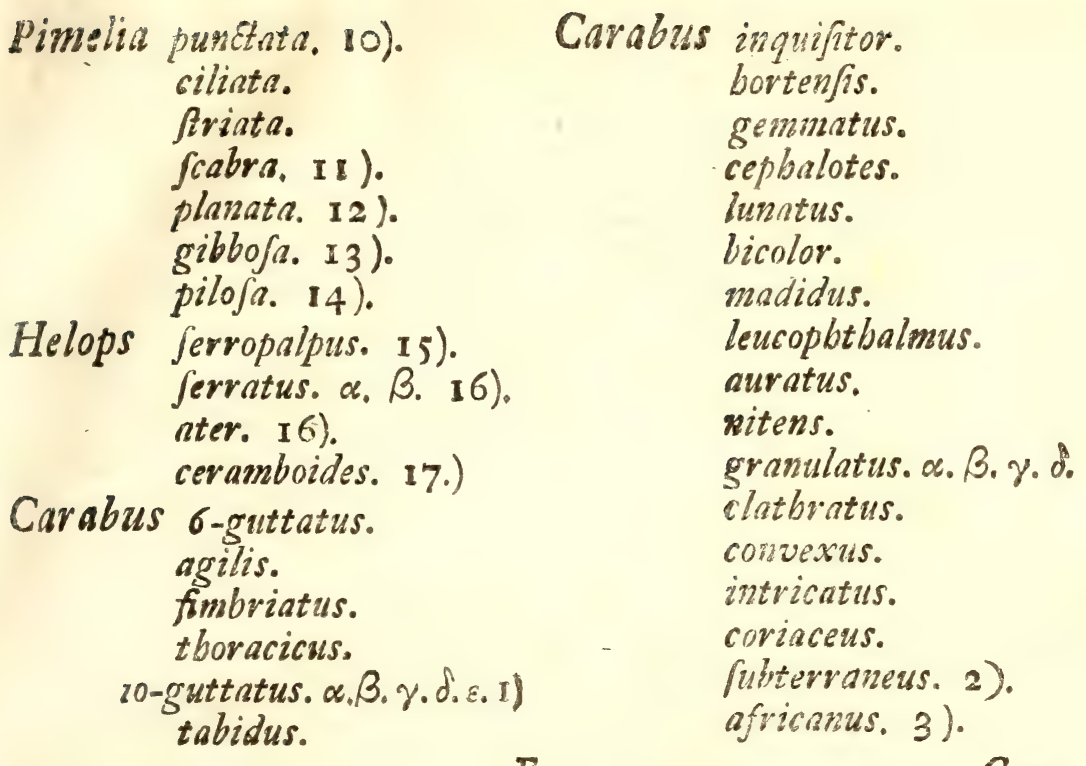

lævibus nitidis punctis marginalibus elevatis. Fıg. 18. Ex Italia Dom. Doct. P. Afzelius.

II) Fig. 24 .

12) Oblongus, gibbus niger lavis elytris ftria utrin. que unica elevata crenata. Fig. 22.

I3) Oblongus gibbus ater, elytris fulcatis fulcis lævi. bus fubrugofis. Fig, 23 .

14) Ovatus, gibbus niger levis elytris poftice pilofis. Fig. 20.

I5) Lineari-oblongus lævis niger thorace fubquadrato. E Finlandia Prof. Hellenius \& Gróndahl.

16) Helops Fabric.

17) Chryfomela Linn.

1) Car. 10-guttatus \& 4-guttatus Fabric.

2) Scarites Fabric.

3) Oblongus depresfus ferrugineus, elytris ftriatis. 


\section{7 ) $50($}

Carabus jycopbantba. collaris. a. B. 4).

afer.

femoralis.

marginatus.

melanocepbalus.

cyanocepbalus.

6-punetatus.

mitidulus.

latus.

cupreus.

vulgaris.

pilicornis.

cervlefcens. a. B.

trilineatus.

2-maculatus. $\alpha . \beta, \gamma \cdot \delta$.

bamatus. 5).

prafinus. 6!.

vaporaviorum.

crepitans.

obtufus.

Carabus 4-puffulatus.

uftulatus.

meridianus.

guttulus. 7).

2-guttatus.

fajciatus.

4-maculatus.

cruciatus. a. B. 8).

crux. 9).

dentellus. 10).

bilineatus. «. $\beta$.

multipunctatus.

2-punciatus.

piceus.

Jpinipes.

velox.

truncatellus.

excifus.

ferrugineus. colon. II). dorfalis.

$C a:$

4) Niger thorace margine elytrorum pedibusque flavis. Fig, 12. 13. E Vermelandia Holmer.

5) Capite thoraceque cyaneis, elytris nigris: lunulis duabus apicum flavis.

$6)$ Carabus violaceus. Act. Nov. Upfal. Vol. 4. p. 20.

7) Niger nitidus pedibus panctoque apicis elytrorum pallidis.

8) Carabus crux minor Linn.

9) Carabus crux major Linn.

I4) Niger margine elytrorum linea flava dentata. Ha. birat in Svecia.

II) Fufco-ferrugineus elytris punctis duobus rubris. 


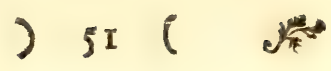

Cicindela gigantea. 12).

japonica.

jyluatica.

bybrida.

tridentata. I3).

carolina.

catena.

riparia.

flavipes.

4-maculato.

aquatica.

capenfis.

campeftris.

germanica.
Bupreftis gigantea.
cbry/is.

fernicornis.

vittata.
Bupreftis ignita.

fafcicularis.

birta.

8-guttata.

9-maculata. a. 3 .

cbryfoftigma.

mariana.

plebeja.

fibivica.

splendens.

minuta.

anea.

ruffica.

4-punctata.

tenebrionis.

viridis.

2-guttata.

2-fafciata. ${ }^{*}$ ).

trifafciata.

11) habitat in Svecia fimillimus Car. ferrugineo, cujus forfan fola varietas, a quo tamen differt puncto finguli elytri rubro.

12) Manticora Fabric.

I3) Cicindela biramofa. Fabricii.

*) Sequentes fpecies omnes novæ e Cap. Bon. Spei. bifafciata: elytris integris nigris: fafciis tribus albis, thorace nigro.

ivifafciata: elytris integris nigris: fafciis tribus albis, tho: racis marginibus flavis.

Io guttata: elytris integris nigris: maculis decem luteis. caffra: elytris crenatis violaceo luteoque variegatis, corpore æneo. 


\section{翟) $52($ )}

Buprefis ro-guttata. a. B. Bupreftis oculata. caffra. cornuta. aurata. rugofa. marginata. notata. acuta. atomaria. ruficollis.

furcata. I). acuminata. 2).

Elater minutus.

siger.

ater. 3).

6-guttatus.

pulcbellus. clavicornis. ceneus. $\alpha . \beta$.

Elat

cornuta: elytris integris albo nigroque lineatis, thora. ce tuberculato.

auratı: elytris integris viridibus: futura aurea, thorace punctis duobus impresfis, corpore æneo.

rugofa: elytris ferratis rugofis fufcis: apice luteo-irroratis. marginata: elytris integris violaceis, thoracis lateribus rubris.

notata: elytris integris violaceis, thoracis lateribus albis, abdomine albo-maculato.

scuta: elytris bidentatis acuminatis cæruleis, corpore nitente.

atomaria: tota ænea elytris tridentatis apice violaceis. ruficollis: elytris integris fulcatis nigris, capite thoracisque lateribus aureo-fulvis.

oculata: elytris bidentatis punctatis nigris, thorace $u$. trinque macula rubra.

I) fupra æneo-fufca elytris acuminatis, fubtus cuprea. Habitat in Svecia.

2) Tota atra lævis elytris integris, thorace punctis im. presfis: in Svecia fatis vulgaris.

3) Elater aterrimus Linn. 


\section{$* x) 53(2$}

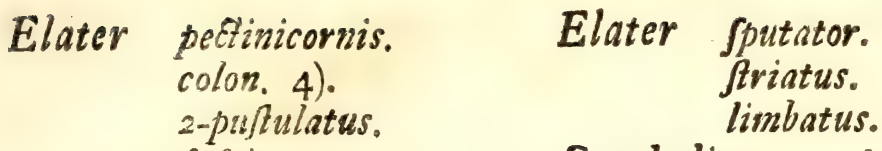

fafciatus.

murinus.

tesfellatus.

oculatus.

ferrugineus.

fangvineus. $\alpha$. $\beta . \gamma_{0}$

Stapbylinus oculatus.

politws.

erytbropterus.

elongatus.

melanocepbalus.

brunneus.

deuftus.

marginatus.

linearis.

caftaneus.

livens.

mefomelus. $\alpha . \beta$.

cruciatus.

baltentus.

triftis.

cingulatus. 5).

feftivus.

dermeftoides?

bupreftoides?

obscurus.

ruficollis.

noctilucus.

rufus.

riparius.

cbryfomelinus.

lunulatus. zmxillofus.

mburinus.

marginatus.

brumripes.

caraboides.

2-puftulatus.

2-guttatus. a. B.

nitidulus.

rufipes.

piceus.

atricapillus.

capen/is. 6).

boleti.

flavipes.

flavefcens.

$F_{3}$

Stra-

4) Ater punctis duobus rubris in medio elytrorum. 5) Ferrugineus capite fafciaque elytrorum nigris. E Finlandia Dn. Gróndahl.

6) Ater aitidus, abdomine albo-tomentofo. 


\section{$\Rightarrow \quad) 54(2$}

Staphylinus lacurofus. 7). Cantbaris bicolor. fulcatus. 8).

fubterraneus.

obfcurus. 9).

olens. 10).

filpboides.

Forficuláa auricularia.

minor.

Necydalis major.

minor.

Malacbius bumeralis.

flavescens. $\alpha$. $\beta$.

ccerulescens, $\alpha$. $\beta$.

viridis. $\alpha . \beta . \gamma$.

rufus.

Cantbaris navalis.

dermefoides.

variabilis, $\propto . \beta . \gamma . \delta . \varepsilon, 1 \mathrm{I})$.

coccinea. $\alpha$. $\beta$.

pectinicornis. $\alpha$. $\beta$.

melanura. $\alpha . \beta . \gamma$.

flavipes.

pallipes.

livida. a. ß. $\gamma$.

bilinenta.

trilineata.

teftacea. a. $\beta$.

lepturoides.

cerulea.

viridisfina. «. $\beta . \gamma$.

fufca.

obfcura.

atra.

ruficollis. 12!.

altica.

oculata. $\alpha . \beta . \gamma$.

collaris. $\alpha . \beta$.

cuprea. a. $\beta$.

argentea.

violacea. $\alpha$. B. 13 ):

cbryfomeloides.o.,.,I4)

7) Fufcus pedibus rubris, thorace fovea depresfa.

8) Niger elytris pedibusque piceis, thorace fulco lon. gitudinali.

9) Ater capite, thorace elytrisque violaceis.

I0) Totus piceus opacus. alis albo-ferrugineis.

II) Ovata fufco-flavefcens, elytris diaphanis; vulga$r$ is in Svecia.

I2) Cantharis cærulocephala. Thunb. Act. Nov. Upr. Vol. 4. p. 18 .

I3) Cantharis fmaragdula Fabr. 4) Thorace convexo rubro, capite elytrisque cyaneo-fufcis. 


\section{* $) 55($ \%}

Cantbaris melooides. Is).

ferraticornis. 16). birta. 17). guttata. 18).

Lampyris marginata. $\alpha . \beta$. maculata. 22). corrufca. 速果 italica.

4-guttata. 19).

fafciata. roffrata. a. B.

\section{anea.}

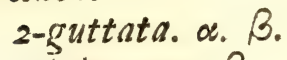

minina. $\alpha . \beta$.

pulicaria.

pedicularia.

nigra. 20).

cyanea. 21).

Lampyris noctiluca. 含东 Splendidula.

bicolor.

cbinenfis,

japonica. $\alpha . \beta, \gamma . \delta$.

proufta. 23).

fangvinea. $\alpha_{0} \beta_{0}$

compresfa.

pectinata.

Calopus Serraticornis.

lineatus. I).

aquaticus. ...... $. \delta . \varepsilon .2)$

15) Thorace convexo corporeque rufis, elytris viridibus.

16) Thorace rubro, corpore violaceo, antennis ferratis.

17) Tota cæruleo-nitida hirta, antennis obfcurioribus.

18) Tota nigra elytrorum apice fafciaque media pedibusque albis.

19) Thorace flavo macula nigra, elytris violaceis maculis quatuor albidis.

20) Tota nigra opaca villofa.

2 I) Tota cærulea nitida hirta, ore rubro. ¿E Veftro. gothia Dn. Gyllenhahl.

22) Ovata fufca clypeo antice elytrorumque macula laterali alba. E Cajana.

23) Ovata flava elytris diaphanis apice nigris. E Svecia.

I) Thorace pedibusque flavis, elytris luteis punctis impresfis nigris.

2) Multum variat magnitudine, facie \& coloribus: 


\section{औ) I6}

Calopus fimplex. ж. B. 3). Leptura dubia.

Cucujus planatus. a. B. 4).

muticus.

ferrugineus. 5).

terebrio. 6).

Leptura viress.

virginea. a. B. 7).

collaris.

rubra.

teftacea.

fangvinolenta.

melanura. a. $\beta, \gamma$.

tabacicolor.

marginata.

nigra.

parifina.

2-puftulata.

obfcura. 8).

elongata. 9).

4-fafciata.

attenuata.

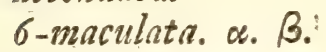

flexuofa.

detrita. $\alpha . \beta$.

myftica. verbajci. alni.

plebeja.

arietis. $\alpha . \beta . \gamma . \delta$.

floralis.

arcuata.

friata.

trifalciata.

liciata.

ruftica.

bajulus.

undata.

frigma.

Sangvinea.

teftucer.

caftanea. variabilis.

violacea.

aulica.

feranica.

Sa:

3) Fufcus elytris aneis pedibus inermibus flavis. $\mathrm{Ha}$ bitat in Svecia.

4) Cerambyx planatus.

5) Thorace lævi totus ferrugineus. Ex India Orient. Doct. Hornftedt.

6) Tenebrio depresfus.

7) $\beta$. Thalasfina dicta.

8) Attenuata tota nigra villofa. In Svecia.

9) Attenuata nigra elytris emarginatis flavis: fafciis tribus punctisque tribus nigris. 


\section{竍) 57 (.)}

Saperda oculata.

juponica. 10).

ferruginea. II).

cylindricas.

Linearis.

fcalaris. \&. B.

tremula.

curdui.

populnea.

carchavias. os. $\beta$.

proufts.

purcinta.

iuters. 12).

6-notatio*?

4-oculata. 13).

fulo. 14).

nebulolo.
Cerambysc longimanus. $\alpha . \beta$.

maxillojus. $\alpha . \beta$.

coriaceus.

faber.

dep farius.

cinnanomeus.

Rubus. 2. B.

fuccinetus.

cerdo, a. B.

textor.

alpinas.

mofobatus.

virens, a. B.

8-oculatus. I6).

cupen/is.

ethorops.

G

C:-

10) Lutea thorace immaculato, capire antennis elytrisque excepta bafi nigris. Fig. 3 .

ii) Tota ferruginea antennis nigris, abdomine bafi albido cingulogue in medio nigro.

I2) Corpore cylindrico lareo, antennis elytrisque apice bidentatis nigris.

13) Corpore cylindrico nigro, thorace flavo punctis quatuor nigris, capite elytrisque bidentatis fiavis.

*) Corpore cylindrico virefcente, elyeris maculis marginalibus fex favis. Fig. 2.

I4) Corpore cylindrico nigro, thorace rubro margio nibus nigris, capite elytrisque rangyineis,

15) Lamia nebulora Fabric.

16) Lamia oculator Fabric. 
Cerambyx caffer. 17). mimofa. 18).

farino/us.

feftivus. 19).

curculionoides.

nebulofus.

araneiformis. $\alpha . \beta_{\text {. }}$

triftis.

fuliginator. $\approx . \beta$.

Kableri. a. B.

edilis. 송 우

bifafciatus. 20).

noctis.

torisator.

futor. $\alpha . \beta . \gamma . \delta . \varepsilon$.

mordax. a. $\beta .21$ ).

inquifitor. $\alpha . \beta$.

4-maculatus. a. $\beta$.

lamed.

interrogationis. «. B. $\gamma$.

pedeftris. $\alpha . \beta . \gamma$.

bispidus.
Cerambyx pilofus. 22).

meridianus. $\alpha . \beta . \gamma . \delta_{0} \varepsilon_{0}$ curfor.

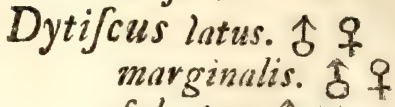

fulcatus. 令 $f$

friatus.

cinereus.

maculatus.

ovalis.

ovatus.

palufris.

granularis.

minutus.

erytbrocepbalus.

2-puftulatus. a. $\beta, \gamma, \delta$. uliginofus.

coftalis.

limbatus.

6-pufulatus.

grifeus.

17) Thorace fpinofo niger, elytris fafciis duabus lunu. laque apicis rubra. Fig. I.

18 ) Lamia hotrentotta Fabric.

19) Stenocorus feftivus Fabric.

20) Rhagium bifafciatum Fabr.

2I) Cerambycis inquifitoris major varietas elytris fu. fco-cinereis: fafciis duabus luteis.

22 ) Totus hirtus niger, thorace fpinofo lateribus albo, elytris fafciis duabus albis. 

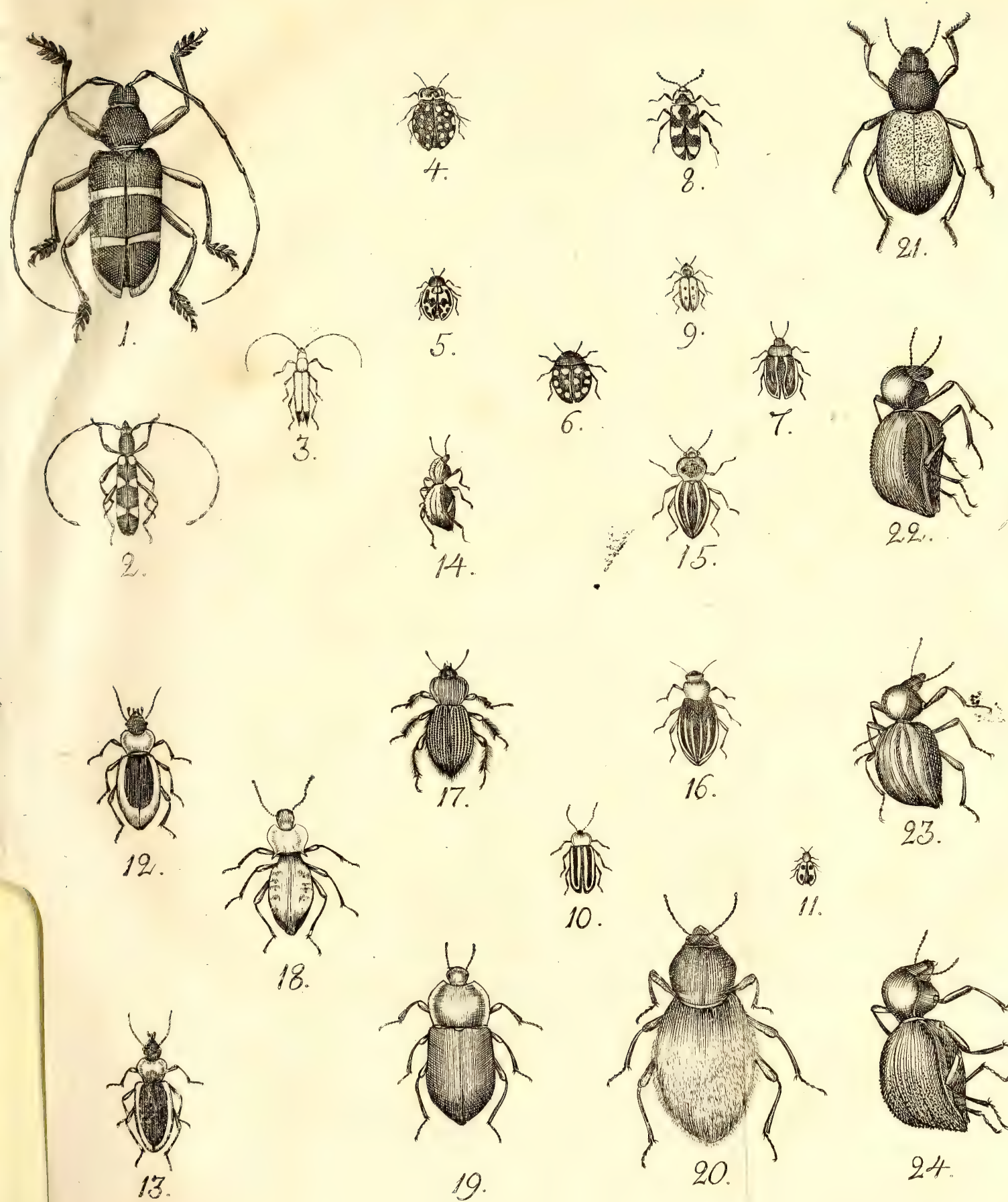

18.

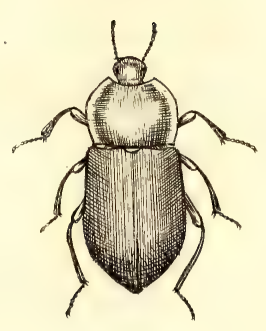

19.
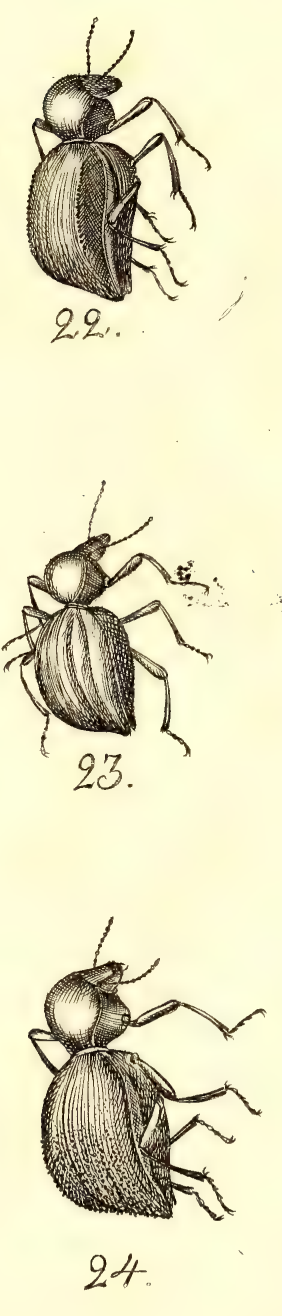

I.N. Athl delinet So. 

D. $D$.

\section{MUSEUM NATURALIUM ACADEMIE UPSALIENSIS.}

cUJUS

\section{PARTEM QVINTAM.}

CONSENSU EXP. FAC. MED. UPSAL.

PR IESIDE

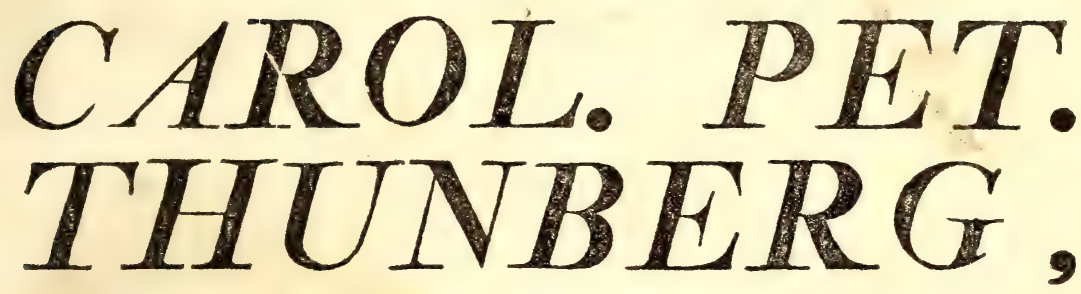

Equite Aurat. Reg. Ord, de Vasa,

Medic. Doct. Frofess, Med, et Botan, Reg. et Ord., Acad. Casar. Nat. Curios. Reg. Scient. Holmiens, Societ. Scient. Upsat. Patr. Holm. Berol. Nat. Scrut. Lund. Harlem. Amsteld. Zeland. Nidrosiens. Medic. Edimburg. et Nat. Studios. Ibid. Membro; nec non Acad. Monsfeliess. A gricult. Paris. Fiorentin.et Batavin. Ind.

Or. Correspond.

\section{Publico exnmini fubjicit,

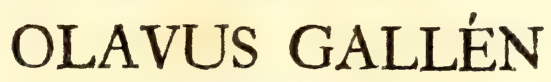 Helfingus.}

IN AUDIT. GUST. MAJ. D. V. DEC, MDCCLXXXVIr. H. A. M. S.

\section{U P S A L I $\not$,}

LITTERIS DIRECTOR. JOHAN. EDMAN. 



\section{DONATION. THUNBERGIANÆ 1785. CONTINUAT. III.}

Truxalis nafutus. Acrydium fubulatum.

$$
\text { 2-punctatum. }
$$

Gryllus Spumans i). elepbas 2). miliavis. morbillofus. flavus. Aridulus. carulefcens. migratorius. pedeftris.

rufus. grosfus. viridulus. biguttulis. objcurus. carolinis. fuccinetus? canejcens 3 ):
Locufta citrifolia. pupa. vituta 4). lanceolata. criftata. viridisfima. ver rucivora. a. $\beta$. bracbytera. laurifolia. myyrifolia. Acbeta gryllotalpa. capentis. campeltris. domeftica. monftrofa. Pneumora 6 -guttata. Maculata. immaculata.

Blatte americana. 15 . erytbrocepbala 5). fufca. 8 \%.

G 2 deulta. Blat-

I) Thorace convexo rugofo, rufo nigroque varius, hemelytris abbreviatis, femoribus muticis.

2) Apterus thorace carinato, totus f́pinofus, viridi-fufcoque varius, femoribus utrinque fpinufis.

3) Thorace carinato denticulato, elytris canefcentibus lineis furcis.

4) Thorace fubquadrato lævi, alis niveis fufco fafciatis.

5) E capite bonæ fpei. 


\section{少 60 (}

Blatta regptiaca. orientalis. 合.

Petiverisna. germanica. tapporica. gigantea. irrorata. madera.

Mentis gigas.

necydaloides.

Fulgore candelaria. laternavia. eurustica.

Cicada aurata. populi. exclnmationis. lineata. Alava. leucopbtbalma. pbtifica. labiata. maculata. faufta. nafuta. ficcifolia. gongylodes. pauperata. $\alpha . \beta$. guttata $\sigma$ ). lobata. oratoria. $\alpha$. $\beta$. cruentata. undulata 7). ruficollis 8). tibicen. Septendecim. bamatodes. crenata 2). Aridula. aurita. cornuta. tuberculata 20 ).

Cica:

6) Thorace marginato crenulato, elytris viridibus macula alba.

7) Thorace albo-lineato, hemelytris hyalinis fafcia nigra undulata abrupta ordineque punctorum nigrorum.

8) Nigra thoracis abdominisque incifurarum marginibus rufis, hemelytris hyalinis.

s) Thorace albo-lineato, hemelytris nigris poftice albis maculis nigris, venis crenatis.

10) Thorace bicorni nigra, fcutello fubulato longitudine abdominis, hemelytris brunneis. 


\section{* ) 6I (}

Cicada fulcicornis "). punctata.

Cime:s nobilis. a. $\beta$.

vittata.

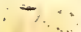

Aockerus. $\alpha . \beta$.

vubra i2).

viridis.

Scarabosides.

fruvipes. a. $\beta . \gamma . \delta$.

acaroides.

nervofa.

ulmi.

coleoptrata.

sumaria.

leucocepbala.

Ariata.

bifafciata.

interrupta.

fangvinolenta.

lateralis. $\alpha . \beta$.

flavicollis.

variegata. a. $\beta . \gamma . \delta$.

reticulata.

filpboides.

fuliginofus.

guttigerus.

maurus. $\alpha . \beta$.

lineatus 14).

liligerus.

grandis.

diopbtbalmus.

multipunitatus.

ocellatus. $\alpha . \beta$.

Julcatus.

tibialis.

cincta.

Notonecta glauca.

obliqua 13).

minuta.

Ariata. $\alpha . \beta$.

Nepa grandis.

rulfica. a. $\beta . \gamma$.
cimicioides.
linearis.
cinerea.

fullo.

punctatus. a. $\beta$.

reticulatus.

commia.

bamorrboidalis.

rufipes.

bidens. ㄱ. 오.

grifeus. a. $\beta$.

baccarum.

11) Thorace bicorni nigra, cornubus bifulcatis, fcutello fubulato longitudine abdominis, hemelytris albis. 12) Cicada rubra Linn. cruenta Fabr.

13) hemelytris nigris lineis duabus obliquis abbreviatis cinereis.

14) Cimex nigrolineatus Fabr. 


\section{* ) 62 (}

Cintex transverfos. bumeralis. fubulatus. criftutus. annulatus. calcaratus. bispidus. villo/us. perfonatus.

crux.

fufcus. thoyacicus.

- analis. unipunctatus. pungens. muricatus. trigonus. acantbaris. not at us. clavicornis. fordidus. marginatus. rbombeus. valgus. a. $\beta . \gamma . \delta$. femoratus.

Jpinifex. pilnfus.

jaculus. clavatus. acutus. j»vanus.

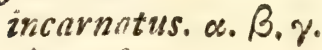
cbivenfis. janus. $\alpha, \beta$.
Cimex brunneus.

rutilus. ancbora. fimbriatus. $\alpha$. $\beta$. venolus. $\alpha$. $\beta$. variegatus. $\alpha . \beta$. interfinctus. ornatus. olevaceus. §. bicolir. biguttıtus. cofiatus. acuminatus. dum'fus. bivtus. lencocepbalus. morio. littoralis. melaleucus. niger. a. B. $\gamma$. ceruleus. corticalis. betule. abietis. cardui. cornutus. juniperinus. prafinus a. $\beta . \gamma$. campeftris. laniarius. firiatus. ulmi. kalmii. populi. 
* ) 631 (

Cimex praten/ss. gotbicus. minutus? filicis. bafticornis. a. $\beta$. aulicus. $\alpha, \beta$. equeftris. byofcyami. a. $\beta$. fuperftitiofus. koenigii. suturalis. apterus. $\alpha$. $\beta$. feltivus. $\alpha . \beta, \gamma$. augur. $\alpha . \beta_{0} \gamma_{0} \delta_{0}$ albidus. bexopbtbalmus. colon. carnifex. deuftus. andrea. purpureus. pini.

arenarius. rolandri. coryli. jemifiavus.

ater. caffer.

nemorum.

clavatus. nebulofus. pabulinus. virens.
Cimex crasficornis. rugofus. lavigatus. nugax. erraticus. dolabratus. lectularius. vagabundus. tipularius. clavipes. lacuftris. Atagnorum.

Cbermes aceris.

fraxini. caltbe. abietis. graminis. falicis.

Coccus besperidum. betula.

Tbrips physapus. monuta. fafciata.

Papilio Priamus.

Sarpedon. EEneas. Iysander. $\alpha . \beta$. Hector. Pammion. Polydamas. Belus. Memnon. Pavis. 


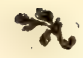

) 64(

Papilio Polytes.

Helenus.

Troilus.

Agenor.

Palamedes.

Acbilles.

Menelaus.

Macbson.

Xutbus.

Tboas.

Leilus.

Agamemnon.

Demoleus a. $\beta$.

Patroclus.

Protefilaus.

Pbiloctetes.

Idomeneus.

Teucer.

Ulysfes.

Neltor.

Fabius.

Podalivius. $\alpha . \beta . \imath$ )

Apollo.

Mnemosyne.

Erato.

Crategi.

Pieva.

Ricini.

Pfidii.

Papilio Melpomene.

Antiocbus.

Calliope.

Mopfa.

Polynnia.

Clio.

Thalia.

Euterpe.

Horta.

Viole.

Cyparissa.

Elatbea.

Sinapis.

Hellica.

Glaucipte.

Daplidice.

Brasfica.

Rapa.

Napi.

Monufte.

Calypso.

Hecabe.

Rbamni.

Palano.

Hyale.

Cardamines.

Marcellina.

Cbryfippus.

Lucindus.

1) Podalirius $\beta$. uti \& Galathea $\beta$. Mægera $\beta . \&$ \&geria $\beta$. benigne communicati funt ab Adj. med. Dn. Dre. P. Afzelic. 


\section{* ) $65($ )}

Papilio

Papilio Hero.

Pampilus.

Pbadra.

Eudoxa 2).

C. aureuns.

Lena.

Dyndiment.

Hyperantbus.

Cas/us.

Fatbropbe.

Laomedia.

Semele.

Tipbon 2).

Circe.

Iris.

Feronia.

Almara.

Afterie.

Megera, a. B. $\gamma$.

Fanira.

Furtina.

Brifeis.

Cardui.

Aviadue.

Mera.

Ligen.

jo.

Denone.

Oritbya.

Ageria.

Dejaniva.

Leda.

Hefione.

Lucinn.

Aglaja.

Adippe.

Similis.

Asfimilis.

Demophons.:

Populi

Obrinus.

Antiopa.

Cinsia.

Maturne.

Latbonia.

Eupbrofyne. Atbalia 2).

Didyma 2).

Dirce.

Bolina.

Atalanta.

Polycbloros.

Uitica.

C. albums.

Levana.

Prorja.

Pbarufa.

Sibilla.

Nipbe.

Papbia.

2) Efper. 
7) 66 ( )

Papilio Niobe.

Vanille.

Disfinilis.

Opbione.

Ilitbuin.

Leucotboë.

Dido.

Galatbea e. $\beta$.

Boëticus.

Timeus.

Pblaeas.

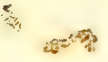

Arcanius.

Hippotboë.

Virgaurea.

Proteus.

Bixce.

Casfius.

Carica.

Dantoetes.

Rojinon.

Qvercus.

Pruni.

Betule.

Cupido.

Syricbtus.

Argiolus.

Argus.

Arion.

Rubi.

Idas.

Tbyra.
Papilio Comma.

Augias.

Metis.

Tbrax.

Malva.

Tages.

Sefoltris 3).

Pompeus.

Pelias.

Pborcas.

Pirantbus.

Syliphus.

Licomedes.

Alcionea.

Ly/imnia.

Porjenna.

Electra.

Alcyone.

Evefimus.

Dardanus.

Salonse.

Beroë.

Virgilia.

Cissa.

Orplire.

Aonis.

Harpalyce.

Coryca.

Perimele.

Camilla.

Colombina:

3) Papiliones a Cramero aliisque auctoribus recenfiti requentes funt. 


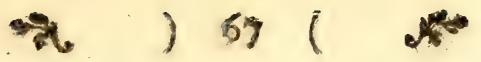

Papilio Petreus.

Eumena.

Argyrius.

Chrone.

Melaneus.

Folus.

Clarus.

Niveus.

Etolus.

Celone.

Cleobes.

Orleus. 1

Taumas.

Titanius.

Spbinx Carolina.

Didyma.

Elpenor.

Porcellus.

Liguftri.

Tbyelia.

Tantnlus.

Stellatarum.
Sphinx Ocellata.

Atropos.

Fuliformis.

Apiformis.

Caliciformis.

Tipuliformis.

$V$ espiformis.

Pectinicornis.

Pinafivi.

Populi.

Tilice.

Eupborbice.

Galii.

Convolvuli.

Filipendula.

Caffra.

Cerbera.

Feneftrata.

Polymena.

Statices.

Pilogella.

Pbegea.

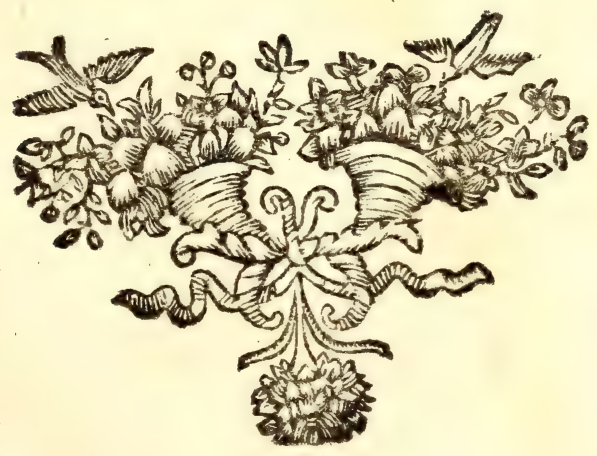




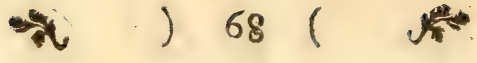 THESES.}

1. Caput retractum, cormutum, rotratums; thovax convexus, marginatus, cordatus, transverjus; elytra flexilia, abbreviata; pedes faltutorii, napatorii in cosnoicendis Generibus Infectorum, notas minime fpernendas fepe afferunt.

2. Colores infectorum variare licet quandoque deprebendan: tur, Jpecies tamen difinguere optime valent.

3. Aninalia, quo minora, co magis sese multiplicare folent.

4. Infecta numero individuorum Ev fpecierum cetera animalia terreftria fuperant.

5. Paricia infectorum species grandes funt, plurima omniun minime evadunt.

6. Grylli genus, ob magnam in antennis diverfitaten, in qlura genera mevito \& necesfarie dividi debet.

7. Preter Locufan crifatan, plures alice Grylli fpecies Pariis nationabus ejculentce junt.

\&. Tetram odoren Cinnices fere omnes fporgunt. 


\section{D. $D$. \\ MUSEUM NATURALIUM ACADEMIÆ UPSALIENSIS.

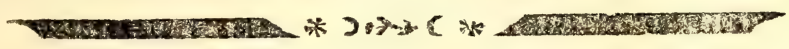 \\ CUJUS \\ PARTEM SEXTAM.}

CONSENSU EXP. FAC. MED. UPSAL.

PR Æ EIDE

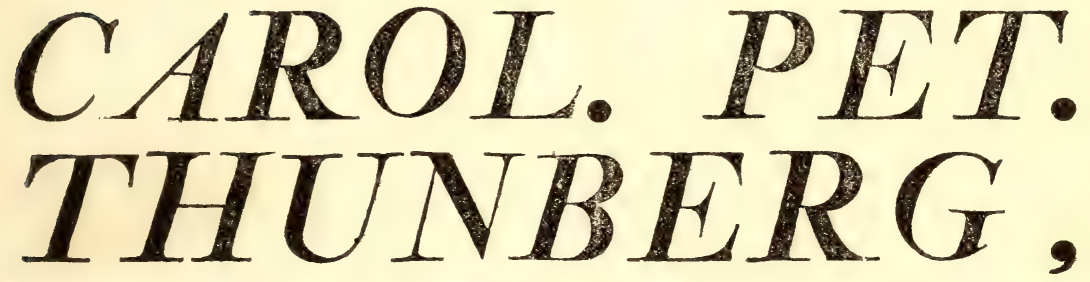

Equite Aurat. Reg. Ord. de Vasa,

Medic. Doct. Frofess, Med, et Botan, Reg. et Ord., acad. Ceesar. Nat, Curios, Reg. Scient, Londinens. Holmiens. Societ, Scient, Upsal. Patr. Holm. Berol. Nat. Scrut. lundens. Hariem. Amsteid. Zeland. Niprosiens. Haiens. Nat. Scrut. Medic. Edmeurg. et Nat. Studios. Ibid. Membro; nec non acad. Scient. Paris. Monspeliens. Agricult, Paris. Florentin. et Batayin. Ind. Or. Correspond.

Publico examini fubjicit, CAROLUS GUSTAVUS SCHALÉN Stockbolmienfis.

IN AUDIT. GUST. MAJ. D. XVII. MAJ, MDCCIXXXVIII. H. A. M. S.

$=-9)^{2}(r=9)^{2}(6=0)^{2}(6=0$ UPSALI $\mathbb{E}$, LITTERIS DIRECTOR. JOH. EDMAN。 

Bombyx atlas a. ㅅ․ 우. B. Bombyx mori.

besperus.

cecropia.

cytberea, a. B.

mylitta。 $\alpha . \beta$.

prometbea. a. B.

luna.

militaris.

difcolor. $\mathbf{1})$.

pavonia major.

cyntbia. 1).

minor. 含. 오.

tars.

quercifolia.

illicifolia.

capenfis.

quercus. $\alpha . \beta$.

potatoria.

pini.

dimeti.

verficolora. S. \&.

rubi. 疋. 早.

vinula. 1. ?.

laneftris. 다. ㅇ.

populi.

catux.

everia.

procesfionea. neuftria.

caftrenfis.

bieracii.

cosfus.

dispar. 令. q.

pudibunda.

falcelina.

bucepbala.

cerulocepbala.

decora.

ziczac.

dromedarius.

coryli.

monacba.

palpina.

camelina.

aulica.

lubricipeda.

mendica. I. 우

leporina.

falicis.

crategi.

rusfula.

jacobea.

granmica. ㅅ․ㅇ․

plantaginis.

villica.

L

Bom-

I) Crameri bicolora \& cynthia. 


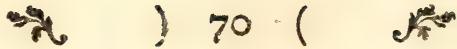

Bombyx bebe.

cuija.

crotalaria.

bera.

antiqua. ‥ 오.

gonoftigma. 궁. 웅

pylotis.

graminis. a. $\beta$.

fuliginofa.

cribrum.

grifea. 2).

ferrata. 3).

lugens. 4).

furcula.

templi.

tremula.

bunnuli. 5. . 9

lupilina. $\hat{\text { of: }}$ ?

becta.

anaftomolis.

curtula.

reclusa. 5)
Bombyx cbryforrboea.

cygnea. 6).

afculi. 7).

Noctur bubo.

odora. a. B. 8).

ppiralis.

dio/corece.

materna.

afrea.

4-punctatin:

pallens.

vaccinii.

quadra. $\propto$. $\beta$.

ficus. $\alpha$. B. carica.

manlia.

batis.

rufina.

jcuto/a.

glypbica.

dipfacen.

ni.

roboris.

2) Infect. Svec. P. I:a.

3) An Bombyx fagi Linn?

4) Alis atris, pofticis macula fulva, abdomine flavo trifariam nigro-punctato.

5) Bombyx reclufa Fabr.

6) Alba alis anticis puncto nigro, ano flavo.

7) Phalrna pyrina Linn. Faun. Svec. p. 306. Hepialus afculi Fabr. Spec. Infect. p. 208. Bombyx æfculi Fabr. Mantils. p. I 16.

8) Communicata a D. D:r Swartz cum multis aliis pulcherrimis ex America calidiore. 


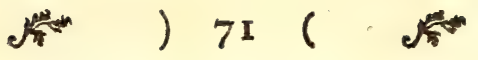

Noctua albicollis.

italica.

folida.

trapetzina.

Sponfa.

napta.

pacta.

fraxini.

pronuba.

retufa.

maura.

libatrix.

plecta.

C. nigrum.

gotbica.

Erasfice.

exclamationis.

fulvago.

citrago.

cbryfitis. $\alpha . \beta$.

feftuce.

gamma.

divergens. 9).

interrogationis. $\alpha . \beta$.

meticulofa.

oo.

derafa.

jerici.

fatellitios.

diffinis.

abfintbii.

Noctua occulta.

delpbinizi.

perfpicillaris.

linurie.

pij.

oxyacantbe.

atriplicts.

pyramidea.

typica.

lucipara.

olevacea.

leuconzelas.

triangularis.

pinaftri.

firigilis.

aprilina. 10).

licbenes.

p pio

cbi.

aceris.

litura.

perficarice.

tragopoginis.

polyodon.

nigricans.

oculea.

flnvicornis.

triplacia.

rumicis.

exoleta.

verlafici

L 2

No.

9) Habirat in Lapponia D. Adjunct. Winbom. 10 Runica Fabric. 


\section{哓 $) 72($ )}

Noctua umbratica.

putris.

comma.

myrtilli.

arbuti.

ludifica. II).

evidens.

argentea. 12?.

fontis. 13).

carnea. 14.)
Noctua maforeta. is).

pteridis 16).

porphyrea. 17).

ancilla. …‥ 18)。

typhe.

parthenias. †).

confpicillaris.

Arigula. 19).

mestbaftri. 20).

cordigera. ${ }^{*}$ ).

No.

\section{II) Linn. 12 ) Artemilix Fabr.}

13) Alis deflexis nigris, anticis margine poftico ci= nereis punctis lineaque apicis nigris. Habitat in Hallandia D. Osbeck, in Wermelandia Med. Cand. Holmer.

r4) Alis deflexis brunneis, ftigmate poftico flavo, antico fafciague intra marginem cinereis; pofticis fufcis margine rufefiente Hab. in Hallandia Doct. Osbeck.

is) Alis incumbentibus albis, anticis lineola punctis. que fex aggregatis arris. Habitat in Svecia D. Paykull.

16) Alis deflexis dentatis ferrugineis, fafcis duabus ru: fis maculisque tribus albidis; pofticis fufcis. D:n. Wildenow.

17) Alis deflexis ferrugineis, anticis macula alba lunata margineque pallide dentato. Habitat in Svecia.

18) Alis fufcis, anticis maculis tribus albis diaphanis, abdo:nine flavo, trifariam nigropunctato.

t) N. vidua fabric.

19) Criftata alis deflexis ferrugineis poftice albo-ftriatis, fafciis tribus undatis albis, lineola punctis duobus ftigmatibusque albis. Habitat in Svecia. Misfa ex Hal. landia, fub nomine Noctuæ arnicæ, a D:no D. Osbeck. 2n) Alis flavis nigro-pundtatis, anticis fafcia e pun. ctis nigris, abdomine luteo tri-fariam nigro-punctato.

*) Alis anticis nigris fafciis undatis albis, ftigmate al. bo; pofticis Alavis margine nigro. Hab. in Svecia. Holmer. 


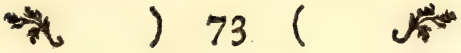

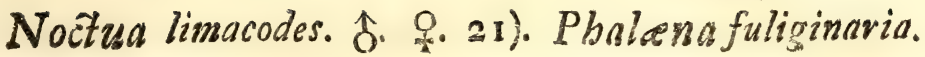

polynsita.

lota.

nictitans.

depuncta.

tritici.

Pbaleena tbymiaria.

lactearia.

vernaria.

putataria.

papilionaria.

pufaria.

falcataria.

ficuluria I).

fambucaria.

prunaria. a. B. S. 是.

melanaria.

pennaria.

nlniaria. a. $\beta . \gamma$.

Jyringaria. $\alpha . \beta$.

defolitivia. ․․․․

pulveraria. a. $\beta$.

elingvaria.

corylaria. 今. ?. 4-fofctaria.

lacevtinaria. $\alpha . \beta \gamma .2$ ).

punctaria. $\alpha . \beta$.

pendularia. $\alpha . \beta$.

profapiaria.

Scopularia.

pictaria. a. B. 含. ․ 3).

fugaria.

betularia.

zorpoiria.

birtaria.

catilinaria.

tripunctaria.

piniaria.

vejpertaria. $\alpha . \beta$.

deleataria.

purpuraria.

cultraria.

anataria.

vibicaria.

atomaria. 4).

dolabraria.

marmoraria. 5).

$\mathrm{L}_{3}$

Pba:

21) Alis ferrugineis, anticis fafciis duabus nigris di vergentibus.

I) Cultraria. Fabric,

2) $\gamma$. dentaria. Infect. Svec. I,

3) Lichenaria Fabr.

4. Varietates plures.

dentatis.

5) Alis cinereis nigro-irroratis, fafciis duabus fufcis 


\section{" ) $74($ 奖}

Phalena vittaria. 6).

Pbalena fulvata.

notata.

grosfulariata.

prunata.

albicillata.

baftata.

triftata.

violata.

fuccenturiata.

oblongata.

Aratiotata.

potamogata.

nympbicata.

leminata.

ulmata.

populata.

comitata.

miata.

bilineata.

chenopodiata.

4-fafciata.

plagiata. t).

crenata.

cuspidata. 7).

teftata.

innotata. 8).

didymata.

spartiata.

immutata.

rectangulata.

albeniillata.

brumata. 含. ‥

remutata. 9).

immorata.

plunata. 10)

finuata.

variata. $\alpha$. $\beta . \gamma$.

trifafciata.

furcata. .

undulata.

repandata.

dubitata.

juniperata.

averfata.

cratagata.

emarginata.

ferrugata.

fluctuata.

deallata.

ocellata.

nebulata.

6) Alis fufcis, fafciis duabus undulatis divergentibus nigris. Habit. in Svecia. Printz.

†) Duplicata. Fabr.

7) Sociata Fabr.

8) Alis omnibus fulcis puncto nigro.

9) Centrata fabric.

10) plumbaria fabric. 
\# $) 75($ (

\begin{tabular}{|c|c|}
\hline 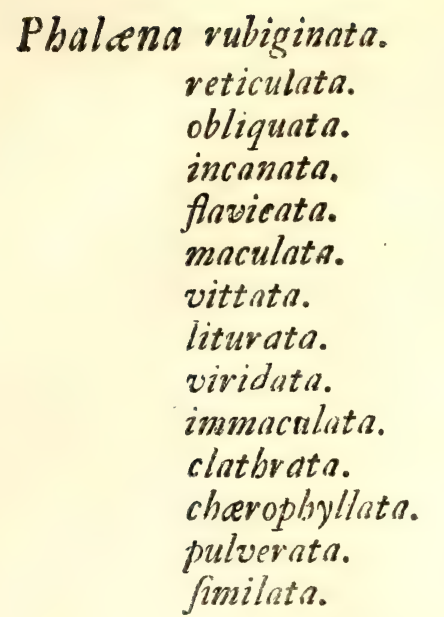 & 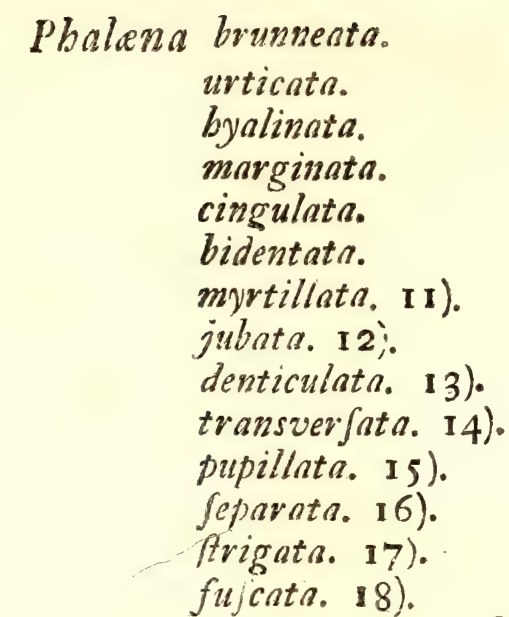 \\
\hline
\end{tabular}

Pbo.

II) Alis cinereis, fupra puncto fafciisque obloletis fufcis. Habitat in Svecia.

I2) Alis albis poftice nigro-irroratis, anticis apice fufcis fafcica alba, omnibus fafcia punctoque nigris. Misfa mihi e Germania a D. D. Wildenow.

13) Flava alis omnibus ocello fafciaque denticulata fufca, margine nigro punctato.

14) Alis nigris farcia lata media alba, ftriga nigra interrupta, punctogue in pofticis albo. Habitat in Svecia Cand. Holmer, \& Printz.

15) Alis fufcis medio ferrugineis: ftrigis variis undais albis; ocello duplici, altero pupillato. Habitat in Svecia D. Printz.

16) Alis anticis albis, fafciis tribus duplicatis nigris, tertia divergente. Hab. in Svecia D. Delèn.

17) Alis flavefcentibus poftice ftrigisque tribus fufcis. Habitat in Svecia Printz.

18) Alis fufco-cinereis, fafcia flava, punctis tribus marginalibus nigris. Habitat in Svecia. Holmer. An Pho liturate varietas? 


\section{牙 ) $76(\quad)$}

Pbalcena paludata. $\dagger^{\text {). }}$

Pyralis punctalis.

pinguinalis.

verticalis.

forficalis.

facralis. 19).

nemoralis.

rofralis.

probolcidalis.

tentacularis.

barbalis.

glaucinalis.

farinalis.

ocellaris.

Julpburalis.

frumentalis.

purpuralis.

recurvalis.

nivealis.

reticularis.

minialis.

fordialis.

duplaris.

atralis.

coftalis. 20).

bamalis. 21).

Tortrix Banckinna.

Strcmiona.

Lediana.

Scballerinna.

rivellana.

cynosbana.

refinana.

Wabllomiann.

fasciann.

coronana.

Haftiana.

lunana.

punctata.

mundana.

Xylofterina i).

Uddmanniana.

Solandriona.

Cruciana. 2).

Logiana.

Alfromeriana.

Yeations.

beracleana.

t) Alis canis, anticis fafciis quatuor nigris, tertia dentata, quarta reverfa. Hab. juxta paludes turfofas Weftro. gothiæ, menfe Julio. D. Gyllenhah!.

19) Phaicena facraria fabr.

20) Purpurana Thunb.

2I) Alis anticis fulcis, fafcia media flexuofa punctoque albis. Habitat in Svecia. Printz.

1) Weftriniana. Thunb.

2) Gyllenhaliana. Thunb. 


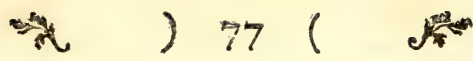

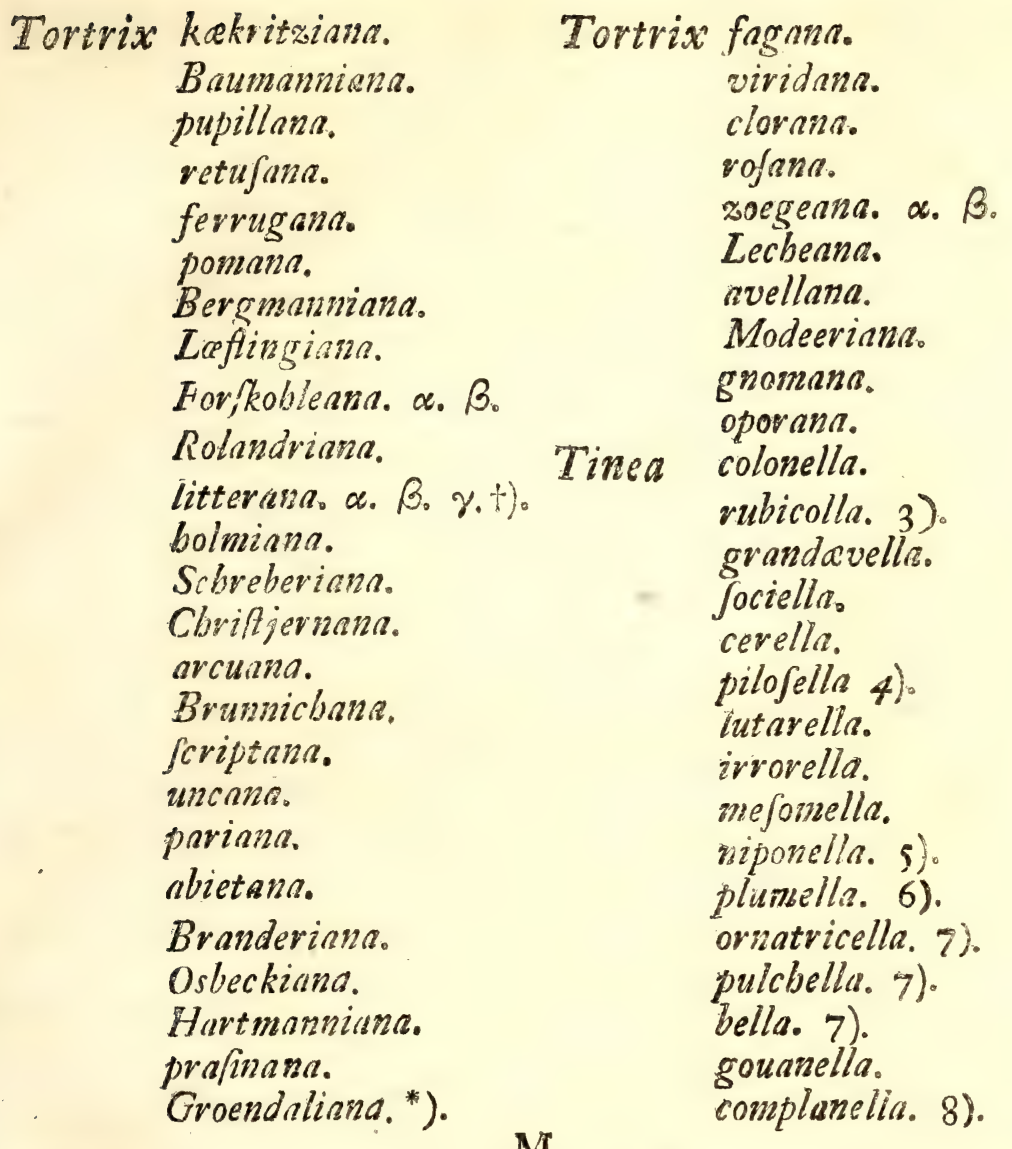

M

Ti-

†) B. fquamana; $\gamma$. fcabrana.

*) Alis anticis ferrugineo-aureis lituris fex argenteis maculisque quinque flavis. In Srecia D:n. Groendal.

3) Bombyx rubicollis.

4) Cinerea fafciis punctisque obfoletis fufcis.

5) Flavercens margine poftico punctato.

(vo.

6) Flavefcens capire, thorace elytrorumque margine fla-

7) Bombyces Fabr. 8) Noctua quadra \& complana Fabr. 


\section{st}

Tinea ferruginella. 9).

aridella. 10).

derafella. I1).

pascuella.

culmella.

pratella.

rorella. I2).

bamella. 13).

parentbesella.

nemorella. 14).

Sparganella. is).

pinetella.

ebiella. 16).

fimbriella. 17).

atrella. 18).
Tinea atropunctella. 19). dealbella. 20). angulella. 21). mellonella. Serratebla. Podalla. Degeerella. Clerckella. Rajella. Roefella. Lirneella. Wilkella. Gadartella. Merianella. Geoffroijella.

9) Fufco-ferruginea immaculata.

I0) Cinerea fafciis duabus obliquis fufcis.

I I) Flavefcens linea nigra antice derafa, poftice ramola, apice fafciis duabus argenteis.

12) Cinerea punctis nigris irrorata, linea alba ramofa, arcu medio obfoleto pofticoque fufco.

I3) Fulca linea hamata argentea, arcu maculaque apicis nigris.

14) Cinerea albo-lineata puncto utrinque nigro. impresfis.

I5) Aureo-ferruginea punctis utrinque tribus fub

16) Alucita 2 punctella.' Fabr.

17) Alis aureis margine omni niveo.

I8) Atra tota immaculata.

19) Nigra tota punctis alarum fex atris.

20). Argentea linea intra marginem fufca ramofa.

21) Cinereo-albida puncto poftice utrinque nigro. In Svecia Pfeif. 
Tinea fuefella.

compofitella. capitella. bratteella. leucatella. cinctella. punctella. tessella. Jarcitella. oxyacantbella. fulphurella. 1). pedella.

Leuroenboekella. Minutella. Atipella. fcabrella. lappella. betulinella. lucella. gemmella. arbutella. argentella. pruniella. alpevella. sequella. pellionelia. xyloftella dentella. vittella.
Tinea granella.

picavella. tertianella. mercuella. populella. fylvella. rbomboidella. bicofella. cucullatella. purpurella. 2), albella. 3). elongella. Rigmatella. Rbediella. Albinella. Petiverella. a. $\beta$. trigonella. firobilella. fenella. Bjerkandrella. tapetzella. ungvicella. a. B. 4). ramella. tadella.

Fungiella. a. $\beta$. Salicella. Reaumurella. Swammerdaniella. Frifchella.

M 2
1) Alucira fulphurella. Fabr.
2) Alis cinereis linea purpurea.
3) Nigra ciliata margine extimo albo.
4) Ungvicana. Fabr. 


\section{) 801 और}

Tinea cinerella.

gelatella. 1. 오.

fufcella.

evonymella.

padella.

ftannella. 5).

veftianella.

dodecella.

Swederella. *). caltbella.

Mouffetella.

Nisella.

Harisfella.

Broronella. 6).

joniperella.

porrectella.

curvella.

guttella.

ruficella.

rubiella.

interruptelia.

aiboflavella.

figuratella.

fraxinella.

proletella.

multipunctella.
Tinea cembrella.

occultella.

Alucita didactyla.

pterodactyla.

tetradactyla.

tesferadactyla.

pentadactyla.

bexadactyla.

monodactyla?

Libellula 4-maculata.

depresfa. a. B.

bifafciata.

flaveola.

indica.

rubicunda. 令. .

ferruginata. $\alpha$. $\beta$.

vulgatisfima.

vulgata.

cancellata.

fafciata.

equeftris.

anea.

carolina.

forcipata.

grandis.

misuta. 7).

Liber-

5) Plumbea immaculata, capite luteo.

*) Alis anticis cupreo-aureis: maculis duabus aureoAlavis; poftice cinereis ciliatis. Habitat in Anglia \& Gallia. Conc. Reg. D. Swederus; in Svecia Printz.

6) Alis ferrugineo-aureis: anticis fafciis tribus, pofticis duabus aureis. 1). Doct. Swartz.

7) Forfan eadem cum Lib, variegata. Fabr. 
* ) 81 ( 壮

Libellula virgo. a. ß. $\gamma$. puella.

unimaculata. 8)。

Ephemera vulgata.

venofa.

bioculata.

boravia.

culiciformis.

diptera.

fufcata.

Pbryganea phalonoides.

grandis.

friata.

flavilatera.

mininuta.

Weneri.

longicornis.

reticulata.

umbrofa.

analis?

bilineata.

filola.

nigra.

azurea.

albifrons.

grilea.

rbombica.

pilofa.

palipes.
Pbryganea marginata.

variegata.

bicaudata.

Hemerobius perla.

cbryfops.

albus.

hivtus.

pbalenoides.

bumuli.

6-punctatus.

pulfatorius.

2-punctatus.

Myrmeleon libelluloides.

lutteum.

formicarinim.

formicalyn.

capenfe.

italicum.

Panorpa communis. I. f. $\alpha, \beta$

japonica.

capenfis.

grandis.

Coa.

byemalis. \%.

Rapbidia opliopsis.

mantispa.

Termes fatalis.?

capen/is. 9).

8) Alis albis bafi macula magna fufca. De Geer Inf. Vol. 3. p. 558. Tab. 26. fig. 5. e Ceilona.

9) Hemerobius marginatus? Iinn. De Geer Infect. Vol. 7. Tab. 38. fig. 3. 


\section{* ; $82($ 登}

Termes teftacea. 10).

Formica berculeana. $\alpha$. $\beta$.

Mutilla atrath.

rufa. a. $\beta$.

rubra.

nigra.

fufca.

objoleta.

caspitum.

bamata.

atrata.

gulora.

cepbalotes.

Sexdens.

bematoda.

maculata.

ammon.

afvicana. I1).

colon. 12).

Mutilla occidentalis.

europea a. B.

maura.

aurate.

formicaria.

diadema.

fcabra. 14).

ruficornis.

Apis maculuta.

centuncularis.

longicornis.

biftrio.

variegata. 15).

4-dentata.

conica.

maxillora. annulata.

tumnulorum.

vuficornis. $\alpha$. B. $\gamma$.

clavicornis.

cinerea.

fubterronea. acervorum.

florifomnis. tridentata. fuccincta. anea. corulefcens.

Iо) Hemerobius teltaceus. Linn. De Geer Infect. Vol. 3. Tab. 27. fig. 4.

I I) Villofa teftacea, capite nigro, abdomine cylindrico. e Cap. b. fpei.

I2) Nigra abdomine villofo teftaceo: punctis duobis nigris. e Cap. b. Ipei.

13) M. formicaria Linn. Ichneumon formicarius. Fabr.

14) Mut. formicaria. Fabr.

I5) Nomada. Fabr. 


\section{武 ) $83($ )}

Apis tricolor.

trincorum.

cariofa.

retulia?

volirata.

lucorum.

pratorum.

byprovum.

mufcorum.

Syluarume.

lapidaria.

bortorum.

terreftris.

violacen.

caffira.

belvola.

lagopoda.

cunicularia.

mellifica. 少. 平。

manicata.

bicornis.

rufa.

rybyenfis.

transverfa. 16)。

Vejpa cincta.

affinis.
gallica.
bifafciata.
americana.
$V$ espa arcusta.

petivlata. conica. olivacea. I7).

Crabro. vulgaris. parietum.

mutraria?

jpinipes.

bidens. trifafciata. campeftris. coaretata. ruta. biglumis. uniglumis.

Spbex fabulofa.

viatica.

fufca.

pirifex.

variegata.

colon?

leucoftonia.

figulus.

fispes.

gibba.

fosforia.

crebraria.

aresaria.

Spbex

16) Thorace capiteque flavo-maculato, abdomine nigro cingulis margine flavis, primo fegmento rubro, fecundo linea utrinque flava.

17) De Geer. 3. 582. 
Sphex viga.

$$
\text { myltacea. }
$$

pectinipes.

capenfis. $\alpha . B$. ichneumonea.

clavipes.

Cbryfis ignita.

aurata.

fulgida.

fafciata.

Chryjis cruciata. viridula. cyanea.

Sirex gigas.

marifcus. juvencus. $\alpha . \beta$.

jpectrum. camelus. columba.

\section{THESES.}

1. Plurima funt Lepidoptera, quorum noxam longe magis cognitam nobis babemus, yuam quidem eorune in Poli. tia nature utilitatem.

2. Apis mellifica, cera artificiofi fui domicilii \& dulcisfimo melle, ufum nobis, tam medicum, quam oeconomicum conciliat, vere magnum.

3. Ex omnibus forfan Infectis, Bombyx Mori, telis fuis rericeis innumeros bomines veftiens \& ornans, fine dubio utilisfima eft cenfenda.

4. Infecta cevte innumera animalibus majoribus, inprimis vero avibus in efcam E alimentum cedunt. 

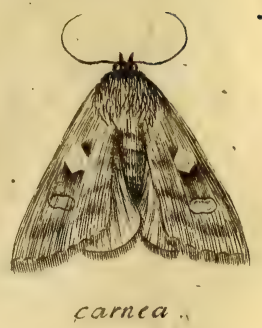

nom

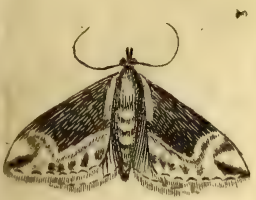

fundis.

Masplate

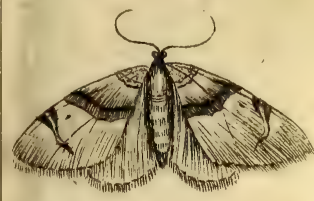

Separata.

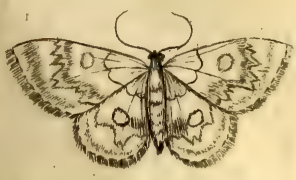

denticulata.

omiram 14ti

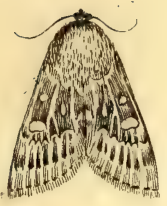

Striqula

Sisata

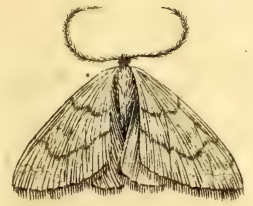

vittaria

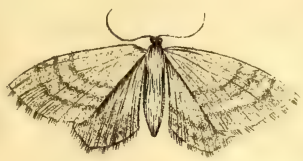

Erigata.

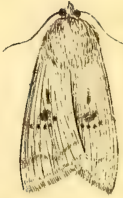

maforeta.

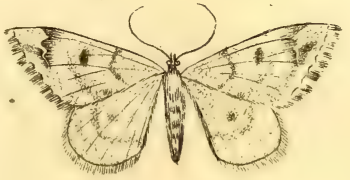

jubatata :

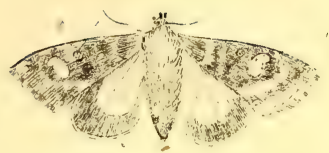

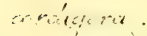

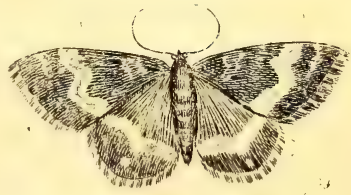

ipanuerfata.

Zutacta
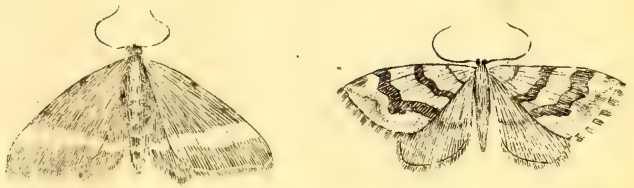

iesala.

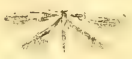

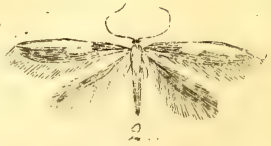

hunatis. 



\section{D. \\ MUSEUM NATURALIUM \\ ACADEMIE UPSALIENSIS. \\ - \\ cujus \\ PARTEM SEPTIMAM. \\ CONSENSU EXP. FAC. MED. UPSAL. \\ PRESIDE

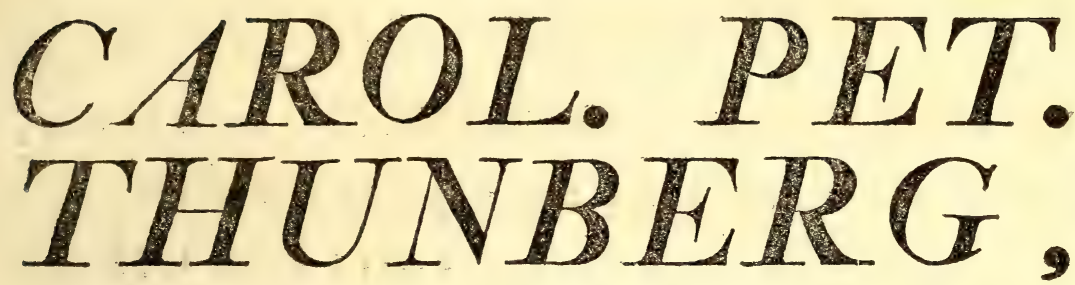

Equite Aurat. Reg. Ord. de Vasa,

Medic. Doct. Frofess. Med. et Botan. Reg. et Ord., Acad. Ceresay. Nat. Cyrios, Reg. Scient, Londinens. Holmiens. Societ, Scient, Upsal. Patr. Holm. Berol. Nat. Scrut. lundens. Harlem. Amsteld. Zeland. Nidrosiens. Halens. Nat. Scrut. Medic. Edimlurg. et Nat. Studios. Ibid。 Membro; nec non acad. Scient. Paris. Monspeliens. Agricult. Paris, Florentin. et Batavin, Ind, Or. Corréspond。

\section{Publico examini fubjicit, JOHANNES BRANZELL}

Verl. div. min. Vermelindus.

IN AUDIT. GUST. MAJ. D. VII MART. MDCCLXXXIX. H. A. M. S.

UPSALIE, LITtERIS DiRector. JOH. EDMAN. 
Frain Mott till Elefant, frin Gli till Grönlands Hvalar, Alt pa vir runda Boll, on Skaparns ara talar.

\author{
Tribeir Orar. 1778.
}


SACRAM REGIAM MAJESTATEM

SUIUIIE FIDEI VIRO,

Regis Regnique Sviogothici SENATORI,

Regine Augustissime SUMMO MARESCHALLO

Regi Collegir Metallici PR $\mathbb{E}$ SIDI,

Ominium S:a R:e Mitis Ordinum

EQUITI ET COMMENDATORI,

ILLUSTRISSIMO EXCELLENTISSIMOQUE

\section{COMITI AC DOMINO}

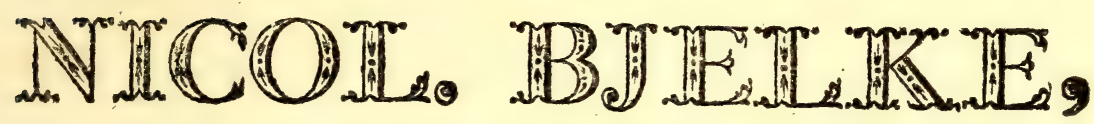

M ECENATI SUMMO,

$S A C R U M$. 


\section{THESES.}

1.

Entonrologia tum in aliis fcientiis, tum in ipfo facre Theologie Studio exiniiuns prebet urum.

\section{2.}

Opera Dei, etiam minima, piis atque eruditis /crutatoribus magnane adferunt voluptatent.

3.

Multorum, que jam inutilia judicantur, utilitatem baud exiguan pols bec obfervatio, experimentis junfin, pro. trabet in lucers.

4.

In toto Nature fyftemate nibil per fe noxion eft, nibill prorfus inutile.

5.

In ordine Infectorum dipterom ab uns parte Mufce incredibile nobis inferunt damnum, ab alteva in Natura politio utilisfone funt cenfenda.

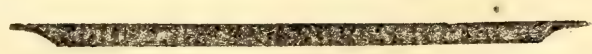




\section{DONATION. THUNBERGIAN $\mathbb{E}$ I $785^{\circ}$ CONTINUAT. V.}

Tentbredo femorata. a. B. $\gamma$. Tentbredo nigra.

lucortim. $\alpha . \beta$.

annevinu.

sitellina.

lutea. a. B.

marginata.

mitens. $\alpha . \beta$.

fafciats.

oblcura.

juniperi. 1. . ․

alces. I).

dorfita. $\alpha . \beta . \gamma . \delta, \varepsilon_{0}$

cnodis.

ufulata.

clavicornis.

annulata. $\alpha_{0} \beta . \gamma$.

roje.

germanica. $\alpha . \beta . \gamma$,

ovata.

almi.

cerafi.

padi. 2).

rapa.

cynosbati.

gonagra.

atra. livida.

caprea.

ruffica.

rufipes.

cinct $\alpha$.

bicincta.

jcropbularia. a. $\beta$.

fyluatica.

punctum.

mefomela. a. $\beta$.

nassata.

falicis.

viridis.

I2-punctata.

reticulata.

erythrocepbals. 念.

betulia.

flavicormis. \&. B. 3). Jeptentrionalis.

abietis.

vafra. a. B. $\gamma$.

lucorum.

Icbneumon fugillatorius.

raptorius.

culpatorius.

$N_{3}$

Icbneu:

I) Nigra tibiis pallidis, antennis ramofis villofis. Habitat in Svecia.

2) Morio Fabric,

3) T. flavicornis \& luteicornis Fabric。 


\section{*) $86(\%$}

Icbncumon Sarcitorius. extenforius. biguttorius. 4). ambulatorius. faturatorius. infractorius. snolitorius. $\alpha . \beta$, crippatorius. motatorius.

pisorius.

bidentorius. annulatorius. luctatorius. $\alpha . \beta$. volulatorius, $\alpha . \beta$. vaginatorius. $\alpha, \beta$. perfuaforius. $\alpha . \beta$. fulorias. deliratorius. tripunctorius. 5). fosforius. lituratorius. reluctator. Icbreumon comitator. peregrinator. clavator. 6). violator. 7). incubitor. migrator. profligator. defertor. denigrator. vubiginator. 8). fomentator. bospitator. infidiatur. capitator. 9). ornator. latrator. elongator. irrorator. venator. maculator. moderator. faltator.

bo annulatis.

4) I. fcutello e-guttato totus niger, antennis al-

5) I. fcutello e-guttato thoraceque maculato, totus ater, In Veftrogothia Bjerkander.

6) I. torus luteus, femoribus pofticis incrasfatis. E cap. b. fpei.

7) I. ater no tibiisque pofticis elongatis flavis. E cap. b. Spei.

8) I. Canguineus totus, alis nigris macula rubra in India orientali Hornftedt.

9) Habitat in Infula Sumatra, Thunberg. 


\section{) 87 (}

Icbneumon pugillator.

necator.

oculator. 10).

inculcator.

jaculator.

asferator.

prevogator.

- titillator.

delujor.

compunctor.

rutilator.

manifeliator.

extes jor.

mandator.

exarator.

refinelle.

turionella.

frobilelle.

glomeratus.

globatus.

coarifatus. I I)

luteus.

luteolus. 12).

glaucopterus.

ramidulus.

circumflexus.
Icbneumon cinctus.

bedeguaris.

ramicornis.

cynipidis.

lavvarum.

puparum.

gallarum?

apbidum.

ovulorum.

pedeftris.

agilis.

acarorum.

Cynips bascarum.

vamuli.

inferus.

pedunculi.

folii.

caprex.

rofe.

bicornis. 13).

Oefirus. bovis.

torandi.

nafalis.

biemorrboidalis.

ovis.

Tipuls pectinicornis. $\hat{\imath}$. ․․

Tipula

10) Cynips inanita. Linn. Syit. Natur. p. 920.

11) Juteus abdomine clavato: primo fegmento coarctato. Habitat in Suecia. Gyllenhahl, In capite bonæ fpei. Thunberg.

12) luteus abdomine cylindrico. Habitat in Sve. cia. O̊dman.

13) atra abdomine cyaneo, (cutello bicorni, 


\section{* ) 88 ( )}

Tipula rivora.

4-maculata.

crocata.

atrata.

variegata.

bortorum.

terreftris.

oleracea.

pratenfis.

contaminata.

lunata.

ocellaris.

Anvefcens.

2-maculata.

3-punctata.

6-punctata.

annulata.

fafciata.

relegationis.

plumipes. I4).

cornicina.

monoptera.

arundineti.

forilega.

nigra.

plumbea.
Tipula plunora.

vuficollis. 1.5).

tbome.

Fobannis.

femorata. 16).

bortulana, $\alpha . \hat{\delta} . q \beta$.

$\gamma$. marci.

d. pomona.

e. felrilis.

brevicornis.

pennicornis.

notata.

rufipes. 17).

culiciformis.

littoralis.

phalceroides.

bivta.

monilis.

parifienfis.

motitatrix.

8-punctata.

2-punctata.

Jericea.

fafciculata. vividula,

flexilis.

Stra-

14) T. cinereo-fufca pedibus omnibus pilofis. Habitat in Veftrogothia. Gyllenhahl.

15) Habirat in Japonia. Thunberg.

16) atra hirta alis nigris, pedibus pofticis elongatis compresfis, Hab. in Japonia.

17) Linn. Faun. Suec. N:0 1778. Fabric. mantisf. pag. 325. N:0 46. 


\section{9 ) $89($ 鹿}

Stratiomys cbameleon.

microleon.

bydroleon.

bypoleon.

trilineatus.

pantberinus.

uliginofus. 18).

tigrinus.

clavipes.

marginelius.

Syrpbus levcopus.

conopfoides.

icbneumoneus.

cinctus.

bicinetus.

arcuatus.

Scolopaceus.

tringarius.

Spbegeus.

fufcatus. 19).

tardus.

Bibio plebeja.

anilis.

bottentotta.

Jatyrus.

morio.

miaura. \&. $\beta$.

atbiops.
Bibio lugens. 20).

Mufca bombylans. mytacer. offraceas. intricaria. lappona. inanis. pellucens.

brunnea. 21\%. vulpina. meridiana. carnaria. lardaria. maculata. domeftica. cadaverina. mortuorum. vomitoria. cyanea. rajar. canima. ruffica. elata. furta. coleoptrata. . noctiluca. afliformis. femorato.

18) S. muticus Fabric.

19) Tipula Fufcata. Linn.

20) B. tota atra, abdomine bafi ferrugineo pellucido. Hab. in cap. b. Spei. 21) Syrphus inanis Fabric, 


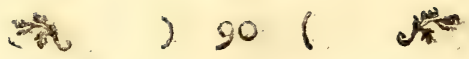

Mufca pipiens.

podagrica.

slongatis.

japonica. 1).

glisucia.

feftiva.

diopbibalina.

vejpiformis.

transfuga.

pendulia.

florea.

mutabilis.

arbuftorum.

ทยmor

tenax.

mentbofuro

mellina.

firipta.

rivefii.

pyyrafisit.

faltatrix.

thicorams.

giblos 3.2$)$.

rotuindata.

fera.

- Titeralis.

Segnis.

fyluarime.

fallax:

clivipes.
Mufca ruficornis.

Sepulcralis. viduata. frit.

putris.

pluvialis. feneftralis. ameditabunds. brasficaria. Gucularia. metallina. flavicornis. tremula. criftata. ruffifrons. marginata. combinata. cupraria. devia. polita. zingulata. voralis. coenziteriorum. pubera. jubsultans. grosfa. Jarvarum. radicums." fcybalaria. fimetaria.

I) M. antennis ferariis, nigra, abdomine clavato bafi-pellucido. Habitat in Japonia.

2) Hippobofca cincta. Thunb. act. Upl, nov. 


\section{- ) $95($ 㬐}

Mufar Aercoraria.

corrigiolata.

Mufca arrogans.

Ferrata.

tigrina.

ciluria.

canalicularis.

luvida.

gava.

petronella.

roftrata.

urtice.

folfutialis.

beraclei. 3).

Borefcentio.

cerafi. 4).

capenfis.

faltunis.

byofcyami.

parietina.

arnice.

cardui.

germinationis.

cynipfen.

vibrans.

virens. 5).

grosfificutionis. a. B.

feminationis.

umbellatarum.

Tabanus bovinus.

bromius.

autumnalis.

tarandinus.

ruficus.

stbiopicus. $\alpha . \beta .6$ ).

roftratus.

atratus?

pluvialis.

cacutiens.

lugubris.

Uarbatus. 7).

ferrugineus. 8).

calens.

ferveus.

$\mathrm{O}_{2}$

Tabanus

3) Tusfilaginis Fabric.

4) Frondefcentiæ Linn.

5) M. antennis fetariis virens, alis hyalinis apice nigris. Habitat in Svecia.

6) $\mathrm{T}$. pubefcens thorace nigro lateribus albis, abdomine ferrugineo, dorfo apiceque nigro. Hab. in capite bonæ fpei. Thunberg.

7) T. ore pectoreque barbato, abdomine nigro fegmentorum marginibus albis.

8) T. pubefcens corpore coftaque alarum ferrugineis. Habitat in Cajenna americæ. 


\section{$7) 92($ )}

Tebanus pellucens. bidentatus.

Culex pipiens.

bifurcatus. 오.

pulicaris.

reptans.

annulatus.

lutefcens.

Empis borcalis.

pennipes.

livida.

Atercorea.

flavipes.

moura.

cinevea.

Stomoxys calcitrans.

irritans.

Conops vificularis.

aculeata.

macrocepbala.

favipes.

ferriginea.
Conops buccata.

atra.

Bombylius major.

medius.

minor.

capen/15.

atropos. 9).

maurus. $\alpha$. $\beta$. 10).

variegatus. II).

Afilus rabroniformis.

giblofus.

gilvus.

flavus.

marginatus.

forcipatus.

germantcus.

tipuloides.

oelindicus.

cinctus.

leucopterus. 12).

crifatus. 13).

Hippo.

9) B. alis albis bafi atris, corpore hirto nigro albo-maculato. Habit. in Cap. b. Ipei.

10) B. alis nigris apice maculaque majori albis, cor pore ferrugineo nigro-maculato. Habit. cum priori.

II) $\mathbf{B}$. alis ferrugineis albo maculatis, corpore ferrugineo nigro-maculato Habit. cum prioribus.

12) A. cinereus thorace nigro-lineato, alis lacteis. Habit, in Cap. b. Spei.

Habit. cum priori.,

13) A. cinereus hirtus, thoracis crifta nigra pilofa. 


\section{$\Rightarrow$ ) 931 (}

Hippobolca equina. avicularia. bivundinis. ovina.

Lepifma Jaccharina. polypoda. terveftris.

Podura plumbea. arborea. fimetaris. aquatica.

Pediculus bumarus.

pubis. corvi. apis.

pica. anferis. tinnunculi.

Pulex irritans. penetrans.

Acarus ricinus. veduvius. crasfipes. mufcarum. coleoptratorum.

- rupeftris. geniculatus. bolojericeus. aquaticus.

Aranea avicularia. diadema. fenica. tavantula.
Phalangiunn opilio. reniforme.

Cancer minutus. pelagicus. $\alpha . \beta . \gamma$. vocans. menas. depurator. pagurus. araneus. longimanus. tuberculatus. dorfipes. Bernbardus. Diogenes. Eremits. Arctus. fuviatilis. bomarus. carcinus. norvegicus. gammearus. Squilla. onantis. foyllarus. pulex. Aagnalis.

Scorpio afer.

maurus. americanus europaus. cancroides.

Onifcus oceanicus. oeftruns.

$\mathrm{O}_{3}$ 
Onifcus entomon.

ayuaticus.

ceti.

afellus.

armadillo.

corallinus.

Monoculus polypbessus. Fulus pulex.

concbaceus.

apus.
Scolopendra morfitans. forficata. pbospborea. eleffrica. marina?

lagura.

complanatus.

terrefiris.

fabulofus.

indus.

भiser

To, 


\section{D. $D$. MUSEUM NATURALIUM ACADEMI $\mathbb{E}$ UPSALIENSIS.

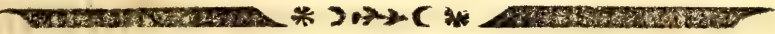 \\ CUJUS \\ Partem Octavam.}

CONSENSU EXP. FAC. MED. UPSAL。

PR FESIDE

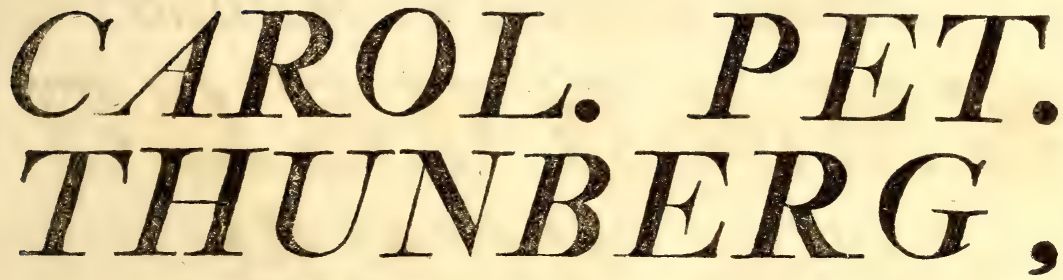

Equite Aurat. Reg. Ord. de Vasa,

Medic. Doct. Profess. Med, et Botan. Reg. et Ord., Acad. Cersar. Nat. Curios. Reg. Scient. Londinens. Holmiens. Societ. Scient. Upsal. Patr. Holm. Berol. Nat. Scrut. lundens. Harlem. Amsteld. Zeland. Nidrosiens. Halens, Nat. Scrut. Medie. Edimburg. et Nat. Studios. Ibid, Membro; nec non acad. Scient. Paris. Monspeliens. Agricult. Paris. Florentin. et Batayin. Ind. Or. Correspond.

\section{Publico examini fubjicit, CAROLUS ERICUS RADEMINE, Stockbolmienfis.}

IN AUDIT. GUST. MAJ. D. XIII. JUN. MDCCLXXXIX. H. A. M. S.

\section{U P S A L I E,}

LITT, DIRECT, JOHANN, EDMAN. 
SACRAM REGIAM MAJESTATEM SUMMIE FIDEI VIRO,

REGIS REGNIQUE SVIOGOTHICI SENATORI, ORDINIS DE STELLA POLARI EQUITI ET COMMENDATORI, ILLUSTRISSIMO EXCELLEN'TISSIMOQUE LIBERO BARONI AC DOMINO

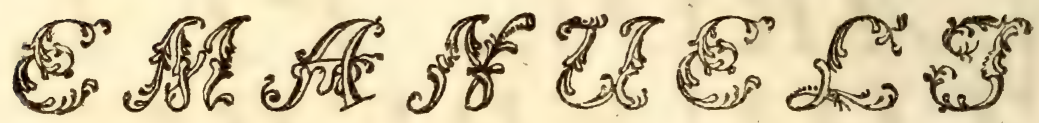

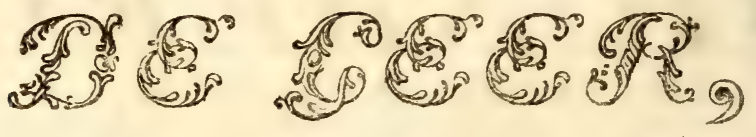

\section{MACENATI MAXIMO.}




\section{S:A R:无 M:TIS \\ $M A G N$ FIDEI VIRO, \\ IEGIE CANCELLARIAE CONSILIARIO,}

REGIXE ORDINIS DE STELLA BOREALI EQUITI AURATO,

GENEROSO ATQUE NOBILISSIMO

DOMINO

AND. SCHÓNBERG,

I. ACAD, SCIENT. ET SOCIET.PRO-PATR. MEMBRO,

SACRATUM VOLUIT, DEBUYT

GENEROSI ATQUE NOBILISSIMI NOMINIS

humillimus cultor CAROLUS ERICUS RADEMINE. 
$\mathrm{O}_{\mathrm{m}}$ Snillet mera vårdigt tyder

En kånfla i ett tackfamk bröft, Ån om Naturen hjertat lyder, Hur of âll! då med denna rỏit Jag ER blott detta offer 1kanker; Ett offer fom med viljan likt Skull bli fa evigt och fă rikt, Att Skald få hỏgt ej nånfin tånker!

Faft ofullkomlighetens drag Sig uti hvarje rad förråder, Dock hjertat till den fidfte dag Då ófver Mennfkan ódet råder Utaf den omma kårleks ho̊gd Fullkomligheten ftads fkall ro̊ja, Som gör att jag till Skaparn bo̊gd För EDRA dar en fuck fkall höja. 


\section{DONATION. THUNBERGIAN止 I $785^{\circ}$ CONTINUAT. VI.}

\section{VER MES.}

a. Inteftina.

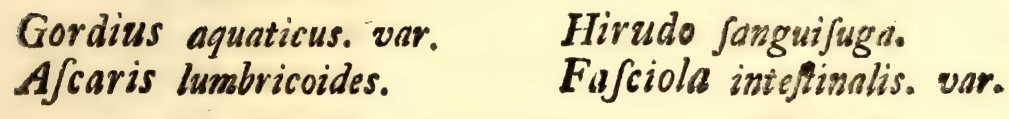

B. Mollugea.

Triton in Lepade anatifera.

r. Teftacea.

a. multivalvia.

Afterias rubens. levigata. opbiura. pectinata. multiradiata. minuta. medufe.

Echinus efculentus. rofaceus. $\alpha . \beta$. placenta. gratilla. I). faxatilis. cidaris. $\propto$. B. I). lacunofus. I). orbiculus, 2).
Ecbinus pomwm. 2).

Cbiton punctatus. aculeatus. fquamofus. cireneus. ruber.

Lepas anserifera. anatifera. balanus. balanoides. tintinabulum. co. $\beta$. tefudinaria. sitella. ecbinata.

I) Incompleti absque fpinis.

2) Petrefacti vel filicei. 
- ) 96 (

Pbolas dactylus.

crijpata. navalis. 3).

B. bivalvia.

Mya arenaria 4). crifpata. 4).

Tellina balibica. a. $\beta . \gamma . \delta$. truncata 4). albrda. a. 6) B. 4). cornea. a. $\beta$.

Unio *) margaritiferus. 5). pictorum. corrugatus.

Solen vagina. vuljella. 6).

filiqua. enfis. legumen. vadiatus. guineen/is. firigilatus. papyraceus.

Tellina virgata. $\alpha . \beta . \gamma . \delta$. planata. radiata, trifafciata. $\alpha_{0} \beta . \gamma$. carnaria. bimaculata. 6). donacina. balauftina. dedicatula. lingua. fragilis. lacuftris. divaricata. 6). roftrita. $\alpha$. $\beta$. incarnata. crifpa. lactea. digitarin. 6). inequivalvis. 6).

Cardium cardisfa.

medium 6). ecbinatum. aculeatum. ciliave. 6). ifocardia. 6). unedo.

Car:

3) Teredo navalis. Linn.

4) Fosfilis.

5) cum margaritis variis.

6) unica tantum valvula.

") Unio: tefta bivalvis, æquivalvis, æquilatera. Dens ani in valvula dextra folidus fubintrufus; in finiftra duplex : omnes orenulati. 


\section{म ) 97(}

Cardium murieatum. 6).

flavum. 6).

levigatum.

Serratum.

edule. $\alpha . \beta$.

tuberculatum. 6).

retufum. 6).

magnum. 6).

ruficum. 6).

pectinatum. 6).

fragum. 6).

bemicardium. 6).

Mactra glabrata. 6).

corallina. 6).

fultorum.

folida. 6).

violacea. 6).

plicataria. 6):

Donax trunculus.

Ariata.

cuneata.

fcripta: variet, plur. irus.

denticulata.

rugofa.

Venus dione.

papbia. 6).

dyjera.

verrucofa.

cafina. 6).

cancellata.

erycina.

iflandica.4).
Venus cbione. $\alpha . \beta$.

caftrenfis. a. $\beta$.

meroë.

deflorata. a. $\beta$.

fimbriata.

veticulata. 6).

tigerina.

proftrata.

exoleta. $\alpha . \beta . \gamma . \delta$.

pectinata. a. $\beta . \gamma . \delta . \varepsilon$.

foripta.

edentula. a. $\beta$.

decusfata, $\alpha, \beta, \gamma$.

borealis.

rotundata. 6).

virginea. $\alpha . \beta . \%$.

meretrix.

punctata.

geometrica.

leta. $\alpha . \beta .6$ ).

litterata.

gallina.

maculata. var. plur.

Spondylus gederopus.

plicatus. 6).

Cbama arcinella.

cor.

gigas. $\alpha . \beta$.

bippopus. $\alpha . \beta$.

antiquata. 6).

calyculata.

gryphoides. $\alpha ! \beta$.

trapetzia.

fatiata. 6). 


\section{- $) 98($}

Arca tortuosa. granosa.

nox.

barbatio.

lactea. ${ }^{*}$ ).

Antiquata. †).

undata. *).

glycymeris.

piloja. a. $\beta ., t)$.

rofirata. $\alpha . \beta . \dagger$ ).

numımaria. $\left.{ }^{*}\right)$.

fenilis. a. B. †).

inequivalvis. $\dagger$ ).

modiolus.

pella. a. B. †).

Oftrea maxima.

jacobar.

pleuronectes.

radula. *).

plica. a. $\beta$.

pallium. *).

nodofa. *).

pellucens. ").

fanguinea. *).

varia. 1). opercularis.
Oftrea Alvicans. ").

lima. a. B.

malleus.

folium.

edulis.

orbicularis.

fornicata. ").

fafciata. $\dagger$.

pufio. *).

ziczac *).

felina. *). 2).

borealis. $\left.\left.\left.\alpha^{*}\right) \beta^{*}\right) . *\right) \cdot \gamma \dagger$.

Mytilus crifta. "). modiolus.

margaritiferus. $\alpha . \beta$. barbatus *). edulis. a. B. $\gamma$.

cygnaus.

birundo.

anatinus.

difcors.

pboladis. (). 3).

diluvianus. $\left.{ }^{*}\right)$ †).

exuftus. ${ }^{*}$ ).

bilocularis.

Melina

") valvula unica.

†) fosfilis vel petrefacta.

I) Plurimæ varietates.

2) lingua felis:

3) cum nido calcareo.

4) Melina: telta bivalvis, æquivalvis, rudis; cardo edentulus fulcis quatuor transverfis in utraque tefta. 


\section{- ) $99($}

Pinna muricata.

Terebratula 5) pectinata. Y). pecien. t).

plicatella. f).

Iacunofa. $f$ ).

crispa. f).

terebratula. a. B. f).

truncata. $\alpha . \beta . \dagger$.

byjteropborus. $t)$.
Terebratula reticularis. †). novenzfriata. *). t) grypboides. $\dagger$.

Placenta 6) orbicularis.

Crania 7) brattenburgenfis. a.

Anomia 8) cepa. [*). B. †) electrica. epbippium. patellifor mis.

$\gamma$. uni-

5) Terebratula: Tefta bivalvis, inæquilatera, fubinæquivalvis, ad cardinem hians.

Cardo. Valvulæ inferioris margo pofticus ab utroque hiatus latere replicatus \& intra marginem dente fo. lido prominente inftructus.

6) Placenta: Tefta bivalvis, æquilatera fubinæquivalvis valvulis integris claufis.

Cardo edentulus, fed in una vel utraque valvula cal. li duo lineares, acuti, in angulum acutum convergentes.

7) Crania: Tefta bivalvis, fubinæquilatera, fubinæe. quivalvis, orbiculata, valvula inferior callis tribus intru. fis deciduis; val\%ula fuperior eminentis duabus fixis infra cardinis marginem.

Cardo edentulus.

8) Anomia: Tefta fubtrivalvis, inæquivalvis, inæquilatera. Valvula inferiore plana perforata: fuperiore con. vexa, tertia minuta pede animalis affixo.

Cardo. In valvula inferiore Callus linearis obliquus in margine lobi dextri. In valvula fuperiori Callus parum exfertus, ambitu fimilis intra marginem, utroque ligamento juncto. 
$\gamma$. univalvia.

Argonauta argo. \&. $\beta$.

Nautilus pompilius.

spirulto.

salcar.

beccarit.

sapbanus.

rupbanifos uns.

rugofus.

cripus.

ratatius.

Conas nusfitella.

marmoreus. a. $\beta_{0} \gamma_{0} \delta_{0}$ imperialis.

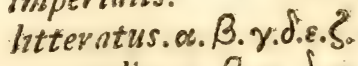

generalis. $\alpha_{0} \beta_{0} \gamma_{0} \delta_{0} \varepsilon_{\text {. }}$

canitaneus.

miles. a. B. $\gamma$ 。

omiralis fummus.

figulinus. $\alpha . \beta$.

ebraus. a. $\beta$.

mufcarum. $\alpha . \beta$.

varius $\alpha . \beta$.

firietus.

textilis. $\alpha_{0} \beta . \gamma_{0} \delta_{0} \varepsilon$.

geograpbus.

tuslipa.

granulutus. \& $\beta_{0}$

betulinus. $\alpha . \beta$.

clavus.

sulicus.

minimus. a. $B$.

zaercetor, $\alpha . \beta$.
Conus bullatus.

ruficus. gl ucus.

magus.

Spectrum.

virgo. $\alpha . \beta_{0}$

Cypran mappa

exantbems. $\alpha . \beta$.

arabica. $\alpha . \beta, \gamma$.

lititi.

errones.

ftolida.

avgus.

teftudinaria.

ftercoraria. $\alpha_{0} \beta_{0}$

carneola.

zebra.

lurida.

vanelli.

ferpentis.

mauritiana. $\propto . B_{0}$.

vitellus.

suus.

tigris. $\alpha . \beta$.

lynx.

ifruella. a. B.

birundo. a. B. .. .. E. S.

afellus.

moneta. $\alpha . \beta, \gamma \cdot \delta$

anmulus.

talpa.

classdefina.

cy. 


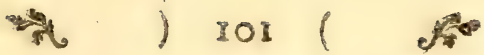

Cyprea ocellata.

ametbyltea.

fapbylea.

ziczac.

fragilis. $\alpha . \beta$.

flaveola.

caurica. $\alpha . \beta$.

erofa.

spuria.

belvola.

poraria.

pediculus, $\propto . \beta$.

nucleus.

globulus.

cribraria. a. $\beta$.

fuccincta. a. $\beta$.

cervus.

Bulla oevim.

verrucasa.

gibbofa.

naucum.

amprilla. \&. B.

ficus. a. B. $\gamma$.

fontinalis.

bypnoruns.

terebellum.

bydatis.

aperta.

ampluftre.

pbyjis.

cypraa.

Lignaria.

Spelta.

Voluta plicaria.
Voluta porplyrea.

oliva. variet. phur.

ifpidula. var. plur. monilis.

perficula. ce. B.

glabella. a. $\beta . \gamma$.

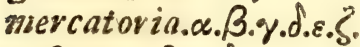

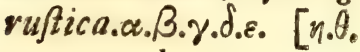

parpercula.

pertufa.

mitra: papalis. a. B. epifcopalis.o..B.

mufica. \&. B. $\gamma$.

vespertilio. $\alpha . \beta$.

capitellum. a. $\beta$.

turbinelliss.

ebrea.

jacabricula.

miliaria.

cymbium. of B. $\gamma$. olla.

mendicaria. $\%$. $\beta$.

cancellata.

coffea.

faba.

tornatilis.

cornicula.

fanguifuga.

pyrum.

craticulitta.

dactylus.

Buccinern glans.

olearium. a. $\beta$. $\gamma$.

perdix. 


\section{* ) 102()}

Busccinum doliums. ecbinopborum. rufum.

tefticulus. arcola. \&. B. $\gamma . \delta$ glaucum. $\alpha . \beta^{\beta}, \gamma_{\text {, }}$ viluex. papillofum. neriteum. $\alpha . \beta$. barpa. $\alpha . \beta . \gamma . \delta$ 。 patullum. lapillus. Spiratum. $\alpha_{0} \beta_{1} \gamma_{0} \delta_{0} e_{0}$ undatum. nitidulum. bevigatum. cornutum. vittatum. mancinella. decusfatums. galea. $\alpha . \beta$. maculatum. fubulatum. arcularia. pomum. $\alpha . \beta_{0}$ dimidiatum. Arigilatum. plicatum. glabratum. smutabile $\alpha . \beta$. pullus. \&. $\beta$. srenulatum $\alpha . \beta . \gamma .3)$.
Buccinum Tramaftoma. becticum. gibbofulum. atrum. flammeum, $\alpha . \beta$. Strombus pelecani. $\alpha . \beta$. vittatus. $\propto \beta_{\text {o }}$ urceus. fcorpius. lentiginofus. Diance. $\alpha . \beta$. Inbuanus. gibberulus. Zucifer. epidromis. a. . . millepeds. conarium. fuccinetus. lambis. $\alpha . \beta . \gamma$. gallus. $\alpha . \beta$. fufus. marginatus. pugilis. Jpincfus. 3). fisfurella. 3\}. cbiragra. onifcus. tuberculatus.

Murex fyracufanus. barfellusn. «. $\beta$. tribulus.

3) fosflilis. 


\section{角) 103(}

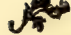

Murex brandaris. a. $\beta$.

trunculus.

vanuofus. Q. B. $\gamma \cdot \delta . \varepsilon$.

Murex lotorius.

fcorpio?

femoralis.

babylonius.

colus. a. B.

delpeEtus.

tritonis. $\alpha$. B.

tulipa. a. B.

corneus.

tropezium.

vertigus.

aluco. a. B. $\gamma_{0}$

evinacets.

anus.

pufio.

rubecula.

rapa (Bulla.

rana. $\propto$. B. $\gamma . \delta$

antiquus.

fufcatus.

nodus.

melongens.

morio.

cocbilidium.

frrobilator. $\alpha$. B.

reticularis. (Buccin.

ricinus.

Spirillus.

clatbratus, \&. $\beta$.

faxatilis.

limse.

by/tix.

perficus. (Buccin.

neritoideus.

gyrinus.

bezonr.

cbryfoftomus.

radula.

cornutus.

craticulatus.

capitellum.

pileare.

cingulatus.

perverfus.

lampas.

Trocbus miloticus.

maculatus. $\alpha . \beta_{0} \gamma$.

cingulatus.

perpectivus.

cinerarius.

magus. a. $\beta$.

veftiarius. $\alpha . \beta$.

labio.

conulus.

telefcopium, $\propto . \beta$.

punctatus.

zizypbinus.

modulus.

varius.

viridefcens.

pharaonis. a. A.

umbilicaris.

pagodus.

fangutinarius.

bybridus. 


\section{勿 ) 104 ()}

Trocbus cicercula. obtufatus.

Turbo littoreus. cimex.

pullus. a. $\beta$.

petbolatus. co. $\beta$.

pagodus.

calcar.

marmoratus.

argyroftomus.

delpbinus. a. $\beta$.

fcalaris.

clatbrus.

terebra a. 3. 3).

variegatus.

bidens.

muscorums.

neritordes.

fanguineus. a. $\beta$.

correus.

crenellus.

rugofus.

politus.

lacteus.

nautileus.

cbryfoftomus.

pica. w. $\alpha$.

uva.

replicatus.

acutangulus.

gemematus.

lincina.

engulinus.

reflexus. $\alpha . \beta$.
Turbo confervalis. cocblus.

Helix Spirorbis.

lapicida.

Jcavabeus.

complanata.

vortex.

cornen.

bijpita.

orbu/torum.

jantbina.

vivipara.

nemoralis.

fignalis. putris.

tentaculnta. auriculnvia. pomatia. perverfe: algiva. lacuftris. lucorums. albella. compressam bifpana. OETOR. baliotoidea. virginea. c. B. $\gamma$. fragilis. a. $\beta$. caracolla. parcellana. $\lim \cdot \mathrm{s}$. citrinio. a. 队ि. dolibrata. 


\section{* ) 105()}

Helix pupa.

achatina. a. B. $\gamma$. mide.

decollata.

Nerita canrena. a. B.y.d.

glaucina. $\alpha . \beta$.

рира.

albumen.

littoralis.

virginea.

polita. $\alpha . \beta_{0} \gamma_{0} \delta_{0}$

peloronta.

biftrio.

exuvia.

lisufivis.

mannmalla.

vitellus.

viridis.

bidens.

corona.

grosfa.

chameleoir.

plicatia.

Haliotis tuberculata.

afinino.

minuta.

friata.

Patella faccbarina.

granatina.

carulea.

mammilaris.

puftula.

nubecula.

fisfira.
Patella nimbosa.

porcellana.

fornicata.

compresja.

cornea.

militaris.

barbara.

ungarica.

gromularis.

cymbium.

perforata. $\alpha . \beta$.

graca.

unguis.

noucbina. a. B. 3).

lacinioja.

roftrata.

tefudinaria.

cbinenfis.

Derstalium elepbantinum.

dentalis.

Serpula spirorbis.

triquetra.

contortuplicata.

glomerata.

filograna.

lumbricalis.

exuvia.

angvina.

Sabella penicilas.

fcrupofa.

alveolata. 
d. Litbopbyta.

Madrepora pileus.

fungites. damicornis. fafigiata. turbinata. ramea. nuricata. oculata. porites. cespitofa. polygams. organum.
Madrepora favofa: Tubipora mufica. penis. Millepora cellulosa. polymorpba. coriacea. reticulata. aspera.

Cellepora pumicofi. ciliata. spongites.

e. Zopbyta.

Pennatula phojplorea.

Ifis bippuris.

Gorgonia placomus. verrucofa. ceratopbyta. antipatbes. verticillaris. elongata. abies. $\alpha . \beta$.

Alcyonium exos.

Spongia tubulofa.

Fluftra membranacea. foliacen. pilosa. trancata.

Corallina offacinalis. opuntia. Sertularia falcata.

Helwimbolithu. oribocerotes. androdamos. judaicus. Ecbinites. tubiporus. a. B. $\gamma$.

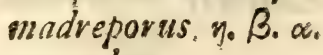
entrocbus. afteria columaris. car vopbyllites. belemnites.

Helmintbolitbus epitonium. alveatus.

Pbytolitbus litbopbyllum.

Phytolitbus filiis. Graptolitbus fcalaris. 


\section{THESES。}

I.

Teftis Conchyliorum \& Coralliis Calx fuam imprimis originem deber.

2.

Variis morbis tollendis infervit ufus calcis medicus, maxime recentis \& rite præparatæ.

$$
3 \cdot-
$$

Plura adhuc nobis ignota funt Conchylia, quorum effigia impresfa vel domicilia petrefacta in Mufeis Curioforum fervantur.

4.

Ut corpora Vermium plerorumque ftruftura fua a ceteris Animanibus maxime difcrepant, ita eorum pro. pagandi fe methodus valde fingularis.

5.

Neque in Oeconomia calcis, ex habitaculis Conchy liorum ortæ, utilitas exigua antimari oportet. 



\section{D. \\ MUSEUM NATURALIUM ACADEMI E UPSALIENSIS.}

CUJU'S

\section{PARTEM NONAM.}

CONSENSU EXP. FAC. MED. UPSAL.

\section{PR IESIDE}

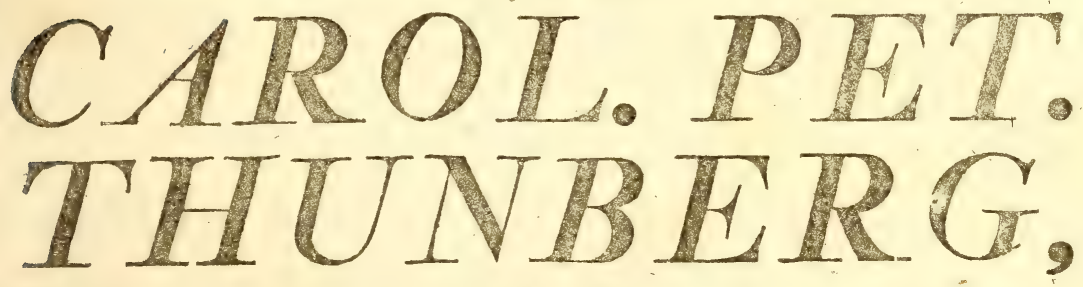

Fouite Aurat. Peg. Ord, de Vasa,

Medic. Doctr. Frofess. Mer. et Botan. Reg. et Ord., Acad. César. Nat. Curios, Reg. Scient, Londinens. Hormens. Societ, Scient. Upsal. Patr. Holm. Berol. Nat. Scret. Lundens, Harlem. Amasteld. Zeiand. Nidr osiens. Haiens, Nat. Scrut, Linn, Londin. Medic. Edimburg. et Nat. Studios. Ibid. Membro; nec non Acado Scient. Paris. Monspeliens. Agricult, Paris. Florentin. et Batavin, Ind, Or, Correspondo

PRO PILEO DOCTORALI

\section{ET}

ADSEQUENTIBUS PRIVILEGIIS.

Publico examini proponit

JOHANNES MARTINUS EKELUND,

Fjerdhundrensis,

Reg. Coll. Med. Membr. Part. Septent. Prov. Westrob. Med Ordo et ad Cohort. Reg. Ped. Westrob. Chilir. Primarius.

In Audit. Gust. MAJ. D. Xxx Martir. I 791. Horis ante et pofz mevid. folitis.

\section{UPSAL, I E,}

Apud Direet. Jomand Erman, Reg. Acal. Typogr. 


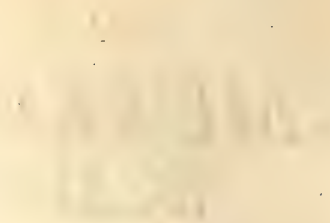

$\checkmark$

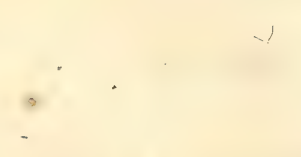




\section{- DEN HO์GVÁLBORNE HERREN}

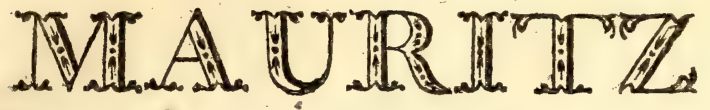

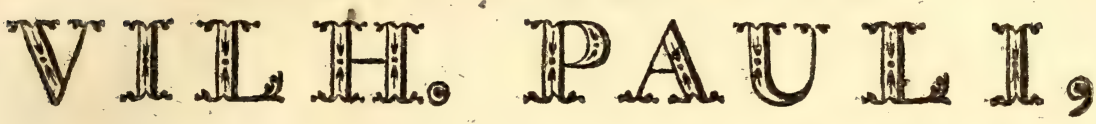

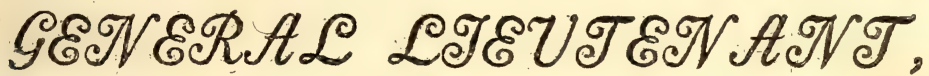

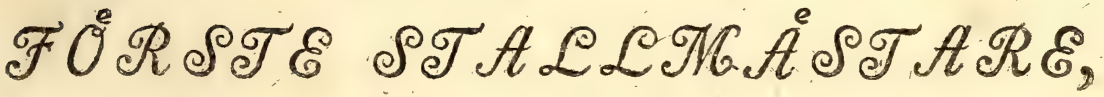

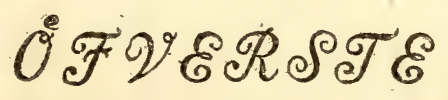

ZूóR KONGL.WÅSTERBOTTNS REGIMENTE,

\section{GOMGTEENDEV R}

AF KONGL. SVÅRDS-ORDEN,

OCH

RIDDARE AF KONGL, SVÅRDS-ORDEN MED STORA KORSET:

FOR

DTGD OCH TAPPERHET

AF

KONUNGEN ÄLSKAD,

AF

NATIONEN VÖRDAD,

AF

H五LTAR BEUNDRAD,

GENOM

HÖERTATS GODHET, VAंLGORRANDE MANNISKA. 



\section{Nådig.fe Herre!}

Pet âr af EDERT Namn jag vâgar gifva detta cirbetet lyffer: Det air af EDER Naid jag air lyckilig; att voorda EDRA befallemingar. 



\title{
DONATION. THUNBERGIANE.
}

\author{
CONTINUAT. VII. \\ unnowana \\ VEGET ABILIA
}

Monandria.

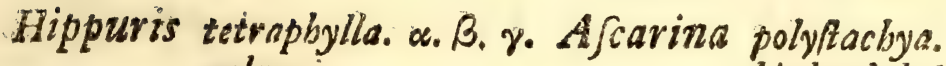

Salicornia berbacea. a. $\beta . \gamma$. Nipa fruticans. I-5.

fruticofa. a. $\beta . \gamma$. Thalia geniculate. a. $\beta$. virginica.

arabica.

eafpica. a. B. $\gamma$. foliata. $\alpha . \beta$.

cupresfina. procumbens.

auftralis. frobilina.

cruciata.

Cbara vulgaris.

flexilis.

bifpida.

tomentofa.

Cynomiorium coccineum. jamaicense.

Balanophora fungofa. Brofimum alicaftrum.

spurium.

Hippomane mancinella. biglandulofa. cannaformis, fol.

Globba nutans.

marantina.

japonica.

Amomum singiber. $\alpha$. $\beta$.

zerumbet. $\alpha . \beta . \gamma . d$.

compnetum.

cardimomum? $f$.

mioga.

Sylvefire.

curcuma. $\propto$ B.

Coftus arabicus. glabratus.

Maranta comofa.

arundinacea.

Kempferia galanga. rotunda. speciofa. erubefcers. fol. 


\section{$\rightarrow$ ) $134($ )}

Canna indica. a. B. $\gamma$ anguftifolia. glauca.

Alpinia racemosa. a. $\beta$. petiolaris. occidentalis.

Pbilydrum lanuginofum.

Cafuarina equifeta. $\propto_{0} \beta_{0} \gamma_{0}$. nodiflora.

Piftia fratiotes.

Najas marina.

Pbyllacbne uliginofa.

Dablia crinita. \$. ‥

Ceratocarpus arenarius.
Blitum capitatum. virgatum. cbenopodioides.

Mniarum biflorum. Laciftema myricoides. Corifpermunn bysopifolium. (a. 3. fquarrofum.

Callitricbe autumnalis. verna. tenella.

FEgopricon betulinum. a. B. Zannichellio paluftris. Radermacbia integra. of. incija. S. $\alpha . \beta . \gamma$.

Diandria.

Syringa perfica. a. $\beta_{\text {. }}$ vulgaris. $\alpha . \beta$. suspensa.

Cbionantbus compacta. virginica. ceilanica. incrasfatios.

Olea odorats. fragrans. excelfa. mesricana. apetale. europea. a. B. capenfis, $\alpha . \beta$.
Pbillyrea angufifolia, $\alpha . \beta$. media. latifolia. a. $\beta$. arborea.

Liguftrum vulgare. $\alpha . \beta_{0} \gamma_{0}$ Fasminum glaucum. japonicum. a. $\beta$. anguftatum. trifiorum. undulatums. birfutum. Jambac. a. B. $y_{0}$ fruticans. capenfe. azoricues: 


\section{* ) $135($ )}

Fasminum multiflorum. ceilanicum. didymum. bumile. odoratum officinale. grandiforwm.

Veronica falicina. floribunda. foutellata. capenfis. alpina. acinifolia. a. $\beta$. ferpyllifolia. $\%$. B. faxatilis. fruticulosa. $\alpha . \beta$. nummularifolia. peregrina. becrabunga. bellidioides. ciliata. 1). tetrapbylla fol. officinalis. $\alpha . \beta$. montana. bonarota. 2). agreftis: arvenfis. proftrata. romana. ancgallis.

Veronica incana. cataracte. Spicata. villofa.

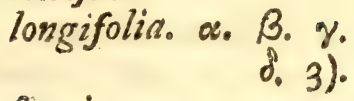

Spuria. virginica. a. $\beta$. Jabirica. chamadrys. a. B.\% latifolia. teucrium. $\alpha . \beta$. bederacea. $\alpha . \beta$. tripbylla. a. $\beta$. verna. $\alpha . \beta$. bybrida. auftriaca. $\alpha . \beta$. multifida. pinnata. $\alpha . \beta$.

Pinguicula vulgaris. alpina. villesa.

Utricularia acicularis. bifida。 cerulea. obtusa. inflexa. vulgaris. minor.

1) V. aphylla $L$.

2) Bonarota. L.

3) Veronica Marittima, variet, 


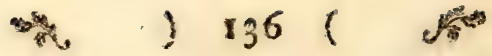

Litopbila muscoides.

Fraxinus americana.

$$
\text { ornus. } \alpha . \beta . \beta .
$$

Dialium indum.

Anciftrum fanguiforbs.

Circaa lutetiana.

$$
\text { alpira. \&. B. }
$$

Forftera Jedifolia.

opbrys apbylla.

monopbylla. $\alpha . \beta$.

loe felii.

paludosa.

dipbylla.

oliganthe.

clypeola. 4).

refupinata. 4)

lucida.

fpicata. $\alpha . \beta$.

ftacbyoides.

mu/cofa.

plantaginea.

umbellifera.

porrifolia.

alpina.

atrata.

nidus. $\alpha$. $\beta$.

bicolor.

inver $\int a_{0} \alpha, \beta$.

Opbrys volucris.

caffra.

ovata.

cordata.

tripbylla.

monorchis. $\propto . \beta . \gamma$.

alata.

lancea.

corallorbiza.

aracbnites.

myodes. $\alpha$. $\beta . \gamma$. antropopbosa. $\alpha . \beta$.

Spiralis.

torta. 5).

biplumata. 6).

Aretbufa capenfis. \&. B.

jecunda.

alaris, $\alpha . \beta$. villofa.

crifpa.

Serapias fquamata. aculeata. melaleuca. o. $\beta$. patens. a. $\beta$. regularis. cordigera. tabularis. polyftacbya. Alava fol.

4) Epidendr. Forft.

5) Satyrium fpirale Svartz.

6) Arethufa Lin. Bipinnula Commerfon. 


\section{$\Rightarrow \quad>137(\approx$}

Serapiss pedicellata.

falcata.

xipbopbylla.

enfifolia.

rubra.

erecta.

lonchopbylla.

longifolia. $\alpha . \beta . \gamma$

latifolia. $\alpha . \beta$.

alta.

tuberofa. flos.

Orclis abortiva.

monopbylla.

japonica.

nigra.

byperborea.

biflora.

fufcefcens.

fatyroides.

plantaginea.

repens.

picta.

adnata.

birtella.

cucullata.

Secunda.

viridis. $\alpha . \beta$.

albida.

monorbiza.

foliofa?

babenaris.
Orcbis bircina.

coriopbore.

odorata.

paluftris. :

conopsea. $\alpha . \beta$.

pyramidalis.

globoja.

maculata.

mafcula.

latifolia.

Sambucina, a. B. 7).

morio.

pallens.

triloba.

speciofa.

isfulata.

fufca.

militaris. $\alpha . \beta, \gamma, \delta$.

fancta.

bispida.

papilionacea.

ciliaris.

Sufanna.

radiata. 8). pectinata.

Satyrium grandiflorum. $\alpha, \beta$.

fecundum.

ferrugineum. rufefcens. $\alpha . \beta$.

excelfum. $\alpha . \beta . \gamma$.

bifidum.

7) Incarnata et mixta.

8) Orchis fufanna. Flor. Japonica. 


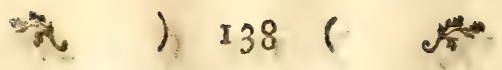

Satyrium fagittale.

draconis.

cernuum. $\alpha . \beta, \gamma, \delta$.

Epidendrum autumnale. tenellum. rigidum. fol. tuberofum. tortusa. epigogium. cylindvicum.

- cornutum. a. B. $\gamma$. flexuofum. Spatbulatum. a. $\beta$. barbatum. $\alpha . \beta$. bicorne. $\alpha . \beta . \gamma . \delta . \varepsilon$. orobancboides. $\alpha . \beta$. pumilum.

friatum. bicallofum. bracteratuns. a. $\beta$.

Epidendrum ferrulatum.

teretifolium.

teves fol. globofurn.

ranoofum. fol.

fruticulofum. a. $\beta$. teftafolium.

proliferum.

veftitum.

diffufum.

graminoides.

lineave.

vomiforme. montrnum. anceps. fol. jecundum. mmbellatum. nodofums. polybulbon. fragrans. cocbleatum. fesfile. fol. nutans. flabelliforme. variegatum. undulatum. triquetrum. vanilla. fol: fanguineum. furvam. labiatum. nervofum. 9). ophioglosfoides, I0). fiviatum. enfigolizum. gentinnoides. II). tricbocarpon. ecbinocarpon. glaucum. tribuloides.

9) Ophrys nervofa. Flor. Jap.

8) Arethufa Lin.

Ii) Limodorum Svartz. 


\section{2) 1391}

Epidendrum corniculatum. Linodorum fubulatum fol.

alpeftre.

laxum.

racemiflorum.

vufcifolium.

Limodorum funale.

filiforme.

fafciola.

pufillum.

utricularioides.

rugofum.

anabile.

ä̈reuns. 12).

iriffe. \&. B.

giganteum.

barbatum.

falcatum. 13).

longicorne.

enfatum.

bians.

speciofum.

latifolium.

coriaceum.

utriculatum. fol.

palmifolium.

lanceola.

jertularioides.

fatyrioides.

noniliforme.

crumenatum.

biflorum. crifpatum.

Cypripedium japonicum. bulbofum. calceolus. $\beta . \gamma . \delta$. $\alpha$. dipbyllus.

s. flavus.

2. ventricofus.

Dife maculata.

\%. grandiforus.

longicornis.

Lemna trifulca.

minor.

gibba.

polyrbiera.'

Cecropia peltata. I-5.

Salix bermapbrodita. triasdra. 8. pestandra. a. 占. 9 . B. 金. ㅇ. pbylicifolia $\hat{\delta}$. c. $\beta . \gamma . \delta$.

japonica. . q. $\alpha . \beta . \%$. capenfis. ?.

mucronata. + . vitellina. 9. amygdalina. Â. 9. bafiata. 占. a. $\beta$. q. $\alpha . \beta$. agyptiaca. of $\alpha . \beta$. $q$.

12) E. flos aëris.

13) Orchis falcata. Flor. Japon. 


\section{\# ) $140($ 塔}

Salix fragilis. 亦 $\alpha . \beta$. t. $\alpha, \beta$. babylonica. of. $\alpha . \beta$. purpurea. 唡. $\alpha$. $\beta$. belix. \&. myrfinites. of. 9 . arbufcula. S. $\$$. $\alpha$. 3 . berbacea. 余. retufa. 5 . reticulata. \$. myrtilloides. 8 . 9 . integra. glauca. 수오. aurita. 余. 9 . lanata. 3. . birfuta. $t$.

Salix alpina. of.. . pyrenaica. 杰. ․․ arenaria. S. क. a. B. incubacea. . 9 . flor. repens. S. 9 . fufca. $\hat{\text { in. }}$. rofmarinifolia. 1. a. B. Q.a.B. $\gamma$. viminalis. ‥ a. $\beta$. . . alba. $\hat{\delta}$. ㅇ. caprea. a. androgyna. B. $\hat{\delta}$. $q$. $\gamma$. cinerea. 命. fol. a. $\beta$.

Gunnera perpenja. a. fo.

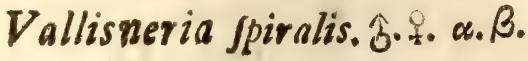

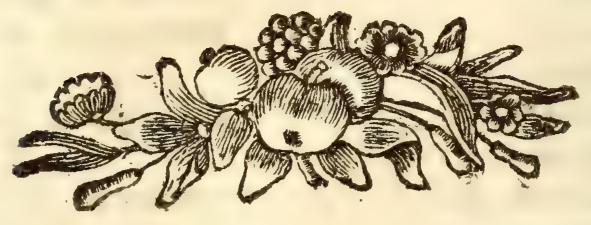




\section{THESES.

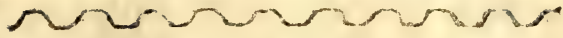 \\ Th. I.}

E Scrtaminers radices et femina imprimis in Pharmacopolia introducta fuerunt. Omnia hrec Aromata acria, calida et fragrantis ftimulant, calefaciunt et corroborant, æque ufui diatetico ac medico infervientia.

Th. II.

Orchidearum Bulbi carnofi, quorum princeps fine dubio eft, quæ ex Perfia adfertur, Radix Salep, mucilaginofi et nutrientes acria involvunt et langvidis vires reftituunt.

\section{Th, III}

Cinchon fpecies, quæ Americæ natales fuos de. bent, omnes vi gaudent fummopere febrifuga. Officinalis e montibus Peruvianis diu æeque ufitata ac laudata fuit. Anguftifolia ex infula Domingo et Cariban e Caribæis infulis allatæ fuas omnino merentur laudes, uti et Corymbofa ex infulis maris auftralis; Rulre autem, fic dictæ Cinchonæ, ignotæ arboris Americanæ cortex, ceteras omnes vi antecellit. Omnes refinofæ, amarisfimæ $\mathrm{Fe}$ bres intermittentes et remittentes feliciter tollunt et Gangrænæ fortiter refiftunt, neque in Tusfi ferina et Rachitide vi fua corroborante parum utiles.

$$
\text { Th. IV. }
$$

Oleum, ut Indiæ orientalis incolis dicitur, Culit $\mathbf{L a}$ van e variis corticibus et radicibus Laurorum, in primis Lauri Sas/afras deftillari folet. Oleum hocce brunneum, fubopacum, acre, calidum et aromaticum unctionibus variis externe infervit, etiam fedat dolores Athriticos. Odontalgiam tollit et Infecta nociva pellit.

Th. V.

Camphora, prreftantisfimum remedium, e variis plantis fuaveolentibus, ut docent experimenta 111. GAUBIr, elici 
elici poteft. Commanisime e Lauri cmmbare ligno et radice, in Japonia codtione folet cxirahi, poftea, ut præ. ftantior evadat, in Europa fublimanda. Nativam autem, quæ præltantisfima et longe nobilior, ignota adhuc arbor Borneenfis exfudat, in Noftris Pharmacopoliis huc usque certe rarisfima. Optime omnium relolvir, Spasmos fedat et tranfpirationem auget, a Practicis frequentisfime prafcripta.

\section{Th. VI.}

Cajoputi, malaico nomine nobis notum, fuaveolens oleum ut Camphora fuida non incongrue confiderari poteft. Tenuisfimum et omnino avolans hocce e foliis $\mathrm{Me}$ lalencæ leucadendra foliis oleum deftillatum odore gaudet Therebinthinæ cum Camphora mixtæ, Infectis et Vermibus maxime inimicum, unctionibus præcipue externis infervit, Inflammationes eximie refolvit et intonfas dentium atque arthriticos dolores fæpe momento citius fedat tollitque.

\section{Th. VII.}

Remedia, quæ leviter alvum ducunt, Eccoproticn dici folent, quæ fortiter agunt, Purgantia vocantur, et quæ vehementius adhuc ftimulant, Dreftica audiunt. Hæc præterea vel dulcia funt, vel acida vel amara vel etiam acria.

\section{Th. VIII.}

Sudorem promovent et augent eadem remedia, qux urinam pellunt, pro diverfa conditione ægri.

\section{Th. IX.}

Sic quoque Emerica fæpe alvum fimul laxant, et Laxantia quandoque vomitum excitare deprehenduntur. Th. X.

Pro diverfa fua qualitate, Laxantia alia funt, qua ventriculum et inteftina corroborant atque alvum fere obftipant, alia quæ debilitant et fluxum nimis protractum excirare valent.

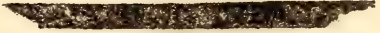


D. $D$.

MUSEUM NATURALIUM

ACADEMIE UPSALIENSIS.

* $\sin (\cos )$

cujus

Partem Decimam.

CONSENSU EXP. FAC. MED. UPSAL.

PR $\mathbb{E S I D E}$
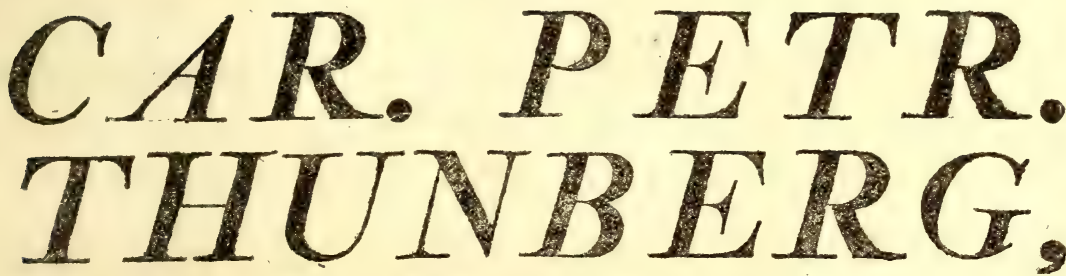

Equite Aurat. Reg. Okd. de Vasa,

Medic. Doct. Profess. Med, et Botan, Reg. et Orin, Acaz. Cresar. Nat. Curios, Reg. Scient. Londinens. Hoimiens. Societ. Scient, Upsal. Priladelpe. Patr. Holm. Berol, Nat. Scrut. Lundens. Harlem. Amsterd. Zeland. Nidrosiens. Halens. Nat. Scrut. Linm. Londin. Medic. Edima burg.et Nat. Studios. Irid.Membro;necnon.Acad. Scient. Paris.Monsperiens. Agricult, Pakis. Medic. Londin. Florentin. et Batarin. Ind. Or. Correspond.

PUBLIBO EXAMINI SUBJICIT,

HARALDUS KUGELBERG, SMotandus.

In Audit. Gust. Maj. B. xxmi Nov. MDcexci. H. A. M. S.

\section{UPS A L I E,}

LITTERIS VIDUE DIRECT. JOH. EDMAM. 


\section{THESES.}

I.

Plantarum vires Botanicus perfpicit, Chemicus examinat, Medicus prudenter experitur.

II.

Vix inter gramina planta venenata reperitur;

III.

Semina enim nuda Graminum, imprimis Cerealium, maxime falubria funt \& nurrientia.

\section{IV.}

Oeconomo non parum utilis erit cognitio graminum, quibus prata fæpe cum fiuftu conferuntur \& emendantur.

V.

Diætam vegetabilem carnex in non paucis præferendam merito cenfemus.

$$
\text { VI. }
$$

Neque in foro medico fua carere utilitate reputand funt genera quædam graminum. 


\section{DONATION. THUNBERGIANÆ}

\section{CONTINUAT. VIII. \\ nonnomon \\ Triandria.}

OSyris alba. 앙ㅇㅇ japonica.

Maba elliptica. Boerbavia fericea.

birfuta. chinenfis. erecta. diffufa. virgata. fcandens. tetrandra. ciliata.

Valeriana celtica.

locufa. 1. 2.

rulisa. 1. 2.

fupina.

cormucopice. faxatilis. \&. elongata. montana. tripteris. villogia. ecbinata. pyrenaica. phu. fibirica. tuberosa. calcitrapa $\alpha . \beta . \gamma$.
Valeriana dioicn.

officinalis. capen/is. bexandra.

Witfenia maurn. Antbolyza ringens. plicata. cunonia. lucidor. atbiopica. nervofin.

Gladiolus montanus.

flexuefis. recurvus. falcatus.

biflowes. dicotbonous. crifpus. triftis $-1-17$. communis. a. $\beta$. wat fonius. merianellus. merianus. Spicatus. alnpecuroides. a. B. alatus. $\alpha . \beta_{.} \gamma . \delta_{0}$ bicolor. $\alpha . \beta$. anceps. $\alpha$. B. $\gamma$. 


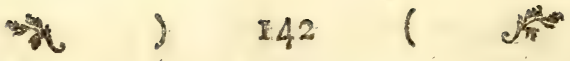

Gladiolus junceus.

Galaxia graminea. $\alpha_{0} \beta$.

fetifolints. ovata. $\alpha . \beta$. marginatus. a. $\beta, \gamma$. Dilatris corymbofa. nugufus. $\alpha . \beta_{0} \varepsilon_{0} \gamma_{0} \delta_{0}$ vifcosa. longiflorus, a. 5. $\gamma$. Moraa melaleuca. tubiflorus. plicatus. I = II. jpatbaceus. gramineus.

spiralis. africaias. $\alpha, \beta$. pufilla. bermudiana. $\alpha . \beta . \gamma . \delta_{0}$

Ixia fraticosa. as. $\beta$. ixioides, minuta. \&. $\beta$. latifolia. bulbocodium. $1-8$. bumilis, $\alpha, \beta, \gamma$. pilofa. bivits.

fectndn. a. B. crilpa. a. B.

cinnamonisea. corymbosa. $\alpha . \beta$.

linearis. capillaris, a. B. $\gamma$. fetater. a. $\beta_{1}, \gamma_{0} \delta$. fcillaris. a. B. arifitata. $\alpha . \beta . \gamma$. bulbifera. a. B, . d. s. pendula. erefia. I - 5 . maculata. I-9. crocata. 1-4. lances. pentandra. $\alpha . \beta$. falcata. $\alpha . \beta . \gamma . \delta$. excifa. gladiata. apbylla. filiformis. Spatbacel. Aexioja. $\alpha . \beta . y_{0} \delta$. collina. I- 4 . polyantbos. $\alpha$. $\beta$. coerulea. a. $\beta$. zimbellata. crifpa. ividioides. cbinenfis. undulata.

Iris ciliata. minita. pumila. fufiane. florentina? biflora. a. $\beta$. variegata. germanica. jambucina. 
Irisi fqualens.

compresja.

tripetala. a. B. $\gamma$.

tricuspis. II-s.

plusiaria.

gnelini.

xipbiuar.

pjeud-acorus.

fectida.

virginica.

verficolor.

ocbroleuca.

Spatbacea.

samoja.

fryvinclium.

perfica.

ongufta, a. B.

fetacea.

tenuifolia.

graminea. $\alpha . \beta . \gamma$.

Spuria.

fibirica I - 4 .

pavonia.

crifpa. a. $\beta$.

papilionacea $\alpha . \beta_{0} \gamma$.

edulis. a. $\beta . \gamma$.

triftis. $\alpha . \beta$.

polyfacbya. .

vifcaria.

bituminofa.

Wachendorfia tbyrfifora.
Wacbendorfia tenella. graminifolia.

Hippocratea volubilis, a. B. connofa. ceilanica. elliptica.

Comocladia integrifolia.a. $\beta . \gamma$. Leefingia bispanica. Tamarindus indica. Hervandi ovigera. Sonora. $\alpha . \beta$.

Rumpbia amboinen/s?

Cneorum tricoccon. a. $\beta$.

Xyris capenfis.

Pboenix dactylifera. 1-8.

Ficus nymphoides. foll. religiofa. $\alpha . \beta, \gamma$. aspera. bengalenfis. cordata.

fipulata fol. falcata. fol. punctata. pumila. erecka. trigona. pertufa. nitida. benjamina. reflexa. 


\section{- I ) 144}

Ficus racemofa. drupacea. indica. a. $\beta$. obliqua. granatum. fol. prolixa. tinctoria. fol. reticulata. $\alpha . \beta$. finuata. bifpida. a. $\beta$. beteropbylla. carica. a. B. $\gamma . \delta . \varepsilon$. toxica.

Stilago bunius. $\alpha . \beta$.

Tragia mercurialis. a. $\beta$. cbamalea. urens. votubilis. fcardens. capenfis. villofa. cufpidata. involuciata. a. $\beta$. connabina.

Typha latifolia. anguftifolia. minor.

Sparganizbm erectum. $\alpha . \beta$. natans.

Ompbalea caulifora. nucifera. cordata. fol. axillaris.

Polycbnemum arvenfe. $\alpha . \beta$. monandrum. falfoloides.

Agyneja pubera.

Wildenowirs compresfa. $\alpha . \beta$. teres. ?.

Ariata. 9 .

Elegia juncea. 3. a. B. $\gamma$. Q. $\alpha . \beta$.

Refio imbricatus.

vaginatus. aviftutus. of. q. cernuus. $\hat{\jmath}$. umbellatus. $\hat{\delta}$. spicigerus. 尔赵. teEorum. $\alpha$. $\beta$. acuminatus. $\hat{\text { s. }}$ parviforus. $\hat{j}$. erectus. $\hat{\delta}$. argenteus. $\mathrm{A}$. jabiofus. $\delta$. $\alpha . \beta$. thomnocbortus. $\hat{\alpha}$ a. $\beta$. fruticofus. fimplex. triflerus. tetragonus. $\hat{\text { E. }}$. triticeus. है. q. glomeratus. incurvatus. digitatus. verticilaris. ‥ $\alpha$. $\beta$. $\int \operatorname{cop} a$. 
Refio virgatus. t. paniculatus. 1 . dicbotomas. ‥ $\alpha . \beta, \gamma$.

Scboenus lanceus. lavis. Scleria Latifolia. flagelluns, birtella. filiformis.

Scboenus aculeatus. $\alpha . \beta$. pufillus. filiformis, a. $\beta$. frintus. capitellum. foariofus. nigricans. littoreus. fpicatus. trifsacbyos: mucronatus. ferrugineus. fufcus. cuspidatus. arifiatus. compar. flexuofus. capillaceus.

Carex dioica. of. ㅇ. tbermalis. glomeratus. $\alpha . \beta$. umbellatus. tricbotomius. corymbofus. $\alpha . \beta_{0} \gamma_{0} \delta_{0}$ cyperoides. capitata. $\alpha, \beta$. pulicaris. leucoglocbin. uncinati. a. $\beta$. cyperoides. cepbalotes. baldenfis. capenfis. a. B. $\gamma_{0}$ avenaria. glomerata. sbordorróiza. leporina. $\alpha . \beta$. vulpina. $\alpha . \beta$. brizoides. chavata. muricata. ufulatus. bulbofus. inanis. marifcus. a. B. capillaris. debilis. gracilis.

albus. Aellatus.

remota $\alpha . \beta . \gamma . \delta$. loliacea. $\alpha_{0} \beta$. elongata. crnefcens. praniculata. a. $\beta$. brunnea, microftackya. beleonaftes. profirata. 


\section{$-x$}

)

Carex fava. a. $\beta$. depauperatr. digitata. montana. tomentofa. globularis. filiformis. triftacbya. faxatilis. atrata.

limofa. copillaris: pallefcens. panicea. folliculata. peudocyperus. capitoja. diftans. joponica. agaftacbys. a. B. $\gamma_{0}$ leptoftacbys. birta. pilulifera. pumila. veficaria. $\alpha_{0} \beta, \gamma$. acuta.

lafocaspa. $\alpha . \beta, \gamma$. drymeia. incurva.

Scirpus acicularis.

capitatus. $\alpha$. F. beotbryon.

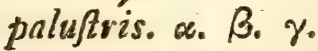
cespitofus.
I46

Scirpus fuitans. a. $\beta$. pilofus. monoftacbyos. junceus. fetaceus, $\alpha . \beta_{0}$ mutatus. geniculatus. plantaginoides. articulatus. tricufpis. byftrix. $\alpha . \beta$. vaginatus. ciliaris. $\alpha . \beta$. laciniatus. membraysaceus. radiatus. mucrobatus: $\alpha, \beta$. marginatus. triftacbyos. truncatus. bottentoterts. farigiatus. argenieus. fupinus. Squarrofus. monander. antareticus. \%. B. borbatus. intricatus. romanus. $\alpha . \beta$. navittimus. \&. $\beta$. bolojcbanus. lacuftris. a. $\beta . \gamma_{0} \delta$. autumnalis. 


\section{被) 147 ( 燶}

Scirpus piluliferus.

hustila. $\alpha . \beta$.

ferrugineus, $\alpha . \beta$.

ditbotomus. $\alpha$. $\beta$.

fpadiceus.

miliaceus, o. B. $\gamma$.

jylvaticus. a. $\beta_{0}$

Cyperns articulatus.

prolifer.

monti.

textilis. a. $\beta$.

spatbaceas.

tenellus.

minimus.

monoftacbyos. $\alpha . \beta$. lavisatus.

squarrofus.

flavejcens.

fufcus.

pannonicus.

filiformis.

polyfacbyos. o. F. $\gamma$.

pumilus. a. $\beta$.

triflorus.

compresfus. a. B. $\gamma_{0}$

bexaftacbyos.

tenuis.

frigofus.

brunneus. $\propto$. $\beta$.

confertus.

glomeratus. $\alpha, \beta$.

ligularis, $\alpha . \beta$.

ivia.

bajpan. $\alpha . \beta$.
Cyperus odoratus?

difformis. $\alpha . \beta$. vifcolus. $\alpha$. $\beta$.

pulcber. $\alpha . \beta$.

gliber.

rotundus. $\alpha . \beta$.

lanceus.

longus. d. B. $\gamma$.

efculentus.

procerus. a. $\beta$.

elatus. $\alpha . \beta$.

diftans.

elegans, $\alpha . \beta \gamma$.

papyrus. a. $\beta$.

alternifolius. $\alpha_{0} \beta_{0}$

Eviophorum alpinum.

vaginatum. $\alpha . \beta$.

polyfachyum. a. $\beta_{0}$

cyperinum. $\alpha . \beta$.

Fuirena paniculata.

Nardus thomea.

Aricta.

arifato.

Lygeum spartum, $\alpha . \beta$.

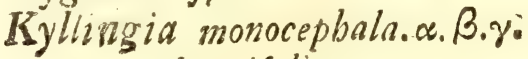
brevifolia.

triceps.

filiformis.

panicea. $\alpha . \beta . \gamma$.

umbellata. $\alpha . \beta, \gamma, \delta$.

Pommereulla cornucopice.

Zea mays. $\alpha . \beta . \gamma$. 


\section{武 ) 148 ( )}

Olyra paniculata. $\propto . \beta_{\text {. }}$. pauciflora.

Coix lacryma. a. $\beta$. gigantea.

Cornucopice cucullatum. Panicum plicatum.

friatums.

ciliatun.

turritume:

cylindricum.

colonum. $\alpha$. $\beta$.

brizoides.

corvi, $\alpha, \beta$.

galli. $\alpha . \beta, \gamma$.

birtellum.

compofitum. $\alpha . \beta$.

pilofum. a. $\beta$.

grosfarium. $\alpha . \beta$.

laxums.

cespitofum.

fangvinale. $\alpha, \beta . \gamma . \delta$.

lineare?

filiforme. $\alpha . \beta . \delta . \varepsilon . \xi . \eta$.

daitylon. $\alpha . \beta \gamma$.

oryzoides. a. $\beta$.

deufums.

truncatum.

miliaceum.

pubefcens. a. $\beta$.

micrantbum. a. $\beta$.

caudatum. $\alpha . \beta . \gamma$.

semorofum.

brevifoliuns.
Panicum patens, $\alpha . \beta$.

molle.

flavejcens.

fufcum.

fafciculatum.

latifolium, $\alpha . \beta . \gamma_{0}$ glutinofum.

tricboides. $\alpha . \beta$.

diffufum.

pallens.

ransofum. $\alpha . \beta$.

coloratum.

capillare.

bifpidum.

ferratum. $\alpha . \beta$.

arundinaceum.

polygamum.

virgatum.

multifhorum.

rigens.

ramofum. $\alpha . \beta$.

dicbotonums.

arborefcens.

divaricatum. \& $\beta$. y. cartbaginenfe.

acurainatum.

lanatum. $\alpha . \beta_{\text {. }}$

Ariftida americana.

adfcenfonis. $\alpha . \beta_{0} \gamma$.

veftita.

tripilis.

by/trix.

plumofa. a. $\beta . \gamma$.

lanata. 


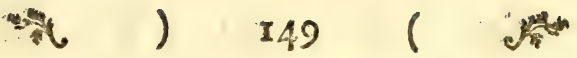

Antboxantbum odoratum. a. Paspalum conjugatums.

\section{arinitum.}

Alopecurus pratenfis.

agrefiss, o. $\beta$.

geniculatus.

bulbofus.

cupenists.

echinatus. \&. $\beta$.

puniceus. ") a. $\beta . \gamma . \delta$.

$$
\varepsilon . \xi . \eta \text {. }
$$

Phlewign protenle.

rodofum. $\alpha . \beta$.

alpinum. a. $\beta$.

avenavium.

Manifuris myurus.

Phalaris phleoides.

afpera. $\alpha . \beta$.

dentata.

nquatica. $\alpha, \beta, \gamma$.

bulbofa.

utriculata.

minor.

capen/is.

canarien/is.'

paradoxa. o. $\beta$.

arundinacea, $\alpha, a . b, \beta$.

bifpida. erucaformis.

Paspalum filiforme. $\beta$.

diffufum.

vaginatury.

disfectum.

glomeratum.

decumbens.

forobiculatum. $\alpha_{0} \beta_{0} . \gamma_{0}$

villofunt.

virgatum.

verticillatum.

paniculatums.

Milium lendigeruns.

villofumb.

globósun.

punctatum.

cimicinum. $\alpha . \beta_{0}$

effufum, $\alpha . \beta$.

digitacum.

paniceum.

compresfum.

Agroftis minima.

Spicata.

virginica:

alba.

indica.

tenax.

mexicana. $\alpha . \beta$.

capillaris.

Aolonifera, $\propto, \beta, y$.

$\alpha . \gamma \cdot \beta$.

purpurascens.

\section{$\mathrm{X} 2$}

Agro=

*) Alop. monfpelienfis idem, 


\section{吼) $150(1)$}

Agroftis cornucopice. $\propto . \beta$. tenuifolia. $\alpha . \beta$. cinna. ciliata. ovata. bromoides. a. B. ferotina. interrupta. canina. arundinacea. rubra. miliacsa. a. $\beta$. venti. a. $\beta$. avenacea.

Spicata. arifella. pernatio.

- juncea. copillata. \&. B. $\gamma_{0}$ tenax. $\alpha$. $\beta$. capenfis. argueis.

Ligurus ovatus.

Andropogon caricofun. contortum. crinitun. diffacbyon. virginicum. birtum. $\alpha . \beta$. barbatunz. a. $\beta$. ifcbemum. «. B. villofum. bicorne. a, $\beta$.
Andropogon brevifolium.

ifcbomoides. cymbarium. foftigiatum. faccbaratum. gryllus. nutans. ciliatum. $\alpha . \beta$. fsboenantbus. a. $\beta . \gamma_{0}$ $\delta . \varepsilon . \xi_{0} \eta_{*}$ ferratum. cotuliferum. $\alpha . \beta$.

Holcus Spicatus. $\alpha . \beta$. avenaceus.

fericeus. ferratus. afier. odoratus. mollis. lanntus. o. B. capillanis. caifrorum. bicolor. balepeniss. forgbum. faccbaratus.

Saccbarum Spicatum. a. $\beta$. falciculatum.

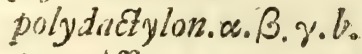
teneriffe. fpontaneum. raverna. officinarum. $a . b . c$. Leer- 

新
)
IS I
(
蓇

Leerfia monandra.

bexandra.

oryzoides. $\alpha . \beta$.

Aira minuta.

aquatica. $\alpha . \beta$.

carulea.

spicata.

alpina.

canefcens. $\alpha$. B. $\gamma$. pracox.

carycpbyllia. a. $\beta$. montana.

cespitofa a. B. flexuofia. $\alpha, \beta$.

capillaris.

ontaicica.

Melica minutar.

uniffora.

nutons.

pyramidalis.

altisfima.

racemofa.

dectmbens.

capenfis.

geniculata. a. $\beta$.

ramofa.

gigantea.

cilicita. a. B.

Egilops ovata.

triancialis.

Ifchawum muticum.

murinum.

ariflutums.

Ifchernum involutum.

Apluda zeupizis.

mutica.

tripolitana.

paradoxa.

arilata.

Tripfacum difacbyon.

bermapproditicum.

dactyluides. a. B.

Uniola paniculaźa.

Spicata. o. B.

bipeninato.

Briza capen/is.

geniculata.

fisiata.

minor.

wivens.

media. $\alpha_{0} \beta, \gamma_{0}$

maxima. a. $\beta$.

Poi Jpinofa,

jubulata.

Spicata.

crifata. a. B. $\gamma$.

farmentofa. o. B. $\gamma . \delta$.

glomerata. a. $\beta$.

cyperiodes.

racenofa.

rigiala. \&. B.

alpina. a. $\beta$.

bulbofa.

arenaria. $\infty . \beta$.

filiformis. $\alpha, \beta, \gamma$. 


\section{$\Rightarrow$}

)

Poa abysfanica.

flava.

glutinofa.

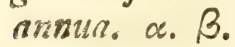

compresfa.

pratenfis.

nnpufifolia.

iverialis.

paluatris.

aquatica. $\alpha . \beta$.

ferruginea.

barbata.

birta. a. B.

virginica.

latifolia.

capillaris.

prolifera.

multiflora.

ansabilis.

pilofa?

joponica.

diftans?

mallabarica.

cbinerifis.

tenella.

eragrofits. $\propto$. $\beta . \gamma$.

Fefuca bromoides. $\alpha . \beta . \gamma$. ovina. $\alpha$. $\beta$.

rubra.

ametbyfina.

duriufcula.

vigidiufcula. dumetorum. elatior.
Fefucuca inyurus.

phenicioides.

pauciflora.

mifera.

fluitans.

calyciza.

criflata.

varia.

marittima.

Jylutica.

finnats.

cambrica.

lolincea.

fibivica.

Dactylis cynofuroides.

cilianis.

lagopoides.

bifpida.

villosia.

glomerdta. $\alpha_{0} \beta_{0} \gamma_{*}$ ferrata.

levis.

Bromus criftatus.

rigens.

fcopavius.

vamisus.

Squarrofus.

japonicus.

bifidus.

fecalinus, $\alpha . \beta$.

arvenfis.

mollis.

nanus.

littoreus. 


\section{$\rightarrow \quad 15311$}

Bromus tectorum.

multiflorus.

ciliatus.

pectinatus.

evectus.

fevilis. \&. B. $\gamma_{\text {. }}$

afper.

giganteus.

inermis.

racemofus.

rubens.

madritenfis.

purgans.

diftacbyos. $\alpha . \beta$.

pinnatus. $\alpha . \beta . \gamma . \delta$.

Avenn fragilis.

bromoides.

alpina.

forgbums.

antaretica.

ariftidoides.

trifeta.

loeflingiana?

purpurea.

liapulina.

bijpida.

elepbantis. I. 2.

pallida. a. a. 6. B.

filiformis.

flivirica.

elatior.

flave fcens.
Avena Selquitertio.

pubefoens.

nuda.

fativa.

fatua.

Aterilis.

frigafa.

orientalis.

pratenfis.

Arundo bambos. I-7.

avenaria.

epigejos. a. B.

calamagrofitis.

donax. I-2.

pbragmizes. co. $\beta, \gamma . \delta$.

confpicua.

thenax.

Antbiftirin ciliata.

Rottboellia repens. fol.

dimidiata. $\alpha . \beta, \gamma . \delta$.

incurvata.

articuliata.

exaltata. 1).

coeloracbis.

egipbiloides.

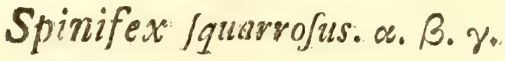

Secale reptens.

oriertale.

creticum.

villofunt.

cereale. $\alpha_{.} \beta . \gamma . \delta . \varepsilon . \xi . \%$

i) Rottb. procera. Swartz, 


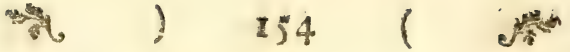

Triticum unilaterale.

tenellum.

inioloides.

mavittimun.

jwnceum.

repens. $\alpha . \beta$.

diftucbum.

Spelta.

monucocerns.

turgidun.

bybermum.

a?ivum.

polonicum.

compofitum. a. B.

Hordeum nodofum.

jubatum.

pratense.

copen/e.

muเทีนm.

bulbofun.

difficbon.

seocriton.

bexaftichon.

vulgare. 2. 3. 4 .

Elymus arenarius.

europaus.

tener.

caninus.

pbiladelpbicus.

ranaden/2s.

virginicus.

fibiricus.

reflexus.

Elymus bystix.

Lolium teme.

\section{medufe.}

perenne. a. $\beta$.

temulentum. $\alpha, \beta$.

difintyon.

coelorichis.

Chloris petren.

cruclita.

cilinta. poly?acbya.

radiata.

Cynofurus uninle.

falcatus. crifutus. a. B. difachios.

carulcus, a. B.

lima.

paniculatus.

durus.

cegyptius. a. $\beta, \gamma, \delta$. coracanits. a. $\beta$.

indicus, $\propto, \beta, \gamma . \delta . \varepsilon$.

virgatus. a. B. $\gamma$. $\delta$. domingenfis. $\alpha . \beta$. ecliniatus.

aureus.

flexifolius.

Cencbrus capitatus. alopecuroides, $\alpha, \beta$. Spicatus. $\propto \beta$ diftucbus. $\alpha$. B. $\%$. citiaris, $\alpha . \beta$. 


\section{* ) $155($ )}

Cenclorus geniculatus. 1.) boydeiformis. 2.)

tourpurafiens. a. B. 3.) viridis. a. $\beta \gamma . \delta$. $\varepsilon$. gliacus. $\alpha . \beta . \gamma$. Setof: $t s$. verticillatus. 4.) a. $\beta$. y. a. b. c. $\delta$.

E. a. b. 1, b. 2, c.

$\xi$ a. b. भ.

fiamentufus.

ecbin tus.

tribuloides. $\alpha . \beta$. racemofus. $\propto . \beta . \gamma$. Picramnia antidefma. \& $q$. Qveri a bippanica.
Axyris cerataides.
canadem is

byibrida.

amarantboides.

Koenigia iflandica. Holofteum dinndrum. cordatum. umbellatum. $\alpha . \beta$ magellanicum. 5).

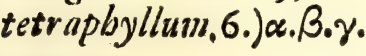

Lec bea minor. major. Eriocaulon triangulare.

I) Panicum hordeiforme. œ. Flor. Japon.

2) Panicum hordeiforme. B. Flor. Japon.

3) Panicum hordeiforme. $\gamma$. $\delta$. Flor. Japon.

4) Huc pertinent Panicum italicum, germanicum, fio biricum, marittimum, melfrugum Auctorum.

5) Donatia.

6) Polycarpon. 


\section{औ ) 156}

Pbyllanthus verrucofus.

urinaria. $\alpha . \beta$.

bacciferus. $\alpha, \beta, \gamma$

maderafpatanus.

neтrorum.

smblica. a. B.

rrflexus.

fcandens.

nigrefcens. $\alpha, \beta$. conami. $\alpha . \beta, \nu . \delta$. verticillatus.,

grandifolius.

glaucus.

ramifforus.

laurifolius, $\alpha \cdot N \cdot \gamma, \cdots$

\section{Tetrandria.}

Protea decumbers.

florida.

cyanoides.

patula. a. B.

Spharocepbala.

ferravia. $\alpha . \beta . \gamma_{0}$

triternata.

glomerata.

pbylicoides.

lagopus.

Spicata.

fceptrunt.

crinite.

conocarpa. $\alpha . \beta$.

elliptica.

bypopbylla. a. $\beta$.

cucullata. $\alpha . \beta$.

tomentofa. $\alpha . \beta$.

beteropbylla.

pinifolia.

racemesa.

incurvata.

caudata.

bractersta.
Protea comofa. purpurea. prolifera. corymbofa. nana.

lanats.

torta.

alba.

aulacea.

umbellata,

linearis.

foolymus.

mellifera.

repens.

proftrata.

obliyua.

parviflora.

pallens. a. B. .. d.s.

conifera. a. $\beta$.

levifanus. a. $\beta$.

Atrobilina.

imbricats.

Sericea.

Saligna. 


\section{\#) $157($ )}

Protea argentea. $\alpha$. . $\beta$. acaulis. $\alpha . \beta$. myrtifolia. grandiflora. glabra. speciosa. a. B. $\gamma$. totta. birta. pubera. $\alpha . \beta . \gamma_{0}$ divaricata. Spatbulata. cynaroides. cordata.

Bank/ia Jerrata. fol. Ammnannia sangvinolenta. octandra. latifolia.

Spicata. baccifera. ramojior.

Fagara euodia.

trifolia. emarginata. tragodes. a. $\beta$. fol. pterota. coperafis. $\alpha . \beta$. piperita. armata. fol. borrida. Spiuofa.

Curtifan faginea.
Otber a japonica.

Orix a japonica. Samara coriacea. Ptelea trifoliata. pinnata.

Scbrebera Scbinoides. I). Aucuba japonica. a. $\beta$. Serpicula repens. 令. cos 6. T. verticillata. $\hat{\delta}$.

Embotbrium coccineum. umbellatum.

Epimedium alpinum. Monetia barlerioides. Skimtuia japonica. Potbos fcandens. violacea. monopbylle. I. 2:

Trapa natans. \&. $\beta . \gamma$. Cornus fvecisa. $\alpha$. $\beta$. cavadenfis. florida. alternifolia. $\alpha . \beta$.. . marcula. alba. Sericea, $\alpha$. B. -janguinea. japonica.

Montinia acris. $\mathrm{f}$. 오. Ludwigia alternifolia. $\mathrm{Y} 2$ 
$x$

Ludwigia repens.

oppofitifolia.

Allionia incarnata.

Knautia orientalis.

propontica.

Scabiofa bumilis.

trifida.

fonliris:

sinerea.

fucsifa.

Syriace.

rigida.

integrifolia. $\alpha . \beta$.

amplexicaulis.

fyluatica.

tuturica.

anven/ss.

alpina. $\alpha . \beta \gamma$.

decurvens.

uf tulata.

transfyluanica:

leucantba.

cretica. $\alpha \beta, \gamma$.

graminifolia. a. $\beta . \gamma$. prolifera. $\alpha . \beta$.

pillafina. $\alpha . \beta$.

ncrulis.

africana. $\alpha . \beta$.

fiellita, o. $\beta_{0}, \gamma_{0} \delta . \varepsilon_{0} \xi_{0}$

ficula. \&. $\beta$.

divaricata?

mavittima. a. $\beta$.

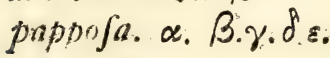
atropurpures.
158

Scabioja argentea.

ucranica. $\propto$. B.

gramuntia. $\alpha$. $\beta$.

columbaria. a $\beta$. $\gamma$. d.

ifetenfis. $\alpha$. $\beta$.

ocbroleuca. \&. B.

Stilbe pinaftra.

evicoides. $\alpha . \beta$.

cernua:

Dipfacus fulbonum.

fitinfus.

laciniatus.

Alypum longifolium.

Globularia nudicaulis.

cordifolia. $\alpha . \beta$.

vulgaris. ๙. $\beta . \gamma$.

alypum.

bifnagarica.

Cephalanthus occident lis. Hydropbylax marittima. $P$ avetta indica. a. B. $\gamma$. d. caffra.

Wallenia laurifolia. a. $\beta$.

Catesbea parvifora.

Callicarpa americana. $\alpha . \beta$.

ferruginea.

tomentofa. $\alpha_{0}, \beta$.

japonica.

Aggipbila elata.

foetida.

Petefia carnea.

racemofa.

I. 
Plantago incurvata.

Ixora alba.

coccinea. $\alpha . \beta$.

Ernodea littoralis.

Hoffmannia pedunculata.

Cocco/ipfilum repens.

Hedyotis polygonoides.

peploides. r).

rupeftris.

birta.

auvicularia.

verticillata.

fruticofa.

Oldenlandia capenfss.

temuifolia. $\alpha . \beta$.

firicta.

foetida.

bifora.

triflora, a. $\beta$.

longifforas

repens.

lineavifolia.

verticillata.

umbellata. a. B. $\gamma_{\text {. }}$

paniculata.

birfuta.

Scoparia arborea. a $\beta . \gamma$. dulcis. $\alpha . \beta$.

Polypremum procumbens. Plantago lacufiris.

fubulata.

alpina.

bellardi.!

dubia.

Y 3

pjyllium.

pilofa.
polymorpba:
loeflingii.

marittima. $\alpha . \beta, \gamma \cdot \delta . \varepsilon$.

cretica.

cylindrica.

albicans. $\propto . \beta$.

Jagopus.

birfuta. $\alpha . \beta$.

capenfis.

lianceolata. $\alpha . \beta . \gamma$.

media.

major: $\alpha . \beta . \gamma_{0}$

afintica.

maxima.

lufitanica.

spinofa.

macrorbiza.

virginica.

ferraria.

cornuti.!

coronopus. $\propto . \beta . \gamma$.

gquarroja.

pumila.

cynops.

indica.

afra:

Budleja incomta. virgata. $\alpha . \beta$. globoja. occidentalis: americana. San-

r) Peplis terandra Linn. 
Sangviforba officinalis.

nedia.

canaderfis, $\alpha \cdot \beta$.

Manettia lygiftum.

Labatia fesfilifhra.

Pence a myrtilloides.

fruticulofa. $\alpha, \beta_{0}$

mucronata.

squamofa.

marginata.

fucata.

lateriflora.

tonnentofa.

Houfronia carulea.

Rubia galioides.

petiolaris.

tinctorum.

cordifolia.

Galiumn rubioides.

palufire. $\alpha . \beta$.

trifidum.

montanum.

tinctorium.

aliginofum.

spurium.

jaxatile.

pyrenaicum.

minutum.

pufillum.

smucronatum.

expanfum.

asperum.

glabrum.
Galium capense.

verum. $\alpha . \beta . \gamma . \delta$. mollago.

fylvaticum.

ariftatum.

bierofolymitamu

glaucum.

purpureum.

rubrim.

fulpbureum.

megalospermun.

spinulojum.

rotundifolium.

boreale.

marittimun.

aparine.

parifienfe.

Valantia bypocarpa.

cruciata.

muralis.

glabra.

cucullata.

articulata.

aparine.

hispida.

Asperul. levigata. \&. B. $\gamma$. odorata.

pentandra. arvenfis. \&. $\beta$.

tauvina.

tintoria.

cynancbica.

Sherardia muralis. 


\section{औ) $16 \mathrm{r}(1)$}

Sberardia arvenfis. a. B. Salvadora perfica.

fruticofa.

Spermacoce firita.

Lauropbyllus capenfis.

tenuior.

verticillata.

bivta.

bifpida.

villofa.

orymoides.

Knoxia ceilanica.

Diodia profirata.

fimplex.

foandens.

famentofa.

Nigrina Spicata.

Alcbemilla valgaris. $\propto . \beta . \gamma$.

capenzis. a. B.

alpina.

monandra.

apbanes.

pentapbylla.

Campborofma monpelienfis.

glabra?

pterantbus.

Nepenthes deftillatoria.

Cometes furattenfis.

Crucianella marittima. a. $\beta$.

mappia.

agyptiaca.

monspeliaca.

procumbens.

anguftifolia.

latifolia.

patula.

Scabrita fcabra. a. B.

Cavanilla Jandens. ․ 오.

Hippopbrë vbamnoides. 令. 9 .

Tropbis americana.s.9.\%. aspera.

Rivina lavis.

buanilis.

octandra.

$B$ at is marittima. 食.

Vifcum capense.

japonicunn. I).

opuntioides. $\alpha, \beta, \gamma_{0}$

rotundifolium.

panciflorun.

obfcurum.

rubrum.

album.

verticillatum. a. $\beta$.

rotundifolium. 2).

antarcticunt.

flavers.

obliquum.

racemofun.

Dorftenia cordata.

Dor-

I) Vifcum opuntioides. Flor. Japon.

2) Cadaba. Forn. 


\section{औ}

)

Dorftenia lucida.

pubefcens.

contrajerva.

Parietaria capenfis.

urticafolio.

officinalis.

judaica.

micropbylla.

lufitanica.

debilis.

Boebmeria saudata.

cylindrica.

littoralis.

triplinervis.

bivta. .

romiflora.

Jpicata. a. 3. 3).

Urtica membranacea.

depres 5 .

diffufa.

elata.

fesfilifora.

rufa.

divaricata.

urens, $\alpha$. $\beta$.

dioica.J.Q. Q. B.\%.

laxa. $\alpha . \beta$.

repens.

Aolonifera.

betulefolia.

rugofa.

ferrulata.
162

Urtica nummularifolia. ciliata. berniarisides. triantbemoides. micropbylla. lucida. reticulata. nudicaulis.

radicans. cuneifolia. pilulifera. grandifolia. $\alpha . \beta$. cannabina. balearica. macropbylla. dodartii alienats. niven.

frutefcens. villoja. interrupta. ruderalis. cenaden/s.

japonica. lappulacea. aftuans. a. $\beta$. Atimilans. capen/is. a affra.

Isnardia palufiris. Elargnes anguftifolia. umbellate. 
* ) $163(1)$

Elcagnus multifora.

$$
\text { cripa. }
$$

pungens.

macropbylla.

glabra.

Brabejum fellatum.

Gonatocarpus micrantbus.

Hypecoum penduluns.

erectum.

procumbens.

Buffonia tenuifolia.

Cufiuta americana.

europar.

epitbymum.

Honnamelis virginica. $\alpha$. $\beta_{\text {. }}$ Scbafferia completa. $\mathfrak{1}$. 옹. latevifora. ․․․․․

Antbospermum galopina. 1). lancenlatum.

fcabrumb.

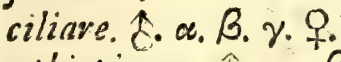
atbiopicum. $\alpha . \alpha, \beta$.

$$
\gamma \cdot \text { 早. }
$$

Morus tinctoria. $\alpha . \beta$.

nigra.

alba.

rubra.

tatarica. $\alpha . \beta$.

indica. $\alpha . \beta$.

papyrifera. $\alpha . \beta, \gamma$.
Betula pumila.

nigra.

excelfa.

pyrifolia.

antaretica.

japonica.

alba. $\alpha . \beta . \gamma . \delta$.

lenta.

alnus.

incana.

tripbylla.

laciniata. fol. $\alpha . \beta$.

bybrida, $\alpha . \beta$.

\section{Argytbarnnia candicans.}

Cranzia levigata.

$B u x u s$ virens. $\alpha . \beta . \gamma . \delta$.

Myrica gale. $\$$.. .

cerifera. 念. ㅇ.

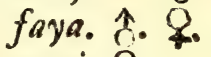

nagi. + .

pimenta. S. 9 .

cordifolia. I. ?.

atbiopica. Jै. $\alpha . \beta$.

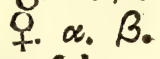

quercifolia. fol.

alperifolin. flor. 1 .

Ilex crocea. fol.

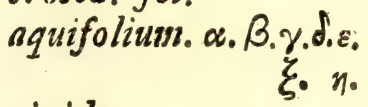

rigida.

japonica.

latifolia.

Betula nans. $\alpha$. $\beta$.

I) Galopina circæoides. Nov. Gener. Plantar. 


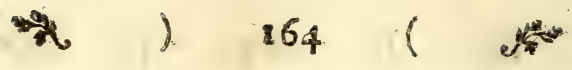

I t $x$ prinoides.

rotunda.

integra. $\alpha_{0} \beta$.

crenata.

casfine.

ferrata.

emarginato. afiatica.

Coldenia procumbens. Sagina evecta. a. B. procumbens. apetala.

Myginda latifoliz. pallens.
Myginda rbacoma.

Potamogeton compressum. lucens. ferratum. fol. matans, $\alpha . \beta$. crifpusn. pectinatum. gramineum. smarinuss. pufillum. denfum.

Ruppia marittissa. «. $\beta$. Pomax umbellata. 


\section{D.}

\section{MUSEUM NATURALIUM ACADEMIA UPSALIENSIS.}

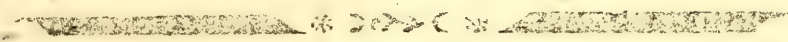

$$
\text { PART. XI. }
$$

CONS. EYP. FACULT. MED. UPS.

PRRSIDE
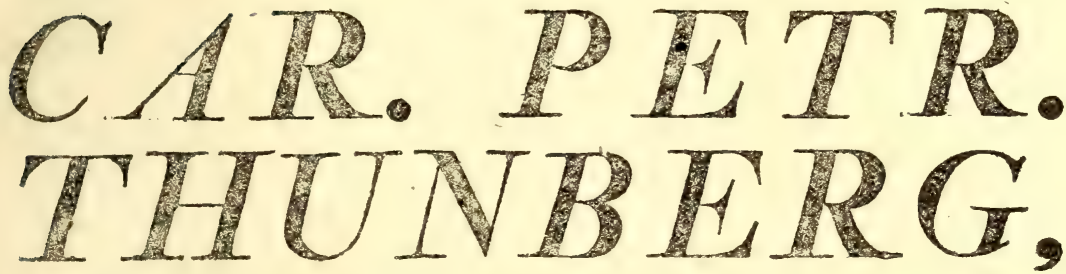

Foute Aurat. Reg. Orbo de Vasas

Mfenc. Doct. Frofess. Minde Botan. Reg. et Ord., Acab. Cesar. Nat, Curios. Reg. Scient. Londinens. Holmiens. Societ, Sciant. Upsat. Puit adgleg. Patr, Horm. Beror. Nat. Scrut. Lundens. Harlem. Amstely. Zitand. Nidrosiens. Haiens. Nat. Scrut. Linn. Londin. Medic. Edim. yurg.et Nat.Stumios Tbid. Mearera; nec non acad Scient, Paris.Monspelizns. Agricult, Paris. Medic. Londin. Florentin. et Batavin, Ina.

OR. CORRESPOND.

PUBLICO EXAMINI SUBMITTIT ๑OHANWIS PETRUS S\%ÓBERG. STOCKHOLMIRNSIS.

In Audit. Botanic, D. Thunir m d C X Xil. H. A, M. S.

UPS A L I Æ,

Litreris Vidua Direct. Jok, Edman 
TROTJENARE, SAMT STALLMÁSTARE

VID

UPSALA ACADEMIE,

$V$ ALBORNE HERR

C.

Tillagnas 'desfe blad, fafom prof af den agtning, hvarmed jag har åran fórblifva

\section{VÅLBORNE HERR STALLMÅSTARENS}

O̊dmjukafte Tjenare JOH. PET, SJO̊BERG. 


\section{DONATION. THUNBERGIAN.}

\section{CONTINUAT. IX. \\ Pertandria.}

Mirabilis longifora.

$$
\begin{aligned}
& \text { jalappa } \\
& \text { dicbotoma. }
\end{aligned}
$$

Weigela japonica.

Plumbago europara. a. $\beta$. $\gamma$.

cillanica.

rojes.

capenfis.

fcandens.

Cerintbe major. $\alpha . \beta$. minor. $\alpha . \beta$

Mesferfibinidia argusia.c. $\beta$. fruticofit. \&. $\beta$.

Echium coudotum.

fricutum. altisfinnum. levigatun. glabrum.

incanum.

tricbotomunt.

bifpidum. paniculatum

trigonum. candicans.

capis atum.

creticum.

italicum.

Infitanicum.

violaceum.
Ecbium plantagineum. vulgare. $\alpha . \beta . \gamma$. Arictum. fruticofum. argenteum.

mordax.

Heliotropitim curasfavicum.凶. B. $\gamma$.

fruticofum.

gnapbaloides.

lineatum.

jupinum. a. B. $\gamma$.

europicum.

indicam. $\alpha$. $\beta$.

parviflorum.

peruvianum.

Pulmonaria maritima. anguftifolia. officinalis. \&. B. $\gamma$. fibivica. $\alpha . \beta$. virginica. $\alpha . \beta$.

Litbospermum officinale. $\alpha . \beta$. arvense. $\alpha . \beta . \gamma$. purpureocaruleum. fruticofum. dispernum. bifiidum. ciliatum. 
Litbofpermum foabrum.

papillofum.

Onofina fimplex.

ecbioides.

n orientalis.

Sympbytum officinale a.r.2.ß.

tuberofuns.

Borago officinalis.

indica. of $\beta$.

orientaits.

africana. \&. B.

Lycopfis pulla.

veficaria.

variegata.

arvonisis. a. B. $\gamma$.

augufitif:lia.

orientalis. a. B.

Ajperugo procumbens.

divaricata.

Cynoglos sum officinale.

virens.

virginicums.

cbeirifolium.

apenninum.

lavigatumi. a. $\beta$.

lufitanicum.

linifolium. $\alpha . \beta$.

ompbalodes.

japonicum.

jaoanicum.

ceilanicum.

bifpidum.

birfutum.
Cynoglosfum ecbinatum. muricatum.

Ancbufa officinalis, o. ß. $\gamma . \delta . \varepsilon$. capenfis.

euguftifolia. italica. a. B.

undulata. $\alpha . \beta$. tinctoria. a. $\beta$.

lanatn.

sempervivens.

Myofoitis fcorpioides. a B. . . d. lapputa. lquarrofa. apula. fruticofa. rupeftris. alpina. pirenaica. Spatbulata. virginica, a $\beta, \gamma$.

Nolana profirata.

Scbeffjeldia repens.

Pennarstia corymboja.

Pifonia fubcordata. inermis. coccinea. aculenta. o. F.a.ß. $\gamma$. grandis fol. umbellata.

Coris monspelienfis. a. $\beta$. Hydropbyllum canadenfe. virginicund. $\alpha . \beta_{\text {. }}$ 


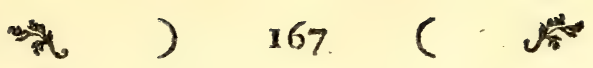

Cortuses gmelini. matbioli.

Anagallis tenella. linifolia. nionelli. a. B. centunculus. punita. arvenfis. carulea. latifolia.

Ly/imacbia Aricta. vulgavis. \&. $\beta_{0}$

Epbemerum. atropurparea. fulicifolia. tbyrfiflora.

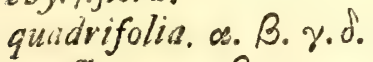
punetata. $\alpha . \beta . \gamma$. Rellate. nevonfa. nemorum. $\alpha . \beta$.

Nummularia. japoriza.

Cyclamen europaum. a. $\beta$. Dodecatbeon Meadia. Soldanella alpina. Primula cortufoides. cuneifolia. integirifolia. verticillata. veris $\alpha$. $\beta$. farinoja. auricula. a. $\beta$.
Primula minima. Androface villofa. carnea. a. B. lacter. Septentrionalis. elongata. maxima.

Aretia vitaliana. alpina. belvetica.

Hottonia paluftris. indica. Menyanthes capenfis. indica. nyrspbides. trifoliata.

Dorena japonica. Allarnanda catbartica.

Spigelia antbelmia. marilandica.

Ophiorbiza mungos. mitreola. umbellata.

Retzia capenfis. Lifiantbus arboreus. cordifolius. latifolius. umbellatus. chelonoides. ex/ertus.

Datura arborea. framonizm. 
Datura ferox. tistislia. metel.

faftuofa. inermis.

Hyofcyamus niger. a. $\beta$. aureus, $\alpha . \beta$.

albas.

pufillus.

pbyfaloides.

muticus.

fcopolia. a. B.

Nicotiana ruffica.

glutinofa.

tabacum.

fruticosa.

paniculata.

Verbafcum pblomoides.

lycbnitis.

nigrum.

blattaria.

pbaniceum.

Barbavii.

Ofbeckii.

myconi.

tbapsus.

finuatum.

jpinofum.

Convolvulus spinofus. a. G.

cneorunt.

argenteus.

cantabricus.

dorycniwm.
Convolvulus fcoparius.

cenotberoides.

foridus.

lineatus. a. $\beta$.

meflatanus, $\alpha, \beta$.

perficus.

tricolor. a. $\beta$.

ficulus.

bivtus.

foldanella.

Falckia.

brafilienfis.

capree.

Sagittatus.

baftatus.

edulis. a. B. fol. batatas. fol.

verticillatus.

jericeus.

crenatus.

trinervis.

aureus.

glomeratus.

canarienfis.

umbellatus. a. $\beta$.

turpetbum. fol.

peltatus.

grandifiorus.

geneellus.

nervo/us.

biflorus.

farinofus. cordifolius. 


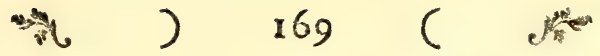

Convolvulus fibericus.

fommonia.

ayvenfis.

Sipizm.

mediun.

purpureus.

bederaceus.

carolinus.

panduratus.

trilobus.

mil. o. B. $\gamma$.

puellaris.

multifidus.

angularis.

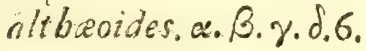

disjectus.

quinguefolius.

pentapbyllus.

Azalea glauca.

vifcofa.

mudiflora.

indica.

rojisarinifolia.

procumbens.

lapponica.

Epacris rofmarinifolia. fol.

pumila.

longifolia.

juniperisn.

fajcicularis.

acerofa.

Ayenia levigata.

pufilla.
Ayenia Sitinofo fol.

Carrsfa corandas. a. 3 .

Spinarmen.

facquinia armillavis.

verzofis.

linearis.

Laugeria tomentofa.

Cbironia tetragona.

mudicanlis.

jafminoides.

linoides.

lycbroides.

baccifern.

frutefcens.

trinervis. 1. 2.

angularis.

Diapenfía pyrenaica.

lapponica.

Pblox filberica. $\alpha . \beta$.

fubulata.

pilofa.

carolina.

glabra.

paniculata.

maculata.

divaricaía.

Polennonium caruleum. $\alpha$. $\beta$.

repians.

roëlloides.

campanuloides.

Ipomeen fimplex.

lescantba. 

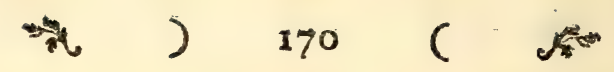

Ipomeea coccinea.

lucunofa.

folaniflora.

noctis.

paniculata.

tamifolia.

glaucifolia.

triloba.

bepaticafolia?

trigridis.

tuberofe. fol.

Varronia mirabiloides.

cturasfavica.

lineata.

glabofia. a. B.

martinicenfs.

Ardifia ferrulata. tinifolia. lateriflora. parajitica.

Cordia gerafcantbus.

callococca.

tetrasdra.

polygama.

calopbylla. fol.

niyxa.

micrantbus.

Sebeftena.

viburnoides.

Carpodetus ferratus.

Myrfine africana.

Bladhia japonica.
Bladbia crifpa. villisa.

Proderiafetida. a. B. Raumolfia nitida. a. B. canejcens.

Cerbera thevetia. mangbas. a. B. glabra: abovaj.

parviflora.

Gynopogon alysin. for.

fellatwn. forndens.

Arduina bifpinofa. a. $\beta$. Ebretia tinifolia. virgata.

bourveria.

Tournefortia bicolor.

cyszofa.

fetida.

birfuta. a. $\beta$.

volubilis. a. $\beta, \gamma$. argentca.

Bumelia retusa.

nigra. I. 2.

montann.

falicifolia. a. B. $\gamma_{\text {e }}$ rotuundifolia. pallida.

Cbry Sopbyllum cainito. glabrum? monopyrenum. microcarpons. 
Cbryfopbylluns rugofum. mbaucaco. fol.

Sideroxylon lycioides. fol. decandrum. inerme. a. B. spirofum. folo nigricans. argenieum. stelanopbletum. 1.2 .3$. cymofum.

Cefrrums montanun. jamsicense. rugofus. nocturnum.

vefpertinun. diurnum.

vonenatum.

Figreas ceilanica. x. 2.

Strycbros vomica. I-4potatorum.

Opbioxylonferpentinum.a. $\beta . \gamma$. Cafpicum finenle. grosfunis. frutefcens. baccatum. ативичт.

Solasium tuberculatum. dipbyllum. $\alpha . \beta$. pleudocaprieum. bavanense. jcandens. fellatum. racerrofum. $\alpha_{0}$ B. B
Solanum verbafcifoliums, $\propto$, $\beta . \gamma$. jubinerne. melongena.

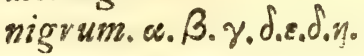
macrocarpum. a-angulare. a. $\beta$. bonariens? dulcamara. o. $\beta$. quercifoliuns. aviculare. lyratums. tuberofum. a. B. lycoperfscum. perqvianum. lycioides. urbamense. igneum. «. $\beta . \gamma$. aquartia. niveuns. in fonum, fanctum. iomentofuan. $\alpha$. $\beta$. torvum. $\alpha$. $\beta$. jamaicense. ferox. indicuns. paniculatum. coccineum. mammofum. $\alpha$. B. $\gamma_{0}$ campecbienfe? virginianum. fodomeum. marginatum. trilobatum. capense. Reramonifolium. 


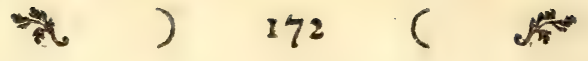

Pbyjalis profirata. fomnifera. cbenopodifolia. flexuoja. arborefcens. \& $\beta, \gamma$. tomentofia.

curasfavica.

vifcosa.

pen/ylvanica.?

alkekenzi. a. B.

peruviana. $\alpha$. $\beta$.

angulata. $\alpha . \beta . \gamma$. pubefoens.

pruinofa.

barbadenfis.

Atropa mandragora.

belladonnt.

pbysalodes.

folanucen.

race mofin.

arbavefcens.

frutefcens.

Ellifia nyctelea.

Lycium barbatwin.

japonicum. a. B. $\gamma$. afrum.

vi idum.

tetrandoun.

barbirum.

europoum.

cineveum. $\alpha . \beta$.

borsidum. a. B. $\gamma$.

Ignaiia amsara.

Ceropegia candelaurum.

fagittata. tenuifolia.

Nerium ceilasicum. oleander. $\alpha . \beta$. divaricatum. antidy)entericum.

Echites fusculenta. bijpinofa. asperuginis. floribunda. $\alpha . \beta$. coftata. torulofa. umbellata. corymbofa. caudata. angularis. fipbilitica.

Pergularia japonica. eduhs. I. 2.

Plumieria altisfina. rubra. a. B. $\gamma$. alba.

Cameraria latifolia. Tabernamontana citrifolia.

dilcolor. a. B. $\gamma$. elliptica. amfonia. Iourifolia.

Vinca pujella. minor. 
Vinca major.

$$
\text { rofea. a. B. } \gamma \text {. }
$$

Scavola plumieri.

Samolus valevandi.

Virecta vireits.

Bellonia fpinofa.

Macrocnemum jamaicenfs.

Rondeletia virgati.

$$
\begin{aligned}
& \text { incana. } \\
& \text { tomentofa. } \\
& \text { umbellata. } \\
& \text { tbyrjifora. }
\end{aligned}
$$

Cincbora angulifolia.

caribes. Q. B

fluibunda. $\alpha . \beta$.

oficinatis.

corymbofin.

bracbycarpa.

Portlandia grandiflora. I. 2.

tetrandra.

coccinea.

Solandra grandiflora.

Roëlla ciliata.

Squarrofa.

muscoja.

Spicata.

Pbyteurna crifpa.

pancifora.

bemi/pharica.

comofa.

orbicularis. $\alpha . \beta$.

fisicata.
Tracbelium tenuifolium. \&. $\beta$.

diffufum.

coruleum.

Dentella repens.

Morinda citvifolia. $\alpha . \beta$.

Rayoc. a. B.

lauvifolia.

numbliata?

Cepbaëlis elata.

PJychotria borizontalis.

nutans. fol.

tenuifolia.

marginata.

uliginofa.

grandis.

crocer.

birfuta.

pavetta.

pedunculata.

pubefcens.

fatens.

ferpens.

afiatica. $\alpha, \beta, \gamma$.

bracbuata.

berbacea.

myrtifolia.

alpina.

patens.

corymbora.

laurifolia.

glabrata.

titipulacee.

fetofa. 
Pfycbotric involucrati. fol. pendula.

Coffea guianenfis. fol. occidentalis. a. $\beta$. arabica.

fambucina.

Cbiococca racemoja. o. B. y. . . barbata.

Campanula graminifolia?

capillacer.

linearis.

fesfilifora.

adpresfa.

fubulata.

bispidula. o. $\beta$.

paniculata. a. $\beta$.

rinerea.

unidentata.

fafciculata.

tenella. $\alpha . \beta$.

perficifolia.

patula.

rotundifolis. a. B. .

Scbeucbzeri.

uniflora.

rapunculus.

Aberica.

alpeftris.

bononien/is.

tripbylla.

americana.

undulata. 1. 2.

capentis. $\alpha, \beta$.

nemoralis.
Campanula cernua. a. $\beta . \gamma_{0} \delta$. cervicaria. lobelioides. marginata. porofa.

rbyrjoidea.

glomerata. a. B. barbata. alpina.

aurea. $\alpha . \beta, \gamma$. mollis. edulis. pulla. conifia. medium.

tracbelium. $\alpha$. B. $\%$ latifolia. a. $\beta$. bederacea.

Allioni.

lilifolia. a. B. carpatica. peregrina. glauca. tetrapbylla. pyramidalis. perfolinta. $\alpha . \beta$. rbomboidalis. procumbens. $\alpha . \beta$. faxatilis. rapunculoides. bybrida. Jpeculum. $\alpha$. $\beta$. erinus. canarienfis. 
Gardenia Porida. o. B. $\gamma$. d. Hamellia axillaris.

radicans.

genipa.

Tbunbergia.

Rotbmannia.

marittinan.

mus fanda.

pinofa.

micrantbus.

pedunculato.

nimata. fol.

Randia. fol.

Lonicera bubalina.

alpigena.

Sempervirens.

media.

caprifoliunn.

periclymenum.

grata.

glauca.

japonica.

xylofeum. $\alpha . \beta, \gamma$.

pyrenaica.

carulea.

nigra. $\alpha . \beta$.

Jympboricarpos. $\alpha . \beta$. dievilla. tatarica. involucrata.

Triofteum perfoliatun.

Musfexda concolor.

$$
\text { frondofa. }
$$

Hamellia ventricofa. $\alpha . \beta . \gamma$.
Sabicea birta.

cbryfantba.

Eritbalis fruticosa.

Nauclea orientalis. occidentalis.

Brunia nodiglora.

paleacea.

lanuginola.

verticillata.

abrotanoides.

Stavia radiata. a. $\beta . \gamma$.

glutirsofa.

Walkera obtufata. fol.

Sauvagefia erecta.

Itea virginica.

cyrilla.

Roridula dentata.

Claytonia virginica.

fiberica.

Hovenia dulcis.

Ceanotbus capfularis.

americanus. a. $\beta$.

afaticus. a. $\beta$.

africanus.

Ruyfcbia furubea.

Celaftrus filiformis.

micrapbyllus.

lucidus

laurimus.

roftratus. a. $\beta$. 
Celaftrus alatus.

tetragonus.

foandens. punctatus.

Ariatus.

acusrinstus. a. $\beta$.

cermuss.

myrtifolius?

procumbens.

undatus.

orbiculatus.

crenatus.

edulis. I.)

americanus. 2.)

europaus. a. $\beta . \gamma_{.}$. .)

latifolius. 2.)

leprofus. 2.)

tobira. 2.) buxifolius. $\alpha . \beta, \gamma . \delta$. rotundifolius. fol. pyracantbus. a. B. integrifolius. linearis. japonicus.

Buttneria micropbyilla.

Cedrela odorata.

Diofma rubra. $\alpha . \beta . \gamma . \delta$. birjuta. bijpida. $\alpha . \beta$. pubefcens. ciliata. a. B.
Diofma ericoides.

oppofitifolia.

capitata.

villosa.

lineuris.

enfata.

lanceolata?

rus:ofa.

ovita. $\alpha . \beta$.

uniflura. $\alpha . \beta$.

pulcbella, a. B.

cresata.

betulina.

margisata. $\alpha . \beta, \gamma$. imbricata.

tetragona.

barbigera.

Calodendrum capense.

Mang? fera Jylveftris.

Corynocarpus lavigata.

Rbarnnus lotus.

paliurus. $\alpha . \beta$.

Cbrifti.

cenoplia. $\alpha . \beta$. obliquus.

jujuba?

zizopbus.

capen/is.

mbucronatus.

lycioides.
r.) Catha fornkåht.
2.) Euonymus. 
Rbamnus infeetorius.

faxatilis.

dauricus. fol.

catbarticus.

divaricatus.

theerans.

circumfcisfus.

celtifoluus.

tetragonus. a. B.

volubilis.

farcomplailus.

- solubrinus.

alaternus, $\alpha . \beta$.

objcurus.

spberospermus.

alpisus.

latifolius. a. $\beta$.

frangula.

lineatus.

idears.

Hirtella triandra. $\alpha . \beta$. anevicana.

Vitis quadrangularis. I.) fifyoides. a. B. I.) indica.

cordifolia. I.)

vitiginea. $\alpha . \beta$. I.)

capenfis. $\alpha . \beta$.

vulpina.

Labrif $a$. a. B. $\gamma_{0}$

vinifera.

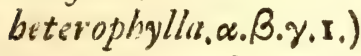

$V i t$ is tripbylla 1).

trifoliata. fol.

cbinenfis.

cirrbofa. a. 1.)

ceilanica.

pentapbylla.

japonica. 1.)

arborea.

Aqquilicio fambucina. $\propto$. B.

Ribes reclinatum.

crifpum.

diacantba. $\alpha . \beta$.

grosfularia.

nigrum. $\alpha . \beta, \gamma$.

vubrum.

cynolbati.

alpinum.

Efchalonia myrtilloides.

Hedera quinquéolia. $\alpha . \beta$.

pendula.

belix. a. B. $\gamma . \delta$.

Plectronia ventofa.

Pbolic pinifolia.

nauctonsta.

eviopbores.

villoía.

lancerilata.

evicoides.

parviflara.

fipularis. 
Pbylica inaberbis.

Arigola. $\alpha . \beta$.
plumofa.
capitata.
buxifolia. a. $\beta$.
callofa.
jpicata.
racemofa.
cordata.

Sirelitzia augulta.

Regina. I. 2.

Heliconia pfittacorum.

bibai, I, 2 .

Argopbyllum nitidum.

Lagrecia cuminoides.

Conopharpus racemofa. a. $\beta$. evecta. $\alpha . \beta$.

Acbyrantbes muricata.

altisfima.

aspera. $\alpha . \beta . \gamma$.

lappacea. $\alpha . \beta$.

brachiata.

patula.

profirata.

alternifolia.

corymbiofa. $\alpha$. B. $\gamma$. balinuifolia.

Irefine

Amarantbus gangeticus.

mangoftanus.

lividus.

olerciceus.

blitum. a. B.

polygonoides.

fcandens.

bybridus.

paniculatus.

Jangvineus. c. $\beta$.

retrofiexus. a. $\beta$.

flovus.

bypocbondriacus.

cruentus.

caudatus.

Celoja trigyna. 1.2.

Jpinofus. $\alpha$.
trigyna. 1.
marilandica.

criftata. $\alpha$. B. $\gamma$.

margaritacea. $\alpha . \beta$.

caftrenfis. .

argentea.

nodiflora.

pyramidalis.

paniculata. a. F.

lanata. $\alpha . \beta$.

Illecebrum vermiculatum.

viridis: $\propto . \beta$.

grazizans.

melancbolicus.

tricolor.

polyganus.

paronycbia. a. $\beta$.

verticillatum.

javanicusn. a. $\beta$.

acbyrantba.

tenerum. 


\section{叫) 8 (79 (}

Illecebrum monfonianum. divaricatum. floribu:dwn. polygoroides. cymofum. juffraticolum. ficoideam. fangviriolentsms. arabicum. fessile. alfinifolium.

Glaux marittima. $\alpha . \beta$. Ceratonia filiqua. 今. o. Thefium line tum.

fragile. $\alpha . \beta$.

Jpinolun.

imbricatuss. frunale. $\alpha$. $\beta$. $\gamma$. ftritum. $\alpha . \beta . \gamma \cdot \delta . \varepsilon$. paniculatum.

fquarvoiush. capitatums. $\alpha . \beta$. alpizunz. ce. B. $\gamma$. linopbyllums. frifea. triflorum. eupborbioides. colpoon.

Pbocama pendula.

Gouania tomentofa. Nyssa aquatica. I. 9 . Stapelia pilifera. mammillaris. 


\section{$\Rightarrow 180,()$}

Apocynum venetum. «. $\beta$. Gentiana exacoides.

cannabinum.

frut $\int$ ens. $\alpha . \beta$.

reticulatum.

baftaturs.

Afclepias apbylla.

filiformis. a. B.

verticillata.

frutic $\int$ s. $\alpha . \beta$.

fiberica.

caniflora.

nigra.

vincetoxicum.

curasjavica.

incurnata.

viminalis.

tuberofa.

rubra.

nena.

nivea.

purpurafcens.

crifpa. a. $\beta$.

undulata. $\alpha . \beta$.

acsminata.

grandiflora.

fyriaca. $\alpha . \beta$.

gigantea.

arborefcens.

volubilis.

Spertia corniculata.

rotata.

dicbotoma.

perennis.

allens. $\alpha . \beta$.

dubia.

spicita.

Japonaria.

nivalis.

amarella. $\alpha . \beta$.

verna.

acaulis.

pyrenaica. $\propto . \beta$.

campeftris. o. $\beta$.

tenella.

pulcbella.

vifcofa.

rivularis.

pallens.

decumbens.

marittima.

faxofa. pumiln.

pannonica.

utriculoja.

bavarica.

ciliata. $\alpha$. B.

afclepiadea. $\alpha$. B. $\gamma$. verticillata.

montana:

purpurea.

punctata.

aquatica. $\alpha . \beta$

lutea.

Cenínurium.

cruciata.

Gen- 
Gentiana filiformis.

pueunonantbes.

aures.

Cresfa cretica.

Hydrolea Jpinofa.

Porana volubilis.

Rocbefortis vata.

cuneata.

An:bafis Jpingofa.

appylla: $\alpha . \beta$.

foliofa.

tamaivififolia: $\alpha . \beta$.

Velezia rigida.

Nanna janaicenfis. ceilanica.

Linconia alopecuroides.

Heucbera americana.

Saljola aiffita. a. B. birfuta. alicsfima. a. B.

tragus.

kali. $\alpha . \beta$.

fativa.

foda.

tamarifcinn.

rolacea. a. $\beta$.

falla.

Salfole inermis.

byssopifolia. a. B. g. D.

divaricata.

fruticofa. a. $\beta$.

vermiculata.

muricata.

apbylla. a. B.

Cbenopodium littorale. $\alpha . \beta_{\text {. }}$

tataricum. 3.)

$\gamma . \delta .3$.

micropbyllum.

coriaceum.

pedunculatum. 3.)

portulacoides. \&. B.3.)

cinereum. 4.)

ba.'imus. 3.)

veftıtum.

verruco (um. 3.)'

roleum. 3.)

bortenfe. 3.)

afiracaners(e. 3.)

dubium. 3.)

baftatum. a. B. 3.)

patulum. 3.)

laciniatum.

triandram.

benricus.

urbicum.

fcoparia.

proftrata, a. $\beta, \gamma . \delta$.

fidoides.

mucronatum.

atriplicis.

rubrim.

ericoides.

C 2

Clee-

3.) Atriplex Auctorum.

4.) Atriplex glauca. Linn. 
） $\quad 182 \quad(\quad)$

Cbenopodium Serotinum.

album.

viride.

bybridum.

purpurafcens.

botrys. a. B. $\gamma$.

ambrofioides. $\alpha$. $\beta$.

antbelminticum?

glaucum.

vulvaria.

polyspernum.

finense.

fcoparia.

marittimum.

arifatum.

Beta cicla.

vulyaris. 1. 2. 3.

Humulus lupulus. 0 .

Cannabis chinenfs. 2 . ․․ Sativa. 歺. 文.

Herniaria fraticofa. a. $\beta$.

glabra.

birfuta.

lenticulata.

Gompbranainterrupta. $\alpha . \beta$. globofa. $\alpha . \beta$.

Bofea yervamora. ㅅ. ㅇ. Ulmus pumila. I. 2. 3 . americana. fol. campeftris. 1.2.

Pometia pinnata.

Celtis aculeata. micrantbus.
Celtis vimimlis.

lima.

orientalis.

occidentalis.

Nepbelium lappacesm. 唡. ?.-

Coprofina fetida. lucida.

Vablia capen/is.

Bumalàa trifoliata.

Breynia, difticta.

Plyyllis nobla.

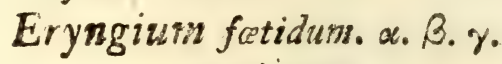

aquaticun.

plamtin. a. B. $\gamma$.

pufillun.

tricupidatum.

marittinum.

campefire. $\alpha . \beta$.

Burgati.

ametbyftinum. alpinum. a. B.

Dicbondra repens.

fericea.

Hydrocotyle vulgaris. $\alpha_{0}$..$\gamma$. americana. mofcbata. bir uta. afiatica. ranunculoides. javanica. glabra. 
Hydrocotyle villofa. a. B. $\gamma$. tomestofa. $\alpha . \hat{\beta}$.

tridentata.

linifolia.

virgata.

Panax fimplex.

quinquefolia.

arberea.

Cussonia tbyriffora.

spicata.

Sanicula europen. a. B.

canadenfis. $\alpha$. $\beta$.

marilandica.

cilinta.

Arctopus ecbinatus. a. $\beta$. Aftrantia cilinis.

major.

minor.

carniolica.

Heracleutn pyrenaicum. 1. 2. alpinum. 1. 2.

angufifolizm. fol.

fpbondylium.

panaces. I. 2.

fibiricum.

aufriacum. 1. 2. 3.

Oenantbe exaltata.

interupta. a. $\beta$.

ferulacea.

cracata. a. $\beta . \gamma$.

fitulofa. $\alpha . \beta$.

globulosa.

pimpinelloides, a. B.\%.

Ecbinopbora jpinofa.

Oenant be carvifolia.

critbmifolia.

prolifera.

inebrians. I. 2.

temuifolia. a. $\beta$.

tenuifolia. a. $\beta$.

Caucalis grandiflora. $\alpha . \beta$. arucoides. $\alpha . \beta_{1} \gamma_{0}$ africana. latifolia. pumila.

leptopbylla. $\alpha . \beta$. platycarpos. $\alpha . \beta$.

Artedia jquamata.

Daucus bifpanicus. 1. 2. 3 .

gingidium. $\alpha . \beta$.

muricatus.

vifnaga.

ciarota, $\alpha . \beta$.

mantitanicus.

Tordylizun fyriacum.

latifolium. I. 2 .

maximun.

rodulofum.

officinale.

antivifous.

Laferpitiam angultifolium. peucedanoides.

Peucedarum copiliceum.

dewrithliums. gevicislatum.

officinale. 
Peucedarum japonicum. flaus.

$$
\begin{aligned}
& \text { aljaticum. } \\
& \text { cofatei. }
\end{aligned}
$$

Annmi glaucifolium.

copticum. I. 2.

majus. a. B.

creticum.

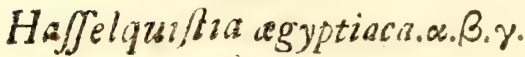
cordsta.

Conium rugofum.

rigens. $\alpha$. $\beta . \gamma$.

africanum. $\alpha . \beta$.

maculatum. I. 2.

jibiricum. I. 2. 3.

Bunium bulbocaftanum.

Atbamanta annua.

fibirica. oreoselinum. 1. 2. 3. cervaria. I. 2. creten/is.

jicula.

meum.

condenfata.

libanotis. $\alpha . \beta$.

Bupleurum rotundifolium.x. $\beta$.

longifolium.

fellatum.

petraum.

angulofum. a. $\beta$.

falcatum. I. 2.

odentites.

gerardi.

Bupleurum Semicompofitum.

decompofitum.

a. $\beta$.

racunculoides.

rigidum.

temuis.imum. $\mathbf{x} .2$.

junceum.

ciliatum.

giganteum. 1. 2. 3.

capitatum.

quinquedentatum.

villofum.

difforme.

fruticefcens.

Spinofum.

falicifolium.

arborefcens.

fruticofum. $\alpha . \beta$.

Sium filifolium.

anguftifoliuss.

grandiflorum.

paniculatum.

patulum.

asperum. $\alpha . \beta$.

bifpidum.

repens. a. $\beta$.

decumbens.

falcarica. I. 2. 3.

latifoliums.

angufifolium. $\alpha . \beta$.

nodiflorum.

fifarum. $\alpha . \beta$.

ninfi. fol. 


\section{औ ) 185 (}

Sium vigidum.

ficulum.

japonicum.

villofum.

Selinum pyrenaicum.

carvifolium.

snonnieri.

paluftre.

divaricatum.

carvfolia.

Cuminusn cyminum.

Ferula glauca. fol.

Critbruum mavittimum.

pyrenaicun.

Bubon gallonum. $\alpha . \beta$.

macedonicum.

gunn:iferun.

Cacbrys odontalgica.

libanotis.

ficula.

Levifticum ligufticum.

gingidium.

anfiriacum. I. 2. 3.

peloponnefiacum. a. $\beta$.

froticum. I. 2.

Angelica lavigata. I. 2.

Razulii.

lucida. 1. 2.

verticillaris. 1. 2. 3.

ntropurpurea.

fylveftris.

arcbangelica.

Sifon verticillatum.

Sifon Segetum.

aknmi.

amomum. I. 2.

canadense. I. 2.

inundatum.

Atbuse meum.

bunius.

cynapium. 1. 2.

Coriandrum fativum.

tefficulatum.

Sefeli filfolium.

frisiatum.

cheropbylloides.

ammoides. $\alpha . \beta$.

turbitb.

glaucum. $\propto \beta$.

montanum a. $\beta . \gamma$.

tortuofum.

anพuนm. $\alpha . \beta$.

articulatum. $\alpha . \beta$.

elatum. $\alpha . \beta$.

pyrenaicum. 1. 2.

tripolitanum.

bippomanantbum. I.2.

pinspinelloides.

pumilum. $\alpha . \beta$.

Scandix auftralis.

antbrifcus. 1. 2.

nodofa.

infelta.

odorata. $\mathrm{r}$. 2.

pecten.

cerefolium. 


\section{$x) 186(1)$}

Charopbyllum aromaticum. Apium petrojelinum. I-3.

\section{I. $2 . \quad$ graveolens.}

aureum. I. 2.

bisfutum. $\alpha . \beta$.

tomisleniumi. I. 2 .

bitlon uris.

Syluelire.

arzifutum.

fraibum. o. $\beta$.

culoutum. 1. 2.

capenle.

Pbellandrium mutelina. 1.2. aquaticum. I. 2.

Imperatoria oftrutbium.

Cicuta virofa.

maculata.

Smyrnium perfoliatum. I - 3. olufatrum. 1, 2. aureums.

integrum.

laterale. $\alpha . \beta$.

Carum bunius. I. 2.

Thapfia trifoliata. I-3.

villofa. 1. 2.

garganica. I. 2.

Paftinaca fativa. I. 2.

opopanax. I -5.

lucida. I. 2.

Anethum fericulum.

graveolens. a. $\boldsymbol{\beta}_{\text {. }}$

Segetum.

Agopodium podagrario. \&. 2. pimpinella faxifraga. 1-3.

magna. 1.2.

intermedia.

rubens.

dioica. 令. 早.

disjecta. 1. 2.

glauca.

orientalis.

anifuns.

capen/is.

Xanthorbiza apiifolis. 1-3.

Xantboxylon clava. I-3. trifoliatum.

Viburnum prunifolium. casfinotdes. tinus.

nudurn.

levigatum.

pyrifolium.

deritatum. $\alpha . \beta . \gamma . \delta$. lantinn.

tomentofum.

opulus. a. $\beta$.

lentago.

virens.

jervatum. $\alpha . \beta . \gamma . \delta$.

crofuns.

birtum.

dilataum.

macropbyllum. a. B. 
Viburnum cufpidatum.

villojum.

Sambucus laciniata.

ebulus.

japonica.

canadenfis. $\alpha . \beta$.

nigra.

Rbus atruns.

\section{racemosa.}

cotinus. $\alpha . \beta$.

anguftifoliunn. a. $\beta . \gamma$.

lanceum. $\alpha$. $\beta$.

pubelcens.

lievigutum.

toxicodendrum. a. B. $\gamma$.

radicans.

cirrbiforum. T. 2.

lucidum a. $\beta$.

villofum. $\alpha$. $\beta$.

tridentatun.

dentatum.

tossentofum.

finuatum.

cuneifolium. fol.

insifun.

digitatum.

metopium. fol.

fuccedoneum.

vernix.

canadense.

copallinum.

procerum.

coriaria.
Rbus glabrum.

typbinum.

carolinianuss.

jovanicum.

pauciforum.

Turnera pumila.

cifroides.

ulmifolia.

Sayotbra gensianoides.

Alfine media. $\alpha$. B. $\gamma$.

fegetalis.

mucronata. a. $\beta$.

Telepbium imperati.

Corrigiola littoralis. a. $\beta . \gamma$. telephifolia.

Pbarnaceum fruticofum.

mollugo.

cerviena. a. $\beta$.

lineare.

dicbotomum.

glomeraturs. a. $\beta$.

teresifolium.

albens.

incanum.

micropbyllum. qaadrangulare. $\alpha . \beta$.

depressum.

marginatum.

Serpyllifoliun.

umbellatum.

cordifolium.

Piftaciá cappadocica. \$. 9. 
Clutia ericoides.

Piftacia lentifcus. 走. ㅇ․ therebintbus. fol. trifolia.

Cesfine capenfis $\alpha$. B. $\gamma$.

barbara.

colpoon. I-3.

peragua.

maurocenia.

Pasfiflora punctata.

ferratifolia.

minimsa.

fuberola. a. $\beta$.

taurifolia.

vubra.

angufifolia.

bivfuta.

perfoliatd.

cuneata.

quadrasgularis. 1. 2.

lutea.

fetida. $\alpha . \beta$.

musucuja. \& $\beta$.

pomifcro. fol.

aurantiaca.

pallida.

bolofericea.

vefperitito.

incarsiata.

rotunaifolia.

carulea.

Andracbne telepbium.

fruticofa.

Clutia alaternoides. \&. $\beta$.

trubejcens. A. ?.

tomentorn. of. ?.

retufa?

beteropbylla.

pulcbella. \&. ?.

acuminata.

birta. 3.?

Spatbelia fimplex. I. 2.

Stapbyle trifoliata.

pinnata.

occidentalis.

Tamarix gallica a $\beta$. iridica.

germanica. $\alpha . \beta$.

Drypis jpinofa.

Statice armeria.

pleudasvmerin. a. $\beta$.

ppeciofa. a. B.

purpurata.

monopetala.

finuata.

mucronata. $\propto$. $\beta$. ecbinus.

aurea.

Juffruticofa. $\alpha . \beta$.

cordata.

ecbioides.

menuta.

ferulacea.

reticulata.

pruinosa. 
Statice tatarica. $\alpha . \beta$.

incana.

limonium, $\alpha$. $\beta$.

Al:xuofn?

olectolia. a. B.

anuculafolia. $\alpha$. $\beta$.

bellidifolian.

longifolia.

linitolia. $\alpha . \beta$.

Fabra.

tetragons.

Commerfonia ecbinata.

Xylopbylla falcata.

latifolia.

arbufcula.

montana.

angufifolia.

longifolia.

Bajella alba.

rubra.

lucida.

Parnasfia paluftris.

Evolvulus nummularifolius. emarginatus.

gangeticus.

tridentatus. a. $\beta$.

alfinoides.

linifolius.

Spinacia oleracea. I-5.

Aralia Spinofa. r. 2.

pentapbylla.

capitatis.

arborea. $\alpha . \beta$.

vacemofa. $\alpha . \beta$.

mudicaulis.

cordata.

degitata. I. 2.

cbinewis.

Acrida camabina.

Crasfula umbellata. a. $\beta$. $\gamma_{0}$ decumbens. $\alpha$. $\beta$. $\gamma$. aquatica. 4.)

verticillata.

tillas. 5.)

miujcosa. a. B.

glonerata. $\alpha . \beta$.

natens.

proftrata.

pellucida.

dichotoma. a. B.

inanis.

vetroflexa. $\alpha, \beta$.

pulcbella.

tetragana.

faiffoutionsa.

muricata.

pruinofa.

tbyriflora.

Spicata.

D 2
Cras-
4) Tillæa, Linn.
5) Tillæa mufcofa, Linn. 


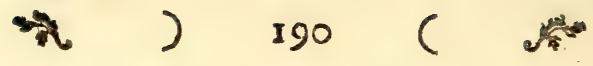

Crasjula rabens.

fubulata.

ramosa.

cymofa.

mollis.

pubefcens.

forabra.

cepbalopbora.

punetata.

montana.

turvita. fol.

alpefiris.

capitella.

perfoliata.

obvallata.

centauroides.

marginata.

tomentofi.

crenulata.

perfoliata.

pyramidalis.

deltoider.

cultrata. \&. $\beta$.

ciliata.

coccinea.

rupeftris.

colysedonis.

argentea.

lacer.

tects.

veflita.

corallina.

columnaris.

Crasfula orbicularis.

Septas. 6.)

bemijpherica. $\alpha, \beta$. barbata. minima. cordata. . Jpatbulata. dentata.

Antidefma alexiteria. fol.

Gifekia pbarsacoides. Ferwillee a cordifolia. triloba.

Linum africasum. etbiopicum. quadrifolium. anguffifolium. arboreum. monogynum. gallicum. firvictum. companulatum. fuffruticofum. ce. $\beta, \gamma$. tenuifolium. a. $\beta$. catbarticum. vifcofum. alpinum. flavum. bivjutsm. mavittinum. nodiflorum. narbonease. perenne.

6) Septas capenfis, Linn. 


\section{啁) 174 ( )}

Linum ufitatisfimum.

virginicums.

radiola. $\alpha . \beta$.

Aldrovanda veficularis.

Drojera acaulis.

cumeifolia. $\alpha . \beta$.

rotundifolia.

longifolia.

Drofera capenfis.

lufitanica.

ciffiflora. $\alpha . \beta$.

indica.

Sibbaldia procumbens.

atraica.

erefta.

Tb. 1.

Enumeratio Naturalizum collectionis publica, $\mathbf{c u}$ juscunque fit natura Regni, licet primo intuitu minoris momenti videatur, non exiguum tamen adfert ufirm.

\section{Th. 11.}

inter omnes Hifforice naturalis partes, ea oinnino primum locum obtinet, que Regni Vegetabilis incolas familiares nobis reddit.

Th. III.

Clasfis Pentandria E quod ad numerum civium, So quod ad ufum medcum, pra reliquis maximam noftram attentioneta meretur.

Th. IV.

Hac quoque ipfa Clasfis maltas venenatas plan. tas continet.

\section{Th. $V$.}

Pentandria Digynia, Jeu Umbellatarum fic dio ciarum ords difficilius admittit notas Cbaracterifticas. 
nenten 


\section{D.}

\section{MUSEUM NATURALIUM ACADEMILE UPSALIENSIS.}

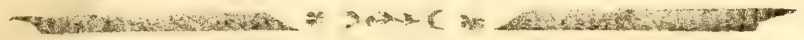

CUJUS

PARTEM DUODECIMAM.

CONS. EXP. FACULT. MED. UPS.

$P R$ RIDE
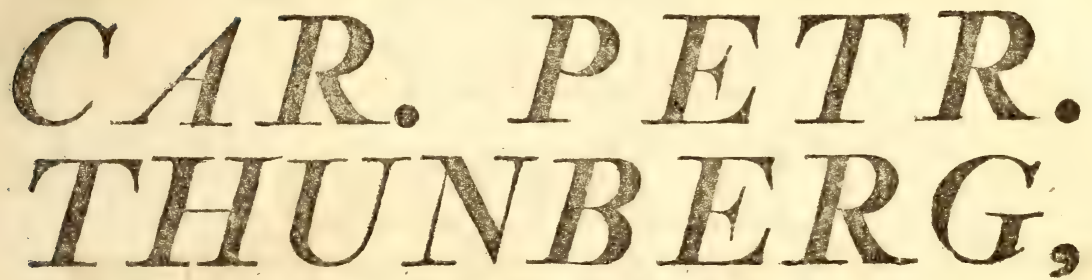

Equite Aurat. Reg. Ond. de Vasa,

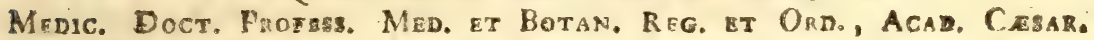
Nat. Cukios, Rig. Scient. Londinens. Hoimizns. Societ. Scibnt, Ursal.

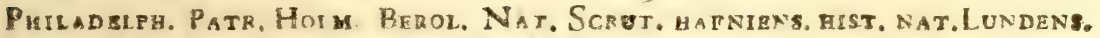
Hartem. Austerd. Zeinand Nidrosigns. Haiens. Nat. SCrut. Linn. Londin. Medic. Edimeusg. et Nat.Studios. Inid. Memero; nec non Acad Scient, Pagab.

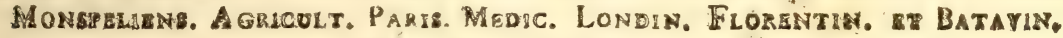
Ind. Or. Corrastond.

PURLICO EXAMINI SUBMITTIT CAROLUS ALEX. LINDBLADH, SUDERMANNUS.

IN AUdit. Botanic, D. VIII DECEMB, MDCCXCII. H. A, M. S.

\section{U P S A L I \&,}

Litteris Vidue Direct, Joh. Edman: 
SACRAM REGIAM MAFESTATEM

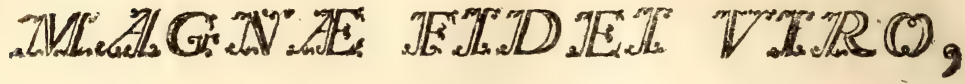

PROVINCIE SUDERMANNICAE

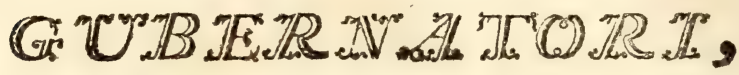

GENEROSISSIMO AC NOBILISSIMO

\section{DO 16 E 0}

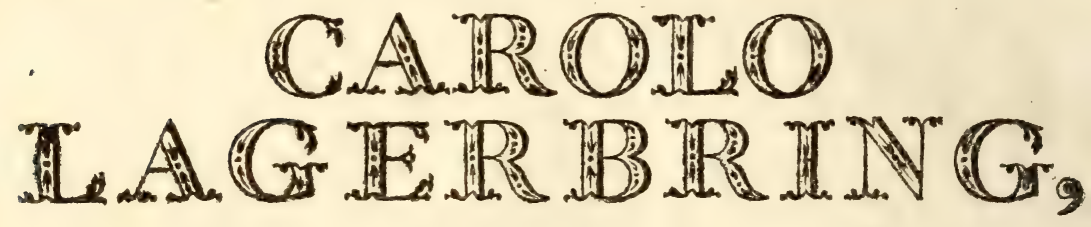

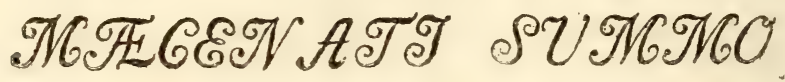




\section{VIRIS}

\section{ADMODUM REVERENDIS ATQUE PR IECLARISSIMIS,}

S:R R:A M:TIA SACR. ECCLES. NYCOPENS.OCCID.ET ORIENT,PAST。

ITEMQUe PR REPOSITIS dignissamis,

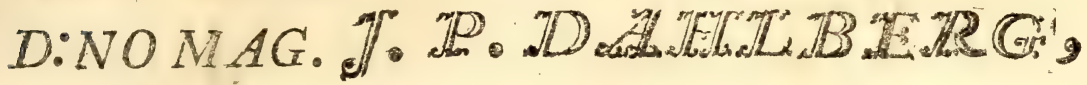
- ET

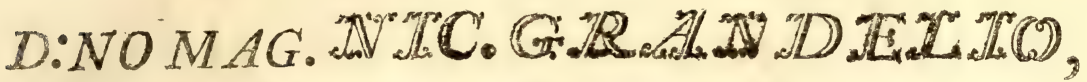
PATRONIS BENIGNISSIMIS,

\section{VIRIS}

AMPLISSIMIS ET CONSULTISSIMIS,

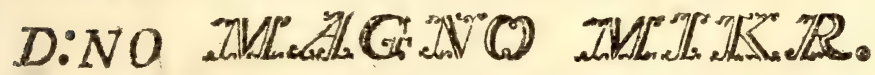

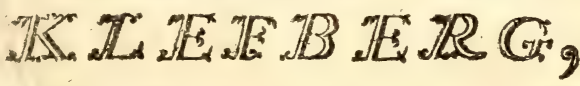
CONSULI NYCOPENSIUM FUSTISSIMO, ET

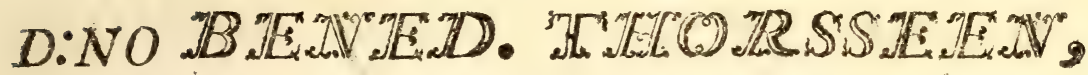
CONSILIARIO ET CONSULI VICARIO ALQUISSIMO, FAUTORIBUS OPTIMIS

Tesferam hanc mentis venerabundæ \& gratisfi$m \mathfrak{m}$ offerre voluit, debuit NOMINUM VESTRORUM 
RADMANNEN OCH FABRIQUEUREN ADEL OCH HÖGAKTAD HERR

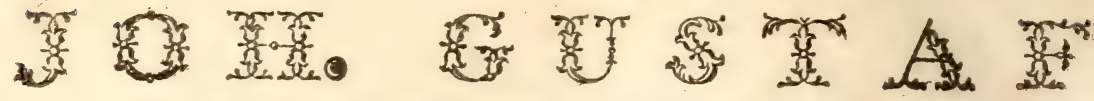

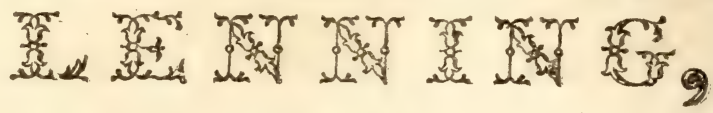

\section{SAMT \\ $\ddot{A} D L A F R U N$}

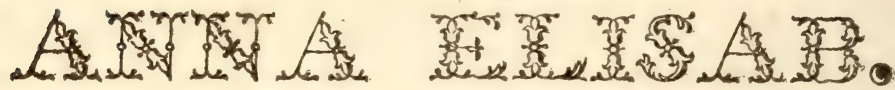

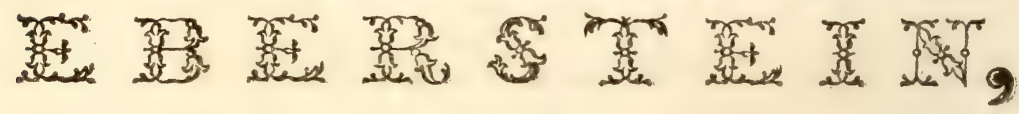

\section{HANDELSMÁNNEN \\ $\bar{A} D L E$ OCH HÖGAKTADE HERRAR,

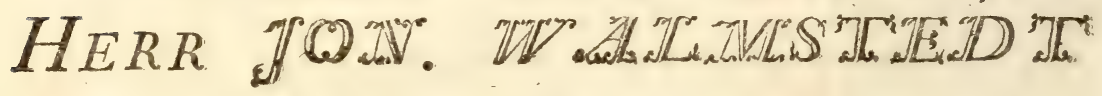 $\mathrm{OCH}$

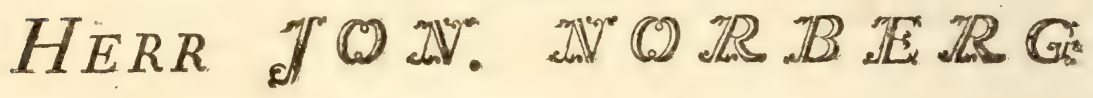
Tillagnas detta odmjukaft 


\section{DONATION. THUNBERGIAN. continuat. $\mathrm{X}$. Hexandria}

Bromelia ananas. I. 2. pingvinalis. lingulata for. bracteata.

Licuala spinofa. I-5. Chamserops bumilis, I-2. excelfa. $\mathrm{x}-2$.

Thrinax parviflora. 1. 2. Cocos nucifera. I-6. Mauritia flexuofa. flor. Tillandfia ufneoides.

recurvata. tenuifolia. polyfachyd. priniculata. I. 2 . utriculata. fol. pendula. fafciculata. monoftacbya. anguftifolia. canefcens.

Hepetes angufifolia. Burmannia juncea. dificicba.

Commelina radicifora. tubernfa. virgisica.
Commelina diffusa. Zanonia. rudiflora. nudicaulis. parviflora. erecta. communis. lengbalenfis. africana. Speciofa. I). Spatbacea. 1). criftata. 1). malabarica. I). cordifolia. I). multiffora. I). monandra. 1). wnbellata. 2).

Burfera gunnifera. Canarium commune. 1.2. Gleditfcia inermis. fol. triacbanta. I. 2. Frankenia pulverulenta. $\alpha . \beta$. bivfuta. $\alpha_{0} \beta . \gamma \cdot \delta_{0}$ levis. notbria.

Lorantbus incanns. tetrapetalus.

E 3 Lo-

I) Tradefcantiæ auctor.

2) Tradefcantia virginica. 


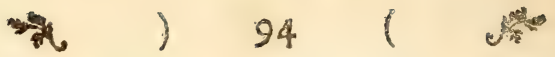

Loranthus europens.

americanus.

jpicatus.

Sictis.

pauciflorus.

parvifolius.

esmarginatus.

pentandrus.

tonzento/us.

Hillia longiflora.

tetrandra.

Ricbardia fabra.

Berberis faberica.

vulgaris. I-3.

ilicifolia.

parvifalia.

finerafis fol.

cretica.

Leontice leontopetalum.

Prinos verticillatus. a. $\beta$.

fideroxyloides, $\alpha_{0} \beta$. montana.

glaber.

Tectona grandis. $\alpha . \beta$.

Nandina domeftica.

Acbras fapota.

$$
\text { disfecta. }
$$

Mufa paradifiaca. I-3.

Corypha umbraculifera. x. 2.

Galantbus nivalis. $\alpha . \beta$.

Leucoinum firumofurn.

vernum.

Leuscoium cofivum.

autumnale.

Niarcisfiss poëticus. a. B. bulbocodium. ferotinus. minor?

bicolor. \&. $\beta$.

pleudonarcisfus.

odores.

orientalis.

tasetta. $\alpha . \beta$.

jonquilla.

Pancratium littorale.

illyricum.

cariboum.

marittimum.

ceilanicum.

Amaryllis regine.

lutea.

atamensca.

formosa.

Hernantbus fpiralis. a. $\beta$.

undulatus.

farnienfis.

falcatus.

toxicarius. I. 2.

orientalis. 1-4.

ciliaris. $\mathrm{I}=4$.

pubefcens.

puniceus. 1. 2.

belladonna.

coccineus. I. 4.

longifolius. I. 2 . 


\section{\#) $93(2)$}

Crinum anguffifolium.

lineare.

obligvum.

Speciofum.

afsaticum.

americanum.

latifoliunz.

Alfiroemeria pelegrina. I. 2. Ligta.

Getbyllis ciliavis.

villofat.

fpiralis. I. 2.

lanceolata.

Hypoxis fafcicularis.

ereats

decumbens. $\alpha, \beta$.

villoja. a. $\beta, \gamma$. d.

plicatilis.

ferrata. $\alpha . \beta$.

alba. a. $\beta . \gamma . \delta$.

Aellata. $\alpha . \beta, \gamma$.

ovata.

minuta:

Agave americana, $\alpha . \beta$. vivipara.

Tulbagbia alliacea. cepacea.

Massonia latifolia. lanceolate. undulata. ecbinata.

Pontederia cordata. limoja.
Pontederia axures. fol. rotundifolia. bafiata.

Bulbocodiums vernum.

Apbyllanthes mon/pelienfis. Hewreracallis fulva. a. $\beta$.

flava.

japonica. a. $\beta . \gamma$.

cordate.

Polionthes tuberofa.

Maublia enffolia.

linearis. a. B.

Aletbris byacintboides.

uvaria.

capersis. $\alpha$. B.

farinofa.

Spicata.

Aloë retufa. fol. dicbotama. fol.

Spicata. porfoliata. $\alpha . \beta$. aracbnoides. pumila. a. $\beta$. dificicba. I. 2. naculata. $\alpha . \beta$.

variegata.

vifcoja. Spiralis. lingva. fuccotrina.

Asphodelus Tuteus. $\propto$. $\beta$. ramofus. fiftulofus. 


\section{\#) 96 (}

Ornitbogalum bulbiferum.

reticulatum.

prlosum.

gramenifoliums.

juponscum

viutans. $\alpha$. $\beta$.

umbellatum. $\alpha$. $\beta$.

narbonense.

pyranilale.

pyreraicuns.

ments.

lut $u$ uns.

unforum.

albuc ides. ¿. $\beta$.

bracteatum.

maculatum. ò. $\beta$.

tbyrfoides, ci. $\beta$.

arabi nin.

altisfimum.

crenulatuns.

ovatum.

ciliatum.

cupenfe. fol.

puntiatum. $\alpha \cdot \beta \cdot \gamma \cdot 3$ ).

parvung. 3).

Antbericum fcabrum. I. 2.

lagopus.

favofum. I: 2.

cuudatum. $\alpha, \beta, \gamma$.

bijpidum.

undulatum.

fimbriuturn.

borsutum.
Antbericum muricatums.

falcation. comofus latifnlium. $\alpha$. B. ciliatum.

japanicun.

cirrhatum.

adenintbera.

cripum.

minginatums.

filifoliums.

ferotinum.

iriquetrum. à B. $\gamma_{0}$ d. fpircle. $\alpha . \beta$.

filiforme. $\alpha . \beta$.

revolutum. $\alpha$. $\beta$.

flexifolium.

arnuum.

afphodeloides.

brevifolumo.

alooides.

graum.

liliago. à. A.

ramofum.

fritefetns.

liliafir uns.

calyculatum.

os/ifrogum.

incureum.

nutans.

contorturm. a. $\beta$. $\gamma$. pruciflurum. à $\beta$.

Lanaria plumosa. 


\section{- ) 97 (}

Hyacintbus nonscriptus.

cernutus.

brevifolius.

Serotinus.

ametbyftinus.

orientalis. a. $\beta . \gamma . \delta$.

corymbofus.

convallarioides.

flexuo/us.

revolutus. à. $\beta$.

mujcarius.

racemojus.

botryoides.

comofus. a. $\beta$. $\gamma$.

miniturnfus. ¿. $\beta$. $\gamma$.

Lachenalia contaminatı. $\alpha . \beta$.

orcbroides. a. $\beta . \gamma$.

birt\%.

pailian a. B.

$r$ : fl:xa.

tricolin. $\alpha$. B. $\gamma$.

pendula.

viridis.

Phormizm tenax.

Sanjevieria tbyrffora. 4).

cetbiopica.

fragrans. 5).

Convallaria majalis.

bifolia.

polygonatum. à. $\beta$.

latifolia. $\alpha . \beta, \gamma$.
Convallaria cersua. $\alpha . \beta . \gamma$. vacemofa. $\alpha$. verticillata. multiflora.

Jpicata. Rellata.

Ariftolocbua fipbo. rotunda. longa. $\alpha . \beta$. piftolocbia. indica. arborefcens. peltate. odorata. triloba. balabiata. Jerpentaria fol. clematitis. Jempervirens. angvicida. fol.

Allium ampeloprafum.

porrum.

liseare. rotunduns.

vilforialis. birfutum. a. B. magicum. obliqvum. ramofim. roseum. tataricmm.

4) Alethris hyacinthoides.

5) Alethris frägrans. 


\section{) 98 ( )}

Allium Sativam.

fiorodoprafum.

arenariums. $\alpha . \beta$.

carinatuin. $\alpha . \beta$.

spberocepbaluse.

descendens.

mofcheicum

flivum. $\alpha . \beta$.

pallens.

olevaceum.

nutans.

ferefcens. $\alpha$. $\beta$.

odorim.

angulofune. ot. $\beta$.

пigи иm. $\alpha$. $\beta$.

urginkm. $\alpha$. $\beta$.

cepa. $\alpha . \beta$.

moly.

butl): $: 5 m$.

fiftulisuin.

fcboenoprafum, $\alpha$. $\beta$.

cajpicum.

Scille italisa.

marittina. I. 2.

amoena. $\alpha . \beta$.

lufitanica

bifolia.

japonica.

peruviana.

autumsalis.

Cyanelle alba, $\alpha . \beta$.

lutea.

capenfis, $\alpha, \beta$.

Albuca major. de. B.

minior.

fuftigiata. a. $\beta$.

vifc'fa.

piralis. à. B.

Uvularia cirrboja.

amplexifolin. fol.

perfolieta.

Jesfilif:lar. a. $\beta$. birta fol.

Fritillaria iuperialis. $\alpha$. $\beta$. meleasvis, a. $\beta \gamma$. pyrenaice. à. $\beta . \beta . \delta$. per/caca.

Liliuss martagon. à. $\beta$. pbiladelpbicum. kamichateum. condidum. \& $\beta$. bulbif-v⿴m. $\alpha$. $\beta$. cbalcedonzcum. pomponicuin. ennadenje. o. $\beta$. japonicum.

Erytbroniums canis.

Juperbum.

Gloriofa Juperba.

Tulipa gefneriana. $\mathbf{x}$.

Jylveftris. 1-2.

biflura.

breyniana.

Yucca filamentofa. fol. gloriofa, a. $\beta$. 
Yueca aloifolia. draconis fol.

Ebrbart digyna.

Dracena draco. fol. terminalis. à, $\beta . \gamma . \delta$. Pbarus enfifolic. graminifolia. bemicliry, a.

Ajporagus acutifolius.

afinticus.

officinalis.

declinatus.

flixususus.

afjhy? Iis.

virticillatus.

fubulatws.

foniters. à. $\beta$.

Lingeus. a. B. 6)

fals tus.

retrofrictus.

allus.

dependens.

capentis. a. B.

frintus.

evectus.

volubilis.

undulatus. $\alpha$. B.

medonlordes.

curdifolius. 7).

Pollia japonica. javanica.

mencmatein.

aristato.

natans.

latifolius. $\alpha . \beta$.

Izncus bufonius, $\alpha$. $\beta . \gamma$.

jayvini.

pedeformis.

acutus. $\alpha . \beta$.

punctorius.

capenfis.

cepbalotes.

ferratus.

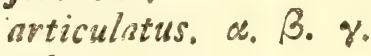

jylvaticus.

pilofus. ò. $\beta . \gamma$

auveus.

trifidus.

biglumis.

triglumis.

Spicatus.

congloneratus.

inflexus. ¿. $\beta$.

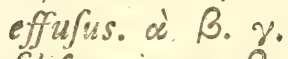

filifurmis. \&. B.

ziodous.

capitatus. $\alpha . \beta$.

niveus. \&. $\beta$.

fupinus.

anglicus.

6) Afparagus falcatus. Flor. japon.

7) Medeola afparagoides, Linn. 


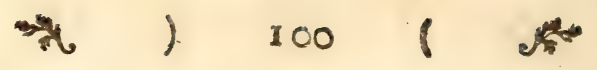

Iuncus paluftris. a. $\beta$. tenageia. campeftris. a. $\beta$. bulbofus. a. B. $\gamma$. Aygius.

fquirrofus. rufefceris.

Orontium aqvaticuss. jeponicum.

Acorus calamus.

$$
\text { graminif lius. }
$$

Lindera unbellata.

Calsmus rotang. I-5.

Tamnus communis. 墭. Peplis portula. a. $\beta . \gamma_{0}$

Gabnia procera.

Oryza fativa. $\alpha . \beta$.

Zizania aquatica.

Colcbicums montanum. fos. autunnale. 12.

Melantbium pumilum. capens.

vurmbea a. B. $. \delta . \delta . \zeta$. viride.

cilistum. \&. B.

triquetrums. $\alpha . \beta$.

luteum.

vivginicum.

lotum.

fibiricum.

albunn.

nigrwm. I-3.
Medeola virginica.

Helonias ápbodeloides. bullata. Trillium fesfile. kamt fcbateum. cern:um.

Triglocbin mavittimum. palufive. bulbofim.

Rumex vabariarum. polmatus.

vibes. rbaponticus. coinpozity. la ceolatis. by trippitium. d. tirus. minittinits. agyptzus. perficuricides a. $\beta$. patientla. a. B. crispus. a. B. brittinnicus. Sangvineus. Sortbulatus. a. B. divaricatus. bucepbalopborus. aquaticus. pulcber. obtuffolius. verticillatus. arifolius. nenolapatbum. 


\section{翟) 101 ( )}

Rumex alpinus.

lanavia.

digynis.

rofeus.

veficerius.

Jizino/us. a. fo.

laxsrians.

acutus.

fout itus.

t:aberofiss.

Sigitzatus.

acetoss.

acetofella. a. B.

tingitanus.

Bores fus flabelliformis. I-5.

Scbeucbzeria palufris.

smagellanica.

Smilax berbacea. 5. 9. excella.

aspera.

ceilantica.

farsaperills. ㅎ․ ?

china: 1.2.

taninoides?

caduca?

pfendocbiva I-5.

laqueans.

ripogomum.

oblongata. folo
Diofcore tripbylls. S. . . B.

oppofitifolia. fol.

japorica. ?.

fativa. fol.

villofa. 옹

balbifera.

alata. A. $\alpha . \beta$.

aculeata.

Rigonia angufifolia.

$$
\text { 5-pbylla. \$. \&. }
$$

ovats.

baftata. fol.

5-obs. f. $\alpha . \beta$, , 定

7-loba. o.

yuinata.

bexapbylla.

Flagellaria ivdica. a. B.

Petiveria alliacea.

octandra.

Alifma plastago, a. B. $\%$

natans.

cordifulia. a. B.

parnasfifolia.

damajonitim.

graninifolia.

flava.

ranunculoides. «. $\beta_{0}$.
Trientalis europer.

Difandra profirata. AEsculus povia. bipiocafamm, I-3.

Heptandria.

Gvettarda elliptica. memlirinacea.

Speciofa. scalla. 


$$
\Rightarrow \text { ) } 102 .(1)
$$

Siururbs cermus, o. $\beta_{\text {. }}$ Limeum copenfe. Limeuth africalsum. atbiopicums.

I.

In minutisfimis maxima Summi Numinis cernitur gloria.

II.

Ingens plantarum copia \& varietas methodum ftaBilem Botanicis enixe commendat.

\section{III.}

Non ex fola utilitate Medica aut Oeconomica pretium fcientiæ Botanicx eft fatuendum.

\section{IV.}

Non inutile prorfus judicandum, quidquid nondum certum \& confpicuum prabuit ufum.

$$
\text { V. }
$$

Latinitas Botanica, nofro in prinis ævo adauteta, Lexicon peculiare fibi deporcit. 


\section{D.}

\section{MUSEUM NATURALIUM ACADEMIE UPSALIENSIS. PARs XIII.}

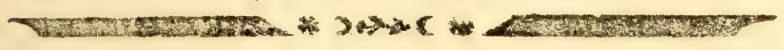

QUAM

CONS. EXP. FACULT. MED. UPS.

$P R$ RSIDE
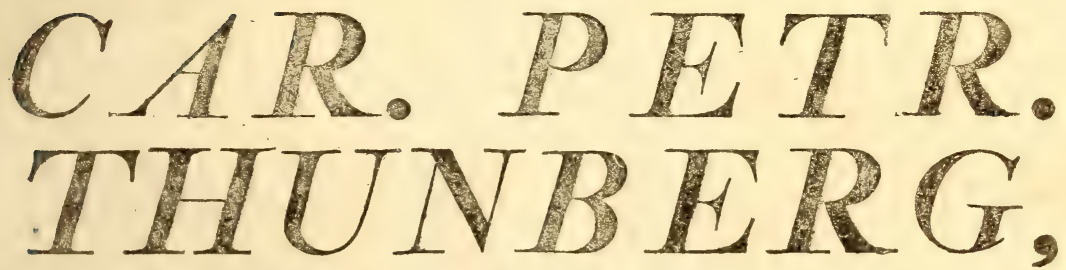

Evurts Aurat. REG. ORd, de Vasa?,

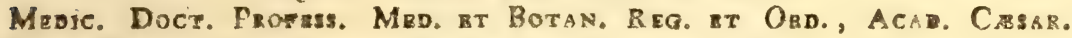
Nat. Cunios. Rrg. Scient. Londingne. Holmiens. Societ. Scient. Urzat. Puiladelz. Patr. Holm. Berol. Nat. Scrut, hafmigns. mist, nar. Lundens. Harlem. Amstrid. Zzrand. Nidnosians. Halene. Nat. Scrut. Linn. Longin. Medic. Edimarzg, it Nar.Stodios. Ibid. Membro; nge nom Acad. Scient. Parse.

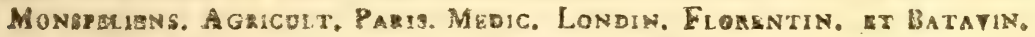
IND. OR, CORRESPOND.

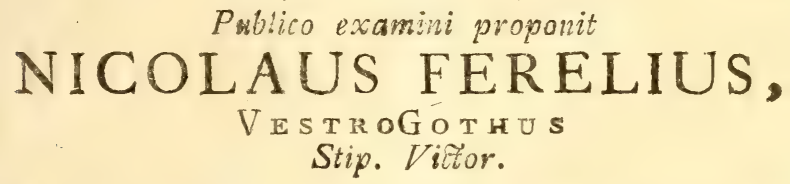

IN AUdT. BOTANIC. D. XX DECEMB, M DCX CII.

H. A, M. S.

U P S A L I $Æ$,

Littrais Vidua Direct. Joh. Edman. 


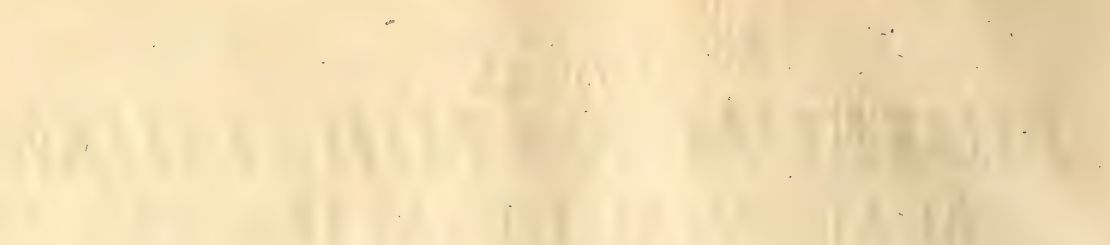
(1:. sin 
ADMODUM REVERENDIS ATQUE PRAECLARISSIMIS,

\section{DOMINO MAGISTRO}

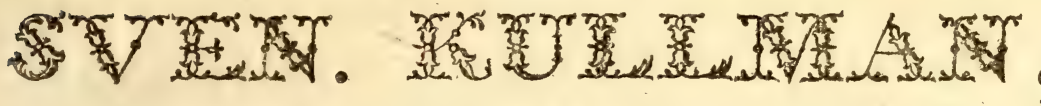

ECCLESIE IN ALGOTSTORP PASTORI ET PRAEOSITO VIGILANTISSIMO,

\section{AVUNCULO OPTINIO}

вт

\section{DOMINO MAGISTRO}

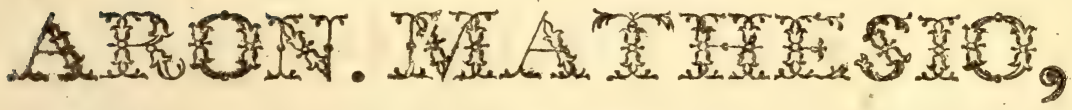

ECCLESIA SVECAN瓜 LONDONENSIS ANTE HAC PASTORI MERITISSIMO,

\section{PATRONO FAVENTISSIMO,}

Innumerorum memor beneficiorum, pagellás hafce gratisfimi animi pignus, piis adjectis votis, venera. bundus offert 
THE REVEREND M:R

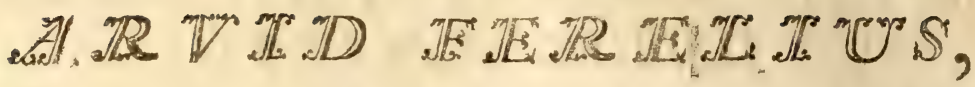

DEAN OF SKO̊FDE,

FORMERLY MINISTER OF THE SWEDISH CHURCH

IN LONDON

AND

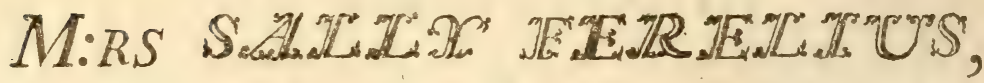
BORN KU LLAGA

MY DEAREST PARENTS!

Offering these premices of my Situdies to the best of Parents, my gratiful heart, melting in reverence and love, would pour out on Your beloved hands the overflowing tenderness of a happy Son.

May the Almighty long preferve Tour precious days to the blesfings of a thoufand hearts! prays MY DEAREST' PARENTS!

YOUR

ever grateful, and ever dutiful fon NICHOLAS FERELIUS. 


\section{DONATION. THUNBERGIAN. CONTINUAT. XI. \\ Octandria.}

Diospyros tetrafperma. virginicts. lotus. a. $\beta$. kaki. $\alpha . \beta$. ebenum: $\alpha$. $\beta . \gamma$.

Vaccinium uliginofum. ๔. $\beta$. meridionale. fol. corynbofum.

frondofum. arboreum.

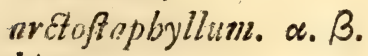
birtuns.

mucronatum.

Aamineum. \&. $\beta$. refinofum. fufcatums. amoemum. cilintum. $\alpha \beta$. bracteatum. alividum. ideun. cereum. cylindricum. corymbofum. frond ofuns. exaltatum. myrtillus. oxycoccus. ligustrinum.
Erica marifolia. dalcecia. $\alpha . \beta$. tenuifolia. bracteata. Thunbergii. tetragona. unabellata. a. B. petiolata. bryantba. fucata. $\alpha . \beta$. axillaris. imbricata. a. B. \%. \&. . melantbera. \&. B. leucantbera. Spumofa. $\alpha . \beta$. capitata. a. $\beta$. q. pasferine. totta. $\propto . \beta$. virgata. \& $\beta . \gamma . \delta$. cilinris. $\alpha . \beta$. bispida. Petiveri. a. $\beta \gamma$. Plukenetii. $\alpha . \beta$. cepbalotes. pulcbella. a. B. veftita. pinea. coccinea. $\alpha . \beta$. 


\section{* ) 104 ( )}

Erica cylinarica.

curunfor $\theta_{0} \propto . \beta_{0}$

tubigina.

glardulofa.

cerintionics. cis. $\beta$.

Sparmani.

Misfini.

sentustofia.

faftigiata. $\alpha . \beta, \gamma$.

com? $a$.

destutia.

vijcuris. a. $\beta . \gamma_{0}$

meciterranea.

nult ifora.

vagans.

berbacea.

cubica.

rosenagra.

simintacea. $\alpha . \beta$.

baccans.

phisyodes.

cermua.

retorta.

obliqua. $\alpha . \beta$.

vulgaris. $\alpha$. $\beta$.

ginplitodes.

miscofa.

corifolia.co. B. $\gamma, \delta . \varepsilon$.

calycina.

triflara.

fcopuria. $\alpha_{0} \beta . \gamma \cdot \delta_{\text {. }}$ Bergiana.
Ericas formofa.

rubers. c. B.

incapknta.

cincrea a. $\beta$.

aufiralis.

qe filuta. I-8.

wiveria.

firia.

pilibifera.

tetralix. a. $\beta$.

irsfiata.

abietina. $\alpha$. B.

mamiriofa.

empetrifolia.

spicata.

octoploylla.

glutinofa.

lutea. a. B.

depressa.

bulicicalon.

Monfoniana.

nişrita.

reger minans.

urealaris. $\alpha$. B. $\gamma$.

birta.

bicolor.

articularis.

wirefins.

plinifulia. $\alpha . \beta$.

pitbefcens, $\alpha . \beta, \gamma . \delta . \varepsilon$ birjuta. I). articulata. x).

\section{1) Blæriæ。}




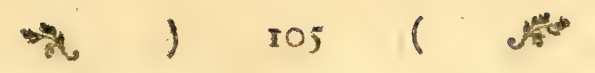

Eriga glabra. 1).

falciculata. r).

carteca, x).

ferreta. x).

blaeria: 3).

jurpurea. a. B. I).

pusilla. a. B. $\gamma \cdot 4)$.

plumofa I).

aliterss a. B. $\left.\gamma_{0} \mathrm{I}\right)$. penisuleta. I).

Grubbia rofnurinifotia. a. $\beta$.

Oenotber a longifora. «. $\beta$.

mollisfina.

biennis.

fruticofa.

smuricata.

pinnata.

pumila.

octovalvis.

Memecylon capitellatum.

Limsonia leuis.

inernis

fpirofa.

Awryris gileaden/is.

$$
\begin{aligned}
& \text { kataf. } \\
& \text { maritiona. } \\
& \text { fylucteris. }
\end{aligned}
$$

Melicocca bijuga.

Zumbolifer a pedunculata. \&.

Guarea trichilinides. a. $\beta$.

Hedmigla baljomefsra.

ofbeckia chinenfis.

$$
\text { ceilanica. }
$$

Rhexia miriona.

Gaura biennis.

in glabellum.

muntanun, $\alpha . \beta, \gamma_{0}$ paluftre. $\alpha . \beta$.

parviflorum.

angufifolium. a. $\beta_{\text {. }}$

alpinsm. ๔. $\beta$.

latifolium.

tetragonam.

roferim.

birfutum. a. $\beta$.

dubium.

gelidum.

perennis.

Allopbyllus rigidus.

ceilasicus.

raichojus.

cominia.

colbe.

Anticborus depresfus.

purpurea.

Oenother a rofen. a. B. $\gamma$. grandiflora.

perviflura.

$\mathrm{H} 2$

Conbretum loxum.

Backea frutejcens.

Tropecolum majus.

Tro-

1) Blæria ericoides Linn.

4) Erica nudiflora? Linn.

3) Blæria ericoides Linn. 


\section{* ) $106($ )}

Tropecolum minus.

bylridum.

Pasferianamiandra. eviocepbatis. peregrinuss.

Mimu uops kauki. eiengi.

Cblora perfuliata. a. B.

Fucblia cocinea. involucrata. excorticata.

Ximenia elliptica.

Dirca palufris.

Dapbne oleoides.

indica.

mezereum. 1. 2.

cheorum.

alpina.

gnidium. $\alpha . \beta$.

tartonraira.

villofa.

dioica.

calycina.

tbymelea.

Laureola.

foetida.

japonica.

odora. $\alpha . \beta$.

tinifolia.

occidentalis.

Stellera pasferina. a. $\beta$.

cbamajajme.

Pasferina cepbalopbora.

Setora.

ftricta.

Strutbiola nana. 5).

nervoja.

glonierats. $\alpha . \beta$.

grandiflora.

tinctoria.

capitata. $\alpha . \beta$.

Spicata.

linoides. a. $\beta$.

cilinta.

uniforn. $\alpha$. B. $\gamma$. antbylloides. $\alpha . \beta$. laxa. birfuta. \&. $\beta$. longifolia. cornucopie. levigata. a. B. axillaris. involucrata. gnidia. proftrata. villofa. pilasa. virgata. $\alpha . \beta$ s). evecta. $\alpha . \beta . \gamma$. ovata.

Gnidia dapbnafolia. capitata. Sericea. racemofa. a. $\beta$. tomentofa. carinata.

Gni-
5) Struthiolæ. 


\section{* ) $107($ ( )}

Gnidia fcabra.

argented.

imbricata.

filamentofa. \&. $\beta$.

biflore.

pinifolia. a. B.

oppofitifolia.

Hypelate trifoliata.

Dodonea vifcofa. a. $\beta$.

angufinfolia.

Populus tremula. beteropbylla. S. 9 . ballamefera. 0. aiba. 1. t. nigra. $1 . ?$ ?

Corylus avellana. maxima. fol.

Galenia procusubens. africina, $\propto \beta$.

Codia montana.

Moebringia muscofa.

Valentinia ilierfolin.

Werumanmia trifolinta. $\propto . \beta$. parviflora.

racemoja.

birts.

glabra.

tomentufa.

Acer monfpesfulanum. a. B. faccbarinum. $\alpha . \beta$. platanoides. montanum. penfylvanicum.
Acer tutaricum. trifidum. jol. creticum. negundo. a. B. $\gamma$. collinfonia. fol. campeftre. a. B. Feudoplatanus. a. $\beta$. vubrums a. $\beta . \gamma$. palnzatum. a. $\beta . \gamma$. pictum. fol. 7-lobtm. fol. japonicum. dissectuin.

Paulinia cartbaginenfis. $\alpha . \beta$. divaricata, fol. curasfavica. caribaa. bavbadenfis. pinnata. japonica. polyphylla.

Cardiospermusugrandiforum. corindunt. balicucabrive.

Sapindus cbinenfis. $\propto \beta$. Saponeria.

Coccoloba temuifolia. excoriata. 1. 2. nivea. uvifera. $\alpha . \beta . \gamma_{0}$ Polygonum ochreatum. erectum.

$\mathrm{H}_{3}$ 


\section{\# ) 108 (}

Polygonum bydropiper.

perfecaria.

filiforme.

b. platum.

oricstaie. a. B.

penfyluanicun.

arifolitam.

perfoliatum.

cbinense. a. B. $\gamma$.

tetaricum.

fagolyrums.

contuivulas.

dumetorum.

feandens.

Sericeum.

baccatums.

fibivicum.

viviparun. o. B. mesitiflorum. co. $\beta$. boftatsin.

undulatum. articulatum.
Polygonum misus.

frutefcens. $\alpha . \beta$.

virginicus, $\infty$. $\beta$. sipinum.

anguftifolium

aviculare. $\alpha$. $\beta$. $\gamma$.

maritimum. $\alpha . \beta$.

acidum.

crocatum.

fagittatum. a. B.

bistorta.

anppibium.

atrapbaxis. a. B. 6).

Adoxa moscbatellina.

Paris quadrifolia. $\alpha . \beta$.

Elatine bydropiper.

alfinafirums. a. $\beta$.

Myriopbyllum ceilanicum.

fpicatwm. verticillatum.

Msrgaritaria nobilis.

\section{Enneandria.}

Areca catechu. r-5.

Lauris benzoe, a. $\beta$.

indica. a. $\beta$.

foetens. $\alpha . \beta$.

affivalis.

chloroxylon. \&. B.

cinnamomum. $\mathrm{I}-9$.

casfia. fol. $\alpha . \beta$.

perfica.
Laurus borbonia. a. $\beta$.

pendula.

patens.

juatana.

coriacea.

exultata.

floribunda.

campbora. $\alpha . \beta$.

nobilis. I. 2.

6) Atraphaxis undulata. 
* ) 109 ( )

Laurus triandra. a. $\beta$. fasfafras. I-4. perviflura. calycina. paniculata. pedunculata. glauca. Iucida. umbellata.

Qvercus pbellos. a. B. robur. $\alpha$. $\beta . \gamma$. marylandica, fol. nigra. dentata. glauca. cuppidata. acuta. Servata. glabra. elonguta.

Quercus ilex. a. B. $\gamma_{0}$ reptans. palufris. fasber. fol. prinos. $\alpha$. B. fol. rubira. a. $\beta$. ajculus. fol. coccifera. cerris. as. B. alba.

Cas Jyt ba filiformis. cornuta. Mercurialis amman. ․ 웅 tomentofa. S.? perennis. 5. 早. procumbers. amabigus. androgyna. Butomus umbellatus. Hydrocharis rana.

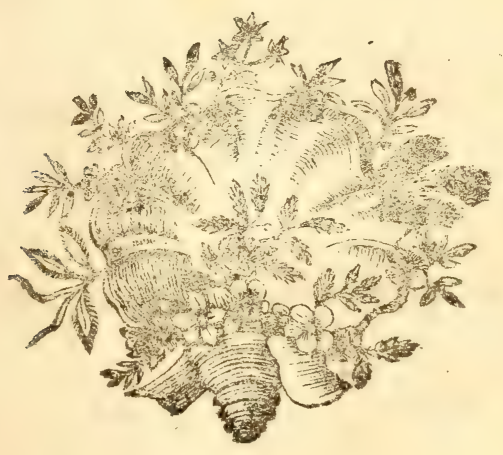




\section{THESES.}

\section{I.}

Cumb Staminum "umarys quandoque varict, fimillina nlias Genera, Bluria Ev Evica, ab iroicems Sparari non dibent.

II.

Nequ? Rbeum a Rumice leparare pportet, licet Stamina interdun novem, interdun ofo, interdum Sex.

\section{III.}

Sexum Plantarum noverunt Veteres, genevaliorem es/e perspexerunt Recentiores; uviverfalem esfe affirmavit Linnceus; in Mufcis demonfravit Hedvigius; infungis tefatur Hydnora, in Filicibus Zamia \& Cycas.

\section{IV.}

Flores pleni semina nulla proferunt.

V.

Exigua eft differentia Bulbun inter \& Genmam.

\section{VI.}

Genus Plante naturale, qoousque evitari potef, in plura artificialia dividi non debet, imprimis fi partium numerus non confuns fit. 


\section{D. \\ MUSEUM NATURALIUM ACADUMNE UISALIENSIS.}

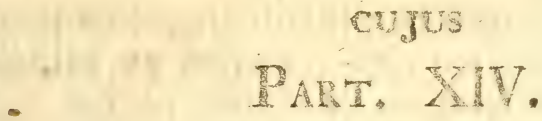

CONS. ENP. RACULT. HED, UPS.

\section{PRASTDE}
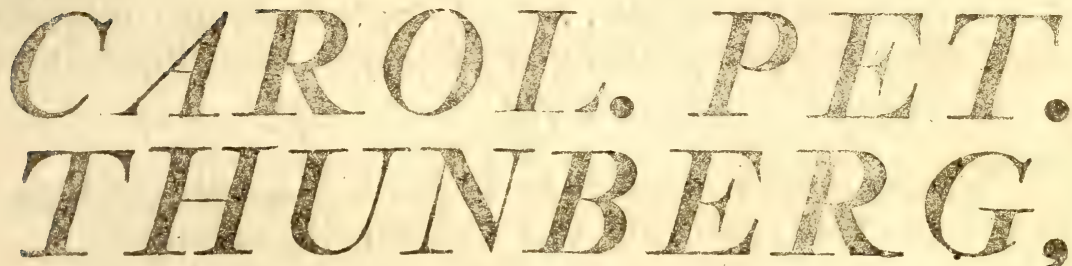

Equite Aurat. Reg. OKD. de Vasa,

Mrdic. Doct. Profess. Med. et Botan. Reg. et Ord, acalo Cresar. Nat. Curios, Reg. Scient, Londinens, Holmiens, Societ. Scient. Utsat.pmilab. Patr. Holm. Beror. Nat. Scrut, Paris, et Haeniens. Hist. Nat. Lund. Hartemo Amsterd. Zeland. Nivrosiens. Ticinens. Jenens. Halens. Nat. Scrut. Linn.Londin. Medic. Edimburg. et Nat.Studius.lbid.Membro; nec non Acad. Scient, Paris. Monspeliens. A gricuit. Paris. Medic. Londin. Florentin. ex Batavin, Ind. Or. Correspond.

\section{PUBLICO EXAMINI SUBMITTIT}

\section{NICOLAUS MATHESIUS,}

F $\mathrm{INO}$

IN AUDIT. BOTANIC. D. XXI DEC. MDCCXCIII. H. A. M. S.

\section{U P S A I FE,}

IITTERIS VIDUA DIRECTOR. JOHANN. EDMAR. 


\section{THESES.}

I.

Magnam fane prodere videntur ignorantiam atque leviratem vix ignofcendam, qui omnem ex Hiftoria $\mathrm{Na}$ turali Philofophiam in extilium abigere volunt.

II.

Hinc conficitur, valde falfum esfe cujusdam in $\mathrm{Hi}$ ftoria Naturali fcientiam, ex majori vel minori numero nominum plantarum, infectorum \&c. qux tenet, dijudicare.

III.

Herbaria inter ceteras in Hiftoria Naturali collectio. nes, maximam cum voluptate conjungunt utilitatem.

IV.

Cum tempus plantarum florendi vegetandique quam maxime variabile fit, differentiam fpecificam haud conftiruere posfe videtur.

V.

Naturæ convenientisfimum videtur, ut nomina generica fpecificis priora fcribantur.

VI.

Color, odor \& fapor, qualitates plurimorum vegeta. bilium produnt.

\section{VII.}

Vegetabilia æque ac animalia vivere, licet non fenCationibus gaudeant, pluribus conftat argumentis. 


\section{DONATION. 'THUNBERGIAN. CONTINUAT. XII. \\ Decandria.}

DiCtamnus allus.

Kleinbovia bofpita. :. 2.

Anagyris fetida.

Cercis faliquaforum.1.2.3.

$$
\text { canadenziss. }
$$

Sopbora alba.

$$
\begin{aligned}
& \text { aufralis. 1. } 2 . \\
& \text { tinEtaria. 1. 2. } \\
& \text { lupinoides. } \\
& \text { alopecwroides. } \\
& \text { occidentalis. } \\
& \text { tomentofa. 1. 2. } 3 . \\
& \text { beptapbylla. } \\
& \text { monofperma. } \\
& \text { japonica. } \\
& \text { tetraptera. }
\end{aligned}
$$

Baubinia divaricata.

variegata.

toment 0 a.

acuminata.

aculeata.

Spicata.

fcandens.

ungulato. fol.

Hymencea courbaril.

Parkinfonia aculeata. 1. 2.
Cefalpinia lijuga. fol.

crifta.

veficaria.

Jappan.

coriaria. t).

pulcberrima. t).

aculeata. *).

elata. 8. 2. t)

Gvilandine bonduccelle.

bonduc.

nuga. $\mathbf{1 . 2 .}$

moringa.

Casfia abfus.

viminea.

tora: r. 2. 3 .

bicapfularis.

emarginata.

falcata.

occidentalis. I. 2.

planifrliqua.

fericea.

fifula. x. 2.

piloja.

Senna. I. 2.

biflura. I. 2.

Serpens.

liguftrina.

t) Poinciana.

*) Poinciana bijuga. 


\section{$\Rightarrow \quad) \quad 121$ (}

Cassia marilandica.

fopbora.

alata.

berpetica. fol.

javanica. fol.

cbamacrita. 2. 3. 3.

glanduloja.

bineata.

virgate.

capenfis.

nictitens.

procumbens.

Adenantbera falcatari fcandens.

Cynometra caulifora.

Profopis fpicigera.

Hemsertaxylons campecbian. 1.2.

Misrraya exotica. 8. 2

Bereera kenigir.

Ruta graveolens. I. 2. 3.

montana.

sbalepanfis.

legitima.

pinnata.

paliens.

Tribulus maximus.

linifolia.
cifroides.

terreftsis. x. 2. 3.4.

lanuginofus. I. 2.

Schoria afra.

Grajacum officinale. 3. 2. janetinn. fol.

Frgonia indica. fol.

cretica.

Quisqualis indica.

Melafoma glabra.

Eksbergia conjerfis. I. . .

Trichilia mofchata.

birtio.

alliaces.

fpundioides.

follida.

Turve? puhecens.

Melia azedarach.

asudiracbete.

vivers.

Sroittenia mabagoni.

Helicteres ifsura. x. 2.

jomaicenfis. 1. 2.

angufuritolite.

glabreta.

levigate. 1. 2. 3.

racemora.

abicans.

argentea. fol.

gytedrungular is.

file deris.

wisg insa.

fornders.

montena.

jordifisod.

vitida.

impetinlaris.

at intervin.

rubers. 


\section{$\rightarrow$ ) $213($ N}

Melafioma purpurafcens. tetrandra.

prosera.

fafcicularis.

micrantbus.

tamonea.

capillaris.

umbrofa.

malabatbrica.

tibourbina.

bipida.

bivfuta.

bivelle.

potens.

pilofa.

Prigillofa.

El indulofa.

fabrofa.

boloferices.

Zygobyllum fomplex.

cordifolium.

fobrgo..

alhish.

wowg Jina.

fesfilfolum. a. $\beta$.

Spinofums.

macrophyllum.

retrofractum.

Heiperis coccrinea

Limoria matrobylla. I. 2.

ministis.

lisitis.

acida

trifgituta. i. 2.
Qvasfia amars.

ceilanica. fol.

excelfa.

fimaruba. 1. 2.

crocea.

Cletbra arborea.

alnifolia.

pubejcens.

occidentalis.

pariculatis.

Pyrola unifora.

rotundifolia. 1. 2.

minor.

Secunda.

umbcllats.

maculata.

Ledum polufive.

latifolium.

tbymifoliwm.

Monotrope bypopitbys.

unifiora.

Yusferia ereda. 5. 2.

repens. I. 2.

fuffruticofa.

nevvofa.

inclinetata.

Inocarpers edulis. I. A.

Styrasi occidentole.

oficinale.

grandifolism.

Anacardum occidentale. $\$-4$

Codore Roijeni.

Androgueda ovata. 


\section{*) $) 114$ ( औ}

Andromeda mariana. a. B. Rhododendron birfútum. anaftomofans. paniculata. calyculata. 1-5. tetrayona. polifolin. carulea. a. $\beta$. byproides. rupeftris. japonica. $\alpha . \beta$. fafciculatia. jamaicenfis.

racem fil. pilulifera. axillaris. arborea. laurifolia. diffufa.

Arbutus micropbylla.

ur $/ \mathrm{i}$. alpina. rigida. a. $\beta$. pumila.

unedo. andracbne. procera.

Rbododendron laurinum. douricuin. chryfantb. ponticum. maximum. ferrugineum. cbanseciftus.

Kalmia glauca. latifolia. r. 2.

Epigar repens. anyulfifolia.

Gaultberia procumbens. antipoda.

Copaifera officinalis. fol. Terminalia arbufcula. latifolia. cat appa. b'enzoè. fol. mauritiana.

quercifolia. fol. Bucida buceros. Samyda parviflora. villofa. spinefcens. glabrata. tenuifolia. decandra. Serrulata. sylveftris. Augea capenjis. Dais oetandra. difperma. fol.

Sclerantbus perennis. 1. 2. annuus. 1. 2. polycarpos.

Triantbema anceps. bumifufa. 


\section{* ) $185(10$}

Triantberna pentandra.

$$
\begin{aligned}
& \text { monogyna. } \\
& \text { decandra. }
\end{aligned}
$$

Cbrysopplenium alternifolium.

1. 2 . oppofitifolium.

Carpinus betulus. I. 2. oftrya.

Roijetsa glabra. x. 2. pallens. lucida. villola. birfuta. 2. 1. 2.

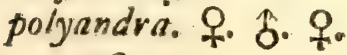

Cunonin capenfis. Hydrangea arborefcens.

Tiarella cordifolia. Mitella dipbylla.

Dianthus prolifer. 1. 2. 3. armeria. 1. 2. deltoides. x. 2. 3. alpinus. I. 2. atrorubers. japonicus. burbatus. 1-5. cartbufianorum. I. 2. ferrugineus. caryopbyllus: monftropomeridianus. glaucus. I-5. cloinen/is. 1.2. monjpelienjis. I. 2. bujpanicuis.
Diantbus plumarius."

Superbus. r: 2. 3. crenatus. arenarius vir gineus. cefpitofus. Jcaber: arboreus. 1. 2. fruticofus. pungens.

Arenaria flaccida. fusciculata. verna. procumbens. imbricata. maritima. $\mathrm{I}-3$. linifhra. ceraftoides. peploides. tetrinquetra. bifiora. laterifora. trinervia. ciliata. balearica. multicaulis. Serpyllifolia. 1-3. triflora. montana.

medisso faxatilis. bispida. tentifolia. laricifolia. 


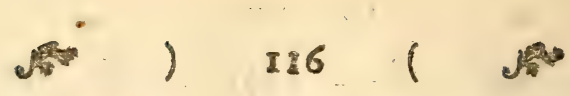

Arenorio friar.

$$
\begin{aligned}
& \text { हैimationa. } \\
& \text { ablinara. }
\end{aligned}
$$

Stellaria mintulals.

$$
\begin{aligned}
& \text { boleftea. } \\
& \text { bypericifolis. } \\
& \text { cerafuides. 2. 2. } \\
& \text { sliginoja. } \\
& \text { biflora. } 3.2 . \\
& \text { nemsor usis. I. } 2 . \\
& \text { Scoperia. } \\
& \text { graminea. 1. } 2 . \\
& \text { craslifolia.: } \\
& \text { radicans. }
\end{aligned}
$$

Saxifraga tricufpidata.

bulbifera. bypnoides. 1. 2. 3 . farmentofa. I. 2. tridactylates. I.5. petraa. penfylvanica. granulata. flor. plerso. umbrofa. broncbialis. odorata. ajpera. fibirica. cafía. 1. 2. mutata.r.2. adrcendens. punctata. rotundifolia. I- $\hat{3}$. burferiana. clufii. 1. 2.
Samifraga fuperba. bivfuta. cutylecion. 2. 2. 3 . cafpitofa. r. 2. 3. ardirofesea. branides. ojugifolia. bitora. mulcoides. aizoides. curreifoliz. I. 2. aizoon. pedemontana. lingulata. mojcbata. Aellaris. 3. 2. 3. feaioides. autumnalis. I. 2. vivulavis. nivalis. 12. oppofitifolia. geranioides. crasfifolia. cordifolia. cormua. birculus.

Silene cretica .I 2. conica. conoider. elongata. acaulis. 1. 2. vallefia. I. 2. noctiflora. I. 2. 3. nocturna. 1. 2 . 
क्षै

Silene anglica.

lusfitanica.

5-vwinera.

cernua. \&. B.

gallica.

ceraftoides.

mutabilis.

nutans.

amcena.

paradoxa.

frutico/a.

crasfifolia.

viridiflora.

bellidifolia. I. 2.

beben.

fricta.

pendula. I. 2.

virginica.

atocion.

antirrbina.

rubella.

inaperta.

portenfis.

muscipula.

armeria.

rupeftris. 1. 2.

faxifraga.

fibirica.

tripolitana.

nis aen/is. 1. 2.

auftralis.

alpeffris.

Cberleria Sedoides.
17

Deutzia fabra. a. B.

Garidella nigellaftrum.

Erytbraxylon bavanesse. areolatum. I. 2.

bypericifolizw:

scbinus mollis.

indica. $\mathrm{x} . \mathrm{x}$.

areira.

Gypjopbila paniculata. perfolinta.

faxifraga.

muralis, r. 2. 9.

profivata. 1. 2.

altisfima.

corymbofa.

Arutbium.

dicbotoma.

adfcendens.

rigida.

repens.

fuftigiata.

Saponaria lutea.

illyrica.

porrigens.

vaccaria.

cretica.

ocymoides. I. 2.

orientalis.

officinalis.

Cucubalus beben. I 4 . catbolicas.

tataricus. I. 2 


\section{*) 118 ( 残}

Cucubalus alpeftris.

fabirius.

vifco us.

baciferus.

maritinus.

fellatus. I, 2.

cblorantbus.

reflexus.

glutinofus.

otites. 余. 옹.

fibiricus.

mollisfimus,

reflexus.

italicus.

regytius.

dauricus.

Kiggelaria africana. I. 2.

Spergula nodofa.

pentandra. I. 2.

fubulata.

Saginoides.

arvenfis.

Agroferma coronaria.

jovis.

calirafa.

gitbago.

nicaenfis.

Pentborum sedoides. a. $\beta$.

Lychnis coronata.

4-fida.

4-dendata.

dioica. S. 9 .
cuculi. a. B.

Lycbnis minima.

apetala.

alpina. 1. \&.

vifinia. $\alpha . \beta$.

chalcedonica.

speciofa.

Bergia capenfis.

glomirata.

Oxalis monopbylla.

acetofella. 1. 2.

minuta.

punetata.

nistnus.

compresfa:

purpurea. 1. 6.

coprina. 1. 2. 3.

cernua.

lanata. I. 2.

fericen.

violacea.

repens.

bifida.

glabra. I. 2.3.

birta. I-4.

verficolor. I-4.

incarnata.

corniculata.

fritta.

flava.

comentosa.

senfitiva.

Malpigbia faginea. fol.

lucida. fol. 


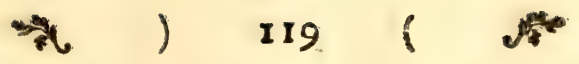

Malpigbia nitida.

urens. I. 2.

coriacea.

glabra.

verbufcifolia. fol.

excelfa.

bunisifterioides.

coccigera.

punicifolia. fol.

craslifolia. flor.

aquifolia.

Banniferia bengalenfis.

$$
\begin{aligned}
& \text { fulgens. } \\
& \text { laurifolia. } \\
& \text { longifolia. }
\end{aligned}
$$

Triopteris jamaicenfis. 1. 2.

rigida.

citrifolia.

Ceraftium dicbotomum.

manticum. 1. 2.

lineare.

latifolium. r. 2.

alpinum. I. 2. 3 .

pentandium.

perfoliatum.

fernidecandrium.

arvenfe. I. .

Arictum.

tomentofun.

vifcosuns.

rbaticum.

vulgatum. r. 2 .

repens.

fuffruticofun.

Ceraftium aquaticum.

lanatuns.

Sedum ceruleum.

ansuxm. 1. 2.

pubefcens.

violaceun.

tslepbium.

giaucum.

anglicum.

bifpanicums. I. 2 .

cederi.

fpinofum.

villofum.

populifalium. I. 2.

rupefite.

fellatum. 1. 2.

bybridum.

cepar.

atratum.

anacampferos. I. 2.

aizoon.

dajypbyllum.

albusnt.

6-angulare.

acre.

veflexum.

liseare. 1. 2.

$R$ hodiola rojea. $\hat{0}$. $q$.

Cotyledon orbiculata.

cuneata.

triflora.

paniculata.

baenspbarica. fol.

purpuren. 


\section{$\Rightarrow 12012$}

Cotyledon teretifolia:papillaris. suammallaris. I. 2. cacalioides. reticulata. mulizonia. bifpanica. umbilicus. laciniata.

Carica Spinofa. I. 2. popna.

Spondias dulcis. papaja. 1. ?. myrobalarus.

Averboa carambola. bilimbi.

Coriaria farmentofa. myrtifolia. I. 2. rufcifolin.

Suriana maritima.

Neurada procumbers.

Pbytolacca octandra. 3. 2. decandra. disica. icofandra.

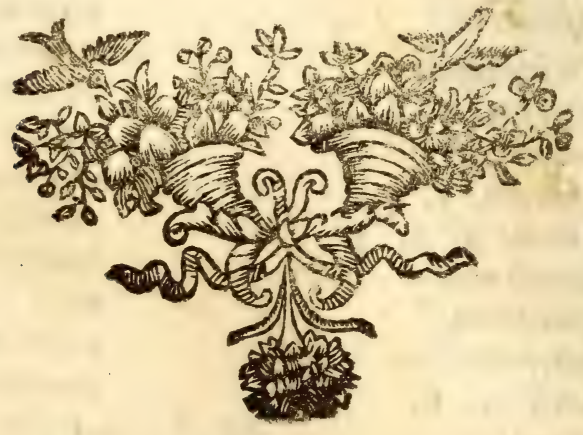


D. D.

\section{MUSEUM NATURALIUM ACADEMIE UPSALIENSIS.}

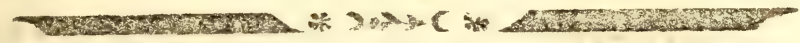

\section{cujus}

PART, XV.

CONS. EXP. FACULT. MEDIC.

PRESIDE
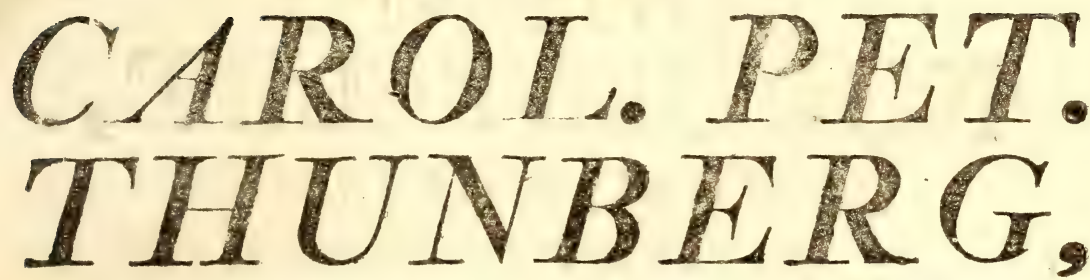

Equite Aurat. Reg. Ord de Vasa,

Medic. Doct. Profess. Med. et Botan. Peg. et Ord. Acad. Casar Nato Curios. Reg. Scient. Londin. Holm. Socfit. Scient Uspat. Philad. Patr. Horm. Berol. Nat. Scret. Paris. et Hafniens. Hist. Nat. Lun . Hartem. Amsterd. Zeland. Niprosiens. Ticinens. Ienens. Halens. Nat. Scrut. Linn. Londin. Medic. Edimburg. et Nat. Studros. Ibid. Membro; Nec Non Acad. Scient. Paris. Monspeliens. Agricult. Paris. Mfdic. Londin. Florentin. et Batavin. Ind, Or. Correstond.

\section{PUBLICO FXAMINI SUBMITTIT}

\section{MAGNUS HEDREN;}

STIP. REG. VERMELANDUS.

IN AUDIT. BOTANIC. D. XVI. APRIL. MDCCXCIV. H. A. M. S.

U P S A L I E,

ITTERIS VLDUE DLRETOR. JOHANN. EDMAN. 


\section{VIRO}

\section{GENEROSO ET NOBILISSIMO,}

\section{DOMINO}

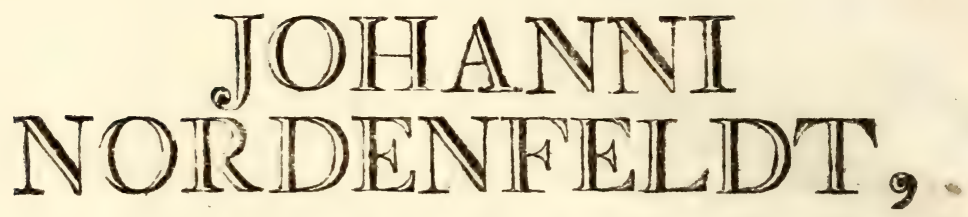

CAPITANEI LOCUM TENENTI, BOTANOPHILO SUMMO, PATRONO MAXIMO 


\section{DONATION. THUNBERGIAN. continuat. XIII. \\ Dodecandra.}

Tbeligonum cynocrambe.

A Soruth europeum. cunadenfe. virginicunt.

Cytinus bypociftus. Ceratopbyllum fubmerjum.

$$
\text { demerfums. }
$$

Bosconia frutejcens. Huafonia ericoides. Tomex japonica. Apactis japonica. Rbizopbora mangle. gymnorbiza. cylindrica.

Halefia tetraptera. Crateva gynandra. marmelos. tapia. religiofa.

Garcinia mangoftana. Eurya jnponica. I. 2. Triumfetta lappula. procumbens. urticafolia. Semitriloba. bartramia.

Peganum barmala. gmelini. dauricum.

Dodecas furinameinis.

Nivaria foboberi.

Canella alba. Portulaca anacampseros fol. triangularis. pallens.

meridiana. oleracea I. 2. fruticoja. pilofa. trigona $\mathrm{x}$. 2. quadrifida.

Lytbrum tbymifolium 1. 2. bysfopifoliums I. 3. tenellum. fruticofum. parfonfia. ciliatum. cordifolium. melanium. cupbara 8. 2. verticillatum. pempbis. virgatum 8. 2. falicarin. 1. 2.

Blakea trinervia. triplinervia.

Befaria aftuans. Basfáa longifolia. 


\section{-}

Decumaria barbara.

Euclea lancea.

capenfis है. ?.

undulata. $\delta$ 1. 2 9.

Agrimoni repens $1 \cdot 3$.

eupatoria.

agrimonioides.

Datifca cannabina.

Re feda sapenfis.

fesamordes. I. 2.

glauca.

odorata.

luteola.

caneficens.

lutea.

undulata.

phyteuma.

alba.

fruticulofia.

Heliocarpus americana. fol. Menifpermum trilobstum. I.

japonicum. s. 9 .

acuminatum. fol.

canadente. 소. ㅇ.

carclinum.

glabram. \&.

os biculatum. ㅇ. 웅

Juglans alba. 1. 2.

oblonga.

nigra. I. 2.

vegia.

biccata.
Fagus pumila.

caftanea 1. - 3 .

fyluatics. 1. - 3.

Espporbsa armata.

antiguoruas.

canarien/ss.

mammillaris.

officisar urs.

rudiata.

fa/ciculata.

coronata.

medufe I. - 3o

mauritanica.

punicea.

falicina. foll.

nevirfolin.

tithymaloides.

cotinifolia.

muricata.

tirucalli.

maculata.

rubra.

origanoides.

byperisifolia. 1. 2.

birta.

pilulifera.

thymifolia. 1. 2.

parvifora.

canefcens.

cbamxjyce.

peplis.

polygonifolia.

granivea. 


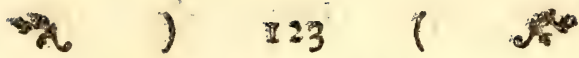

Eupborbia ipecacumbe. portulacoides. myrtifolia. obliterata. atoto. linifolia. glabrata. terracina. pubefcens. retufa. peplus. 1. 2.

falcate. exigua. tuberofa. elliptica. latbyris. 1. 2. illyrica. glauca.

geniftifolia. r, 4. jpinoja r. 2. epithymoides. dulcis. pitbyufa. portlandica. faxatilis. paralias. pinea. Segetalis. beliofcopia. I. 2.
Eupborbia serrata. verrucofa. corollata. I. at: coralloides. I. 2. pilofa. orientalis. platyphyllos. efula. cyparisfias. I. 2. Friata. mysfonites. palueris. byberna. jylvatica. cbaracias. prateness.

Vifmea monerera. Pallaja caspica. 1. 2. Tacca pinnatifida. 1. 3. Glinus ononides. lotoides.

Sempervivum tortuofum. ar acbrites. globiferwan. fol. teEtorums. arboreum. $x-3$. montanums. birtum.

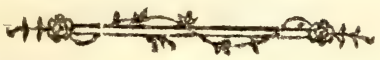




\section{) $124(2$}

Icosandria.

Cactus mammillavis.

grandiforus.

triangularis.

Alagelliformis.

tetragonus. caul.

perefkin. fol.

pbyllantbus. fol.

pentagonus. caul.

ficus.

curasfnvicus. caul.

peruvianus. $f$.

tuna. caul.

bexagonus.

opuntia.

cocbenilizfer.

pendulus.

Eugenis uxifora.

recemofa.

acutangula.

malaccenfis.

jambos. I. 2.

caryopbyllata. 1. 2.

Pbiladelphus inodorus.

coronarius.

Pfidium pyriferum.

pomiferum. 1. 2.

myrtifolium.

montansm.

Myrtus moururi.

levis.

communis, $\alpha . \beta . \gamma . \delta . \varepsilon$. brafilienfis.
Myrtus anguftifolia. 1. 2.

alpine.

caryopbyllate."

ceilanica.

difticba. a. $\beta$.

unbellata.

cumini.

dioica.

pimenta.

biflora.

punctata. fol.

crenulata.

splendens.

monticola.

glabrata.

cordita.

acris.

legufrina.

virgultoja.

lineati.

greggii fol.

parvifora.

buxifolia. $\propto . \beta_{2}$

aprica.

procera.

fragrans.

elliptica. latifolia.

Calyptrant bes cbytraculia.

zuzygium.

rigida.

Punica granatum.

nana. 
Amygdalus orictutalis. fol. communis. I. e) perfica. I. - 3. nama. 1. 2. 3. puimila. 1. 2.

oresus spberocarpa. virginica. 1. 3. padus. penfylvanica.

\section{nana.}

infititia. Iufitanica. laurocerafus. cerafus. $\alpha . \beta, \gamma, \delta_{0}$ armeniaca. mabaleb. 1. 2. pumila. glandulora. aspera. tomenives. japonica. paniculata. elliptica. incifa. cbamecer afus. domeftica. avium. $\alpha . \beta$. Spinofa. T. 2. occidentalis. fol.

Cbryjobalanus icaco.

Sonneratia acida. Ilini a peduncalata. acida.
Crategus arbutifolia, a. $\beta$. lavis. bybrida. monogyna. 1. 2. 3. torminalis. azarolus. oxyacantba. indiea. glabra. a. B. aria. 1. 2. villofa. dulcis. I. 2. tomentoja. corcinea. lucida. cuneifolia. viridis. punEtata. calearata. plicata. galli. rotundifolia. pyrifolia. maura. acerifolia.

Sorbus áucuparia. domeftica. 1. 2. bybrida. americana.

Sefuvium portulacaftrum.

Spiraa cbomadvifolia. a. $\beta$. falicafolia. ulmaria. $A_{3}$ incifa. 
Spirea palmata.

bypericifolia. x. 2.

lavigata. \&. 2.

trifoliata. 1. 2.

crenata. a. B.

forbifoiia.

alba.

lobata.

tomentora.

opulifolia.

aruncus. 1 - 3.

filipendula.

Tetragonia expanfa.

fruticofa.

birfuta.

berbacea. 1. 2.

Jpicata.

procunzbers.

evecta.

Aizoon canariense. bispanicum.

rigidum.

fecundum.

glinoides.

paniculatum.

fruticofunn.

farmentofuns.

perfoliatum.

Mezembryantbemum lineare.

criniflorum.

digitiforme.

teficulare.

truncatum.

moniliforme.
Mizembryantbe-

mum fafigiatum.

nodiflorwm. I. 2 .

cryfiallinum. 2. 2.

copticum.

geniculiflorum.

nofifirum.

decusfintum.

jplendens.

unliellatum. $\alpha . \beta . \gamma_{0}$ expanfum.

tripolium.

emurcidum.

avticulatum. I. 2.

ciliutum.

corallinum.

fafcisulutum.

fili orme.

caliniforme.

opotaliams.

ovaturs.

linceums.

cordifoliums.

beililifolium.

deitoideum.

barbatum.

bispidum, $\propto . \beta, \gamma$. capillare. $\propto . \beta$.

Sesfille.

tricbotonusn.

scabrum. 1. 2. 3.

emarginatums.

uncinatum.

Jping uns. $\propto$. ß. $\gamma$. 


\section{减) $127(12$}

Merembryantbe. Mespilus japonica. muses tenuifoliunt. \&. B. Pyrus japonica. ftpulaceum. crasfifolium. loreum. fol. acinaciforne. pomeridianum.

fabulofuns. diff urme. alvidum. vingens. o. B. $\gamma$. dolabriforme. 1. 2. pugioniforme. 1. 2. pruisofum. aureum. lave. vervuculatum. angwlitum, $\alpha$. B. $\gamma$. edule. bicolor. ferratum. fol. . micans. papulafum. tetragonum. glaucums. tortuofuns, a. $\beta$, fol. pinnatifidum.

Mespilus arbutifolia. phonopyrum. pyracintba. canadenfis. anelanchier. I. 2. cbameme/pilus. germanica. cotonenfter. I, 2. baccata. cydonia. I. 2. polveria. coronaria. communis. malus. butryapium.

RaJ a Perfica. eglanteria. cimnamomea. $\alpha$. $\beta$. arventis. pimpinellifolia 1. 2. ppino/isfima. rugofa. virginica. carolina. villosa. Sempervirens. rubigino a. centifolia. gallica. pumila. collina. finica. alpina. pendulina. alba. a. B. carina. indica. multiflora.

$R u b u s$ cahus. tripbyllus. cotonenfler. r. 2. 
villofus.

palmatus.

trifidus.

incifus.

jonaticenfis. o. B.

dalibardus.

multifurus.

Idous 1. 2.

fruticofus.

odoyatus.

indicus.

cbinenfis.

parvifolius.

fvaveolens.

faxatilis.

cbamemorus. 合. ․ auftralis.

arcticus.

moluccanus.

occidentalis.

Tormentilla eveta.

reptans.

Dryas octopetala.

pentapetala.

geoides.

Fragaria Monopbylla.

vefca. 1. 2. 3.

Potentilla tridentata.

niven.

grandiflora. a. $\beta$. monspelienfis.

nolvegica.

seta.

fibirica.

nivalis.

Aipularis.

glutinofa.

valderia.

alba.

alchemilloides.

clufiann.

caulefcens.

argentea.

birta.

canadenfis.

reptans.

opaca.

verna.

aurea.

intermedia.

fericen.

multifida.

fruticofa. I. - 3 .

anjerina.

bifurca.

pimpinelloides.

fupina. penfyluarica.

rupeftris.

fragarioides. 


\title{
X. ) $129 \quad(1 \%$
}

Goum japonicum.

rivale. $\alpha . \beta . \gamma . \delta$. montanum. $\alpha . \beta$. virginicum. urbanum. $\alpha . \beta$. capense.

\author{
Geum alpinum. \\ canadense. \\ reptans. \\ Comarum paluftre. \\ Calycantbus precox. \\ floridus. $\alpha . \beta$.
}

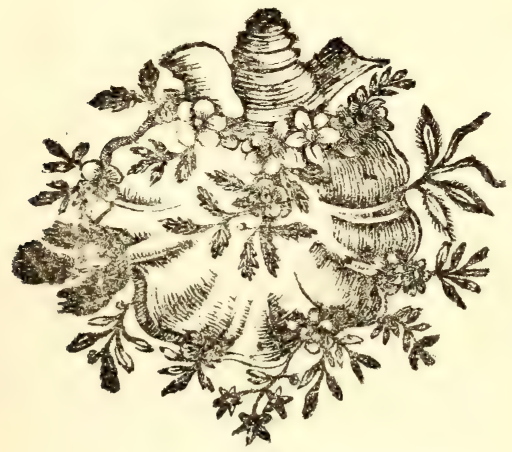




\section{THESES.}

I.

Liber naturæ, foliis conftans infinite multis, ad crea. torem venerandnm attentos ducit obfervatores.

II.

Tria, qux ftatunntur naturæ, regna non ubique certos oftendunt limites.

III.

Herbarum cognitio in quocunque vitæ genere prodeft.

IV.

Botanica \& Entomologia fibi invicem ita inferviunt, ut unam perfecte callere nequeat, qui in altera fic hofpes.

V.

Nullum datur animal aut vegetabile, quod fit abfolute venenatum.

\section{VI.}

Quid medicinæe ftudiofo fit fcitu necesfarium optime peritus judicat medicus.

VII.

Multa exiguam aut nullam oftentant utilitatem, qua tamen re vera utilia funt.

\section{VIII.}

Chemix in botanica imprimis applicata non exiguus eft ufus.

\section{IX.}

Mos, qui jam diu invaluit, herbas a certis nominandi cultoribus, non vanam ambitionem, fed gratum erga Botanophilos prodic animum. 


\section{D. $D$. \\ MUSEUM NATURALIUM ACADEMIE UR SALIENSIS. \\ Qung \\ cujus \\ PAET, XVI. \\ VENIA EXP. ORD. MED. UPS PR ES IDE
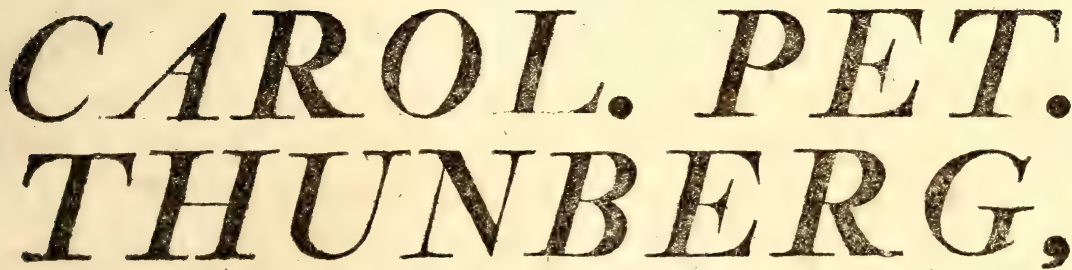

Equits Aurat. Reg. Ort. de Vasa,

Mrdic. Doet. Profess. Med. et Botan. Reg. et O d Acad. Cesar. Nat.

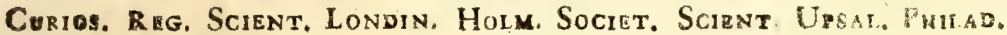
Patr. Hotm. Beror. Nat. Scrut. Paris. et Hafniens. Hist. Nat. Lund. Hartem. Amsteld. Zeland. Nidgosigna. Ticinens. Ienens. Halens. Nat. Scrut. Linn. Londin. Medic. Edimburg. et Nar. Studios. Inin. Membro; Nec Non Acad. Scient. Paris. Monspeliens. Agricult. Paris. Medic. Londim. Florentin. et Batavin, Ind. Or. Cormespond.

PUBLICO EXAMINI OFFERT

\section{SVEN ALGURÉN, \\ SMOLANDUS.}

IN AUDIT. BOTANIC. D. XIX. JUNII MDCCXCIV.

H. C.

$$
\text { U P S A L I F, }
$$

ITTERIS vIDUE DIRECTOR. JOGANN. EDMAN, 


\section{THESES。}

I.

Nugas antiguorum, generationem fintuentium equivocam, diu non fone causfa a perfpicacioribus explofas comperimus.

II.

Zoologic cognitionem bumano es fe generi admodum neces: faviam, eum credimus flatim fore confesfurum, qui ufuum ac incomasodorum, ad nos ex animantibus redundantiun brutis, leviter meniznerit.

\section{III.}

Hiftoriam naturalem fcientice dofribendo nomine, in que corporum naturalium cognitio certo ac determinato eft ordine tradendie, band male nos facere autunamus.

\section{IV.}

Quam profecto in muserum fcientiarsm, fatis firmis nos: convincere valentium rationibus, immen fa reperivi Numen potentice, rilatan volumus.

\section{V.}

Cwjus ideo cruditione prieditum non lubrica, in caftra tranfilire posfe atbeiftarum, magna adeft dubitondi ratio.

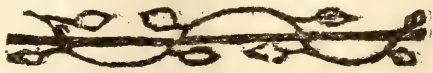




\section{DONATION. 'THUNBERGIAN. CONTINUAT. XIV.}

Polyandria.

Marcgravia umbellata. Posfira finplex.

Pariana campeftris.

Capparis spinofa.

frondufa. I. 2. 3.

Breynia. a. $\beta$.

filiquora.

cysapballopbara.

borrida.

Sepiaria.

capenfis.

ferruginea. a. $\beta$.

morifoni.

longifolia. fol.

formofa.

grandis.

tripbylla. a. B.

ACtea japonica.

Spicata.

vacemofa.

cimicifuga. 1. 2.

Sangvinaria canedenfis.

Podopbyllum peltatum.

dipbyllum. fol.

Cbelidonium japonicum.

majus. $\propto . \beta$.

bybridum. x. 2.

corniculatum. I. 2.

glauciwm.
Papaver bybridum.

alpinum.

nudicaule. 1.2

vbeas. .7. 7 .

dubium.

orientale. cambricuns.

fomnifer um. I-6.

aculeatum.

Argemone mexicana. I. $x$. tomentofa.

Cambogia gutta. fol. Muntingia calabura.

Sarracenia purpurea.

Alava.

Nympbea lutea.

albe.

lotus.

capenfis.

nelumbo. 1-5.

Chryfitrix capenfis.

Bixa orellana. 1. 2.

Mammed americana.

Barringtonia speciofa.

Ochna jabotap.

Sguarrofa.

nitida.

Grias caulifiora. 


\section{* ) $133($ ( )}

Inopbyllusn calopbyllum. x. 2. Lecytis ollavia. calaba. 1. 2. nagasfarium.

Sparrmannia africane. I. 2. Clufia pediceilata. jesfilis fol.

Tilia europar. a. $\beta$. pubefsens. fol. americana.

Ligbtfootia indica. 8. ferrata.

Letise guidonia.

Acidoton uretis. 5. ? Hedyo/mum arborefcens. Delima farmentofa. Ternftrcemia mendionalis. nutans.

Grenoia orientalis. I. 2. occidentalis. x. 2. mallococca.

microcos. latzfolin. tomentofa. triflora. acuminata. afratica. 1. 2.

Marila racemofa. falvifolia.

Eleocarpus ferrats. dentatn.

Legnotis elliptica. casfipurea. japosica.

Melaleuca jeoparia. diffrufa. perforata. lucida, fol. virgate. aftuofa. cillata. forida. leucadendra. I. 2 . curdata.

Eucalyptus obliqua.

Vateria indica.

Looja bifpida.

Xylopia muricata.

frutescens.

Lagerftrainia indica, 8. 2. javanica.

Thes boben. x. A. viridis.

Eroteum ibeoides. sndulutuin. 1.2.

Ciftus libanotis. monspelienfis, a. B. 2 . laduniferus. crippus. albicans. X. 2. latus. 


\section{$* 2) \times 34(1$}

Ciftus vaginatus.

lauvifoliss.

falvifolius, $\alpha, \beta$.

villofus.

creticus.

incanus.

populifolius.

halimifolius. a. $\beta$.

funana.

levipes.

umbellatus.

canus.

marifolius.

rofers.

celandicus.

calycinus.

guttatus.

tuleraria.

canadenfis.

pilopelloides.

lavandulifolius. $\alpha . \beta$.

lanceolatus.

lunulatus.

falicifoliss.

ledifolius.

aegyptiacus.

pilofus. $\alpha$. B. 2.

Squamatus.

numsmularius.

glutino/us.

racemofus.

arabicus.

birtus.

apenninus.

Cijtus beliantbemum.

furejanus.

thymifolius.

Cycas circinalis. 1-6. 9. ‥ revolute. I. 3. fol.

Zarnis pumila.

caffra. 合. ㅇ. x. 4.

Caryota urens. I-5.

Poterium /pinofum. x. 2. bybridum. fanguifirba.

Liquidambar Ayraciflusm.

peregrinuns.

Corcborus flexuofus.

ferratus. fol.

feandens.

japonicus.

tomsentofus.

clitorius.

trilocularis.

tridens.

aftuans.

cap/ularis.

birfutus. $\alpha, \beta$.

birtus.

filiquo/us.

Mimofa funveolens.

mangium.

unguis. $x .2$.

latifolia.

B 3 


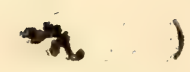

Mimofa viva.

Senfitiva.

pudica. 1. 2.

inga. I 2. 3.

fagifolin: fol.

nodofa. fol.

Burgoni.

Entad.

virgata.

punctata.

pernambucana.

erborea.

Speciosa. T. 2.

grandifiora.

comofa.

latifiliqua. fol.

mangen/is.

jaligera.

nilotica.

farmefiana.

borrida.

tortwoja.

caffra.

ajperata. 1. 2.

cafia.

pennata.

tenuifolia.

tamarindifolia.

Uvaria paniculata.

discreta.

birfuta.

japonica.

ceilanica x. 2.
I35

Gnetum gnemon.

Annona triloba.

glabra.

reticulata.

paluftris."

fauamefa. I. 2.

muricats.

Cliffortia evicafolia. teretifolia. ㅇ․ 오. jumiperina. 5.8 filifolia. S. cinerea, \&.

Atrobilifera. 1.8 . graminea. ₹. ?. vucifolin $\hat{f}$. $q$. ilicifolia of. Serrata. A. odorata. 5 . pulcbelia. . 9 . srenatas 8.9. obcordata. 9. trifoliata. 8 . 9 . ternata. S. 9. falcata. 숭. farmentofa. I.

Hedycaria dentata. Xylofma orbiculatuss.

Peconia albifora. tenuifolia. fibirica. anomala. officinalis.

Fotbergilla gardeni. 


\section{) $136(1 \%$}

Begonia grandis.

macropbylla.

foandens.

birfuta.

Homalium racemofum.

Pandanus odorata. 1-3. 1.?

Nigella bijpanica.

Aquilegia vi cofa.

cronadenfis.

alpina.

viridiflora.

vilgar is. I-4.

cblorantba.

Wablbonia indica. damajcena.

Sativa.

orientalis.

arvenfis.

Delpbinium anebiguum.

confolida.

ajacis. 1-5.

aconiti.

elatum. $\alpha . \beta$.

atropurpureums.

peregrinum.

grandiflorums.

altaicum.

Reaumuria vermiculate:

Stratiotes aloides.

alifmnides.

Zoftera oceanica.

marina.

Arum ringens.

ferratum.

virginicum.

carnafolium.

lingulatum. fol.

pictum.

peregrinum.

puniceum.

ftaphifagria.

Aconitum japonicum.

lycoctonum. 1-3.

antbora.

volubile.

arifarum.

purpureum. colccafin. fol.

macrorbizon.

efculentum. arhorefcens.

pyrenaicum.

variegatum.

cammerum.

uncinatum.

orientale.

napellus:

fagittefolium. fol.

maculatum.

trilobatum

tripbylluz. $\alpha . \beta$.

ternatum.

auritum. fol.

mufcivorum, I, 2. 3. 


\section{- ) 137 (}

Arum dracontium. dracunculus. 1. 2.

Dracontium jpinojum. I. 4. polypbyllum. pertufum.

Caltba paluftris.

Houtuynia cordata.

Platanus orientalis. occidentalis. I. 2.

Wintera aromatica.

punetata. axillaris.

Dillenia retula.

dentata.

integra.

Ipeciofa.

enfata. fol.

Illicium anifatum. x. 2. for adanum. atbiopica. I. 2.

Anemone bepatica. ¿. $\beta$. patens. $\alpha . \beta$. alpina. $\alpha . \beta$. fulpburea. $\alpha . \beta$. pratenfis. x. 2. pulf.tilla. 1. 2. vernalis. 1. 2. valdenfis. coronaria. I-4. bortenfs. 1. 2 . pilmata. trifolia. nemorofa, a. $\beta$. ranunculoides.

apernina.

dicbotoma. penfylvanica. Jylvejtris. 1. 2. virginuca.

narcisfiflora. 1. 2. 3. thaliEtroides.

Liriodendrumtulipiferum.1.2. Atragene indica. Magnolia grandiflora. 1. 2.3.

glauca. 1. 2. 3 . acuminata. 1.2. tomentofa. birfuta. tripetala. 1. 2. obovata. fol. fericea.

Micbelia shampaca. t fjampaca. cernua. japonica.

alpina. 1. 2.

ceilanica. 1. 2.

capenfis.

tenuifolia. I. 2.

trifoluata. fol.

Clematis erecta.

integrifolia. fol. x.2. paniculata. cirvbofa. viticella. 
Clematis viorna.

crifpa.

orie talis.

florida.

juponica.

bexipetala.

virginica.

bracbiata.

triloba.

dioica. I. 2.

vitalba.

flammula.

japonica. fol.

cbinenfis.

Thaliatrum japonicum.

alpinum.

fatidum.

tuberofus.

corwuts.

dioicuns.

minus. 1. 2.

majus. I. 2.

fibiricum.

purpurafeens.

angufifolium. 1-g.

flavum. 1. 2.

fimplex.

aymilegifoliums.

petaloideum.

contortum.

Adonis aftivalis.

autumnalis.

appennina. T. 2.

vernalis. $\mathrm{I} .2$.
- Adonis flammen.

capen/is.

veficaturia. 1-3.

atbiopica.

Ranunculus reptans.

myofurus.

nodiflorus.

lingua.

gramineus.

pyrenaus.

flammula.

amplexicaulis. 1. 2:

parnasfifolius. x. 2.

ficaria.

bullatus.

plantaginifolius.

Tora.

muricatus.

alpeftris.

lapponicus. x. 2.

lanuginofus.

sapen/s.

ternotus.

japonicus. $\alpha . \beta$.

Sceleratus.

bederaceus.

illyricus. 1. 1.

platanifolius. 1-3.

nivalis. a. $\beta . \gamma$.

byperloraus.

creticus. 1. 2.

casfubicus. 1. 20

villnfus.

birjutus. 


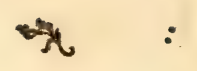

Ranunculus glacialis.

pbilonotis.

penfylvanicus,

auricomus. I. 2.

abortivus.

aconitifolius.

pubejcens.

repens.

bulbofus. a. $\beta$. .

polyant bemus.

arvenfis. I. 2 :

parviflsirus.

acris. I-3.

aquaticus.

falcatus.

orientalis.

rutefolius.

millefolius.

Trollius europeus. 1. 2.

afiaticus. I. 2.

Sagittaria lancifolia.

obtuffolia.

fagittifolia. 1. 2.

Ifopyrum fumarioides.

aquilegioides.

tbalietroides.

Helleborus trifolius.

niger.

viridis.

byenatis.

fatidus. I. 2.

Caltba paluftris. \& $\beta$.

Fiper betle.

malamiri.

amalago.
Piper furiboa. longum. I-3. decumanum. aduncum. 1. 2. cubeba. capenle. latifolium. vetujum. pellucidum. geniculatum. bipiciutu. obtufifolium. peltatum. diftacbyon. umb:llatum. excelfum. alpinum. nitidum. 1. 2. verrucofun. amplexicaule. trifolium. discolor. glabellum. a. $\beta$. monopbyllum. inequale. yuadrifolium. reflexum. pallidum. fellatum. cordifolium. Jerpens. rotundifolium. bifpidulum. tenellum. nigrum. 


\section{D. \\ MUSEUM NATURALIUM ACADEMIIE UPSALIENSIS.

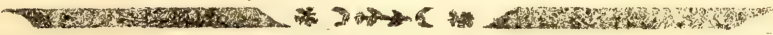

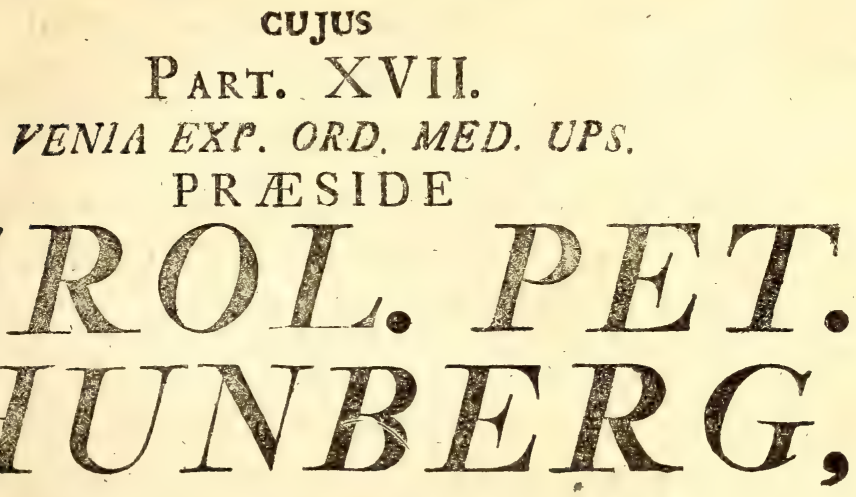

Equite Aurat. Reg. Ord. de Vasa, Medie. Doet. Profess. Med. et Botan. Reg. et Ord. Acad. Chesar. Nat. Curios. Reg. Scient, Londin. Holm. Socret. Scient. Ussai. Puilad. Patr. Holm. Berol. Nat. Scrut. Baris. et Hafniens. Hist. Nat. Lund. Harlem. Amsteld. Zeland. Nidrosiens. Ticinena. Isnens. Halens. Nat. Scrut. Linn. Londin. Medic. Edimeurg. ex Nat. Studios. Ibin. Membro; Nec Non acad. Scrent. Paris. Monspelinns. Agricult. Paris. Medic. Londiw. Froremtin. et Batavin. Ind. Or. Correspond.

PUBLICO EXAMINI SUBJICIT

G A B R IEL S A D T TEN, SMOLANDUS.

In Audit. Botanico die Io Dec. I794. H. A. M. S.

\section{UPS A L I $Æ$,}

IITTERIS JOK, FRED. EDAAF, REG, $A C A D$, TYPOGE, 


\section{THESES.}

\section{I.}

Veram Hiftoriæ naturalis cognitionem, Sacrarum interpreti litterarum, magno esfe ufui, nemo eat infitias.

II.

Inter tria Naturæ regna juftos certosque determinare limites, ob nimiam affinitatem, arduum admodum esfe videtur.

III.

Corporum mineralium nonnulla fuisfe aliquando fluida, experientia haud difficulter evincimur.

IV.

Eam cum animalibus vegetabilia proprietatem habent communem, ut vita gaudeant \& ope fexus utriusque propagentur.

\section{V!}

Scientiæ botanicx, cujus ftudium pariter utile eft atque jucundum, perplurima in vita communi debemus commoda.

\section{VI.}

Tempus durationis animantium, tempori accretionis plerumque accommodatum deprehendimus. 


\section{DONATION. THUNBERGIAN. CONTINUAT. XV.}

Didynamia.

Perilla ocymoides. Lycopus vireinicus. europeus. exaltutus.

Leonurus cardiaca. jibiricus. taturicus. marrubiaftruin.

Glecboura bederacer. Hysfopus officinalis. o. B. lopbantus. nepetoides.

Byftropogon canariense. pectinatum. $\alpha . \beta . \gamma$. plumofum. I. 2. integrifolium.

Mentbe cervina. ז. 2. capen/is. corymbofa. pulegium. 1. 2. 3. viridis. I. 2. canaden/s.

piperita.

fativa. gentilis. crijpa. rotundifolia. I. 2.
Mentba aquatica. bivguta. arven/is. auricularia. niliaca. JylveAtis. I. 2. Sideritis canarienfis. canüicans. cretica. fyriaca. perfoluata. montana. I. 2. elegans. I. 2. romana. decumbens. rugof $x$. fruticuln 5 . incana. bysfopifylia. 1. 2. 3 . feordioides. I. 2. 3. Spinoja. birfuta. I. 2. 3 . pillida. plumosa. lonata. ciliata. Lavandula carnofa.

A 2 


\section{武) 141 ( )}

Lavandula Spica. 1. 2.

Alocchas. 1. 2. 3. 4.

dentata.

pinnata. 1. 2.

multifida.

Teucrium ehaticum. 1, 2.

fordizim.

regitum.

multijorum.

flavum.

masfiliense.

erubefeens.

canejcens. 1. 2. 3 .

canadense. 1. 2.

virginicum. 1. 2.

inglatuns.

villofüm.

arduini.

bivcanicum. I. 2.

fcorodonia. I. 2.

tataricum.

pyrenaicum.

purnilum.

creticum.

gnapbalodes.

capitatum. 1 - 6 .

polium. 2. 3. 3 .

Supinum.

montanum.

marum.

fruticans. 1. 2. 3.

izpa. 1. 2. 3 . parviflorum. cbamapitbys.
Teucrium misfoliantsm.

africanum.

capenfe.

cbannedrys.

hucidum.

cuberee.

Spinofume.

campanulatum. botbrys.

Ajug pyramidalis. I. 2. orientalis, I. 2. reptans. I. 2. 3. alpina. I. 2. genevenis. decumbens.

Phlornis capenfis. fruticofa. nisfolit. lycbnitis. I. 2. 3. famia. venti. I. 2. 3 . laciniata. tuberofa. ceilonica. obliqua. indica. nepetifolia. moluccoides. leonurus. leonotis. cbinenfis.

Monard fifulosa, 1. 2. didyma, I. 2. 


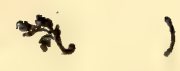

Monardo clinopodia.

pructuta. 3. 2.

Betonica officinalis.

orientalis.

alopecurus. I. 2.

alpina.

Arict:

Lamium oryalm, I. 2.

lavigutam.

garganicum.

maculatum. I. 2.

slbum. $\alpha \beta$.

purpureum. $\alpha . \beta$.

virgineum.

Pollicbia galleobdolon. amplexicaulis.

Galeop/is ludanum.

tetrabit.

bifpids.

Stachys ecbinata.

circinata. I. 2.

fylatitica,

palufitris. 1. 2.

alpina. 1..2. 3.

germaxica.

lanata. I, 2. 3 .

cretica. $\alpha . \beta$.

Spiso/a.

orientalis.

palafina.

maritima.

retbiopica. I. 2.

recta. 1. 2.
142

Stacbys annua. 1. 2 .

patens.

birta.

arvenfis. 1. 2.

fatids.

Nepeta cataria. a. $\beta$.

pannonica.

nuda.

violacen. $\mathrm{r} .2$.

ucranica.

angufifolia. I. 2.

crifpa.

tuberofa.

virginica.

italica.

nepetella.

malabarica.

frordotis.

lavandulacea.

multifida. I. 2.

incana.

Verbenanodiflora. I - 4.

javanica.

capen/is.

foecbadifolia.

bonarienfis.

indica.

jamaicenfis.

prisinatica. I. 2.

mesicana.

lappacea.

carolina. I. 2.

urticefolia. I, 2. 3. 
Verbena baftata.

aubletia.

officinalis.

Jpuria.

japina. I. 2.

tripbylla.

Satureja tbymoides.

juliana.

tbymbra. I. 2.

greeca.

montana. I. 2.

bortenfis.

capitata.

viminea.

Ametbyftea carulea.

Ballota nigra. I- 4 .

alba.

suaveolens. I-3.

difticba.

ma opporum.

lanuta.

Marrubium alysfow.

peregrimum.

candidum.

Jupinum. I. 2.

vulgare.

africanum.

crifpunt.

bijpanicnm.

pjeudodictamnus.

acetabulofum.

alysfoides.

indicum.

Moluccella levis. I. 2.

Jpinofa.

frutefcens.

tubernfa

Scutellaria minor. I. 2. baftifolia. 1. 2. galeviculata. $\alpha . \beta$. byssopifolia. cretica.

peregrina. albidn. 1. 2. lateriflora. 1. 2. indice. 1.2. virginica. altisfima.

- altaica. lupulina. alpina. 1. - 3 .

orientnlis. 1.2. Tbymus serpyllum. I 6. vulgaris: 12. sygis. T. 2. acinos. I. 2. alpinus. 1. 2. piperella. cephalotes. mafticbinus. micans. misrtifolius. Bromnit.

Zizipbora acinoides. cafitata. 
Zizipbora tenuior.

bifparica.

Plectranthus fruticofus. 1. 2.

Ocymuin rugi,um.

c"ifpum. 1. 2.

acutum.

racemofunt.

tomentojum.

paniculitum.

tbyrifloru'n.

monachorum.

gratis/inum.

album.

bafilicuin.

minimum.

farctum.

tenuiflorum.

polyftacby n. I. 2.

mentbindes.

Scutellavioides.

inflexum. I. 2.

virgutum.

Scalbum.

diffuliam.

Cunila miriana.

pulegioides.

tbymoides.

Salvia agyptiaca.

jvaveolens.

dentata.

pblomoides.

cretica.

officinalis. $\alpha, \beta$.
Salvia pomifera.

urtic folis.

fylveftris.

nemsorofa.

viridis.

occidentalis.

nivea.

rigida.

aurea.

paniculata.

africana. T. 2.

canarienfis.

spinofa. I. 2.

borminum.

mexicana. 1.2.

bispanica.

triangularis.

difermas.

glutinofa. x. 2.

corylifolia.

abys/inica.

formofa.

coccinea.

Serotina.

dominica.

nutans. 1.2.

nilotica. 1. 2.

verticillata. I - 3 .

fclarea. I. 2.

virgata, $\mathbf{1} \cdot 3$.

pratenfis. I - 3 .

indica.

amplexicaulis. 
Salvia fetida.

viscofa.

fyriaca.

bematodes.

verbenacea. 1. 2.

lyrata. I. 2.

rugofa.

triloba.

gurita.

byzantina.

napifolia. I - 3.

obsufata.

clandeftina. I. 2.

fcabra.

runcinata. I. 2.

atbiopis. x. 2.

ceratophylloides. I 3 .

ceratopbylla.

pinnuta.

japonica. 1. 2.

Prunella bysfopifolia. 1. 2. vulgaris. 1 - 4. grandiflora.

laciviata.

Rosmarinus officinalis.

Cleonia lufitanica.

Tricboftemia dicbotonum.

brachiatum.

Collinfonia pracox.

Dracocephal.virginicum. 1.2.

peregrinum .I. 2.

peltatum. $\mathrm{x}, 2$.

Dracocepbalum. canefcens. grandifloruns. moldavicum. tbymiflorum. altajenfe. canariense.

sutans. pinnatum. Ruyjcbianum. fibiricum. aufriacum.

Origanum egyptiacum. vulgare. 1. 2. creticum. dictamnus. onites.

hipyleum. finyrnatuss. beracleoticum. majorana. mavu

Clinopodium vulgare. 1. 2. 3. incrnum. I. 2. rugofum. I. 2. fruticofum.

Tbymbra Spicata. Melittis japonica. melisfophyllum. I. 2. Melisfa grandiflora. nepeta. calamintba. 1. 2. fruticoja. officinalis. 
Melisfa cretica.

Horminasps criflatum.

Pbryma leptofachya.

Prajutat majus. I. 2.

$$
\text { minus? }
$$

Cafileja fisfofolia.

Tanaciunn claviculatun. Por.

$$
\text { parofiticum. I. } 2 .
$$

Orabancbe uniflora.

$$
\begin{aligned}
& \text { virginica. } \\
& \text { major. I. } 2 . \\
& \text { puppurea. as. B. } \\
& \text { capenifis. } \\
& \text { fquariofa. } \\
& \text { minor. } \\
& \text { americana. } \\
& \text { cermus. } \\
& \text { lavis. } \\
& \text { raninfit. I. } 2 .
\end{aligned}
$$

Aledva copenfis.

Lathren clandefina.

fquamuria.

Burtila coccinea.

$$
\begin{aligned}
& \text { alpina. } \\
& \text { pallida. } \\
& \text { vifcosa. }
\end{aligned}
$$

gymmandra. ${ }^{4}$

Euphrafia longifora.

$$
\begin{aligned}
& \text { lutea. } \mathbf{1 .} 2 . \\
& \text { latifolia. } \\
& \text { cuneata. } \\
& \text { vifcoja. }
\end{aligned}
$$

Eapbrafie oficinalis.

odontites.

Rbinantbus foaber. I. 2.

capenatis.

elepbas.

trixago.

crifta. 1. 2.

virginicus.

Melampyrurn criftaturs.

nemzarofum.

arvenge.

pratenfe.

fylvaticum.

Barleria buxifolia.

prionitis.

birfuta.

longifulia.

noctiflora.

criftata.

pungens.

Gmelina afintica.

Torenia afiatica.

Acamibus mollis.

Ipinofus.

ilicifolius. I. 2.

cardrifolius.

furcatus.

capen/is.

procunsbens.

integrifolius. 1. 2.

maderafpatanus.

Crelceratia cucurbitina. 
Crefcentia cujette. 1. 2. Premna int grifolia. vilioja.

Halleria lucida. elliptica.

Selago faljolvides. carymbasa. cunefcens. divaric ata. geniculata. fruticola. articulata. triquetra. bijpidis. diffufa. a. B. fabibida. glomerita. paniculata. ovatı. a). anguftifilia. b). poniggaloides. oinerea. fpuria. rapunculoides. $\alpha, \beta$. beteropbylla. pufilla.

fafciculatio. verbenacea. birta. cepbalopbora. cordata. decumbens.

Selogo cilinta. rotundifolia.

Lippia cymnfa. Bucbnera cordifolia.americana. ceilanica. afiatica. bilabiata. cermua. cuneifolian. pinnatifida.

Manulea micropbylla. linifolia. integrifolia. revoluta. carulea. capenfis. incana: cuneifolie. divaricats. capillaris. beterophy!la. plintaginea. capitita. a. $\beta$. alitirrbinoides. virgata. cepbulutes. toment of i. bixta. cbeirantbus. a. B. thyriff ra. $\alpha$. $\beta$. coryunbufa. $\infty . \beta$. 


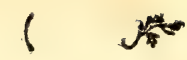

Manulea altistina.

rubra.

argentea $\alpha . \beta . \gamma \cdot \delta_{0}$ bifiza a. B. $\gamma$. $\delta$.

cordita.

pimntifila.

Jetrea volubus. I. 2.

Sibtborpla europaea, a. B.

Eninus alpimus.

filuginoides.

abiopicus.

wiofis.

oustitimis.

finditix.

lychidd us.

of icanus. a. B. y.

patens.

tomenojus.

inci/us.

triagis.

Hebenfretia integrifolia.

dentatio \&. B. $\gamma . \delta$.

cilieta.

fpicuta.

capitata.

fititicofa. c)

erinoides.

corduta.

Lantana sculata $\propto$, $\beta$.

invoincrata.

cumbara.
Lantana trifolia a $\beta$

and? $1 \mathrm{tat}$.

frictis.

odos ata.

coccinea

ajricunia \& 3 .

crippa.

rumofa.

enjen/is.

falvifilat.

Calceolaria pluntugines.

pimnat.

Alicunnianitida.

tonentolus.

refinifera.

Tozzia alpina.

Limofella aquatica.

capenjas.

Bromallin demisf.

elata.

Lindernua pyxidiria.

capenfis.

diantbera.

joponica. a. $\beta$.

landellia diffufa.

Sibvenckia americana.

Myoporum tenuifolium.

pubejcens.

Ruellia jpinejcens. angufifolia. fol.

B 2

Ruel.

c) Eranthemum parviforum. Berg. 
Ruellia striculata.

jponica.

fimilis.

eupbrafioides.

iancea.

erecta.

vingens. $\alpha . \beta . \gamma . \delta$.

maderafpatana.

piloja.

depresfa.

bumilis.

cordica.

frepens.

busbata.

blecbnum.

blecbioides.

clandeftina.

paniculata.

arifata. $\alpha . \beta$.

digitalis.

f: uticola.

ovata.

obluyar. $\alpha . \beta$.

repanda.

perfica.

tetragona.

anagallis.

antipoda.

Jerrata.

difformis.

fregrans.

crippa.

reptans.

tuberola.
Ruellia rupefiris.

Fuficicia adbatoda.

ecbolium.

betonica.

picta.

infundibuliformis.

Spinofa.

acicularis.

armata.

orcbioides.

gandarusfa.

vertisillaris.

tranquebarenfis.

byssopifilia.

moretiana.

spatbulata.

conglutinata.

repandd.

Eufacbiana. martinicen/s.

adjurgenss.

bicnlyculata.

falicifolia.

capenfis.

longifolia.

fugax.

nemorofa.

cerulea.

japonica.

crinita.

fetofa.

acaulis.

criftata.

procumbens. 
Fufsicia reptans.

gangetica.

ciliaris.

bumifula.

tetvanira.

pectoralis.

comiara.

purpurea.

cbinenfis.

clavata.

obliqua.

Jexangularis.

naputa.

Capraria bitora. $\alpha . \beta$.

durantifolia. $\alpha . \beta$.

longiflura.

lanceubata. $\alpha . \beta$.

vigida.

undulata.

Digitalis purpurea. «. $\beta . \gamma$.

$$
\begin{aligned}
& \text { minor. } \\
& \text { thapfi. } \\
& \text { lutea. } \\
& \text { ambigua. } \\
& \text { ferruginea. } \\
& \text { canarienfis. } \\
& \text { cceptrum. }
\end{aligned}
$$

Bignonia fempervirens.

grandiflora.

tomentofa. I. 2.

longisfina. fol.

catalpa. $\alpha . \beta$.

ungvis. $\alpha . \beta$.
Bignonia paniculata.

cupreolata.

aquinoctialis.

pentapbylla.

leucoylon. fol.

radicans. I. 2.

fans. I. 2.

fraxinea.

capenfis.

cbelonoides.

javanica.

indica. I. 2.

Cbelone glabra.

obliqua.

pentfemon. $\alpha$. B. $\gamma$ -

ruelloides.

Gratiola officinalis.

monnieri.

bysfopioides.

virginica.

repens.

tilleoides.

Sefamum orientale.

indicum.

proftratum.

Ge/neria pumila. acaulis.

tomentofa.

grandis.

jcabra.

calycina.

exjerta.

craniolaria.' 


\section{- 1}

151

Gesneria pulcbella.

mucrogamilis.

Sternodra maritima.

Scropobalario mellefera.

Jombu fina.

vernalis.

cinina.

altaca.

pacifar.

mavilasdica.

nodola.

auriculista.

forvadonia.

betonirifilia.

orient.his

frutejcens.

aquatica.

trif liata.

lucita.

peregrina.

Antirrbinuin apliyllum.

cymbataria.

pilosun.

elatine.

cirrbojum.

Sptrium.

asyptiacum.

triphyll:m. a $\beta$.

triorntbopborum.

purfurelim.

verficolor.

repens.

alpinum. sintirrbin. monfres lanum.

Jpurtum.

tilfe.

Jis in lind.

areves?/s.

pilaterinum.

Jîntrite.

z. Jut ju.

milicitele.

glinguruma.

nloinian.

origun iflium.

calenl:

f) tings.

bicerme.

b:s butiam.

frutirans.

fo bithn.

lonsicorize.

j)ismitu:n.

gandins.

birkits.

gevififolium.

jusceus.

liniria. a. $\beta$.

peleseli.

linafilium.

chalifingle.

reflexum.

jedisculitim.

curtatum.

majius.

orontium. 


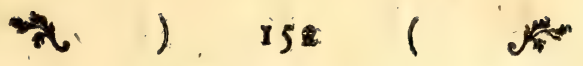

Antirrbinum papilionaccum. Pedicularis vofirata.

molle.

afarina.

bellidifolium.

canaden/e.

balian.

majoranoides.

bexandrum.

valezianum.

tbymifolium.

bengalenje.

Heqnimeris montana. a. B. $\gamma$.

fibuloja.

diffufa.

unilabiata. $\alpha . \beta$.

macropbylla.

Columnea bumilis.

bijpida.

rutilins.

birfuta.

Gerardia creito.

purpurea.

tubulofa.

glutinga.

lanceolita.

nigrina.

jeabra.

japonica.

delp inifolia.

fivor.

pedicularia.

Pedicularis palueris.

Jyluatica. fceptrum.

verticillata.

refupinata.

recutita.

firmmea.

bir/uta.

rojea.

lapponica.

comoja. $\propto . \beta$.

filioja.

tuberola.

gy: oflexa.

venofa.

Mimnlus ringens.

Dodertia indica. $\alpha$. $\beta$.

orzentalis. $\alpha . \beta$.

Hortynia annua.

perennis.

l'edulumm murex.

Linna boveulis

Bontia dapbroides.

Cofvetia jyramidata.

Cierodendr.trichotimum. I.2.

in ortunatum. 1.2.

phirmidis.

fortunatum.

colamitofun.

paniculitume.

Volkameria icuminata.

jupenica. I. 2.

aculeata. 
Volkameria inermis.

ferrata.

Citbarexylon crassifolium.

melanocardium.

evectum.

caudatum.

fervatum.

quastangulare.

villojatiz.

Ovieda mitis.

$V$ ite $x$ ovata.

capitata.

unbrofi. 3. 2 .

trifoliata.

pinnata.

cafta. $\mathbf{x} .2$.

negundo. 1. 2.

Duranta ellifia.
Befleria longijora. criftata. cymofa. biflriva. lutea.

Brunsfellia undulata. americana.

Hyobancbe fangvinea.

Tourrecia lappacea.

Cymbaria datica.

Taunberga millabarica. guvanica. intica. copenfis.

Meliantbus major, 1. 2. minor. comofis.

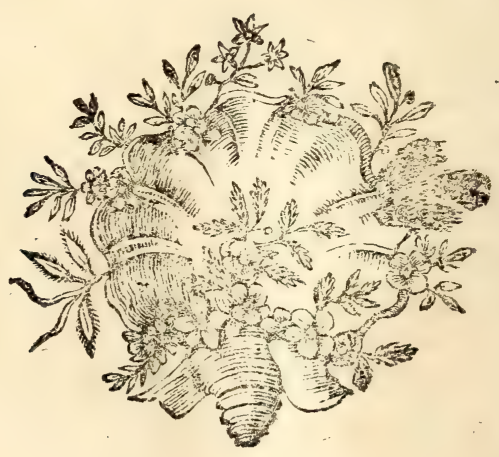




\section{D. $D$. \\ MUSEUM NATURALIUM ACADEMIA UPSALIENSIS.}

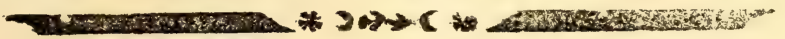

\section{cuJu's}

PART. XVIII.

VENIA EXP. ORD. MED. UPS. P R E S IDE
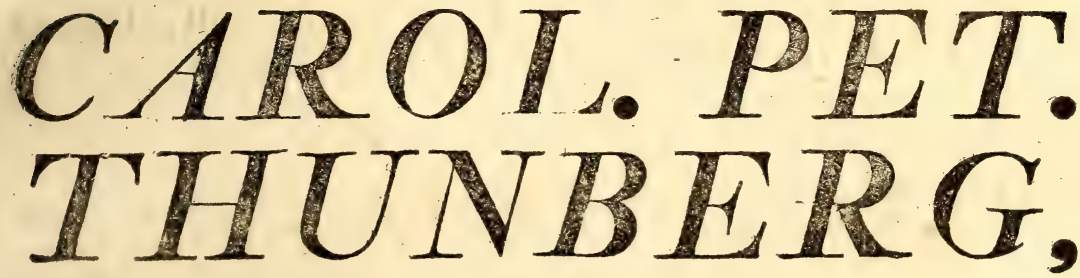

Equite Aurat. Reg. Ord. de Vasa,

Medic. Doct. Profess. Med. et Botan. Reg. et Ord. Acad. Cuesar. Nat. Curios. Reg. Scient, londin. Holm. Societ. Scient. Upsai. Puilad. Patr. Hoim. Berot. Nat. Scrut. Paris. et Hafniens. Hist. Nax. Lund. Haried. Amsteld. Zeland. Nidrosiens. Ticineng. Ienens. Halens. Nat. Scrut. Linn. Londin. Menic. Edimbura. et Nat. Studios. Irit. Memero; Nec Non Acad. Scrent. Paris.

Monspeliens. Agricult. Paris. Medic. Londin. Fiorentin. et BATAVIN. Ind, Or. CORRESPOND.

PUBLICO EXAMINI SUBJICIT

CAROLUS ZETTERSTRÓM,

JEMTLA N D US.

- In Audit. Botanico die 17 Dec. 1794.

H. A. M. S.

U P S A I Æ,

LITTERIS JOH. FRED. EDMAN, REG. ACAD. TYPOGR, 


\section{OBSERVATIONES.}

I.

Genera \& Species Tetradynamix Clasfis, quippe qux maxime eft naturalis, difficilius dignofcuntur.

II.

At ejus virtutes Medicx in eo conveniunt, interne vel externe adhibeantur, ut fimulent.

III.

Hic ergo validior diuretica \& epifpaftica inveniuntur.

\section{IV.}

Quxeque contra Scorbuticas affectiones habentur remedia, præter quod fint tutisfima, etiam præ $\mathrm{fe}$ ferunt illud commodi, ut \& medicaminum \& cibi inftar praberi posfint.

\section{V.}

Talem vero vim hifce herbis inesfe, nif recentes, fruftra exfpectaveris. 


\section{DONATION. THUNBERGIAN. CONTINUAT. XV!}

\section{Tetradynamia.}

Vella arnua.

plevdocytifus.

Myagrum faxatile. $\alpha . \beta$.

auftrincws.

mono fper numb.

orientale.

perfoliatam.

fativum.

panirulaturz.

rigidum.

perenne.

rugofums.

bifporicum.

egyptiacuss.

Subularia aquatica.

Draba verme. $\alpha$. $\beta$. $\gamma$.

muralis, $\alpha, \beta$.

incana.

birte.

Bellatio.

alpina.

pyrenaica.

aizoides.

Iberis faxatalis.

gavexiana.

gibraltarica.

rotund folia.
Lberis andicaulis.

carni.ja.

pinnista.

Senperforens.

fimpervirens.

linifilia.

ambellita.

amara.

Alysfum alpeftre.

deltordeun.

bulimifolum.

montanum.

creticum. 1. 2.

veficaria.

clypeatum.

utriculatum.

byinerboreum.

calycinum.

Jpinofum.

campeftre.

finuatum.

incanum.

faxatile.

niinutum.

minimum.

Clypeola josthlajpi.

mavityma.

tomentofia.

A 2 
Peltaria capenfis. Bifcutell didyma. coronopifolia. fempervirens.

lavigata. apula. intermedia. grata.

Anafatica bierocbuntica.

$$
\begin{gathered}
\text { fyriaca. } \\
\text { Thlasp: } \\
\text { campefire. } \\
\text { faxatile. } \\
\text { birtum. } \\
\text { alliaceum. } \\
\text { perfoliatum. } \\
\text { arverse. } \\
\text { alpeftre. } \\
\text { montanum. } \\
\text { burfa. a. B. }{ }^{2} \\
\text { ceratocarpon. }
\end{gathered}
$$

Lepidium perfoliatum. I. 2 . veficarium. procumbens. alpisunt. petraum. cardomines. Spinufum. I. 2。 capenfe. pinnatum. bipinnatum. fotivum. I. 2.
Lepidium latifolium. I. 2: fubulatum, 1. 2 . graminifolium. linoides. flexuofum. didymum. ruderale. I. 2. 3 . virginicum. iberis. bonariense. Thlaypidinides. frondulum. I. 2 . pifcidium. montanum.

Eryfimum barbarea. officinale. alliaria. repandum. I. 2. 3. bieracifolium. cheiranthoides.

Cocblearia officinalis. dassica. granlandica. anglica. corompifolia. armoracia. I. 2. glaftifolia. draba.

Lunaria pinnata. diffusa. elongata. 
Lundria fcabra. aัnua, I, 2: rediviva. 1. 2 .

Ricotian cespptiaca. Rapbaness ripbanifrum. faitivus. condutus. fibivicus. tenellus. oleifor.

Hesperis verna. ofricana. matronalis. a. $\beta$. diffufa. triftis. 1. 2. inodora.

Cheirantbus eryfimoides.

friclus. callofus. carriofus. limenvis. gramincus. elongitus. torilofus. argutus. I. 2. montanus. temifolius. alpinus. I. 2. guadrangularis. littoreus. falimus.
Cbeirantbus maritimus.

cloizs.

fruticulerus.

cbeivi : -3 .

aвъиия.

incanus $1-5$. fenefirvalis. trilobis. trifis.

vancinatus. fol. fincutus. tricuppidatus.

Arabis dentata. I-3.

turrita. canadenfis. balleri. beteropbylla. bellidifolia. pendula. I. 2.

Turritis alpira. alpind $1-3$. glabra. birfita. I. 2.

Dentaria bulbifera. pentapisylla.
Brasfica cbineryis.
ovientalis. I. 2.
campeftics.
arvenfis.
napus.
rapa. 1.2.

A 3 
Bras $26 a$ erucaftrum.

evuca.

veficaris.

oleracea I-IO.

Sinapis cermua.

japanica.

pyrenaica I - 3 .

juncea.

recurvata.

incane. I. 2.

allioni.

cbinenfis.

orientalis.

arvenfis. $\mathbf{1}, 2$.

braficata.

nigra.

erucoides.

levigata.

bifpanica.

Heliopbila pufilla.

filifolia $\mathbf{x}-3$.

amplexicaulis. 1. 2.

incana.

integrifolia,

quinquefida.

beteropbylla.

pinnata.

tripartita.

digitata.

runcinata.

circcooides 8).
Bunias Spinosa. câftica. erucago. orientalis. kakile. myngroides. agyptiaca. fibirica. baldearica.

Crsmbe fruticofa. orientalis. marittima. bifpanica. 1. 2.

Cordomine Jcututa. bellinifolia. petraea. trifolia. debilis. reledfilia. cbelidonia. impatiens. parvifura. bevjuta. priateriss. amara. virginica. ouncinata. graca. Ifatis tivictoria. 
- ) 161

Sifymbrium nafurtiun.

fylveftre. 1. 2.

Sifymbrium apetalum.

iflindicum.

ampbibiume. a. $\beta$.

pyrenaicums.

tanacetifolium. $\alpha, \beta$.

ternuifolium.

fupinum.

polyceratium.

burfifolium.

murale. a. $\beta$.

snonense.

Barreleri.

parra.

Salsuginofum.

Atrictum. 1-3.

Serratum.

indicum.

Sopbia.

mullefoliums.

arenofum。

afperuma

altum.

irio.

la eliti.

orientale.

catbolicum.

aufriacun.

erucaftrum.

Arigo/um. $\alpha . \beta$.

capenfe.

bippanicum.

album.

Cleorne apbylla.

armata.

laxa.

virgata.

juncea. $\alpha . \beta$.

procumbens.

monopbylla.

fruticofa.

arabica.

tenella.

violacea. $\alpha . \beta$ :

ternata.

lingva.

arabica.

tripbylla.

dodecandra.

pentapbylle.

vifcofa.

beptapbylla.

fpinofa.

gigantea. 


\section{THESES.}

I.

Si Sacerdos præter munus, quod ejus proprium eft, de Auditoribus etjam in is, qua eorum valetudinem fpeetant, bene mereri cupit, primum e re erit, ut præjudicatas in Medicinam opiniones evellere fudeat.

II.

Deinde quo porisfimum modo fanitas confervetur morbique pracaveantur, eos inftruat.

III.

Morborum, frequentisfime obvenientium, cam faltim cognirionem habeat, ut is obortis, prout rei ratio poftulat, arte peritum confulere, vel etian, ubi hoc vetaret munus, ejus partes iple agere posfit.

\section{IV.}

Siquidem remedia, quantumvis licet apta, fine debito delectu adhibita, fæpisfime noxam inferrent, quædam de eorum vi \& ufu in diverfis cafibus cugnitio maxime esfet commendanda.

$$
\text { V. }
$$

Qux publica vel privata auctoritate in vulgus edun. tur remedia, ccetui fuo V. D. Minifter nota faciat, idque eo magis quo plura corum eos fpectant cafus, ubi promptisfimis medelis opus fit.

\section{VI.}

Contra ea qua rära \& minus obvia ducit, vel morbos vel eorum curam fpectent vel alia ejusmodi, de his Medicum provincialem faciat certiorem. 


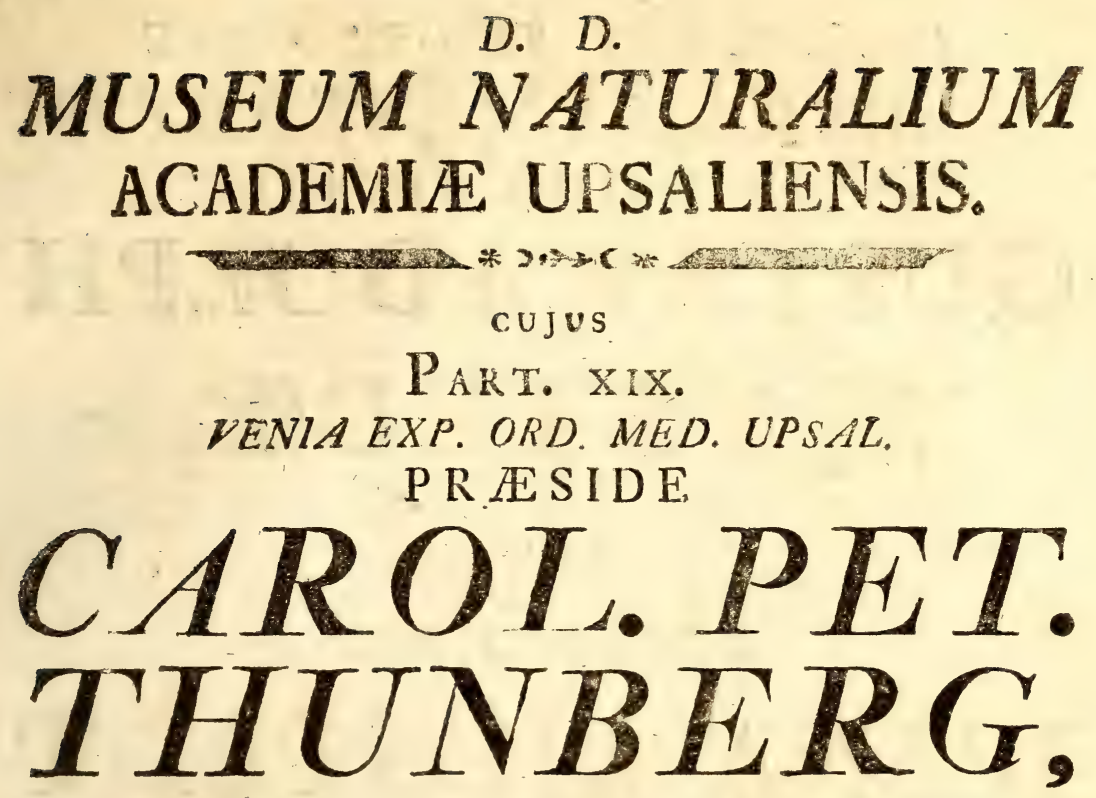

Equite Aurat. Reg. Ord, de Vasa,

Medic. Doct, Profess Med. et Botan, Reg. ex Ord. Acad. Ceesar. Nat. Curios. Reg. Scient, londin. Hoim. Societ. Scient. Upsai. Philad. Patr. Holm. Berol. Nat. Scrut. Paris. et Hafnifens. Hist. Nato Lund. Hariem. Amsteld. Zeland. Nidrosiens. Ticinens. Ienens. Halens. Nat. Scrut. Linn. Londin. Medic. Edimeurg. et Nat. Studios. Ibin. Membro; Nec Non Acad. Scient. Paris. Monspeliens. Agricult. Paris. Medic. Londin. Florentin. et Batavin. Indo, Or. Correspond.

PUBLICO EXAMINI SUBJICIT.
SVENO
ERICUS
$A L B O M$,
WERMELA N D U S.

IN AUDIT. BOTAN: D. X. JUN. MDCCXCVI. H, A. M. C.

\section{U P S A I 死,}

utr teris Joh. Filir. Euman, klg, aAd, typcgr. 


\section{KONUNGENS TRO-TFENARE}

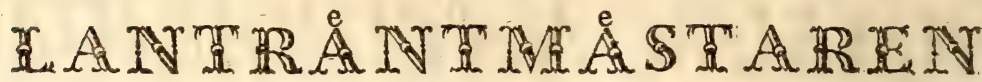

$H O O G A L E$ HERR

GUSTAF ADOLPH

$\mathbb{S} J \dot{O} \mathbb{S} \mathbb{E} \mathbb{N} \mathbb{T}_{9}$

SAM T

HUGADLA FRU

JEANA SJÓSTEDT FODD A

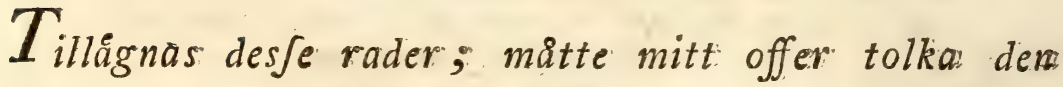
vôrdnad hvarmed jag framliardar

\section{$E D E R$}

o̊dmjukafte tjenare

S. E: $\triangle \mathrm{LBOM}$. 


\section{HANDELSMANNEN \\ ȦDLE осн HO̊GACKTADE}

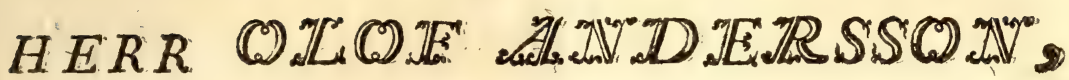

S. A M T

$$
V \dot{A} L \dot{A} D L A \quad F R U
$$

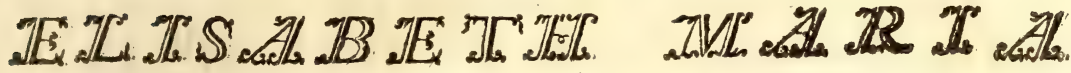

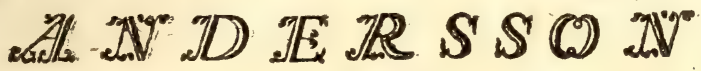

$$
\begin{aligned}
& \text { FO̊D WENERLOF, }
\end{aligned}
$$

MINA HULDA FÓRALDRAR!

Tillåten mig att hărmed ådagalågga den årkănfla ocha tillgifvenhet hvarmed jag framhårdar

\section{EDER}

lydigafte Sow:

S. E. A L R O M. 
I.

Cognitio Botanices non eft mere hiftorica.

II.

Scientia Botanica \& Zoologica aliis bene multis adjuvantur fcientiis, iisdem illæ vicisfim inferviunt.

III.

In Clasfibus herbarum \& Animalium conftituendis characteres potisfimum adhibentur illi, qui ubique \& facile in oculos cadunt.

IV.

Nimis operofum Naturæ fcrutinium Medico \& Oeconomo non omnino necesfarium; moderatum utilisfimum.

$\mathrm{V}$.

Jucundum eft \& utile in Mufæis naturæ contemplari miracula, imprimis dum naturam ipfam intueri loci aut temporis ratio vetat.

\section{VI.}

Juniperus ægris \& valentibus eximia præeftat commoda \& remedia falubria.

VII.

Plurimæ plantæ monadelphæ mucilaginof $x$ funt \& involventes.

\section{VIII.}

Nonnulla quoque fibras textiles præbent. 


\section{DONATION. THUNBERGIAN: CONTINUAT XVII.}

\section{Monadelpbia.}

Securinega durisfima.

Myriftica mofcbata ô $q$ a. $\beta$. Sebifera. tomientofa.

Cupresfus vireus.

$$
\begin{aligned}
& \text { japonica a. } \beta . \\
& \text { tbyoides. } \\
& \text { juniperoides. } \\
& \text { difticba. } \\
& \text { pendula. fol. } \\
& \text { colmmnaris. }
\end{aligned}
$$

Stillingia fylvatica.

Hydnora africane.

Funiperus virginiana. 오 bermudiana fol. berbadenfis. o pboenicen. ?

lycia. † communis. ㅇ. 오. Sabina. '. cbinentis fol. oxycedrus.
$T b u j a$ articulata. orientulis. occidentatis.

Cisfampelos fruticofa. ․ capenfis. 舌. 오. pareira s. a. $\beta$. crapela. birsuta. tomentofa.

Melocbia lapulina. nodiflora. venosa. depres 5 . pyramidats. tomentofa. odorata. corcborifloria.

Ocbioma lagopus. Ephedra giguntea. diftacbya. monoftucbya.

Plukenetia vólubilis.

Gymrantbes elliptica. \&. \&. Aitonia capenfis.

lucida. o.

Thuja cupresfoides. \&. apbylla. dolubrata. fol.
Connarus decumbens. monacarpus. Hermannia filifolia. 


\section{$4166-(12$}

Hermann. Lavendulifolina. $\beta$. Geranium chamadryoides.

bispida.

involucrata.

Galvifolia.

cernua.

recticillata.

cilnaris.

trifurca.

denudata.

glabenta.

bysopifilia. $\alpha . \beta . \gamma$.

tripbyllo. $\alpha . \beta$.

venofa.

vefita.

olnifolia.

incana.

altbaifulia, $\propto . \beta$.

bumilis.

beteropbylla.

bifirrata.

grosfularifolico.

sulcbella.

procumbens.

diffufa.

pinnata.

myrrbifolia.

Grielum bumifufum.

tenuifolium.

Geranium fpinofum.

emarginatum.

anensoides.

monfonia.

speciofum: jangvineum.

profratum.

fibiricum.

tuberofum. $\alpha . \beta$.

columbinum.

pu/llum.

molle.

pyrenaicum. $\alpha . \beta$.

disfectum.

incavum.

canefcens.

bumile.

bobenicurs.

cineneum.

argenteum. $\propto, \beta$.

friatum.

nodnsum.

reftexum.

pbaum.

aconitifoliurs.

pratenfe.

paluftre.

fylvaticum.

nacbrorbizum.

maculatum.

lucidum.

maritimumi.

rotundifolium.

robertionum. $\alpha$. $\beta$.

palmatum. $\alpha . \beta$.

gruinum. 
Geranium pubefcens.

expanfum.

fericeuns.

trifoliatum. $\alpha . \beta_{\text {. }}$

malachoides.

glancopbyllum.

cbium.

incavantum.

peirerum. a. B.

rupelve.

romañum. $\alpha . \beta$.

cicutarium. $\alpha . \beta$.

mojcatum. $\propto . \beta . \gamma$.

precox.

ciconiums.

laciniatum. $\alpha . \beta$.

zonale, $\alpha$. $\beta$.

tetragonum.

peltatum.

iabulave.

elongatum. os. $\beta$.

alcbemilloides. $\alpha . \beta$.

aphanoides.

ciliatum.

enfatum.

beterophyllum.

angufifolitum.

auritum. $\alpha . \beta$.

trilobum.

lanceolatum. $\alpha . \beta$.

glaucum.

ovatum.

betulinum.

owale.
Geraniuin bybridum.

acetofum.

cordifolium.

cucullatum.

odoratum.

cotyledonis. fol.

Servituan.

fidefolium.

echinatum.

lanatuns.

subens.

birfutum.

lobatum. $\alpha$. B.

Aipulaceum.

srticulatum.

variegatum.

africanum.

altbeoides.

acerifolium.

inquinans.

papilionaceum.

vitifolium.

sapitatum.

grosfularioides.

quercifolium. \%. B. \%

bifpidum.

fulgidum.

lavigatum.

graveolens.

exfipulatum.

vijcojum.

crat gegifolium.

tricuspid at ums.

tottum. 


\section{7) $168(15$}

Geranium ternatum. adulterinam. bermannifolium. crispum. divaricatum. abrotanifolium. tectum. acuminatum. plicatum. flexuofum. acaule. pingve. longicaule. tomentofums. lacerum. aftragalifolium. capillere. villofiuu. frutefcens. tenuifolium. proliferum. flos. minimum. appendiculatum. radula, $\alpha, \beta$. triffe. doucifoliums. coriandrifolium. betonicun. myrrbifolium. ferulaceun. carnojum. gibbojum.

Plagiantus divaricatus.
Anacardium occidentale. 1. 2. Fatropba divaricata. manibot. I. 2. bugbes. fol. gos/ypifolin. curcas. multifida. fol. urens.

Dryandra oleifera. fol. cordata. $\alpha . \beta$.

Hygonia myftax.

Brovnea coccinea. fos.

Sterculia tementoja. I, 2. fatida. I, 2. platanifolia. balangbos. I, 2.

Alevrites triloba. Acalypha decumbens. glabrata. cor enfis. tomentofa. elliptica. anguftifolia. betulafolia. lavigata. jcabiofa. reptans. bernandifolia. dioica. pectinata. ciliata. virginica. $\alpha . \beta$. virgata. 


\section{$=169(1)$}

Acalypla indica.

Cuponia glabra.

Japonarioides. tomertofa.

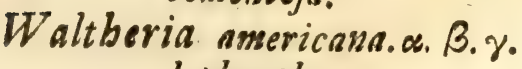
lopbantbus. indica.

Xantbium ecbinatum.

frumarium. ambrofioides.

spinojum.

Croton cajcarille. $\alpha . \beta$.

- variegatum. fol. cuspidatum.

balsamiferum. $\alpha, \beta$. bumile.

flavens.

lacciferum. lucidum.

inopbyllum. fol.

glandulojum.

acutum.

tiglium.

elutberia. $\alpha . \beta$.

fesfiliforum.

pallens.

globofum.

populifolium. fol.

gvianen/e.

maturense.

nitens.

divaricatum.

laurinum.

Croton reticulatum. \&. $\beta$.

spiciflorum.

jebiferum.

moluccanum.

macropbyllum. fol.

nutans.

peltatum.

orbiculare.

tinctorium. $\alpha . \beta$.

aromaticum.

japonicum.

capenfe.

lobatum.

Taxus verticillata. fol.

nucifera. fol.

baccata. $\delta$. ㅇ.

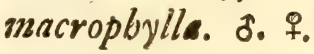

Pinus sylueftis. ‥ ठ. ๔. $\beta$.

canaden is. q.

picet. $\delta$.

pinea. f. $^{\circ}$.

maritima. $\delta_{\text {. }}$

nigra. fol.

frobus, fol.

lavix. d. $q$.

balsames. ․ $\delta$.

cedrus. $\delta$.

pinafter. ㅇ.

palufiris. fol.

tadn. fol.

abies. $\delta$. ․ $\alpha^{\prime} \beta$.

cembra.

Gordonia lafiantbus. 


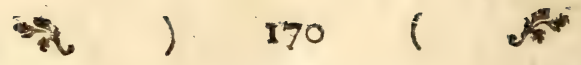

Gordonia bamatoxylon.

Sida carpinifolia. Mycodia turbinata. Mefua ferrea. fol. Bombax beptapbyllum. pentandruins.

Guftavia Augufta. flos. Adanfonia digitata. fol. Sida Jcabra.

levis.

repens.

fcetida.

Spinofa:

vifcola.

ztrens.

jamaicenfis.

anguftifolia.

linifolia.

arguta.

rbombifolia.

falicifolia.

ternat $a$.

malvifolia.

pimpinellifolia.

procumbens.

periplocifolia. «. $\beta . \gamma$.

pallida.

dumofa.

altbeifolia.

paniculata.

palmata.

multifida.

Aellata.

triquetra. ciliaris. cordifulia. retuja. filiformis. umbellata. sliva. peruvinna. planiflora. mollistima. topulifulia. afintice. occidentatis. grandiflora: abutilon. crispa. indica.

Triguera labata.

Adelia acidoton. fol.

vicinella. fol. bernardia.

Ricinus communis. inermis?

freciofus. mappa. globofus.

Hura crepitans. $\alpha$. $\beta$. Stemartia franklinia. malacodendrum.

Dalechampia ternata. 
Dalechampia fcandens. volubilis.

Camellia japunica. $\alpha . \beta$. fafanqua. $\alpha . \beta_{0}$

Gosfypium arboreum.

barbadenfe.

berbiceum.

veligiojum. 1. 2.

birjutum.

Urena, Typbalea.

excisa.

lobata.

finuata.

palinuta.

procumbers.

americaua.

Acbania malvarifcus.

Pavonia Jpicata.

piloja.

cancellata.

spinifex.

paniculatd.

arifiata.

cerlonica.

urens.

Assonia populnea.

Dombeija ovata.

$$
\begin{aligned}
& \text { parnetata. } \\
& \text { pbanicea. } \\
& \text { decantbera. } \\
& \text { acutangula. } \\
& \text { palmata. }
\end{aligned}
$$

Hibijcus fyriacus. $\alpha . \beta . \gamma . \delta$. byftrix. vijcofus. fabdarisfa. elatus. clypentus. pedunculutus. birtus. pbaniceus. Juratten/is. manibot. abelmofcus. trionum. efculeretus. tricuspis. vitifolius. cannabinus. bifurcatus. tubulnfus. palmatus. f.ru uineus. ficulneus. pentacurpos. liliflevus. clyperitus. atbiopicus. mutabilis. fororius. falicifolius. urens. mo/ceutos. tiliaceus. finerfis. $\alpha . \beta$. braflienfis. 
Hibijcus paluftris. Crosfoftylis biflora. Ruizia lobata. palmata. laciniata.

Thespefia populner. Anoda baftata. triloba. criftata.

Malva rotundifolia. alcea. Setosa. anguftifolia. froparia. elegans. fylveftris. peruviana. Spicata. coromandiliana. americana. bispanica. Scabra. proftrata. fragrans. tournefortiana. Sberardiana. limen/is. toment of $a$. bryonifolia. caroliniana. parvifora. mauritiana. verticillate.
Malva crispa. mefcata. agyotia. fellata. villofa. plicata. fruticofa. calycina. triloba. virgata. capen/is. abutiloides.'

Lavatera gallica. maritima. cretica. arborea. I. 2. 3. micans. olbia. triloba. lufitanica. tburingiaca. trimeftris. $\alpha . \beta$.

Altbea narbonen/is. corymbosa. officinalis. cannebina. birfuta. Ludvigii.

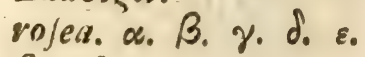
ficifolia.

Malope malacoides. Malacbra Altbaifolia. Alcbornea latifolia. a. $\beta$. 


\section{D. $D$. \\ MUSEUM NATURALIUM ACADEMIE UPSALIENSIS. \\ - ex \\ CUJUS \\ PAKT. XX. \\ VENIA EXP. ORD. MED. UPSAL. PR E I I E
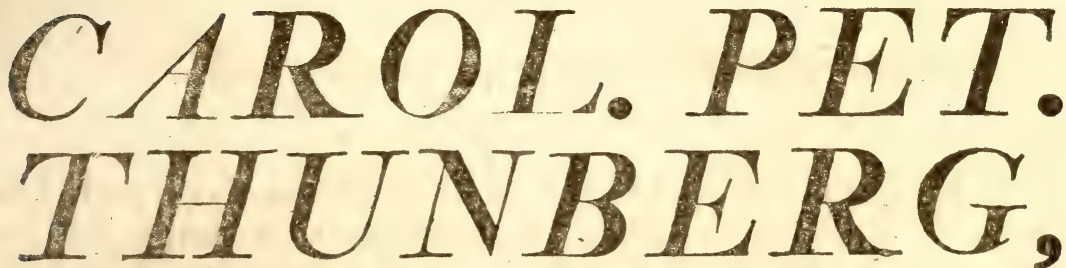

Equite Aurat. Reg. Ord, de Vasa,

Medic. Doct. Profess. Men. et Botan. Reg. et Ord. Acad. Ceesar. Nat. Curros. Reg. Scient. Londin. Holm. Societ. Scient. Ursal, Philad. Patr. Hoim. Berol. Nat. Scrut. Paris. et Hafniens. Hist. Nat. Lund. Harlem. Amsteld. Zeland. Nidrosiens. Ticinens. Ienens. Halens. Nat. Scrut. Linn. Londin. Medic. Edimburg. et Nat, Studios. Ibin. Membro; Nic won Acad. Scient. Paris. Monspeliens. Agricult. Paris. Medic. Londin. Fiorentin. et Batavin. Inn, Or. Correstond.

PUBLICO EXAMINI SUBJICIT

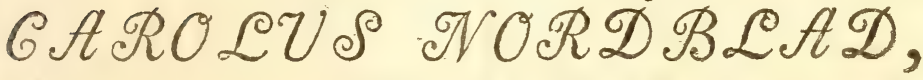
GESTRICIUS. .

IN AUDIT. BOTAN. D. X. JUN. MDCCXCVI. H, P. M. C.

\section{U P S A L I $A$, , i}

IITTERI JOH, FREDR. EDMAN, REG, ACAD, TYPOOR, 


\section{THESES.}

Vix ulla clasfis herbarum facilius dignofcitur. quam Diadelphia.

II.

Ex hac clasfe major in Oeconomia, quam in Medicina utilitas percipi poteft.

III.

Fructibus rubris acidum plerumque ineft,

IV.

In quovis Gymnafio fi esfet fudiof juventuti Hiftoriam difcendi Naturalem occafio, non huic tantum fcientiæ, fed Oeconomix etiam Patriæ optimæ confuleretur.

\section{V.}

Ufus licet multarum, quæ cognitæ funt, herbarum adhuc later, nullam tamen' esfe inutilem contendimus.

\section{VI.}

Botanices \& Entomologix ftudium in quavis vivendi ratione non modo delectationem adfert, fed etiam utilitatem.

\section{VII.}

E Regno vegetabili cibus defumitur fvavisfimus idemque faluberrimus.

Cognitio fiectuum, quos in corpus edir anima, admodum eft Medico necesfaria.

VIII.

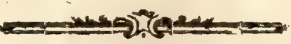




\section{DONATION. THUNBERGIAN.}

CONTINUAT, XVII.

Diadelpbia.

Monnieria trifolia. fol. Saraca indica. $\alpha$. $\beta$.

Funtaria minor.

grasedis. - . $\beta$.

Arumofa.

Spicata.

cucullaria.

virens.

luter.

imp:tiens.

bullboja. a. $\beta$.

officinalis. $\alpha . \beta$.

capnoides.

capreolata.

vefiaria.

claviculata.

enneapbyila. 1. 2.

nobilis.

Polygala acutifolia.

rupeftris.

incarnata.

amara. a. $\beta$.

vulgaris.

morjpeliaca.

filivica o. $\beta$.

paniculata.

lutes.
Polygala verticillata.

major.

bracteolata. $\propto, \beta, \gamma, \delta$

oppofitifolia.

mixta.

fipulacea. a. $\beta$.

alopecuroides.

trinervis.

teretifolia.

myrtifolia. \&. $\beta$.

tbymifolia.

beilteria.

virgata.

paucifiora.

cernus.

umbeliata, a. $\beta$.

Jinoja.

crotularioides.

tb ezans. fol.

diver folia.

micropbyila.

squarrija.

pencea.

cbamabuxus.

lenega.

vivilefcens.

ciliaris.

fanguizea. 


\section{*) 196 (}

Polygala crucinta.

Puticnia evicoides.

dapbnoides.

villoja.

Platylobium parviflorum.

formofun.

Dablbergia monetaria. lanceolaria.

Securidaca evecta.

$$
\begin{aligned}
& \text { virgata. } \\
& \text { volubilis. }
\end{aligned}
$$

Nisfolia arborea?

$$
\text { fruticola? }
$$

Abrus precatoritus.

Pterocarpus ecaftaphyllum.

$$
\begin{aligned}
& \text { lunatus. 1. } 2 . \\
& \text { buxifolius. } \\
& \text { fantnlinus. } \\
& \text { fcandens. }
\end{aligned}
$$

Amerimnon Broznei.

Spartium radiatum.

decuinbens.

purgans.

Spbarocarpum.

eleritum.

complicatum.

virgatum.

Jupranubiuns.

apbyllum.

mannjpermum. contaminatum.

Sepiarium.

junceum.

friatum.
Spartium fcoparium.

Jpinojum. Spurium. fericeum. cytifoides. angulatum. fcorpius.

Erytbrina crita. berbacea. fol. corallodendron. pieta. fol. caffra. bulbofa. monofperma.

Pifcidia erytbrina. Borbonia lievigata. trinervita. lanceolsta. cordata.

Afpalatbus carnosa. ciliaris. genifoides. galioides. vetroflexa. uniflora. arancofa. canejcens. quinquefolin. tridentati. anclyilloides. callora. cretica. capitata. perfica. 
Afoalatbus sinosa. mucronata.

byfivix.

sericea. beteropbylle. afparagoides. lotoides. vervucofa. glomerata. aftroites. cbersopoda. albens. tbymifolia. evicifolia. nigra. argentea.

Crotalaria retusa. cujpidata. verrucofa. juncea. nana. linif lia. biflora. Jagittalis. incana. virenta. cordifulia. lunaris. laburnifolia. incanejcens. juponica. fricea. quinquefolin.
Crotalaria undulata. foi. perfoliata.: crenata. amplexicaulis: suneifolia. triflora. I. 2. retrofracta.

filifolin. Spicata. I. 20" cordata. calyptrata. pedunculata. biflora. a. Sericed. geniftoides. I. 2. capentis. I. 2. imbricata. ternata. trifoliata
Ulex europeus.
Genifa fiberica. pilosa. linifolia. triangularis. tinctoria. florida. myrtifolia. candicans. I. 2. canarienfis. Iufitalick. germanica. anglica. tridentata. 


\section{\#) 178 (}

Genifta fagittalis. Unonis pubescens?

variegata. ornitbopodioides.

alba.

tridentata.

littoralis,

minor.

spinofa.

cberleri.

mitis.

repens.

antiguorum.

reclinata.

vifcosa.

pinguis.

natrix.

crispa.

dentata.

rotundifolia.

alopecuroides.

fruticofa.

arvenfis.

mauritunica.

umbellata.

proftrata.

argentea.

villosa.

Antbyllis bermannia.

lotoides.

tetrapbyllia.

vulneraria. $\alpha . \beta . \gamma . \delta$.

montana.
Antbyllis cytifoides.

erinacea.

barba.

involucrata.

cornicina.

Arachis tripbylla. fol.

bypogea.

Ebenus cretica.

Lupinus integrifolius.

luteus.

varius.

birfutus.

perennis.

albus.

anguftifolius.

rbaseolus latbyroides.

trilobus.

vulgaris. $\alpha . \beta_{\text {. }}$

capen/is.

radiatus.

rufus.

inamanus.

nanus.

max.

lunatus.

mungo?

Dolicbos lineatus. cultratus. incurvus. en/ifformis? umbellatus. birfutus. lablab. 1. 2. 


\section{A ) 178 ( )}

Dolichos finenfis. $\alpha$. B. $\gamma$. Glycine apios. unguiculatus. tranquebaricus. tetragonolobus. fesquipedalis? altisfinus. pruriens. zurens. minimus. capen/ls. fcarabcoides. bulbofus.. trilobus. ariftatus. purpureus. regularis. lignofus. Soja.

biflorus. a. repens. proftratus.

roferis. luteus. lutenlus. polyfachios.

Amorpba fruticofa. 1. 2. Teramnus volubilis. uncinatus.

Glycine villofa. javanica. parviflora. reticulata. monoica. frutescens. pbaceoloides. comofa. Subterranea. nummularia. bituminofa. monopbylla. trilaba.

Cylifta comosa.

Clitoria marilandica. multiflora." galactia. ternatea. virginica.

Pifum fativum. 1. 2. 3. maritimum. I. 2. arvenfe. ocbrus.

Orobus pyrenaicus. tuberofus. vernus. angufifolius. Syluaticus. niger. luteus. latbyroides. capenfis.

Latbyrus coccineus. bengalenfis. articulatus. odor atus. 1. 2. 3. bir/utus. 


\section{\#) $180($ 唇}

Latbyrus angulatus. ampbicarpos.

Cytifus capitatus. pratenfis. tuberofus. Setifolius. clymenum. nisjolia. tingitanus. apbaca.

cacer.

fativus.

inconfpicuus.

annuนs. cblymenum. fylveltris. latifolius. I. 2: beterophyllus. palutr is. pifformis.

Cicer arietinum. Liparia Jpberica. graminifolia. opposita. villega. tomentna. umbellata.

Cytijus volgaricus. cajan. I. 2. fuрiпus. glutinnfus. auftriacus. vi/co/us. argenteus. candicans. glabratus. birfutus. fesfilifolius. nigricans. labarnuns. sericsus. biflorus. pendulinus. alpinus.

Geoffroija inermis. Robinia Spinosa. balodendron.

firox.

feprum. violacea. Speudacacia. bispida. altugana. mitis.

Coluten orientalis. alleppica. arborefcens. frutefcens. berbacea. Glycyrrbiza lavis. ecbinata. glabra. 


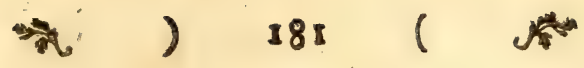

Glycyrrbiza aspera. birfuta.

Coronilla Jecuridaca. cmerus. valentina. juncea. varia. glauca. coronata. minima. argentea. cretica.

Ornitbopus durus. compresfus. pufillus. fcorpioides.

Hippocrepis comosa. multifiliquora.

Scorpiurus vermiculsta. muricuta. fulcata. fubvilloga.

Aefchynomene afpera. Jeshani. 2.34.
Jpinulofi.
americana.
indica.
pumila.
pallida.
Jenfitiva.
coccinea.
grandifiora. 1. 2.
Stylofantbes procumbens. vifcola. elatior.

Hedyfarum albagi. x. 2. bupleurifolism. numsmularium. I. 2. moniliferum. vaginale. I. 2. linefolium. glumaceum. Ayracifolium. reniforme. fororium. T. 2. vefpertilio. gargeticum. maculatum. triquetrum. $x_{4} 2$. Atrobiliferum. I. 2. crashfolium. dipbyllum. 1. 2. ungulatum. spartium. pulcbellumo I. 2. lineatum. albicans. retroflexum. umbellatum. I. 2. polyanthos. biarticulatum. beterocarpon. canadenfe. conefcens. frutefcens. 


\section{- ) 182}

Hedyfarum tauricum.

junceums. mud florune. triflirum. 1. 2. vifcofum. tomentofum. pilosum. virgatune. friatum. fericensm. micropbyllum. caudatum. racemofum. fruticofume. barbatum. lagepodioides.

gyrans. retufurn. adfcendens. Scorbiurus. incrnum. tortunjum. Ppirale. jupinum. tetrapbyllum. eretaceum. I. 2. argentatum. a. $\beta . \gamma$. grandiflorum. alpinum. 1. 2. coronarium.

A:xu: funt. bumile. spino/um. pumilun. onobrycbis. jaxatile. caput. crifta. crinitum. lanceunn. incanum.

Indigofera enneapbylla. farmentofa. Secicea. ovata. pforaloides. procumbens. denudata. candicans. erecta. ceilanica. femitrijuga. angulata. beteropbylla. filiformis. digitata. pinnata. fricta. frutefcens. glabra. cytifoides. lotoides. birfuta. indica. anguftifoliso.' Anil. tinctoria. 


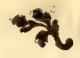

)

Indig ofera argen:ea. birta.

Galega villofa. I. 2. proftrata. officinalis. sinerea. littorslis. virgirica. pifidia. puytrerea.

Pbaca alpina. I. 2. falfola. proftrata. oxypbylla. lanata. myriopbylla.

Afragalus nlopecuroides. I. 2. melalotoides. pilojus?

aliginofus. onobrycbis. cbriftinus. galegiformis. fulcatus. arenarius. micropbyllus. canadenfis. annularis. glaux.

Sefameus. I. 2. bamofus. $\mathrm{r}, 2$. contortuplicatus. beticus.
183

Afragalus Laxmanni. alpinus. Jyriacus. pentaglottis. epiglottis. bypoglottis. dacireus. sicer. glycyplyllus. suftriasus. finicuis. chinenfis. amiodytes. tragacantba. tragacanthoides. trimeftris. montanus. exfcapus. verticillaris. caprinus. uralewhs. laguroides. pbyjodes. depresfus. I. 2. monspessulanus. campefiris. 1. 2. tragoides. bybridus. fatidus. tenuifolius. bullarius. unilocularis. I. 2. Biferrula pelecinus. 


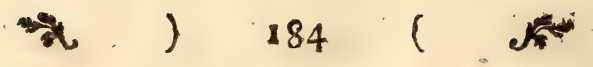

Visia latbyroides.

PSoralea bituminofa. geridi.

pyrenaica.

pannocrica.

dumetorum.

monantbos.

nisfoliana.

narbonenfis.

bybrida.

fativa.

onobrycbioides.

bitbynica.

flvatica.

casfubica.

crassa.

biennis.

bengalenfis.

butea.

peregrina.

sepiums.

capenfis.

pififormis.

faba.

Ervum monantbos.

Seloniense.

lens.

tetraspermum.'

birfutum.

ervilia.

Pforalea tetragonolobr.

glanduloja.

americana.

paleftina.

pinnata.

aculeata.

Spicata.

decidue.

tenuifolia.

birta.

repens.

axillaris.

rotundifolis.

aplyylla.

capitata.

corylifolia.

Trifolium fubterraneums.

bybridum.

fuffocatum.

Spadiceum. anguffifolium.

Ariatums.

rubens.

ocbroleucum.

agrarium.

cberleri.

ornitbopodioides.

lnpinafter.

repens.

lappaceum.

incarnatum.

anguftifolium.

Aellatum.

clypeatum.

spumo fum.

montanam. 


\section{$x$}

)

Trifolium arvenfe. 1. 2. pannonicum. tomentofum. Jcabruns. fquarrofuns. refupinatuns. glomeratum. filiforme. fragiferum. I. 2: comolum. alpeftre. cervileum. indicurn. $\alpha$. $\beta$. mes farènse. polocricunt.

italicum.

creticum.

Medicego arborea.

marina.

lupulina.

profirata.

circivata.

falcata.

fativax.

radiatio.

obscure.

polymor, orbicularis.

intertexta.

coronata.

Scutellata.

ciliaris.

minima.

turbinata.
185

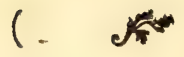

polymor. tornata. laciniata.

Trigonella rutbenica. monfpeliace. indica.

fanum. I. 2. bomosa. tannëafis. corniculata.

spinofa. laciniata. polycerata. platycarpos.

Lotus filiquofus. jacobceus. birfutus. conjugatus. glaucus. $\alpha . \beta$. edulis. rectus. dolyctus." meritimus. corniculatus. arboreus. angufus. cytifoides. peregrinus. arabicus.! ornitbopodioides. creticus. dorycrium. tetragonus. maritimus. 


\section{) 186 (
Polyadelpbia.}

Tbeobroma cacao.

Ambroma angufta. speberi.

Citrus japonica. trifoliate. medica. aurantium. decusnunus. I. 2.

Durio fatida. Hopea tbeaformis. martinifenfis. octopetale. guianen/ss. iucida. tinctoria.

Hypericum longifolium. virginicum. orientale. ericoides. bratliys. coris. arbareumi. fol. erectunt. monogynum. maculatum. I. 2. calycinum. linarifolium. canariense.
Hypericum olympicum. balaaricum. fatidum. agypticum. elodes. reflexum. cayanense. guadrangulare. tomentofum. guineense. kalmionun. birfutun. crispum. gramineuns. bunnifufum. afcyron. androjemum. bircinum. patulum. canadense. montanum. nummulariums. mutilum. atbiopicum. barbatum. perforatum. 1. 2. prolificum. I. 2. pulcbrum.

Afcyrum bypericoides. Andrece. 
D. $D$.

MUSEUM NATURALIUM ACADEMIE UPSALIENSIS.

D

cujus

PART. XXI.

DENIA EXP. ORD. MED. UPSAL.

PR ESIDE
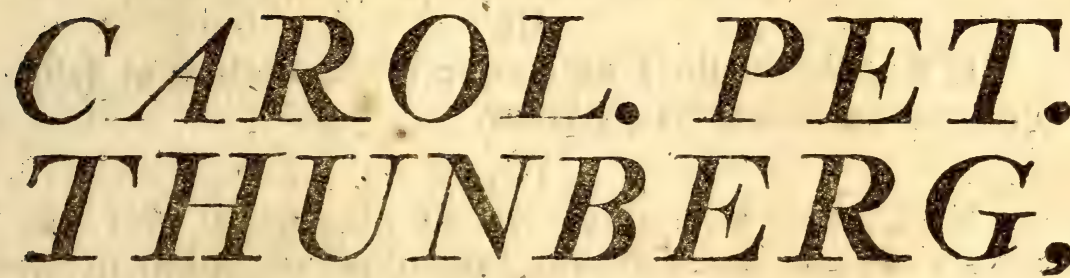

Equite-Aurat. Reg. Ord. de Vasa,

Medrc. Doct: Profess. Med. et Botah. Reg. et Org. Acad. Cesar. Nat. Curios. Reg. Scient, londin. Hoim, Socret. Scient, Ursal. Phirad. Pati. Holm. Beror. Nat. Scrut, Taris, ét Hafniens. Hist. Nat. Lund. Haklem. Amsteid. Zezind. Nidrosiens. Ticinens. Ienens. Halens. Nat. Scrut. Linno Londin. Medic. Edimberg. et Nat。 Studios. Irid. Membro; Nec non Acad. Stento Paris, Monsy eliens. A gricult. Paris. Menzc. Londrn, Froreantim. et Batavin, Iño, Cr. Correstongio

PUBLICO EXAMINI SUEJICIT

IOHANNES G.BERNDTSON.

STIP. VRED. STOCKIOLMIENSTS.

IN AUDIT, BOTAN. D. IIT, MART. MDCCXCVM. H. A M. S。

\section{U P S L I $\mathbb{E}$,}

UITTERIS JOH, F. EDMAN, REg. ACAD, TYPOGR. 


\section{THESES.}

I.

$\mathrm{H}_{\text {aud exiguam \& in ceconomia \& in medicina utilita }}$ tem habent plurimæ lichenum fpecies.

II.

Quos inter primum merito occupat locum Lichen Islandicus, nutriendi ille an medendi caufa adhibeatur.

III.

E fructibus rubris eorumque fuccis optima in febribus potiones diluentes parantur.

\section{IV.}

Multis licet nominibus carneis vegetabilia fint præferenda cibaria, promifcuum tamen eorum ufum naturæ humanæ maxime esfe convenientem credimus。

\section{V.}

Animalia carnivora phytophagis fagacitate \& ferocitate omnino preftant.

\section{VI.}

- Animal quodpiam perfeetioribus fenfibus externis fimul fumtis homini antecellere, vix credere posfumus.

\section{VII.}

Variorum, quos in corpus humanum variæ excitant plagx, effectuum cognitio Medico quam maxime necesfaria eft.

\section{VIII,}

Ex quibus effectibus disfirbilitudinem hominum, ઘquam in diverfis orbis terrarum partibus invenimus, præb. cipae oriundam esfe cenfemus. 


\section{DONATION. THUNBERGIAN. CONTINUAT. XIX.}

\section{Syngenesia.}

Lapfana Z zintba.

communis $1,2,3$.

r bagnciolus.

fill ta.

Kalpinia.

Hyoferis virginica.

minsma \&, 2,3.

fatida.

rediata.

fcabra.

lucida.

rbagadioloides.

cretica.

bedypnois.

Borkhaufia bispida. Prenantbes farmentofa.

dentata.

lyrata.

jquarrofa.

bafata.

cbinenfis.

multifora.

bumilis.

debrlis.

integra.

joponeca.

muralis. purpurea.

viminea.

alta.

tenuifolia.

pinnuta.

Cbondrilla juncea.

nudicawlis.

Crepis birta.

dentexis

lavigata.

filformis.

bavbuta I-4.

veficaria.

alpiza.

rubre.

- fcitida. a. B.

a pera.

fiberica.

eetorum. $\mathrm{I}=\mathrm{j}$.

biennis.

virens.-

diofcoridis.

negleta.

Janctu.

Hieracium incanum.

veriofum. 


\section{- 286 (}

Hieracium pilosello.

dubium.

slpinum I-5.

pumilum.

auricula.

pramorfum.

cymofursi.

aurantiacum.

Gronovii.

fabsudum. I.4.

paludofum.

lyratum.

posrifolium.

murorum. $\alpha . \beta$.

Sprengerianum.

villofum.

amplexicaule.

cerintboides.

pyrenaicum: biatina

rioides. $\alpha . \beta$.

aufviacum.

belveticum.

unbellatum. a. $\beta$.

g? andiforum.

Lactuca capenfis.

fativa.

jeariola.

gnercina.

virofa. 1-4.

faligna:

canadenfisa.

perennis.

Sosscbus fruticofus:

alpinus.
Soncbus lapponsicus

maritimus. $\alpha . \beta$.

plumievi.

floridanus.

fibiricus.

canaderfis.

arvenfis.

tener.

agreftis.

Squarrofus. a. B

olevaceus. $\alpha . \beta$.

Carduus paluftris. ariipus. a. B. acanthoides. polyantbemus. pycrosepbalus.

lanceolutus. stabicus. cyanoides: argerstatus. carus. defloratus. I, 2. monspesfulanus. panronisus.?

nutans.

tuberofus. $\alpha, \beta$.

indicus.

limearis.

cofabonse.

Barrelieri.

neglectus.

medius. Is

Relletus.

mollis. \& $B$ 


\section{ㄱ. ) $187(\%$}

Carduus eriopborus.

ferratuloides.

\section{ciliatus.}

marianus.

Syriacus. od. $\beta$.

beteropbyllus. a. B. $\gamma$. belenioides. $\alpha . \beta$.

iataricus.

acaulis. $\alpha . \beta$.

Arctium lappa. i- 2. perfonatum. 1. 2.

Atradiylis gummifera. bumilis, a. B.r. cancellata. $\alpha, \beta_{1}$ lanceolata. ovata.

Cnicus Jpinofisfimus.

oler aceus.

erifit bales.

centaurosdes.

fernx.

aCArnR.

cernuss.

Carlina actulis. $\alpha_{0} \beta_{0} \gamma_{0}$

lanata.

pyrenaica.

vulgaris.

vacemoja.

atractyloides.

xerantbemoides.

Cynara bumilis.

$$
\text { acaulis. }
$$

Cartbaunus tinciorius. carulexs.

arborefcens:

mitisfimus.

carduncellus.

lanatus.

creticus.

tournefortis

integifolius.

Onopordon acaule.

acanthium.

illyricum. 1.2.

arabicum.

Picris jinponica.

flexuofa

bier scioides.

eibioides.

asplenioides.

Leontondon birtuns.
aureum.
bafile.
proteifornse.
toraxacuns.
fcabrum.
pyrenaisum.
bulbofum.
bijpidum.
autuessale.
tuber ofum.
afivale.

Scorzoryera brevicaulis.

undulata.

eriojpermas:

purpures.

rejedifolia. $\alpha, \beta$. 


\section{* $) 887(5$}

Scorzonera torihentosa.

bumilis.

bispanica.

angufifolith.

laciniata.

picroides.

orientatis.

tingitana.

Tragopogon pratenfe.

sndulatism.

villo/um.

parvifnlium.

dalecbampis.

picroides.

orientabe.

crocifolium.

Geropogon glabrum.

Cicbormuns endivia.

intybus.

spinofum.

Catanancbe Intea.

carulea.

Hypocbaris glabra. a. Aิ.

iradicate.

Arignas.

maculata. a. $\beta$.

Seriols lavigata.

ationenfis.

urens.

Scolymus hipani us. snaculatss.

Andryals la iniata.

firsuta.
Andryale rucinata.

integrifolia.

ragufinat

lanita.

pinnatifida.

cbeirantifolia.

glandulofa.

Ageratum conyzoides, a. $\beta$.

Spilantbus uligincfa.

olevacen.

Jalivaria

acmella.

pleudoacbmella.

Etbulia frucbium

conyzides.

divaricata.

Jparganopbora.

Serratula falicifolia.

fariofa.

alpina.

amiara.

centauroides.

japonica.

Spicata.

glauca.

tinetoris.

coronata.

multifora.' $\alpha$. B. $\gamma$.

Squarrofa. fol.

Cacalia Kleinia.

a arvenfis. \&. B. $\gamma$.

repens.

ficoides.

suniefulia. fol. 


\section{- $) 189(40$}

Cacalic anteupborbium. fol. Eupatorium bys sopifolium. articulata. tomentofa. poropbyllum. radicans. ecuulis. cinerea. fcandens. buftata. fuaveoléss. atriplicifulia. fonchifolia. a. $\beta$. forracenica. silpina $\alpha$. $\beta$. $\gamma$. alhifrons. $\alpha$. $\beta$. bipinnata.

Cbryjocome aurea. tomento $a_{a}$. patula. cernus? ciliatr. scabra. linolyris. a. $\dot{\beta}$. graminifolia. biflora. villofa: purparea. spicata. dicbotonna.

Kubnia ewpatorioides. Eupatorium parvifloram: dalea. fcandens. Houftonis.

baftatum. ceilnnicum. finense. jesfilifolium. albuns: diffusum. altisfinium. trifoliatum. joponicum. cannabinums. cinereun: maculatum. purpureum. a. $\beta$. villofuns. perfoliat um. micr opbyllum. aromaticum. ageratoides. caleftinum. molle

montanum. cordifolium odorntum. ivcefolium. arvenfe. disuricatum. fyriacum.

Santolinid dentata. rosmarinifolin. chamceyparis/us. antbemoides. terrefsris.

Cales 


\section{*) $) 190(\%$}

Calea oppofitifolia.

jcoparia.

Jobata.

jamaicen/is.

leptopbylla.

Atbanafia filformis.

geniftifolia.

pormila.

jesfilifura.

tinifolia.

cinerea.

longifolia.

vefita.

pubefcens.

canefcens.

capitata.

maritinen.

Squarrofa.

uniflorn.

crenata

dentata.

trifurcata.

fidentata.

bir/uta.

parviflora.

annua.

critbmifolim.

pinnata.

millefolia.

Bidens: niver.

birfuta.

fcandens.

minima.
Bidens bullata.

bifinanta.

pilufa.

tripartita.

cernua.

Pteronia campborata.

fexicaulis. $\alpha$. $\beta$.

cepbalotes.

Jpinofa.

cinerea.

fafciculata.

fucculent a.

fouriofa.

glabrata.

inflexs.

glomerata.

retorta.

memibranacea.

vifcaja.

villoga

oppofitifulia.

pallens.

birfuta.

Tarchonantbus lanceolatus, dentatus. camphoratus. pauciflorus. recessofus.

Schamia paniculata. Artbemifia capillaris. abrotanum. arborefcens. orgentea. 


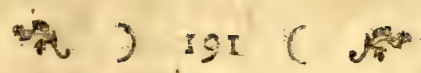

Attemifar arbiopica. judaica. tenuifolia. campefiris sritbmifolia. glacalis. maritime. Jantonica. paluftris. rupeftris. Jpicata. fericen. glauca. abfintbiums. pontica. aufreiaca. vulgaris. fiversiane. pectinata. tanacetifolia. anmua. carulefcens. japonica. dracunculus. integrifolia. minima.

maderafeatama. cbrysantoss. Cookii.

Cotula turbirata. antbemoides aturea. coronopifolia. tontasotifolia.

Cotusles capenfis. fericea. qvinqueloba. firicta.

Baccbaris dioica. ivafolia. a. B. indica. dinfcovidis. balimifolia.

Carpefium abrotanoides. cernuum.

Tanacetum annuкm. nngulatum. argenteum. vulgare: $\infty . \beta$. inconum. balfamita. fibivicum?

Conyza purpurafcens. aurita. $\alpha . \beta_{0}$. finen/s. rigida. arborefcens. \&. linifalia. aferoides. cinerea. smontan a: lobata. sopulifolia: eppendiculate. decurrens. wirgata. lacere. fquarroja. 


\section{* ) 192 (}

Conyza candida.

faxatilis. $\alpha . \beta$.

rupeftris.

nivea

antbelmintica.

feandens.

birfuta.

odorata.

Gnapbalium eximium. difcolorum.

arboreum.

ericoides.

grandiflorum.

teretifoliums.

muricatum.

umbellatune.

milleflorum.

corasatums

appendiculatum.

undatum.

petiolntum.

patulum.

dentatum.

repens.

bispidurse.

ftacbas.

ignefcens?

orientale.

ar enariam.

rutilans.

cymo/um.

albefcens. luteoalbum. nudifoliún.
Gnapbalium fangvineum. undulatum. frtidum. beiiantbemifolium.

Squarro/im. obtuffolimn. indicum. purpureum? margaritaceum. plavtagineum. dioicum. alpinum. hlvaticum. norvegicum. verticillatum. fupinum. oculus. glomeratum. uliginofum. nudum. involueratum. japonicuns devium. paniculatum. candicans. polifolium. Spatbulatum. acuminatum. crasfifolizm. latifolium.

Xerantbeinum annum. bellidioides. veftitum. imbricatum. 


\section{- ) 193 (}

Xerantbernum proliferans.

jpeciocis/inum.

retortum.

virgatum. avgenterm.

Jefamoides. a. B.

jquamofum.

paniculatum.

Rabelina.

variegatum.

lpinofum.

fulgidum.

recurvatums.

Anacyclus creticus.

\section{aureus. \\ valeitinus.}

Perdicium le mifofculare.

magellanicum.

tomsent ofum.

brafilienfe.

Bellis annus. a. $\beta$. perennis. a. $\beta$.

Unxia campborata. Bolionia afteroides. Matricaria Fartbenium.

argentea.

maritima.

fvaveolens.

cbamomilla.

proftrata.

Chryfantbemum monspeliense
Segetum.

montanum.

leucantbenam. $\omega$. B.

Serotinum.

myconis.

fruteicens.

coryinbosum.

inodorum.

acuilleos.

alpisum.

balfamita.

indicum. $\alpha . \beta$.

Hofculofum. $\alpha . \beta$.

ar Etium.

pinnatifidum.

coronariuss.

pectinatum.

millefoliaturn.

bipinnatum?

japonicum.

lacerum.

Doronicurn perdaliancíes.

axfiviacum. $\alpha$. $\beta$.

bellidiaftrum. $\alpha . \beta$.

plantagineum. $\alpha . \beta$.

uniflorum.

altaicum. $\alpha . \beta$.

Ainica montana.

ciliata.

crocea.

tabularis. $\alpha . \beta$.
Arnica 


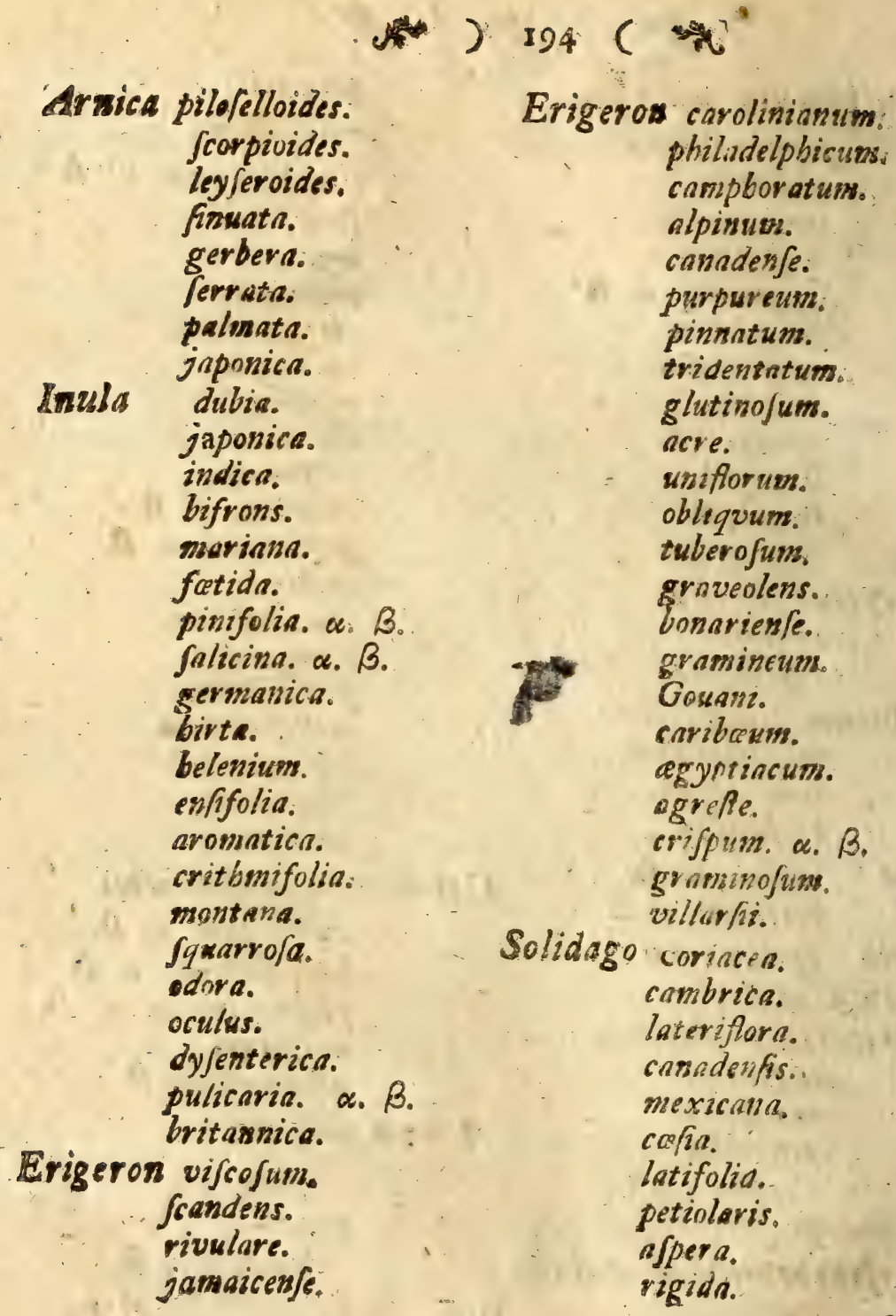

Soli- 


\section{( ) $195(\lambda$}

Solidago bicolor:

Serotina.

virgaurea.

Sempervirens.

flexicaulis. a. $\beta$. gigantea.

nemoralis.

minuta:

altisfsma.

norbonen/is.

Cineraria japonica.

lanata.

repanda. a. $\beta$.

snoritima.

slatn.

geifolia.

difcolor.

glabra.

glauca.

conadenfis.

amelloides, $\alpha$. . $\beta$.

fiberica.

elongeta. a: $\beta$.

cacrilioides.

denticulats.

perfoliata.

braftifolia.

tomentrsa.

cymbalarifolis.

lobata.

cruenta.

fili olia.

aipina. a. B.

integrifolia.

1) Cacalia. Linn.
Cineraria palufiris.

fonchifolis.

apperdiculata. a. B.

capitlaced.

$\left.\gamma_{0} I_{0}\right)$

Iusfilago pumila.

albicans.

anandria. o. $\beta$.

alpina. a. $\beta$.

difcolor.

nutans.

farfara.

japorica.

petafites. 2. 2.

alba.

bybrida.

frigido. 1, 2.

fpuria. 1. 3.

paradora.

trifurcata.

Stnesio verbencfilius.

bieracifolius.

japoniews.

vulgaris.

peucedonifolius.

virgatus.

purpureus.

perficifolius.

replicatus

triflorus.

agyptius.

trilobius.

wizcosus. 


\section{$20) \cdot 196(2$}

Serrecio fluaticus.

elegans. $\propto$. B. .

Sigualidus.

erucifolits.

jacobeus.

abrotanifolius.

inconus.

diffufus.

carindenfis.

aureus.

lyratus.

linifolius.

ro/marinifolius. $\alpha$. $\beta$.

longifolius, $\alpha . \beta$.

cruciatus.

juniperinus.

byzantinus.

erofus.

illicifolizts. a. B.

rigidus.

paludofus.

nemorenfis.

Sarracenicus.

Doria.

doronicum.

glaftifolius.

marginatus.

angulatus.

balimifolius. a. B.

maritimus.

lanatus.

cordifolius.

aquoticus. \&. $\beta$.

myrrbifolius.
Serecio Sesfilifolius.

undulatus.

incifus.

crifpus.

beter opbyllus.

repandis.

Jerritus

glutino/us.

littoreus.

muralis.

Squamoysus.

arenavius.

fujcus. $\alpha$. $\beta$.

pinnulatus.

pulber.

carnojus.

fpirceifolius.

abruptus.

lavigatus.

After vellexus.

fruticulofus. bysjopifolits. dume us. ericoides. linavifolius. linifolius. tenuifolius. acris. umbellatus. $n$. angliae. grandiforus. undulatus, $\alpha$. $\beta$. tripolium. vernus. 


\section{- $) 197($ \%}

After amellus.

fibericus. $\alpha . \beta$.

alpinus.

tenellus. a. B.

pilofus.

divarientus.

cordifolius. $\alpha$. B.

puniceus.

annuus as $\beta$.

Jaber.

indicus, a. $\beta$.

mifer.

levis.

n. belgii.

trade canti.

tardiflorus.

bolofericeus.

mutabilis.

finenfis. a. B.

rnnunculoides.

macropbyllus. a. B.

bifpidus.

Vaillantii.

Bellium bellidioides.

minutum.

Pectis ciliaris.

linifolia.

bumifufa.

Tagetes erecta. patula.

Helenium autumnale.
Leyjera griapbaloides. paleacea. arefotoides.

Sigesbeckia orientalis.

Ecclipta latifolia. profirata.

fissilis.

evedta.

paluftris.

Anthemis arabica.

nobilis.

maritima.

altisfima.

tinctoria. \& $\beta$.

cota.

pyretbram.

mixta.

alpina.

tementofa.

arvenfis.

valentina.

montans.

Acbillea ageratum.

alpina.

ptarmica.

clavenne.

ferrata.

coronopifolia.

macropbylla.

ligufica.

magna. 


\section{4) 198 (}

Acbillaea regytiaca.?

cretica.

nasa. $\alpha . \beta$.

micrantba.

impatiens?

atrata.

pubejcens.

robilis.

tomentofa.

- millefulium.

abrotanifolia.

odorata.

Genipi.

imbricata.

Bupbtbalmum aqvaticum.

frutefcens.

arborefcens.

grandiflorum.

jalicifolium.

Jpinofum.

graveolens.

Amellus lycbritis. \&. B. umbellatus. diffufas.

Robria bifulca.

fulcata. pettinata.

Squarrofa.

lewceolata.

siliaris.

fetora.

bijpidk.
Robria monantbos.

armata.

carlinoides. cartbomoides.

decurrens. cruciata. obovata.

curreatn.

incarn.

grardiflora.

fpunolisfima.

palmata.

Verbefina alata.

Ginenfis.

mutica.

calendulacen

biflora.

baccata.

Lavenia decumbens. evecta.

Staebelina imbricate.

duba.

fruticosa.

gnapbaloides.

chamapeuce.

arborefcens.

corymbofa.

Centaurea crupina.

galactites. mofcbata. amberboi. erucifolia. Lippii. 


\section{$15) 290(x$}

Centaturea alpinn.

orrestalis.

argentea.

repens.

jacen.

amara.

allin.

Splendens.

glinfifolia.

conifera.

nudicaulis.

falmantica. «. $\beta . \gamma$.

muricata.

crocadylium. a. $\beta$.

linifolia.

cysnus. $\alpha . \beta$.

montana.

falicifolino.

uniffora.

capillata.

pbrygin,

pettinats. a. $\beta$.

jempervivens.

nieva. \&. $\beta$.

pullata. $\alpha_{0} \beta$.

fibirica.

ragufina.

foncbifolia.

paniculata. $\alpha$. $\beta$.

ovina.

totarica.

cineraria.

fcalioja. $\alpha$. B. $\gamma$ d. cejpitaja. calcitrapa.

calcitrapoides.

tripolitana.

lanata.

Jeridis.

spbaerocepbala.

ifnardi.

napifolia.

alpera.

beredicta.

eviophora.

folfitialis.

melitenfs.

ficula.

Verutum.

centauroides.

collins.

cespitosa.

Gorteria linearis.

perfonata. $\alpha . \beta$.

uniflore, $\alpha . \beta$.

rigens. $\alpha . \beta$.

iscija.

pinmata.

otbonnites.

diffusa.

integrifolie.

cliata. a. $\beta$.

cernua.

pettinata.

Zinnia pauciflora.

elegans.

verticilltata. \&. $\beta$.

Didelta tetragoniafolia. 


\section{$20) 100(=x$}

Coreopfis coronata. odorata. verticillata. auriculata. $\alpha . \beta$. lanceolata. $\alpha . \beta$. alternifolia. tripteris. reptans. baccata. leucastba. cbryjantba.

OJmites Priata. nervofa calycina. afterifcoides. a. B. campborina.

Filago montana. arzenfis. leontopodium. acaulis. leontopodioides. germanice. pyramidata. gallica.

Micropus eregus. lupinus. Milleria biflora. quinqueflora.

Baltimora recta. Otbonna denticulata. pectinata. sbeirifolia.
Otbonna lingua. cacalioides. coronopifolia. frutefcens. ericoides. beteropbylle. bulbofia. pinsata. linifolia. digitata. atbanafie. tenuisfima. a. B. capillaris. laterifloia. tagetes. s-denta. paniculate. cilata. a. B. munita. abrotanifolia. imbricata. amplexicaulis. rubens.

Zagea leptaurea. Rudbeckia alata. a. $\beta$. amplexicaulis. birta. angufitfolia. oppofitifolia. fulgida. purpurea, \&. B. trilobn. lacinista. x. 2. 


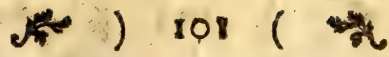

Helianibus annuus. x. 2. 3. Calendula bybrida.

indicus $\%$. $\beta$.

tuberojus.

multiflorus.

frondo/us.

divaricatus.

decapetalus.

altisfimus.

gigantess.

lavis.

angufifolius.

frumofus.

Ofteofpermum pevfoliatum. calendulaceum. berbaceum.

junceum.

polygaloides.

piciferum.

cliatum. $\alpha . \beta$.

imbricatum.

Spinofum.

niveum.

or Etotoides. $\propto . B$.

iriquetrum. $\alpha . \beta$.

illicifolium.

corymbofum.

woniliferum.

pizsuatifidum.

Hippia lufitanica.

Calendula arvenfis.

offictralis. $\alpha . \beta$.

fruticofa.

pluvialis. nudicaulis.

pumila.

gramenifolia.

amplexicaulis.

comentofa.

Atellata.

Aretotis palacer.

antbemoides, $\alpha_{0} \beta$.

pilifera.

anguftifolia.

diffuja.

paradoxa.

ferrata.

afpera. a. $\beta$.

acaulis.

lanata.

interipte.

brevifcapa.

argentea.

decumbers.

elongata.

grandis.

facabra.

levis.

undulata.

petislata.

fericea.

linearis.

glanduloja.

pinnata.

candide.

denudata.

caulefserss. 


\section{- 2 ) $x 02(\ldots$}

Arctotis calendulacen.

triftis.

Superba.

punctata.

pinnatifida.

cernua.

plantaginea.

dentata.

Eriocepbalus africanus.

racemofus.

Polymnia carnoja.

$$
\text { Spinoja. }
$$

tetragonotbeca.

Uvedelin.

caradenfis.

Ambrofia triloba. a. $\beta$.

elatior.

artemiffolia.

maritims.

Partbenium byferopborus.

Iva

\section{annus.}

frutefcens.

Melampodium bumile.

Trixis tberebintbinacea.

Silpbum laceniatum.

afterifcus.

connatums.

perfoliatum.

trifoliatum.

trilobatum.

tberabinthinnceuts.

Oedera prolifera. a. $\beta$. aliena.

Strebe atbiopica. a. $\beta$.
Stcebe profinata.

gnapbialioides.'

dificba.

vbinocerotis.

veflexa.

fabre.

ericoides.

gompbresoides.

cersuna.

proscumblens.

Ecbinops vitvo.

Arigofus.

Spherocepbalus.'

Jpinofus.

Tetrantbus luttoralis.

Elepbantbopus tomento/us.

jeuber.

jpicatus.

birtus.

angufifolius.

Spberantbus indicus.

mollis.

africanus.

Fafione fenegalents.

Crafpedia unifora.

Seripbizms ambiguun.

fufcrum, cinerenm. plumofam.

fafciculatum.

Corymbium Kiforme.

glibe won. mervifum.

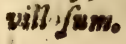




\section{D. \\ MUSEUM NATURALIUM ACADEMIÆ UPSALIENSIS.}

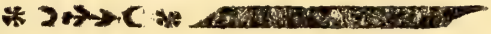

cujus

PART. XXII.

YENIA EXP. FACULT. MED. UPSAL.

P R AES I D E
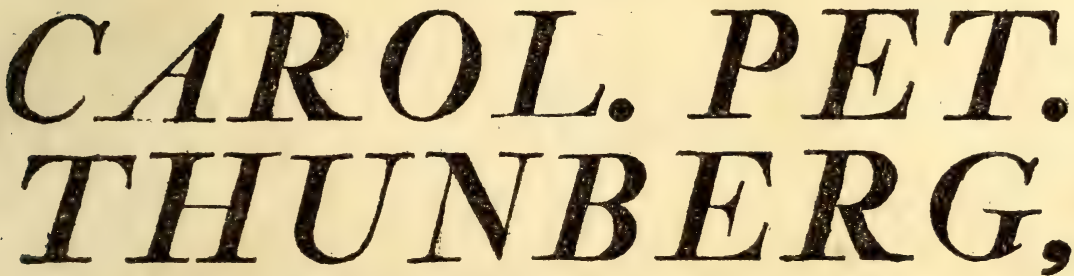

Equite Aurat. Reg. Ord. de Vasa,

Medic. Doct. Prneess. Med. ex Botan. Reg. ex Ord. Acad. Cesar. Nat, Curios. Reg. Scient. Londin. Holm. Societ. Scient. Upsal. Philad. Patr. Holm. Berol. Nat. Scrut, Paris. et Hafniens. Hist. Nat. Lund. Harlem. Amsteld. Zeland. Nidrosiens. Ticinens. Ienens. Halens. Nat. Scrut. Linn. Londin. Phys. Góttingens. Medic. Edimburg. et Nat, Studios. Ibid. Membro; nec non Acad. Scient. Paris. Monsteliens. Agricult. Paris. Medic. Londin. Florentin. ex Batavin, Ind, Or. Correspond.

\section{Publico examini proponit \\ GEORG. WAHLENBERG, VER M E L A N D US.}

IN AUDIT. BOTAN. D. III MAJI MDCCXCVII.

H. A. M. S.

\section{U P S A L I 压,}

uitreris Joh. Fr, Edman, Reg. acad. typogr. 



\section{DONATION. THUNBERGIAN. CONTINUAT. XX.}

\section{Cryptogamia.}

Equijetum arvenfe. $\alpha . \beta$. Lycopodium tenellum.

limofum.

fuviatile.

paluftre.

filvaticum, $\alpha . \beta$.

bienale.

Lycopodium nistum.

belveticum.

japonicuss.

ferratum.

dichotomum.

latifoliums.

faftrofum.

fcariofund.

Squarrofums.

cermuum. $\alpha . \beta . \gamma$.

bryopteris.

myrtifoliam.

volutile.

pblegmavia.

alpinum.

felaginoides.

rupeftre.

issundat wers.

Selago.

flabellatum. a. B. $\gamma$.

Aoloniferumb.

apodum. complanatum.

annotinum.

Clavatums.

ornitbopodioides.

circinale.

fangvinolentum.

plamofum.

lenticulatuns.

Onoclea fenfibilis.

polypodioides.

Opbioglossum reticulatum.

lufstanicum.

vulgatum.

volubile.

forndens.

flexuofü.

podatum. 1. 2.

Osmunda ceilanica.

japonicum. $\alpha . \beta$.

obtufata.

Jpicant. a. B. $\gamma$.

polypodioides.

cervina. \&. $\beta$.

birta.

capenfis. a. $\beta$.

lineat $a . \alpha . \beta$.

Lancen.

japonica, $\alpha . \beta$. 


\section{(*) 200 ( th}

Osmunda regalis. aurita.

cinnamomen.

firutbiopteris. $\alpha$. $\beta$. lunavia. $\alpha$. $\beta$.

rstacea. adiantifolia.

birfuta.

crispa. \&. B.

ternata.

virginica.

tburifera.

peltata.

Acrofticbum spicatum.

lanceolatum.

lingva.

Squamosum.

muscofum.

petiolatum.

fimples.

latifolium.

villosum.

ferrulatum.

graminextls.

baftatum.

pectinatum.

lineare.

aureum. $\alpha . \beta$. $\gamma$.

feptentrionale.

ureyniz.

polypodioides.

sorniculatum.

bslene.
Acrofticbum mante.

trifoliatum. $\alpha . \beta$.

forbifolium.

janctums.

thelypteris.

thaliaroides. $\alpha . \beta$.

julpbureums.

calonsilas.

barbarmm.

ilvense.

rufum.

aufrale.

digitatum.

dicbotomums.

Pteris pilofelloides. $\alpha . \beta . \gamma$. anguftifolia. lanceolata. lineata.

pedata. «. $\beta$.

vitata.

tricuppis.

latifolia.

grandifolia. a. B.

rotundifolia.

tricbomanoides.

longifolia. $\alpha . \beta . \gamma . \delta$. beteropbylla.

finsata.y

neroosa.

caudata. $\alpha . \beta$.

aculeata. $X, 2$.

biaurita. $\alpha$. $\beta$.

denticulate. 
Pteris cretica. a. B. Cenopteris abizopbyille.

crenata.

decurrens, $\alpha . \beta$.

atropuspurea.

mutilata.

comans. I. 2.

bafiata.

aquilina. $\alpha . \beta$.

femipinnata. $\alpha . \beta$.

efculenta. $\bar{\alpha}, \beta$

bewnilis.

Bieclnum virginicum.

occidintale.

crivinale.

anitrale.

japonicum. \&. B. $\gamma$.

radicans.

Hemionitis parafitica.

lanceclata.

linesta.

reticulata.

palmata.

Loncbitis asrita.

japonica.
pedata.

jerrulata.

villoja.

adfcenfonis.

ternufolia.

Caropteris flaccida.

odontites.

auriculata.

rutafolia.

myriopbylla.

vivipara

japonica.

cicutaria.

Afplenium lancesm.

vizopbylium.

ferratum. $\alpha \beta$

fcolopendrium. $\alpha_{0} \beta$.

midus. $\alpha . \beta$.

plantagineum.

bemionitis. $\alpha . \beta$.

bifolium.

pumilums

tenerum.

polyodos.

obtujatums.

pygmeum.

crofum.

obli.jum.

lucidwm.

cultrifolium. $\alpha . \beta$.

dentatums.

granidifoliurn.

dividiatum.

Salicifolium.

nodofum.

trichomanoides.

viride.

incifum.

gavinum.

anceps.

ceterack. 


\section{$x)=21$}

Aplenium casdatun. disjectum. lanceolatum. pramorjwo. - enopteris. balbiferum. fragrans. frintuss. rbirophorum. nigum. murariars.

Polypodium articulatum lanceoletuins. lycopodioides. lingva. angultifoliun. vigidum. acrolticboides. marginellum. enfoitum. gerpens. pilnelloides. pyllitidis. repens. ж. B. $\gamma$. crasfifolium. pifillare. gramineum. lineare. pertufums. pbymatodes. a. B. $\gamma$. haflatum. enifforse. incifurs. Loriceum.

Polypodium fcolopendroides. Evifoliaturs. $\alpha$. $\beta$. $\gamma$. pica. $\alpha . \beta$. ঠ. e. $\xi$. trilobum. aureum. $\alpha . \beta$. quercifolium. foandens. ellipticum. myofuroides. pendulum. otites. pectiratum. taxifolium. vulgere. vivininicum. cilistum. disfimile. pufulatum. tricbomanoides. fuspenfum. auriculatum. borridum. $\alpha . \beta$. marginale. fetofum. fagittatum. a. $\beta$. triangulare. reptans. falcatum. \&. $\beta$. marginatum. fopboroides, 1. 2. obliteratum. $\alpha$. $\beta . \gamma$. exaltatum. 


\section{( ) $212(4$}

Polypodium rbisopbyllum. polufsre. $\alpha . \beta$.

creinatum.

tenellum.

birfutum.

surcculatum.

ferra.

loncbitis. $\alpha . \beta$.

femicordatum. $\alpha . \beta$.

unitum.

oreopteris.

thelyptevis.

crifratum.

pribefcens.

acsleatum, a. $\beta$.

tetragonum, $\alpha . B$.

janctum.

extenfum.

elongatum.

reflexum.

lunatum.

cicutarium. I. 2.

arenatum.

mutilatum, $\alpha . \beta$.

patens.

invifum. $\alpha . \beta$.

asperism.

Spinofam. a. B.

mas. 1.2.

noveboracenfe.

cambricuns.

edultum.

pbegopteris.

lecerum.
Polypodiurs rboticum.

regium.

lepsopbylluns.

dryopteris.

furcatum.

excelfuns. $\alpha$. F. $\gamma$.

veftitum.

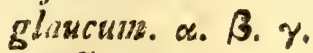

punctatum.

dicbotomum $\alpha_{\text {. }} \beta_{0} \gamma_{0}$

ঠ. $\varepsilon_{0}$

erboreum. a. $\beta$.

villofuse.

pungens.

bivtum.

lineatum.

aculum.

fragrans.

latifolium. I. 2.

fragile.

efculentum.

glaucefcens.

sudum.

caperfe.

effu/um. x. 2.

umbrofsim. x. 2.

affine.

axillare. $\alpha . \beta$.

muscofum.

ariftatum.

adiantiforme.

corinceum.

denticulatum.

reticulatum. 


\section{*) 21315}

Merattia fraxinea. alaza. Adianturn pedatum. capillus. Ariatum. $\alpha . \beta$. rasnofum. ferrulatum. tenerum. radiatum. macropbyilums. deltordeum. fragite. frictam. villofum. $\alpha$. B. pulverulentum. pumilum. atbiopicum. trapeziforme. coudatum. lunulatum. fiabellulatum. criftatum. cuneatum. reniforme. capenfe.

Dickfonia culcita. Squarrofin. frigofa. a. B. flaceida.

disfecta.

Tricboinanes birfutum. cbinenfe. didymum. claziatum.
Tricbomanes canarienfe. repens. folidum. $\alpha$. B. spipbyllum. gibberofurn. elatum. aculeatum. undulatum. ajplenioides. polyantbos. lineare. dilatatum. demisjum. bivalve. multifidums. fucoideum. dendroides. bivtellum. ciliaturs. Sericeum. Sangvinolentum. tunbrigenfe busnile. vigidum. fcasdens. crijpum. crinitum. lacens. pyxidiferum. pufillum. tricboideums. menbranaceum, $\alpha, \beta$. reptans. $\alpha . \beta$. fisfum. 


\section{惜) $214(x$}

Marfilea tetrapbylla. corovinndelica. natays.

Pilularia globulifira. ijoëies laculiris. coromindelica.

Sphagnum paluafre. a. B. $Y$ bajoum curviculluin.

jubulatuss. cu/pidatum. patens. crilpum. pilif ram.

Buxbaumia apbylla. folioga.

Fontinalis minor? antipyretica. falcato.

Splacbrum mnioides. anguftatuen. faftigiatum. luteum. a. $\beta$. rubrum.

Spbericum. vafculofum. ampullaceum.

Polytrichum urnigerwm. bercynicum. aloides. ванит. alpinsurs. commutse. $\alpha . \beta . \gamma$. convolutums.
Polytricbum piliferum.

undulatum. jwniperinums.

Jungergnannia concatenato rivularis. fluviatilis. tbujifolia. palmonta. tricbomanes. concinnata. inflata. $\propto . \beta$. uliginofa. bifida. birotusda. tenuis. adunca. afplenioides. adiantoides. criftata. proftrata. Spbagni. diffusa. viticulo/a. polyantbos. convexa. lanceolata. perfoliata. bidertata. emarginata. fetiformis. bicuspideas: connats. 5-dentata. texera. 


\section{) $215(-2$}

Fungermannia pallens, «. B. Fungersmannia alpina. fimplex. undulata. nensorofa. refupinata. ferrulata. juniperina. albicans. capillaris. cupres/ina. trilobata. reptans. maltiflicre. cocbleaviformis. complanata. transverfalis. fava. cavifolia. folonifera. bracbiata. dilatata. tamaviscifolis. atrata. filiformis. platypbylla.

filicine. ciliaris. vaginata. tomentafa. tricbopbyilla. varia. vifaria. pufilla. julacea. rupeftris.

multifida. epipbylla. finuato. fucoidea. bipinnata. furcaita. dicbotoma. linearis.

Funaria bygrometrica a B.\% Timmia megapolitana. Tetraphis pellucida. androgyna,

Dicranum purpureum. taxifolium. fcoparium. bryoides. beteromalliana adiantoides. glascums. pellucens. pulvinatum. $\alpha, 8$. aciculare. fciuroides. polycarpon. pufillum. Atrumiferum. cerviculaturs. Jetaceuns. ovatum. virens. polyfetum. crijpurn. varium. 


\section{武) 216 ( 情}

Dicronume Alexuofure.

Celfii.

palmaturn.

polvpodioides.

asplenioides.

calycinum.

lycopodioides.

Mafia longifeta.

Pterigynandrum gracile.

julaceum.

filiforme.

Octoblepbaris albida. Encalyptra afiva.

para/fitica.

cirrlata.

ciliata.

extinctoric.

crispa.

Ianceolata.

Tricboftomum lanuginofum.

B. $\gamma$.

canefcens.

beteroficbon.

glauce/cens.

firiEtum.

Tortula muralis.

vuralis.

acuminata.

fallax.

fululata.

mucronulate.

rigida.

convoluta.

Tortula agravia.

pufilla.

Didymodion capillaceun. inclinatum.

Gymnofomum pyriformse.

ovatum.

truncatum.

pennatum.

microftomum.

cilintum.

lopponicum.

Grimmia apocarpa. alpicola. cribrnja.

Weifá recurviroftra.

beteromalla.

acuta.

controversa.

Ortbotbricbum friatum..$\beta$. 3 .

crijpum.

anomalum.

Poblia elongata. inclinata.

Bartbramia pomiformis. \&. $\beta$. Halleriana.

Bryum pyriforme.

nutans.

paluftre.

argenteuns.

carneum.

alpinums.

pulcbellum.

fontanum. 


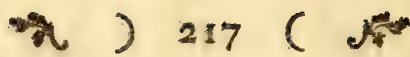
Bryum fquarrofum. crudum. ventricofum. turbinatum. annotinum. rubrum.

vimum. capillare. cespiticium. Serpyllifolium. cuspidatum. rofeums. ligulatum. verticillatum. tomento/uns. Spbarocarpon. dendroides. paterss.

microcarpon.

Spiniforme.

cirrbatum.

Neckera crifpa. viticulosa. dendroides. curtipendula. difticba. paradoxa. glabella. ormitbopodioides. filiformis. pennata. polytricboides. campofita.
Neckera bypnoidea. fulgens. filicina. macropoda. capillaris. albicans. tricbomanoides. complanata. fericea. paludosa. fubtịlis. polyantbos. undulato.

Hypnum denticulatuns. fpiniforme. filvaticum. undulatums. firamineum. vigojum. fluitans. aduncum. triquetrum. friatwm. piliferun. filicinum. prolifirum. delicotulum. parietinum. precionguns. criffa. abietinum. plumofums. cupresifforme. 


\section{म $) 218$ ( )}

Hypнum scorpioides. patulum.

fquarrofum. loreums. reptans.

fafciculatum. tamarijci. alopecurum. flexile. nigrefcens. nigrum. denfum. purum. riparium. cufpedatum. tomentofuns. depresfum. Scbreberi. trichopbyllum. velutinum. micropbyllum. calpitorum. purgens. congefium. tetragonum. torquatum. cirrbofum. paluftre. albicans. Halleri. Jetaceum. molle. jerpens. rutabulum.
Hypnum rotatum. dicbotomunn. coturum. radiaturn. roftratum. penneforme. uncinatum.

Marcbantia japonica. birfuta. polymorpba. S. ‥ 3. androgyna. cbenopodiata. triandia. tenella. conica. beniifpherica.

Blafir pufilla.

Riccia reticulata. fluitans. pyramidata. cryfallina. minima. glauca. natans.

Targionia bypopbylla. Antboceros lavis. crifpus. punctatus.

Ulva compresja. pavonia. montana. latisfima. a. B. reticulata. 
Ulva intefinais. lumbricalis. rugofa. linwa? lactuca. crifpa.

Conferva rivularis?

Lisben pallido-niger.

rubens. albo-cafius. odoratus. Wefrungii. Svartzii. pinicola. fontinalis? fluviatilis. bullora? canali ularis. anit bibia. feticulofa. t: menetisa. nigra?

reticulata. gelatinofa, a. $\beta$.

fericea. polymorpba? rupetiris. $\alpha . \beta$. Ag gagropila. corallinoides. globifera. flabelliformis. diapbiana.

Licben antiquitatis. jolitbus.

favus. byprorum. auretus. denigratus. foriptus. geograpbicus. - ugajus. atrovirens. bysfordes. elveloides. fungoides. bxomyces. icmadopbila. fabuletor um. teftaceus. pertufus. rupicola. graniformis. levcopbeus. granulatus. confluens. fongvinarius. exiguus. fufco-ater. vernalis. calcareus. incanus. botryoides. lutejcens. villojus. atro-albus. albo-ater. niger. Oederi. 


\section{근 220 (}

Licben ventofis.

fagineus.

lentigerus.

angulofus.

candelavius.

frigidus.

impresfus.

fangvinolentus.

petrofus.

inmerfas.

abietinus.

punetatus.

murinus.

concolor.

decipiens.

virefcens.

cevinus.

vitellinus.

forupofus.

beematomina, a. $\beta$.

ruber.

pannofus.

gosfypinus.

brinneus.

aruginofus.

tartareus.

ater.

citrinellus.

fubfufcus.

aurantiacus.

pallescens.

varius.

parellus.

corallinus.
Licben atratus.

Upfaliencis.

fulgens.

verrucofus.

fubimbricatus.

minutis/imus.

levcoltigma.

pufillus.

livitus.

mur orum.

caperatus.

muralis.

rubiginofus.

cafius.

luridus.

ohfourus.

crasfus.

acetabulum.

cartilagineus.

incurvus.

plicatilis.

verrucarius.

fcalaris.

centrifugus.

confperfus.

pictus.

laciniatus.

ompbalodos. \&. $B$.

ciliatus.

orbicularis.

fablunenfis.

Aygius.

Aellaris. 


\section{) $221(\%$}

Licben olivaceus. pulverulentus.

faxatilis. $\alpha . \beta$. cocoës.

parietimus. pbyfodes. ambiguus. micropbyllus. crippus. criftatus. nigrefcens. fafciculavis. rupeltris. palmetus. licbenoides. cbryfopbtbalmas. Burgesfii. ciliaris. tenellus. islundicus. $\alpha . \beta$. nivalis. $\alpha$. $\beta . \gamma$. pulmonarius. hottentottus. plumbeus. Sepincola. furfuraceus. favinaceus. canaliculatus. gilvus.

linearis. filix. calicaris. polymorpbus. prunaftri.

Licben fraxineus. fulformis. faftigiatus. juniperinus. caperotus. pinaftri. crocatus, a. $\beta$. glaucus. tremelloides. damecornis. ceratopbyllus. diaphanus. marginellus. veficulofus. disfectus. lutofus. tomentofus. difcolor. pollinaris. cucullatus. fcrobicularis. leetevirens. fluviatilis. refupinatus. venofus. aphtbofus. arcticus. antarcticus. caninus. polydactylon. borizontalis. perlatus. faccotus. croceus. 


\section{荟 $222(\forall$}

Lichen perforatus.

grifeus.

hirjutus.

pellitus.

polyrbizos.

glaber.

cylindricus.

miniatus. $\alpha$. $\beta$.

velleus.

puftulatus.

deuftus.

erofus.

viridis.

corrugatus.

cocciferus.

pyxidatus.

fimbriatus.

gracilis.

radiatus.

cancellatus.

cornutus.

furcatus.

fubulatws.

flammeus.

alcicornis.

incaufus.

ventricofus.

torulofus.

papillaria.

botrytes.

triftis.

rangiferinus. $\alpha, \beta$.

spinafus.

unciails.
Licben fubulifamis.

globiferus.

pafcbalis.

ramulofus.

aculeatus.

fragilis.

aggregatus.

Rocceila. $\alpha . \beta$.

murfcicoliz.

ocbrolevcus.

farmentofus.

floridus.

dentriticus.

plicatus.

flavicans.

arenarius.

bicolor.

jubatus.

lanatus.

pubefcerss.

chalybeiformis.

hirtus.

barbatus.

vulpinus.

aurantiaco-ater.

ufnea.

capenfis.

cincloonse.

Fucus uvarius.

lendigerus.

natons.

turbinatus.

ferratus.

veficulofus. 


\section{为 $223($ ) 22}

Fucus divaricntus.

ceranoides.

inflatus.

Spiralis.

nodofors.

pyriformis. a. B. $\gamma_{0}$

Fucus volubilis.

fangvineus.

ciliatus.

ornatus.

crijpus.

crijpatus.

iliquofus.

elongatus.

nliatus.

rubens.

Joreus.

feniculaceus.

granulatus.

felaginoides.

concatenatus.

dificbus.

triqueter.

trinodis.

furcellatus.

faftigistus.

ppevdoceranoides.

dentatus.

birfutus.

aculentus. $\alpha$. $\beta . \gamma$.

lycopodioides.

tendo.

confervoides.

corneus. $\alpha . \beta$.

fericeus.

plumofus. $\alpha . \beta, \gamma$.

pisnntifidus.

cartilagineus, $\alpha . \beta$.

gigartinus.

Jpermopborus.

papillofus.

coefpitofus.

venofus.

vittatus.

ramentaceus.

digitatus.

laciniatus.

palmatus. buccinalis. faccbarinus.

acerofus. pinnatus. multifius. ovalis.

pygmaus. prolifer.

Bysjus feptica. $\alpha . \beta$.

flos aque. pbospborea.

velutina.

petraa.-

jangvinea.

asurea.

candida. $\alpha . \beta$.

bombycina.

Cryptarum.

cellaris.

aruginofa. 


\section{婇) $224($ 塔}

Agaricus mufcarius.

fulvus.

campefris.

proeenfis.

nitens.

pallor.

mutabilis.

integer.

Xerampelinus.

violaceus.

fragilis.

friatus.

multifirmis.

cinnamomeus.

vifcofus.

cervinus.

flibellifornis.

deliciofus.

lactifluus.

piperatus.

croceus.

flavo-floccofus.

forinacus.

manzm? ofus.

clavius.

androfaceus.

collarintus.

umbelliferus.

grifeus.

papilionacers.

campanulatus.

pulverulentus.

tener.

devtatus.
Agaricus pilofus.

aruginofus.

galericulatus.

ocbraceus.

finetarius.

fquarrofus.

equeftris.

extinitorius.

lithorbizus.

foliolum.

amet byfinus.

crinitus.

coccineus.

Squansofus.

ehurneus.

bypri.

jecorinus.

fincicularis.

fragrans.

ocbropus.

varius.

fordidus.

ceraneus.

mufcoides.

Merulius cantbarellus.

alneus.

labyrintbiformis.

betulinus.

quercinus.

friatus.

mufcigerus.

Boletus perennis.

vifcidus.

luterus. 


\section{D) $225(\mu$}

Boletus bovinus.

rubiginofus.

fafciatus.

Peziza Alava.

microporus.

bydroides.

paradoxus.

fomeratarius.

cimnabarinus.

fangvineus.

villofus.

membranaceus.

abietinus.

nitens.

verficolor.

fuaveolens.

birfutulus.

papyraceus.

medulla.

obliquzs.

pecinatus.

decipiens.

diraidiatus.

articularis.

Hydnum inbricatum.

repandum. aurifcalpium. forveolens.

refupinatum.

fericeum. parafiticam. tomentofum.

Peziza cupularis. cyatboides.

nigrefcens. fcutellata. polymorpbo. ouricula. jufca. fcabra. cornucopioides. cocblearice.

Helvella mitra.

revoluta. cocbleats. influts. rivofa. atrats.

Cyatbus levis. Ariatus. Tbelepbora carnea. mejenterica. atrata. verficolor. pallida. mefenteriformis. bir fwes. fericen.

Clatbrus recutitus. Pballus efculentus. caninus.

Clavaria fpatbulata. piffillaris. $\alpha . \beta$. Spatbulavis. opbioglosfoides: 
2 ) $226 .(20$

Clavaria viridis.

fufcra.

vermicularis.

coralloides.

muscoides.

bypoxylon.

crifpa.

elegans.

elveloides.

palmata.

Lycoperdon fragile.

equisum.

bovifta.

corcinomale.

ftellatum.

pedunculatums.

gregarium.

epidendirum.

Afrobolus pezizoides.

Atcidium cancellatum.

epipbyllum.

tusflaginis.

Spberia digitata.

mucofa.

nivea.

bypoxylon.

carcbarie.
Tuber guloforum.

Miucor mucedo. eryjipbe.

Stemonitis embolus.

fqueroceponda.

Licbenoides.

furfuraces.

fulva.

Spberobolus fellatus. Tremella gramulata. prusiformis. mejenteriformis. bemifpbarica. adnats. difformis. arbovea. orbicularis. rufa. nofioc. juniperina. verrucofa. varioloja. purpures. alba.

Rbizomorpbabippotrichoides. fragilis. 
Plantæ cryptogamicx, qux quamvis minoris formce aut ignobilioris habitus videantur, prae multis tamen aliis magnum adferunt ufum, præcipue Oeconomicum, cujus exempli gratia nominare fufficir familiam Lichenum.

II.

Ordines hujus Clasfis naturalisfimi quidem funt; ta"men non femper ubique certos oftendunt limites.

III.

Mulci, a quibusdam vilisfimi exiftimati, præ cæteris admirandum fuis fcrutatoribus præbent fpectaculum.

\section{IV.}

Fruftificationis Fuci generis maxime fingularis de. tectio novum adfulget lumen Hiftoriæ naturali, qua fru: Etificatio fub aqua demontrabitur.

\section{V.}

In Fungis, ultjmo articulo Regni vegetabilis, adbuc fufpenfi hæremus, an magis ad ufum noftrum directe pertineant, an ad Oeconomiam naturæ. Maxima pars eorum venenatam produnt indolem, qua proter cos in efcam commendare nequimus. 


\section{MUSEUM NATURALIUM $A C A D E M I Z U P S A L I E N S I S$,}

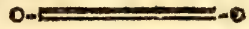

Cujus

PARTEM XXII. VENIA EXP. FAC. MEDICA UPSAT.

PR 巴S I D E

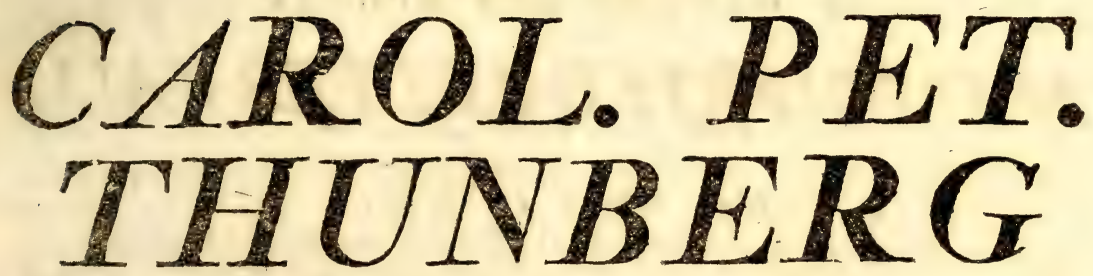

Equite Aurat. Reg. Orb. de. Vasa,

Med. Doct, Prof, Med. ex Bot. Reg. et Ord, Acad. Cesara, Petroptz. et Nat. Curins, Reg. Scient, Lond. Hozm. Societ. Scient, Urs. Philad. Patz. hol a, et Cir, Oecon. Finz. Berol. Nat. Scrut. Paris. Hagn. ex Halems. Hist. Nat. Lund. Harlem. Amsteld. Zeland. Nidrosiens. Ticinens, Innens. Linn. Londin. Phys. et Peytourapu. Gättingens. Nanciens. Med. Menspe l. Med. Matritens. Medic. ex Nat. Studios. Edimburg. Memero mec non Acad. Scient. Paris. et Ingtitut. Nation. Monsteliens. Agricert. Pakis. Medic. Lonijin. Scandinay. Fiorgntin. ist

EataVin. Ind, Or. CORRESPOND.

PUBLICE DEFERT CENSURE

ICOBUS WILHELMUSRUDOLPHI NYCOPIA SUDERMANNUS.

IN AUD. BOTANICO DIE XXIII MAJI MDCCCIV

H. A. Mi, S.

U P A L I E,

TYPIS EDHAIN NANIS。 


\section{aे MONSYTR}

\section{WEAN JAQUES RUDOLPHI MEDECIN ET CHIRURGIEN}

DE L'HOPITAL ET DE LA VILLE DE NYKÓPING

ET

aे MADAME SON EPOUSE

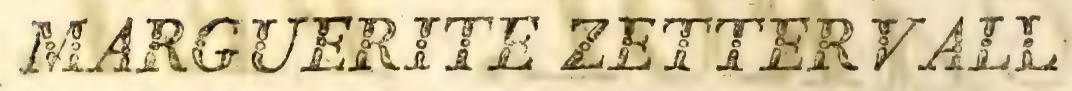

Mes Pere et Mere!

Si ce premier ouvrage, giue je vous offre, ne prouve pas, quie j'ai profité de léducation, que vous m'avez donné; il fervira au moins de preuve des fentinents de lo palus tendre reionnoisfonce, aree laquelle je fersib toste ang vie

Mis cheres Pere Es Mirs

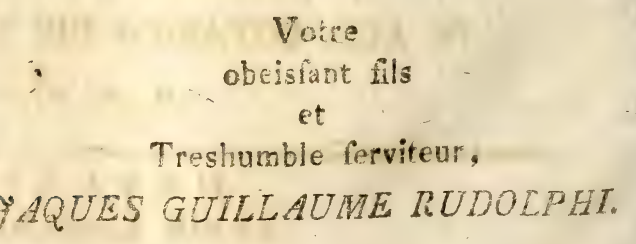




\section{$P$ rö $m i u m$.}

$\mathbf{S}_{\text {cientiis }}$ Philofophicis feculum quoddam proxime præ terlapfo felicius fingi vix potelt, vix, ac ne vix quiden, tantos Reipublicæ Litterariæ cives ulla protulit ætas, raro, fi umquam, progignet to fan æquales.

Primam, ni fallor, hujus ævi reportabit Svecia no. ftra gloriam; plerasque heic excultas fuisfe fcientias videmus, quasdam ad fummum evectas culmen.

Hanc patriam habuit memoria dignisfinus ille $P_{0} t$. heimer, cujus obitum deploravit Architectura. Vidit Phyfice illuftrisfinum heic natum Klingen/jerna, qui ipfius fuit enulus Newotoni; hine ortum duxit Ihro, hiftorix Scandinavica parens; inprimis vero Scientiarum nobilisfima, artem dico Machaonis, hinc duxit cultores; ad quodnam

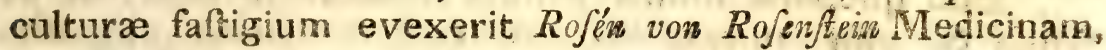
nemini forfan latet. Heic vixerunt, ne dicam amplius, auctores Chenaiæ, Wallerius \& Bergman. Hinc aër, maria, montes \& totus, quantus, orbis terrarum immortales duxerunt Scrutatores, quorum nomina, cuneta, quotqvot venerint, frcula referent venerabunda; non eft quod hos commemorem; quis noftrum, fama faltim, Botanicorum fibi non habet cognitum principem fummum illud lumen Car. a Linmé, cujus gloriam omnes referent freculoruin annales; ut ceteros, quos in arte hac progenuit Svecia illufteres \& quorum memoria apud ultimam revivifcet pofteritaten, omittam. 
Vidimus, qui luesunt faculo praterito in Septemtricne noftro Scientiarum progresfas, hoc quoque loco æquisfunm fit nemoriam vel leviter tangere hujus æi Trincipum, quorum p acipua heic floruere Mufa gratia. Evidentisfima line indicia clementiæ, qua eft amplexus Academiam hance ADOLPHUS FRIDERICUS quando hares Regni Cancellariatum adfumfit Academiae, quo. nam munere depofito fceptrum etiam tenens larga in feientiarum cultores effudit beneficia. Fuit vero \& Con. jux digna Marito, Regina Regni LUDOVICA UDAL. RICA limma fautrix. Mufarum, qux non minus in alis multis, quam, quod jam noftrae elt confiderationis, argumento, luculentisfima tam eruditionis \& ingenii, quam erga fcientias favoris dedit fpecimina. Illuxit tandem Sveciae ætas Augufi aurea, quando habenas regni fumfit beatisfimus memoria GUSTAVUS III. qui hæreditate Maximis a Parentibus acceptam erga Pierios clementiam alumnos fingulari auxit liberalitate, cujus aterna hocce Mufarum delubrum fervabic monumenta.

Inde quoniam omnis pendet hominis felicitas, ut de Peiplo \& phanomenis naturæ rectam habeat cognitionem, Hiforiæ Naturali, præmisfis ejus fulcris, primum inter fcientias philofophicas tribuendum esfe locum cenfemus. - Nihil Atheum, nifi totius hujus Mechanismi confpectus, convincet. - Cuilibet, qui vitam voluptate, qua eft capax, degere cupit, necesfariam plane arbitramur quamdam hiftorize naturalis peritiam. - Si ad commoda, quæe non minus publicæ ac privatæe oeconomia adfert hiftoria.naturalis, eidem primum facile concesferimus locum. - Multa, qua antiquorum temporum fuperftitiofa plebs, velut ex inferis orta, perhorruit, maximam in ampla oeconomia \& politia Naturæe adferre uti- 
litatem hodierna fatis fuperque teftata eft eruditio. Unicuique igitur, hinc concludere fas eft, aliquam hiftoria naturalis cognitionem fore utilem; maxime vero accura. tiorem Naturæ disquifitionem artis Salutaris cultoribus esfe necesfariam lubentes fatemur, fi ad commoda refpexerimus qux reformatis Pharmacopoeis generi hominum adflicto adtulerunt recentis ævi naturæ fcrutacores, quando plurima, antea ufitatá medicamina accuratius disquifica invenerunt plus dami quam emolumenti adferen tia, quadam in fe quamvis bona variis collecta temporibus variisque locis majorem minoremve exlerentia vim. Sxpisfime in id fomma eft incumbendum opera, ne natu major cum minori confundatur herba, \&c.

Hæe \& ejusmodi plura naturæe inquifitionibus debem mus commoda; multa vero adhuc fuperfunt detegenda; nox abiit, non tamen orta dies. - Optandam igitur eft, ut in hanc fcientiam colendam omnes operam navent litte. rarum ftudiofi; quaecumque enim eosdem in futurum manferit vitæ fors, non operam oleumve perdent; inprimis vero qui artem tractabunt falutarem.

Attamen hanc fientiam probe \& optato cum fuccesfu nemo discere fibi credat, nifi Mufea eidem fuppe. tant confulenda; numquam enim tam clari \& diftincti reddi posfunt caracteres Syftematici, ut non ex ufu melius percipiantur. Quam manca fit ex libris, hanc fcientiam tractantibus, haufta eruditio, facile perfpexerit, qui res ex hac examinet naturales; ad maximum tamen ha funt disperfa tenebræ, hæ evanuerunt difficultates per rerum naturalium collectiones, quxe hatt minimum hocce fublevant ftudium.

Inter prospera Scientiarum fata primum fibi vindicat locum gratia principum. Vafta fuerunt fæcula bellis 
tantummodo facra; laurigeris inimica Caftalibus; tandem requievit ventus $\&$, una com tranquillitate, Templi hujus Mufarum cacumen illuftravit aurora. Solimn tancem patria variis quasfatum procellis occupavit $G U$. STAVUS I. hine æetas redit Augula, hine felicisfimum incipit Academia avuin, hinc artes floruere. Beneficis in littecarum cultores Regum hujus Dynaftize alter alterum fuperare eft conatus, \& quo magis ex culta pendeat hominis beatitas, eo major erga principes hofce Mufarum evadit obligatio.

Inter faufta, quorum memoriam Svecix litterarix refervabunt annales, id inprimis fortuitum numerari de. bet, quod anno proxime p: ar rapfo inter alia his conti. git Mufis, quando Sacræ Regiæe Majeftati, Regi noftro Auguftisfimo, GUSTAVO ADOLPHO, clementisfine placuerit. Collectione illa infigni, pulcherrma \& pretiofa, Infectorum, Conchyliorum, Coralliorum, Plantarum, quam olim Regina Regni magna \& immortalis, faut: ix Mufarum LUDOVICA UDALRICA, fummis funtibus, in Arce Regia Dottningholm inftruxerat, Acadeniam hance donare. Hinc variis Regum alio umque donationibus adeo creuit Mufeum Naturalium Upfalienfe, ut vix ulli fecundum omia fere, qua adhuc noftra vidit zetas, fuperet, \& non minus liberalitaten \& beneficentiam teftetur donatorum, quam in futurum Rei litterarize pollicetur emolumenta.

Gazas hafce Regias poftquam feliciter huc transportats fuerunt, cura \& labore indefesto in ordinem redigere ftuduit Cel. Præés, ut Catalogus, quem mibi publici juris facere contingit, cum orbe Erudito communicari posfet. 


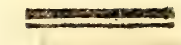

\section{Donatio, 1803.}

\section{GUSTAVI, ADOLPHI,}

Regis Auguft. Svecia Eัc. E̋c. Ëc。

Cervus dama. elapbus. 3 .

Felis Len: $\delta$ junior.

Geotrupes Adtaon. o.

Aloëus.

Atlas. 0.

Didymus.

Endymion.

gidenn.

bercules.

oronedon.

Titarus.

Scarabaus longinsanus.

nafscornis.

rbinoceros, a. $\beta$.

Copris capucinus.

emerginatus.

gigas.

hisponus.

molosfus. a. $\beta$.

panificus.

plinlarius.

fecer.
Copris Schefferi.

feraipunctatrs.

typboeus.

volvens.

ur Jws.

Pasfilus incerrupters.

Lucanus carinatus.

cevous.

dama. $\hat{\theta}, 1)$

Melolontha erytbrocepbole.

fullo. berjuta. a. B. .. d. $\varepsilon_{0}$ birte.

lanigera.

punctata.

Cetonia axrata. fiztisa. $\alpha . \beta$.

capenfis.

cbryfis.

fafcicularis.

indica.

nitida.

Trichius fafciatus.

s) Capreolus, Muf. Reg. p. 32. 
Necrophorus grasdis. 2). Casfida cyamea.

Brentus ancborago. dispar.

Brachycerus cornutus.

emeritus.

Cordyle bernipterus. palmarum.

Curculio annulatus.

argyreus.

depresfus.

indus.

Melanocardius.

mucoreus,

pufio.

fexpunetatus.

Speciofus.

Jpengleri. 3)

ftigma.

Vaginalis.

Meloë majalis.

projcarabaus. a. $B$.

Mylabris Algirica.

sicborei.

impar. a. B.

Blaps gages.

Etirychora Jpinofa.

Erodius gibbus.

imipresfus.

variolojus.

Chryfomela tenehricosa.

Sepidium caraboides a. $\beta$.

Carabus decenguttatus, faftigiatus. bortenfis.

Clerus fipylus. Lampyris corrufca. 4)

Elater nocilucus. oculatus. fyriacus.

Saperda Zonaria. Cerambyx Batus.

defertus.

ferrugineus. beros. mo/cbatus. fuccinctus, $\alpha, \beta$. virens.

Prionus Cervicornis. «. $\beta_{\text {. }}$. cinnamomeus. longimanas. $\propto . \beta$. Spiatbarbis. Bupreftis Barolinenfis. cuprea. falcicularis. gigantea. birta. Aernicornis. ftricte.

2) Silpha indica. Muf, Reg. 3) Abbreviatus. Mrof. Reg. p. 62.

4) Pyralis, Mur, Reg. p. 
Pafma gigas.

Ej. Larva.

pbtbifica.

ej. Larva.

Mantis bicornis.

Gorgylodes : lay w。

guttata.

precavia. a. $\beta$.

religiofa.

tricolor.

Pnevmora imbmaculata. $\alpha, \beta, \psi$.

Ej. larva.

maculatis.

ij. larva.

Truxalis nafutus.

Locufta ecuminata.

quiline.

sitrifolis.

coriacer.

laurifolia. $\propto$. B.

Melanoptera.

ocellata.

pupa. a. B.

rugofa. 5 ).

ficcifolia.

triops.

verrucivora. $\alpha$. F.

viridisfima.

Gryllws Æggyptius.

coerule feens.

criftatus. $\alpha . \beta . \gamma$.
Gryllus favus.

migratorîts.

miliaris. a. B.

morbillojus. \&. $\beta$.

obfcurus.

Serratus, $\alpha . \beta$.

frridulus.

tataricus. $\alpha, \beta$.

Acheta campeftris.

capenfis.

gryllotalpa. \&. B.

Fulgora candelaria. $\alpha$. $\beta, \gamma$

laternarie.

Blatta agyptiaca.

africana.

smevicana. $\alpha . \beta$.

gigantea.

maderce.

orientalis.

Nepa cinsrea. a. $\beta$.

fufca. $\alpha$. $\beta$.

grandis, $\alpha . \beta$.

rubra.

Cicada capenfis.

ciliaris.

crux.

fornicata,

lanata.

firidula.

Tïbicen.

Violacea.

5) Femoratav $F$. 
8

Cimex acutus.

agyptius.

andrea.

arabs.

sulicus.

bipunctatus.

deufus.

fullo.

indus.

peregrinator.

rubicollis.

ferratus.

rexpunctatus.

Stockerus.

ftolidus.

valgus.

variegatus.

viridulus.

Papilio Acefta.

acbilles.

acbins.

aness.

eropus.

Agansemtson. $\propto . \beta, \gamma$.

Agenor.

alimena.

almana. \%. B. \%.

anacardii.

anchifes.

inthemon.

Apollo.

osfimilis.

Bixa.
Popilio Boliza. a. B. Brasfice. Calliope. Carica. casfie.

Clory/rppus. Cleopatra. Cupido.

Cytherea.

Doplidice.

Deiphobus. «. $\beta$.

Demolews. a. B.

Demropbon.

Dido.

Diomedes.

Disfinilis.

Erato.

Eurypulus.

Euryta.

Evterpe.

galathen.

gliciria.

Hector

Helenn.

Helenus. $\alpha$.

Horta.

Hyperbius.

Fatropba.

Idea.

Idomeneur.

lllioneus.

Lampetia.

Leilus. 
Papilio Lemonias.

Leucotboë.

Lyfippus.

Macbaon. $\alpha . \beta$.

Marfyas.

Melpomene.

Melanéus.

Memnot.

Menelaus.

Metis.

Mnemolyne.

Napi.

Nauplius. 6).

Nireus.

Oenone, $\alpha$, B. $\gamma$.

Oritbya. a. F. $\gamma$.

Orontes.

Pammon.

Paritbous. a. $\beta$.

Paris.

pberufa.

phereclus.

phidias.

Philocles.

Pbiloctetes.

Piera.

pipleis.

plexippus.

podalivius.

polydamsas.

polymnic.

polytes. a. $\beta$.
Papilio Porjerna.

Prismus.

Protefiliaus. a. $\beta$.

Proteus, $\alpha, \beta . \gamma, \delta, \varepsilon, \xi$.

Pfidii.

Pyrrhus.

Quercus.

Ricini.

Sarpedon.

Sophoræ.

Spio.

Stelenes.

Telamon:

Teuser.

Thamyras.

Vanille.

Venilia.

Ulysses.

Xantbus. œ. F.

Zeuxo.

Sphinx Alecto.

atropos. $\alpha . \beta$.

Caffra.

Capenfis.

Caricæ.

Carolins.

Convolvali.

Ello.

Elpenor.

Eupborlice.

Ficus.

Labrufcx.

B

Sphinx

6. Clio. Mur. Catal. 
Sphinx Ligufvi.

Megæra.

Ocellata.

Rhegea.

Popzili.

Tantalus.

Ibyelia.

Tilice.

Tifiphone.

Vitis.

Bambyx Atlas.

Cesropic.

decora.

fafcelis

fulvia.

heliconia.

besperus.

LeÊrix.

Euns.

Lunus.

Militaris.

ornatrix.

paphia,

nellex.

virgo.

Noshtua erepufcularis.

frasini.

occidus.

odora. a. R.

patrocius.

punetigers.

zetorta.
Noctud Strix.

Phalana faveolata:

Janata.

Jatropharia.

Sambucaria.

tricinctaria.

tripunctarin.

Hemerobius Libellulojdes. *.6.

Libellula caroliza.

Apis aftuans.

caffra. 7).

tropich.

violaces.

Sphex xgyptia.

capenfis.

cornuta.

fervens.

flavicornis.

grandis.y

indice.

Indoftana.

Vespa affinis.

Canadenfis:

olivacea. a. B.

tropica.

Androna cordata.

dentatr.

cincta.

Bembex figrat .

Mutilla indica. 8).

Bombylius barbatus.

q) Eftuns var。 Muf, Reg. p. $4^{1} \sigma_{a}$

8) diadema, $F$. 
Bombylius roftratus. Aranea avicularia. $\alpha . \beta$. flavisfima.

Trombidium caudatum.

Phalangium reniforme.

Acarus ægyptius.

Monoculus polypbemus. a. $\beta_{0}$

Cancer aneus.

araneus.

calappq.

cornutus.

craniolaris,

criftatus.

fachino. 9\%。

feriatus.

heparicus.

horridus.

lactatus.

Cancer maculatus.

Menas.

longinaus. ㅇ․ ㅇ․․ 10).

pelagicus.

philargus.

raninus.

fcrupofus.

fpinifer.

Arigofus. 11).

fuperciliofus.

tetauicruftatus, 12).

velutinus.

Hippa dorfipes.

Pagurus Bernbardus.

Affacus cancharus.

bomarus:

norvegicus.

9) dorfipes. Linn.

II) chabrus, Mar. p.
I0) Macrochelos. Herbft,

j2) grapfus, Lin. 


\section{THESES}

I.

Excurfones Botanicas utilitatem in vira communi indirecte non tantum adferre; verum etiam directe varios ad morbos fanandos \& avertendos admodum esfe idoneas contendimus.

\section{II.}

Ad fudium rerum naturalium fublevandum haut minimum confert quxdam Phyfices cognitio.

\section{III.}

Univerfa, qux exftant in natura, generi humano, quodam modo nobis quanquam fapius incognito, directe vel iadirecte esfe utilia probabile ducimus.

\section{IV.}

Qvamvis omnia ad vitam degendam necesfaria ex codem non defumantur Regno naturæ; plurimam tamen nobis prabere utilitatem regnum vegetabile verum cenfemus.

\section{V.}

Omnes, qux hodie exfant, rerum" naturalium fpecies inde a prima mundi creatione ducere origiaem falfo contenditur.

\section{VI.}

Si oeconomiam Amphibiorum, qua adhuc maxime in obm fcuro latet, accuratius perfcrutemur, aliquid huic miverfo eadem adferce utilitatis forfan invenerimus. 


\section{MUSEUM NATURALIUM $A C A D E M I Z E$ U PSALIENSIS, -}

\section{CUJUS}

PARTEM XXIV. VENIA EXP. FAC. MEDICE UPSAL.

\section{PR ESI E}
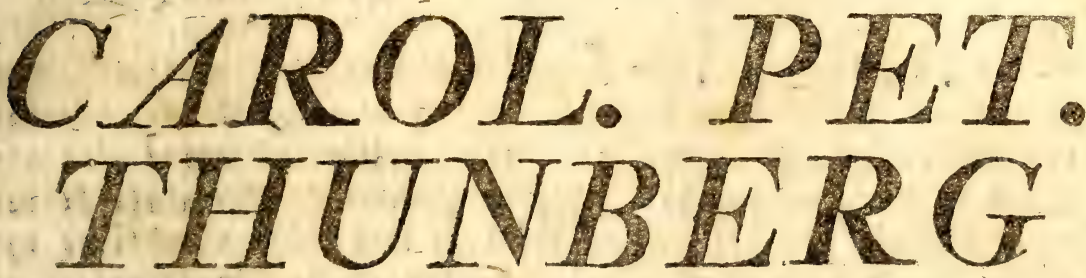

Equite aurat. Reg. Orb. de Vasa,

Med. Doct. Prof. Med. ejt [Bot, Reg. et Ord. Acad. Cesar. Petrofol. et Nat, Curios. Reg. Scient, Lond. Holm. Societ, Scient. Ups. Puiladi Ratr. Hotmo et Civ, Oecon. Finz. Berol. Nat. Scrut, Paris. Hafn. et Halens. hist. Nat. Lunn. Haberm. Amsteld, Zeland. Nidrogiens. Ticinens, Ifnens. Linn. Londin. Pays. et Paytourapu. Gótrtingens. Nanciens. Mro Monspel. Med. Matritens. Medic. ex Nat. Studios. Edimburg. Membro zec nen Acad, Scient. Pakis. et Inspitut. Nation. Monspeliens. Agricult. Parjs. Medic. Londin. Scandinav. Fiorentin. ext Batavin, Ind. Or. Correspond.

PUBLICO SUBMITTIT EXAMINI

FOH. GUSTAVUS HENTZELL WESTROGOTHUS.

IN AUD. BOTANECO DIE XXX MAJI MDCCCIV.

I. A. M. S.

UPSALIF,

TLYPIS EDMANNIANIS. 


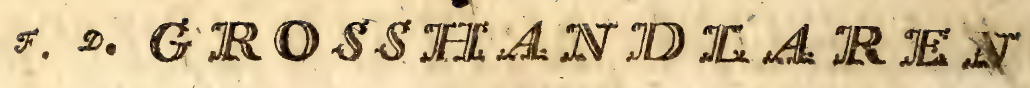
$\because O ̈ G A D L E$

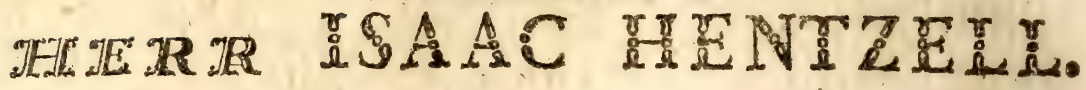

\section{MIN VÖRDADE FARBROR!}

IE dert ådelmod har frìn de spådare âren varit mitt stód. Om kinuslan af min förbindelse vore még gtig at wutrycka min vơrdnad och tacksamher, skulle jag anse mig lycklig; men ord åro nog svaga vedermallen derutaf, jag ofverlámnar derfóre detta àt den tysta kinsslan, - och at framtiden at med mitt formallande up. daga; - ofvertygad at et tomit fmicker skulle afven fiwaktas af Eiler, son mu endast, $i$ ett pa fóresats gruqudadt válgơrande niuten lifuets being. - Mí dá Himlen b lona Edra dygder!Wh sollheter foula Edra spir! - - -

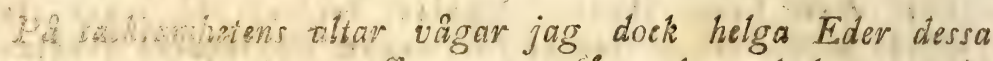

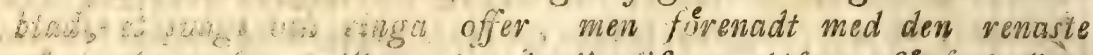

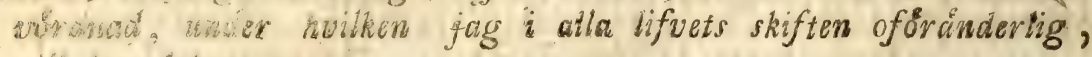
will Jrontefua

EDER,

AI $N$ VORDADE $A R B R O R$ '

Lydige Bror - sow

J. G. HDN TZELE. 


\section{Donationis}

\section{REG. GUSTAVI IV:TI ADOLPHI Continuatio Is:ma.}

Echinus cidaris diademe. efculentus. globulus. grotilla. lacunofus. lixula. lucunter. mammillatus. placenta. rofaceus. pexatilis. Spatagus. Sphreroides. Afterias aradciaca. glacinlis. medufa. orpbiura. pappola. quadrifika.

Chiton Jquemofus. Lepas anotifere. balains. mitella. teftrudinacin. timisnnebulim.
Pholas caindidus. prifilius.

Unio lutzaria.

$$
\begin{aligned}
& \text { margaritiferus. } \\
& \text { pieterums. } \\
& \text { vulfella. }
\end{aligned}
$$

My a truncata. Solen aratikus.

culrellus. enfis. vadiatus. filiqua. frigilatus. vagina

Tellina atbida. foliacea. gari. lavigata. lingua folis. radiata.. roftrata. Scobinate. virgate.

Cordism aculeatum. cardisfa. coflaturs. 


\section{4}

Cardium echinaturs. edule.

frogum.

Ravum.

hesnicardium.

ifocardia.

levigatum.

magnum.

medium.

menicntum.

pectinatums.

: 2 s vuficum.

tuberculatum.

snedo.

Mactra Pultorsm.

Donax euneasa.

pubelcens.

. rugofa.

fcortun.

fripta.

trunculus.

Venus cafina.

caflrenfis.

cbione.

decusjata.

dionse.

dyfera.

edentula.

exoleta.

erycina.

fimbrieta.

flexuoja.

Fonus litterata. meneulate. marica.

meretrix.

suercenaria.

meroe.

penfylvanica.

perulca.

perfinats.

puerpera.

punctata.

proprata.

reticulata.

rotundars.

feripts.

tectrix.

tigerias.

verrucofa.

Chama calyculata. 1)

cor.

giges.

Hippopus.

lezarus: var. plur. femiorbiculata.

Spondylus gaderopus. plicatis.

regius.

Area asitiquate.

barbata.

glycymeris.

gramofa.

soce.

7) Oblovga, Muf, Regin. 
Arca ovata.

pillons.

pe.zunculus.

piloge.

tortwofas.

Ofrea edulis.

fafciats.

flavicens.

foliusn.

- glabra.

jasobar.

imbricata.

lima.

galless.

maxima. nodosa.

obliterata.

opercularis.

plice.

radula.

varia.

ziczac.

Anomia epbipsiuns. Placenta orbicularis. Mytilus anatinus.

bilocularis.

crific.

cygmus.

edulis.

exufus.

frons.

byotis.

birsudo.
Myrilus lisbopbegus:

margaritiferus.

modiolus.

ruber.

ungulatus.

viridis.

Mellina epbispiwm.

ifogonum.

Pinna digitiformis.

lobata.

muricata.

nobilis.

rotundate.

rudis.

Caccata,

Argonavta argo. a. B. $\gamma$. Nautilus pompilius. $\alpha . \beta$.

spiruia.

Conss acbatinus.

ammiralis.

aulicus.

aurifiracus:

betulinus.

bulletus.

sapitarieus.

clavus.

ebraus.

figulinus.

fuliginosus. .

generalis.

geruarus.

geograpbus.

glaurus. 
6.

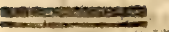

Conus granulatus. imperialis.

litteratus.

marmoreus.

mercator.

miles.

monasbus.

mufcorsmo

vobilis.

zusfitelta.

princeps.

Reginæ.

ruthicers.

Spectrum.

Priatus.

terebellum.

textile.

thalasfinus.

varius,

wirgo.

Cyprcea ametbyifen var. arals.

annulus.

nrabica.

argus.

a fellus.

carizeola.

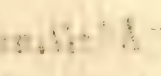

Cyprea birundo.

jJabella.

lata.

lynx.

mappa......

mastitonica.

moneta.

mus. -

эucleus.

oryx.

ocsllats.

pedisulus.

poraria.

regina.

Terpentis.

Rapbylar.

Aolida.

fucrinets.

talpa.

tefrudinaciv.

tigriss

varelli.

sitellus.

asbra.

ziczos.

Bulla ampulls.

caurica.

cribraria.

erofa.

-xanthema.

fragilis.

gobulus:

Belvola.

amplistre.

canaliculyte

gibbofa.

nачсив:.

оขยม.

pbyfis.

werr ucofor

nolise. 
Bulla volva.

Voluta ctidopica.

capitellwrit.

cartinalis.

ceramics.

caffra,

dactylus.

eluraa.

epifcopalis.

fabr.

glabella

jods.

ifpidula.

lapponica.

wercatoria.

midx.

mitra.

monitis.

mufica.

olive.

papalis:

panperculco.

perfisculá.

peritusa.

plicarin.

porpbyrea.

fangvifuga.

fenuricula.

(capha. 2)

twrbinellus.

vespertilio.

vulpecula.
Buccinum areola.

cornutum.

coltatum.

cresulatums.

decusjatum.

dimidiaturs.

doliuns?

duplicatum.

ccbinophorum.

erinaceris.

flammexim.

glabratum:

glans.

glauswire.

barpe.

lancenturs.

maculatunz:

olearium.

papillofurs.:-

patulum.

perdix.

perficusts.

pomum.

rufurs.

fentionfum. 3)

fpiratum.

Arigilatuss.

fribulatum.

tevebelluns. 4)

teficulus.

tuberofum.

sndatum.

Buccinum arcutaria.

2) Cymbium. Muf. Begia.

3) Murex,

4) Conus. 
Buccinum undofum. vibex.

Strombus caneriums. sbiragra. dentatus. Dima. epidromis. fusus. gellws. giblerulus. gigas. Tambis. latisfirmus. lentiginojus: lividus. lucifer. lubianus. mitlepeda. pelecani. pugilis. fcorpius. fuscindus. arceus. vittatus.

Murex aluca.

anfatus.

anus. areanus: asper. babylonius. brandaris. bufonius.
Mures cansaliswlatus. cocblidium. colus. cornutus. femoralis. ficus. bauftellu\%. bippocoftanum. by/zix. lampas. lotorikex. maculofus. mavcinella. melongens. morio.

meritoideus. orodus. paluftris. perverfus. polygonus. pyrum. ramofus. vana. rapa. 5) roticularis.

vicinus. rimberuls. faxatilis. fcorpio. ferobilator. syracufanus. trapersium. 
Murex tribulus. tritonis. trunculas. tulipo. vertagus.

Trochus calcar. consins. dolabratus. hybridus. labio.

maculotus. magus. muricatus. perspectivus. porrsonis. rugiofus. faber. folaris. telefropium. umbilicaris. vefiarius. zizypbimus.

Turbo argyrofomus. cbryfopomus. cletbrus. cochlus. crenatus. delphinus. diftortus. duplicatus. exoletus. margaritaceus. maraoratus. -btusatus.
Turbo pagodus.

petbolatus.

pice.

replicatus.

fealaris.

tectum.

terebra.

นขa.

variegatus. Ais?

Helix arhatina.

albellas.

amerule.

ampullacea.

arbufiorwm.

arietis.

caracolla.

citrine. decollata. glauca.

grírea. baliotoidea. hemeftoma. jantbines. lapicida. Incorum. Iufitanica. militaris. memoralis. meritoidëa. oblonga. odtorra. oculus. perfpicua: perver $\int a$. 
Paílla equefris. fisfura. fuloa. granatina. granularis.

grace.

lacinioja. virginea. vivipera.

Nerita Albicilla. albansen. eterrims. canrena.

corone. exwoia. glaucina: hiftrio. mammilla. plisata. polita. pulligera.

radula. virginea. vitellus.

Haliotis afinim. marmorate. mide. partid.

fribta. tuberculata. veria.

Patella antiquata. barbara. cbinernis.
- lutea.

momillatis. nimbofa. nubecuba. vulice. Jacbariwa. cefindinea. unguis. vulgata.

Serpula anguine. Arenaria. contortuplicata. glomerats. hrimbricalis. penis. triquetra:

Dentalium dentelis. - sepbantinsum. entalis.

Umbilicus, veneris. Tubipora mufice. Madrepora agaricites. celpitofia. dameicornis. fafrigiate. fungites. Madre. 
Madrepora musicata. muficalis. aculota. rasmer.

Millepora aleicornis. Ijis hippuris. ochracea.
Antipathes fpiralis.

Gorgonia antipatbes. elongita. flabelium. vertalina.

s... verrucofa. Spongia diebotoma.

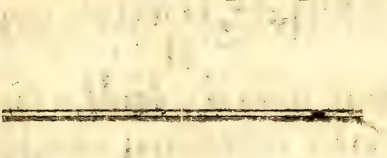




\section{THESES}

$\mathbb{H I}$

1.

qui fuis fubjicere velit disquifitionibus, a propofito ne declinet, quum latisfimum fe aperiat fpatium, quod ei non nifi viribus et corporis et animi indefesfis fit decurrendun; fibi enim perfvadeat, nullan fere litterarum partem, cultori fuo, et majorem utilitatem, et jucundius plabere oblectamentum. II.

Quum ex omnibus naturæ regnis, e thefuro velut vitæ, omnia haurimus commoda nobis necesfaria; non ille fane oleum et operam perdit, qui nobili illa fcientia fum inbuit animum; quinetiam neminem notitis infructum, eam prorfus negligere oportent, omni contendimus juze.

\section{III.}

Scrutatori vero hiforiz nituralis non folum fufficit, prolixa ornatum esfo thëoria: omnem etian, fi veritatem perfequi velit, occafionem amplefatur, in practicis fuam exercendi cognitionem; quocum nudio, in ferutanonis via, veccillinti minus pede pro. greditur, eque fuis firmum parat notitiis fundamentum.

IV.

Seientia botanica xque animo ac corpori fulutaris eft; prxconceptas vero opiniones, car hujus Audii anore tam pauci fuerint capti, in causfa esfe, experientia obnixi judicamus,

$$
\text { V. }
$$

Botanica quamvis fcientia nunc perbene fit illuftrata; ñovas tamen plantarum fpecies, vel earum mixtione qux jam funt, vel varii influxus foli, in quo nutrimenta flbi arripiant, adhuc oriri posfe non negamis.
VI.

Omnes licet in regnis naturæ res occurrentes primo intuitu non videantur nobis quid afferre utilitatis, lucri noftri cupidini con. venientis; minime tamen eas esfe rejiciendas, fed accuratiore ingenii acumine potius perferutandas pucamus.

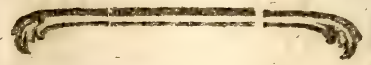




\section{MUSEUM NATURALIUM $A C A D E M I Z$ U P SALIENSIS。}

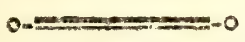

\section{eujus}

PARTEM XXV. TENIA EXP. FAC. MEDICE UPSAP.

PR ESIDE

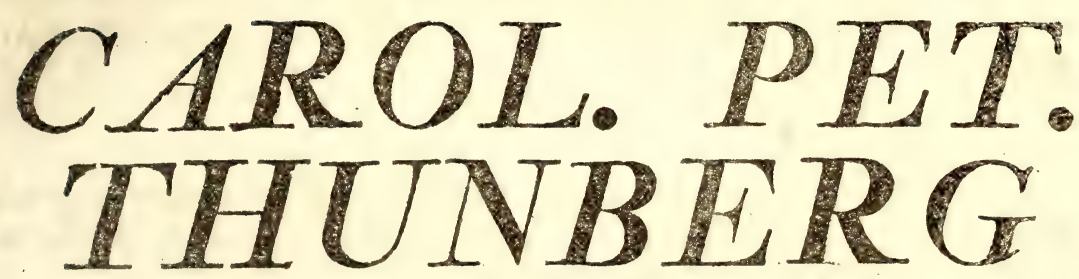

Equite aurat. Reg. Ord. de Vasa, Med. Doct. Prof. Med. et Bot. Reg. ext Ord. Acad, Casaro Petrofor. ets Nat. Curins. Reg. Scient, Lond. Holm. Societ. Scient, Ups. Philad. Patr。 holm, et Civ, Oscon. Finl. Berol. Nat. Scrut, Paris. Hafn. et Halengon Hist. Nat. Lund. Harlem. Amsteld. Zeland. Nidrosiens. Ticineng, Ienens. Linn. Londin. Phys, et Paytourapis. Góttingens. Nangiens. Med。 Mnnspe i. Med, Matritens. Medic. et Nat. Studios. Edimburg. Membro nec non Acad. Scient. Pakis. ext Institut. Nation. Monspeliens. Agricult. Paris. Medic. Londin. Scandinav. Fiorentin. et Batavin, Ind, Or. Correspend.

PUBLACO SUBMITTIT EXAMINI

\section{CHRISTIANUS LEWIN \\ WESTMANNUS.}

IN AUD. BOTANICO DIE II JUNII MDCCCIV.

H. A. M. s.

U P S A L I 2 ,

TYPIS EDMANNIANIS。 


\section{THESES}

I.

Quemvis Hiftoria Naturalis in Medicina, jus fit monnen. si, ut partium illius nullam ignorare debeat Medicus folidus E' expertus, flatuere tamen audemas, Botanicam, Ip omnium moxime negesferiom esfe.

II.

Eundem, pra ceteris Hiftoria Naturalis partibus, eminen. siorem locum in Oeconomia Rurali, Sciontia botanica adjudicemus.

III.

Peregrinationes folidiori doarino of folicior ingenio infrucis, in diverfas terras fufcepta, ad emolumentum Scientiorum in gessere plurimum conferunt.

IV.

Eam tomen fpecialioren utibitatem pro peregrinationibus in alias regiones, adferunt in Palceftinam itinera, quod ine terpretations Sacri Codicis plurimum lucis affundant.

\section{V.}

Unusquisquc enim, qui Sacram Scripturams rite explica. re Ef interpretari voluerit, cognitions phyfica $\mathcal{E}^{2}$ geographica inprimis vero Hiftoria Naturali Terre Sanda, carere ommienequit.

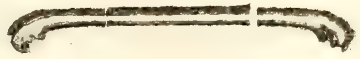




\title{
ÅDEL OCH HO์GAITAD
}

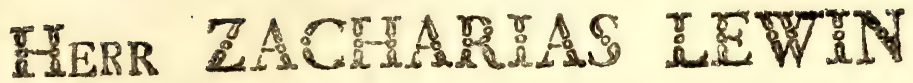

\author{
SAMT
}

\section{ÅLA FRUN}

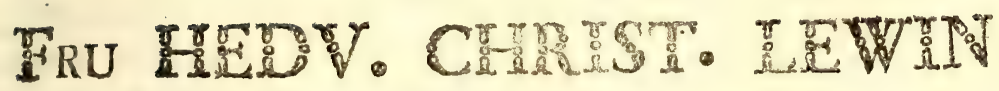 झIODD $2 M T \cdot A . A . S$.}

\section{MINE HULDASTE FORÁLDRAR!}

$D_{\mathfrak{a}}$ jag anser vorrdnad och tacksambet mot en Huld F A R, en Óm MOR fór den forrsta af mina pligter; har jag ej kunnat ne. ka mit hjerta den tilfredsstillelsen, at ofienteligen ådagalågga huru hơgt jag år EDER fơrbunden. EDER helgar jag således dessa blad. Emottagen dem med samma ófverseende godher, af hvilken jag altid ro̊nt så många och fôr mig så dy rbara vedermålen。 Mina tysta vålsignelser skola altił folja EDER, och jag skall med sonlig vơrdnad och årkåksla $\mathrm{i}$ ḋơden framhå:da at vara

\section{EDER *}

lydigste Son 
VIRO

ADMODUM REVERENDO ET PRECLARISSIMO

\author{
DOMINO MAGISTRO \\ JOH. WILH. ÅHLSTRÓM, \\ AD FODIN. ARGENT. SALENS. A SACRIS \\ FAUTORI PRORENSISSIMO
}

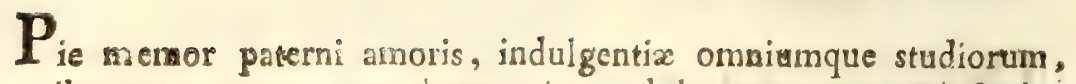
quibus ne puerum complexus, jam adolescentem compleeteris, venerabundus semper agnosidm, quantum Tibi debeam. Nimirum enim me litteris institutum, ad honestatem imbutum volui-. sti. Merita Tua et virtutes reverentia prosequar, quas imitando Tibi gratissimu videbor. 


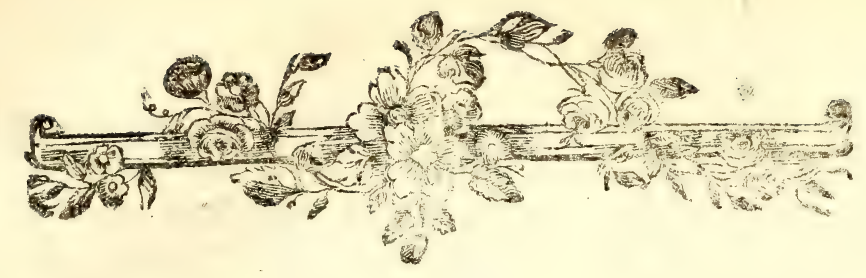

\section{PROOEMIUM.}

A caput erigere occeperunt, rebus naturz perficutandis \& defcribendis decora haud defuere ingenia. Tam late nimirum patere tamque copiofe ac varie abun. dare Hiftoriz Naturalis campum, ut neque pabulum nec voluptatem menti non fubminifret curiofa, fatis fuperque conftare atbitranur. Inter illos vero, qui quo. dam quafi genio in Naturæ mira \& arcana baud fine fuccesiu fuerunt abrepti, infignem ex noftratibus locum fibi vindicat FREDERICUS HASSELQUIST. Ille enim in ipfam fcientiam amore ductus, exigua pera inftructus, moleftum fufcepic irer in AEgyptum, Syram \& Palæltinam. Qua indefesfa diligentia, quo impigro fudio finem infituti itineris adlequi ftuduerit, teftatur ipfus is tinerarium, teltanru abunde nagnæe \& multiplices in nominatis regionibus ab illo collectæ \& in Patriam de. mum transvectre naturæ opes, quas infuper Academia Upfalienfis jan luas putat, ex fingulari clementisfimi Re gis noftri munificentia. qui nulli unguam pepercit rei, qua litterarum florem eatur p omotum. HASSELQUIST noffer jamdudum ad plures quidem abiit, prematura morte in medio magnarum ladura curfu procul a patria abreptus, ait honos manebit illus, quandiu pretium fuum Hiftoria Naturali ftabit. Neque ingrati animi crimen incurrant

cro. 
crorum interpretes, neminem enim fugere poteft, quantum momenti Orientis Hifroria Naturalis conferat cognitio ad Sacram Scripturam recte explicandam \& intelligendam, \& hæc ipfa biftoria ante noftrum HASSELQUIST valde fuit incognita, vaga \& incerta, illius vero induftria nova eidem adfulfit lux, cuique religionis amico gratisfima. Ut vero documento conftet nos non temere laudare, utque adpareat, quid laudato Scrutatori \& Regize munificentix referamus acceptum, Flores bujusce collectionis typis exhibere apud animum conftituimus.

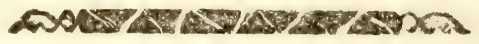




\section{Donationis}

\section{REG GUSTAVI IV:TI ADOLPHI \\ Continuatio II:ed\%.}

Herbarium a Celeb. HASSELQUIST collectum.

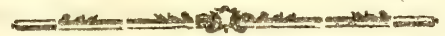

Olea europoca. a. $\beta$. Fasminum fruticans.

Veronica agreftis. anagallis. hederafolia. maritima.

Pingvicula vulgaris. Salix agyptiaca. babylonica.

Momordic balfamina.

Bryonia cordifolia. Rufcus hypophyllum. Boerhavia erecta. Valeriana anguntifolia dioica. locufta.

Gladiolus commenis.

Carex hijpida. paludofa.

Scirpas paluftris. romaneus. fylvaticus. Cornueopice cusullatnem. Posiseun coloratum.
Panicum viride.

Phataris arundisacea. bulbofa. canarientis. paradoxa.

Agrofis interruptio. matrellc. miliaces.

Lagurus ovatus. Andropogon ifchemusu. Holcus forghum. Saccharum officincirtm. Melice nutans. Egilops ovatio Poa coumua.

Bulbofa. cafirs. rigida. trivialis.

Fefuce bromoides. Ductylis glomeraing. littoralis.

- Bromus alopecarus. diflackyos. 
Bromus feoparins.

fterilis. tectorum.

Avena fatua. madritenfis.

Arundo phragmites.

Tritieum repens.

Hordeum bulbofum. murinum.

Elymus arenarius. europaus.

Cynofurus aureus. echinatus.

Holoficum tetraphyllum. Ptelea trifoliata. Cornus fangvinea. Scabiofa pappofa. fitellata. Syriaca.

Globularia orientalis. Plantago lagopus. lanceolata altis/. major. maritima.

Rubic tinctorum. Galium aparine. $V$ alantia cruciata. cucullaria. hifpida.

Crucianella anguftifolia. Camphorosma monfpelienfiso Urtica pilulifera. romana

Eleagnus orientalis. Hypecoum prockmbens.
Morus alba.

Mirabilis dichotoma.

Cerinthe minor. Echium vulgare. Heliotropium europaum. Supinum.

Onofma orientalis. Symphytum officinale. Borago officinalis. Anchufa italica. Sericea. undulata. Anagallis arvenfis. latifolia. monelli.

Lysmachia vulgaris. Cyclamen europoum. Hyofcyamus albus. $V$ erbafcum thapfus. Convolvalus althaifolius. hederaceus. . Scammonia. Scoparius.

Solanum diculcamsara. incanum. nelongena. nigrum.

Nerium oleandor. - Campanula dichoroma. Jpeculum. trachelinm.

Rhamnus lotus. patiurus. 
Yitis vinifera.

Hedera helix

Lagoecia cuminoides.

Impatiens noli tangere. Achyranthes afpera.

Illecsbram paronychia.

Glaux maritima.

Ceratonia filiqun.

Afslepias giganter.

Gentiane centaurium.

Spicata.

Velezia rigida.

Chenopodium ambrofoides. polyfpermum.

Ulmus campeftris. a. $\beta$.

Eryngium tricuspidatum.

Caucalis latifolia.

orientalis.

Artedia fquamata.

Tordylium nodofum.

Ammi majus.

Hasfelquiftia agyptiacâ.

Bupleurum odontites.

rotundifolium.

Sifon ammi.

Scandix anthrifcus.

Cherophyllum xgyptiacum.

Smyrnium xgyptiacum.

$$
\text { perfoliatum. }
$$

Pimpinella anifum.

$$
\text { faxifrago. }
$$

Viburnum opulus.

Sambusus ebulus.

Alfine media. \&. $\beta$.
Piftacia lentifus.

Pasfifore carulea.

Tamarix gallica.

Statice monocephala.

Linum catharticum.

hirfutum.

narbonense.

modiflorum.

Mufo paradifiaca fot.

Narcisfus tazetta.

Paneratium illyricum.

Polianthes tuberofa.

Asphodelus ramofis.

Hyacinthus botryoides. comofus. orientalis.

Convallaria bifora. polygonatum.

Allium chamemoly.

moly.

migrum.

pallews.

Scilla amoena.

urfinum.

Ajparagus acutifolius.

Tamus communis.

Oryza fativa.

Triglochin maritimum.

Rumex bucephalophorus,

$$
\text { rojerus. }
$$

spinofus.

Alifmo plantago.

Chlor o perfoliata.

Adox mofchatellina. 
Quereus ilese.

Mercurialis ambigua. tomentofa.

Anogyris foetida.

Cosfia chamaerifta.

$$
\begin{aligned}
& \text { fiftula. } \\
& \text { occidentalis. } \\
& \text { senna. }
\end{aligned}
$$

Ruta graveolens.

Tribulus terveftris.

Zygophyllum fabago.

Arbutus condrachne.

$$
\text { unedo. }
$$

Chryfospleniam alternifolium. Arenaria rubra. silene behen.

\section{conica. supeftris.}

Saponaria orientalis. Cucubalus behen.

Oxalis cormiculate. Cerafium perfoliatum. Sedum libanoticum. Peganum tarmala. Portulace oleracea. Lythrum cuphoea. Refeda agyptiaca.

$$
\text { lutea. }
$$

Juglans regio. Euphorbia chamafyce.

$$
\begin{aligned}
& \text { heliofcopio. } \\
& \text { peplis. } \\
& \text { paluefris. } \\
& \text { vinwinalis. }
\end{aligned}
$$

Glinus lotoides.

Myrtus communis. Punica granatum. Amygdalus communis. perfica.

Cratagus oxyacantha. Me embry an themum nodiflorew. Rofa alba.

faxatilis.

Rubus fruticofus.

Chelidonium glaucium.

Papaver argemone.

Nymphac Intus.

Ciftus aralicus.

Poterium fangviforba.

Mimosa lebbek. spinofum. nilotica.

Paonia officinalis. Nigella damafcenc. Delphinium peregrimum. Reaumuria vermieulata. Arum arifarum. dracuracules.

Platanus ovientelis. Anemone coronaria. hepatica. Clematis orientalis. Ranunculus ficaria. repens. Menthe rotumelifolic. Tesicrium cayitatum. chunsedrys。 cretienm. maresm. 
Ajuga orientalis. Phlomis fiuticofa. Verbena fupina. Salureja capitata. Marrubium peudodiciammus. Moluccella Jpinofa.

Ocymum bafilicum. Selvia horminum.

$$
\begin{aligned}
& \text { napifolia. } \\
& \text { milotica. } \\
& \text { officinalis. } \\
& \text { Syriaca. } \\
& \text { verticillata. }
\end{aligned}
$$

Rosmarinus officinalis. Dracocephalum canejeens. moldavicum.

Origanum marim.

Thymbra $\int$ picata.

$$
\text { fmyrneum. }
$$

Orubanche lovis. Acanthus arboreus: Scrophularia peregrina. Antirrhinum majus.

Dodariia orientalis.

Cupresfus virens. Thuja aphylla.

Ephedra diftachya. Geranium chium.

$$
\text { lucidum. }
$$

malacoides. roturdifolium.

Ricinus commenis. Gosfypium herbacanm.

\section{Hibifcus manihot.}

trionus.

Malva agyptia.

crifpa.

mauritanica.

130) chata.

roturitifolico

sivefiris.

Lavatera thuringiaca.

trimefiris.

Althe ficifolia.

hirfuta.

rofea.

Fumaria bulbofa.

capnoides.

capreolata.

officinalis.

spicata.

Polygala vulgaris.

spartium angulatum.

junceum. mono/permum.

Afpalathus cretica.

Spinofum.

Ononis natrix.

$$
\text { vifcojis. }
$$

Lupinus anguliffolines.

Pifum jativum.

Lathyrus aphaca.

- Cicer arietinum

Scorpiurus fulcosia.

Hedyjarum alhagi.

Vicia fabc. 
Vicia hybrida.

Ervum less.

Trifolium anguftifolium, procumbens. Atellatum.

Medicago orbiculata. radiatu. Scutellata.

Trigonella hamefa.

Hypericum hiverutum.

repens.

Lapfana communis. rhagadioloides. Atellata.

Prenanthes muralis. Hieracium fanctum. umbellatum.

Laciuca feriola.

Sonshus oleraceus.

Carduus fyriacus.

Carthomus lasatus. Onopordon acanthium. Leonicdon autusnnale. taraxacum.
Scorwonera hifpanica, humilis. orientalis.

Cichorium intybus. Artemefla arborefcens. Baccharis Diofcoridis. Conyza faxatilis. Gnaphalium fongvineum. foechas.

Bellis annia.

Matricaria chamomilla.

Chryfanthemum coronarium. Senecio triflorus. Achillac ptarmica. Buphthalmum fpinofum. Centaurea crupina. cyanus. panieulata. verutum.

Calendula officinalis. Adianthum capillus. Polytricham commune. Bryum trunsatulum. 


\section{MUSEUM NATURALIUM ACADEMI E UPSALIENSIS,

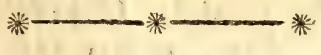 \\ cujus}

PARTEMI XXVI. VENIA EXP. FAC. MEDICAE UPSAL. P R $巴$ S I D E

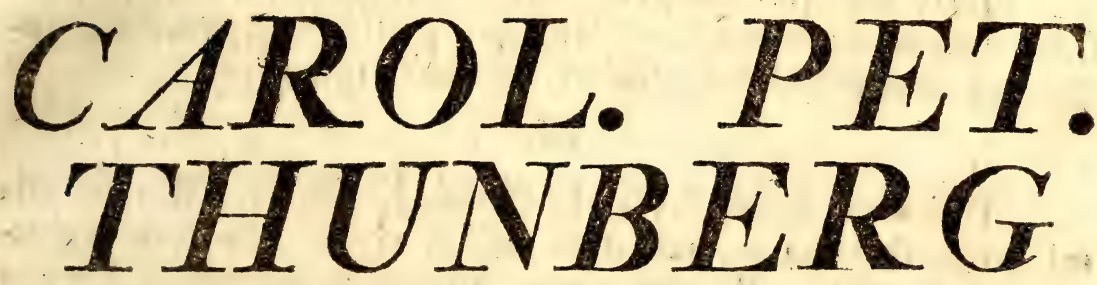

Equits Aurat. Reg. Ord, de Vasa,

Med. Doct. Prof. Med. ext Bot. Reg. et Ord. Acad. Cassar. Petropot. et Nat, Curios. Reg. Scient. Lond. Holm. Societ. Scient, Ups. Philad. Patr. Holm. ex Civ, Oecon. Find. Berod. Nat. Scrut. Paris. Hafn, et Halens. Hist. Nat. Lund, Harlem. Amsteld. Zeland. Nidrosiens. Ticinens. Irnens. Linn. Londin. Reg, Scient. Pays. et Phytourapiz. Górtingens. Nanciens. Men, Monsper. Med. Matritens. Medic. et Nat. Studios. Edimburg. Membro nec non Acad. Scient. Paris. ext Institut. Nation. Monspeliens. Agricuit. Paris. Medic. Londin. Scandinav, Fiorentin. et Batavin, Ind, Or. Correspond。

PUBLICO SUBMITTIT EXAMINI SVENO ABRAH. WESTMAN, VESRTOGOTHUS, IN AUDIT. GUSTAV. MAJ. D. XYII APR. MDCCCV. ม. A. M. S.

\section{Upfalia, Typis Edmannianis.}




\section{THESES.}

I.

Qua gaudemus homines, cupiditatii novitatis, nulla in alia fcientia nobis plus fatisfacere contingit, quam in Hiftoria Naturali; ideoque jure æquo posfumus contendere: ei, qui in natura inveftiganda verfatur, campum offerri amplisfinum.

\section{II.}

Quod autem Hiftoria Naturalis hactenus plus forfan ceperit incrementi extenfionis, quam certitudinis refoctu, curiofitati huic haud injufte tribumus.

HII.

Multa \& haud levia Hiftoria Naturalis afferret adminicula fterilitate incidente annonæa ad miferiem egen-tium levandam, fi detegendo; quem unaquæque rerum e penu naturæ hauriendarum præberet ufum 2 asfiduam ejus cultores darent operam.

IV.

Non modus eft ullus inveftigandarum rerum Natux; quin imo, fi femel inceperis, quærendi defatigatio turpis eft, cum, quod quaritur, emolumento generi hu: mano fit maximo.

\section{V.} moris.

Phyfici non eft asfiumere: ponderis aliquid esfe mo 
GRPSSHANDLAREN

HO̊GÅDLE

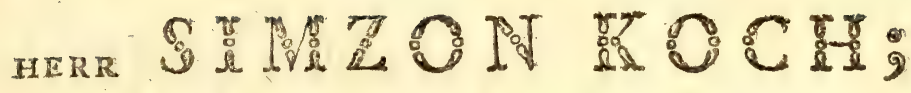

HO̊GÅDL FRU

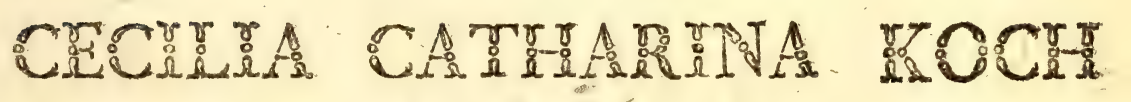

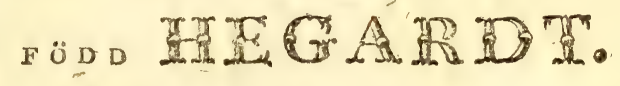

Emottagess detta offer, sisom ett ringa prof af den vơrdnat och ahtning, huarmed jag har diran framhard EDER

\author{
o̊tmjukaste Tjenare \\ SVEN ABR. WESTMAN.
}





\section{REG. GUSTAVI IV:TI ADOLPHI \\ Continuatio III:tia.}

Herbarium Celeb. KALM collectum.

Feronice anagallis.

marilandica

serpyllitolia

virginita.

Utricularia vulgaris.

Circaa lutetiana.

Verbena urticefolia.

Salvia lyrata.

Monarda clinopodia.

$$
\text { fiftuloja. }
$$

punctata.

Gratiola virginica.

Iris verficelor.

Eriophorum alpinum.

Scirpus fetacens.

polyfachyum.

Cyperus alternifolius.

Anthoxantum odoratum.

Arundo arenaria.

$P$ anicum clandeftinum.

Cenchrus tribuloides. Mollugo verticilllata.

Qveria canadensis.

Lechea minor.
Galium bermudianuan.

itrifidum.

Plantago lanceolata.

virginica.

Cornus florida. Sanguinea.

Ifnardia paluftris.

Hamamelis virginica.

Myofotis fcorpioides.

Lycopfis virginica.

Pulmonaria virginica.

Cynoglos fum virginicum.

Convolvulus fepium.

fpithanæus.

Campanula-perfoliata.

Phlox glabra divaricata. macrilata. fubulata, $\infty . \beta$.

Lyfimschia tiliata.

Lonicera diervilla. quadrifolia.

Claytonia virginica. Ajclepias tuberoja. 


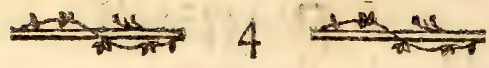

Azalea nudiflora. vifsofa.

Gentiana quinquefolia. faponaria.

Heucher a americana.

Evonymus americanus. Ceanothus americanus. Hydrophyllum canadense. Phyfalis vifcofa.

Hedera quinquefolia.

Vitis labrufca.

$$
\text { vulpina. }
$$

Chenopodium anthelminticum.

Thefium umbellatum.

Panax quinquefolia.

Aralia mudicanlis.

Hydrocothyle umbellata. vulgaris.

Thapfia trifoliata. $\alpha . \beta$.

sifon canadense.

Heracleum panaces.

Sa icula marilandica.

Asopodium podagraria.

$\checkmark$ ibunum acerifoliun.

dentatum.

prunifolium. a. $\beta$.

Sambucus canaderfis.

Rhus radicans.

vernix.

Ulmus campeftris.

Staphylea trifoliata.

Drofere rotundifolia.

Xarthoxylon clava.
Tradefcantia virginica. Allium carsodenfe.

Convalla ia multiflora. sacemoja.

Uvularia perfoliata. jesfilifolia.

Hypoxis erectio. Eryihronium dens. Lilium philadeljaincum. Aleihris farinefa. Antherium calyculatum. Helonias bulleta. $\alpha . \beta$. Melanihum virginicunu. Prinos glaber. verticillatus.

Leontice tisilict oides.

Trillium cernuum. Furicus comper as. finfer is.

Saururus sirimis. Oenother a fruibera. $V$ aecinium cortubofum. ficiod um. hispidulum.

Dirce paluetris. Andromeda arborea. latifolia. mariana.

Polygonum articulatum. dunuctorim.

Laurus aftivalis.

$$
\text { farfafras a. } \beta \text {. }
$$

Sophora tinctoria. Rhododendrum maxinum. 


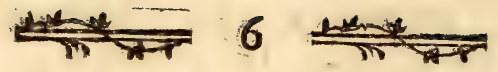

Chelone glabre.

hirfuta.

Pedicularis canadenfis. Anttrrhinum canadenfe. Gerardia flava. pedicularia.

Bignonia catalpa. purpurea.

Draba verna.

Cardamine virginica.

Dentaria enneaphylla.

Arabis canaden/is.

Eryfimum officinale.

Bunias kakile.

Sida Jpinofa.

Polygala fenega. verticillata. viridefcens.

Hedyfarum violaceum.

Crotalaria jagittalis. Robinia psendo-acafia. Lupinus perennis. Clitoria virginica. Trifolium repens.

Citrus medice.

Hypericum perforatum.

Hyoferis virginica.

Hieracium gronovii.

Serratula fpicata.

Cacalia fvaveolens.

Gnaphalium plantaginifolum. Coreopjis bidens.

After annunes. cordifolins.
Zinnia multiflora.

Silphium therebinthinacetum.

Rudbeckia purpurea.

Helienthus divaricatus.

Lobelia inflata.

fiphilitica.

Viola canadenfis. canina.

lanceolata. paluftris. pedata.

Impatiens balfamina. noli tangere.

Arethufa balbofa.

Cypripedium calceolus. Arum triphyllum. virginicum fol.

Dracontium foetidum. Sifyrinchium aneps.

Carex acuta.

loliacea.

Atellulata.

vulpina.

Morus rubra.

Betula alba.

incana.

lenta.

triphyila.

Urtica canadenfis.

Amaranthus gracizans.

Juglans alba.

Platanus occidentalis.

Liquidambar Atyracifuum.

Quercus aquatica. fol. 


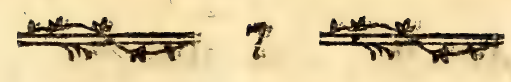

atba.

difcolor. fol.

nigra. o* $\beta$. fol $\gamma$. fo

mibrat a. Bo $\gamma$. fol o phellos. folo.

prinus.

Serrata.

Pinus balfamea. fol.

cannadenfis.

larix. fol.

pinea. foí.

fylveftris: fol. \&. $\beta_{\text {o. }}$.

trobus.

Cupresfus thyoides:

Myrica cerifera.

Smilax herbaced. laurifoliato.

Diofcorea villos $\sigma_{0}$.

7 uniperus Säbina fol.

Eagus caftanea.
Fagus pumila.

fylvatica.

1.Taxus baccata.

Diofpyros virginica.

Valantia cruciatit.

Nys a aquatica.

Gleditfchic triacantha:

Fraxinus americana. fol".

Acer negundto.

penfyluanicum:

rubrum. a. $\beta$.

facchärinum. tol.

Acnida cannabina.

Veratrum hutenw:

$$
\text { album: }
$$

Ofmunda cinnamomea.

$$
\text { regalis. }
$$

Sphagnum paluftre.

Marchantia polymorpha.

Eucus faccharimus.

\section{Trâdforter, Ligna.}

Svart valnọttrâd. Fuglans nigra.

Alm. Ulinus Campeftris.

Cyprefs. Cupresfus virens.

Boke Fagus fyluatica.

Oxel. Cratcegus aria.

Lind. Tilia europea.

Aptl. Piyrass malius.

Pil. Salix fragilis.

Afp. Populus tremula:

Gran. Pinus: abies.

Hág. Prunus padus.

Malur. Betule albar, radix.

Kork. 


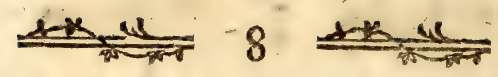

Kork. Quercus fuber.

Körsbar. Prunus cerasus.

Tall. Pinus fylveftris.

Brakved. Rhammus frangnla.

Hvitbok. Fagus fylvatica.

Sasfafras. Laurus fasfafras.

Fikontrad. Ficus carica.

Plommontrdd. Prumus domeftica.

Spanfk Ginft. Genifta hifpanica.

En. Funiperus communis.

Knơl af vildapel. Pyrus malus ferus, tuberculum.

Knól af ronn. Sorbus ancuparia, turbercuhum.

Hvalbjörk ell. Getapel. Rhammus catharticus.

Starkbdrstrdd. Prumus fpinofa.

Tamarinde. Tamarindus indica.

Knoll af afk. Fraximus excelfior, tuberculum.

Knool af ahl. Betula alnus, tubercuhum.

Folfer. Salix pentandra.

Hasfel. Coryhus avellana.

Run ell. Ro̊nn. Sorbus auenparia.

Wide. Salix pentandra.

Hvit fandel. Santalum albam.

Paron. Pyrus communis.

Mandeltrad. Amygdalus conmunis.

Ek. Quercus robur.

Buxbom. Buxus fempervirens.

Lignum Nephriticum.

Lignum Sanctum. Gvajacum fanctum.

Rofentrdd. Rofa.

Hvit Afp. Populus alba.

Arre. Betula incana.

Apricos. Prunus armeniace. Alfvembirstraid. 


\section{MUSEUM NATURALIUM ACADEMIE UPSALIENSIS.}

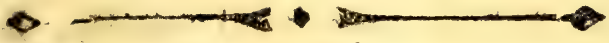

C U J U S

PARTEM XXVII.

VENIA EXP. FAC. MEDICAE UPSAL.

\section{P R ES I D E}
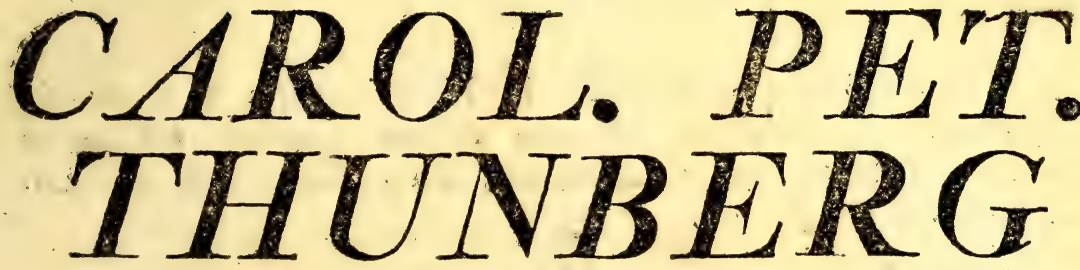

Equits aurat. Reg. Oki). de Vasa,

Med. Doct. Prof. Med. et Rot. Reg. et Ord. Acad. Caesar. Petropol. et Nat Curios, Reg. Scientr. Lond. Holim. Societ. Scient, Ups. Philad. Patr. Horm, et Civ, Oecon. Fint. Berol. Nat. Scrut, Paris. Hafn. Moscou. et Halens. Hist. Nat. Gorenkens. Lund. Harlem. Amsteld. Zeland. Nidrosigns. Ticinens. Ifnens. Linn. Londin. Reg. Scient. Phys.et Peytograpit. Góttingens. Wetteraw. Nanciens. Men. Parts. Emul. Mongpei. Men. Matritens. Medic. et Nat. Studios. Edimburg. Membro nec non Acad。 Scient. Paris, et Institut. Nation. Monspeliens. Agricuit. Paris. Medic.

Londin. Scandinav, Florentin. et Batavin. Ind, Or. Currespond.

PUBLICA CENSURE COMMITTIT

$$
\text { ERICUS HASSELHUN }
$$

BOTHNIENSIS.

IN AUDIT.BOTANICO DIE XVII APRIL. MDCCCX. H. A. M. $S$, 


\section{THE SES.}

Non manet diu fudium cognofcendi naturam, fi ejusdem co. gnitio fic comparatur, ut a mojori minorive memorix facultate pendeat omnis. - Hiftoriz Naturalis quoque Audium, ut eft jucundisfrmum, fi rite inftituatur, fic nefcimus an esfet aliud odiofius, fi amplam nominum rerum naturalium in memoria congeriem folummodo conftitueret.

\section{II.}

Quamvis Hiftoria Naturalis in Medicina ejus fit momenti, ut partium illius nullam ignorare debeat Medicus folidus et expertus, fatuere tamen audemus, Botanicam, ipf onniun maxime necesfariam esfe.

\section{III.}

Eumdem, præ ceteris Hiftorix Naturalis partibus, eminentiorem locum, in Oeconomia Rurali, fcientia Botanicx adjudicamus.

\section{IV.}

Varietatem colorum, qux in corollis cernitur plastarum? ex effectis lucis et aëris, nec non varia earum textura oriri, credimus.

$$
\text { V. }
$$

Inftante difficultate annonx, fi ufus Lichenis Istandici cogaitus esfet, utilitate quam maxime inferviret communi. 


\section{Donationis}

\section{REg. GUSTAVI IV:TI ADOLPHI}

\section{Continuctio IV:ta.}

Tråd-Sorter, famlade af Prof. Kalm,

ifrän Norra America.

Caprifolium.

Letteholtz.

Sweetgum. Liquidambar fiyracifua.

Lind. Tilia Americana?

Benjamin. Laurus Benzoin.

Annona. Annona triloba?

Ródblomiga Maple. Acer rubrum.

Mullberri. Morus rubra?

Willowmarsh. Salix.

Hvit afk. Fraxinus Caroliniana.

Platanier. Platanus Occidentalis.

Viburnum foliis Serratis. Viburnum lentago.

Viburnum fol. rotundis. Viburmum prunifolium.

Afp. Populus.

Spiraa opulifolia.

Toxicodendron fol. pennatis. Rhus vernix.

Lesfer Kalmia or Iwy. Kalmia anguftifolia?

Ahl. Betula alnus.

Spruce or Silfver fir. Pinus picea.

Stort Lingon trdd. Vaccinium arboreum?

Prinos. 
Andromeda arborea.

Diofpyros virginiana.

Svart Larix. Pinns laricina.

Striped Maple. Aier friatums.

Bignonia radicans.

Sumach. Rhus glabrtum?

Cephalanihus occidentalis.

Säcker Lơnn. Acer Jaccharinum.

Beechplum: Amygdalus perfica?

Cornus alba.

Gleditfchia triacantha.

Lotus arbor. Celtis occidentalis.

Mourtain Elm. Ulmus Americana:

Betula.

Aralia Spinofa. Zanthoxylon clava Herculis?

Thymelea. Dirca paluftris.

Cornus femina. Carnus fanguinea.

Viburnum.

Toxicodendron.

Svart Mourtain Maple. Acer penfylvanicum.

Chionanthus virginiana.

Örter utaf Kalms Americanjka Herbarium.

Orchis bifolia.

Salix viminalis.

Phoenix dactylifera. foll.

Holeus mollis.

Myhlenbergia diffufa.

Andropogon nutans.

Uniola Spicata.

Asperula lavigata.

cymanchica: $\gamma_{s}$. 


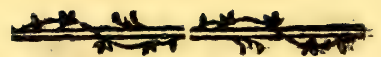

Galium paluftre.'

Claytonia virginica

Betula populifolia.

$$
\text { incana. fol: }
$$

$$
\text { pinnata. }
$$

Evonymus europens.

Trientalis europcea.

Liquidambar Atyrarifues.

Comptonia asplenifolia.

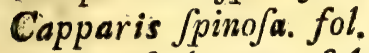
Arum efculentum. fol.

Thymus virginicus.

Mnium hygrometricum:

Fucus veficulofus.

$$
\begin{aligned}
& \text { nodofus: } \\
& \text { filum. } \\
& \text { felaginoides. }
\end{aligned}
$$

Conferva agagropila.

\section{Djur och Enfkildte Delar deraf:}

Elefantis Afiatici Dens molaris.

Hippopotamus Dentes I. . .

Rhebäcks horn, invdxte i trdd.

Rhinoceros horn: et ftort trubbigt.

s.t mindre trubbigt.

et Aort Jpitfigt.

et poleradt.

st med 3 fpetfar.

et litet.

Balana Priapus.

Gingiva.

Monodon Monoceros: Dens: 


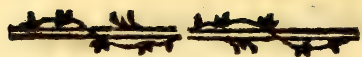

Scelet of 2:ne Simia med Angiolog. prapar. en felis.

Mammalia 9.

Foglar 9.

Mandibula af Buceros.

en Motacilla canarienfis.

Ramphaftus, 4 Species.

Et Amphibium.

Et Mdnnifko-Scelett, med myolog. och angiol prapar.

- Cranium Mammalis.

Teftudo: caput mandibulis ferratis.

carinata; fkal.

Grace; Jkal.

Marginata; Jkal.

Pida ; Jkal.

Serpentina; torkad hel.

tabulata; ftort fkal.

- en mindre.

- en dn mindre.

tricarinat a ; Jkal.

Lacerta Dracana; mutilerad.

monitor.

ftellio.

frumosa.

Diodon hyftrix. $\beta$.

Oftracion cubicus.

Balliftes maculatus.

Squalus Acanthias. $\beta$.

Squali mandibula. 1. 20.3. 40

Dentes.

Priftis Roftra. 1. 2. 3. 4.

Monoculus polyphemus, maximus.

Et ftort Getingbo, holt.

Et - oprodt.

Et mindre. 


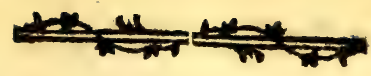

\section{StErre parad/nåckor:}

Chassa gigas, 2 fkal, myoket fora.

En mindre, zfkalig.

Choma hippopus.

En Dito, mindre.

Mytilus margaritiferus, polerad et par.

Lepas balanus, en flor Grupp.

Büccinum tuberofum. 2:ne.

olearium.

Strombus gigas. 3:ne.

chiragra.

millepeda. 2:ne.

lucifer. 2:ne.

Murex tritonis.

ramofus. 2:ne.

Voluta Cymbium. 2:ne.

neptunus.

Turbo olearius. 2:ne.

Sabella.

En polerad.

Coraller.

Madrepora fungites; zo varieteter, fïrre och mindro. meandrites.

muricata. 1. 2. 3. 4. 5. 6. 7. 8. 9.

oculata.

organum.

pileus. I. 2. vor.

porites.

ramea. 1. 2. 3. 4. 5.

reticulate. 
Millepora Alcicornis. 7. 2. 3. 4. 5. 6. 7. 8. 2:ne med Madr. maardrites.

Ifis nobilis.

$$
\text { ochracea. }
$$

hippuris. $x .2$.

Tubipora mufica. 1.2.

Gorgonia flabellum. 1. 2. 3. 4. 5. 6. 7. 8.9. antipathes. 1. 2. 3. 4. 5. 6. 7. verrucofa.

Antipathes jpiralis. fetofa.
dichotoma.

Spongia dichotoma.

\section{Varia.}

Blommor fammanfatte utaf Sndck/kal. Bilder ut af Snackor, Jkadade. Leijon utaf Sndckor, ftórre.

Tdnder, fundne $\vec{i}$ in Mindr.

Et litet aflangt fóndrigt dgg.

Strutsigg.

Et laingt fogelndfte utaf grds-ftra.

Et litet af grds med tomentum blandadto

En Mumia kruka Nlltapt.

En Mumia kruka opnad.

Macererade Blad utaf atfkillige văxter. Mafkdtit trdd, 2:ne varierande Aycken.

En Ramulus of trad, med biada dndar faftwaxt: Ramus.

En vrefis rot, arbetad till en Gubbes bild. 蛋n påk of trdd, mycket knylig. 


\title{
MUSEUM NATURALIUM
}

\section{ACADLMIE UPSALIENSIS.}

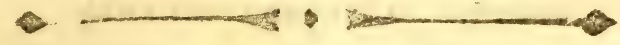

\author{
CUJUS \\ PART. XXVII.
}

VENIA EXP. FAC. MIED. UPSAL.

PR $\mathbb{E S I D E}$
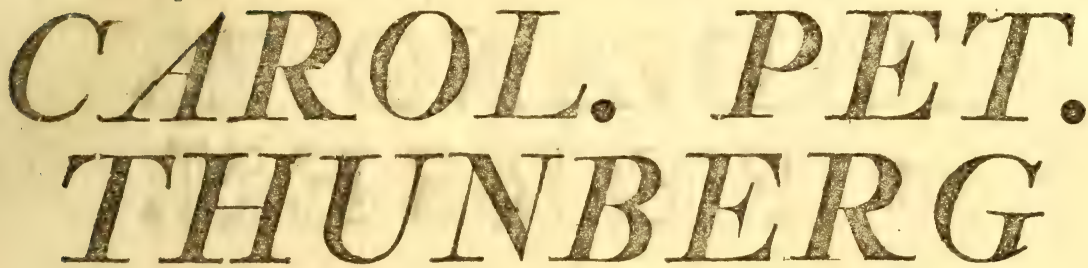

Equite Aurat. Reg. Ori). de Vasa,

Med. Doct Prof. Med. ex Bot. Reg. et Ord, Reg. Coll. Med. Membo Hon. Acad. Casar. Petropnl. et Nat. Curios. Reg. Scient, lond. Holm. Societ. Scient. Ups. Philad. Patr. Holm, et Civ, Oecon. Finl. Berol. Nat. Scrut, Paris. Hafe. Moscou. et Halens. Hist. Nat. Gorenikens. Luni, Harlem. Amsteld. OEcon. et Inst. Lit t. Zeland. Nidrosiens. Ticinens. Innens. Linn. Londin. Reg. Scient. Phys. et Pejytograpia, Góttingens. Wetter aw. Nanciens, Med, Parts. Emul. Monspel, Med, Matritens. Medic. et Nat. Studios. Enimburg. et Petrop. Medico. Chtr. Membro nec non Acad. Scient. Paris. et Institut. Nation. Monspeliens. Agricuit. Paris. Medic. Londin. Scandinav. Florentin. et Batavin, Ind, Or, Correspond.

PUBLICAE SUBMITTIT CENSURA.

GUST AVUS ELGSTROOM STIP. PIPER, VERMELANDUS.

IN AUDIT, BOTANICO D. IV DECEMB. MDCCCXI

FI. A. M. C.

UPSALI 压,

Excudrbant Stenhammar et Palmblad. Reg, Academie Typographi. 


\section{VIRO}

SUMMA IN SACRAM REGIAM MAJESTATEM FIDEZ,

INTER PROCERES REGNI UNI,

SUPREMO A. H. CUBYCULARIO

A T Q U E

AD REGIT CUSTODIAM CORPORIS CAPITANET LOCUM TENENTI; REG.ORD. ENSIFERORUMEX GRAND. CRUCIS INSIGNI COMMENDATORI

ILLUSTRISSIMO ET EXCELLFNTISSIMO COMTTI, DOMINO
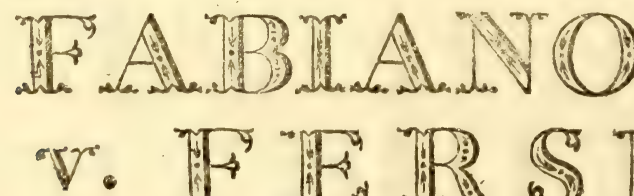
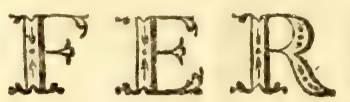

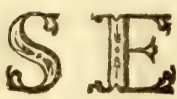


V I R O

SUMM IN IN SACRAM REGIAM MAJESTATEM FIDET; SUPREIMO CUBICULARIO,

A. H. GUBERNATORI,

REGII DE STELLA POLARI ORDINIS COMMENDATORI

-

A TQUE

REGTI ORDINIS ENSIFERORUM EQUITI,

ILLUSTRISSIMO AC GENEROSISSTMO LIBERO BAIRONI, DOMINO
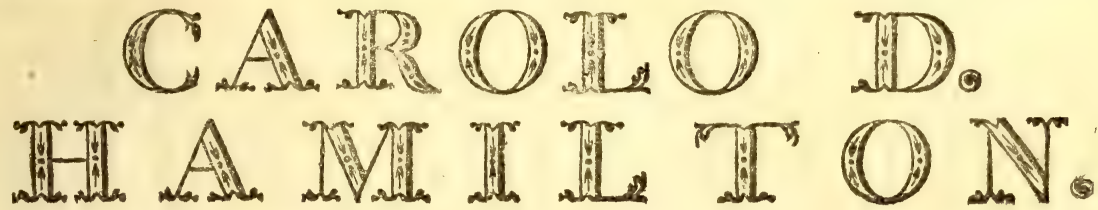

C R U M

debuit

veneraburdus

ALGSTROM. 

SUMMA IN SACRAM REGTAM MAJESTATEM FIDEI,

INTER PROCERES REGNY UNI,

SUPREMO A. H. CUBÝCULARIO

$$
\text { A T Q U E }
$$

AD REGII CUSTODIAM CORPORIS CAPITANET LOCUM TENENTI, REG. ORD. ENSIFERORUMEX GRAND. CRUCIS INSIGNI COMMENDATORE

ILLOSTRISSIMO ET EXCELLINTISSIMO COMITI, DOMINO

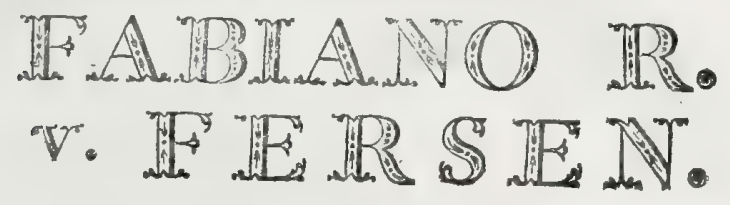

SUMME IN SACRAM REGIAM MAJESTATEM FIDET, SUPREMO CUBICULARIo,

A. H. GUBERNATORI,

REGII DE STELIA POLARI OIDINIS COMMENDATORI

$$
\text { A TOUE }
$$

REGII ORDINIS EISIFERORUM EQUTTI,

LLLUSTRISSIMO AC GENEROSISSIMO IIBERO BARONI, DOMINO

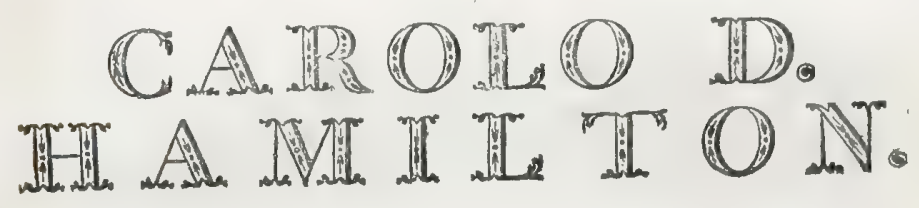


$-$ 


\section{MUSEUM NATURALIUM \\ ACA D. UPSAL。}

\section{\. I.}

(1) nithologiam in Svecia primus Omnium, initio Seculi preterlapfi, coluit illuftris Runвескіus, plurimasque Patriæ Aves coloribus vivis illuftrandas curavit, ftudio et opera Studiofi Medicinæ Ноцтzвом, qui variis Stipendiis Regiis fuffultus, varias Regni Provincias per agravit, Avesque indigenas penicillo excellentisfimo pulcherrime delineavit. Volumen, quod avium figuras comprehendebat, poft mortein RuDBECKII fub halta publica veniit, jamque in Bibliotheca Dom. Baronis De Gefr ad officinam ferrariam Leufftad lervatur. Adeft et copia horum iconum, minus tamen completa, in Mufeo animalium Grilliano ad So̊derfors.

\section{§. II.}

Aves quidem, xque ac cetera animalía, laudatus a Lrnné. ordine fyftematico digesfit, et fvecicas in Fauna recenfuit; paru'n tamen hanc Hiftoriæe animalium partem curavit, nullamque ejus collectionem habuit.

Deinde circa medium præteriti Seculi Ornithologiæ ftuduit Celeb. Profesfor LeChe, licet nibil publici juris fecerit præter Disfertationem de Commoratione hyber- 
nali et peregrinationibus hirundinum, Aboæ habitam 1764. Vir hicce, bene meritus, adeo cum Avibus Patrix fuit familiaris, ut ex ipfa voce et volatu, e longinquo illarum fpecies diverfas difçernere potuerit.

\section{III.}

Huic fcientize Celeb. Præefes, fub curriculo Academico non minus, quam aliis Zoologiæ Clasfibus, operam dedit fidelem et indefesfam; fed imprimis, poft peracta novem annorum itinera, et felicem in Patriam reditum, Aves Patriæ facile omnes quæefivit, collegit, Mufeum Academicum inftruxit, ac fpeciebus non paucis ditavit. Quodque ipfe intra limites Regni, tam longe lateque patentes, efficere non valuit, per Difcipulos fuos dilectos, fuoque exemplo exhortatos, efficere fuit conatus. Fue. runt $\mathrm{Hi}$ iroprimis Doct. Lundmark, Tengmalm, Gevalin, Fulin, Hornfledt, Eklund, Sacklén, Lönnberg, Riflachi, Fahlander, Forsftrom, Quenfel, Alii; præeter eximium Celeb. Præefidis laudatisfinum amicum, Regiæe Cancellar. Confiliarium Nobilisf. Dom. PAYKULL, qui in prædio Valloxfaby locupletisfimum et pretiofisfimum Volatilium Mufeum, fumtibus ingentibus et zelo vix imitando collegit ac adornavit.

\section{IV.}

Poft medium elapfi Seculi, Vir eximius, in Dicafterio fupremo Wafenfi Præeles Nob. Dom. Carlson, Mu. feum avium mille fere omnis generis fpeciebus conftans; adornavit; qure quidem rara collectio, exoticis multis a Dom. Comite Ruuth, Prof. Sparrman, Doct. Horn- 
Stedt, Gróndahu et Dom. Præefide communicatis infigne habuit augmentuin.

Generosf. Dom. Comes Ruvth, e Patriis Avibus etiam elegantem habuit collectionem, uti et Dom. Baro Alstroemer fat amplam, ad Gåfevadholm, cum ceteris pretiofis naturæ gazis fervatam.

$$
\text { ๑. V. }
$$

Peregrinator illuftris, Dom. Profesfor Sparrman', ex itinere fuo ad terras Auftrales et Caffatiam, non paucas domum attulit Avium pnlcherrimarum fpecies, quibus non modo locupletavit collectionem Carlfonianam, fed etiam defcriptionibus et iconibus coloratis nitidisfimis exornavit quatuor illos, qui prodierunt, fafciculos Mufei Carlfoniani, Jam in eo occupatur meritisfimus Vir, ut defcriptionibus et iconibus coloratis illuftret Species Avium Sveciæ indigenarum.

\section{§. VI.}

Illuftris Comes Wachtmeister, inter alias lucubrationes, fcientiis Upfaliæ dicatas, etiam Ornithologix ftudium amplexus fuit, atque eximius Ornithologus collectionem infignem Avium Svecicarum congesfit, jam in Mufeo Academiæ Lundenfis confervatam.

Etiam in Alingsås Vir laude dignus, Dom. Pentz infignem fibi collectionem Avium Svecicarum acquifiverat, quæ dein in posfesfionem venit Dom. Baronis SILFVERSKOீLD; et Gothoburgi adhuc majorem copiam Avi- 


\section{) 4(}

um collegit Dom. John Hall, poft infaufta Posfesforis fata divenditam.

\section{§. VII.}

Inter alias Naturæ gazas, quibus fuperbit Mufeum Animalium Grillianum ad Sóderfors, eminet etiam am. pla et elegans collectio Avium, æque Suecicarum, ac exoticarum, quam opera fatigari nefcia, adornavit Dom. Asfesfor Lindroth.

Sed reque in hac Hiftoriæ Naturalis particula fua laude orbari debet Vir doctisfimus et in multis aliis fcientiis Polyhiftor Celeb. Dom. Doctor O̊nman, qui, dum Mufeum fibi non inftruxit ipfi, fpecimina fua Avium, imprimis aquaticarum Patriæ, vel Academiæ Scientiarum Holmenfi, vel Celeb. Præefidi dono dedit, Hiftoriam et Oeconomiam earum illuftravit multumque amplificavit.

\section{§. VIII.}

Ex Africæ illa regione, quæ Sierra Leona dicitur, rediens feliciter Dom. Doctor et Botanices Demonftrator Afzelius, nonnullas quoque, illas quidem rarisfimas Aves, inter alia animalium fpecimina, in Patriam attulit,

Avium, tam exoticarum, quam indigenarum, quadringenta circiter fpecies, eleganter admodum ornatas, præmatura Nobis morte ereptus Dom. Doctor RislachI collegerat, cito forfan diftrahendas. 


\section{) 5( \\ §. IX.}

Hæc fata fuerunt Ornithologiæ in Svecia, hæc Mu. fea tam ornatiora, quam ampliora Privatorum.

Infignis illa collectio Avium, quam in Mufeo fuo adornaverat illuftris Ornithologus Dom. CarLSon, poft mortem Posfesforis vendita fuit, ac hinc inde diftracta, e qua 185 felecta individua, numerata pecunia fibi acquifivit Academia Upfalienfis. Ut igitur ejus exftaret qua. liscunque Catalogus, fpecimen editurus primum Academicum, non inutilem duxi meam fore operam, qua hafce fpecies curiofis notas redderem Ornithologis.

Falco gyvfalco. magniroftris.

Lanius cayaneus. pomeranus.

PSittacus camis. pertinax. verficolor.

Corvus corax: albomac.

Trogon currucui. erythrorynchus. \& $\beta$.

Merops erythropterus. Galbula paradifea. Bucco niger. tamatia.
Cuculus orientalis. tenebrofus.

Gracula barita.

Crotophaga major. Alcedo alcyon.

americanus.

bicolor.

Oriolus baltimore.

cayaneniss. criftatus. gvianenfis. homorrhous. melancholicus. \&ิ. คे. minor. 5 .

Ori. 
Oriolus perfiens.

$$
\text { textor. ㅇ. }
$$

xanthornus.

Picus erythrocephalus.

flavicans

hivundinaceus.

lineàtus.

undatus.

varius.

Certhio criftata.

muraria.

omnicolor.

pella.

Sperata? q.

fpiza. a. $\beta$,

todagennfis.

Trochilus amethyftinus.

criftatus.

maculatus.

Rynchops nigra.

Aptenodyta demerfa,

Pelecanus fiber.

Anas agyptiaca.

clbida.

ferina.

frenata.

galericulata.

hiftrionica.

sufinc. को.

Spsctabilis, \&.

Larus glaucus.

$\int p o n / a$.

Sterna cappica. .
Sterna ninuta:

mubilofa. abjcura.

Podiceps parotis. $\gamma$.

Colymbus glacialis.

a. B. Ardea alba.

garzetta.

Indoviciana.

minuta. ठ. \&. ohula. purpurea.

Tringa cincta gawbetta. grifea. macularia. punctata.

Tantalus loculator. Scolopax Semipalmata. Fulica cethiops,

fufca.

martinicenfis.

Rallus cayanen/s.

carolinus.

forrugineus.

fuscus.

philippenfis.

Otis tarda: pullus.

Phafianus pictus: junior.

Penelope marail.

Columba aromatica.

carolinenfis. a.

dafypus. $\beta$.

livia: maculata. 


\section{)$: 8$}

Columba gibraltarica. malaccenfis. Speciofa.

Tetrao gibraltaricus. perdix. B. $\gamma$.

Numenius guarauna.

Pipra erythrocephala. rupicola. 3 '.

Tanagra atrata.

$$
\begin{aligned}
& \text { cayana. } \\
& \text { criftata. } \\
& \text { jacapa. } \\
& \text { magna. }
\end{aligned}
$$

Ampelis cayana. pompadora.

Colius erythropus.

Loxia amandava.

$$
\text { Senegalenfis. }
$$

aftrild.

aurantiaca.

macroura.

madagafcarienfis.

maja.

molucca.

oryzivorce. ․․

rubicunda.

Julphurata.

Turdus athiopicus.

brafilienfis. a. B.

ceilonicus.

columbinus.

cyaneus.

dominicus.
Turdus hifpaniolenfis. labradorus. perfpicillatus. polyglottus. Jaxatilis.

Fringilla bengalen/is. cana.

cyanoides. petronia. finica.

Sturnus capenfis. Parus candatus. $\beta$. Alaude alpeftris. capenfis. nigrita. objcura. tabira. tatarica. teftacea. trivialis. zundata. Motacilla aftiva. canadenfis, carolinen/is? ceilanica. T. cinerea.

critata. erithacus. horten/is. Beueorrhoea. maculata. pasferina. feialis. 
) 8(

Motacilla ftapazina.

Ariata.

Emberiza americana.

$$
\begin{aligned}
& \text { capenfis. } \\
& \text { criftata. } \\
& \text { molbyenfis. } \\
& \text { oryzivora. } \\
& \text { ferena. }
\end{aligned}
$$

Mufcicapa crinita.
Mufcicapa hamorrhots.

maculata.

philippen/is.

jurinamenfis.

tectes.

torquata,

tyrannus.

Hirundo javanica.

\section{THESES.}

\section{I.}

Characteres Avium e ftructura pedum defumti, longe certiores funt, quam qui a roftri figtira habentur.

\section{II.}

Secundum vitæ genus diverfum, diverfimodo variant in Avibus non modo roftrum, collum, lingva, pedes et cauda; fed etiam interdum ipfun corpus.

\section{III.}

Non modo pennæe, fed et ipfa osfa in Avibus aëre replentur, ut volatui aptiores fiant.

\section{IV.}

Pauciora remedia Nobis fubminiftrat Clasfis Avium; vix ulla abfolute necesfaria. 


\section{ACADEMIÆ UPSALIENSIS。}

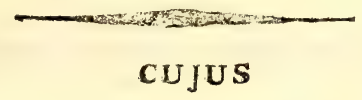

PARTEM XXIX.

NONNULLIS ADJECTIS APHORISMIS.

VENIA EXP. FAC. MED. UiPSAL.
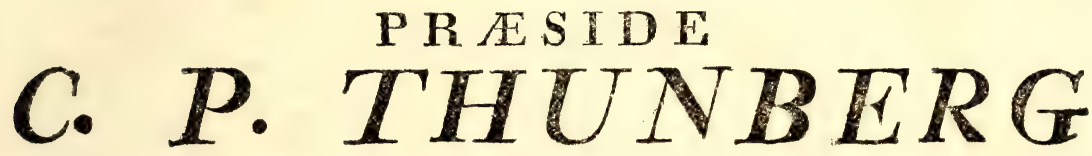

COMMENDATORE RES, ORD. DE WASA,

Med. Doct. Prof. Med, et Bot, Reg. et Ord. Reg Coll. Sanit. Memb. Hon. Acad. Cesar. Petropnc. ex Nat Curios. Reg. Scignt, lond. Holm, Agric. Med. et Evang. Socret. Oecon. upsal. Pazr. Holm. et Civ. Oecon. Finl. Honor. Wermel. Westm. Catm. et órebr. Berol. Nat. Scrut. Paris. Hafn. Moscou. et Haiens. Hist. Nat. Gorenk. Philad, lunis. Harlem. Amsteld. Oecon. et Inst. Lit t. Zeland. Nidrosiens. turicens Ifnens. Linn. Londin. Reg. Scient. Phys. et Puytogsapli. Góttingens. Scient. Gothob. Monach. Ertang. Wetter. Nanciens. Marpurg. Med. Paris. Emul. Monspel. Med. Matritens. Med. et Nat. Srudios, Enimburg. eq Petrop. Medico Chir. Membro nec non Acad. Scient. Paris, et Institut. Nation. Monspeliens. Agricult. Paris. Medic. Londin. Scandinav, Fiorentin. et Batavin, Ind, Oro Currestond. P. P.

\section{ZACHARIAS SJO̊STRO̊M.}

GESTRICIO - HELSINGUS.

IN AUDITOR, BOTANICO D. XXVI. MAJI MDCCCXIX. fr. A. M. S.

U P S A LI

EXCUDEDANT REGIAE ACADEMIAE TYPOGRAPHX. 


$$
\begin{aligned}
& 18512131470
\end{aligned}
$$

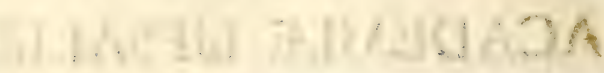

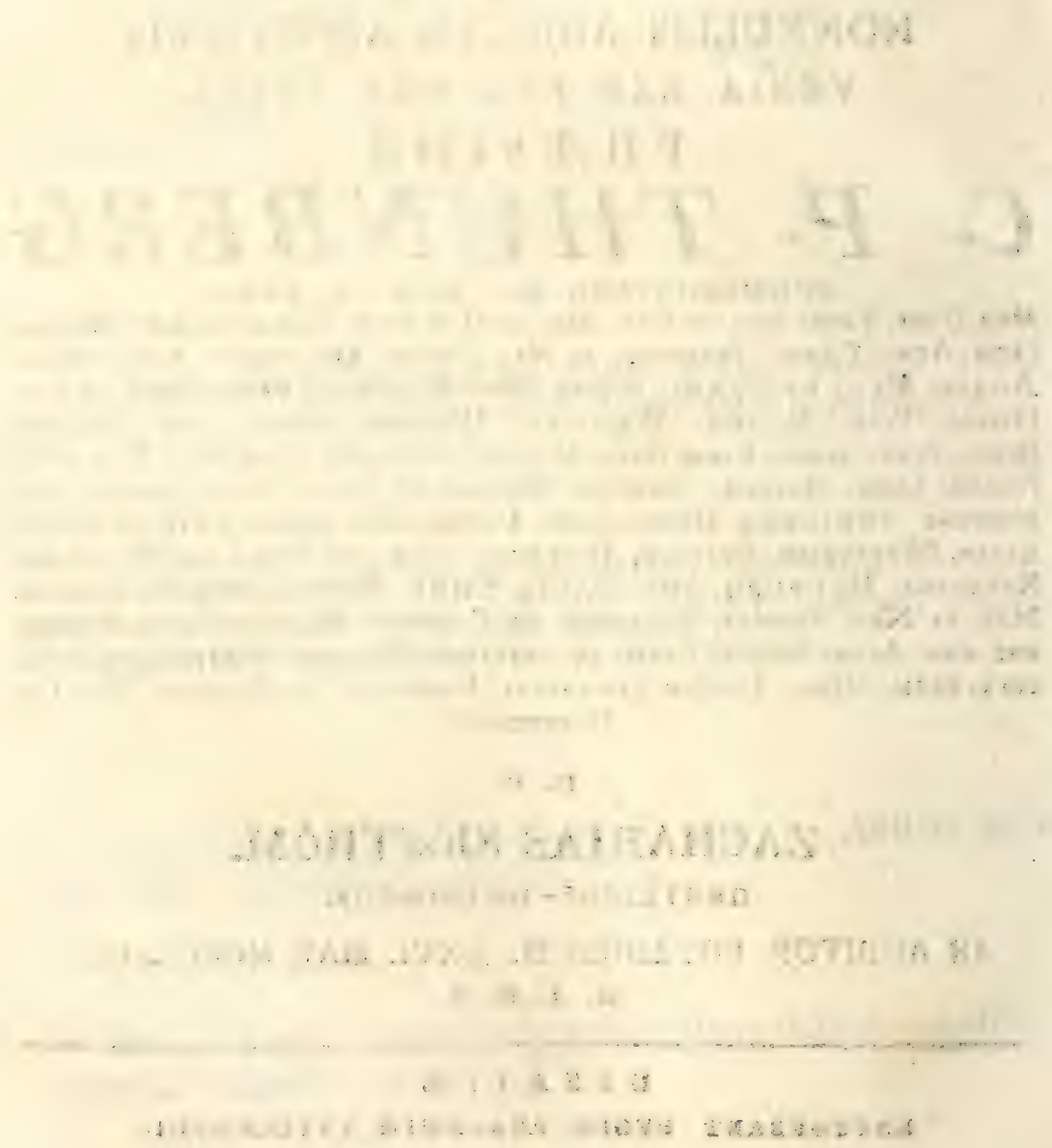




\section{GROSSHANDLARUN I GERLE STAD}

\section{$\mathrm{OCH}$}

RIDDAREH AE KONGI. NORDSTJERNE-ORDEN

$$
\text { HO̊G ÅDLE }
$$

пERR

Helgas Vördnadens, Tacksamhetens och Hjertats offer

DESS

ódmjukaste tjenare ZACHARIAS SJÓSTRỎM. 
ÅDEL OCH HO̊GAKTAD

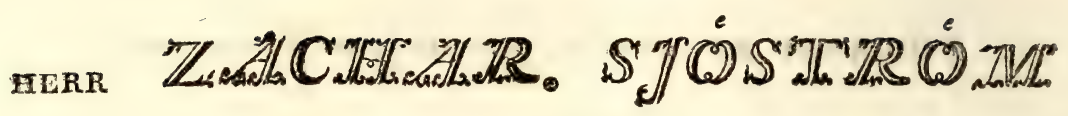

Min Huldaste Farbroder!

Den Faderliga Kärleken

Helgas sonlig vördnad, tacksamhet och kärlek

af

DESS

lydige

ZACHARIAS. 
Ann. 1815--1819.

\section{P}

anditur heic thesaurus animalium \& plantarum, quæ ia fecundisfimo Brafilix folo fub ejusque calidisfimo cælo nara maximâ collegit industriâ Conful Generalis, Vir de Hiftoria Naturali meritisfimus, nobilisfimaque munificentia hujus Academiæ Mufeo Naturalium donavit, Cel. Do minus Weftin:

Continet hæc collectio naturalium præclara fpecies rarisfimas, quarum defcriptio, exceptis quibusdam jam expofitis plantis, adcuratioris disquifitionis operæ fit relinquenda. Quare Deum Optimum Maximum, omnium rerum Moderatorem, Cel. Domino Præfidi ad hoc ceteraque opera immortalia perficienda multos adhuc lætos addere annos flagrantisfimo ftudio precamur.

Nos tantum nunc ad nomina harum, quæ fequantur. naturalium enumeranda progredimur. 
Simia albitrons.

niêtitans.

seniculus $\alpha$. $\beta$.

$V$ espertilio foricinus.

Myrmecophaga tetradactyla.

Dasypus multicinetus.

$$
\begin{aligned}
& g \text {-cintus. } \gamma . \\
& 7 \text {-cindus. } \beta \text {. }
\end{aligned}
$$

Felis tigrina.

$$
\text { onca. }
$$

Viverra canadensis.

masua. $\beta$.

Mustela quiris. B. brasiliensis.

Ursus labradorius.

brasiliensis.

Didelghis cayopollin. ㅇ.

gigantea.

opossum. $\beta$.

Sciurus cestnans. $\alpha$. $\beta$.

Cervus mexicamus.

Sus

Vultur aura. ㅇ.

Falco albicollis.

aquilinus. I.

aurantius.

furcatus.

plumbeus.

sparverius, \$.

sufflator.

rubitings.
Strix Erasiliane. cayanzusis. minuta. semitorquate.

Lanius atricapilins. cayanns. $\beta . \gamma$. canadensis.

formosus. pitagna. sulphuratus.

Psittacus agilis. + .

aracanga. $\sigma^{3}$. jun. carolinensis. passerinus. \&. pulverulentus б. क. severus. 8 . ㅇ․ squamosus. ㅇ. tuipars. ㅇ.

Ramphastos dicolorus. $\alpha . \beta$. toco a. B.

Corvus cayanensis. flavus. pectoralis. Trogon curucui. ${ }^{\circ}$. rufus. $0 . \%$. viridis. $\delta$. I.

Oriolus annulatus. homorrhous. B. niger. picus.

Cuculus cayanensis. ơ. gvira. 
Cuculus vetula. $\delta$.

Gracula quiscula. + . scandens.

Bucco fuscus.

tamatia.

Picus cayanensis.

flavefcens. of \&.

insignis.

lineatus. B. $\gamma$.

melonochlorus.

minutus.

passerinus. 8.

principalis.

rubricollis.

Todies brachycerus.

cinereus.

leucocephahis.

varius.

Trochilus dominicus.

glaucopis.

hirsutus.

mellisugus. \&.

striatus.

superciliosus.

tobaci.

vividis.

Certhia cayana. $1,23$.

cyanea. B. $\gamma$.

flaveola.

guianensis.

spiza. $\gamma . \delta$.
Galbula obscura.

Alcedo amazona.

bicolor. $\beta$.

torquata. ¿. $\alpha . \beta$.

Ardea cinerea. $\beta$.

cocoi.

lineata.

nytuicnrax.

pileata. o. $q$.

tigrina.

virescens. ठ.

Tantalus cayanensis.

Scolopax cayanewsis.

Charadrius vocifertus.

Tringa cayanensis.

brasiliensis.

Tinnamus variegatus.

Podiceps domingensis.

Plotus surinamensis.

Fulica martinica. $\beta$.

Penelope cumanensis.

Tetrao brasiliensis.

Perdix gvianensis. ๖. ₹.

Columba cyanocephala. $\delta$..+ bantamensis. $\beta$. martinica. ठ. passerina.

Alauda rufa.

Turdus alapi. .. 2. 
Turdus bambla.

brasiliensis. $\gamma$

canadensis.

cardinalis. $q$.

cinnamomeus.

cirrhatus. \$.

coraya.

eoreo.

jamnicensis.

lineatus.

minur. ฮ. ․

nemoralis.

plumbers. f.

trichas. d. 9.

Cotinga alba. o.

regulus. o.

speciosa.

virescens. J.

Ampelis collaris. o.

cotinga.

elegans.

fasciata.

magnana.

pompadora. B. $\gamma$. viridis, 8 .

Loxia grossa.

Tanagra brasiliana 2.2 . brasiliensis. ฮิ. ㅇ. cayana..+ cristata. $\delta$. episcopus. $z$. majoramensis.
Tanagra melanopis. $t .2$.

misisippensis.

nigra.

punctata.

silens. ठ. + .

Pipra caudata. ¿.

cephaleucos.

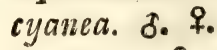

flavicans. + .

forficata. $q$.

leucocapilla. ठ. lineata. 万. 3 .

superba.

Fringilla melanocephala. tricolor. $\delta$.

Motacilla audax.

barbata.

cayana.

cayanensis.

chloroleuca. ․

cyanea.

fusicollis.

guianensis.

ludoviciana.

olivacea.

pratensis.

septencolor.

tgrannus.

Muscicapa acadia.

aurantia.

brunnea

cayanensis. 
Muscicapa cinerea.

coronata.

ferox. a. B.

Iudoviciana.

olivacea.

petechia.

Hirundo dominicana.

Caprimulgus guianensis. 9. ?. rufus.

Tapera brasiliensis.

Testudo discolor. $\beta$. tabulata. \&. $\varepsilon$.

Ostracion quadricornis. $\beta$.

Ballistes tomentosus.

Loricaria plecostomus $\beta$.

Holocentrus tasciatus?

sogo

virescens?

Scarus cretensis. viridis.

Chactodon bicotor.

Blatta varians.

Fulgora laternaria. $\beta$.

Pompilius lunatus. Alurnus marginatus.

Cassida irrorata.

Geoirupes alö̈us. $\delta$.

Buprestis gigantea. $\gamma$.

Haltica $\sigma$-guttata.

Cerambyx bivittatus. subocellatus.

vittatus.
Trachyderes rufipes.

Brentus longimanus.

Lycus fasciatus.

Prionus maxillosus.

Syrphus cyaneus.

Papilio potycaon. $p$.

clytemnestra. p.

Agavus $p$.

Pyrrha p. S.

Pygmalion. $p$.

Philea $p$.

Sara. p. I.

Iote. $p$.

Crassius. $p$.

Eleus. p. S.

Pansanias. $p$.

Cresphontes. $p$.

vitreus. p. s.

Biblis. f.

pellucens. p.

Dolicaon. $p$.

Pylocus. $p$.

Potistes sctiach.

Epidendrum dendrobioides.

clongatum.

Cymbidium crispatum.

pedunculatum.

Eriocaulon Freyreisii.

Xyris caroliniana.

Triopteris floribunda.

Spermacoce verticillata. $\beta_{\alpha}$

Lobelia macropoda.

westiniano. 
Plumbago scandens. Atropa arborescens. Solanum paxiculntum. $\beta$. vellsum. aureum.

Echites brasiliensis. 2. 2. Berberis laurina.

Andromeda coriifolia.

Rhexia imbricata. Meiastoma floribunda. spicata.

Goilandina bonduccella. 3. Pytholacca decandra. B. Begonic rufa. Mimosa síaminea. striata.

Ocymum flexuosum. Cleome triphylla. Croton divaricatum.

Malpighia spicata. Xylophylla obtusa. Polygala panicula? Hedysarum diphyllum. $\beta$. Stevia urticcefolia. Baccharis genistelloides. Eupatorium conyzoides. Musirale.
Eupatorium Freyreisii. Cacalia asclepiadea.

Elephantopus carolinianus. Trichomanes floribundum. Asplenium auritum. erosums. resectum.

Ptevis vespertilionis, $t, 2$. stricta. candata.

Adianthum subcordatum. $\propto . \beta$. platyphyllum. pulverulentum. villosum. $\varepsilon$. acuminatum.

Polypodium pertusum. concinnum. elegans. B. loriceun.

Gymnogranum charophylla. Gymnogramma tomentosa. Blechnum asplenioides. brasiliense.

Dancea rhizophylla. Aspidium pectinatum. Lindsea elata. portoriensis.

\section{APHO.}




\section{APHORISMI RESPONDENTIS.}

\section{I.}

Qvamvis perfe ditio, rerum naturæ fcientia fublimioris philofophicæ disquifitionis rationem exquirat, hâc tamen deftituta Hiftoria, quæ dicitur, Naturalis in dispofitione naturalium atque eferiptione Immortalis Linnei roftrique ævi Botanicorum Summorum opera \& ftudio incredibiles fecir pro. gresfus.

\section{II.}

Sxculo nuper præterlapfo, quo homines exiam fcien. tiis addicti theoreticum fcientiarum pretium minoris facerent, atque omnia ad practicam referrent dignationem, Hiftorix tamen Naturalis cultores fcientifica dueti cognitionis amore ftudia fua atque disquifitiones fuas funs erfecuti.

\section{III.}

Tamerfi multum utilitatis adferat Oeconomica \& Me: dica naturalium cognitio, non eo tamen infolentiæ procedere debet utilitatis ratio, ut ftudium quoque naturalia nova inveftigandi disponendique intermittatur, quo pacto ufui etiam rerum practico male confulatur.

\section{IV.}

Practicam vero \& imprimis Medicam fi refpiciamus utilitatem, maxime huic rei Botanicen conferre adjumen. ta reprehendimus. Chemica tamen plantarum cognitio, quæ 
8

qux ad qualitat"s inveftigardas atque cognolcendas ma. xime confert, hucusque vaide fuit neglecta.

\section{V.}

Vegetabilum autem Chemiam, fi quogue diligentius colatur, non posfe magnos facere progresfus fuspicamur.

VI.

Mineralia facillime atque diutisfime fuas retinere formas, ideoque facilius Chemicr fubjici posfunt folutioni.

\section{VII.}

Res contra Organicss difficiliori modo Chemice fol. vi atque examinari posfe contendirius.

\section{VIII.}

Non poteft ars ita vincere raturam, quominus plantæ, tametfi in fecundius traductre folum, caracteres fuas genericas atque fpecificas retineant.

IX.

Triplex atque Quintuplex foliorum partitio in plantis phænogamis maxime vulgaris eft. Ceteræ tantum funt ex proportione compofitæ vel fimpliciter decompofitæ formæ.

\section{$\mathrm{X}$.}

Ut Hiftoria civitatis humanæ, fic quoque diverfa ta. metfi ratione fuam habet Geographiam Hiftoria Naturalis. XI.

Plantarum vero \& Mineralium Geographia fecum conjunctam adcuratiorem exoptat Geologiam.

\section{XII.}

Scientia Hiftoriæ Naturalis, quæ ut Syftema Naturæ merito falutari velit, in naturalibus disponendis naturam non minus fequi debet quam artem. 


\section{MUSEUM NATURALIUM ACADEMIZE UPSALIENSIS}

CUJUS

PARTEM XXX.

VENIA EXP. FAC. MED. UPSAL;

P R ESIDE

C.
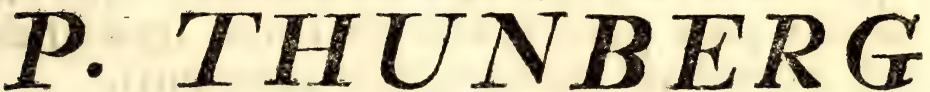

COMMENDATORE REG. ORD. DE WASA,

Med. Doct. Prof. Med. et Bot, Reg. et Ord, Reg. Coll. Sanit. Memb; Hon. Acad. Cesar. Petrofnl. ej Nat. Curios. Reg. Scignt. lond. Holm. Agric. Med. ext Evang. Societ. Oecon. upsal. Patr. Holm. et Crv. Orcon. Fint. Honor. Wermel. Westm. Calm, et Órebr. Berol. Nat. Scrut. Paris. Hafn. Moscov. et Halens. Hist. Nat. Gorenk. Philad. Lund. Harlem. Amsterd. Oecon, et Inst. Litt. Zeland. NiDROSIBNS TURICENS IENENS. LINN. LONDIN. ET HORTICUTUR. Reg. Scient. Phys. et Phytcgraply. Góttingens. Scten's. Gothob. Munacho Erlang. Wettrir. Nanciens. Marpurg. Med. Paris. Emul. Monsper, Med. Matritens.Med. et Nat. Studios. Emimburg. et Petrop. Medico Chir.et pharmac. Membro nec non Acad. Scient. Paris. et Instituto Nation. Monspeitens. Agricuit. Paris. Medic. Londin. Scandinav. Flo. rentin. et Batáin. InR, Or. CuRREstond.

\section{P. P.}

\section{J. E. ÅK E R M A N,}

SUD. INER.

IN AUDIT. BOTANICO D. V JUNII MDCCCXX. H. A. M. S.

\section{U P S A L I $\mathbb{E}$}

IXCUDEBANT KEGIAE ACADEMIFE TYPOGRAPHI. 


\section{T H E S E S.}

\section{I.}

In cupiditate nimia voluptatis rerumque ad ufus vitæ et commoda proxime fpectantium, tam Hiftoriam Naturalem, quam omnem in patria Scientiam negligendi magna quidem ex parte causfa eft quærenda.

\section{II.}

Sicut Natura, ita Scientia Naturalis Totum quoddam organicum conftituit: quxvis igitur hujus pars principalis Naturæ fcrutandæ æque eft necesfaria ejusdemque momenti.

\section{III.}

Cuique fane notitia Hiftorix Naturalis jucundisfima eft atque utilis, iis vero, qui in ftudiis litterarum progresfus facere cupiunt, fummæ necesfitatis.

\section{IV.}

Qui autem Efculapii fefe minifterio dicant, quorumque Macrocosmi mutuam et Microcosmi inter fe rationem intelligere \& explicare intereft, ab his univerfalis præfertim et abfoluta Naturæ eft cognitio poftulanda.

\section{V.}

Deficiens autem in Scholis ac Gymnafiis ad operam juventutis initio fublevandam, excellentisfimæ hujus Scientiæ eruditio progresfibus ejusdem nec parum offecit.

\section{VI.}

Conchylia etfi inter animantia ufu oeconomico inferiorern locuin teneant, operam tamen, quam notitia eorum hactenus defiderata exigit, largiter compenfant. 


\section{DONATION. REG. CAROLI XII.}

\section{AnNo I8I5.}

ARGONAUTA: betulinus:

Argo. muricata.

nodosa.

NAUTILUS.

beccarii.

calcar.

corn. Ammon. petrif.

crifpus.

legumen.

pompilius.

radiatus.

raphaniftrum:

raphanus.

Spirula.

umbilicatus.

CONUS.

achatinus,

Amadis.

Ammiralis: american.

anglic.

petrceus.

regins.

Stummurse

arachnoideus.

furinamenfis.

araufacus. Chersss.

architalasfus.

armineus.

Aulicus.

ouftralis. bifafciatus.

bullatus.

\section{- - marmor.}

capitaneus.

$$
\text { - philippin. }
$$

catenulatus.

cinereus.

clandeftinus.

clavus.

coccineus.

coffere.

contra. Ammirelis.

coronatus.

colfatus.

ebraus.

elongatus.

figulinus.

- fafciatus.

filipunctatus.

Alammeus.

franciscants.

fulmineus.

fufus.

Generalis.

Geographus.

glaucus.

Glaria maris major:

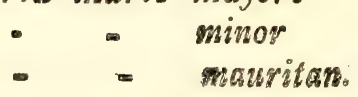

granulatus. 
Senator:

imperialls.

Sinenfis.

indicus.

infularis.

lactus.

levis.

leoninus.

Leofcandens.

leucoftictus.

lineatus.

litteratus.

Magus.

marmoreus.

- - fafciatus.

mercator.

miles.

milliespunctatus.

minimus.

monacus.

nebulofus.

nobilis.

nubecula:

nus atellw.

papilio.

papyrus.

pellis hyana.

pennaceus.

polyzonias.

princeps arabicus:

- - Sumatra.

punctatus.

radiatus.

rudis.

rufticus.

Societas cordium.

spectrum.

- - Sumatra.

Spurius.

Stercas culianm.

- mufcarum.

- pulicums

ftriatus.

terebellum.

rextilis.

- violaceum.

Thome:

Tulipa.

varius.

ventricofus.

vexillum.

virgo.

vitt nigra:

zeilanicus.

adufa.

CYPRAE.

amethyftea.

annulus.

arabica.

argus.

afelles.

atomaria.

carneola.

caurica.

chinenfis. 
cicererta.

clandefina.

coftata.

cresja,

cribiaria.

cruenia.

erofa.

erronea.

exanihema.

felina

feminea.

ferruginoja.

fimbriate.

flammea.

flaveola.

fragilis.

givbulus.

tavif.

guttate.

helvola.

hirundo.

hiftrio-

indica.

ifabella.

leucogafter.

livida.

lote.

lurida.

Lynx.

madagafcarienfis.

mappa.

mauritanica.

miliaris. moneta.

mus.

nucleus.

ocellata.

oculaia.

onyx.

ovum.

pediculus:

plumbea.

poraria.

psilla.

punctulata.

pyrum.

regina.

reticulum.

rubiginoja.

rufejcens.

fanguinolent 6.

jerpentis.

fimilis.

fpurea.

jquatina:

fraphyla.a.

fercorea.

ftolida.

fubjava.

juccincta.

talpa.

tefudinaria.

tigris.

translucens.

turbinala.

andara. 
urcellus.

vanelli.

varioloja.

venerea.

vitellus.

zebra.

ziczac.

zonaria:

BULLA.

ampluftre.

ampulla.

- longior.

aperta.

birofris.

cylindrica.

cyprac.

ficus.

flammea.

gibbofs.

hyalina.

hydatis.

hypnor um.

lignaria.

Milleri.

Naucum.

oblonga.

oliva.

ovum.

phyfis.

rapa.

- nicobarica.

Spelta:

fperma. terebellum:

undaia.

velum.

verrucofa.

volva.

zebra.

athiopica.

\section{VOLUTA:}

ampla.

annulata.

aurantia.

auris cati.

- juder.

- mida.

barbadenfis.

biplicata.

brafilienfis.

bullata.

caffra.

cancellata.

capitellum.

cardinalis.

carneola?

ceramica.

clatirus.

cofis.

Golocynthis.

conzes:

cornicula.

coronata.

coftata:

craiciculata.

crenulata. 
cymbium? dactylus. decusfata. digitalis. discors. cbraa. epifcopalis. exalperaia. faba.

fafciata. filofa.

fiammea. Siava: glabella. glans. globulus.

granofa.

hiaticula. indica. ifpidula. lavigata. leucofoma. leucozorias. livida.

marmorea. mendicaria. mercatoria. miliaria. minula. mixta. monilis. mosio. mucronata. mufica:

nasfa.

navicula.

nig?n.

niver.

nodulofa.

ocellaia.

oliva.

ulle:

pallida.

papalis.

pauperculas

- adornata.

perficula.

$$
\text { - fafiata. }
$$

- vejacul.

pertufa.

plicaria:

porcellana.

porplsyrea.

proputium

prunum.

pufilla.

py) um.

seticulata.

rufina.

rugoja.

rupefiris.

ruftica.

fanguifuga.

fcabricula.

fcapha.

foutulata. 
Spadicea.

Spiralis.

frigofa.

fubdivifa.

fulcaia.

tringa.

turbinellus.

turrica.

utriculus.

variegala.

vejpertilio.

vexillum.

virgo.

volva.

vulpecula.

\section{BUCCINUM.}

abbreviatum.

acicula.

aciculatum.

acus.

affine.

alaitum.

anglicum.

arcularia.

- sminos.

areola.

armillarum.

bizoar.

bilabre.

caudaium.

-alarracta. shalyteatum.

cical scojums

cilialum.

cintreum.

cloinraium.

cummaculatum.

cornulum.

coronalum.

cofalun.

cisnulutum.

dicusfitum.

digitcllus.

dimidiatum.

dolium

duplicatum.

cchinophorum:

edentulum.

erincoceum.

exile.

fafciolatum.

fimoria.

fermmeum.

Auviatile.

foliorum.

fornicatum.

galea.

gibbofulum.

gibbum.

Slaberrimum.

glabratum.

glaciale.

glans.

glaucum. 
hamafloma.

harpa.

haftatum.

hauftoriun.

hecticum.

indicum.

lave.

lavigatum.

lavisfimum.

lanceatum.

Raplllus.

maculatum.

maculofum.

mollianum.

monile.

muricinum.

murinum.

mulabile.

nanum.

neriteum.

nitidulum.

niveum.

nodulofurn.

obliqvum.

oblongum.

ocellatum.

ochrolevicums.

olearium.

orbita

pap llofum.

patulun.

pennatum.

perdix. perficum.

phallur.

plicaislum.

plicatum.

plunatum.

pomum.

porcatum.

prorofum.

pugio.

pullus.

punciulatum.

pyramidale.

recurvirofrum.

reticulatum.

Rudolphi.

rufum.

ruficum.

feala.

Jenegalicum.

fpiratum.

ftolatum.

Sirigaturs.

frigilatum.

Arigofum.

fubalatum.

fuccinctum.

tesfellatum.

teliculus.

textum.

:ranquebaricum.

trifafciatum.

tubsrofum.

umbilicatum. 
urdatum.

undojum.

undulatum.

verrucofum.

vo ficior.

vibex.

vinculatum.

vilialum.

vulgatum.

\section{STROMBUS.}

aculeatus.

alatus.

ater.

auris Afuri.

- Dianta.

auritus.

camelus.

canarium.

$$
\text { - floseulos. }
$$

chiragra. clavicula. coftarus. dendalus. epidermis.

fafciatus.

fisfurclla.

fujus.

gallus. gibberulus.

- - imperfectus.

Gigas.

Taciniatus.

Lambis.

latisfimus.

lentiginofus.

Lucifer.

Luhuanus.

$$
\text { - - alatus. }
$$

marginuius.

millepeda.

minimus.

onificus.

paluftris.

papilio.

Pelecani.

pugilis.

rana.

jcorpio.

jpinojus.

fuccinctus.

tridentatus.

tuberculatus.

ungula.

ureeus.

vexillum.

vittatus. 


\section{MUSEUM NATURALIUM \\ ACADEMIE UPSALIENSIS}

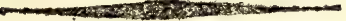

CUJUS

PARTEM XXXI

VENIA EXP. FAC. MED. UPSAL。
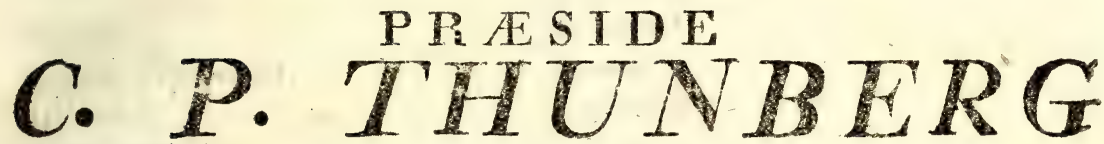

Commendatore Reg. ORd. de Wasa

Med. Doct. Prof. Med at bot. Reg. et Ord. Reg. Coll. Sanit. Memb. Hon. Acad. Cesar. Petrofol. ez Nat. Curios. Reg. Scignt. lond. Holm. Agric. Med. et Evang. Socret, Oecon. upsal. Patr. Hoim. et Crv,

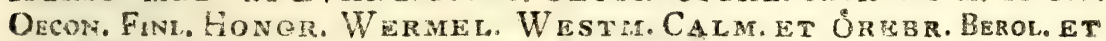
Lips. Nat. Scrut. Paris. Hafi. Mosccu. et Halens. Higt Nat. Gorenk. Puilad. Lund, Hariem. Amsteld. Oecon.et Inst. Litt. Zeland. Np. DRosiens. TURICEAS IENRN. Linn. LONDIN. ET HORTICUTUR. Reg. Scient. Ehys. et Peytograpu. Gótringens. Scten'r. Gothob. Munach, Erdang. Wetter. Nanciens. Marpurg. Meb. Paris. Emul. Monspele Mer Matritens, Med, et Nat. Studios, Emimburg. et Petrop. Medico Chir.et pharmac. Membro nec non Acan. Scient. Paris. et ingtirut. Nation. Monspeinens. Agricuit. Paris. Medic. Lonnin. Scandinave Feq.

rentin. ex Batavin. Ind, Ok. Correspond.

\section{P. P. \\ NICOL. JOH. LJUNGBERG}

STIP. REG. SMOLANDUS

IN AUDIT. BOTANICO D. XXIX NOVEMBR. MDCCCXX H. A, M. S.

\section{U P S A L I FE}

EXCUDEBANT REGIAE ACADEMIA TYPOGRAREY 


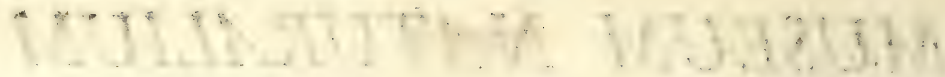
ERKMHIA?T3 GHUMIAOA

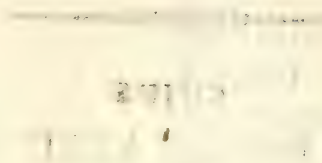

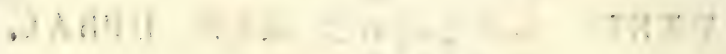

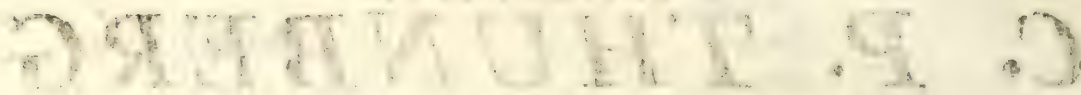

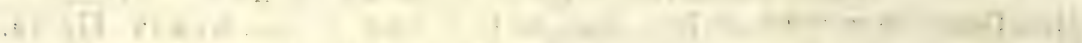

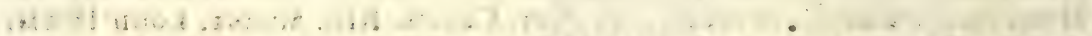

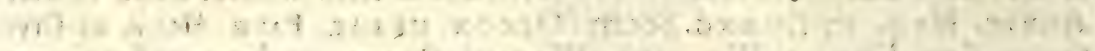
a.

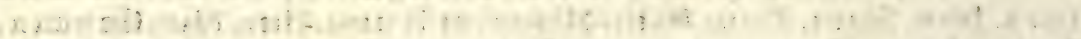

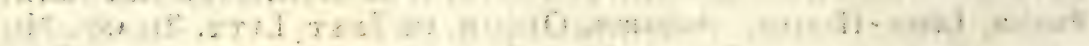

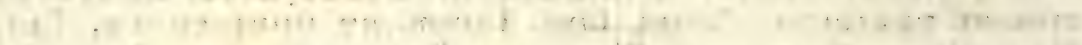

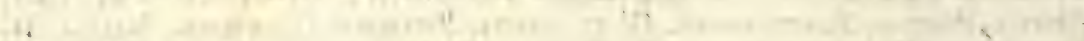

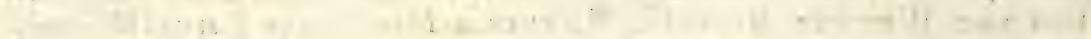

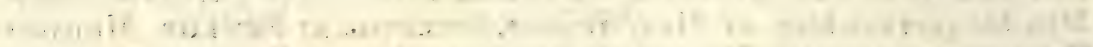

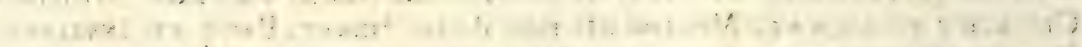

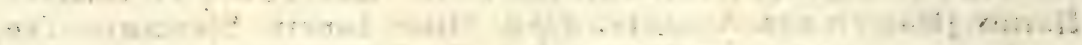

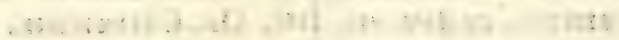

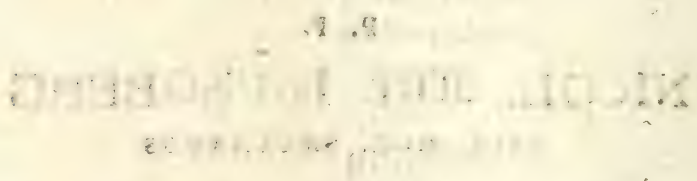

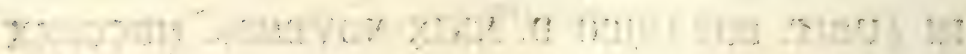

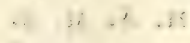

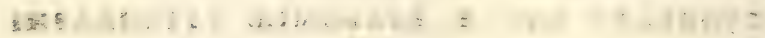




\section{DONATION. REG. CAROLI XIII:}

\section{Continu at. 1 .}

\section{MUREX.}

affinis.

alatus.

aluco.

anjatus.

antiquus.

anus.

argus.

argus fafciatus.

aţraius.

babylonius.

babylonius fpurius:

brandaris.

bufonius.

bulbus.

canaliculatus.

candidus.

carica.

caudatus.

cingulatus.

clathratus.

cochlidium.

columbarius.

colus.

conjul.

cornelis.

corviuius.

coronatus.

crufiula.

cruftula affinis.

cuiaceus. despectus.

diaphanus.

dolarium.

ericeus.

crinaceus.

femoralis. -

feneftratus.

foliatus.

fucus.

fufcatus.

galea.

gibbofus.

granulatus.

granum.

gyrinus.

gyrinus notabilis.

harpa.

hauflellum.

hexagonus.

hippocaft anwm.

hyftrix.

javanus.

islandicus.

lampas.

lanceus.

lingua.

lolorium.

maculosus.

mancinella.

maroccenfis.

melongena. 
monachus.'

morio.

morum.

marum parvum.

nassa.

neritoides.

nodulofus.

nodus.

pardalis.

perverfus.

pilearis.

plicatulus.

plicatus.

polyzonus.

porsum.

purpera.

pyrum.

pyrum nodofum.

qvinquangularis. radula.

ramojus.

rana.

rapa.

reticularis.

ricinus.

rofarium.

rubecula.

rugofus.

facellum.

faxailitis.

foorpio.

foripius.

frobilator jentícofus.

finenfis.

fordidus.

fpirillus.

friatus.

Julcatus.

fyracufanus.

terebellum.

ternatanus.

trapezium.

tribulus.

triqueter.

tritonis.

trunculus.

tulipa.

undarus.

verficolor.

vespertilio.

vertagus.

- fajciatus.

\section{IROCHUS.}

agyptius.

alvearis.

apiarius.

aigyroftomus.

calaius.

calcar.

canaliculatus.

capensis.

carnezs.

cinerarius.

cingulatus. 
eitrinus.

conulus.

- - iris.

- tranquebaricus.

conus.

cookii.

corallinus.

coronatus.

confperfus.

coflatus.

croceus.

cruciatus.

deprefsus.

diaphanus.

divaricatus.

dolabralus.

eryihroleucos.

feneftratus.

foveolatus.

fufcatus.

globulus.

grifeus.

guineenfis.

rybridus.

imbricatus.

inaqualis.

inermis.

iris.

jujubinus.

labio.

bovigatus.

maculatus.

magus. mauritianus.

modulus.

niloticus.

nodulojus.

nodulus.

obliquatus.

pantherinus.

perverfus.

petholatus.

pharaonis.

planus.

pumilio.

punctatus.

py amidialis.

pyramis.

radiatus.

resius.

rojeus.

fcaber.

Schrơterí.

folaris.

- orientalis."

spengleri.

fpinojus.

fellaius.

framineus.

friatellus.

friatus.

frigojus.

tctum.

telefcopium.

tentorium.

tefsellatus. 
tuber.

iuberculatus,

umbilicaris.

uva.

varius.

vernus.

verrucofus: veftiarius. virgatus. virgineus. viridulus. vittatus. zizyphinus.

TURBO.

acttangulus. afer. ambiguus. annulatus. argyroftomus. aurijcalpium. bidens lavis. - friatus. calcar. cafianeus. chryfojtomes. cidails. cimex. clathrus. cochlus. confervalis. cornutus. delphinus. dentatus.

dubius. duplicatus. exfoletus. imbricatus. imperialis. labio. lacteus. ligatus. lincina. littoreus. margaritaceus. marginellus. marmoratus. mespilum. muricatus. mufcorum. neritoides. nicobaricus. nodulofus. objoletus. obrujatus. pagodus. perverfus. petholatus. pies. pollus. quinquedentaiss. reflexus. seplicatus. rugolus. jansuineus. formaisus. 
fcalaris. fetofus.

Sparverius.

jpinofus.

ftellaris.

fellatus.

Ariatulus.

tectum.

terebellum.

terebra.

ungulinus.

นขa.

variegatus.

viridis.

HELIX.

adjperfa.

agophithalma.

alira.

amarula.

ampullacea.

aperia.

arbuftorum.

aspera.

atra.

auricularia.

cancellata.

cai acolla.

carthufiana.

caflainea.

cepa.

cicatricoja.

circinata.

citrina. coccinea.

complanata.

compressa.

contorta.

contraria.

cookiana.

cornea.

cornu arietis.

corrugata.

crenulata.

oryfallina.

decollata.

exilis.

extenja.

fragilis.

fruticum.

fujcaia.

glauca.

globulus.

glutinofa.

hamaftomo.

haliotoidea.

hepatica.

hispona.

zispida.

hortenfis.

janthina.

involvulus.

laciea.

lavigata.

lampas.

lapicida.

leucas. 
ligata.

limofa.

lubrica.

lucernea.

lucorum.

lugubris.

maculoja.

marginella.

microfcopia.

minuta.

nevia.

nemoralis.

neritoidea.

nitens.

nitidula.

nivea.

obvoluta.

octona.

oculus capri.

- communis.

paluftris. pediculus. perfpicua. perversa.

pifona.

planorbis.

planala.

pomatia.

pupa.

puipurea.

putris.

radiata.

fcarabaus
Jepium.

flagralis.

flagnorum.

friatula.

'frigofula.

fubconica.

jubcylenditica.

tentaculata.

umbilicalis.

undata.

indulata.

vermiculata.

vitiata.

vivipara.

volvulus.

vortex.

zonaria.

aculeata.

NERITA.

ala papilionis major.

albicilla.

albumen.

antillarum.

arachnoidea.

ajcenfionis.

airaia.

bidens.

bifalciata.

concellata.

canrena.

chameleon.

cornea. 
cruentata.

dubia.

exuvia.

flammea.

fluviatilis.

fulgurans.

fulminea.

glaucina.

grofsa.

hiftrio.

- frriata.

lacufris.

larva.

littoralis.

magdalrence.

mammilla.

maroccenfis.

maxima.

melaftoma.

mulievis.

multorum punitorum.

orientalis.

papilla.

porimentum.

pellis tigrina.

peloronta.

penmata.

pes Elephantis.

pica.

piperina.

plicata.

polita.

pulligera. pupa.

rufa.

rugofa.

ruma Lupi.

Spadicea.

fercus mufcarum.

tesfellata.

textilis.

turrita.

undata.

undula! $a$.

veneris citrina.

- fufcata.

verficolor.

virginea.

viridis.

vitellus.

vittata.

zebra.

- fuviatilis.

a.finina.

HALIOTIS.

biffriaia.

gigantea.

slabra.

guineenfis.

Iris ?

malmorala.

mide.

ovina.

parisa.

plicasa. 
rugofoplicata.

friala.

tuberculata.

varia.

vifginea.

aculeata.

PATELIA.

anguloja.

aniquata.

avellana.

barbadenfis.

barbara.

caffra.

cancellata.

capenfis.

chloroficta.

citrina.

cochlea.

carulea.

compres $\int a$.

contaminata.

crenata.

crepidula.

cucullata.

cypria.

deauraa.

depresfa.

equeftris.

forruginea.

fissura.

fornicata.

graca.

granatina. granularis.

grifea.

hungarica.

jamaicenfis.

leucopleure.

lufíanica.

luca.

mamnillaris.

melanogramma.

melanoleuca.

militaris.

mitrula.

monopis.

mytilina.

neriloidea.

nimbofa.

noachina.

notata.

nuberula.

- ctoradiata.

pectunculus."

pellucida.

perfoliate.

perverja.

plicaria.

porcellana.

porphyrzonias.

punctulaia.

puftula.

repanda.

reticulata.

rojea.

ruftica. 
Jaccharina. Janguinolenta. foutellum. fincnfis. furinamenfis. tectums. tenuis. teftudinalis. teffudinaria. tramojerica. tricarinata. trigona.

tuberculat $\mathrm{a}$. umbella. umbellata. unguis. virgata.

\section{DENTALIUM,}

aprinum. dentalis. entalis.

fafciatum. ftriatum.

\section{SERPULA.}

afra. anguina. arenaria. clibanus. decusfata. echinata. filograna. glomerata. gorenfis. granulata. lumbricoides. penis. protenfa. jpirillum. jpirorbis. triquetra.

\section{TEREDO.} navalis.

\section{Umbiliti Veneris.}




\section{2 \\ TH E SES.}

I.

Px methodo recentiornm Zoologorum anatomico phyfologica, ubi Teltacea in Ordinem Syftematicum funt redigenda, non parum exinde oritur impedimenti, quod multa ejus animalia generis, qualia utpote adhuc viventia fint, plane funt ignota.

\section{II.}

Caufa forfan gravior, cur morbi Incitationis \& nummerofiores \& implicatistimi facti fint, in mollitie generis noftri progredientis ad morum urbanitatem viræque elegantiam, quærenda eft; qua re efficitur, ut Methodus morbos curandi, multis antea ignotis impediatur difficultatibus.

III.

Perplures morbi chronici a Dizeta perverfa, \& non miuus ab incitamentorum necesfariorum penuria, quam a nimia eorundem copia, originem ducunt.

\section{IV.}

Non e phrenomenis foluen, fed e porentiis etiam nocentibus, vers prodit morborum Diagnoûs.

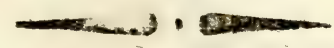




\section{MUSEUM NATURALIUM \\ ACADEMIE UPSALIENSIS}

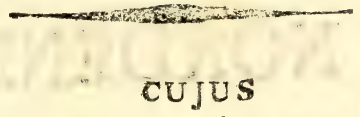

PARTEM XXXII

VENIA EXP. FAC, MED. UPSAL.

\section{PRASIDE}
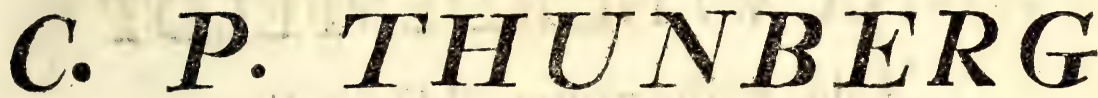

Commendatore Rrou, Ord. de WASA

Med. Doct, Prof. Med. et Bot. Reg. et Oad. Reg. Coll. Sanit. Memb. Hon. Acad. Ceesar. Petrofne, ex Nat, Curios. Reg. Scient. lond. Holm. Agric. Med. et Evang. Societ. Oecon. upsal. Patr. Holm. et Civ. Oecon. Finl. Honor. Wermel. Westm. Calm. ét órebr Berole et Lips. Nat. Scrut. Paris. Hafn. Moscou. et Halens. Hist. Nat Gorenk. Philad, Lund. Harlem. Amstel d. Oecon. et Inst. Lit t. Zeland. Nidrosigns turicens. Ifnens. Linn. Londin. et horticultur. Reg. Scient. Phys. et Phytograpl, Góttingens. Scien'r. Gothob. Munach. Ertang. Wetter. Nanciens. Marpurg. Med. Paris. Emol. Monspel. Med. Matritens. Med. et Nat. Studios, Enimburg. ex Petrop. Medico Chir. et pharmac. Membro nec non Acat. Scient. Paris. et Institut. Nation. Monspeliens. Agricut.t. Paris. Medic. Londin. Scandinav. Flo. kentin. et Batavin. Ind, Or. Correstond.

P. P.

\section{ADOLPHUS FREDERICUS ALTAHR \\ VERMEL.}

IN AUDIT. BOTANICO D. VI DECEMB. MDCCCXX:

H. A. M. S.

\section{U P S A L I AE}

IXCUDEBANT REGIAE ACADEMIE TYPOGRAPHI 


\title{
KONUNGENS
}

TROTJENARE, LO̊JTNANTEN

WÁLBORNE HERR

\section{SOH. N. NORDENFELDT}

\author{
SAMT \\ HO̊GWÅLBORNA FRU \\ ANNA W. NORDENEELDT \\ fódd Friherrinna P OSSE
}

tacksamt och vördnadsfullk

tillägnadt 


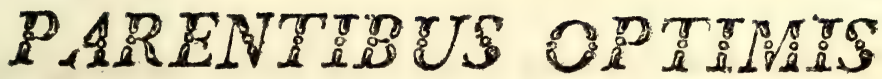

Pietas Filii 


\section{THESES.}

I.

Ut sublime est naturam disquirere, ita, prater animalium, di. gnissma plane disquisitio platarua; quipe que aninalibus proxime cohxrent.

II.

Cam autem dux exstant hujus disquisitionis potiones quarmm altera circa formam versatur, altera vero interiora perscrutatu"; necesse est, ut, quamvis se invicem desiderant, hæc ad scientificam regri vegetabilis cognitionem majoris sit twomenti.

\section{III.}

Et licet illa vitæ communi præbeat utilitatem, minorem tamen oblectationem animo offert.

IV.

Ambas vero has rationes si conjunctim sequimur, non ntilitatem modo invenimus, sed, quod majus est, facilem ad naturæ arcana introitum.

V.

Qui praterea plantas animalibus discernere studet, interioribus consultis, melius progrecitur. 


\section{DONATION. REG. CAROLI XIII. \\ Continuat. II.}

\section{CHITON}

aculeatus.

cimex.

cinereus.

gigas.

magellanicus.

marmoralus.

marmoreus.

piceus.

ruber.

squamosus.

undatus. fpinosa.

fitromie.

tefudinaria.

in inablum.

getruca:

\section{DEOLAS}

cantidus.

cripgenas.

ovieristis.

puflicis.

MYA

LEPAS.

anatifers.

anguftata.

anscrifera.

balanojdes.

balanus.

caretia.

cygnéa.

Diadema.

piongata.

fisfia.

foliacea.

minor.

mitella.

patula.

plicata.

pollicipes,

porosa.

purpurea.
SOLEN.

aretica.

arenaria.

corruga!a.

gibba.

margarilifera.

maroccana.

nicobarica.

nodofa.

oblanga.

pictorum.

truncata.

analinus.

bullatus.

cultellus.

diphos.

ensis.

linearis. 
radiatus.

filiqua.

ftrigilatus.

tellinatus.

vagina.

vespertinus.

virens.

TELLINA.

Adansonit. albicans. amnica. angulata. anguftata. balauftina. balthica. bimaculata. Bornit. brafiliana. calcarea. candida. carnea. coccines. cornea. cruciata. oryfallina. depresja. digitaria. divaricats. donacina. faba. fasciata. fervenfis. flavescens. Aluminalis.
Auminea. fiuviatilis. foliaces. fragilis. gargadio. gari. hyalind. incequalis. incarnat a. inflata. lactica. lacuftris. lata. lingus. muricata. oblonga. opalina. operculata. palliderofeco. pellucida. piliformis. planata. punicea. pufilla. radiata. remies. reticulalas. rofirata. rufefens. rusola. jabulosa. fcobinata. Spengieri. friciula. 
triangrta is.

trifalcinia.

trilaiera.

truniala.

variegeta.

violaica?

virgeta.

virginica.

virvea.

Zonala.

CARDIUM

aculeatum.

albidum.

cancellatum.

cardisfa.

ciliare.

ciliatum.

coflatum.

crasfum.

danaciforme.

echinatum.

edule.

flovum.

flexuofum.

fragum.

glaucum.

groenlandicum.

hemica dium.

ifocardia.

lovigatum.

latum.

levcofornum.

lithocardium.

magnum.

medium. muricatum.

oblongum.

papyraceum.

peotinatum.

pect niforme.

politum.

retufum.

ringens.

rofeum.

ruficum.

ferraium.

friatum.

tuberculatum.

unedo.

MACTRA.

corallina.

cuneata.

cjgnea.

glabrata.

slauca.

lutraria.

maculata.

papyracea.

piperita.

plicaria.

rugofa.

folida.

Spengleri.

friatula.

fultorum:

targida.

violacea.

vitrea.

DONAX.

argentea. 
bicolor.

cunea! a.

denticulata.

faba.

Irass.

pubefcens.

punctaid.

radiala.

rugoja.

fcoitum.

foripta.

ferro.

epinofa.

framined.

Ariata.

trunculus.

VENUS.

affinis.

efra.

albicans.

anus.

borealis.

brunnea.

campefcienfs.

cancellata.

candida.

caf $\sigma_{\text {. }}$

caftren/is.

chione.

concentrica.

contemta.

contraria.

corbicula.

corrugata.

crenate. cruentata.

decusjata.

defiorata.

Dione.

divaricata.

dura.

d)fera.

edeniula.

erycina.

exoleta.

fafciaio.

fimbriaia.

flammia.

flexuofa:

gallina.

gallus.

geographica.

globofa.

guineenfis.

hermaphrodita.

hisfrio.

jamajcenfiso.

japonic a.

incruftata.

islandica.

juvenilis.

Lat

Iameltofa.

lata.

lineata.

litterata.

- radiata.

Iufitanica.

luforia chinenfis.

- lutescens. 
maculata.

marica.

ma! adoa.

mercenaria.

meretrix.

meroë.

nebulofa.

nocturna.

opima.

orientalis.

paphia.

paupeicula.

pectinata.

penfyluanica.

petulca.

phryne.

proftrata.

puerpera.

punitata.

punetulata.

purpurafens.

radiata.

reticulata.

rotundala.

rucra.

rugofa.

feripta.

fenegalenfis.

finenfis.

fpuria.

fquamosa.

ftellara.

textilis.

tigrina.

tripla. tumens.

undulata.

variegaia.

verrucoja.

virens.

virginea

an! iquatus.

SPONDYI, US.

citreus.

croceus.

ducalis.

folivin brasfica.

- pitroselini.

Gaderopus.

maculatus.

nicoba: icus.

pictorum.

plicatus.

variegalus.

antrquata.

CHAMA.

bicornis.

calyculata.

citrea.

cantaminata.

car.

foliacea.

gigas.

gryphojdes.

hippopus.

Lazarus.

maltkiana.

nicobarica.

nux maris.

oblonga. 
Satiata.

thaca.

trapezia.

A RCA.

cquilatera.

afra.

amygdalina.

antiquaia.

barbata.

bicolorata.

campechienfis.

cancellata.

cendida. decusfata. fosflis. glycymeris. granofa.

indica.

lactia.

lota.

magellanica.

marmorata.

mitrata.

modiolus.

multifriata.

Noa.

nucleus.

nummaria.

ovala.

pectinata.

pectureulus.

pilofa.

rhomboidalis.

rhomboidea.

roftrata. fenilis.

torluoje.

undaia.

OSTREA.

aurafiana.

cornucopice.

cri?aia.

croces.

cucullata.

decemradiata.

deniticulata.

diluviana.

dubia.

edulis.

elcgans.

ephippiums.

fajciaia.

flammea.

folium.

fornicata.

forjkolei.

fragilis.

gibba.

slabra.

glacialis.

guttata.

hiftrionica.

hybrida.

jacobaa.

japonica.

islandica.

ifogonum.

lima.

Iutea.

malleus. 
fenegalenfis. maxima. media. minuia. munelina. nodofa. nodulosa. obliteraia. ochrolesca. opercularis. pallium. parafitica. pellucens. perna. pleuronecies. plica. plicatula. porphyrea. punctata. pufilla. pufio. radiata. radula. retufa. Sanguinea. Semiaurita. feñatoria. jenegalenfis. finenfis. finuofa. folaris Spondilvidea. Squamofa. Aellata. fubrufa.

\section{tenera.}

tenuis.

tranquebarica.

trirodiata.

turgida.

varia.

verficolor.

violacia.

vulfella.

ziczac.

PLACENTA.

ephippium.

\section{ANOMIA.}

aurita.

biloba.

cepa.

craniolaris.

cranium.

crifpa.

detruncata.

dorfata.

electrica.

ephippizim.

gryphus.

hyfterita?

muricata.

peftinata.

placenta.

plicaiella.

reticularis.

fanguinolenta.

ferpentis.

fquama.

fquamula.

friatuls. 
liihophagus.

tercbratula.

truncata.

undulala.

vitiea,

MYTILUS.

afer.

affinis.

albus.

anatinus.

argenteus.

auris.

auf?ralis.

avicula.

barbatus.

bidens.

bilocularis.

cinnamomeus.

coralliphagus.

crifla.

cygnour.

dijcors.

edulis.

fajciatus.

frons.

fufcus.

hirundo.

hyotis.

laius.

rugojus. margaritiferus.

modiolus.

murinus.

nicobaricus.

niger.

perna,

radialus.

ruber.

fmarasdulus.

fognalis.

fitialuius.

ungulatus.

verficolor.

vi, idis.

vulgaris.

Zellenfis.

aculeata.

PINNA.

bicolor.

nigrofumigata.

nobilis.

papyracea.

rotundata.

rudis.

fquamofa.

vexillum.

vitrea. 


\section{MUSEUM NATURALIUM ACADEMIF: UPSALIENSIS}

CUjus

PARTEM XXXII, ULTIMAM

VENIA EXP. FAC. MED. UPSAL。

PRASIDE
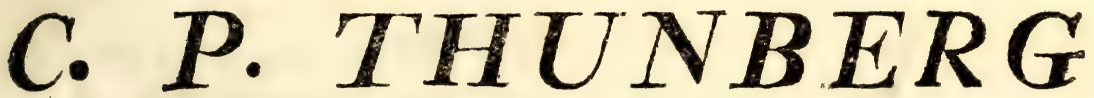

Commendatore Reg. Ord. de Wasa

Med. Doct. Prof. Med. et Bot. Reg. ex Ord Reg Colz. Sanit. Memb. Hon. Acad. Casar. Petropnl. ej Nat. Culzos. Reg. Scient, lond. Holm. Agric. Med. et Evang. Soctet. Oecon. upsal. Patr. Holm. et Cit. Oecon. Fint. Honor. Wermel. Westm. Cazm. et Ortebr. Berol et tips. Nat. Scrut. Paris. Hafn. Moscov. et Malens. Hist. Nat, Goren k. Plilad, lunt. Harlem. Amsteld. Oecon. ex Inst. Litt. Zeland. NiYRTSIENS. TURICENS. IRNENS. LINN. LONDIN. ET hORTICULTUR. REg. Scient. Phys. et Phytograply. Gótringens. Scientr. Gothob. Munach. Erlang. Wetter. Nanciens. Marpurg. Med. Paris. Emul. Mongper. Med. Matritens. Med. et Nat. Studios. Enimburf. et Petrop. Medico Chir.et pharmac. Membro nec non Acab. Scient. Paris. ex Institut. Nation. Monspeliens. Agricuit. Paris. Medic. Londin. Scandinav. Flo. rentin. et Batayin, INd, OR. CuRrestond.

\section{F. P. \\ CANUTUS LUDOVICUS ALTAHR VERMEL。}

IN AUDIT, BOTANICO D. XXIII MAIJ MDCCCXXI? I. P. M. $s$.

\section{U P S A L I IE}

EXCODEBANT REGIA ACADXMIA TYPOCRAPAS 

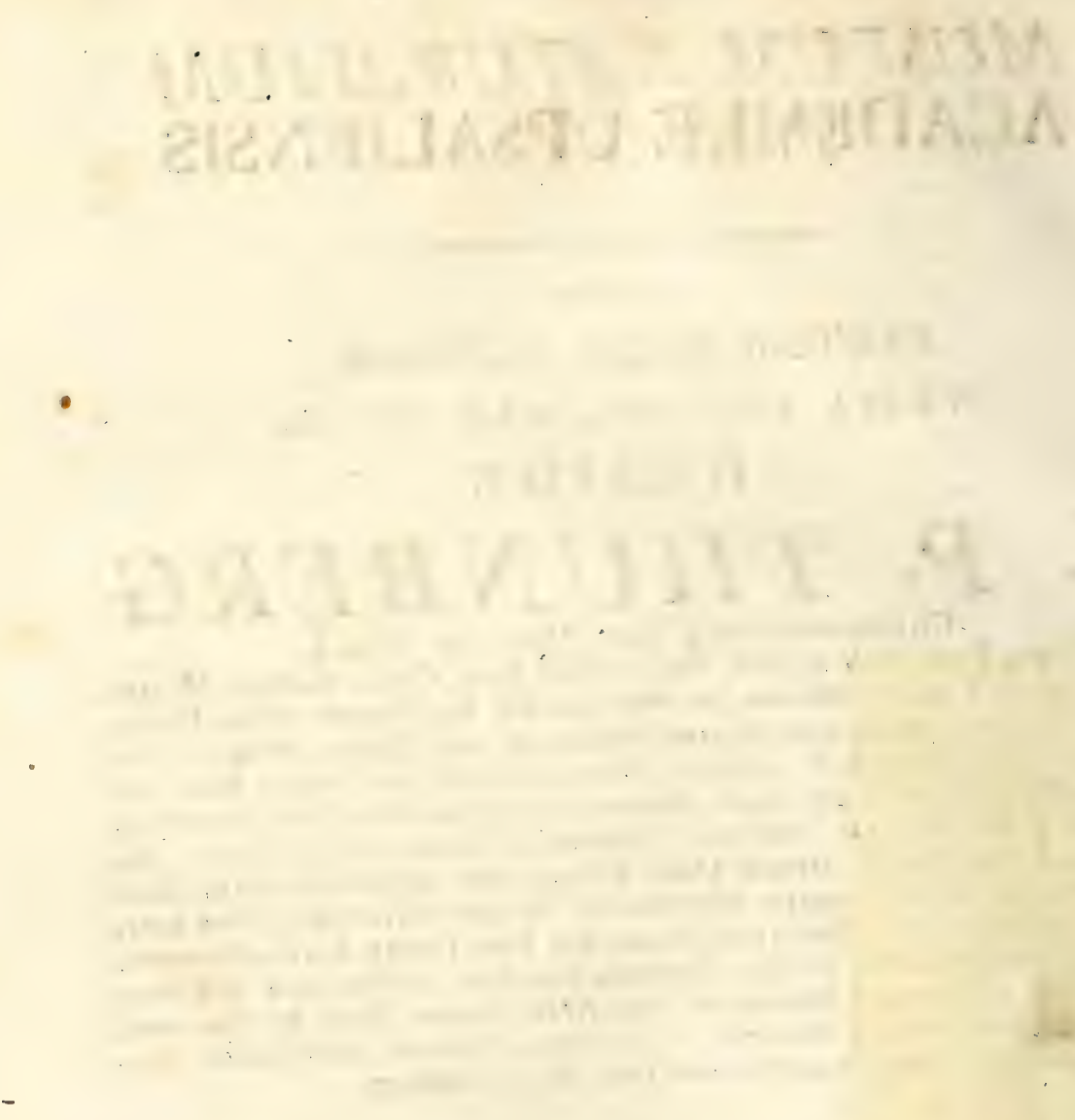
monstevr CHR. REINH. GeIJER atditevt

ET A MADAR: E

MADAME A. E. GFIJEP.

née LENNARTSSON

Mansieur et Madame;

En décernant un hommage public aux vertus que j'admire en vous depuis que j'ai l'houncur d'etre associé á l'édncation de vos enfans, je ne puis exprimer qu'imparfaitement les divers sentimens dont mon àme est agitée. J'aimerois sne tont á célébrer votre indulgence á l'ombre de la quelle mes traraux ont fructifié, votre bienveillance qui n'a cessé de les embellir, et sur tout l'amitiè dont vous m'avez honoré. Mais l'exprešsion de Ia vraie reconnoissance et-celle du respect sont tout ce que je: puis vous offrir; veuillez donc la recevoir ainsi que l'assurane atu devouement avec le quel je suis:

Monsieuret Madame

Yotre trés humble servitear.

C. L. ALTHAR. 


\title{
De Huldaste Fo̊råldrar
}

helgadt

\author{
af \\ En lydig Son \\ KNUT LUDVIG.
}




\section{DONATION. REG CAROLI XIII. CONTINUAT. III. TESTACEA MAJORA.}

Argon Argo: Muricata

- Nautil. pompilius.

- Buccin. Cornutum. dolium echinophorum galea. glancum. olearium. plicatum. rufum.

Voluta colocynthis. Turbo olearius. Serpula lumbricalis Lepas tintinabulum.
Strorib. Chiragra. gallus gigas lambis. latissimus lucifer. Murex defpectus. fensoratus. lampas. nodofus. tritonis.

Chama hippopus. Pinna rotundata.

\section{APPENDIX.}

Braunrolh Achatir. Braun Schildkroote.

Brett - Spiel.

Daum - brett.

Fiffiner brett.

Geflechte kattje.

Geftreifte Bandthute.

Kleine Achatin.

Mennoniten thute.

Arcularia papillosa.

Gelb - Mund.

Glatte Soumechew.

Glatte Schraub.

Nasfa oblonga.
Schildkróten thute.

Svartzbraune thute:

Róthe majerer.

Cornicula Schrooten.

Geflamte Gurke.

Langewunden Voluta:

Svartzbraune Voluta.

Ungevundene Voluta:

Violetbunte Voluta,

Stachliche króter.

Sturm Haube.

Umgevundene Mus.

Mond/chnecke.

Silbermond. 
Geflechte krabbe.

Gehownter Fechter..

Geripte kampfhan.

Gefreifte querband.

Marmo irte kampfhan.

Marmorirte kr.

Sommer fprosfen.

Sonnenftral kr.

Band fpindel.

Baftard Betz:
Tromelstock.

Die Linürte.

Die gevơhlte.

Grosfe mecrehr.

Daumen nagel.

$W_{\text {Eisfe Stein. }}$

Hund-term.

Die Dïnne.

Elelhuf.

Amplisfima hæc et preticfisfina Conchyliorum collectio, a beatisfina nemoria Rege CAROLO XIII, anno 1815 , numerata pecunize fumma, octo millibus Imperialium emta et Mulaeo hujus Academia gratiofisfine donata fuit. Hæc munific ntia glo:iofisfimæ memoriæ Regis mirifice et fummopere ad ornanientum Mufai hu. jus Univerfitatis et utilitaten Patriz; ac eruditionent $j$ ? ventutis Academica inde redundantem, contribuit. Ubique enim, ubi litteræ exhortantur et florent, ibi femper funt refpublica feliciores, fcientiæ augentur, artes perficiuntur, et omne felicitatis genus multiplicatur.

Sed neque hac felfcitas unica eft, qua huic alma Litterarum Natri feliciter contigit. Placuit quogue Re. ginæ, fanctisfinæ apud nos memoriæ, HEDVIGE ELI. SABETE CHARLOTTE gratiofisfime donare rarisfimorum non minus, quam pulcherrimorum Papilionum Collectionem, e Brafilia sibc allatorum.

Er ferenisfimo quoque Principi Regio, Svecia et Norvegixe Haredirario, Domino JOSEPHO FRANSI: SCO OSCARI, Academiæe Cancellario clementisfino ar- 
rifit infigni Coleoptratorum Brafilienfium numero divitias Mufæi Academici benignisfime adaugere, dono aque pretiofisfimo ac ipeciofisfimo.

Gratisfima mente dum hæc vere Regia dona læti, quin immo latisfimi accipimus, optamus vehementer, iterumque optamus, ut semper fint, ficuti vere merentur, magni xeftimata, optime confervata, ac utilia reddita; nec paucis vel nemini accesfa, unquam oblivionis temebris tradita, vel incuriofe neglecta et deperdita.

\section{DONATION. REGINE}

\section{HEDVIG E ELISABET CHARLOTT}

$\begin{array}{cc}\text { Papilio Achilles. p. } & \text { Papilio Laërtes. p. s. } \\ \text { Alcyonia. p. } & \text { Niveus. } p . \\ \text { Casjia. p. } & \text { Orion. p. } \\ \text { Clymenus. p. s. } & \text { Fhyllus. p. } \\ \text { Clytemneftra. s. } & \text { Polydamas. p. } \\ \text { Dancë. p. . } & \text { Rhetenor. p.s. } \\ \text { Dido. J. } & \text { Vanilla. p. } \\ \text { Iphictus. s. } & \end{array}$

I3 DONAT. PRINCIP. REG. JOSEPHI FRANCISCI OSCAR.

Cordyle palmarum. phoenicis.

Rynchemus cupratus. irroratus. pardalis.

Curculio Achatinus. imperialis. a. $\beta$.

Brentus longimanus.
Rutela amazona. confufa. emarginata. fucata. glauca. ver icolor.

Geotrupes aloeas. t. Ateuchus smaragdulus. $\propto$. $\beta$. 
Melolonitha anea. marginata.

Haltica 8-puftulata.

Bupreftis gigantea.

Elater noctilucus.

phosphoreus, $\alpha . \beta$.

Ariatus. $\alpha . \beta$.

Alurnus marginatus.

Saperda 4-maculata.

Cerambyx feftivus a. $\beta$.

Prionus Atigma.

achatinus?
Pasfalus interruptus. a. B. Casfida conea.

б-puftulata.

futuralis.

Cathederes fuccinctus. $\alpha$. $\beta$.

Cicindela albella.

Eumolpus teftacens.

Helea violacea.

Acheta gryllotalpa.

Centrotus marginatus.

Tettigonia tibicers.

Cpinofus.
tibicen
armata.

Xylocopa morio.

Eristalis cyanea.

Præter illa, quæ nondum examinata sunt.

\section{APPENDIX AD MUSEUM ACADEM.}

Ursus arctos.

Equus afmes.

Cervus tarandus: $\delta$. $\beta$. albus

alces. \& donata

ab illufter. et generof. Comit e

D. Brahe.

Felis vulpinus.

Canis vulpes: crucig. $\beta$.

Phoca variegata: foetus.

Ungula Capree arniata.

Cutis rhinocerotis.
Crania Delphini.

Cameli.

Caballi

Elaphi.

Martis.

Vulpis.

Gulonis. $\alpha . \beta$.

Babyrusia.

Melis.

Arctos.

Lutre. 


\section{APPENDIX AD MUSEUM VESTINIANUM.}

Sciurus Carolinenfis.

Sorex braflienfis.

Coluber leminifatus. $\delta$.

Faleo bidentatus.

magniroftris.

pectcralis.

Strix Scops t, $\beta$.

Lanius cajanis, $\delta$.

Pfittalus accipitrinus:

$$
\text { aftivus. } \delta \text {. } \varepsilon . \xi .
$$$$
\text { Tui. }
$$

Ramphaftos dicolorus. $\gamma$

$$
\text { pavoninus }
$$

Trogon curucui. む.

Corvus caijanus.

Alcedo maculata $t$.

Trochilus mango. $\beta$.

fuperiliosus. \&.

Pipra favigafter. $t$.

$$
\begin{aligned}
& \text { pareola t. } t 2 . \\
& \text { virens } . \\
& \text { viridis. } .
\end{aligned}
$$

Cotinga coronata. ठ. む.

$$
\text { flava } \mathbf{6}
$$$$
\text { firiata. } 8 \text {. }
$$

Ampelis pompadora. 5 .

$$
\text { pufilla. }
$$

regalis.

firiata.

viridis. \&,

Molacilla ayanocephala. Mufcicapa barbata.
Picus minutus. む. pasferinus. な.

Todus minutus.

Anas dominica. $\alpha . \beta$.

Ardea maculata.

Platalea ajaja.

Procellaria pusfinus. 3 .

Rhederoftra collaris.

$$
\begin{aligned}
& \text { nigra. } \\
& \text { ochracea. } \\
& \text { ftriata. }
\end{aligned}
$$

Columba passerina.

Penelope marail. $\beta^{\prime}$

Brachyurus gularir. $\delta$. ruber. $\delta$.

Xyris ciliata.

Eriocaulon cespitofum. hirfutum. ramofinn.

Spermacoce tenuior. verticillata. d. elliptica. Arigosa.

Billbergia Specioja.

Loranthus emarginatus. $\beta$.

Bauhinia armata.

Bignonia binata.

elliplica

jafminoides.

Avicennia elliptica.

Acrofticum calomelanos. $\xi$.

Polypodium consinnum. B. 
Rallus caijanenfis.

Scolopax calidris. B.

Melolontha futuralis.

Doryphora reliculata.

Chryfomeia Philadelphica.

Casfida truncata.

Geotrupes motata $\alpha, \beta$.

Alurnus rufus.

Papilio Zacinthus む. $\int$.

Phasma lateralis.

Ponera viliofa.
Scolopendrium officinarum. $\delta$. Blechnum fimplex. Martenfia fpeciofa. $\alpha$. $\beta$.

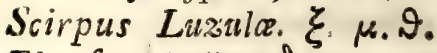
Eleufine indica $\delta$ \%. Baccharis lancea t. 2. tomentofa.

Quibus accedent, quæ huc usque non rite funt explicata vel coguita.

Duæ Collectiones Conchyliorm difparuerunt, adeoque e Catalogo Mulai eliminari debent: Collectio fci. licet Petrejana, cujus in antiguo Horto botanico, a memet derelicto, fpecimina multa fuerunt fpoliata. Qrod reliquum fuit, ad novum Hortum transportatum, in adibus minus confervationi rerum naturalium ab ini tio faventibus, fimul cum collectione Teftaceorum a memet donata, ab humiditate, mucore et fungis, qua magnam partem, læam et perditum evafit. Eorum, quæ hæc fatalis' calamitas non omnino deftruxit, deinde pars quæedam ad Mufeum Academiæ Carolinæ Iundenfis misfa fuit, pars alia pro alio quodam Mulæeo ordinata fuit. 
Ultima hac, fab meo Praefidio, parte col phonem impono Catalogo Mufaei Academia earulu $\mathbf{N}$ ituralium rerum, quæ huc usque examinatæet cognitæ fuerunr, Succesforibus forfan felicioribus cetera, qua nondum rite cognita funt, $x$ tate jam provecta, et tæedio viginti annoruin ingrato confumtus, relinquens.

PRASES. 


\section{THESES.}

I.

F- Scientix naturales, au f fien perent, fi non minus in rebus co jurg ndis, quam in diftingven.. is, ftu. dium et opera poneretur.

II.

Cur vero fcientifico tenor $\mathrm{m}$ inus valeant ha exdem, ratio etiam in perverfitate illa quærenda videtur, qux in natura ftudio non res per $f e$, fed utilitatem tantun, quæ inde ptrcipitur, refpicit.

III.

Omnis etenim utilitass, quæ ex fcientiis hauritur, fi quidem vera eat, fua fponte fe offert, nec ut finis habenda est.

\section{IV.}

Naturæ ftudium in Scholis non tantum non primum esfe, verum etiam ferme neglectum fummo jure queri nobis videmur.

V.

Scientia enim, qua circa res naturales verfatur, ejusdem, si non majoris ad animos juveniles formandos momenti eft, ac alia quævis. 
D. D.

\section{MUSEUM NATURALIUM ACADEMIE UPSALIENSIS.}

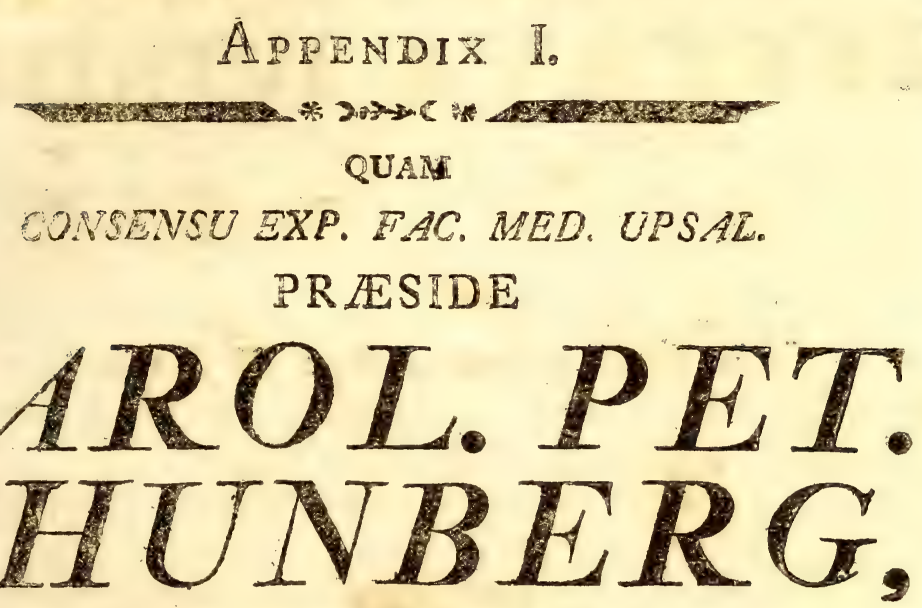

Equite Aurat. Reg. Ord. de Vasa,

Madic. Doct. Profess. Med. ex Botan. Reg. et Ord, acad. Chesar. Nat. Curios, Reg. Scient. Londinens, Holmiens. Societ. Scient. Upsaz. 2atr. Horm. Berot, Nat. Scrut, lundens, Harlem. Amsteid. Zeland. Iidrosiens. Halens, Nat. Scrut. Linn. Londin. Medic. Edimburg. et Nat。 Stujios. Imid. Membro; nee non Acad. Scient. Paris. Monspeliend. Agricult, Paris. Fiorentin. ez Batayin, Ind. Or. Correston.

\section{Publico examini proponit

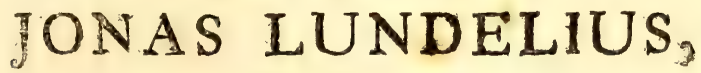

Smolandus.

In Audis. Gust. Maj. Đ. 9. Febr. 179 T.

Horis anite merid. Solitis.

\section{U P S A L I 两,}

Apud Direct. Johann. Edman, Reg. Acad. Typogr. 


\section{MONSIEUR,}

LE COMTE, MARECHAL DE LA COUR,

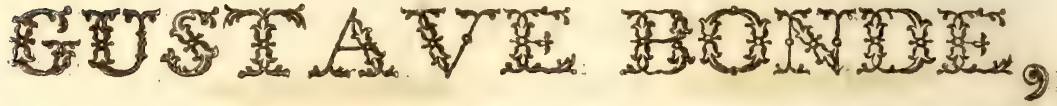

Cés feuilles font dedièes E facrèes.

par Son

tores: humble bु tres obeisfant

ferviteur

Joras LUNDELIUS. 


\section{DONATION. THUNBERGIAN\&,}

\section{Append. I.}

\section{wronomann}

Lernur murgos.

Canis lasopus. *).

Viverra fetens. 1). erminea. B. Alavescens. r. fusca.

Cavia porcellus. $\beta$.

$$
\text { capenfis. }
$$

Mus mufculus. B. albus.

$$
\text { \%. variegatus. }
$$

Sciurus vulgaris $\beta$. bibernus.

Bos caffer: captit.

\section{ช. maculatus.}

Cerous tarandus. 옹 오. capreolus. 9 .

Pfittacus galgulus. cbryjopterus. menftruus. leucocepbalus. eritbacus.

Oriolus cbryjocepbalus. Upupa epops. Plotus abinga.
Certbia armillata. trochilea.

Pbaëton etbereus. Anas jpectabilis. A. Mergus albellus. ‥ 2)。 Alca aretica. ferrator. ?. alle.

Tringa pugnax. 3. var.

Pbaliamus gallus: $\eta_{\text {c crispus. }}$ pictus. colcbicbus.

Numida meleagris. ㅇ.

Coluinba oenas.

Ej. caput: roftris duobus. 3). bantamersis.

Crase alector. Turdus trioftegus. Loxia prafina. 疋. javen/is. 全. ferruginofa. A. ?. Emberiza miliaria. Fringilla petrowia.

R 2

*) Confift. Acad. Prot. I788. Mart.

I) Muftela putorius L.

2) Mergus minutus, Lin.

3) Monftrofum fpiritu vini fervatur. 
Fringilla domefica. ₹. var. ㅇ. Acrocbordus javanicus: pullus. calebs. var. S. S. Raja torpedo: var. s).

Tanagra viridis. cannabina. ㅇ․ miraletus. 5). cbloritica. Mufcicapa paradifi. Motacilla pbcenicurus. var. alba. 9 .

Tefiudo denticulata. mydas: cor. 4).

Raria temporavino a. B. $x$. Lacerta gecko. var. it. var. miliaris. it to var. dorfalis. agilis: var. it. var. palufsis: var.

it. var. omeive: cor.

Colubier folatus. pullntus: rbombentus. bilateralis: omelanocepbaiuso. beteroclitus. corallinus.

Angris colubsina. maculatis. Ampgisluana abo. aquila. 5). fullonica. 5\% Squalus fafciatus. catulus. 5)。 (quitina. 5). musielus. 5): centristo. 5\%

Centrificus fcolopax. 5). Oftracion gibbofus. Pegafus natans. Murcena belena: vrro selulos as picta. asurusista. fajciata.

Opbicbotbus cinereus anguilla. $5 \%$

Stromaters factola. 5). Uranofcopus foaber. 5). Blennius ocellaris. 5). Scisena mbra. 5). cataplonata. s). Cepola rabefcens. Perca picta: var. P. fluviatilis: $\alpha$ 。

4) Cor biauritum Spiritu vini fervatur.

§) Siccati, gypfo fapius farcti \& vernice obducti. 


\section{$\Rightarrow 1131$}

Gaferofezes ductor. 5).

Gobuzs elotris: var.

$$
\begin{aligned}
& \text { it. var. } \\
& \text { niger. s). }
\end{aligned}
$$

Scorpana porcus. 5).

Zeus faber. 5.).

$$
\text { (crof } a .5 \% \text {. }
$$

Loricaria plecollomus.

Plezronecies tridactyhs. zebra.

linguatula. 5).

Sparus fargus. 5).

$$
\begin{aligned}
& \text { buterta. 5). } \\
& \text { exytbrinus. 5). } \\
& \text { cantbarus. 5). } \\
& \text { folpa. 5): } \\
& \text { dentex. 5). }
\end{aligned}
$$$$
\text { esytbrinus. 5). }
$$

Labrus. bimaculatus.

$$
\text { julis. 5): }
$$

Mulluas barbatus. 5).

Trigia lyra. 5).

Clupea aloja. 5.).

Mugil cepbelus. 5).

Cyprinzus auratus: unr.

brama.

erytbropbtalmuso

idus.

Cyprinus ballerus.

biarbus. 5).

bierkin.

afpius.

carasfius.

Hifter japonicus. unicolor. B. q. aneus.

depresfus.

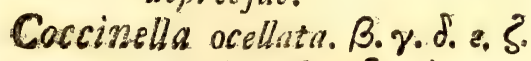

grandis. B. 6).

I I-maculata.

12 - punctata.

3-punetatn. 3 .

1. 3-notata. $\beta$.

22 - punctata.

28-punetatn.

flavicollis. B. $\gamma$ :

pantherina a. $\beta$.

caffra. $\alpha . \beta$.

frontalis. $\alpha . \beta$.

abbreviata.

arcuata.

variabilis. $\alpha . \beta$.

Antbrentis verbafci.

Elopborus pulicarius. 7).

nubilus.

Zucanus piceus. 今. 8).

$\mathrm{R}_{3}$

Scara.

6) Coccinella verficolor. Fabr.

7) Hifter pulicarius Thunb. nov, ast. Upf, Yol, 4o Sphæridium mintitum. Fabr.

3) Lucanus tenebroides. Fabr. 


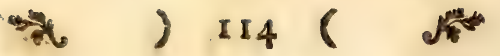

Scarabeus aries.

fyricbtus.

retilus.

piceus.

2-punctatus. a. 1 . oromedon. $\alpha . \beta$.

2-miaculatus.

inquinatus.

arenarius.

porcatus.

teftudinarius."

terreftris.

foeteris.

analis.

fcybalarius.

Copr is midas.

feftiris.

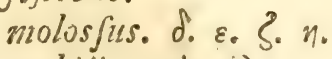

mobilicornis. .

flavipes.

variolojus.

gibbofus.

lemur.

ovitus.

flagellatus.

nutans.

capra. 仓ै. ?.

nemeftrinus.

Melolontba variabilis.
Melolontha birta. 9).

Senipunfiata. 2-punctata. 10).

lanius.

uv/us: $\beta$. vittata.

atomaria. artbritica.

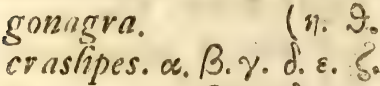
spinipes. $\alpha . \beta_{.} \gamma_{0} \delta$. podngrica. $\alpha$. $\hat{\beta}$. abbreviata. alopex.

fruticoln. of.? pulveralema.

ruficornis.

agvicola? a. $\beta$.

mutabilis?

capicola. $\propto$. $\beta$.

raricola.

villoga.

violacen. I I).

longicornis.

viridis.

cenea.

pallida.

dentipes.

carmelitica.

fullo. ․․ㅅ․ 오.

9) Cetonia hirta. Fabr.

I0) Trichius 2- punctatus? Fabr.

II) Melol. farinofa, Fabr. 


\section{\# ) $115($ )}

Melolontba erytbrocepbils. proboscidea.
verficolor.
cardui.
vittnta.

Tbrox arenarius.

borvidus.

Carculio baccbus.

rbinomacer. I2).

coriarius. 13).

vitzatus.

jota.

ilicis.

cloropus.

atvivofiris.

cbbreviatus.

jaceo:

cynaze.

viminalis.

tremulas.

malve.

tytbri.

4-tuberculatus.

arator.

trifolii?

fraxini.

calcar?

futuralis.

capenfis.

nigrita.

Curculio punctatus.

pruni.

obefus.

frigidus.

aurifer.

cracca.

perlatus.

nitidulus.

planiroftris.

palliatus.

gemmatus.

filiformis.

niger.

barbarus.

corzeus.

ajcanii.

3-guttatus.

allirofteris.

suriatus.

ocellatus.

apterus. $\beta$.

nodolofus. $\beta$.

genninatus.

fisymbrii.

eryfimi.

asfimilis. 14).

globofus.

crifpatus.

emeritus.

roftratus, a. 3 .

Curcus-

12) Rhinomacer attelaboides. Fabr.

13) Rhinomacer curculionoides. Fabr.

If) Brentus. Fabr. 


\section{4) II6 ( )}

Curcuilio retufus. verrucofus.

Attelabus curculionoidss. coryli. $\gamma . \delta$.

Hydropbilus fcarabooides. orbiculavis, 15). minutus.

Spbaridiugn 2-punctatum. eritoma. 16)。 ips $17 \%$ morio. 18). plagiatums.

Byrribus dorficits. (9. 19). Dermefles maculatus, $\delta_{\text {. }} \varepsilon_{0}$ ?. $\eta_{0}$

picipes. lunatus. macellavius. trifafciatus.

Boftricbus tefaceus.

minutus.

jefuita. a. B. 20). eufitatii.
Boftricbus niger. pygineas. micrograpbus. cressazus.

Tritoma vaffrons. Ips bemomboidalis, \&. B. 2. 2. atomaria.

Silpha granulata. reticulatis.

Nitidula anea. 22\% obfcura. 4-guitata.

Bruchus cifti.

rolinic.

crenatus. "). mimofanus.

Hijpa teftacen. Casj3da jamaicenfis. ferruginea. marginella. 2-maculata. 4-maculate.

lavibus.

15) Simillimus Sphæridio fimetario. Differt Elytis

16) Tritoma 2 - puftulata. Fabr.

17) Ips 2 - puftulata. Fabr.

18) Tritoma. Fabr.

19) Habitat in Veftrogothia. Gyllenhahi. - Dermeiftes fafciatus Thunb, huc referendus.

20) Apate Fabr.

21) Ips hæmorrhoidalis. Fabr.

22) Ips Fabr.

*) Boftrichus crenatus. Fabr. 


\section{$-x) 117$}

Casfida zo-maculats.

furcata.

lunata. 23).

equeftris.

punctata.

pallida. 24).

Cbryfomela cuprea. a. B. $\%$. tremula. 1).

jpeciofa. 2).

adonidis.

centaurii.

20-punetata.

Polida.

rumicis.

gigantea. †).

Sopbia.

boleti. $\beta$.

aucta. $\alpha . \beta . \gamma_{0}$

tabida.

vitis. 3).

gloriofr.

iufitanica.

bicolor.

varians.

Bankii.

antericana.
Altica aquinoettialis, «. $\beta_{0}$

nigripes.

dorfalis.

atra.

hemijpharica.

caroliniana. 4).

Mordella abdominalis.

Erodius gibbus.

muricatus. \%

afer.

Meloë vittatus.

10-punetatus:

bicolor.

16-guttatus.

3 -fafciatus.

4-fasciatus. a. $\beta$.

undatus.

3-punctatus. a. $\beta, \gamma_{0}$ oculatus. $\propto$. $\beta$.

14-punctatus.

impar.

cacus. a. B. $\gamma$.

pusulatus. $\propto$. $\beta$.

ro-guttatus, $\alpha . \beta, \gamma$.

Clerus mutillarius.

$S$

Clerus

23) Tota flava punctata, habit, in Svec.

24) Casfida inæqualis. Fabr.

1) Chryfom, populi varieras.

2) Chryfom. faftuofæ varietas.

t) Erotylus. Fabr.

3) Cryptocephalus. Fabr.

4) Chryfom. caroliniana.

*) Pimelia muricata. Fabr. 


\section{- ) 118(}

\section{Clerus niger.}

parifinus.

8 -punctatus.

Spondylis dentipes. a. B. 5). Stapbylinus tricolor.

quadratus.

tricornis. $\delta$. ㅇ․

littoreus. \&. $\beta$.

fanguineus.

emarginstus.

flovalis.

depresjus.

fimilis.

pubefcens.

canaliculatus.

angufatus.

sitidus.

analis.

bypnorum.

tectus.

aneocepbalus.

fulgidus.

affinis.

Stapbylinus limbatus.

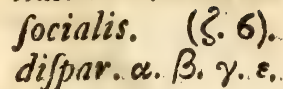

Ptinus longicornis. germanus.

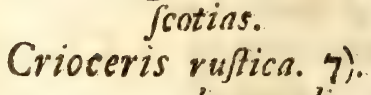

bumeralis. 8).

rufipes: 9).

lepturoides. 10).

lineola.

brunnipes, II).

teffacea, 10).

melanocepbala. s-punctata. fanguinea.

Cryptocepbalus cyaneus. bialenfis. 12). bucepbalus. cbalybeus. pubefcens. atrapbaxidis. flavilabris.

Crypto-

5) Alurnus dentipes. Fabr.

6) Staphyl. chryfomelinus \& filphoides. Lin.

2) Chryfomela ruftica. Fabr.

8) Ciftela humeralis. Fabr. Leptura 2-puftulata.

Thunb. act. nov. Upf. v. 4 .

9) Erotylus rufipes. Fabr.

10) Ciftelæ. Fabr.

II) Crioceris rufipes, Fabr.

12) Chryfomela halenfis. Fabr it. Cryptocephalus, Fabr. 


\section{$\Rightarrow$ ) $119($ )}

Cryptocephalus cbryfocepbal. Bupreftis berolinenfis.

6-maculatus.

pallens.

pufillus.

4-notatus.

Eurycbora acuminata. 13). carinata. 13).

Cucujus depressus.

2-punctatus.

teftaceus.

bifulcus. 14).

curfor. 15).

Bupreftis elegans. carioja. 16).

trifajciata. $\beta$.

nitidula.

cyaner.

favomaculata.

manca.

bicarinats.

fulcata.

prafina.

crevulea.

dimidiata. 17).
6- guttata.

irrorata.

bamorrboidalts:

rutilans.

flabellicornis. 5.9 .18 ).

Elater quercinus.

fufcipes.

undulatus.

rufus.

ignitus.

virens.

gigas. ").

Scarites brunnipes.

gigas.

Tenebrio picipes. $\dagger$ ).

Carabus 4-fulcatus.

arvenfis.

reticulatus.

gigas.

bole/ericeus.

cinctus.

pallipes.

picipes.

13) Pimelia. Fabr.

14) Habitat in Blekingia. D. Rhen.

rs) Tenebrio curfor. Lin.

I6) Bupr. tenebrionis var.

17) B. ralicis. Fabr.

18) Hifpa flabellicornis. Fabr. Elater Bupreftoides. L.

*) Ciftela gigas. Fabr.

t) Hilpa picipes. Fabr. 


\section{है ) $120($ )}

Carabus ancbora. 19).

ruficornis.

azureus:

4-guttatus.

nigricornis.

turcicus.

lepidus. a. B. y. d. e. ? oblongopunctatus. purpurafcens.

indagator.

bruniseus.

retu/us.

irregularis.

metallicus.

Cicindela femipunctata. 6 -lineata.

Dytifcus punctulatus.

2 -punctatus.

feneftratus. a. B. $\gamma$.

bybneri. 20).

bermanni.

cicutarizs.

planus.

depresfus. $\propto . \beta$.

picipes.

Dytifcus inaqualis.:a. $\beta$. crasficornis.

Leptura baftata. 今. 8. livida.

ruficornis.

unipunctats.

Lamia evytoropbala."). praufa. * ). mavilandica. $+j:$

Callidium luridwm. biders. Spinicorne. isenvatumb. femoratum. clavipes.

Saperda annularis. 21). virefcens. vittata. erytbrocepbala. futuralis. nigricornis.

Prionus fuliginofus. lineatus. Spinibarbis. damicornis.

19) Carabus crux minor. Fabr. Carab. cruciatus $\beta$ Mufei Catalog.

20) Dyt. fufcus? Lin.

*) Callidium. Fárr.

t) Stenocorus. Fabr.

2 I) Callidium. Fabr. 


\section{THESES.}

\section{I.}

Ex: Animalium mirifica ftructura \&. Oeconomia, Summi Creatoris fapientia \& potentia elucet.

II.

Humorum circulatione, fenfatione \& motu arbitra. ria imprimis diftinguuntur animalia a vegetabilibus;

\section{III.}

Etenim Animalia non modo crefcunt, ut lapides, \& vivunt, ut vegetabilia; fed etjam fenfu gaudent ac facultate fefe voluntarie movendi.

\section{IV.}

Male generationem in univocam \& $x$ quivocam diftinxere Veteres Naturæ Perfcrutatores, nec ullis hæc illorum diftinctio nititur fundamentis.

$$
\text { V. }
$$

Quemadmodum enim nullæ plantæ ex feminibus fuis non progignuntur; ita ne minima quidem animalcula nifi ex ovis fuis generantur. 

D. D.

\title{
MUSEUM NATURALIUM ACADEMIEE UPSALIENSIS.
}

\author{
APPENDIX $I I$.

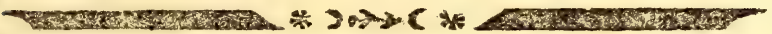 \\ QUAM \\ CONSENSU EXP. FAC. MED. UPSAL. \\ PRESIDE
}

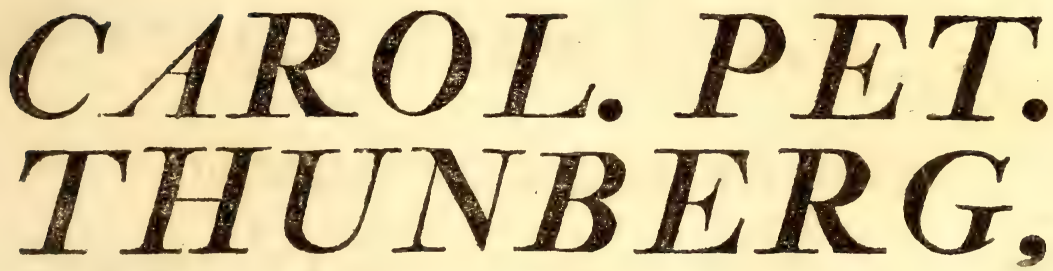

Equite Aurat. Reg. Ord. de Vasa,

Medic. Doct. Profess. Med, et Botan. Reg. et Orn., Acad. Cresar. Nat. Curios. Reg. Scient. Londinens. Holmiens. Soctet. Scient. Ussai. Patr. Holm. Beror. Nat. Scrut. Lundens. Harlem. Amsteld. Zeland. Nidrosiens. Harens. Nat. Ścrut. Linn. Londin. Medic, Ejimburg. et Nat. Studios. Ibid, Membro; nec non Acad. Scient. Paris. Monspeliens.

A gricult, Paris. Florentin. ex Batavin, ind. Or. Correspond.

\section{Publico examini proponit \\ HANS YMAN,}

Smolandus.

In Audit. Gust. Maj. DXX $X_{\text {Aprit. }} 72^{1 .}$

Horis ante merid. Solitis.

U P S A L I $\mathbb{E}$,

Apud Direet. Johann. Edman, Reg. Acad. Typogz. 


\section{RÅDMANNEN OCH GULDSMEDEN, \\ ADEL OCH HÖGACKTAD \\ HER R}

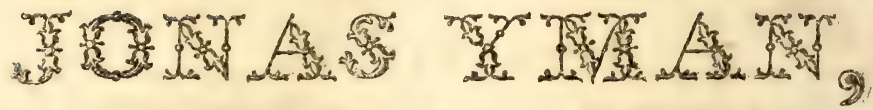

SART

\section{DPGDÄDLA FRUN}

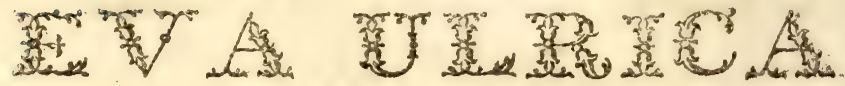

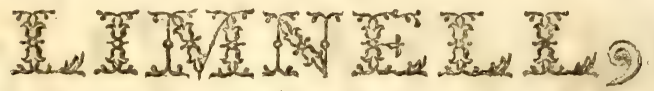

\section{MINE HULDASTE FORALDRAR!}

Den vórdinat, ärkinfle och tillgifvenhet hvartill Mine Huldafle Forrildrars godhet och otaliga villger. ningar mig forbinda; gôr, at jag $i$ edmjukher an. haller, det Mine Huldafte Foneldrar tiskas anfe det. ta, Som et litet vedermaile af min fonliga tackfam. het. Forblifver med vordinad till doden

\section{MINE HUYDASTE FORALDRARS}

lydigfe Son 


\section{DONATION. THUNBERGIANE.}

\section{APPEND. II.}

Cerambyx morio.

bsmeralis. 2).

depresfus.

lineatus. 3).

fartor.

varius.

fasciculatus. 4).

4-maculatus. 5).

12 -maculatus. 6).

Cantharis fanguinolenta.

equefris.

cardiace.

pyralis.

sigripes.

dispar. 3. 옹.

Calolymus 7). barbatus.

$$
\text { javipes. }
$$

morio.

Lampyris minuta. probofcideus.

$v u f a, \propto, \beta$.

obfcura.

cincta.

caperafs, $\alpha . \beta_{0} \gamma_{0}$ palliata.
Forficula gigantea.

dendata.

fiexuofa?

Iruxalis brevicornis. vittatus.

Locufta atbropica.

epbippiger. laxmanni.

Gryllius carinatus.

ferratus.

Cicada banio. quercus. ruftica. banata. $\alpha_{0} \beta_{0}$ per/picillata. pbalenoides. ferruginee. obtula.

Cimex zoftera. irroratus. paradoxus. pallens. 6-guttatus. praternss.

$T_{2}$

2) Leptura humeralis. Fabr.

3) Lamia lineata. Fabr.

4) Cerambyx pilofus. Fabro

5) Stenocorus. Fabr.

6) Leptura. Fabr.

7) Lymexylon. Fabr. 


\section{* ) $124(2$}

Cimex triguttatus: biftrionicus. fmarngdulus. maculatis. levis.

pyri:

podagricus. lateralis. fabricii.

fylvaticus. suelanocepbalus. 8). grylloides. membransuceus. torquatius. Aliabub cbii. tilice. $\alpha$. B. $\gamma$. osilis. $\alpha . \beta$. femipunetarus: firidutus. 9). civitis.

Nepa rubra. grosja.

Cbermes urtice. alni.

Coccus cbaracias. Papilio fyluasus. lavaterce. aracbise. davus. ‥ ㅇ. byfipyle.
Papilio tbypbon. ")

dia.

pyrrbus.

midamus.

blandina. S. 9.

cubule.

maturna. var.

idea.

mara. var. atbalia. dapbne. erinn. amyntbas. opitilete. aracintbus. panifcus. bille. nieve. garbus. Gatbus. xantbe. linea. of. ㅇ. Spbinx carniolica. fulvn. glaucopfis. pruxi. bylas. celerio. letbe. sperbius.

8) Lin. non Fabr.

9) Reduvius. Fabr.

*) Hero. Fabr. 
Spbinx Arigilis.

$$
\text { ruftica. }
$$

Bombyx matronula. capucina. purpurea. aurifuta. jodutta. 10). compressa. flava. morio. ㅇ. fagi. ‥ 우 maculosa.

Noctua paranympba. fimbria. orbona. frugalis. purpures. lucernea. favago. turca. jota.

circunflexa. alni. triquetra. compta. liguftri. rurea. megacepbala.
Noctua abrotani. ridens. ob/cura. deplana.

Pbalana plumbaria. gilvaria. margaritaria.

Osfeata. rbamanata. ornata. equeftrata.

Pyralis bombycalis. Tinea blankardella. bonnetella. cuprella. Scopolella.

Epbemera nigra.

Pbryganea ciliaris. lutaria.

Panorpa byemalis. I. Termes arda. Formica Smaragdina. pubefcens. tuberums. flava. Mutilla americana. compresfa. $\mathrm{T}_{3}$

10) Hepialus jodutta. Fabr. Bombyx vitis ideæ. Lin.

11) Andrena. Fabr.

12) Crabro. Fabr. 


\section{* $126(2)$}

Apis brafilianorum.

albipes.

fauriciana.

bamorrboa.

bicalor.

be murroidalis. II)。

ruderata.

cryptarusn.

prubejcens.

grayjtacea. 12).

battorfana.

fignata. 13.)

antiguenfis.

aftuans.

uniglumis. 12).

radula. 14).

bryorim.

fafciata. 11).

arbuftorum. Is):

disjuncta.

punctata.

olivacea.

mexicana.

labrata. II).

marginata, 81).

Apis forcenfis. forea. cincta. II).

Vespa bumilis.

6-fafciata.

pyriformis.

biglumis. $\beta$.

bicolor.

marginalis.

Spbex argentata.

morio.

flavicornis.

diadema.

evania. 16).

nigra.

exaltata.

penfyluanica.

lunata.

epbippia.

palmipes.

lobatn.

femorata. I7).

crabronea. 18).

fubterranea. 19).

13) Bembex. Fabr.

34) Scolia. Fabr.

15) Apis pratorum Linn.

16) Evania maculata. Fabro

87) Tiphia. Fabr.

18) Crabro fabulofus. Fabro

19) Crabro. Fabr. 


\section{叶) $127($ \%}

Leucoptis dorfigera.

Cbrylis nitidula.

Splendidula.

ametbylina.

fuccincta.

werea.

bidentata. ").

Tentloredo flava.

lebreuborib corrufcator.

extiortater.

molchator.

Mufca allimana, 1).

longipes. 2).

corvina.

Tipula juniperiza.

Consops roffrata. 3).

lineata. 3).

Afilus violaceus.

csliciformis. 4).

toutonus.

linearis.

Podura nivalis.

Pediculus bovis: vituli.
Laplyfá depilans.

Pbolas candidus. 5).

Tellina frobinata. foliacea. 5).

remsies. 5).

tranquebarica.

Cardium coffatum. 5). virgineum. 6):

Venus Aexuofa. 5).

marica.

circinata.

Mactra lutaria. 5).

Donax Jcortumb. 5).

Chavera lazarus. 5).

femiorbiculata. s).

Arcsi decusfatio. 5).

pectunculus.

Ofrea obliterata.

Mytilus litbopbagus. a. B.

unguis.

rugofius.

Melina epbippiums.

Pinsa pectinata.

rudis.

Tene-

*) Chryfis bidentata Linn.

I) Syrphus. Fabr.

2) Syrphus femoratus. Fabr.

3) Rhingia. Fabr.

4) Mufca grosfipes. Linn.

5) Unica tantum valvula.

6) Venus borealis, Catal, Mur. 


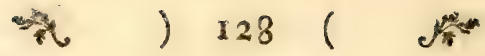

Terebratula Ariatula. 7). Serpentis. biloba. 7).

Argonauta argo. $\gamma$.

Nautilus pompilius. B. picius.

Strombus lividus.

Murex aruanus.

pyrum.

canaliculatus. $\alpha . \beta$.

Jenticosus.

$\gamma$. pufillus.

ortboceros. 7).

Conus aurifiacus.

$$
\text { d. exfculptus. }
$$

genuanus.

princeps?

nobilis.

Cyprace onyx.

$$
\text { cicercula. }
$$

Voluta atbiopica. a. B. $\gamma$.

virgo. $\alpha . \beta$.

vulpecula.

ruffina.

Buccinum erinaceum.

undofum.

prevojans.

tuberojum. a. $\beta$.

glaciale.

reticulatum.

duplicatum.

Strombus latisfimus?

gigas.

dentatus, \&. $\beta$.

cutaceus. $\propto . \beta$.

decollatus.

asper.

javanus.

dolariunt.

bippocaftanum. olearium. $\propto . \beta$.

Trocbus Rriatus. divaricatus. folaris. Scaber. muricatus. cruciatus.

Turbo muricatus. obtufatus. a. $\beta$. tectum, 8). aurifcalsiun. punctatus. 9). Ariatellus. 10). imbricitus.

Helix perspicua. beemafisoma $\alpha . \beta$. ampullacea. $\alpha . \beta$.

Helix
7) Fosfilis vel petrefacta.
8) Tectum perficum. Linn.
9) Trochus. Linn. 10) Turbo, Linn. 


\section{- ) $129($ )}

Helixe fubcylindrica. lavigata. barbara. itala. gualtbierians. oculus. firiatula. planorbis. gingens. contorta. sngulina. zonaria.

Nerita mamillaris, $\mathbb{I} \mathbb{E}), \alpha . \beta . \gamma . d$. albicilla. excifs. pulligera.

Haliotis parva. mida. Patelsa rufica. notnta.
Patella neritoidea.

crepiaula. equet:stis. criftuta. pellucida. lucudr: valgata. a. B. Y. d. pectinata?

Unbilicus venevis: varieta tes plures.

1/2s dichotomiq. sebracea. nobilis.

Gorgonic Setofi. ventalina. fabellums.

Corallina rubens. pinzata. Madrepora prolifera. Millepora alcicornis.

1.5) Helix mammillaris, Linn。 


\section{THESES.}

I.

Pilis te rantur Mammalia; plumis Aves; fquamis $\mathbb{P}$ i. Ices; cure nuda indurata Amphibia;

II.

Cum infeEis cutis fit cataphraeta Vermesque mollufci denudati fape tefas circumferant câlcareas.

\section{III.}

- Osfibus infruuntur ploraque Aninalia; funt tamera - son paucs his orbata.

$$
\text { IV. }
$$

Cerebrum \& Medulla fpinalis, in plerisque Animalibus confpicua, multum differunt a medulia Vegetabiliurn. $\mathrm{V}$.

Qurecumque Clasfis Zoologiz animalia continet phy. thivora manfveta, \& carnivora fera.

\section{VI.}

Non adeo incongrue, pro diverfa ratione vivendi \& prædandi, vel diurna vel nocturna dici posfunt animalia. 


\section{D. \\ MUSEUM NATURALIUM ACADEMIE UPSALIENSIS. Appendix III.

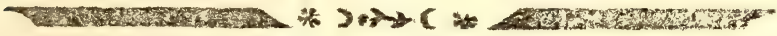 QUAM VENIA EXP. ORD. MED. UPS. PR ES IDE
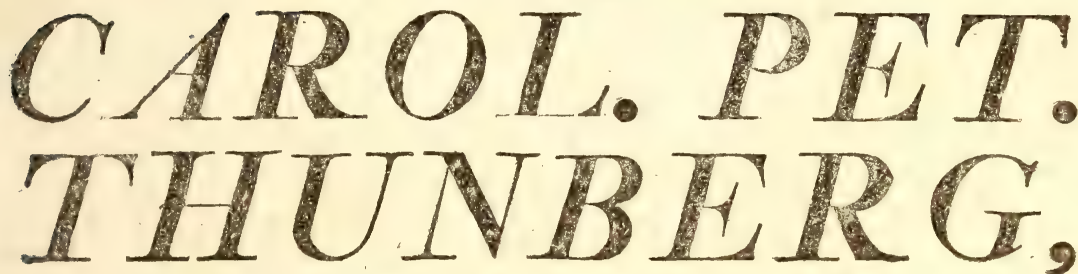

Equite Aurat. Reg. Orb. de Vasa,

Medic. Doct. Profess. Med. et Botan, Reg. et Ord. Acad. Cesar. Nat. Curios. Reg. Scient, londin. Holm. Societ. Scient, Upsat. Philad. Patr. Hot m. Beroz. Nat. Scrut. Paris. et Hafniens. Hist. Nat.

Lund. Harlem. Amsteld. Zei.and. Nidrosiens. Ticinens. Ienens. Halens. Nat. Scrut. Linn. Londin. Medic. Edimeurg. et Nat. Studios. Ibin. Memero; Nec Non Acaz. Scient. Paris. Monspeliens. Agricuit. Paris. Medic. Lonmin. Florentin. et Batayin. Ing, Or. Correspond.

PUBLICO EXAMINI SUBJICIT

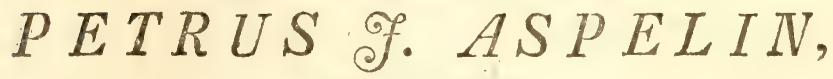
SMOLANDUS.

In Audit. Botanico die 8 Dec. 1794.

H. A. M. S.

\section{U P S A L I Æ,}

LITTERIS JOH, FRED. EDMAN, REG. ACAD. TYPOGR, 



\section{DONATION. THUNBERGIAN.}

\section{APPENDIX III.}

Simia nentefrina. apella: atra.

Myrmecopbaga jubata.

Viverra najua.

Canis lupus.

vuipes.

mefomelus.

famiharis. $\alpha$.

Felis caracal.

$$
\text { catus. a. }
$$

Ur fus gulo.

Dafypus 9 cinetus.

Hyjtrix critata.

Siciurus palmarum.

cineveus.

gizs. 1).

Marmota capes/is.

marittima.

nitela. I)

cricetus.

Mus terreftris. $\beta$.

Ovis aries. $\hat{\delta}$. bifpar. -. S. yuadricornis.

Cervus axis. 9 .

Falco osfifragus.

Strix lapponica.
P/ittaciss pullaris. pondiceriamus.

Corvus sorone. frugilegus.

Picus auratus. criftatus.
auratus.
cbinen/is. baltimbore?

Galbula viridis. Gracula criftrtella.

Certbia cyanea. ceilonica. lotemia.

Trocbilus mango. moncbitus. mellivorus. Alcedo cloryjocepbala. critata. fmyrnen/is. tridactyla. rudis.

Pipra pareola. aureola. Serena.

$A n$ a s clangula. 오. erytbropus. Arepere.

I) Myoxus. 
Anas africana. $\hat{0}$.<smiles></smiles>

dispar. S.

aner. Q fera.

bofolas. 9. fore.

circia. 余.

Pelecanus curalinenfis.

Larus cinerarizs.

\section{trifictylus?}

Ardea cicomia.

not) ficorax.

Scolopax liarofa.

Inpponica.

Recurvirofira nvofetta.

Platalea pygman. leusorodin.

Fulica chloropus. porphyrio.

Charadrias alexandrinus.

Trimg a cinclus. prginax.

Tetrao perdix.

tetrix. 1. var.

rufus.

francolinus.

Pbafianus crifatus.

nyetbemerus.

gallus: ecaudatus. 9 .

ej. pullus rofiris duobus.

- capitibus duobus.

Meleagris gallopavo. $\hat{\delta}$. Alozda calandra.
Alouda crituta. campefiris.

Turdus cyaneus. paradijeus. canorls.

Loxit cbloris. vubicella.

Emberiza cia.

Fringilla butyracen. canaria. vir. triftis.

Mufcicapa atricapilla. q. Motacilla tipbia. albr. .

Tanagra mexicana. tatao.

Colsubr-berus: pullus. petbolatus. var. Ampbisbana alla: grandis. maculata.

Xipbias gladius.

Callionymus japoricus.

Silurus maculatus. Blennius lumpenus.

Cottus gobio. Perca 6-lineata.

fario.

fusca. cirrola. pertufa. 7-faciata. 


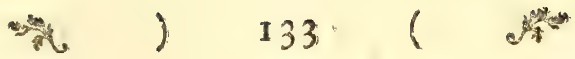

Perca: 3-lineata. fiviatilis.monftrvar.

Cyprinus fareins.

\section{tivea}

Syngnatbus opbiodon.

Hifer jouctutus.

glabratus.

ferrugineus. \&. $\beta$.

ábbreviatus.

fulcatus.

fi. ustus.

4-Priatus.

cafus.

planus. 令.

cruciatus. $\alpha . \beta$.

Semipunctatus.

Corcinella fenegallentis.

fegetalis.

lapponica.

fernica.

artica.

cbinenfis.

$\mathrm{IO}$ : maculata.

9 -notata.

reppentas.

gloliofa.

morginepunctata.

16-notota. 1).

Elopborus elongatus.

flavipes.

Curculio ceraforum.
Curculio crux.

curvirnftyis.

tenuirofirts.

rasa.

bispiailits.

guttula.

aquatus.

varidus. 今. ?.

ocellatus.

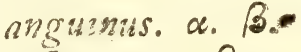

cofiatus. $\alpha$. B.

2. masculatus.

Finenvis.

bordance.

trimacslatus.

corticalis.

confulus.

bypoleucos.

fusciroftris.

btattarie.

planus.

atbiops.

vernalis.

didymus.

napobrasfica.

iateralis.

padi.

litura.

globulus.

troglodytes.

ercticus.

1) 16 - punctara Fabric。 


\section{pubefcens.}

Attelabus pubefcens.

Lucanus dama.

Scarabous asper.

quadridens. 占. \&.

conglagratus.

titanus.

2-punctatis. $r$.

jordidus.

arator.

nigripes.

Copris mobilicornis $\hat{\text { s. }}$. bybneri.

xyphins.

reflexus.

Melolontba floralis, a. $\beta$. atviplicis.

Tbrox crenuta.

Hydropbilus grifeus.

picipes.

lateralis.

emarginatus.

Byrrbus gigas.

cuffriacus.

pulverulentus.

cinctus.

fasciatus.

minutus.

albopunctatus.

Spbrceridium lunatum.

marginatum.

$134<$

Spberidiusn bumerale. ferrugineum. rufipes. nigripenne. ruficolle. chryfomeloides. flavipes. bicolor. laterale. 2. pusctatsin. Boftricbus bifurcatus.

laricis.

cylindricus.

Dermeftes atomarius.

punctatus.

4- maculatus.

lycoperdi.

funatus. $\alpha . \beta$.

nigricornis.

ferra.

finctarii. cellaris. longicornis. nidentatus. cordatus. teftaceus. caftaneus. 3). 20 -guttatus.

Clerus 2-puftulatus. 4). aneus. 5).

Tri-

3) Mycetophagus, 4) Hisfea 2-puftulata, B. 5) Lagria. znea $F^{*}$ 


\section{औ \\ 135}

Tritoma furinamenfis.

filciasa.
bicolor.
2 - puftislata.
contracta.
juglindis.
depressa.
fraxini.
pallipes.

Necropborus japonicus.

mortuorum.

Casfida margaritacea.

aufriaca.

flaveola.

Nitidula marginnta.

imperialis.

fordida.

villofa.

limbata.

10-guttata.

pusfilla.

viridefcens.

Silpba lapposica.

Opatrum grifenm. $\alpha$. $\beta . \gamma$. Melö̈ voblii.
3-maculatus.
lineatus.
fimbriatus.
rugofus.
flavus.
birfutus.

Melö urfus.

lunatiss.

Mordella paraduxa. E. \&.

ferruginsa.

2- maculata.

maculata.

Altica fuscipes. 6).

anglice.

Ips fungorum.

collaris.

4-maculata.

fangvinicollis.

Cbryfomela 6-punciata.

foutellata. dorfalis.

Gyrinus bicolor. ignita.
bicolor.
spinofus.

Brucbus analis.

futuralis.

4-pufulatus.

Notoxus thoracicus.

Ciftela varians. a. $\beta$.

thoracica.

Ctyptocebpolus foripes.

paracentbeja.

tboracicas.

bumeralis.

bivittatus.

Helwoigii.

Cry.

6) Chryfomela. F. 


\section{औ. I 126}

Cryptocepbalus letus. ancus.

Crioceris capenfis. cartharoides.

Sepidium tricuspidatum. lunatum.

Seaurus atratus. Blaps gages.

Carabus ambiguus. a. $\beta$. glabratus. forutator. interyuptus. spinibaybis. atterrimsis. alpinus. veftitus. flavipes. germeanicus.

Cicindela Ariata. uliginofa. Necydalis thoracica. umbellatarum. uffulata. fangvinicollis. Bupreftis lata. lugubris. undata. rubi. porcata. emerginata. decoftigma.
Bupreftis morio. ocellata.

Elater tboracicus. pallipes. beratodes. filffurntis?

Stapbylinus varians. nitidulus. alpinus. Priatus. vuficolis.

Forficula parallela.

Cucujus vonilis.

Leptur a fmarasdina. anguftata. 6. gut ata. A. 옹. 8 - maculata. limbata. S. ?. futuralis.

Lamia Specinfia 7). triffosciata. 8). bieraglypbica.

Saperda lineola. teftacen.

Cerambyx afer. vuficollis. 8) cantbarimus. fulvus. longipes. a. B. 9). Dytifcus transverfalis. 


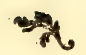

Dytifcus albreviatus. dorjalis. irroratus. impresfus. confuens. lineatus. ferragineus. fiscus. fullginofus. agilis. notaitus. 6-pufalatus. congener. Roëlelii. Fiscisiz. 0 . porcatus.

Locufta fpinzloja. albifrons. conocepbala. falx.

Cicada genifice. bamorrban. leporina. orni. aptera.

Cimex lavatere. nigelle. spiniger. trifasciatus. faxutilis. globus. infidiator. 1) bottentuttus.
$137(2)$

Aps is quercus. Papilio marlyas.

Difa.

minimus. adonis. Corydon. plocibe. Norna. Frigga. Frejia. Ifis.

om ortutina. Delia. dictynna. pailes. Lappona. Embla. Cleobis. bylas. Damon. 오. cleopatra. cupbeno. \$. \&. r. album. cyllarus. Arrino:e. eucharis. achine. Mavianne. argia. gliciria. Remus. Helena. Ierpficore. 


\section{我) 338 ए}

Papilio cbaritonia.

Hild . Amploion. Aretbura. camilla.

eynara. 숭요

medua. 占. evebus.

Sphinx infanfta.

fenefrina.

anothere.

Bombyx oleaginea.

buplewy ario.

fusca.

fignata.

pitbyocampas.

lapponica.

morro. 5 .

bicolora.

pruni.

fulminer:

casfinea.

tritopbus.

plumigera. 余. 오.

anfera.

Noctua euphorbie:

leucoptera. cbalcites.

melaleuca:

lipponica.

L. album.

croceago.

suelanopra.

ceraffo
Noctua fulpburago. aurago. rutilngo. rbizolita. infabilis. promissa. virens. auricoma. flammatra. finulans. munda. rubiginea. linogrifea. petrificata.

figna. cinerea. cinnamomea. ferena. brnetea. gilvago. ferrago. puella. petrorhiza. obelifca. finilago. cacimacula: pulmonaris. cinevea. 앙으. obry/ograpba. disfimilis. egregia. triftis. cleeropodii. 


\section{) $139 ?$ ?}

Noctua binacsilofa.

gracilis.

jerla.

vymplagoge. 余. ․ pifacina.

precox.

advera.

Pbalena bexapierata.

fuscaria.

circuidaria.

angalaria. abruptaria.

fordaria.

zonate.

cognata.

erundinata.

corylata.

flavoficiatata.

birfutaria.

bipunetaria.

limbaria.

eupborbiata.

adufata.

monaria. 令. 9).

efcularia.

plunnaria. a. B.

Pyralis ventilabris.

margaritalis. caspitalis. porpbyralis.

Tortrix applana.
Toytrix Smeatbmanniana.

Perzilana.

Rbenana.

finbriana.

birundana.

Helliana.

vaculana.

decusfana.

cantana.

cmarginana.

palliduns.

Sticknanniand.

Aviatana.

Acbarians.

variegana. $\alpha$. $\beta_{0} \gamma_{0}$

reticulana. $\alpha . \beta$.

pflugiana.

Tbunbergiama.

lundana.

Paijkulliana.

profundana.

Tinea multisuttella.

carduella.

fisciella.

pectinella.

paripunEtella.

boletella.

gigantella.

plismbella.

cofzella.

B 2

9) Bombyx F. 


\section{- $) 140(1)$}

Tinea lactella. 10) tripunctanella. 10). litter ella. abruptella.

ferruginella. forficella.

Paijkullella. II \% Sparmanella. Sulzella. pufiella.

Schafferella. oppofitella. axillellat 4. guttella. 6-guttella. binotella. penicills.

Gyllenbalella. Blancbardella. 2-maculello. Gleicbella. tboracella. trinotella. auropunctella. fungella. flavella. obscurella. fagella. \&. $\beta$. atomella. oculella. virgella. farinella.
Tinea laterella. viduella: firigilella. tetrapunctells. fo ciella. Erxlebella. flivifrontella.

Alucita rbododactyla. Heznerobias pilicornis. deuftus. guttatus. feneftratus. fuscutus. flivicans. fafciatus. longicarnis. maculatus. nitidulus. 4. punctiatus. Myrmelicon barliarum. Fanorpa pectinicornis. Formica triders. bemorruoidalis.

3 . lineata. ferruginea. viatica. ruficollis. armata. ragofa. cingulata. arenaria. 


\section{次 ) 141 ( )}

Mutilla pectinicornis.

capentis.

culon.

2. guttita.

leucosepbala.

rubra.

tricineta.

Maroccana.

vzolacea.

Ap is pilipoda. I).

bivfuta. 2).

verficolor.

gibba. 3 ).

6. $\operatorname{cin} \mathrm{Ct}_{\mathrm{a}}$.

pilipes: 4).

agrorume.

ficalira.

milti unctata.

glauca. 5).

fericea. 5) o

marginata. 5).

excifat 5) o.

fiijca. 5).

arcuata. 5).

fcutellata. 3).

fer ruginata. 3)

Ve/pa suaculita.

purpurajcens.

oculea.

fimilis.

calida. a. B. $\gamma$.
Vespa crax.

campaniformis.

globifor mis. $\alpha . \beta$.

nigra.

birintita.

bilabiata.

baftata.

2 - guttata.

rufogafer.

Pinigera. $\propto$. B.

difineta.

tibialis.

trilineata.

2. maculata.

- lfcura. o. B.

pinczigera.

bivituta.

Spbex lucida.

florida.

longipes.

flavifrons. $\alpha_{*}, \beta_{0} \gamma_{0} \delta_{\infty}$

fiava.

esfimilis.

wniculor.

rufipes.

2. guttata.

ocellata.

lunsta.

corvilea.

fimilis.

nigrita.

B. 3

Splex

I) And zna pilipes, F, 2) Andr. 3) nomada, F. 4) apis F. 5) Bember. 


\section{* ) 14211}

Spbex sexcinta.

conica.

fericea.

I - puinctata.

maderaspatana. a, $\beta$. peEtoralis.

Sanguinolenta.

maura.

illucens.

dimidiata. 2).

maculata.

Scolia thoracica.

flavifrons. as. $\beta$. bemorrboidulis.

4. maculata.

interrupta.

5 . punctata. a. B.

fexcincta.

zxorio.

collaris.

4-notata. \&. $\beta$.

cepbalotes.

capenfis.

5 fajciata.

fulva.

maroccana.

6-faciato. $\alpha . \beta$. ocellata. $\alpha . \beta . \gamma . \delta$. unifajciata.

Cbalcis clavipes. $\alpha . \beta$. guttata.

minuta.
Cory/as' calens. (emaiaurata. 3).

Corynis trifis. $\alpha . \beta$. Testbredo morio.

intercus.

pini.

armillata. tboracica. pafinace. cingulata. pectoralis.

cyanea.

carbonaria.

Icbneumon jecalis. falcatorius. enervator. leucopbtbalmus.

Cynips glesbome. Tipula macrocepbala. virens. cincta.

Strationys epbippium. Rbagio atratus. Bibio filata. Mujca 4.punctata. tetrapuncta. 6 - punetata. 8-prnzata. punctigera. ferratula. dauci. $\alpha . \beta$,

2) Gabro, 3) Splex. Lia. 


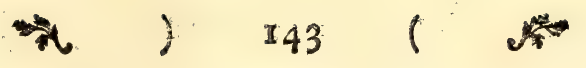

Mufca manfueta.

Tabanus bijpinofus.

marens.

colon.

exelluniss.

guttatus.

tboracicus.

singulatus.

vittatus.

brunneus.

cryjoptbalmus.

Empis marginata.

tboracica.

rufipes.

fufca.

trilineata.

joutellaris.

femoratio.

atra.

gibbojs.

Stomoxys fibirica.

Conops apiformis.

eofitalis.

winuta.

- Bombylius aqualis.

literalis.

fenefratus.

analis.

dimidiatus.

Afilus ater.

crasses.

bifafciatus.
Afilus flavipes: tibialis.

Pedicalus meleagridis.

vespertilionis.

beematopi.

Aranea nidulans.

Pbalangium lunatum.

Cancer curfor.

Seticornis.

roltratus.

dongiroftris. $\alpha, \beta, \gamma, d$.

lunaris.

ruricola $\alpha . \beta$.

rbomboides.

punctatus.

craniolaris.

nucleus.

fornicatus.

lanatus.

cancellus.

depresjus.

granulatus.

cornutus. firigo/us. a. B. Maija.

Solien anotinus.

Tellina pifformis. a. $\beta, 4$ ).

Mytilus ruber 4).

Ojtrea gigas. a. $\beta$.

Ariatula?
4) Unica valvula. 


\section{THESES.}

I.

Quo plures deteguntur res naturales, eo etiam luculen. tior adparet magna illa por totam naturam fesset extendens rerwin cateina.

II.

Anteyuom ii, qui suri manent publicis negotiis non -ccupati, otiofum tempus Entomsulogia tribuant, valde parvos faciet joientia illa progresjus.

III.

Medico non partm, Oeconomso autem plurimum adferset commodi, folida Entomologice Notitia.

IV.

Multum eft a teneris adfvefcere attentos, etiam ad res minimas, oculos advertere.

\section{V.}

Quo brevior eft revum Naturalium defcriptio, fi nimirum pracipuas notas sbaracterifficas cuntineat, eo eft perfe. tior, eo utilior.

\section{VI.}

Anteguam Deconomia infectorem plaribus innotefcat bo. minibus, univerfalis utilites ex illa sor expectunda videtur. 
D. $D$.

MUSEUM NATURALIUM ACADEMIÆ UPSALIENSIS.

- 3 -

C.UJUS

APPENDIC. IV.

VEN1A EXP. ORD. MED. UPSAL.

P R ESIDE
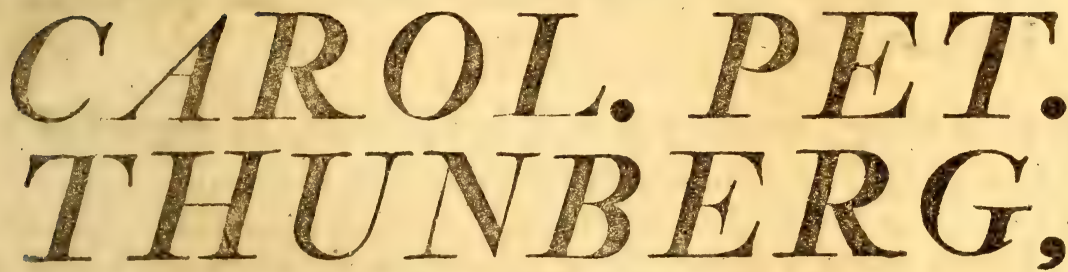

Equite Aurat. Reg. Ord, de Vasa,

Menic. Doct. Profess. Med. et Botan. Reg. et Ord. Acad. Ceesar. Nat.

Curios. Reg. Scient, Londin. Holm. Soctet. Scient, Ursal. Puilad.

Patr. Holm. Berol. Nat. Scrut. Paris. et Hafniens. Hist. Nat.

Lund. Harrem. Amsteld. Zeland. Nidrosiens. Ticinens. Ienens.

Halens. Nat. Scrut. Linn. Londin. Medic. Edimburg. et Nat,

Studios. Ibin. Membro; Nec non Acad. Scient. Paris. Monspeliens. Agricult. Paris. Medic. Londin. Florentin. et Batavin. Ind, Or. Correspond.

PUBLICO EXAMINI SUBJICIT

PETRUS SUNDBERG,

STIP. STRANDB. OSTROGOTHUS.

IN AUDIT. BOTAN, D. XXIII. NOV. MDCCXCVI.

H. A. M, C.

\section{U P S A I I $巴$,}

Litteris Joh. Fr. Edman, Reg. ACAD, typogr. 


\section{T H E S E S.}

1. Empirifmo maxime favet illud. "Wo man Thatfa. chen hat, find Erklárungen fehr entbehrlich" (Vide Brandis: Verfuch ü. die Lebenskraft, p. 24.)

2. Secretiones plantarum, oleum, refina \&c. eodem modo ac in glandulis corporum animalium præparari videntur.

3. Nec aliter, quam per vafa lymphatica, in vegetabilibus perfici abforptionem exiftimanus.

4. Fibris enim vegetabilibus quandam inesfe incitabilitatem contendere haut dubitamus.

5. Ufu quorundam organorum, guftus inprimis \& olfactus, cum bruta fre antecellant, tactu autem, durioris extremitatum integumenti causfa, nobis plerumque inferiora fint; probabilem cenfemus opinionem III. Buffon, quod fenfui huic exquifitiori rectius debeant homines judicium.

6. Sagaciora igitur funt animalia, quibus tactu res explo. rari datum, ex. gr. fcandentia, quæ pedibus anterioribus hominum more utuntur.

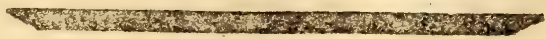




\section{DONATION. THUNBERGIAN.}

\section{APPENDIX IV.}

Vespertilio molos/us.

Felis porealis 1).

Viverra zobellina.

maty?

Hyftrix dorfita.

Sorex fodiens.

Mus agreftis.

Capra bircus.

Falco lugopus.

jpurzerius.

litbofalco S. ?. baliatus. var.

Strix arctica.

Lanius tyrannus.

fabacl. doleatus. 2).

Croropbaga ani.

Bucco caijarenfis.

Ramupbaftos erytbroryizbos. aracact. viridis.

Alcedo anevicanus.

Trocbilus gularis. Anas glaucion.

$$
\text { erytbropus. 9. 3.) }
$$

Colymbus arcticus. ?.

Larus vidibundus.

Alca pica.

Sievna fuliginofa.

Pboemicopterus ruber

Rallus nquaticus. porzana.

Tringa glaveola. pufilla.

Ardea nigra.

Scolopax gallinula. gallinaria.

Otis tetrax.

Columba palumblus. Motacilla lufcinia.

acredula.

Tanagra caijana.

Claviger teftaceus.

Coccinella nitidula.

Arigata.

I I-notata.

14-meculata'

28-notata.

tricufpis.

abdominalis.

8-puftulata.

$A=$ fimilata. Coce

1) Katt-L.o. 2) potius Turdus, 3) Anas albifrons. 


\section{\# ( 145$)$.}

Coccinella verrucata.

ur/2nat.

Pasfalus dentatus. a. $\beta . \gamma$.

Lucanus cornutus.

dama.

capreolus.

Scarabeus juvencus.

aloèus.

monodon.

cborinaus.

contaminat"rs.

conflugratus.

fordidus.

4-pufulatus.

foetidus.

pecari.

ferutator.

Copris camelus.

depresfus.

emarginatus.

canobita. S. 오

reneus.

femipunctatus.

alces.

Melolontba aquinoctialis.

birticollis.

lanigera.

inanis.

Cetonia faftuofa. $\alpha$. $\beta$.

marmarata.

viridis. $\alpha . \beta$.

metallica.

fquamosa.
Cetonia foricola. bombylius. caftanea.

Antbribus dorfalis. Brzicbus undatus. oleiperda. loti.

Curculio Squaviofus. varians. faliceti. punctun. brasfica. peudacori. nsfimilis. varinbilis. esbii. verbafci.

13-punctatus. micans. lassiz. epilobii. fellatus. vorax. forbi. punctiger. inflexus. artemilite. lonicere. pollenofus. marmoratus. culcaratus. lopidopterus. obliquus. 


\section{$\Rightarrow$}

Curculio pictus.

pilofus.

fcanicus.

falicivorus. antiodont nlgicus. pubefcens.

Apate difpar. a. B. dentatus.

lineatus.

Anobium rugofum.

Chryforsela pretiofa. coriaria.

bottentotta. bovifte. rapbani. carnifex.

Cryptocepbalus lobatus. violaceus. bemorrboidalis.

Lagria metallica.

Altica glabratio. brasfice. fulvicornis. ruficornis. euploorbice. nigrita.

rubi. parvula.

Nitidula ferruginea. bemorrboidaiis. Stapbylinus mordax. 1).
Stapbylinus binotatus. 2.)

brunneus. minutus.

fracticornis. pilicornis. fplendens. plagiatus. arenarius. cimicoides. dilatatus.

Cossyphus depressus. Elater viparius.

4-puftulatus. fulgens. tefiaccus. analis. bolosericeus. impresfus. notatus. pilofus. pallipes. rufipes. violaceus. cinereus. latus.

Serropalpus Ariatus. 4-puffulatus.

Carabus bumeralis. foliatus. borealis. parumpunetatus.

1) Oxyporus maxilofus. $F$, 2) Uxyp. 2-puftulatus, F. 


\section{( 148$)$ (}

Carabus gibbus.

4-punctatus.

ovalis.

fecalis. $\alpha . \beta$.

brevicollis.

erytbrocepbalus.

excavatus.

purpurasens.

virefcens.

marginalis.

fylvefiris.

Malacbius teftaceus.

faladonius.

Contbaris pellucida.

pallida.

analis.

collaris. 3.)

angulata.

Pyrocbroa grandis.

Lycus aurora.

Melyris bamorrboidalis.

Tenebrio obfcurus.

fenoralis. 4.)

glaber. 4.)

tboracicus. 5.)

Buprefis punetata.

Prionus bilinentus.

scabricornis.

Cerambyx platipes.

brunzreus. $\dagger$ ).

grijeus.

atomarius. *.)

cruciatus. ${ }^{*}$.)

barbicosnis.

8-maculatus, ".)

Salicis. a.)

indagator, b.)

Lamia as(insilis. c.)

ornota. d.)

thoracica.

Arepens. f).

Leptura luridn.

Mordella ventralis.

Meloë punEtatus.

Apbis corni.

vicie.

pineti.

Acantbia cofata.

3) Malachius ruficollis $F$.

a) Callidium. F.

4) Blaps. F.

†) Saperda F.

b) Rhagium. F.

5) Trogofita.F. *) Lamia F.

c) Callidium plebejum.F.

d Callid. 3-fafciatum. $F$.

f) Stenocorus $F$. 
"

Cimex gramineus. globus. triftis. faxatilis. rivulorum. familiaris. flovalis. 2 punctatus. 4. punctatus.

Locufta camellifoliz.

Cicada taurus.

grossa. teftacen. cufpidata.

Notonecta coleoptrata. Papilio. amatbufa. doris. facra. $\alpha$. B. diaphanus. Clymene. phyllis. Leucippe.

Spbinx eryx. fufanna. clotho. nerii.

ergotis.

Bombyx lunigera. Voetua ononis. texta. eupborbire. lycbnidis. iunavis.
Noctua luoria.

culta.

finzulans. obelifca. augur. crepufcularis.

Pbalana procellata. cervinata. corvaria.

Tortrix Scbulzians.

Pyralis porphyralis. cespitalis. pollinalis.

Tinea Ekebladella. labyrintbelle.

Hemerobius friatulus. Ephemera brevicauda. merginata.

Libellula fluetuans. clavata.

Pbryganea varia. Apis lapponica. Cbry/is lucidula. Corynis filvarum. Andrena circulata. Mutilla balen/ss. epbippium. fridula.

Sirex pyyllius. Icbneumon pedatorius. negatorius. 
Musca nectares.

lycbiridis. onopordinis.

Bibio dentata.

2-punctatu.

fafciata.

Rbagio vermileo.

ferrugineus. bicolor.

penicillatus.

bilineatus.

diadema.

culciformis.

guianen/is.

lacteus.

zonatus.

Tipula flaveolata.

flavipes.

4-punclata.

ferruginea.

pomona.

pyri.

flexilis.

barbicornis.

fafciculata.

pufilla.

Afilus aftuans.

Lepifma villosa.

Trombidium tinctorium.

Cancer marmoratus. erinaceus.
Cancer barbatus. 6.)

cbiragra.

emeritus.

facchino.

princeps.

arameus: bufo.

pelagicus: cedonulli.

tefiudinavius.

bydropbilus.

limaculatus.

porcellanus.

nizytilorum.

granulatus.

dromis.

Pagures bungarus.

miles.

clypeatus.

Aftacus elepbas. a. $\beta$.

bamfius.

innzocuus.

urra.

Ecbinus biforis.

Afterias glacialis.

Mya ovalis.

Tellina biradiuta.

Mactra sangvinea.

Chama exapperata.

Veutus fuccircta. reticula.

6) Herbit non Fabr. 


\section{D. \\ MUSEUM NATURALIUM ACADEMIE UPSALIENSIS.}

*

cousus

Appendic. v.

VENIA EXP. ORD. MED. UPSAL.

P R E S I DE
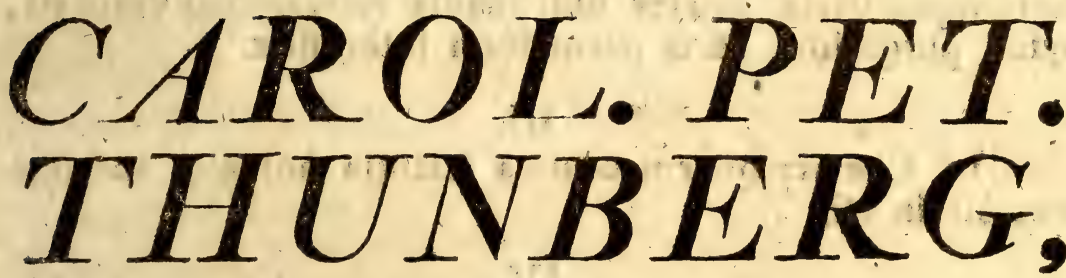

Equite Aurat. Reg. Ord. de Vasa,

Métc. Doct. Profesg. Med. et Botan. Reg. et Ord. Acad. Cesar. Nat.

Curias. Reg. Scient. Londin. Holm. Societ. Scient. Upsal. Phitad.

Patr. Hoim, Berol. Nat. Scrut. Parig. et Hafniens. Hist. Nat.

Lund. Harlem. Amsteld. Zei. and. Nidrosiens. Ticinens. Ienens.

Halens. Nat. Scrut. Linn. Londin. Medic, Edimburg. ex Nat.

Studios. Irid. Membro; Nec non Acad. Scientr. Paris.

Menspeliens. Agracult. Paris. Medic. Londin. Florentin.

et Batavin. Ind, Or. Correspond.

PUBLICO EXAMINI SUBJICIT

ERICUS G ADELIUS.

STIP. PIPER. STOCKFOLMIENSIS.

IN AUDIT. BOTAN, D. III. MART. MDCCXCVII.

H. $\mathrm{P}, \mathrm{M}, \mathrm{s}$.

\section{U P S A L I \&,}

y,tTteris Joh. Fr, Edman, Reg. acad. Trpogt. 


\section{F TESES: \\ 1.}

Cognitioni herbarum vegetationis anatomiam earundem magnam credimus allaturam esfe lucem.

II.

Inter bona, quæ nobis adferunt folia arborum herbarumque varia, præter nutrimenta referendum certe eft, quod purgationi aëris permultum inferviunt.

III.

In Chemia pharmaceutica feientia botanica abfolute necesfaria eft.

$$
\text { IV. }
$$

Herbx in Clasfe Triandria præ cæteris apta pechdibus prabent nutrimenta.

$$
\text { V. }
$$

In variæ coeli temperiei in corpora \& hominum \& cretorum animalium effectibus, folam Noftalgia: causfam quarendam esfe cenfemus.

VI.

Rei rufticæ incumbentibus, cognitio morborum, qui? bus fre laborant arbores arque herba, non poteft non. esfe urilisfira. 


\section{DONATION. THUNBERGIAN: \\ APPENDIX, V.}

Myrifica glomerate. 1. \%. Salicormia amplexicaulis.

Najas minor.

Pbalypar fanguinea. 1.9.

Veronica urticafolia.

orientalis.

tarivice.

Pingvicula lifitanica.

Utricularia Aellaris.

Orcbis psycbodes.

Lemina obeordats.

Salive fisfa. q.

bicolor. 令。

conifera. $\hat{\delta}^{\circ}$

amaligua.

feroting.

Fraximus carolinians. pubefsens.

Fisfilia pfstacowum.

Tragia corniculata.

Cucumsis. glexuojus. colocyntbis. africanus. prophetarkm! anguris.

acutangulas. melo. dudaims.
Cucumis cbate.

Jativus. coromson.

Cusurbita lagenaria.

ovifere.

pepo. verrucosa. melopepo. citrisllus.

Aomordica baljamina.

lanata.

charantia.

opericulats.

luffa.

trifoliata.

siažcrium.

Tricbojantbes anguirs.

cucumerima.

Bryomia dioicas

altra.

grandis.

africante.

fcabre.

japonica:

raceinofia

provetiats.

trilobis.

angulate.

levis.

acutangula. 


\section{*) $104(\%$}

Bryonia s-loba. digitata. dissecta.

Sifyos angulata. garcini.

Rujcus aculeatus. bypoglossum. bypopbyllum ondrogynus. racemofus. volubilis. reticulatus.

Iris juncifolia.

Morea dicbotoma. ovata.

Syena Maijaca. fol.

Scirpus cbinenfis. aufiralis.

Cyperus diffacbyos.

Carcx aspera.

Zea compresfas x. 2.

Cencbrus muricatus.

Pbalaris Zizanioides. Paspalum foloniferum.

Pbleum Gerardi.

Alopecurus vaginatus. Pon paniculata. Feftuca ciliata. Jpadicea. Bromus diandrus.
Avena bulbofa. diffic bophylla.

Elymus Ariatus Secale bulbofum. Hordeum fecalinum. maritimum.

Proted evecta. candicans. villora. odorata.

Bankfia pungens. pyriformis.

Scabiofa vuterfolia. Hedyot is racemnfa. Galium grecum.

tenue. tricbopbyllum. bercynicum.

Simbuleta tenuifolia. Fernelia mauritiana. Mitibella repens. Myonima borbonia. Pyroftria Commerfoni Pencal formofa. Plantago amplexicaulis. nigricans. Agipbila arbarefcens. Trapa bicornis. Dorftenia Houftani. Embotbrium buxifolium.

2) Anhoxanthum paniculatum. Linn. 


\section{\# ) 105 (}

Embotbrium critmifolium. Lobelia tomentofa.

Betula fruticoja.

pinnata. fol.

papyracea.

Pagannea guianentis. fol.

Bofcia undulats. a. B.

Heliotropium marifolium.

jcabrum.

Ancbufa barrelievi.

Cynoglos.um fcorpicides.

Onofina taurica.

Primula maxima

Cyclamen couns.

Weigela coreenfis.

P'blox fetacea.

Convolvulus terreftris.

Campanula elatires.

Ppytbeuma feorzonerifolta.

Lobelia fimplex.

pinifolia.

dortmanna.

depresfa.

anceps.

volubilis.

arborea.

acuminata.

furinamenfis.

phytbeuma.

bellidifolia.

bulbifsa.

triquetra.

longifiora:

recunda.

patula.

cardinalis.

debilis.

fipbilitica.

inflata.

Cliffortiana.

cypbia.

urens.

minuto.

laurentia.

erinus.

erinoides.

ceilanica.

luter.

birfuta.

coronopifolia.

tenedla.

pubeferns.

linearis.

crenata.

cardamines.

incifa.

cinerea.

Ariata.

bifide.

repers.

tbermalis.

javanica.

pygmac.

Godenta ovata. bellidifolis.

Aritha. 


\section{(n) 106()$^{\circ}$}

Godenis beterojbylla. albida.

Rondeletis buxifolia.

PJycbotrin expanja.

Vangueria madagafcarienfis.

Conocarpus emarginata.

Verbafcum pimatifidur. b)

croceum.

creticum.

aveturus.

orientalis.

Zaborofa bonarientes:

Ceftrum Pargui.

Lycium tataricum.

Laugeria refanofa.

Conoboria flavejcens.

Pbylica tricbotoma.

Brusia alopecuroides.

lavis.

$\operatorname{deufta.}$

lata.

micbropbyilla.

Polycardia siadagajarienas. Viole capenfis.

pedata. $\alpha . \beta$.

pinnata.

primsulifolia.

birta.

odorata.

paluitisis.

canina.

montara.

cenifia.
Viola candenfis.

suirabilis.

biflora.

unifiora.

procumbens.

ivicolor. $\alpha$. B. $\gamma$.

grandiforia. a. $\beta$.

calcamatr.

cornuta.

Suffruticosa.

ipecacuarba.

Aricks.

palmaia.

Impatiens capeniss.

bifida.

finenfis.

latifolia.

rofmarinifolia.

Valsomina, $\infty . \quad B$.

triflora.

nolitangere.

Melicytbus ramiforras.

Acbyrantbes Aellata.

Illecebrups capitatum.

Thefium foabrum.

Gymnocarpus decasdra.

Cy ancburs parviforums.

Anabafis cretacea.

Gertian Jeptemfida.

nana.

Bupleurum Gerardi

Tordylium undolefurs.

b) Celfia Species Linno 


\section{- ) tor (}

Caucalis procumbens. aquifolium.

Seliwum Seguieri. cbabraei.

Spanautbe paricilata. Ferula tingitama. Laferpitium fimplex.

filer. gallicum. fol. praténicum. balleri. filaifoliume.

Liguficicum connubiense. Sefeli cheropbylloides.

filifoliam.

Snyrnium apiifolium. Danaa aquilegifolia. Pompinslla nigra.

Rbus excifum.

Viburnums plicatums:

Xantboxylon album.

\section{triossdrun. \\ fimplex}

Drofera'peltata

Hemanbus vaginatus.

Narcisfus vividiflorus: Alium cbomenoly.

poniculatim.

ienellum.

cilisturn.

Masfonio Jcrbora.
Lilizm maculatum. longifor um. lancifolium

Agrve virginica. Hemerocallis sndulata. Prinos lucida. Berberis canadenfis. Gisetrarda crisflifora. Rbexia ornata. Combretums decondrum, Acer dalycarpurm. Michauxia campanulata: Vaccinium bifprdulum Evica pupurafcens. nudefora. abjintboides. pilitera. feariofa. incurve. Paterfonia.

Sropolia compofita. Pasferina longifion. Polygonsm crasfifolinm. Haloragis prolurata. Quercus difcolor. fol.

$$
\text { aquatica. }
$$

Laurus cyatbifera:

Zygopbyllum proftratwors Melajtoma picta.

Cacucio coccinea. Cafearia nitida. 


\section{*) $108(-2$}

Bucida capitata.

Saxifraga decipiens.

Diantbus falinus.

Cucubalus multiflorus

Silene orcbidea.

Arenaria cespitofa.

Hortenlia japoxica.

Poupartia borbonica.

Spergula ceraftoides. Ariftotelia Macqui. Rejeda mediterranea. Eupborbia maritima.

pumila.

Jylveftris.

Blackmellia jusfieuii.

Sempervivum fedoides.

Prunus pygmaea.

Pyrus bybrida.

falicifolia. fol.

Rubus jastetus.

Geum intermedium.

Ciftus annuss.

levis.

cinereus.

linearis.

alpeftris.

ericoides.

canarienfis?

Marua arabica.

Ludia mauritiana.

Flacourtis ransontbi.
Uvaria excelfa.

Teucrium Laximanni

fibivicum.

Antirrbinwm fragans.

Digitalis lanata.

Bignonia latiflora.

Eupbrafía pumila.

Jalisburgenfis.

Salvia babliziana.

$$
\text { aufriaca. } 1,2 .
$$

Orobancbe cerulea.

Vellá tatarica.

Iheris petraa.

Thlaspi precox.

Bifcutella napiformis.

Cbetrantbus odoratus.

ruderalis.

Crambe tatarica.

Geranium anceps.

coccineum. rofeum. feropetalum:

Acalypba cordata.

Securinega duris/ma.

Robinia caragana.

Squamata. fol.

Aftragalus virefcens.

Gallega Aricta.

grandiflora.

Hypericum erberejcens. fot. 


\section{D. $D$. \\ MUSEUM NATURALIUM ACADEMI $/$ UPSALIENSIS.

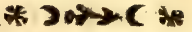 \\ C U.Jus \\ APPENDIX. VT. \\ VENIA EXP. ORD. MED. UPSAL. \\ PRÆSIDE

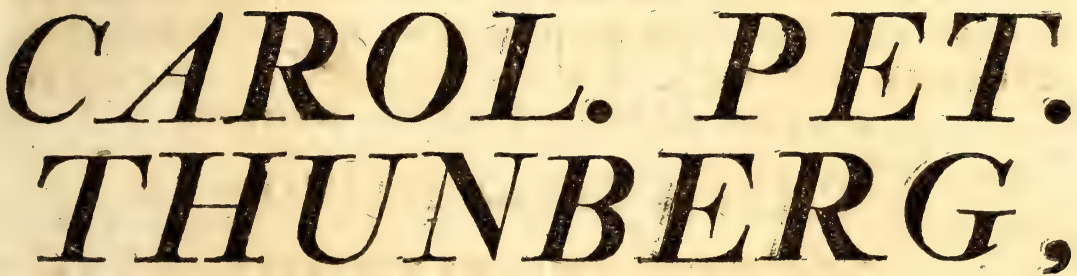

Equite Aurat. Reg. Ord. de Vasa,

Mrdic. Doct. Profess. Med. et Botan. Reg. ex Ord. Acad. Cersar. Nat. Curids. Reg. Scient, Iondin. Holm. Societ. Scient, Upsat. Philad.

Patr. Holm. Berol. Nat. Scevit. Paris. Hafniens. et Halendo. Hist. Nat. Lund, Hariem. Amsteld. Zeland. Nidrosigno. Ticinens, Ienens, Linn. Londin. Pays. Góttingens. Medic. et Nat. Studios. Edmburg. Membro; nec non Acad. Scient.

Faris. Monspeliens. Agricult. Paris. Medic. Londin.

Scandinav, Florentin. ert Batavin, Ind, Or. Correspond.

PROPON I T

fOHANNES ERICUS FORSSTROMM,

DALEKARLUS.

IN AUDIT. BOTAN. D. XXI JUNII MDCCXCVIII-

H. A. M. S.

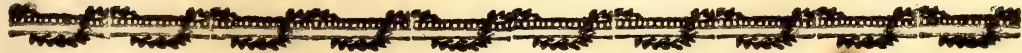

UP S A LI Æ,

Litteris JOHAN. EREDR. EDMAN, Reg. Acad. Typogr. 


\section{IN}

SACRAM REGIAM MAJESTATEM MAGN $Æ$ FIDEI VIRO,

GENEROSISSIMO ATQUE NOBILISSIMO

\section{DOMINO}

\section{GUSTAVO DE PAYKULL,}

SER. SVEC. REG. CANCELLAR. A CONSILIIS : ACAD. ET SOCIET. SCIENTIARUM STOCKHOLM. UPSAL. PETROPOL. FLORENT. SIENENS. NATUR. SCIENT. BEROL. HIST. NATUR. PARIS. ET HAFN. MEMBRO.

\section{ENTOMOLOGO SUMMO,}

\section{$\therefore \quad$ MECENATI MAXIMO.}

IN

SACRAM REGIAM MAҰESTATEM

$$
\text { MAGNE FIDEI VIRO, }
$$

FUDICI PROVINCIALI RQUISSIMO

GENEROSISSIMO ET IOBILISSIMO

DOM. ZACH. STIERNECREUTZ,

MAECENATI OPTIMO.

\section{SA CRUM.}




\section{CELEBERRIMO ATQUE EXPERIENTISSIMO}

\section{VIRO: \\ DOMINo PIRG. MART. HALL;}

M. D. PROV. VEST. MED. ORDIN.

\section{PATRONO OPTIMO!}

Ut aliquod exfaret documentum pia venerationis gratis: Simique animi ob collaia TUA in me beneficia, TIBI, qui primus Soientiam Naturalium amare me docuifti, has: pagellas facratas volui, debui

\section{CELEBERRIMI NOMINIS TUI}




\title{
HOF-PREDIKANTEN \\ $\mathrm{OCH}$ \\ KYRKOHERDEN
}

HÖGÄREVÖRDIGE OCH HÖGLÄRDE

HERR $\mathscr{O H A N ~ E S S E L I U S , ~}$

\author{
SAMT \\ COMMINISTERN \\ VÄLÄREVÖRDIGE OCH HÖGLÄRDE \\ HERR LARS RESTADIUS,
}

MINE O̊MASTE VÅLGO̊RARE!

Upptagen, Băfte Vålgo̊rare! imed vanlig godhet detta offer af den måft lifliga tackfamhet. - EDRA vålgerningar, jag vet det, fơrtjente ett vărdigare - Hvarfờre kan jag icke mer ån blott helga EDER desfa blad, min erkånfla och en fuck fo̊r EDER beftåndiga vålgång!

\section{Framhårdar att vara}

\section{MINE O̊MASTE VÂLGO̊RARES}




\section{DOINATION. THUNBERGIAN.}

APPENDIX. VI.

Simia inurs. \$. 9.

Felis pardus.

jubata.

Viverra vulgaris.

Cavia acoucbi.

Mus ampbibius.

rattus.

Camelus dromedarius.

Falco cyaneus.

Strix nivea.

Corvus lapponicus.

Pbaeton pbanicurus.

Anas albifrons.

bernicla.

clypeata. S.

fegetum.

Larus minutus.
Tringa arenaria.

Scolopax fufca.

$$
\text { calidris. }
$$

Cloaradius cedicnemus.

Glareola aufiriace.

Peneloge criftata.

Turdus rofeus,

Rana bufo.

Lacerta aqvatica.

Angvis lumbricalis.

Pleuronectes bippoglosjus.

Salmo alpinus.

Cyprinus vimba.

Cordyle 6 maculatus.

ferrugineus.

Curculio caricis. I)

articulatus.

Cur-

1) Longiroftris picens, pilis denfis brevisfimis cinerafcentibus, thorace fubrotundo elytris punctato-ftriatis, pedibus, roftro antennisque brunneis. Defcr. Caput nigro brunneum; roftrum thorace capiteque longius, incurvum, glabrum, tenue, brunneum, apice obfcurius; antenne roftro longiores, piceæ, apice fufefcentes; oculi fubimmerfi nigri. Thorax fubcylindricus, antice paulo anguftatus, villis \& fqvamulis ad latera denfioribus, cinerafcentibus. Scutellum minimum. Elytra vix thorace latiora at plus dupplo longiora, fubcylindrica, dorfo planiufculo, - leviter punctato-ftriata, picea, pilis brevisfimis cinerafcentibus, fæpe maculis 4 denudatis piceis, poftice coarCtata, declivia. Ala longitudine elytrorum, hyalinæ nervis obfcurioribus. Sternum $\mho^{3}$ Abdomen picea, pilis brevisfimis cinerafcentibus. Pedes brunnei; $\operatorname{tar} f i$ obfcuriores; femora mutica. Magn. Curc. T. album æqviparat. Locus. habitat racius in Arundine Phragmitide, ad Ángsỏn Ipfe; in Caricỉbus Veftro- 


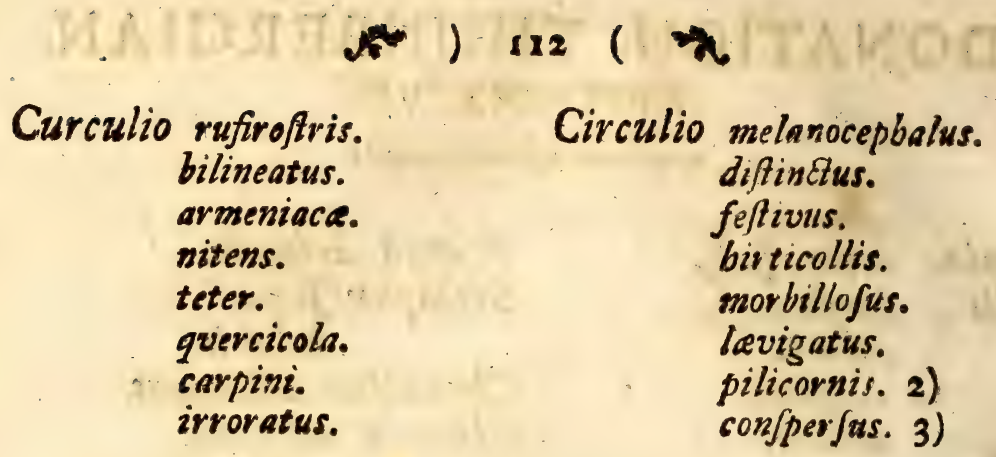

Cur:

gothiz Gyllenhal at prope Alingsås Pentz. O6\%. Differt a Pini quod quadruplo minor, roftro tenuiore, longiore; ab Arundinis quod alatus, thorace brunneo \& magnitudine duplo minori;-a Sifymbrii pedibus brunneis nec non corpore magis coaretato, elongato; \& ab omnibus hujus familize cusculionibus facillime dignofcitur corpore maxime coaretato, oblongo.

2) Breviraftris, niger cinereo-varius, elytris friato-punctatis, antennis pilofis, nigris, pedibus rufis, dentatis. Defcr. Cap. nigrum cinereo-tomentofum; roftrum thorace brevius, cylindricum apice incras. fatum, ad bafin antennarum tuberculis elevatis nigris; antenna nigræ; pilis rarioribus cinerafcentibus, tenues, capitc thoraceque duplo longiores; oculi laterales, nigri, parum prominuli. Thorax niger, cinereo-tomentofus, globofus, punctatus. Scutelinm nullum. Elytra nigra, cinereo-varia, nitide punctato-Atriata, gibba. declivia, thorace duplo latiosa at plus duplo longiora. Sternum É. Abdomen nigra, nitida. Pedes rufi, pilis rarioribus, cinereis; femora omnia acute dentata, rufa; tibioe rufæe geniculis nigris, tarfi picei, tomento denfiori cinerafcente. Magn. Curc. fere fcabri. Locus habitat in falitetis ad Alpes Tranftrandize rarisfime \& non nifi duo inveni exemplaria, quorum alterum Celeberrimo Priefidi alterum Doetori Hall communicavi. Obf. Differt a calcarato F. magnitudine elytrisque non fimpliciter fed punetato-ftriatis.

3) Breviroftris, oblongo-ovatus, obfoure cinercus, roftro carinato, thorace trilineato, elytris puncatata firiatis maculis nigris fparfis. Defcr. Cap. nigrum, pilis brevisfimis cinereis : rofirum thorace brevius, obfolete carinatum; antennce capite thoraceque breviores, rufefcentes, clava nigra; oculi nigri, parum prominuli. Thorax fubglobofus, cinereovillofus, lineis tribus albidis. Elytra thorace paulo latiora-at triplo fere longiora, punctato ftriata maculis quibusdam fparfis nigris. Sternum Co Abdomen nigra, zneo-tomentofa. Pedes cinereo-nigri villofi: fomorn mutica. Magn, Curc. Pini fere aqviparat. Locus Ad Alpes 


\section{to II3 (}

Curculio fulcifrons. 4) Attelabus penfyloanicus. Antbribus fcriptus. Bracbycerus algirus.

bimaculatus. detritus. excifus. gemm tus. globif rus. $\alpha . \beta$. inequalis. exr opacus. papillojus. pertujus. pifferus. premorfus. rugofus. Serratus. tetragonus. vacca.
Bracbycerus vaviolofus. areolintus. Scaraberus Ariatus. cornutus. 5) elivatus. bemifibaricus. bypocritus. pufillus. a. $\beta_{\text {s }}$ ater. $\alpha . \beta$. tesfulatus. didymus.

Tricbius piger. Copris irroratus. vitulus. capucinus. $\alpha . \beta$. panifcus. vandelli. S. . volvens. cedipus.

\section{A 2}

Dalecarlicas rarius hanc reperi fpeciem. Obf. Differt a Murino F. duplo anguftior roftroque carinato; a mœrente $\mathrm{P}$. roftro carinato; a geminato P. quod oblongo-ovatus \& haud globofus, elytrisque thorace triplo fere longioribus.

4) Breviroftris, cinereo-niger, antennis tibiisque rufis, fovea fronti profunde impresfa. Defcr. Cap. nigrum, punctatum; roftrum breve, latius, fovea profunde impresfa; antennae rufefcentes, longitudine capitis, primo articulo obfcuriore; oculi prominuli, nigri. Thorax-cylindricus punctatus, fetis cinerafcentibus confperfus, concolor. Elytra, thorace vix latiora at duplo longiora, fubcylindrica, ftriato - punctata, ciner fcentia. Sternum Ë Abdomen nigra, pube cinerafcente. Pedes: femora nigricantia mutica; Tibice tarfique rufefcentes. Magn. Curc. linexta dunlo minor. Locus Sudermannix ad prædium Nas pasfim. Ohf. Differt a Curc. lineato oculis prominulis, thorace concolore; a lineello Bons.D. thorace elytrisque concoloribus; a viridicolle $F$. fovea capiti impresfa.

5) Cetonia $E$. 


\section{) $114(x$}

Copris frisator?

Cetonia abdominalis. acuminata. aulica. $\alpha . \beta$ : interrupta. $\alpha . \beta_{\text {. }} \gamma_{\text {. }}$ interrogationis.

Iris.

furinama.

Melolontba plicata. pectoralis.

nitens.

lineata.

dèftai.

marginella.

Opatrum cavolinum.

lufitanicum.

Hifter picipes.

purpurafcens:

Elopborus crenatus.

pygmeeus.

Colydiumn filiforme.

Coccinella annulata.

4 punctuta.

brunnea. 6)

Boftricbus bicornis. 7)

somegrapbus.

pubejcens.

ater.

melanocepbalus.
Boftrichus vittatus.

cornutus. variabilis. 8) obfcurus: 8)

Spbaridium Aircoreum. limbatum.

Dermefles chinenfis. tesfulatus. urtice.

Nitidula flavomaculata.

Casfida 6 punctata.

2 guttata.

Spinifer.

bipuftulata.

cribravia.

Erotylus: furinamenfis:

Aücbenia: 14 punctata.

Coryfomela rugoja.

nigrita.

littoralis. 9)

brucboides.

gemellata.

pyritofia.

fervida.

Jalicis: scbacb.

friata.

Crioceris flaviconnis. 9)

Lagria: flavipes:.

Ciftela livida.

6) Rafo-brunnea immaculata fubtus pallidior. Obf. Differt ab omnibus hujus generis, tota brunnea, immaculata, mirima, fcilicet vix? magnitudinem minimi pediculi fuperans. Locus Ad radices arborum.

2) Apate. F. 8), Dermeftes. F。

9) Galeruca. Fo. 


\section{) IIs (}

Ciffela rufisollis.

Cryptoceplalus marginatus. multiguttatus.

Altica ffymbrii. eruce. falicaries.

Anobium rugofum.

rufipes.

friatum. caftaneums.

Ptinus crenatuss.

Ptilinus pectinatus.

flivejcens: myftacinus?

Trox gemmatus.

Hypulus bifafciatus.

Hijpa discolor.

Notoxus: bifafciatus.

trifafciatus:

penjylvanicus.

Ripipborus flabellatus.

Hydropbilus affinis.

bicolor.

truncatellus:.

marginellus.

Mordella ruficollis.

Carabus dentatus.

casfideus.

filpbordes:

Priatus.

violaceus:
Carabus terricola. pelidnus. asfimilis. puncticollis. teniatus. atbiops. ve:pertinus.

Scarites gagates:

Scaurus carinatus.

Meloe brevicollis:

marginatus.

Mycetopbagus fafciatus.

Hypopblicus caftaneus.

Stapbylinus poputi. excifus.

Lytta cinereas.

Cicindela littoralis: Mylabiris: crasficornis. 10) Bupreftris morio. pygmas:

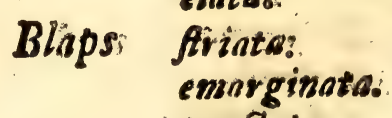
elates. punctata. Tenebrio dentipes. 11 )

firsicus. elongatus: curvipes. tibialis: 12) Cerambyx molitor. 


\section{+) $116(2)$}

Cerambyx rufipes.

Prionus laticollis. ?.

Callidium bolosericeum.

fulcratum.

Sepidium rugofurs.

Eurycbora canaliculata, II)

grandis.

Pimelia cepbalotes.

gibba.

papillofa.

tenebrarides.

rufipes.

Erodius pilula.

variolofus.

planus.

bicoftatus.

impresfus.

Dytifcus pictus.

balenfis.

2 guttatus.

Mantis precavia.

fuperfatiofa.

elongata.

grandis.

maroccans.

Cimex urtice.

fuperciliofus. 13)

punctum.

ferus $\alpha . \beta$.

Reduvius Serratus.
Reduvitus gigas.

Gerris currens.

Apbis cardui.

Chermes epilobit.

Cicada fanguinea. 14)

clandeftina.

Faponica.

tuberculata.

pini.

4 oculata.

bicincta.

coccinea.

transverfa.

trinotata.

Papilio pbilenor.

eurypiylus.

Remus.

Helena.

Vefta.

lucilla.

melas.

alcer.

Ramina.

Bombyx mali. 占. 우. argentina. $\delta$. 9 . centrolinea. $\delta . q$.

Noctua flivicincta. ulula.

Pbalana viridaria. lunaria.

Tortrix Nezeniana.

II) Helops. F.

13) Albomargiratus. F.

I4) Tettig. faugvinolenta. F. 


\section{- ) $1171 \Rightarrow$}

Tortrix fuscana.

Forfterana.

1 Jertana.

2 punctana.

mitterbacbiana.

Blomiana.

Fablbergiana.

Ljungiana.

Tinea limbella. vindella.

Libellula juncea.

Semblis viridis.

Cynips petioli. (querc.) Icbneumon quafitorius. Scbolia bortorum.

2 cincta.

2 maculata.

6 maculata. injubrica.

Tentbredo carulefcens.

$V$ espa oculata.

acuta.

pomiformis.

Mafaris apiformis.

Mutilla corenata.

Crabro 6 guttatus.

Pbilanthus coronatus.
Formica 4 punctato.

Sirex pygmaus.

Bibio fabaus.

Tipula fulvipennis.

minutisfima.

Aranea migratoria.

piratica.

angulata.

- quatica. arenaria. criftata. pallidula. paluftris. cancriformis.

Pbalangium bimaculatum. Trowbidium bolosericeums. aquaticum.

Pediculus fuis. galline. tetraanis. birundinis. porcelli. pavanis.

Cancer aculeatus. Herbp. Gaimmarus maculatus. locufta.

Scolopendra gigantes. viridis. 


\section{I.}

Quantum in Philofophia Naturali proftitit Newtonus, tantum in Hiftoria Naturali effecisfe Carolum à Linbé exiftimamus.

\section{II.}

Ex quo Hiftoria Naturalis majori cum fuccesfu excoli cœepit, rovam qvafi, eamque nobiliorem induere fibi vifa eft taciem ac formam Ars Machaonia.

\section{III.}

Plagas feptentrionales Svecix Meridionalibus esfe fertiliores Infectorum aliorumque Naturalium haud dubitamus.

\section{IV.}

Non adhuc inter Entomologos, qvantum nos quidem - novimus, ratis conftat, quic fit verus atque præcipuus ufus Antennarum Palporumque in Infectis confpiciendorum.

\section{V.}

Haud facile erit dictu ubi major confpiciatur Creator, in toto Syftemate mundano, vaftisfimo ac pæne immenfo, an in Infectis \& cryptozois, etiam minimis.

\section{VI.}

Emigrariones, quas interdum ex Alpibus Borealibus inftituunt Marmotæ Lemmi, aut inopiæ victus, ex nimia veris ficcitate profluenti, aut etiam multitudini, jam nimis alauctx adfcribendas esfe putamus. 


\section{D. $D$.}

\section{MUSEUM NATURALIUM ACADEMIE UPSALIENSIS.}

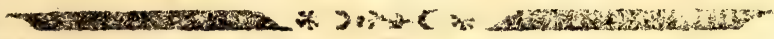

c U JUS

APPENDIX. ViI. VENIA EXP. ORD. MED. UPSAL.

\section{PRASIDE}

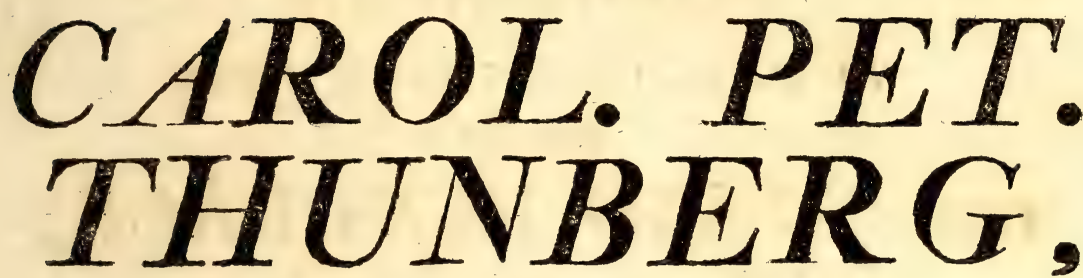

Equite Aurat. Reg. Ord. de Vasa,

Medrc. Doct. Profess. Med. ex Botan. Reg. et Ord. Acad. Cersar. Nat.

Curios. Reg. Scient. londin. Holm. Societ. Scient. Upsal. Philad. Patr. Horm. Berol. Nat. Scrut. Paris. Hafniens. et Halens. Hist. Nat. lund, Harlem. Amsteld. Zeland. Nidrosiens. Ticinens. Ienens. Linn. Londin. Phys. Góttingens. Med. Matritens. Mrdic. et Nat. Studios. Edimburg. Membro; nec non Acad. Scient. Paris. Monspeliens. Agricult. Paris. Medic. Londin. Scandinav. Florentin. at Batavin, Ind. Or. Correspond.

PROPONIT

\section{LAURENTIUS FRED. GRAVANDER,} STIP. NESSEL. WESTMANANNUS.

IN AUDIT. BOTAN. D. XI DECEMB. MDCCXCVIII.

H. A. M. S.

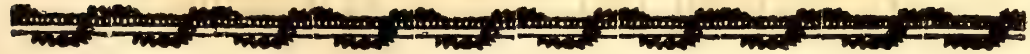

UPS A LI $Æ$,

Litteris JOHAN. EREDR. EDMAN, Reg. Acad. Typogr. 


\section{PROSTEN осH KYRKOHERDEN}

O̊FVER BJO̊RKSTA FO̊RSAMLING

NO̊GÄREVO̊RDIGE OBH HÖGLÄRDE

HERR

MAG. MICHAEL OHMAN,

\section{REGISTRATORN!}

VID KONGL. UTRIKES EXPFDITIONEN

HÖGÄDLE

HERR ARVID LUNDBÅCK,

SAMT

RAD OCH HANDELSMANNEN

ADEL OCH HÖGAGTAD

HERR JOHAN ERIC RAMSTÉN,

Af vo̊rdnad och tackfamhet tillågnadt. 


\section{DONATION. THUNBERGIAN. APPEND. VII.}

Phyllodes vaginalis. Lopezia racemola. Camocludia ilicifolia fol. Fasminum verticillatum. Veronica pyrenaica. paniculata. lacinista.

Fraxinus forifera. fol. bumilior. fol. fimplex. fol.

Circea virginica. Orchis mixta. incarnata.

Linocieria ligufrina. Anciftrum decumbers. Epidendrum coccineum. Limodorum crispatum. Cypripedium bulbofum. Salix triandria. o. ?. acuminata. fol. pyrenaica. 15 . to.

Iris. enfata. orientalis. tuberofa.

Gladiolus grandis. tenellus. levis. brevifolius. laxitus.
Gladiolus inflitus.

haftatns. cordatus. punctatus. gracilis. bracteatus. Fabricii. glumaceus. equitans. undulatus. exfcapus. fecundus. ringens. elongatus.

Ficus americana. Melotbria pendula. Anguria lobata. Cucurbita bispida. Bryonia umbellata.

bifpida. birfuta. laciniofa.

Scbonus reftioides. Scirpus micbelii. caricis. natans. trifpicatus.

Carex obtufata. interisedia. 


\section{(.120)}

Carex curta.

pracox.

indica.

axillaris-

argentea.

alpina.

maritima.

ampullacea.

divulfa.

juncifolia.

Cyperus marginatus.

pumilio.

pangorei.

fapigiatus.

tuberojus.

brizoides.

Nardus indica.

\section{giganteus.}

Coix triticea.

Ariftide capen/is.

Andropogon infulare.

Aire involucrata.

POB nemoralis.

glauca.

ciliaris. $\alpha=\delta$.

Feftuca decumbers.

fufca.

pratenfis.

interrupta.

Dactylis cespitofa. I)

Spartina paspaloides.

Stipa avenacea.
Stipa copillacer. jurcea. Spicata. pennata. tenax. capenfis. ariftati.

Antbifteria imberbis. rimofa.

Rottboellia monandra.

procera.

Acharia tragodes.

Agipbila arborefcens.

martinicenfis.

Rubia angufifolia.

Scabiofa crenata.

Embotbrium buxifolium.....

fericeum. critbmifolium:

Hedyotis mavitima.

Protea apifolia.

Urtica rugofa.

Betula vilcofa.

Brucea ferruginea.

Potamogeton beteropbyllum.

perfoliatum.

Ilex obcordata. fol.

Myofotis palufris.

Sprengelia incarnata.

Solanum aculeatisfimum.

Ardifia elliptica. ovata.

I) Dactylis glomerata e. muf. cat. 


\section{( I 2 I)}

Diosma alva.

obtufata.

virgata.

pectinata.

bifulca.

cupresfina.

latifolia.

glantulosa.

orbicularis.

Pharnaceum Spatbulatum.

Imbricaria cilinta.

Cisenopodium lacidum.

Commelina polysama.

Narcisfus autumnalis.

Hyacintbus romanus.

Allium triquetrum.

Rumex paludofus.

Beckea denfifolia.

Epilobiuin grandifolium.

Laurus cyitbifera.

Strcemia glináulofa.

Styrax glabrum.

Cafalpinia brafiliana.

Stellaria bumifufa.

Cucubalus parriforus.

Arenaria felligera.

Euphorbia beteropbylla

Forkohlea tenux.

Aponggeton montacbyum.

$$
\text { diffucbyum. }
$$

Toxicodendrum cupene. Rofa mofcbata.
Myrtus tomentosa.

Begonia bumilis.

Cara:pa latifolia. fol.

Eucalyptus corymbofa.

capitellata.

obliqua.

piperita.

Fabricis myrtifolia.

Melaieuca ambigua.

flavescens.

lanigera.

leucadendra. $\mathbf{x}$.

linarifolia.

lucida. fol.

perforata.

fcoparia.

virgata.

Metro/ideros anguftifolia.

capitata.

coliata.

coftate.

diffura.

florida.

bijpida.

lanceolata.

aeftuofa.

Weftringia Rosmariniformis.

Teucrium faxatile.

aurerm.

verticillatum.

likanotis.

Filficia can jcens.

Ruella patala. 


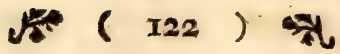

Scutellaria pilesa.

Orobanclée exigua.

Monarda ciliata.

Leonurus crispus.

Pedicularis incarnata.

Lepidium crispum.

Thlafpi peregrinum.

Altbea cbinen/ss.

Oxalis afinina.

Pultenea Aipularis.

Polygala cordifolia. $\alpha$. B. tomentosa. violacea.

Amerimnon ebenus. ASpalatbus aculeatai.

acuminata.

armata.

bracteata.

cephalotes.

comoja.

divaricata.

flexuofa.

bispida.

inconta.

incurva.

juniperina.

lactea.

lanata.

multiflora.

niver.

obtufata.

pinea.

pungens.
Ajpalatbus rubens.

rugola.

spicata.

Spinefeens.

fquarrofa.

trigona.

triquetra.

villoja.

virgata.

vulnerans.

affinis.

fangvinea.

Crotolaria elongata.

lanata.

lineata.

parvifolia.

pilofa.

reflexa.

tomentola.

villofa.

volubilis.

Perfonia carnofa.

Dedomannia lancea.

Wiborgia obcordata.

fufca.

fericea.

Lebeckia pungens.

armata.

denfa.

bumilis.

Rafnia angulata.

axillaris.

diffusa. 
(123) (12

Rafnia elliptica. erecta.

Liparia capitata. teres. tecta. veftita.

Glycine glandulosa. totti.

beteropbylla. erecta. fecunda.

Hedy farum squarrofum. cilintum. $\alpha . \beta$. cordatum. imbricatum.

Ononis Spicats. birfuta. micropbylla. capillaris. elongata. decumbens. excija. racemosa. umbellata. glabra: involucrata. quinata. lagopus. fafciculata. Indigofera filifolia. depresfa. iscana. capillaris.
Colutea proffrata. pubescens. obtufata. linearis. tomentofa.

Galega fericen. friata. mucronata. bumilis. pinnata. capen/is.

PSoralea mucromata. triflora. tomentofa. involucrata. argentea. Ariata. Ancbydis.

Trifolium ftipulacerm. bir futum. lanatum. diffufum. aureum

Trigonella glabra. tomentofa. birfuta. villosa. armata.

Fumaria decumbens. incifa. racemsofa. pallida. Aftragalus poterium. tauricus. 


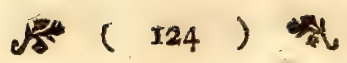

Hypericum verticillatun. Stobaa carlinoides.

Syrnpbonia globulifera.

Lap Jana crispa.

Robria fulcata.

bifitca.

pectinata.

patula.

Squ irrofa.

lanceulata.

decurrens.

lanata.

rigida.

betberopbylla.

pinnata.

pinnatifida.

Scorzonera capen/is.

Ethulia auriculata.

Jetofa.

bulpida.

ciliaris.

obovata.

cuneata.

incana.

fpinofisfima.

monuntbos.

armatu.

pungens.

carlinoides.

grandiflora.

cruciata.

decurrens.

palmata.

Coreopfis formofa.

Zinnia revoluta.

Senecio lividus.

Centaurea ferox.

Hyoferis tenella.

Crepis Ariata.

Soncbus umbeltifer.

glaber.

Stobea glubrata.

Cacalia arbujcula.

Chrofocoma microphylla.

Euputorium retrofraetum.

Schousboea crenota.

Pteronia tomentola.

paniculata.

glauca.

ecbinata.

cilista.

Atbanafia flexuofa.

tomentofa.

glabra.

alpera.

pectinata.

Polypodium femina.

Poiytricbum arficum.

feptentrionale.

Dicranum osmundoides.

secundum. rigidulum. paludofunt. fcbreberianum. longifolium. 


\section{( 125$)$ 米}

\section{Meefis uliginofa. dealbata.}

Didjmod on caeruleum. Gynnoftomum Heimii. Otbotricbum cryfodon. Fryum pallens. avidrogynum. Hypnum Myofuroides. implexum. vivulare. rufefcens. nigricans.
Hypnum rufciforme. revolvens. precox.

Tortula. tortuoja. curta.

Fontinalis fquamsosa. Licben Squamo/us. diffufus. cinereus.

Boletus alni. Clavaria pratenfis. Spbieria gregaria.

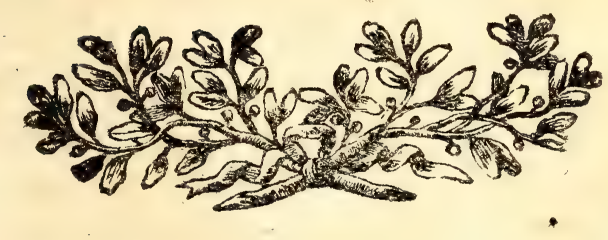




\section{THESES.}

I.

Corium Cervi tarandi crasfitudine \& firmitate multis præcellens in Oeconomia ufum præaliis commendandum præftat.

II.

Oestrorum ova in dorfum Cervi tarandi di. misfa crebris aqux lavacris ablui posfe, atque fic in ipfis incunabulis esfe intermoritura credimus; fi autem hoc non fuffecerit, omnia ulcera crasfiori \& graveolenti quodam unguento, quod caloris aërisque aditum arceat, obliniantur, fic ut Larvæ feu Pupæ in ipfis latentes explicari non poterint.

\section{III.}

In omnibus plantarum defcriptionibus, quæ com. munem ufum Oeconomicum fpeetant, femper \& præcipue adhibendi funt Termini Botanica civitate donati. IV.

Adcuratx provinciarum per patriam defcriptiones, ad ufum potius quam admirationem disfpofitæ ad exemplum defcriptionum Oeconomicarum, quas fuscepit \& jam ferme perfecit Societas Londinenfis Pro . Agricultura (Board of Agriculture) non folum Hiftoriæ Naturali; fed etiam Oeconomiæ, publicæ non minus quam privatæ, maximam lucem adfunderent. $\mathrm{V}$.

Is Vulgi doctor vere falutatur, qui populum docet non tantum in Oraculis Divinis fidei fulcra malorumque folatia quarere; fed etiam mundi fummum Rectorem in rerum natura benevolum, magnum \& admirandum discipulis proponit, feduloque adhortatur, ut ipfum, Moralitatis fupremum Judicem, fincere venerentur. 


\section{D.}

\section{MUSEUM NATURALIUM ACADLMIE UPSALIENSIS.}

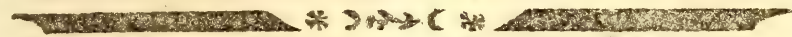

C. U JUS

Appendix Virr.

VENIA EXPER. ORD. MED. UPSAL。 PRESIDE

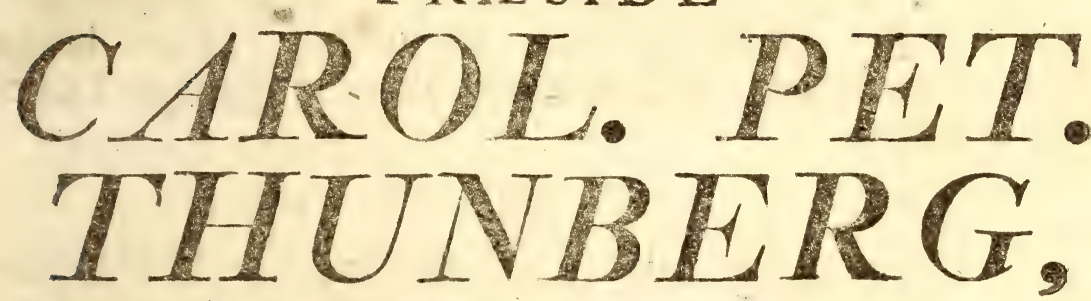

Equitg Aurat. Reg. ORd, de Vasa,

Medic. Doct. Profess. Med e: Botan. Reg. ext Ord. Acad. Chasar. Nat. Curios. Reg. Scient. Londin Horm. Societ. Scient. Uzsat. Phitado Patr. Hoim. et Civ. Berol. Nat. Scrut. Paris. Hafno er Haleng. Hist. Nat. Lund. Hariem. Amsteld. Zeiand. Nidrosieng.

Ticinens. Isnens. Linn Iondin. Phys. Góttingens. Med. Matritens. Medic et Nat. Studios. Edimburg. Membro; mec non Acad. Scient. Paris. Monspetiens. Agricuit. Paris. Menic. Londin. Scandinav. Fiorentin. ext BataVin。 Ind, OR。 Correspond。

PROPONIT

ERICUS MAGNUS JUHLIN WESTMANNUS. IN AUDIT. BOTAN. D. 12 NOVEMB. MDCCC. H. A. M. S.

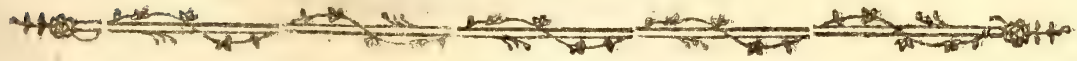
U P S A L I $\mathbb{E}$.

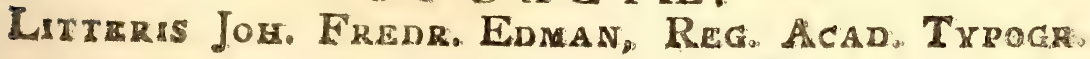




\section{THESES. \\ I.}

Cibariorum penuria vix laboraret patria, fi, alias præter fruges, Solano tuberofo frequentiorem nava. rent operam agricolæ.

\section{II.}

Quandoquidem ad vitam fuftentandam confervandamque, tot tamque varias conferat vires Regnum vegetabile, patriæ utique confultum foret quam optime, fi Botanicam quoque fcientiam, Floram faltim patriæ familiarem fibi redderet futurus quisque Ecclefix Doetor.

\section{III.}

Regnum vegetabile, animali uberiorem Medicinæ materiem præbere, eft, quod quotidiana docet experientia.

\section{IV.}

Quod ufus Nicntianæ tabaci famem pellat, in causfa non eft infita quædam alendi vis, fed inter alias etjam evactatio liquoris, appetitum excitantis.

V.

Poft folidam Scientix Machaonicx cognitionem, prudens erga ægrotos familiaritas, Medico, ut felix fit praxis, maxime eft commendanda. 


\section{DONATION. THUNBERGIAN.}

\section{APPEND. VIII.}

Salicornia indica. a. B.

Hellenia allugbas.

Fraxinus parvifolia.

Serapias lingus.

Valeriana coronata.

$$
\text { difcorder. }
$$

radiata.

Iris Faponice.

Lafling is pentandra. Picus jycomorus. fol. Anguria pedata. flos. Bryonia palmata. cordifolin.

Carex buxbanmii. salligona. clandeftira. conglobata. difticba. fulva. glaucefcens. bamata. leonuroides. Iittoralis. lucida. mutabilis. penfylvanica. pyrenaica. riparis.
Carex fisenfis. cusvata. tepbroftacbys.

Scirpus bybridus. Kyllingia incorspleta. Paspalran dipicbuns. longeforum.

Pbalaris nodofa. Maluupn muritimums. paradoxum.

Syena fluviatilis. Andropogon muricatsm. Agroftis verticillata. Chloris barbata. Egilops caudata. Pois lata. vijcoja. Fefucs terella. aleopecurus. pbleoides.

Saccbarum cylindricum. joponicuras.

Dactylis Arinta. Bromus hifpanica. Mipoides. Avena tuberofa. 


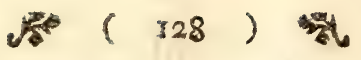

Avena penfylvanica.

Rbamnus ellipticus.

Pic usengia pentandra. $\delta$

napar.

Carta as ramiflorus.

Berula glatinofa. fol.

Eleagnus lutifolia fol.

Coiylas colurna. fol.

Cyroglos Jum patum.

Ecbium orzentale.

Convolvulus japonicus.

Cordza coromandelica.

Solanum radicaiss.

Verbafcum coromandelicum.

1 pomcea guamoclit.

Lycium Bcerbaviafolium.

Ceanotbus japonicus.

Bladbia glabra.

Raamolfia tomentofa.

Varronia filiformis.

Rondeletia birta.

Lonicera flexwosa.

Lobelia denfa.

radicans.

acaulis.

campanuloides.

Campanula pufilla.

Pbylica fecunda.

Rbannus oleoides.

pumilus.

igvaneus. fol.

Celajtrus dilatatus.

Gardenia mitis. I)

Scavola Kanigii.

Impatiens baccifra.

$V$ itis flexuofa.

Gronovia feandens.

Illecebrum lunatums.

Amaranthus deflexus. cbloroft acbys.

Cressa indica.

Gompbrena brafilienfis. 1. 2. Gentiana glacialis. beteroclita. tenera.

Hydrocotyle umbellata. triloba.

Bupleurum Biliardicri.

Daucus divaricatus.

Pevcedanum alpeftre.

Bubon rigidius.

Tordylium apulum.

Thapfia fatida. fol.

Caucalis arvenfis.

nodiflora.

Laserpitium capenfe.

latifolium.

trilobum.

ferulaceum.

I) Randia mitis, 
Piftacia vera. of.

Viburnum acerifoliusn. alnifolium.

Clutia polygonoides. $\delta$. ‥ Bafella cordifolia. Aralia japonica.

Statice trigona. allincea.

Tamarix articulata.

Linum vifcofums.

Crasfula perforata.

Commelina gersiculata.

Lacbenalia rubida.

Ornitbogalum rupeftre. a. B.

Eucomis nana.

undulata.

Convallaria japonica.

Sculla orientalis.

Pbremix guineen/s. for. 2.)

Afparagus japonicus.

Funcus lusuloides. fetaceus. gracilis.

nutans.

Rumex tataricus. undulatus.

Smilax purpurata. Ariftolocbia bilobata. Erica verficolor.
Erica longifora. follicularis. lituiflora. guttofiora. pyramidalis. penicillifornis. campanulatis. fragrans. verecunda. terminalis. taxifolia. Arigoja. juniperifolia. pburetraformis. jasminiffora. vacciniflera. bolojericea. gracilis. fabra. transpavens. glabella. margaritacea.

Oenotbera villosa. Epilobiumn villofum. Melicope ternata. Daphne sericea.

Gnidia levigata. implex. Acer dafycarpon. ठ. +. opulus. fol.

\section{A 2}

2) Eleis. 


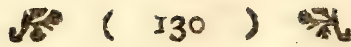

Polygonum Arigofum.

canaden e.

Ousercus valentina.

Ceralopetalum gummifermm. Zygopby!lus fotidurs.

Limonia pentuphylla.

Samyda oefandra.

Silene geniculata.

Spergula Arizta. a. $\beta$.

japnnica.

Turrea vivens.

Aponogeton crispum.

Basfía latifolia.

Euphorbia gerardiama.

Glinus Jetiflorus.

Forfkoblea armata.

candida.

Triumfetta nummularis.

toment ofa.

Rofa inersis.

Rubus villofus.

Mimofa intria. fol.

purpures fol.

gliucis fol.

connigera fol.

peregrana.

fenegualenfis.

r.bombifolia.

finmlex.

difcolor.

cinerea.
Capparis torulofa.

Sioanea dentata?

Carazpe latifolia. fol.

Prockia crucis.

Arum tenuifolium.

Uvaria javanica.

Aneynone cernua.

Clematis trifoliata. fol. jayanica fol.

Tbalictrum lucidurs.

Arum divaricatunis.

Ciftus birfutus.

ferratus.

Sideritis bifpernica.

Ocymsin reflexum.

Salvir cretica.

urticufolis.

Gratiola cumiloides.

Evploralia triculpidata.

Selago fricti.

Jufsicia lancea.

mollabarica.

ecbioides.

bratieats.

Astitivbinum macrocarpon.

Ruellia balfasmina.

Bignonia crucigera.

Scutellaria bavanenfis.

$$
\text { integrifolia. }
$$

Citbarexylon cinerewm. 


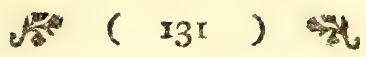

Draba nemoralis. contorta.

Bifcutella lyrata.

Crambe fitiformis.

Arabis thatiana a $-\delta$.

Cheirantbus parviflorus.

Sinapis bifpida. alba.

Geramiun bicolor.

fesbrum.

acuminatum.

Oxalis pulcbella. obtula canefcens cuieata. dentata. grasdiflora.

bumilis. loxula livida. Speciofa. fulpburea. tubiflora. variabilis. verficolor.

Pentapetes poranicea.

Fatropba glauca. fuberifolia. Pbyllantbus rbamnoides. Sterculia javonica. Croton Pentzii. lobatum.
Sida triloba. $\alpha . \beta$. dioica.

Hivifcus pufllus. Gosjypinus.

Malva grosfularifolia. tridactylites. verofa. Melosbia Jupina. Fumaria tenuifolis. Cytifus glaber. Hallia lanceolata. alata. flaccida. Apolatbus cinerco. fufca. siletine. lavicifolia.

Polygala amcena. parvifora. filiformis. pbylicoides. micrantbss. laxa. Priats.

Rafnia arsgufifolit. oppofita.

Ononis beteropbylla. micrantbus. Jecunda. Jerices. Ripulatr. Arigofa. 


\section{然 (132) क्षै}

Boibonia perforata. Hypocalyptus cordatus.

calyptratus. jericeus. glincus. cane/cens.

Robiniapygmea.

Dulichos decumbens. gibbofus.

Hedyfarum exigum.

$$
\text { repens. }
$$

Indigofera trita. punctata.

Liparia villefa. griminifolia. birfuta. tomentofa. umbellifera.

Colutea rigida.

$$
\begin{aligned}
& \text { veficaria. } \\
& \text { excifa. }
\end{aligned}
$$

Galega filiformis.

$$
\text { totta. }
$$

PSoralea racemofa. bractenta.

Phofeolus caracalla. fol. Aftragalus cbikenfis.

Pbaca frigida. carolinianus.

$$
\text { betica. }
$$

Orobus birfutus. Trifolium mauritanicum.
Irifolinm officinale. alpinum. procumbers.

Lotus corniculate. Medicago birjuta. Soncbus polufiris. Carduus vigens. Robria cynaroides. Heracium belveticum. undulatum.

Hyoferis bijpida.

Cicborium divaricatum.

Onopordon macrocantbum.

Cynara glomerata. jcolynus.

Cbryfocoma undulata. Evpatoriusm rigidum. nervofum. odornatuln.

Pteronia faftigiata. aspern. Atbanafia punctata. Relbania reflexa. fantolinoides. pungens. trinervis. pinnats.

Stabelina fafciculata. Tarcbonantbus ellipticus. Artemifia tanacetifolia. Tancetum axillare. 


\section{\%(133) 稀}

Tanacetum grandiforum.

multiflorum.

obtuf turss.

juffruticofum.

Crepis puichra.

Baccharis neriafolia. for.

Cocoplis alua.

Gnupbaliuns adjcendens.

afperum. aurisulatum. capillaceums.

capicellatusn. cephalotes.

cerinutus.

debile.

dealbatum.

decumbens.

divaricatum.

divergens.

erofum.

excijum.

expanfum. \&. $\beta$.

fafciculatum.

faftigiatum.

felivum. a. $\beta$.

frutefcens.

beteropbyllum.

birfutum.

bumale.

javanicum.

moculatum.

maritimum.

micrantbum.

molle. niveum.

odotaluras.

orbiculare.

polyantbos.

profiratum.

pulilum.

pygmeam.

quinguenerve.

revolutum.

rotundifolium.

rubellum.

fanguincum.

fabrum.

feripbioides.

ferrntums.

fplendidun.

Acebelinoides.

Arigofum.

tinctum.

tricoltatum.

trifiduns.

veftitum.

undulatum.

Xerantbemum canefcens.

lancifolium.

radicans.

Atoloniferum.

friatum.

Conyza canefcens.

fordida.

Erigeron birtum.

japonicum.

incifunte. 


\section{(134) ( 34}

Eirigeron pismatifidum.

$$
\text { foabrum. }
$$

Inula cotuloides.

Arnica crenata.

$$
\begin{aligned}
& \text { Jerrata. } \\
& \text { grandis. } \\
& \text { laxata. } \\
& \text { alpina. }
\end{aligned}
$$

Tusfilago cernua.

Dorna bipinnata.

$$
\begin{aligned}
& \text { ernfa. } \\
& \text { incifa. } \\
& \text { nivea. } \\
& \text { pinnatifida. } \\
& \text { ferrata. } \\
& \text { undulata. }
\end{aligned}
$$

Cineraria angulata.

$$
\text { aspera }
$$$$
\text { auriculata. }
$$

Cineraria cordifolia.

coronate.

pandurata.

Facobea reclinata:

onguftifolia.

mucronata.

nivea.

paniculats.

bidentata.

Scabra.

veltuta.

finensis.
Jacobea tomentofa.

Senecio alpinus. criftatus. grandiflorus. muric stus. quercifolius. foldaginoides. Ariatus. breftatus. pjeulocbinenfs.

After crinitus.

cymbalarie. dentatus. diffufus. divergens. elongatus. beteropbyllus. birtus. macrorbizus. muricatus. paniculatus. rotundifolius. a. $\beta$. jerratus. Atrigofus. villofus. obtufatus.

Artbernis cbia. cretica. cotula.

Leyfere callicornia. ciliata. ericoides. 


\section{( 135$)$ 林}

Leysera ounta.

picta.

pilosella.

incana.

Cotula bipinata.

$$
\text { filifolin. }
$$$$
\text { globifera. }
$$

minuta.

nudicaulis

pufilla.

quinquefida.

tripinnata.

Lidbeckia bipinnata.

Rofenia glandulofa.

Bupbtbalmum beliantboides.

Verbefina fruticofa.

Lavenia vijcofa.

Ofmites dentata.

Lapeiroufia calycira.

Silpbium jolidaginoides.

Oedera birta.

Arciotis elegans.

formofs.

incifa.

muricata.

nodofa.

nudicaulis.

- pectinata.

fcapigera.

trifida.

undulata.

critbmoides.
Otbonnd pinmata.

Perdicium nervofum.

Eriocepbalus glaber.

Calendula aspera.

cunenta.

decurvens.

glabrata.

bifpids.

muricata.

pismata.

foabra.

Gundelia Tournefortii.

Cesataurea agyptiaca.

ofeofpermum bidens.

bitinnatum.

filiforme.

birfutturs.

incomum.

junceum.

Scabrumb.

jpinefcens.

teretifolium.

Stcobe afpera.

incana.

siver.

pbylicoides.

Corymbium birtum.

Equifetum alpinums.

telmateia.

Lycopodium javanicums.

obfcurwm.

depresfum.

B 


\section{(136)}

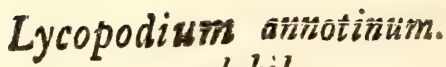
volubile. Acrofticburn birtum. byperboreum. alicorne. $\alpha-\gamma$. furcatum. quercifolium. auritum. $\alpha . \beta$. arifolium.

Ajplenium cordatum. viride. decusjatum. acroltrichuides. ambiguแm. auritum.

compresfum.

falcatum.

furcatums.

Polypodium polycarpon.

ftigmo zum. fellatum. curvatum. teniatum. triferiale. alpirum. ioncbidioides. biferratum. pteroides. disfeEtum. obtufatum. appendiculatnm. $a-\delta$. lobatum. bijpidum.
Polypodium dentatum. fontanum.

Woodwardia orientalis.

Onoclea capenfis. Ofmunda cordata, I'teris auriculata. confluens. argentea. attemuata. tripartita. culpidata. fabellata. incija. tabularis.

Polypodium tottum. Adiantum anviculatum. parvilobum. lacerum. caffrorum. pteroides. fragrans.

Trichomanes incifum. radicars.

Davallia contigua. acuminata. aurita. elegans. multifida tenuifolia.

Danea cuspidata. Lind fea fagittat . Vittaria enfiformis. 


\section{( 137 ) 秋}

Hymenopbylium clavatum. Lichen parallelus.

undulatuns.

birfutum.

emarginatum.

denticulatum.

Fungermannia podopbylla.

Tricboftomum bypnoides.

Hypnum lucens.

applenioides.

Aellatum.

nitens.

atrovirens.

illecebrum.

lucidums.

cordifoliums.

connsutatum.

diaftropbyllu:s.

Weisfia pufilla.

Tortula tenuis.

Bartbramia latifolia.

Dicranums Spurium.

falcatum.

Bryum marcbicum.

elongatum.

Polytricbum pumilum.

Licben albus.

excavatus.

monocarpus.

divavicatus.

filiformis.

belopberus.

notbus.

fellatus. diapbanus. finuofus. macularis. pulveratus. Spurcatus. elatinus. epipbegus. epipaftus. rufefcens. fuscus. leptaleus. bepatizon. antbelinus. aipolius. ulotbrix. diatrypus. diffractus. lanuginofus. vivellus. limofus. vespertilio. flaccidus. fcotimus. lacerus. marginalis. tunceformis. Jpurius. collinus. filula. bellidiflorus. pleolepis. 


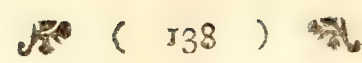

Lichen parecbus.

cervicormis.

belodium.

tupbinatus.

ventricofirs.

digitatus.

deforisis.

caftaneus.

latebrarum.

coscodes.

ropeus.

variegatus.

melalevcus.

epidermidis.

atomarins.

genomatus.

diapbanus.

congetius.

vulgatus.

rubellus.

litterellus.

jerpentinus.

dijcoides.

agelaus.

gibbofus.

oroftbeus.

Ebrbartionus.

Epanous.

luteus.

luteolus.

eryfabe.

decolordius.

vofellus.

impolitus.
Licben Dillenianus. fulphareus. lopicidus. tepbromelus. nomylaceus. parafemus. fophodes. grumsofus. cinereo-fu/cus. cyatboides. cacionufus. uliginofus. mifcellus. filiaceus. incarnatus. clivellus. byperellus. corynellus. gonatodes. cereolus. concolor. glaucocarpus. leucopbraus. bracteatus.

Fuczus acirarius. Boletus verficolor. Clavaria capenfis. fallax. fragilis.

Spharia tremelloides. devifa. difciformis. Ecidium nnemones. 


\section{D.}

\section{MUSEUM NATURALIUM ACADEMIE UPSALIENSIS.}

C U J US

Appendic. IX.

DENIA EXP. FAC. MEDICA UPSAL,

\section{PR R I DE}
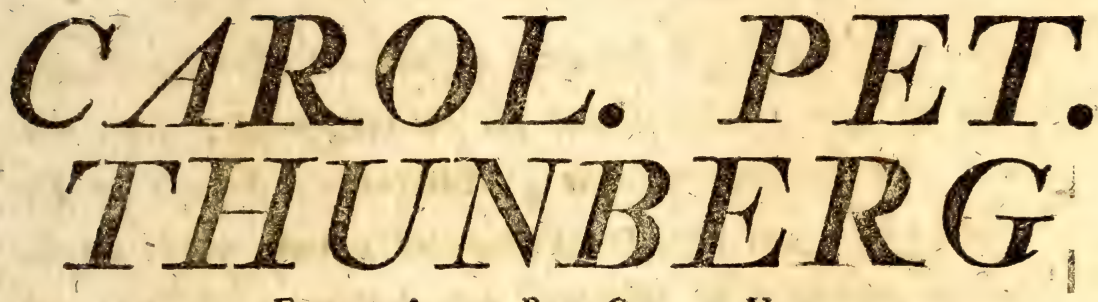

Equite Aurat. Rzg. Ord, de Vasa,

Med. Doct. Prof. Med. ex Bot. Reg. ex Ord. Acad. Cesar. Petrofol. et Nat. Curios. Reg. Scient, Lond. Holm. Societ. Scient, Ups. Piallad. Patr. Holm, et Civ, Oecon. Fint. Berol. Nat. Scrut. Paris. Hafin. Moscou. ex Halens. Hist. Nat. Lund, Harlem. A msteid. Zeland. Nidrosiens. Ticinens, Imnens. Linn. Londin. Reg. Scient. Pirys. ex Phytouraph. Góttingens. Nanciens, Med. Monsper. Med. Matritens, Medic. et Nat. Studios. Edimburg. Membro nec non Acad. Screntr. Paris. ett Institut. Nation. Monspeliens. Agricult. Paris. Medic. Londin. Scandinav. Florentin, ey Batavin, Ind, Or. Correspond.

PROPONIT

\section{CAROLUS ERICUS WULF}

Stip. Reg. Sud. Neric.

IN AUDIT. BOTANICO SUP. D. ITI APR. MDCCCVI.

H. A. M. S. 


\section{$T I L Z$}

$$
\text { KNWFRU }
$$

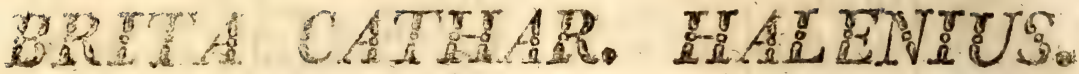

\section{HOGADLA FRU!}

Med glädje iasittager jag detta tillfälle, att offenteligen yttra: min vürdnad ooi tackfainhet för do välgerningar jag, under mist flerariga viffande $i$ Edert huis, atnjutit $-D_{0}$ öka vigten, neve lïtta verkftälligheten af min pligt, att bidraga till Er Sons bildning, och derigenon till hans och: Eder oßkiljaktiga Saillhet:

\section{HOGADLA FRUNS}




\section{DONATION. THUNBERGIAN.}

A P PEND, IX.

Hippuris vulgaris. $\beta$.

Laciftema myricoides. $\beta$.

Blitum tataricum.

Zannichellia paluftris. $\beta$.

Cavolinia indica.

Fafminum verticillatum. $\beta$. '

Olea europera. $\gamma$. $\delta$.

Chionanthes compacta. $\beta$.

Syringa chinergis.

laciniata: perfica.

Font anefia phillyreoides.

Pingvicula alpina. $\beta$.

Utricularia humilis.

media:

vulgaris. $\beta$.

Wulfenia ageria.

Veronica acinifolia. $\gamma \cdot \delta_{0}$ \&. alpina. $\beta$.

arvenfis. $\beta$. auftriacm. $\gamma . \delta_{.} \varepsilon$. beccabunga. B. $\gamma$. hederifolia. $\gamma$. integrifolia. latifolia. $\beta$.

maritima. $\alpha . \beta . \gamma . \delta$. multifida. $\beta . \gamma . \delta$. paniculata. $\beta$.
Veronica peduncularis.

peregrina. $\beta$.

pimnata. $\gamma$.

ponse.

proftrata. $\beta$.

foutellata. $\beta$.

fibirica. $\beta$.

Spicata: bifida.

Spuria. $\beta$.

teucrium. $\gamma$.

triphylla. $\gamma$.

Anciftrum repens.

Spicatum.

Fraxinus heterophylla. fol.

Scabra. t. 2.

Coftus jpeciofus.

Amomum curcuma. $\gamma$.

Malaxis cordata. $\gamma$. umbellifera. $\beta$.

Lepanthes cochlearifolia.

cornicina. pulchella. tridentata.

Stelis micranthus. ophioglosfoides.

Dendrobium myofurus. polytachyum, a. B. 
Epiderdrum anceps.

bifidum.

Cymbidium enfifolism. $\beta$. equitans.

Neottic cernua.

Ophrys myodes. d. \&. ?. ovata. B. $\gamma . \delta$

Orchis bifolia. $\beta$.

brevicornis. conopsea. $\gamma_{0} \delta$ denfiflora. hijpida. $\beta$. latifotia. B. allia.

maculata. $\hat{\beta}$. $\gamma$ rubra.

mascula. $\beta$.

militaris. $\delta$. .

monorhiza.

odorata. $\beta . \gamma$

Satyrium orecitum.

Epipactis pallens.

$$
\text { viride. } \gamma . \delta
$$

serapias enfifolia. paluftris.

Salis alba. I. B.

$$
\text { \& } \beta \text {. }
$$

amygdalina. fol. to 2. arenaria of $\gamma$

babylonica. ?.

cinerea. \&.

fis $\int \mathrm{a}$. ․

fragilis cum ftipulis. 2. $\gamma . \delta$.
Salix

Ofyris

Phoenix dactylifera. 9.

Elate 1. 2. 3.

Rotala verticillaris.

Ortegie dichotoma.

hifpanica. $\beta$ \%

Loeflingia hifpanica. $\beta$.

Boerhavia chinenfis. $\beta$.

diffufa. B. $\gamma$.

scandens. $\beta$.

Valeriana calcitrapa. $\delta$.

olitoria. $\alpha . \beta$.

rubra. $\gamma . \delta$.

ruthenica. a. B. $\gamma$.

faxatilis. 2. 2. 2.

fibirica. $\beta$.

Crocus officinalis. $\gamma, \delta . \varepsilon . \zeta$.

Moraa carulea.

chinerifis. 2. 3.

plicata. B.

Atriata. 1.2.

Wachendorfia paniculata. $\beta$. Iris biflora. $\% . \delta$. 


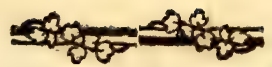

Iris crifata. caulis. \&. $\varepsilon$. germanica. $\beta$. graminea. $\delta$. ochroleuca. B. plumaria. $\beta$. pumila. $\beta$.

fibirica. Afyrinchium. $\beta$.

Spathulata.

verna.

virginica. $\beta$.

xiphium. $\beta$.

Ixia bicolor. $\alpha$. $\beta$.

elliptica.

feneftrata.

frograns. 1. 2. 3. 4.

maculata: coccinea.1.2. viridis, t.2.

radians.

Squatida.

Gladiolus brevifolius. $\beta$.

marginatus.

merianus. $\beta$.

vingens. a. .. S. (B.y.d.)

secnudus.

zundulatus. $\beta . \gamma_{0} \delta$. Watfonius. $\beta$.

Antholyza cethiopica. $\beta$. cunonia. $\beta$. plicata. B.

Bryonia angulata. $\beta$. cordata.
Bryonia hifpida. $\beta$.

laciniofa. B.

racemofa. B.

fcabra. $\beta$.

Momordica trifolia. $\beta$.

Cucumis flexuofus. B.

hifpida. B.

Ficus afpera. B.

erecta. $\beta$.

pumila. $\beta$.

Carex acuta. $\beta . \gamma . \delta$.

ampullacea. $\beta . \gamma$.

aquatilis.

atrata.

Bellardi.

cane/cens. $\beta$.

chordorrhiza. $\beta_{0} \gamma_{0}$

curta. $\beta$.

curvula.

cuspidata.

Davalliana.

difans. $\beta$.

duriufcula.

echinata.

flova. *. B. $\gamma$.

flavefsens.

fulva. B.

frigida.

gibba. $\alpha . \beta$.

glareofa.

globularis. $\beta$.

intermedia. $\beta . \gamma . \delta$. 
Carex, irrigua.

lagopoides.

Iaxa.

linnofa.

littoralis. $\beta$.

livida.

loliacea. $\gamma_{0}$

maritima.

microglochin.

smicroftachya. $\beta$.

mucronata.

nardifolia.

migricans.

norvegica.

ovalis.

pailefcens. B.

paludofa.

panicea. $\beta . \gamma$.

paradoxa.

pedata. $\alpha . \beta$.

penfylvanica. $\beta$.

petreca.

pilulifera. B.

raphioftachys.

rariflora.

repens.

rotundata.

falina.

Spicata.

ftricta. a. B.

tenuiflora.

schoenus aggregatus.

cladium.
Schoenus corymbofus. marifcus. $\gamma$. nigricans. $\gamma$. uftulaus. $\beta$.

Scirpus ceftivalis. agreftis. antarcticus. $\% . \delta$. articulatus. $\beta$. capitatus. $\gamma \cdot \delta$. caricis. $\beta$. ciliaris. $\beta$. eyperoides. dichotomus. $\gamma$. elongatus. fiftulofus.

fufcus. $\alpha . \beta . \gamma . \delta$.. glomeratus.

Halleri. bateralis. luzula. $\delta$. $\varepsilon$. ovatus. pallefeens. polytrichoides. Sylvaticus. $\gamma$ tortuofus. tricephalus. trigonus. triftachyos. $\beta$. polyftachyos. $\alpha . \beta . \gamma$. $X y r i s$ indica. $\beta . \gamma$. pauciflora.

Elegia juncea. ㅇ. $\gamma$. Reftio bifidus. 
Reftio cuspidatus. elongatus.

mutans.

Squamofis. teitorum. $\gamma$ umbellatus. \&. $\beta_{0}$.

Coix lacryma. $\gamma$. Scleria bracieata.

latifolia. $\beta$. lavis.

lithofperma. involucrata. umbellata.

Eriophorum gracile. Kyllingiabrevifolia. $\beta$. triceps. $\beta$. $\gamma$. umbellata. $\alpha$.

Cyperus alopecuroides. arenarius. $\alpha . \beta_{0}$. ariftatus. caftaneus. eruentus. dubius. elegans. $\delta$. eminsens. $t$. 2 . faftigiatus. B. $\gamma . \delta . \varepsilon$. haspan. $\gamma$. $\delta$. hirtus. macrocephalus. maderospatamus. nitens. pannonicus. rigidus.
Cyperus Saintonici.

Secundus.

furinámenfis. tenniflorus. tuberofus. $\beta$. vegetus. $\alpha . \beta$. virefcens.

Crypfis aculeata. $\beta . \gamma$. phleoides.

Pappophorum alopecuroideum. fibiricum. Zoyfia maritima. a. $\beta$. Mühlenbergia diffufa. Cenchrus Burmanni. ciliaris. $\gamma$

Typha latifolia. $\beta$.

Saccharum arundinacersm. cylindricum. B. jpontaneum. $\beta$.

Holous alpimus. mollis. $\beta$. odoratus. $\beta$. pertufus. Sotifolins. Andropogon gryllus. $\beta$. proftratum. 1.2. ftriatum.

Pafpalum conjugatum. $\beta$. diffichum. $\beta$. kora. Atoloniferum. $\beta$. Anthiftiria ramofa. $\beta$. Phalaris arundinacea, $\gamma$. pitta. Phose 
Phalaris capenfis. $\beta$. erucceformis. $\beta$.

minor. B.

phleoides. $\beta$.

Panicum ariftatum.

bifulcatwom. $\alpha . \beta$.

capillare. $\beta$.

clandeftinum.

colonum: $\gamma$.

fufeum. B.

galli. $\delta$.

grosjarium $\gamma$

latifolium. $\delta$.

laxum. $\beta$.

marginatum.

miliacerim. $\beta$.

polyftachyon.

repens.

ftagmineum.

Agroftis alpina.

capillaris. B. $\gamma$.

diandra.

pygmexa.

fabra.

tenax. B. $\gamma$.

verticillata. $\beta$.

Aira cefpitofa $\gamma$.

Poa amabilis. B. $\gamma$.

aquatica. $\gamma$. $\delta$ bulbofa. B. capilllaris. $\beta$. chimenfis. $\beta$. collina.

Poa

coromandelica. criftata. $\delta$. glutinofa. japonica. $\beta$. mallabarica. nemoralis. $\beta$. nutans.

tenella. $\beta$.

unioloides. B. $\gamma$.

Egilops ciliaris.

ovata. B. $\gamma$.

Dactylis ficuta. a $\beta$.

Phleurn fchoevioides.

Avena arifidoides. $\beta$.

fragilis. $\beta$.

pallida. $\gamma$.

purpurea. $\beta$.

fterilis. $\beta$.

verficolor.

Apluda mutica. B. $\gamma$.

tripolitnna.

Ifchomum muticum. B.

Anthoxanthum odoratum. $\gamma$.

Lagurus ovatus. B. $\gamma$.

Milium lendigerum. $\beta$.

Melice afpera. á. $\beta$.

ccerulea. $\beta: \gamma$.

mutans. $\beta$.

unilora. $\beta$.

Chloris barbata. $\beta$.

paspaloides. $\alpha$. $\beta$. petrcea. B.

radiata. $\beta$. 
Cyrofurus agyptins. \&. ?. echinatus. $\beta$. indicus. ?. ท. ovatus. Spherocephalus. virgatus. á.

Foftuca arrindinacea. Halleri. heterophylla. myzurus. $\beta$. ovina. $\delta$. $\varepsilon_{0}$ phálaroides.

Triticum farctum. hijpanicum. junceum.

Hordeum bulbofium. $\beta$. capenfe. $\beta$. diftichum. $B$. nodofum. $\beta$. jecalimim. $\beta$. zeocriton. $\beta$.

Secale cereale. do polyfacts. ह. compof. reptans. B. $\gamma$. Spica elong. Manifurus gramularis. Rottboellia dimidiata. e.
Rottboellia lavis.

Stipa palbacea.

Elymus arenarius. $\beta . \gamma . \delta_{0}$ hordeaceus.

Bromus inermis. $\beta$.

mollis. $\beta$.

smultiflorus. racemofus. $\beta$.

Ariftide capenfis. $\beta$. $\gamma$. Setacea.

Arundo anguftiflora. calamagroftis. $\beta . \gamma_{\text {r }}$ colorata. epigejos. $\gamma . \delta$. Ariễa. á. $\beta$.

Calamagrojtis arundinacea. $\beta_{0}$ Axyris ceratoides. $\beta$. hybrida. $\beta$.

Mollugo hirta. $\beta$. pentaphylla. $\gamma . \delta$. Senegalenfis. verticillata. $\boldsymbol{\gamma}$.

Holofeum cordatum. $\beta$. tetraphyllum. d. Lechea minor. $\beta$. Empetrum album. \&. Minuartia dichotoma. ß 


\section{THE S E S.}

I.

$P_{\text {lures regni noftri provincias haud parum lueri exinde esfe }}$ capturas, credimus, fi equorum, quos jam alunt, numerus minueretur, locoque eorum afinis uterentur.

II.

Si Thex plantationes, ut posfunt, excolerentur in Europa, poft dimidiam tandem frculi partem, vel etiam citius, nihil plane lucri a foliis fruticum fuorum exportandis reportarent Chinenfes.

III.

Omnes Europrorum conatus in fuccedaneo quodam fic dicto Theæ Bohex inter plantas indigenas detegendo, hucusque fuerunt fruftranei.

\section{IV.}

Mirandum fane eft, Europæos, omnium fapientisfimos, alienas inque America remotisfimas occupare terras, ibidem fummo cum labore argentum effodere, in hoc ad Europam trantportando maximum incurrere difcrimen, nec minori denique periculo idem ad alteram terræ partem, Indiam Orientalem trans: ferre, foliorum fruticis cujusdam reportandorum caufa.

\section{V.}

Studium hiforiæ naturalis atati dudum juvenili esfe com. mendandum, merito nobis videmur posfe contendere.

\section{VI.}

Valde falleretur, qui contenderet, cognitionem hiftoriz naturalis non nifi Medicis esfe utilem; noftra fcilicet ex mente nemo eft, cui hre cognitio plus minusve haud afferre posfit vel lucri, vel voluptatis.

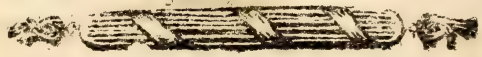


D. D.

\section{MUSEUM NATURALIUM ACADEMIÆ UPSALIENSIS.}

C U J U S

ÁpPENDIC. $\mathrm{X}$.

VENIA EX̃P. FAC. MEDICAE UPSAL.

P R $Æ S I D E$

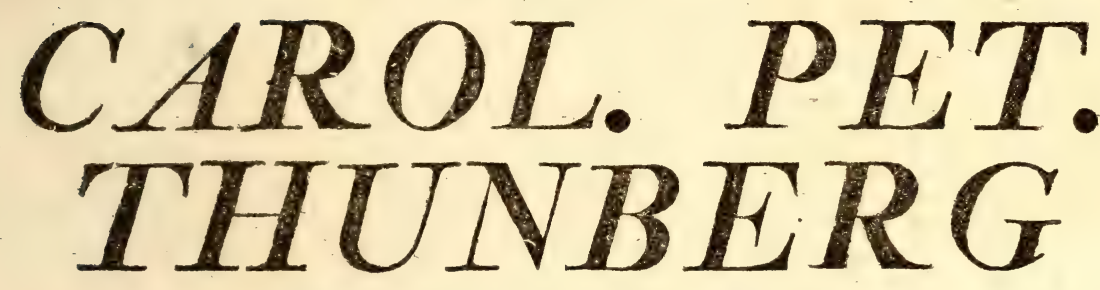

Equite Aurat. Reg. Ord, de Vașa,

Med. Doct. Prof. Med, et Bot. Reg. ext Ord. Acad. Cesar. Petropol, ex Nat. Curios. Reg. Scient, lond. Holm. Societ. Scient. Ups. Philad. Patr. Horm. et Civ, Oecon. Fint. Berol. Nat. Scrut. Paris. Hafn. Moscou. et Halens. Hist. Nat, Lund. Harlem. Amsteld. Zeland. Nidrosiens. Ticinens. Irnens. Linn. Londin. Reg. Scient. Pays. et Phytourapil. Góttingens. Nanciens. Men. Monspel. Med. Matritens. Medic. et Nat. Studios. Edimburg. Membro nec non Acad. Scient. Paris. ext Institut. Nation. Monspeliens. Agricuit. Paris. Miedic. Londin. Scandinav. Florentin. ex Batavin, Ind, Or. Correspond.

PUBLIC瓜 CENSUR E COMMITTIT

$$
\text { GO } O A S \underset{\text { SMOLANDUS. }}{R} \mathrm{P} D I N
$$

IN AUDIT. BOTANICO SUP. D. MAJI MDCCCVI.

H. A. M. S.

Upfalia, Typis Edmannianis. 



\title{
DONATION. THUNBERGIAN.
}

\author{
APPE ND. $X$.
}

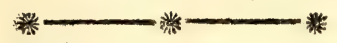

Protea caudata. B.

comofa. $\beta$. daphnoides. decumbens. $\beta$. hirfuta. hypophylla. $\gamma_{0}$ obtufa.

phylicoides. $\beta . \gamma$ pubera. $\delta$. reticulata. rngofa.

ferraria. d. $\varepsilon$. \&. fpathulata. $\beta$. tenuifolia. truncata. verticillata. virgata. $\alpha . \beta$. Embothrium fericenm. $\gamma$. Scabiofa acaulis. $\beta$. arvenfis. $\beta . \gamma_{0} \delta_{0}$ banatica. cretacea. divaricata. $\beta$. integrifolia. $\gamma_{0}$ ifetenfis. $\gamma$. maritima, $\gamma$. $d$. gutafulia. $\beta$.
Scabiofa fpeciofa. a. B.

$$
\begin{aligned}
& \text { ftellata. } \eta \text {. } \\
& \text { tatarica. } \\
& \text { uftulata. } \beta .
\end{aligned}
$$

Hedyotis paniculata. spermacoce latifolia.

fiabra. verticillata. 6.

Scolofanthes verficolor. Ernodea littoralis. Galium album.

aufriacum. boreale. $\beta$. elatum. glancum. B. $\gamma_{0}$ hifpidum. horridum. numifufum. leptophyllum, saxatile. $\beta$. fylvaticum. $\beta$. tomentofum. trifidum. $\beta$. uliginofum: $\beta$.

Houftonia corvilea. $\beta$. Rubia cordifolia. $\beta$. Ajperula cynanchica. $\beta$. 


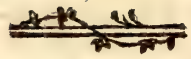

Afperula lavigata. $\gamma$. Cruaianella anomala.

ciliata. decumbens. pentandra.

Ixora coccinea. $\beta$.

Penca acuta. myrtilloides. B.

Budleja occidentalis. Sangviforba offrimalis. $B$. Cornus alternifolia. $\delta$. fanguinea. $\beta$. fvecica. $\gamma$.

Oldenlandia tenuifolia. $\beta . \gamma_{*}$ Ludvigia repens. $\beta$. Sevparia dulcis. $\gamma . \delta_{\alpha}$ Fagara pterota. $\beta$. Ammannic ramofior. $\beta$.

Trapa bicornis. $\beta$.

Nigrina acuminata.

Ncpenthes deftillatoria: $\beta$.

Hippophä̈ rhamnoides. folo. Trophis afpera. $\beta$. Laurophyllus capenfis. ㅇ. Samara coriacea. $\beta$. Schrebera Jchinoides. $\beta$. Crantzia lovigata. Santalum album. Rivina lavis. $\beta$. Plantago cornuti. $\beta$. $\operatorname{cras} \int a_{0}$. dubia. B. filiformis.
Plantago tanceolata. $\delta . \varepsilon_{6}$ lufitanica. $\beta$. maxima. $\beta$. media. $\beta$. minuta. faxatilis. virginica. $\beta . \gamma$. Urtica ceftuans. $\beta$. eaffra. $\beta$. capenfis. $\beta$. glomerata. interrupte. $\beta$. tanceolata.

Bahmeria frutescens. fol. Nerteria depressa.

Hypecaum debumbens. $\beta$. $\gamma$. Morus indica. $\gamma . \delta$. nigra. $\beta$. papyrifera. $\delta$.

Betula alba. $\delta_{0} \varepsilon_{0}$ incana: $\gamma$. pinnata. $\beta$. populifolia. $\alpha . \beta_{\text {. }}$ triphytla. $\beta$. vifcoja. $\beta$.

Hamamelis virginica. $\gamma$. Cufcuta africana. chinernges. Myrica gale: fol. quercifolía. fol. Vifcum album. B. Ilex aquifolium. $\delta$. $\varepsilon$. Anthofpermum siliare. ․ B. Petamogeton gramineun. 


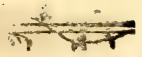

Poianogeton hucai. B.

nuarinush. $\varepsilon$,

matans $\gamma$.

Heliotropium europeum. $\beta$.

ceilanicum.

curasfavicum.

incanum.

perficum.

undulatun.

Lithospermum graminifolinm. officinale. B. tenuifloram.

Anchusa anguftifolia. $\beta$. italica. $\gamma$.

intea.

officinalis. ¿. n.

Onosma cafpicum.

jempervirens. $\beta$.

echioides.

Sericeum.

tataricum.

tinctorium.

Symphytum afperim。

Cynoglosfum cheirifolinm: B.

javanicum.

linifolium. $\gamma$.

rugofun.

Myofot is frorpioides. $\bar{\varepsilon}$ ?. n. fquarroja. of.

Echium afperum.

giganterm.'

plantaginertm.

subrum.

Pasimonaria officinalis. B. $\gamma$.
3

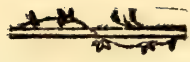

Cerinthe major. $\gamma$.

Borago orientalis. $\beta$.

Tournefortia feetida. $\beta$.

Nolana proftrata. $\beta$.

Soldanella alpina.

Androface elongata. S.

obtuffifolia.

feptentrionclis.' $\beta$.

Primula auricula. $\gamma$. cortufoides. $\beta$.

elation.

farinofa. B.

glutinosa.

marginata.

veris. $\gamma$.

Phlox carolina. $\beta$.

glabra. B.

paniculata. B.

Menyanthes ceilanica. indica. B. $\gamma$.

peltata.

Cyclamen enropaum. hederifolium. perficum.

Iyfimachia dubia.

quadrifolia. $\varepsilon$.

falicifolia. $\beta . \gamma$

verticillata.

vulgaris.

Anagallis arvenfis. B. $\gamma . \delta$.

Convolvulus althooides. $\varepsilon$. $\}$

ท. N. \% $x$

canarienfis. $\beta$.

cantabricus. $\beta$. 


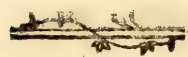

Couvoloulus carolints. 2. decumbens. gosfypifer. a. $\beta, \gamma$. medius. $\beta$. parviflorus.

s- phyltus. $\beta_{\text {. }}$

repens. I. 2. s:mbellatus. $\gamma . \delta$.

Ipomeea noctis. $\beta$.

Azalea indica. B. $\gamma$. pontica.

Plumbago suropaca. lapathifolia.

Theophrafta longifolia. Campanula ajugafolia. betonicaefolia. a. B. $\%$ braiteata.

capenfis. B. fasciculata. $\beta$. glomerata. latifolia. $\gamma$ medium. paniculata. $\gamma$. perficifolia. $\beta . \gamma . \delta$. ह. ?.

pyramidalis. 2. rapunculoides: $\beta$. ruthenica. notundifolia. $\delta . \varepsilon_{\text {. }}$. fiberica. $\beta$. Stellata. trachelinum. do
Campanula undulata. $\beta$. $\%$ zoyfii. bulbofa. B.

Lobelia coronopifolia.

cyphia. 2. depresfa. $\beta . \gamma_{0}$ erinus. $\beta . \gamma . \delta$. hirfuta. $\beta_{0.1}$

linearis. lutea. $\beta_{0} \gamma_{0}$

- patula. $\beta . \gamma$. fiabra. fecunda. B. Setacea. fiphilitica. $\beta$. tenella. $\beta$. triquetra. $\beta . \gamma$. Godenia ramofa. a. $\beta$. Scevola Plumierii. $\beta$. Polemonium cceruleum. $\gamma$. Roëllia ciliata. B. Phytevma campanuloides. Samolus valerandi. $\beta$. Marinda royoc. $\gamma$.

Nauclea oecidentalis. $\beta$. orientalis.

Nigrina Servata. Loniser a coevnlea. $\beta$. tatarica. $\beta$. Genioftoma rupeftris. Corynocarpus lovigata. 


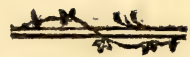

Plearania ventofa

Pilocarpus racemafus.

Egiceras minus.

Myrfine africansa. retusa.

Cinchona floribunda. $\gamma$.

Coffea americana.

Pfychotria Aipulacea. $\beta$. Verbafcum croceum. $\beta$.

bychnitis. $\beta$.

phlomoides: $\beta$.

phoniceum.

Nicotiana paniculata. ruftica. $\beta . \gamma . \delta$. tabacum. $\beta$. undulata. 1. 2, 3.

Datura arborea. $\beta$.

faftuofa. $\beta$.

ferox. $\beta$.

lavis. $\beta$.

Atropa phyfaloides. o.

Phyjalis proftrata. $\beta$.

Capficum grosfum. B.

Hyofcyansus phyforloides.

Solanum diphylhum. $\gamma$.

infanum. $\beta$.

lycoperficum. $\beta$.

nigrum. $\delta . \%$

peruvianum. $\beta$.

quadrangulare. $\beta$.

quercifolium. $\beta$.

tomentofwn. $\gamma_{0}$.
5

Berthieria guineem/is.

Ceftrum parqui. $\beta$.

vefpertinum. $\beta$.

Lycium afrum. $\beta$.

Serisfa capenfis. $\beta$.

Ardifia coriacea.

elliptica. $\beta$.

excelfa.

Cordia callococca, $\beta$.

obliqua.

rebeftena. B.

Varronia lineata. $\beta . '$

Chryfophyllum cainito. $\beta$.

Rcemeria argentea. $\beta$.

Rhamnus alaternus. $\gamma$.

alpinus. $\beta$.

oeltifolius. $\beta$.

colubrinus. $\beta$.

ellipticus. $\beta$.

frangula. $\beta$.

mucronatus. $\beta$.

faxatilis. $\beta$.

theezans. $\beta$.

Zizyphus palinurus. $\gamma . \delta$.

vutgaris. $\beta$.

Phylica glabrata. a. $\beta_{\text {o }}$.

globofa.

hirfuta. a. $\beta$.

lanceolata. $\beta$. $\gamma$.

paniculata:

pinea.

racemosa. $\beta$. 
Phylica rosmarinifolia. $\propto$. B. Stavia glutinofa. B. Ceanothus africanus. $\beta . \gamma$. Celaftrus acuminatus. $\gamma . \delta . \varepsilon_{0}$ Ribes petraum. americanus. B. $\gamma$. buxifolius. z. Viola biflora. $\beta$. crispus. ellipticus. latifolius. $\beta . \gamma . \delta$. laurinus. $\beta$. montanus. $\beta . \gamma$. pyracañthus. $\gamma$.

Diosma ciliata $\gamma$. fcandens. $\beta$. crenata. $\beta$. cuspidata. deufta. excifa. hifpida. $\gamma$. imbricata. B. $\%$. oblonga. $\alpha$. ß. $\gamma$. pubefiens. $\beta$. pulchella. $\gamma$. fucculenta. $\beta$. tetragona. $\beta$. umibellata. uniftora. $\gamma$. uftulata: villofa. $B$.

Brunia capitella. comoja. a. $\beta$. globofa. phylicoides. fgarrofa. ralcarata. $\beta$. cainina. $\beta$. decumbens. B. grandiftora. $\gamma$. d. montana. $\beta$. paluftris. $\beta$. pedata. $\gamma$. fuffruticosa. 3 . - tricolor. \& $\varepsilon$. Impatiens balfamina. $\gamma$. noli tangere. 3.

Hirtella paniculata. triandra.

Simbuleta tenuifolia.

Piftacia trifolia. $\alpha . \beta$. Celtis occidentalis. $\gamma$.

Ulmus prumila.

Paderia fatida. $\gamma$. Gardenia fragrans. Vitis capenfis. B. $\%$. japonica. $\beta$. indica. $\beta . \gamma$. vulpina. Po.

Heliconia bihai. $\gamma$. Achyranthes altisfima. B. $\gamma$. argentea. cephalotes. cuneifolia. 


\section{7}

Achyranthes echinata.

expanfa.

Periploca graca. B.

indrca.

fruticose.

Celofia comofa. $\alpha$. $\beta$.

embella. 1. 2. 3.

criftata. $\delta_{0} \varepsilon_{\text {. }}$

margaritacea. $\beta . \gamma \cdot \delta$. nodiflora. 2 .

paniculata.

Claytonic perfoliata.

Amaranthus caudatus: $\beta . \gamma_{0}$

polygonoides. $\beta$.

melancholicus. $\beta$.

Sanguineus. $\gamma$.

Scandens. $\beta$.

tricolor:

Illecebrum divaricatum. $\beta$.

ficoideum:

javanicum.

lanatum. $\gamma$.

lanceum.

laniflorum.

latifolium.

paronychia. $\gamma$.

Sericeum.

fesfile. B.:

ftellatum.

Julcatum.

Irefine anguftifolia. $\propto$. B.

Oribafia

$$
\text { celofioides. } \gamma_{0} \text {. }
$$

Periploca emetica. efculenta.
Sylveftris.

Vince minor.

Pergularia tomentofa.

Cynanchum cordifoliwm.

Afclepias amoena.

canefcens.

incarnata. $\beta$.

fruticofa. $\gamma$.

nivea. $\beta$.

undulata.

vincetoxicum.

viridifiora.

Apocynum paniculatum.

Herniaria hirguta. $\beta . \gamma$.

Humulus lupulus. t.

Beta cicla. 2.

Thefium frietum. $\beta . \gamma . \delta$

frilea. $\beta$.

Linconia alopecuroides.

Chenopodium ambrofioides. $\gamma$.

glancefcens. 1.2 .

glaucum. $\beta$.

hortenfe. 2. 3 .

hybridum. 2.

littorale. $\varepsilon$.$\} . \eta_{0}$

incidum. 2.

murale. $\beta$.

polyjpermam. $\beta$.

portulacoides $\gamma=\delta_{\%}$ verruco/umi $R_{\text {o }}$ 


\section{T H E S S.}

Th. I.

P falebrofisfimas difficultates Zoologix ftudiofis adferunt: Qux appellationis diverfitas etiam in ceteris hifforix naturalis partibus valet.

\section{Th. II.}

Prout fcientix \& artes increfcunt, ita Botanice etjam majus capiet incrementum, quippe quæ medicis| œconomisque utilisfima, \& cultoribus fui fuavisfima.

\section{Th. III.}

Plantationes lini melius ruri, quam oppidis convenire videtur,

\section{Th. IV.}

Cupiditati, qua flagrant homines, novi quidquam difeendi; optime fatisfacit, fi quæ alia, fcientia hiftorix naturalis.

$$
\text { Th. V. }
$$

Multis modis florum variationes, etjam novas fpecies, producere gaudet alma Venus, quos inter variationes culture, foli, climatum \& artis maxime valent. 


\section{D. $D$. \\ MUSEUM NATURALIUM \\ ACADEMILE UPSALIENSIS. \\ $\rightarrow$ cujus \\ Appendic: XI. \\ VENIA EXP. FAC. MEDICA UPSAL. \\ P.R压SIDE}

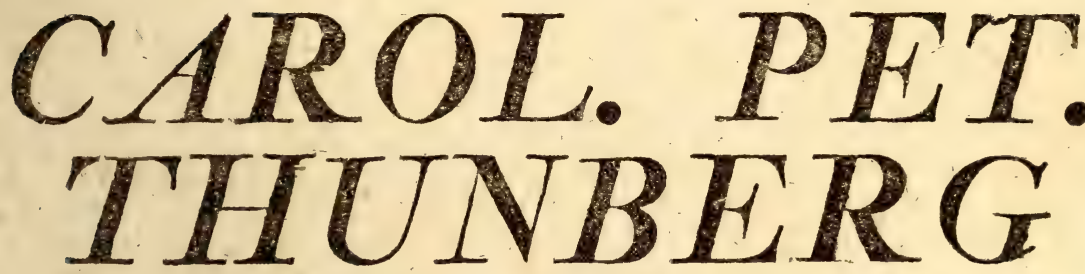

Equite Aurat. Reg. Ord, de Vasa,

Med. Doct, Prof. Med, et Bot, Reg. ext Ord, Acad. Cexsar, Petrofne. et Nat. Curios. Reg. Scient. Lond. Holm. Societ. Scient. Ups. Philad, Patr. Holm, et Civ, Oecon. Fint. Berol. Nat. Scrut. Paris. Hafn. Moscou, et Halens. Hist. Nat. Lunjo. Harlem. Amsteld. Zerand. Nidrosiens. Ticinens. Ienens. Linn. Londin. Reg. Scient. Phys. ext Phytourapu. Góttingens. Nanciens. Med. Paris. Emul. Monsper. Med. Matritens. Medic. et Nat. Studios. Edimburg. Membro nec non Acad. Scient, Paris. et Institut. Nation. Monspeliens. Agricur.t. Paris. Medic.Londin.Scandinay. Florentin. et Batavin. Ind, Or. Correspond.

PUBLICA CENSUR COMMITTIT

PETRUS ELGSTROM SMOLANDUS.

IN AUDIT. BOTANICO SUP. D. VI DEC. MDCCCVI. H A. M. s.

Upalia, Typis Edmannianis. 



\section{DONATION: THUNBERGIAN.}

APPEND. XI.

Humulus hupulus. $\stackrel{8}{+}$ Saljole indica. proftrata. $\varepsilon$. rofacea. $\gamma$. Sativa. B.

Anabafis triandra.

Casnabis chinenfis. 8. 2.

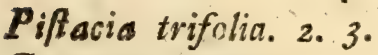

Geniana centaurium. $\beta . \gamma$. chlorantha. $\operatorname{diffu} a . \alpha . \beta$. elongata. filiformis. $\beta$. germanica. a. $\beta$. pannonica.

pneumonanthe. $\beta$. puinctata. $\beta$. Jpicata. B. $\gamma$.

Chironia frutescens. $\beta$. jasminoides. $\beta$. tychnoides. $\beta$.

Panax intemuata. fruticofa. 1. 2. 3. 4. lobata. morototoni. $x, 2$.

Hydrocotyle afatica. B. verticillata.

Spananthe paniculata. $\beta$.
Sonicula europeca. B. Bupleurum falcatum. 3 .

Gerardi. 2. jurceum. 2. odontites. 2 . rotundifolitum. $\gamma$. Semicompofitum. $\gamma$

Cusfonia dentata 2. 3.4. 4 . Serrata. 2.

Eryngium cacruleum. maritimum. 2. Cachrys undulofa.

Sium

Oenenthe crocata. 2. exaltata: 2 . globulofa. 2. Artedia Squamata. $\beta$. Daucus visnaga. $\beta$. Caucalis grandiflora. $\gamma$. Athamantha fibirica. $\beta$. Angelica Archangelica. 2. atropurpurea. 2.

Cicuta virofa. 2. 3 . Aethufa bumins. B. cynapium. 3. Coriandrum fativum. tefticulatum 2. Anethum capense. fol. 
Anethum foeniculum. 2. 3 . graveolens. $\gamma$.

Pimpinella dichotoma. dioica. t. 2. Saxifraga. 2. 3. 4.

Carum Carvi. 2. 2. 3. 4. 5. 6. Paftinaca lucida. 3.

$$
\text { fativa. } 3
$$

Imperatoria of truthium. 2. Selinum Carvifolia. 2. Seguieri. 2.

Liguficam levificum. 2. peloponnefiacum. $\gamma$.

Bubon galbanum. fol.

Peusedanum efficinale 2.

filans. 2. 3 .

Laferpitium gallicum. 1.2.

latifolium. 2.

pruthenicum. 2.

Siler. 2.

Sefeli ammoides. $\gamma$.

\section{annuит. $\gamma$.}

ariftatum. 1.2.

chaerophylloides 2.

elatum. $\gamma$.

pygmaeum.

torulofum. 2 .

Chaerophyllum aromaticum 3.4.5.

$$
\text { aureum. 2. } 3 \text {. }
$$

Standix nodofa. 2. odorata 3 . pecien. 2.

Rhus ailantius.
Rhus

carolinum. 2. coriaria. 2. cotimus. $\gamma$. crenatum. $\alpha . \beta$. dimidiatum. $\alpha . \beta$.

dissectum.

glabrum. 2. 3.

glaucumi.

mucronatum. pauciflorum. 2. sofmarinifolinm. a. $\beta$. fuccedanerum. 2. tomentofum. 2. toxicodendrum. $\delta$. viminate. 2.2.

Statice acerofa. armeria. B. $\gamma$. . cordata. 2 limonium. $\gamma$. Spicata. finuata. 2.

Linum hirfutum. 2. perenne. 2. Drofera cuneifolia. $\gamma . \delta$. indica. $\beta$.

Evolvulus alfinoides. 2. Pharnaceum diftichum.

mucronatum.

Viburnum macrophyllim. $\gamma$. pyrifolium. $\beta$. Serratum. $\varepsilon$. Casfine barbara. 2. 
Casine colpoon. 4. Sambucus canzadenfis. 2. Tamirix tetrandra. Andracbne telephium. 2. Clutic alaternoides. $\gamma$. pubefcens. $\beta$.

Nephelium lappaceum. Aralia piloja.

fciadaphillum.

Commelina africana. $\beta$. axillaris. japonica. mudiflora. $\beta$. vaginata. zanonia. $\beta$,

Bromelia karatas fol.

Chamaerops humilis, 3 .

Elais guineenfis. 1. 2. 3 . Calamus falac, $1,2.3,4$. Gleditfchia triacanthos 3. 4. Loranihus caffer. incanus. 2.

Allium angulofum. $\gamma$. globofin.

mofchatum. B. oleracenum. 2. 3. parviforim. pedunculatum. Sativini. 2. Jcorodoprafum 2. filiricum. 2.

Tulipa biflora: iniflora, gefneriana: Spont. t. 2 . cult $2=20$.
Bulbocodiam trigonum.

Narvijsus anguflifolins. incomparabilis. odortis: 2. orientalis. 2. triandalus.

Leucojum aefivum. Pontederia racemoja.

trincata.

Burmannia biflora.

Convallaria japonica. $\beta$, Hyacinthus Jerotimus. Albuca altisfma. fafigiata. $\beta . \gamma$. viridiflora.

Lilium philadelphicum. 2. pomponicum. 2.

Fritillaria cancafina. meleagris. $\delta . \varepsilon$.$\} .$

Gloriofa fuperba. 2. 3. 4. Hypoxis erecta 2 . Ornithogalum condatum 2.2. circinatum. thyrfoides. $\gamma$. trigonophyllum.

Asphodelus tanricus. Gethyllis fpiralis. 3. Anthericum planifolinm. ramofun. 2 .

Eucomis nana. 2.

Rumex bipinnatus. Funcus extenfus. glauczus. Ppadiceus. 
Ehrharta ramoja. 2. Oryza villoja. Alethris fragrans. 1, 2. Melanthium album. 2. Diofcorea fativa. \&. Asparagus acutifolins. 2. declinatus. 2. fafciculatus. officinalis. 2 . verticillatus. 2.

Alifma parnasfifolia. 2. plantago. $\delta . \varepsilon$.

Gvettarda fpeciofa. 2. Aefculus flava. pavia. $\beta$.

Oenothera parviflora. 2. Epilobium dubium. rofmarinifolium.

Daphne javanica. tartonraira. $\mathbf{3}$. tomentosat.

Pafserina capitata. $\gamma$. eriocephala. $\beta$. hirfuta. $\gamma$. uniflora. $\delta$

Gnidia capitata. $\beta$. fcabra. $\beta$.

Struthiola virgata. $\gamma . \delta$. $\varepsilon . \zeta$. $V$ accinium corymbofum. 2 .

Erica cinerea $\gamma$. macrocarpons. confpicula. corifolia. ?.
Erica hirfuta. $\beta$.

hispida. $\beta$.

mellifura. multiflora B. mudifora. $\beta$. Atricta. verticillata. vulgaris: microph.

Honkeneya ficifolia. Mimufops Elengi. 2. Dodonaea angufitalia. 2. 3 . elaeagnoides.

Lawsonia achronychia. inermis. 2.

Paulinia mexicana. triternata. 1.2. Sapindus rubiginofus. I. 2. Myriophyllume verticillatum. 2.3. Paris quadrifolia: pentaph. Populus alba. tol. argentea. fol. nigra. fol. 1. 2. Fungia ciliata. Allophyllus cobber. B. racemo/us. $\beta$.

Aeer palmatum.2. platanoides. 2. tataricum. 2.

Polygonum acidum. 2. articulatum. 2. divaricatum. 2. 2. 3 . frutefcens. $\gamma$, 
Polygonum glabrum.

hydropiper. $\beta$.

oricntale. $\gamma$.

penfylvanicum. 2.

fibiricum. 2.

Laurus borbonia. $\gamma$.

indica, $\gamma$.

nobilis $\gamma . \delta$.

perfea. $\beta$.

$P$ terocaryamontana.

Aftranthus cochinchinenfis.

Qvercus aefculus. $\beta$.

$$
\text { alba. } \beta \text {. }
$$

dubia.

himilis.
Schinus mollis. 2.

Ledum latifolium. 2. paluftere. $\beta$.

Dionaea muscipula. fol.

Rhexia aehifanthera.

Melaftoma elegans. hirta. malabathrica. 2. 3. racemosa. B. repens. 1. 2. Arigillofa. 2.

Triumfetta procumbens. 2. - Semitriloba. 2.

Lythrum cuphaea. 3 . hysfopifotium. 4.

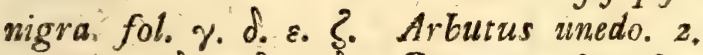
rubra $\gamma$. $. \varepsilon . \zeta$. . .. „. Bocconia frutefcens. 2. Juber. $\beta, \gamma . \delta_{0} \quad$ Mabea taquari.

Cassytha filiformis. $\beta$.

Sophara japonica fol. B.

Theligonum cynocrambe. microphylla. $\beta$.

Cassia chamazcrifta. 2.

falcata. $\beta$.

fiftula. 3 .

liguftrina. 2.

nictitans. 2. 3 .

procumbens. 2.

Gvilandina bonduccella. 2.

Tornex Sebifera.

Samyda ociandra. 2.

flueftris.

Cajearia eliptica. nitida. 2.

Terminalia alata.

Royena anguftifolia.

hirfuta. $\hat{\text {. }}$.

Halefia tetraptera. 2.

Hacmatoxylon campechianum. 3. Hydrangea arbore/cens, 2.

Ccesalpinia pulcherrima. 2.

Schotia 'alata.

Saxifraga crashifolia 2.

Speciofin. $\beta$.

Qvasjia amara. 2.

mutata. 2.

orientalis.

penfylvanica $z$. 
6

Saxifraga farmentofa. 2. tridaciylites. 6.

Gypsophila faftigiata 2. 3. glomerata. 2. 2. muralis 4. proftrata. 2. repens. 2.

Dianthus albens. bicolor. cancajus. fragrans. leptopetalus. plumarius. 2. filveftris.

Menifpermum canadense. fol. japonicum. ઈ. 2. Trianthema anceps $\beta$. decandra. 2 . monogyna. 2 . Refeda alba. 2. glanea 2. 3 . lutea. 2.3 . phyteuma. $z$. undulata. 2.

Euphorbia amygdaloides. cyparisfias. 3 . exigua. 2,3 . genififolia. 5. $\sigma$. Gerardiana. 2. glabrata. 2. hirta. 2 : lanceolata. $\alpha, \beta$.

Euphorbia lathyris 2. 3. micrantha. myrfinites. $\delta$. paluttris. 2. pinea. 2. platyphyila, 2. faxatilis. 2. Spinofa. 2. tirucalli, 2. Arenaria auftriaeá. 2. laricifolia. 2. maritima. 4. 5 . fulcata. temuifolia. 2 . verna. 2.

Stellaria aijpine. graminea. 2. uliginofa. 2 .

Cucubalus catholicus. 2.

fruticulofus. 2 . glutinofus, 2. molitisfmus. 2. multiflorus. 2. vifcofus 2. 3 .

Silene behen. 2.

eermua. $\gamma$. chloraefolia. mutans, 2. pumilio. reticulata. 7.2 . Taxatilis. fupina. 2.2 . 
Deutzia Scabra. $\gamma$.

Prockia indica.

Datifca cannabina.

Fagus pumila. \&.

$$
\text { fyluatica. 4. 5. } 6 .
$$

Cotyledon caefpitofa.

Sedum oederi.

Rhodiola rofea. :

Lychnis capenfis. chatcedonica. e.

Ceraftium arvenfe. 3 . vnlgatum. 3. 4.

Spergula apetala. 2.

Kiggelaria africana. 3 .

Spondias mangifera. $1,2,3$.

Glinus lotoides. 2.

Forfkohlea candida. 2.

$$
\text { tenas. } 2
$$

Neurada procumbens. $\beta$.

Aponogeton diftachyon. 2. 3. 4.

Cactus grandiforus. 2.

pendulinus. $\beta$.

Eugenia racemofa. 2.

$$
\text { p evdopfidium. }
$$

Calyptranthes shytraculia. 2.

Fabricia laevigata.

Myrtus lucida.

tomentofa.

Prunus cerafus. $\varepsilon$. bufitanica. 2 . mahaleb. 3. Semperfiorens. Serotina.

Prockia ceilanica. I. 2.

\section{vitlofus. B. $\gamma$.}

Tilie americana.

Chelidonium majus. $\gamma$.

Capparis Jpinofa. 2.

Calophyllum ellipticum.

$$
\text { excifum. }
$$

Ciftus arabicus. $B$. canadenfis. 2 . formofis.

guttatus. B. $\gamma$. halimifolius. $\gamma$. kelianthemung. $2 \cdot 8$.

hirtus. 2. incanus. 2. ledon.

libanotis. $\beta . \gamma$. oelandicus. $\beta$. racemofus. 2 . Salvifolius. $\gamma$. Surejanns. 2.

Corehorus capsularis. 2.

Mimofa dulcis. $\alpha . \beta$. linifolia. natans. 1.2 . verticillata.

Ochna nitida. 2. 3. fquarrofa. Trewia nudiflora. 1 Grevia afatica. 3. 
8

Voteria indica 2:

Muntingia calabura. $\beta$.

Crataegus calcarata. 2.

Cliffortia juniperina. ^. 2.

polygonifolia

ruscifolia $+\frac{?}{3}$

Atrobilifera.'. 2.

trifolieta. $\beta$.

Paconia albiflora. 2 .

humilis 1.2 .3 .4$.

temuifolia. 2.

Begonis macrophylla.

Delphinium Aaphifagria 1.2.

Aconitum napellus 2.

$$
\text { taurimum, } \text { volubile. } \beta \text {. }
$$

Aquilegi daurica.

Tetragonia axpanja.

herbacea. 3 .

Mefpilus canadenfis. ż. chamaemeepilus. 2.

germanica. 2.

pyracantha. 2.

P.yrus malus. 2.

Mefembryan themum expanfium 2. hifpidum. $\delta, \varepsilon$. sodiflorum. 3 . piunstifdum. 2.

temuifoiinm. 2.

Aizoon pamiculatum. 2.

Spiraed alba. B.

crenata. k.
Spirca filipendila. $z$. hypericisolia. 3 . uimaria. 2.

Nigella damafcena. $\beta$.

Rofa chinenfis.

lutea 1 .

parviflora .. 2.

$m u / c 0 \int a .1 .2$.

parvifolia.

pimpinellifolia. 3. 4. pumila. 2.

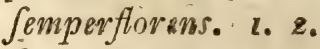
Jpinnfisfima. 2.

Rubus rofaefolins.

Tormentilla evecta: s.pet.

reptans. 2.

Geum virginicum. 2.

Potentilla hirtá. 2.

intermedia. 2

nitida.

norvegica, 2.

recta. 2.

Jupina. 2.

Caltha paluftris.

Arum arborefcens. 2. macrorhizon. 2. peregrinum. 2.

Dracontium polyphyllum. 2. 3. Piper geniculatum. 2. lanceolatum. polyftachyon. fcabrum. umbellatum. 2:

Uvaria japonica. 2. 3 . javanica. 2. 
Annona muricata. 2. 3. 4. Squamoja. 3 .

Clematis erecta. 2. 3. 4. hexapetala. 2. viticella. 2.

Anemone alpina. $\gamma . \delta$. dichotoma. 2 . hepatica. $\gamma$. patens. $\gamma_{0}$ pulfatilita. 3 . ranunculoides. 2. vernalis. 3.
Trollius curopains. $\because$ Helleborus foetidis. 3 . Ranunoulus afiaticus. gtacialis. B. oxyspermus. paluttris. penfylvanicus. 2. polyrhizgs. rutarfolius. 2.

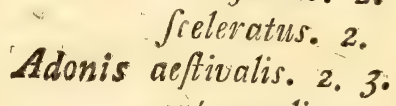
autumnalis. 2. 3, 


\section{THE S E S. \\ I.}

Ab agricultura incipit generis noftri humanitas.

II.

Elementorum ex ratione, plantarum deducimus colorem. IiI.

Aquan aëremqué precipua herbarum alimenta, cenfer liceat.

\section{IV.}

Vitam xt atemque vegetabilium ex gace oxygenio defluere, credimus.

\section{V.}

Vulcanos motusque terre, ab igne quodam centrali derivan: dos, negamus.

\section{VI.}

Montes, faltem plures, via humida esfe progeneratos Juculentisfine comprobant petrificata, in illis obvia. 


\section{MUSEUM NATURALIUM \\ ACADEMI 2 UPSALIENSIS.}

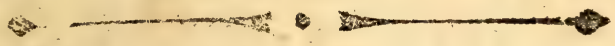

CU J U S

APPENDIC. XII.

VENIA EXP. FAC. MEDICAE UPSAL.

PRÆSIDE
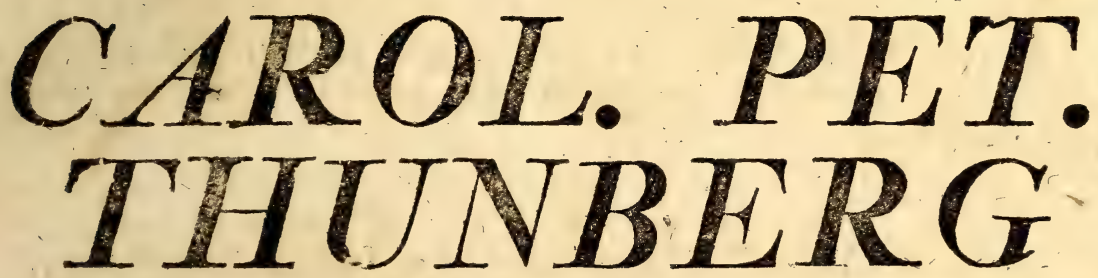

Equitr. Aurat. Rig. Ord. de Vasa,

Med. Doct. Prof, Med, et Bot. Reg. ext Ord. Acad, Ceasar. Petropol. ex Nat, Curios. Reg. Scient, Lond. Holm. Societ, Scient. Ups. Philad. Patr. Hoi m. et Civ, Oecon. Fint. Berol. Nat. Scrut, Paris. Hafn. Moscou. et Halens. Hist. Nat, Lund, Harlem. Amsteld. Zeland. Nidrosiens. Ticinens. Irnens. linn. Londin. Reg. Scient. Phys. et Phytourapia. Góttingens. Nanciens. Men. Paris. Emul. Monsper. Med. Matritens. Medic. et Nat, Stuios. Edamburg. Membro nec non Acad. Scient. Paris. et Institut. Nation. Monspeliens. Agricur.t. Paris.Medic.Londin.Scandinav. Florentin, et Batavin, Ind, Or. Corresponda

\section{P. P. \\ CAROLUS NETHERWOOD, \\ STIP. ORD, EQU. SMOLANDUS.}

IN AUDIT. BOTANICO SUP. D, X DEC. MDCCCVI.

H. A, M. S.

Upfalia, Typis Edmannianis. 


\section{TH E S E S.}

I.

Antiquorum in hiftoria Naturali progresfibus, minorem terræ cognitionem maximas pofuisfe obices cenfemus.'

\section{II.}

Detectionem America, ad id, quo gaudet, faftigium, hiftoriam Naturalem magnam ad partem promovisfe contendimứs.

\&

III.

Inter fructus ab Indiis in Europam transportatos, amplioris, nulli, fructu Solani tuberofi, fuerunt utilitatis.

IV.

Amplisfimam Hiftoriæ Naruralis cognitionem rem rufticam colentibus minus necesfariam credimus.

\section{V.}

Pratorum culturæ melius confultum fore putamus, $f_{t}$, \& tempore vernali \& autumnali, fues $a b$ is depafcendis prohibeantur. 


\section{AP PEND. XII.}

Ajuga orientalis. 3.

Glechoma hederacea. $\beta$.

Lycopus fibiricus.

virginicus. 2. 3.

Leonurus fibiricus. $\beta$.

Hysfopus officinalis. $\gamma . \delta_{0}$

Teucrium botrys. $\beta$. canejcens. $\beta . \gamma$.

flavnu. B.

japonicum. $\alpha . \beta$. montanum. $\beta$.

Mentha arven/s. $\beta$. $\gamma$. rotundifolia. $\gamma$.

Byftropogon plumofum. 1.2. Elsholtzia criftata. 2. Ocymum polyftachyon. 3 . proftratum.

$V$ erbena mexicaura. $\beta$. Nepeta carnlea.

melisforfolia. tuberofa. B.

Salvia clandeftina, 3 . corylifolia. B. gintinofa. 3 . grandiflora. horminum. $\beta$. leucantha. inrata. B. $\gamma$. mexicana. $\beta$. mutans. 3.
Salvia officinatis. $\gamma_{0}$ Spinofa. $\beta$.

Thymus. cephalotes. $\beta$. 2iygis. 3 .

Sideritis rugofa. $\beta$. - Scordioides. 4. Syriaca. $\beta$.

Prunella vulgaris: lutea.

Dracocephalum fibiricum. $\beta . Y_{0}$ Melisfa nepeta. $B$.

Scutellaria minor. 3 . Stachys rethiopiça. 3 . Satureja juliana. $\beta$. Ballota migra. $\beta$. $\gamma$. jivaveolens. $\beta$.

Marrubium indicum. $\beta_{0}$

Monarda didyma. 3 . punctata. 3 .

Lamizm purpureun. $\mathscr{\gamma}$

Galopfis tetrahit. 3 . Rhinenthus crifta. 3 . orientalis.

Bartfia alpina. $\beta$. Euphraifa officinalis. $\beta$. Melampyrum fylvaticum. B. Lathraa pholypea. Orobanche ramofa. 3. 4. Barleria criftata. $\beta$. hirfuta. $\beta$. prionitis. $\beta$.

A 
Torenia afiatica. $\beta$. Acanthus capenfis. $\beta$. Premna Serratifolia.

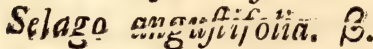
cephalophora. $\beta$. diffula. $\gamma$. paniculata. $\beta$. polygonoides. $\beta$. rapunculoides. $\gamma_{0}$ Scabrida. $\beta$. fivuria. B.

Manulea antirrhinoides. $\beta$. carnilea. $\beta$. capillaris. $\beta$. cuneifolia. $\beta$. integrifolia. $\beta$. thyriffora. $\beta$. Hebenftretia ciliata. $\beta$. Lantana aculeata $\beta$. annua. $\beta_{\text {. }}$ coccinea. $\beta$. trifoliata. $\gamma$.

Limofella aquatica. $\beta . \gamma$. Schivonckia amaricana. $\beta$. Ruellia mollisfima. utliginofa. Fufticia acaulis. $\beta$. bicalycutata. $\beta$. coerulea. $\beta$. gangetica. $\beta$. lithofperinifolia. paniculata. pieta. $\beta$.

repens.
Fuflicia Sericea.

Spathuluta. $\beta$. umbellata. verticillaris. $\beta$.

Capraria biflera $\gamma$. durantifolia. $\gamma$.

Digitalis lutea. $\beta$. purpurea. $\beta . \gamma$. Bignonia capreolata. $\beta_{\text {. }}$ catalpa. $\gamma \cdot \delta$. javanica. 2. 3 . indica. 3. pentaphylla. 2. 3. ftaus. 3.

Chelone campanulata.

Gratiola filiformis. grandijura. trifida.

Calceolaria linearis. violacea.

Hemimeris unilabiata. $\beta$. $\gamma$. Antirrhinum alpinum. E. 3. bicorne. 2. 3. 4. 5. 6.7. 8.9. 10. 11. cordatum. $\beta$. longicorne. $\beta$. majus. $\beta$. $\gamma$. marturum.

purpureum. B. $\gamma \cdot \delta_{0}$ reffexum. $\beta$. repens. 2. 3 . fcandens. Jupinum. B. triphyllum. $\gamma$. 
Peloria

Mimulus ringens. $\beta$.

Martynia perennis. $\beta$.

Pedalium murex. $\beta$.

Pedicularis folioja. $\beta$.

$$
\begin{aligned}
& \text { - jeptrum: } 2 . \\
& \text { fylvatica. } \beta .
\end{aligned}
$$

tuberiofa. $\beta$.

Clerodendrum calamitofum. $\beta$. cordifolinum. phlomidis. $\beta$.

Volkameria aculeata. 2. 3.

inermis. 2.

Citharexylon quadrangulare. $\beta$. Vitex cafta. 3. 4.

$$
\begin{aligned}
& \text { negundo. } 3 \text {. } \\
& \text { ovata. } \beta_{\text {. }}
\end{aligned}
$$

Beferia biflora. $\beta$.

Brunsfelfia undutata. 2.

Thunbergia tapenfis. $\beta$.

$$
\text { javanica. } \dot{\beta}_{0} \text {. }
$$

Melianthus major. 3. 4. 5. .6. Myagrum orientale. $\varepsilon_{0}$. perfoliatum. 2.

Vella annua. $B$.

Draba incana. $\beta$. nemaralis. 2.

Iberis amara. 2.

$$
\begin{aligned}
& \text { mudicautis. } 2 . \\
& \text { petrca. } \varepsilon_{0} 3 . \\
& \text { rotundifolia. } 2 .
\end{aligned}
$$

Alysfum colyoinum. 2 .

$$
\begin{aligned}
& \text { clypeatum. } \beta \text {. } \\
& \text { genonenfo. }
\end{aligned}
$$

Alysfum incamum.

linifolium.

minutum. 2.

montanum. 2. 3.

faxatile. 2.

fimuatum: 2.

fpinofium. 2.

Clypeola maritima. 2.

Peltaria alliacea. $\alpha$. $\beta$.

Bifcutella apula. 2. 3 .

iyrata. 2.

Anaftatica fyriaca. $\beta$.

Thlafpi alpeftre. 2.

arvense. $\beta$.

bur $/ a . \delta$.

hirtum. 2.

perfoliatum. B. $\gamma$.

faxatile 2.

Lepidium frondofum. 2.

graminifolium. $\beta$.

pifcidium. 2.

virginicum. 2.

Cochlearia officinalis. $\beta . \gamma$.

Lunaria annua. 3 .

Ricotia agyptiaca. 2.

Punias kakile. 2.

orientalis. 2.

Dintaria pentaphylla, 2.

Ifatis tinctoria. 2.

pinnata.
ctoria. 2.

C:arabe filiformis. 2.

hifpanica. 3. 4.

maritima. 2.:

orientalis. 2.3. 
Cardamine crantziana. hirfuta. 2. 3 . impatiens. 2.

Arabis -alpina. d. carvlea. pendula. 2. thaliana. $\varepsilon$. ? veroniccefolia. Cheiranthas alpinus. 2. argutus. 2. contortuplicatus.

holveticus.

leucanthuis.

montanus. 2. parviflorus. 2. taraxifolius.

Eryfimum barbarcea. 2. cheirantoides. 2. 3". hievacifolium. 20. officinale. 2. 3 . repandum. 4. Hejperis africana. $\alpha . \beta_{0}$. verna. $\beta$.

Raphanus glabratus. fibiricus. 2. tenellus. 2.

Heliophila digitata. 2: integrifolia. 2 . pinnata. 2. 3.

sifymbrium amphibium. $\gamma$. Barrelieri. 2. indicum. 2. 3 . iflandicum. 2: sajturtium, 2. 5.
Sifymbrium pumilum.

falfugino/um. .2. Sophia. 2.

Sinapis juncea. 2. fylvefte. 3. 4. recurvata. 2 .

Brasfice eruca. 2. 3. orientalis. 2.

Cupresfus difticha. 2. Iuniperus barbadenfis. $B$ : bermudiana. 2 . oxycedrus. 2. fcabrida. 全.

Pinus abies: monftrofa. vininalis. Thuja cupresfoidés. B. occidentalis. $\beta$. Hyanora africana: 4 andros. Cisfampelos hirfuta. + . Ephedra monaftachya. \&. Phyllanthus debilis.

verrucofus, $B$. Waltheria americana. $\delta$. Hermannia alnifolia. 2. citiaris. $\beta$. diffufa. $\beta$. pimnata. $\hat{\beta}$.

Grielum humifujum. $\beta$. tenuifolium. $\beta$.

Geranium capitatum, 2. 3. chinu. 2. 3 . cicutarium. $\gamma$. columinum. $\beta$. cordifoliusn. $\beta$. 
Geranium disfectum. $x$. ellipticum.

gibbofunt. 2: 3*.4.

grimum. 2. 3 .

tucidum. 2.

macitatum. 2 .

malacoides. 2 .

maritimum. $\beta$.

niolle. 2.

mofchatum. $\delta$. palmatum. $\gamma$

paluftre. 2.

papilionacennt: 2。

phoum. $\beta$.

pyrencicum. $\gamma$.

reflexum. $\beta$.

rigidum.

Robertianum. $\gamma$.

fetofint.

fiyluatienm. 2 .

tetragonum. 2 .

trifte. $\beta$.

villofum. $\beta, \gamma \cdot \delta$.

Oxalis cernua. 2.

corniculata, 2:-3. 4 . polyphylla. a. B.

Atricta. 2. 3.

Pentapetes fraberifolia. 2.

Fatropha curcas. 2.

manihot. 3 .

Croton einteria. $\gamma$.

tiglium. 2 .

variegatum, $z_{\varphi}$

Taxus baccata. fol
Taxus elongata.

falcata. fol.

latifolia. fol. towentofa. $\lambda$.

o fol.

Xylophylla tatifolia. 2.

Bombax heptaphylihum. 2. 3.

Adelia bernardia. 2.

Ricinus moppa. 2.

Camellia japonica. $\gamma$.

Urana finuata $\beta$.

typtralca. $\beta$.

Triguera lobata. $\beta$. $\gamma$,

Sida anguftifolia. 2 .

indica. 2. 9

palmata.

periplocifolia. do

vetwija 2.

rhombifolia. 2. 3. 4.

Cpinofa. 2o.

Achania pilosa. 2.

Pavonia Spicata. 2.

Hibifcus ficulnens. $\beta$.

fraterneus.

hirtus. 2 .

manihot. 2 .

merifolins.

muntabilis, 2.

obtufifolius. .

fabdariffa. 2.

finienfis. 2.

furcttenfis. 2 .

fyriacus. $\beta, \gamma$.

trionum. 2. 
Thefpefia populnea. 2. Anoda triflata. 2. 3. 4.

Dilleniana.

parvifora.

triloba. 2.

Malva alcea. 2, 8 .

americana. 2.

coromandeliana. 2 .

cretica.

elegans. 2. 3 .

grosfularifolia. 2.

mojchata. 2.

rotundifolia. 2. 3 .

fpicata. 2. 3 .

Jylveftris. 2,3 .

virgata. 2.3 .

Lavatera panciata.

Kleinhoria hofpita. $z$.

Bannifteria coriacea.

fulgens. 2.3 .

finemarienfis.

Bïtineria microphytla. z.

Pasjigora longifolia.

juter. 2.

rubra. 2.

juberofa. $\gamma$.

Seriana Inpulina.

triternata.

Saraca indica. $\gamma$.

Fumeria capreoata. B. $\gamma$.

veficaria. $\beta$.

Abrus precatorius. 2.

Polygala alopecuroides. $\beta$.

amcena. $\beta$.

bracteata. $\gamma$.
Polygala thymifolin. $B$. tinetoria.

Hypocalypius fericeus. 2. Borbonia perforata. $\beta$. ferrulata.

Viborgia fufca. 2. obcordata. 2.3.

fericea. 2.

Genifta anglica. 2. 3 .

germanica. 2.

hivfuta.

pilofa. 2.

fcariofa.

fibirica.

tinctoria. 2.

tridontata. 2. 3 .

virgata.

Ononis arverifis. 2.

hircina.

parviflora.

spinofa. 2. 3.

Antyllis gernrdi.

vulneraria. $\varepsilon . \xi$.

Lupinus firrfutns. 2. 3 .

luteus. 2.

perennis. 2.

Phafeolus capenfis. 2.

Cytifus aufriacus. 2.

biflorus. 2.

cajan. 3.

sesflifolins. $z$.

Geoffroial inermis. 2. Robinia caragana. 2. fruticofa. $\infty . \beta_{\text {. }}$ 
Robinia hifpida. z. mitis. $\beta$.

pygmea. 2 . fepium. 2.3. violacea, 2.

Coronilla varia. 2. Afchynomene americana. 2.

Hallia alata. 2 . diffufa.

Hedyjarum biflorum. buxbanmii. canadenfe. $\beta$. caput. $z$. cretaceum. $\gamma$. ebemus. $\alpha$. B. gangeticum. 2 . gramineum. humile. 2. lagopoides. 2. movilifarum. $z$. numitariun. 3 . obfcurum. onobrychis. $z$. petrceum. pilofum. 2. rufefcens. triflorum. 2. 3. villofum.

Dolichos capitatus. $\alpha . \beta$. minizuns. 2. polyftachyos. B. $\gamma$. rofeuss. z. fimenfis. $\delta$. tritobus, $z_{\text {. }}$
Teramnus volubilis. $z$ 。

Clitoria ternatea. 2. 3. 4. Stylofanthes grianenifis. Glycine filiformis. a. B. heterophylta. $\beta$. monoica. 2. 3 . phafeoloides. 2.

Orebus lathyroides. 2. Lathyrus angulatus. 2. aphaca. 2. articulatus. $z$. heterophyllus, 2 . latifolins. 3. 4.

$V$ icia onobrychoides. 2. pamnonica. 2. fativa. 2. 3. - fylvatica. $z$.

Lebeckia contaminata. 2. 3. cytifoides. $\beta$. Sepiaria. 2. 3 .

Spartium mono/permsim. $\boldsymbol{z}_{\text {. }}$ purgans. 2. fooparium. $B$.

Crotalaria anthylloides. guianenfis. imbricata. 2. laburnifolia. 2.3. linifolit. 2 . macrophylla. a. B. $\gamma$. paniculata. $\alpha . \beta$. retufa. 2 . Sericea. 2. tomentofa. $\beta$.

Ulex europecus. 2 , 
Cylifta Scariofa. Galega capen/is. 12. caribara. maxima. montara. ternate.

- trifoliata. a. $\beta_{0}$ Indigofer a anguftifolia. $\beta_{\text {. }}$ colorata. pungens.

Aftragalus anguftifolins. aufriacus. 2. canadenjis. 2. exfcapris. 2. Ealegiformis. $2 . \mathrm{a}$ glycyphyiths. $z$. hamofus. 3. halleri. hypoglottis. 2. melilotoides. 2. pentaglottis. 2. - phyfodes. 2. pilofis. 2. pitimofus. fanguinoleutus. temuifolins. 2.

Phaca alpina. 3 . incana.

Ervum lens. 2. monanthos. 2. Colutea linearis. 2. Ajpalathus albens. $\beta$.
Aspalathus labtea. B.

multiftora. $\beta$.

pinguis. pungens. 2 . qivinquefolia. $\beta$. jubulata.

Pforalea bituninofa. 2 . capitata. $\beta . \gamma . \delta$. decidua. $\beta$. hirta. 2. palceftina. 2. 3 .

Trifolium anguffifolinum. 2 . arvense. 3 . fragiferum. 3. incarnatum. 2. indicum. $\gamma$. italicum. 2. pratenfe. $1.2 .3 \cdot 4$ procumbens. 2. rubens. 2. fesfiliflorum. ftriatum. 2. Subterraneum. 2.

Lotus dorycniam. 2.

Trigonella glabra. 2. platycarpos. 2.

Medicago viliaris. 2. 3. 4.5 .6$. elegans.

- lupulina. 2.

minima. 2. iributloides.

Abroma angufta 2. Wheleri. $\beta$.

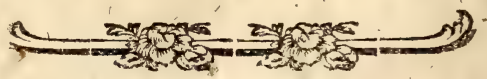


D. D.

\section{MUSEUM NATURALIUM}

ACADEMI 2 UPSALIENSIS.

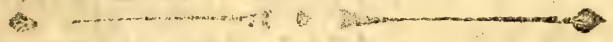

C U J U S

APPENDIC. XIII.

VENIA EXP. FAC. MEDICAE UPSAL.

PR $Æ S I D E$
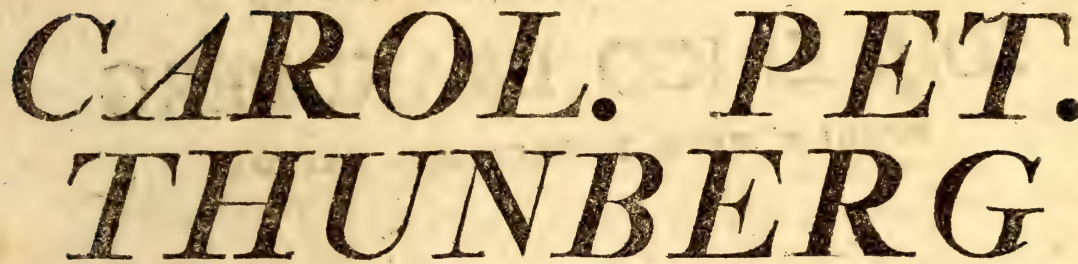

Equits Aurat. Reg. Orj). de Vasa,

Med. Doct, Prof. Med. et Bot. Reg. et Ord. Acad. Cesar. Petrofol. et Net Curios. Reg. Scient. Lond Holm. Suciet. Scient. Ups. Phllad. Patr. Hoim, et Cry. Oecon. Fine. Beror. Nat. Scrut, Paris. Hafn. Moscou. et Halens. Hist. Nat Lund. Harlem. Amsteld. Zeland. Nidrosiens. Ticinens. Ienens. Linn. Londin. Reg. Scient. Phiys. et Phytourafi. Góttingens. Nanciens. Med. Paris. Emul. Monsper. Med, Matritens, Medic. et Nat. Studios. Edimburg. Membro nec non Acad. Scient. Paris. ex Institut. Nation. Monspeliens. Agricutt. Paris. Medic.Londin.Scandinay.

Florentin, et Batavin, Ind, Or. Correspond.

PUBLICAE CENSURE COMMITTIT

ERICUS GUSTAVUS GROTH

DALECARLO WESTMANNUS.

IN AUDIT. BOT ANICO SUP: D. XII DEC. MDCCCV1.

H. A. M. S. 


\section{IN SACRAM REGIAM MAJESTATEM}

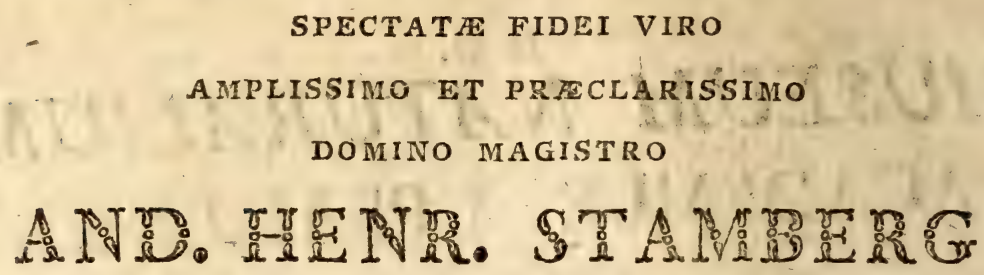

AD. REG. GYMN. AROS. ELOQV. ET POËS, LECTORI AVUNCULO OPTIMO

NEC NON

ADMODUM, REVERENDO DOMINO

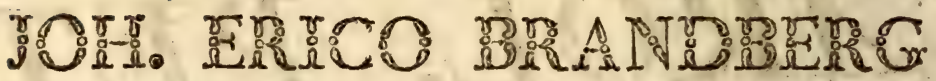

SCHOLAE TRIV. FAHLUNENS. RECTORI

Tesferam hanc mentis venerabundæ, gratisfimæ offerre voluit, debuit Nominum Veftrorum

Cultor humillinus 


\section{VIRO}

ADMODUM REVERENDO ATQUE PRACLARISSIMO

[ECCLESIE SVERDSJO゚ENS. PAST. ET. PREPOSITO DOMINO MAGISTRO

\section{PETRO GROTH}

PARENTI OPTIMO.

\section{PIUS FILIUS}




\section{THE S E \\ I.}

$\mathbb{S}_{\mathrm{i} \text { ab effectu res quxdam fit umquam dijudicanda, quis pretium }}$ fuum, fuarmque laudem Medicinæ, falubri illi valetudinis arti, iret derogatum.

II. Exfticise tamen non fane admiratione invenimus, qui bene. fican hanc artem tanto odio fint profecuti, ut perniciem humani generis \& peftem teterrimsm illam velint habendam.

III. Agmen horum ducere videtur multi ille nominis J. J. Rous. feau, Medicinam adeo exofus, ut medicum non prius jubeat arcesfendum, quam to usque res fit deducta, ut augeri amplius malum deteriusque fieri nequeat.

IV. Nifi tamen nos omnia fallunt contendere haud dubitamus hoc tantum, quo in optimam fcientiarum fertur ille, odium ne. quaquam ex ipfa esfe derivandum, exinde vero unice esfe ortum, quod infanabili ille, fibi foli reliquendo, laborans malo, in inperitas vero delapfus manus, plus ceperit doloris ex curatione quam ipfo ex morbo.

V. Neque dubitamus omnino quin ceteri omnes hujus fcientix ofores, xque futilibus innitantur orationibus longe, vero aliud præ fé ferentes incertum artis queruntur ut plurimum, utpote qux filicet nullis firmis fuperfructa principiis, non nifi cæco cafui debeat falubres fuos, quos interdum edat, effectus.

VI. Apertis tamen oculis aut non vident aut videre non volunt qui alman Anatomices operam fubfidiumqúe, largam $\mathrm{Na-}$ turalis Scientix fupellectilem, qui focias has opes idonei fatis principii vicem gerere negent.

VII. Et quod futurum confidimus aliquando, ubi ad culmen tandem \& fummam perfectionem deducta fint ambo hxc medendi artis amica fubfidia, artem hanc quoque ipfam certam, erroribus non obnoxiam amplius futuram auguramur.

VIII. Qui vero illam rejiciendam omnino jubeant \& explodendam quia erret interdum atque excidat fine, folem e mundo, caloribus quia æhluet aliquando, volunt fublatum.

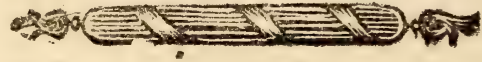




\section{DONATION. THUNBERGIAN.}

\section{APPEND. XIII.}

Hopea martinienfis. B. Hypericum dubium. elodes. $\beta$. hircinum. F. monogynum. $\beta$. prolificum, 3 . pulchrum. B. punctatum. reflexum. $\beta$. tomentofum. $\beta \gamma$. virginicum. 2 .

Trapopogon picroides. $\beta$. pratonse. $\beta$.

Scorzoncra capenfis. $\beta$. hispanica. 2. humilis. 2. laciniata. $\beta$. orimtalis. 2.3 . picroides. 2. 3 . pufilla. tingitana. $\beta$.

Sonchus alpinus. 2. arvenfis. 3. 4. glaber. $\beta$. lapponicus. $\beta$. maritimus. $\gamma . \delta$. olevaceus $\gamma . \delta, \varepsilon . \zeta . \eta_{0}$ ง. ․ $x . \lambda$. fibiricus. 2. 3. 4. tener. 2. 3 .
Lattuca sapenfis. 2. 3. 4. perennis. $\beta$. virofa. 5.6 .

Chondrilla juncea. $\beta$. Prenanthes lyrata. 2. multifora. 2. muralis. '2. purpurea. 2. farmentofa. 2.

Leontodon lyratum. palufte. Apargia hifpida. 2. 3 . Tbrincia hirta 2. 3 . Hieracium amplexicaule. 2. 3. anreum. $\beta$. auricula. 2. caucaicum. cerinthoides. 2. dubium. 2. 3 . Gronovii. 2. 3. murorum. $\gamma \cdot \delta_{0} \varepsilon_{0}$ palludofum. 2. 3. pilosella. 2. rhomboidale. Sabaudum. 2. 3. 4, 5 . fibiricum. $\beta$. 6. 7 Ataticofolium. taraxacoides. 


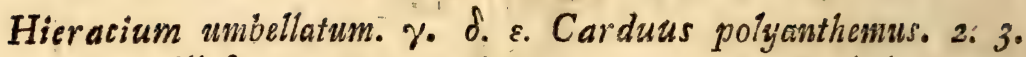
villofum., 2.3. 4. 5.6.7.

Crepis alpina. $\beta$. biennis. 2. 3 . Diofcoridis. Fo. pycnocephahiss. 2. 3 . rubra. B. virens. $\beta$.

Cnicus helenioides. $\gamma$. monspeliacus. oloracenis, $\beta$. paluftris. 2. 3 . Serratuloides. 2.

Helmintia echioides. 2.3. Spino/us. B. Tolpis barbatn. 5 . Andryala integrifniia. $\beta$. laciniata. $\beta$. lanata. $\beta$. pinnatifida. $\beta$.

Hedypnois cratica. 2. 3 . Seriola cethuen/ss. 2. Hypocharis glabra. $\gamma$.

Rohria incana. $\beta$. maculata. $\gamma$.

Lapsana crifpa. 2. foetida. $\beta$.

Rhag adiolus ftellatus. $\beta$. Borchhaufia hifpida. 2.

Cichorium endivia. 2. 3. intybus. 2.

Scolymus hifpanicus. 2. $V$ erronia anthelmintica. Serratula alpina. 2. 3. 4. coronata. 2.

Carduus arabicus. 2.

Cynara foolymus. 2. fyriacus. $\gamma$. tartaricus, 2. 3. 4. Carlina corymbofa. lanata. 2. racemoja. 2. vnlgaris. $\beta$. Bidens bipinnata. 2. cermua. $\beta$. hivfutc. 2 . nivea. 2. nodiflora. pilofa. 2 . tripartita. 2.

Cacalia albifrons. $\gamma_{b}$ arbufcula. 2. atriplicifolia. 2. haftata. 2. Kleinia. B. argenteus. 2. crifpus. $\gamma$. cyanoides. $\beta$. defloratus. 3. mutans. 2. quinquefolia. rigida. falja. fcandens. 2. fonchifolia. $\gamma . \delta . \varepsilon . \zeta . \eta_{\text {. }}$ 
Mikaria Scandens. B.

Eupatorium aromaticum. 2.

cannabinum. 2. 3 .

coeleftinum. 2.

japonicum. 2. 3 .

purpureun. $\gamma$.

finenfe. 2. 3.

Chryfocoma cerua. 2. 3. 4. 5o

linofyris. $\gamma$.

Tarchonanthus dentatus. 2 . lanceolatus. 2. racemofus. 2.

Athanafia capitata. 2. filiformis. 2. 3.'

flexicaulis.

flexuofa. 2,

punctiatas

facbra.

trifurcata. 2. 3. 4. 5. $\sigma_{\text {。 }}$

Relhania geniftexolia. 2.

Pentzia crenata. 2.

Ptironia elongata.

Artemifia arborefcens. 2. campeftris. 2. dracuneulus. 2. madorafpatana. 2. maritima. 2. 3. pontica. 2. rupeftris. 2. fantonica. 2 . tanacetifolia. 2. vulgaris, $\beta . \gamma$.

Gnaphalium arenarium. 2,
Gnaphalium arvense. 2. candidisfimum. capitatum. coronatum. 2.3 . cymofum. 2 . 3 . dioicum. 2. 3. 4. difcolorum. $2.3 \cdot 4$. divergens. 2.3 . cricoides. 2. 3. 4. foetidum. 2. glomeratum, 2. luteo-album. 2. 3. 4.5. margaritaceum. 2 . multiflorum. norvegicum. 2. obtuffifolium.2.3. purpurcum. 2. 3. revolutum. 2, filvaticum. 2. fquarrafum. 2. ftoecas. 2. 3. 4 . teretifolium. 2. 3.4. uliginofum. 2 . undatum. 2 , Elichrysum cane/cens. 2. paniculatum. 2.3.0 proliferum. 2. Sefamoides. $\gamma . \delta_{0}$ Jpeciofum. 2. fqvamafum. 2. fítiatum. 2. virgatuin. 2. 3. Conyza Afgyptiaca. ?. 3. A 2 
4

Conyza arborefcens. $\gamma$.

bifoliata.

cinerea. 2. 3. 4. 5 .

Gouani. 2.

obliqna. 2.

octorata. 2.

purpurafiens. 2.

faxatilis. $\gamma . \delta$.

ficula. 2.

Erigeron acre. 2. 3 .

canaden $\int$ e. 2. 3. 4.

pinnatum. 2.

Baccharis halimifolia. 2. 3 .

Carpefiurn cernuum. 2.

Tusfilago farfara. 2.

frigida: radiata.

fpuria. 2. 3.

Senecio, abruptus. 2.

agyptius. 2.

arenarius. $\beta$.

doria. 2.

doronicum. 2.

clegans. $\gamma . \delta$.

erucifolinus. 2.

grandiflorus. 2.

jacobcea. 2.

juniperinus. 2.

linifolius. 2.

marginatus. $2.3 \cdot 4$.

pinnilatus. 2.3 .

repandus. 2.

rigidus. 2.

rofmarinifolius. $\gamma$.
Senecio fquamofus. 2.

triflorns. 2.

vifcofus. 2.

Facobara peucedanifolia. $\beta$.

vulgaris. $\beta$. After cordifolius. $\gamma$. $\delta$.

cymbalaria. 2. 3 .

dumofus. 2. 3 .

ericoides. $z$.

fruticofus. 2 .

hispidus. 2. 3 .

muiricatus.

paniculatus. 2.3 .

puniceus. 2. 3 .

finenfis. $\beta . \gamma$.

frigofus. 2.

tardiflorus. 2.

tenellus. $\gamma . \delta$.

tripoliwm. 2.

villofus. $\gamma$.

Solidago canadenfis. 2. flexicaulis. $\gamma$. lateriflora. 2. pinnata. 2. 3 . virgaurea. 2.

Cineraria alpina. $\gamma . \delta$. cacalioides. 2. lineata. 2. lobata. 2. 3 . maritima. 2. tusflaginis.

Doria elongata. $\gamma$. Inula bifrons. 2. 
Inula oculsus. 2.

pulicaric $\gamma$.

dorosicum.

Arnica glacialis.

lanata. 2.

fonrpioides. 2.

$B$ ellis perennis. $\gamma$.

Tagetes erecta. 2. 3 .

lucida.

patula. 2.

tenuifolia, $\alpha . \beta$.

Leyfera artiotoides. 2.

sallicornia. 2. 3.

pilofella. 2.3. 4 .

polifolia. 2.

Zinnia elegans. 2. 3.

Cryfanthemure carinatum.

glabratum.

hirtum.

incanum. 2.2 .3$.

indicum. $\gamma . \delta . \varepsilon . \zeta$.

Myconis. $z$.

pygmatum.

fegetum. 2.

Pyrethrum alpinum. 2.

balfamita. 2.

corymbofum. 2. 3 .

frutefcens. 2.

millefoliatum. 2.

Lidbeckia turbinata. 2. 3.

Cotula anthemoides. $\beta$.

aurea. 2.

bipinnata 2.
Cotula coronopifolia. 2. fericea. 2.

Anthemis altisfinia. 2. arabica. 2. 3 . maritima. 2. mixia. 2. 3 . valentina. 2.

Achillaa ageratum. 2.

alpina. 2.

magna. 2.

mililefolium. 2.3 . nobilis. 2.

ptarmica. $\beta . \gamma$.

pube/cens. $\beta$.

fibirica.

tenuifolia.

tomentofa. 2.3.

Ecclipta erecta. 2. proftrata. 2.

Sigesbackia occidentalis.

Verbefina alata. $\beta$.

nodiflora.

Helianthus annuns. 4. 5.

tubeformis.

tuberofus. 2.

Rudbeckia amplexicaulis. 2, 3.

laciniata. 3. 4. 5.

Lavjenia erecta. 2.

Cosmae bipirnata. $\alpha . \beta$.

Coreopfis tripteris. 2,3 .

Centaurea amberboi. 2.

calcitrapa. 2 .

cineraria. 2. 
Centeuria crocodylium. $\%$ yaszus.

crncifolia. 2. 3. 4. 5.

feroix. 2.

galabtites. 2. 3 .

gliftifolia. 2.

jacea. 2. 3 .

ifnardi. 2.

lancita. 2.

Lippii. 2.

mapifolia. 2.

nigra. $\gamma$.

ovina. 2.

pectinata. $\%$.

phrygia. 2.

Scabiofa. $\varepsilon$.

Sempervirens. 2.

folftitialis. 2.

tartarica. 2 ,

Millefia biflora. 2.

Polymnia canadenfis. 2.

uvectalia. 2.

Calendula amplexicantis. 2.

arvenfis. 2.

graminifolia. 2.

hybrida. 2. 3. 4.

officinalis. $\gamma$.

phuvialis. 2.3 .

ftellata. 2.

Arctotis afpera. $\gamma . \delta$.

calendulacea. 2. 3 .

- cernua. 8 .

iparadoxa.
Artot is nudulata. 2 . 2.

Silphium connatum. 2.

laciniainu. 2.

perfoliatum: 2 .

therebintinatum, $z$ :

Oedera prolifira $\gamma$.

Opeofpermum ciliatum. $\gamma$.

junceum. 2. 3. 4.

polygaloides. 2.

Spiruscens. 2. 3. 4. 5.

fpinefum. 2.

Othonna abrotanifolia. 2 .

athana/ii. 2.

capillaris. 2.

coronopifolia, 2.3 .

denticulata. $\beta . \gamma$

multifida.

peokinata. 2.

pinnatifida.

fulcata.

tenuisfima. $\gamma$.

trifida. $\alpha . \beta$.

trifurcata.

verginea.

Eriocephalus africanus. 2. racemofus. 2.

Parthenum hyfterophorus. 2. integrifolium.

Fafione montana. 2. 3. 4. Elephantopus fcaber. 2. 3 . Sphoranthus indicus. 2. 3. Stoebe diftica. $z$.

$\int c a b r a .2 .3$. 
Ambrofia artemiffolia. 2. etatior. 2 . 3 . maritima. 2.

Equifetum arvenfe. $\gamma$. finviatile. $\beta . \gamma$. giganitenn: $\beta$.

Lycopodium clavatum. B. $\gamma$.

curvatum.

dichotomatm. $\beta$.

mutum. B.

plilegnaritu. $\beta$.

plumofun. $\beta$.

Ophioglosjum fandens. 2. 3 . Onoclea crifpa. $\gamma$.

$$
\text { Senfibilis. B. } \gamma \text {. }
$$

firuthiopteris. $\gamma$.

OSmunda adiantifolia. $\beta$.

ceilanica. 2.

vegalis. $B$.

virginica. 2 .

Todea barbara. 2. 3 . Acroftichum curenm. $\delta$. calome binos. 2. conforme.

heterophyllum.

hirtum. $\beta$.

ivenfe. $\beta$.

latifolinm. $\beta, \gamma$.

tburifera. $\beta . \gamma$.

Pteris aculeata. 3 .

atropurpurea. B. $\gamma_{0}$ attenuata. $\beta_{1}, \gamma$. biaurita, $\gamma \cdot \delta . \varepsilon$.
Pteris capenfis.

caudata. B. $\gamma$.

flabellata. $\beta$.

heterophylla. $\beta$.

longifolia. $\varepsilon$.

pedata. $\gamma$.

pillofeloides. $\delta$.

serrarici.

tripartita. $\beta$.

Davallia aculeata. 2.

flaccion.

Dickfonia culcita. z. iniegra.

Menifism trifoliatum.

Diplazium grandifolium. $\beta$.

Woodvardia orientalis. $\beta$.

radicans. $\beta . \gamma$.

Blcchnum anfirale. $\beta$.

occidentale. B. $\gamma$.

orientale. $\beta . \gamma$.

Coenopteris cicutaria. $\beta . \gamma . \delta . \varepsilon$. fiaccida. $\beta$.

Adianthum cethiopicum. 2.

capillus. 2.

eaudatum. 2.

pedatum. 2.

pulverulentum, 2.

tenerum. 2.

trapeziforine. 2.

villofum. $\gamma . \delta$.

Polypodium aursum. $\gamma . \delta . \varepsilon$. connectile.

effusum. 3. 4.5 . 
8

Polypodiam lanceolatum. 2. Afpidium foemina.

phegopteris. 2.3 . phymatodes. $\delta$. pilofelloides. $\beta$. puftulatum. $\beta$.

figmofum. $\beta$. triferiale. 2.

Afpidium aculcatum. $\gamma_{0} \delta . \nu_{0}$ fontanum. 2. anthriscifolium. copense. 2 . criftatum. 2. exaltatum, $\beta . \gamma$. fragile. 2. furcatum. $\beta$. mas. 3. rheticum. 2. Spimulofumı. $2.2,3 \cdot 4$, trapezoides. trifoliatum. 7. \&. truncatum. umbrofum, 2 . villofum, 2.

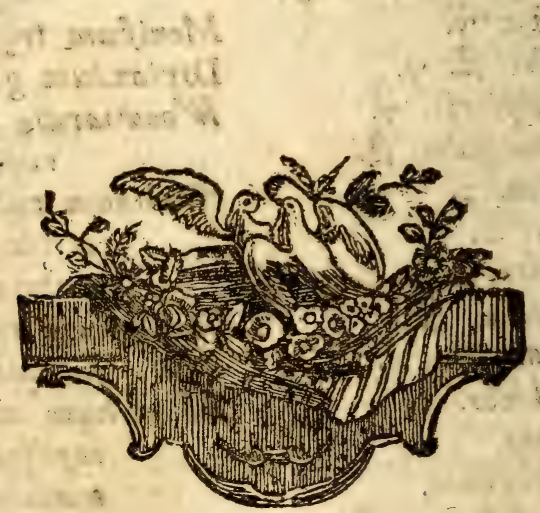




\section{D. $D$. \\ MUSEUM NATURALIUM ACADEMIL UPSALIENSIS. \\ - \\ C U J U S}

Appendic. XIV.

VENIA EXP. FAC. MEDICA THPSAL。

PR ÆSI DE
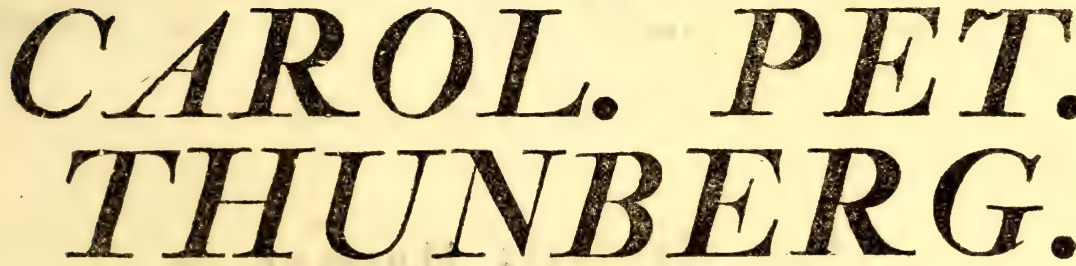

Equite aurat. Reg. Ord, de Vasa,

Med. Doct. Prof. Med, ex Bot. Reg. et Ord. Acad. Cefsar. Petropol. et Nat. Curins. Reg. Scient. Lond. Holm. Societ. Scient. Ups. Philad. Patr. Holm. ex Civ. Oecon. Fint. Berol. Nat. Scrut. Paris. Hafn. Moscou et Halens. Hist. Nat. Luni. Hariem. Amsteid. Zeland. Nidrosiens. Ticinens. Ienens. Linn. Londin. Reg. Scient. Phys. ex Phytourapit. Góttingens. Nanciens. Med. Paris. Emul. Monspel. Med. Matritens. Medic. et Nat. Studios. Edimburg. Membro nec non Acad. Scient. Paris. ex Institut. Nation. Monspeliens. Agricuit. Paris. Medic.Londin.Scandinav. Florentin. et Batavin, Ind, Or. Correspond.

PUBLICAE CENSURA COMMITTIT

\section{JOH. WILH. DALMAN,}

Nobilis.

IN AUDIT. BOTANICO SUP. D. X DEC. MDCCCVII.

H. A. M. S.

UpSalia. 'Typis Edmannianis. 


\section{BRUKS-PATRONESSAN}

\section{HOீGÅDLA}

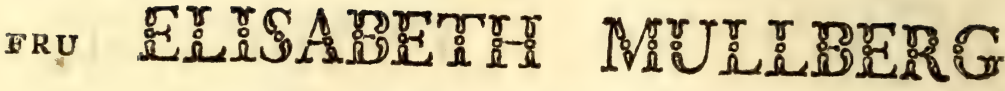 FODDD IUUNVDGREIN.}

\section{Min Huldaste Mormor}




\title{
BERGM ̊ STAREN
}

$$
\text { VÅLBORNE }
$$

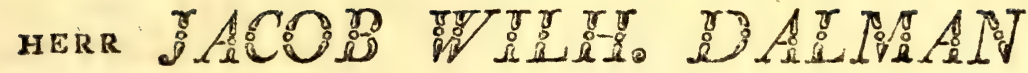

$$
\text { SAMT }
$$

$$
\text { VÅLBOR NA }
$$

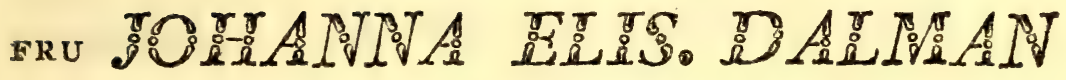
FO์DD IC ÖINTG.

\author{
Mine Huldaste Fórådrar
}

Vordnad och Tackfamhet. 

BRUKS-PATRONESSAN

HO̊GÅDLA

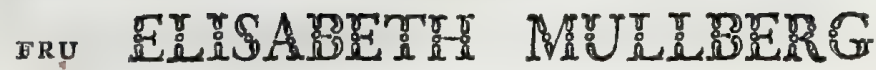
FODD 芸UINDSGIREIN.

Min Huldaste Mormor

Helgas dessa blad af
BERGMÅSTAREN

VÅLBORNE

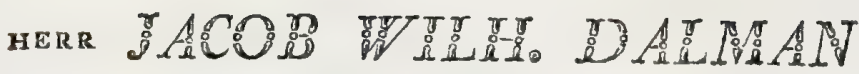

SAMT

VÅLBOR NA

FR TOOP FO์DD KÖNIG.

Vordnad och Tackfanhet. 


\section{Theres. \\ I.}

effosfx, iadubia probent tentmonia, olim animalium fpecies vixisfe, qux hodie forfan non exfant. - Inde quoque non fine probahilitate quadam concludi poteft, adhuc unam live alteram emorituram esie.

II.

Hæipfre reliquire, revolutiones maximas in Teliuris fuperficie olim evenisfe, ctiam indicare videntur.

III.

Infectorum utilitas, quamvis primo afpectu minima feu vix ulla vicleatur, in oeconomia tamen Natura certe maxima eft.

IV.

Inter medicamenta, qux e regno animali colliguntur, non minoris momenti funt qux Infecta prabent.

\section{$\mathrm{V}$.}

Methodum illam (a Cel. Furin \& Cel. Miegen propofitam), qux ad nervos alarum in Piezatis \& Antliatis refpicit, non parvum commodum afferre putamus, fi etiam Glosfatis accommodetur.

VI.

Inter omnes ordines plantarum, fungorum maxime a cete. ris difcedit; multo melius tamen ad regnum vegetabile, quam ad aliud eft referendus. 


\section{DONATION. THUNBERGIAN.}

APPEND. XIV.

Cyathea afpera. $z$. esculenta. 2. $\operatorname{exten} \int a .2$.

Ajplenium bulbiferum. $\beta . \gamma$. lucidum. $\beta$. obtufatum $\beta$. nigrum. $\beta . \gamma . \delta_{0}$ rhizophorum. $\beta$.

Trichomones radicans. 2. reniforme. rigidum. 2. fcandens. 2.

Grammitis aurita. $\beta$. compressa. $\beta$. furcatum. $\beta . \gamma . \delta$. hemionitis. $\gamma \cdot \delta_{0}$ innulata. polypodioides. precmor $\int a^{*}$. pumila. $\beta$.

Marfilea tetraphylla. 3. sphagnum alpinum.

capillaceum. cymbitolium. jovense. latifoliun. fquarrofum.
Phafoum macrocarpotio maticum. nitidum.

Sorratums.

Polytrichum formosum. longifolium. vabellum. Atrietum. Avchenopterum heterophyllion. latifolium. Anictangium aquaticum.

Cinclidium Aygium. Funaria calcaria. Timmia anfriaca. Tetraphis ovata. pellucida. Dicranum africanum. ambiguum. Billiardieri. flagellare. flavidum. interruptum. latifolium. microcarpuns. montanum. fcoparium. 2 . virens. 2. 
Encalypta freptocarpa. Trichoftomum ericoides.

fasciculare. fontinaloides. microcarpon. pallidum.

Tortula tortunfa. $\beta$. Didymodon homomotum.

Weifla rupeftris.

fchifti.

fiareleana.

Hypnum - compofitum. crifpum. curtipendulum. fiuitans. 3 . pufillum. rigidum.

Gymnoftomum recurviroftrum. intricatum. lutefcens. mollufcum.

Grimmia apocarpa. $\beta$. motrate.

recurvata. splachnoides.

Orthotrichum obtufifolinm. pumilum.

Barthramia crispa. gracilis: Bryum excelfum. longicolle. quinquefarium. Rottleri. undulatum.

Leskea attenuata. incurvata. julacea. Weifia aciphylla. cirrhata. crifpula. Dickjouii. fugax. nigrita. rọerts. pralon $\mathrm{xum} .2$. prolixum. umbratum.

Ulva filiformis. filofa. inteftinalis. $B$. lactuca. $\beta . \gamma$. ligulata. lubrica. pavonia. $\beta$. plantaginea. plumofa. protifera. pruniformis. umbellicata. $\alpha$. B. $\gamma$. Conferva albida. atra. atropurpurea. capillaris. chara. ciliata. comata. 
Conferva compacta.

compressa.

confervicola.

crippata.

criftata.

deciminoja.

diaphana.

difuja.

elongata.

ericetorum.

fluviatilis. B.

fracta.

fucicola.

genuflexa.

glomerata.

hirta.

Һiмиo/a.

littoralis.

muralis.

prolifera. $\alpha . \beta . \gamma$.

pura.

reptans.

rubra.

Scalaris.

fooparia.

Setacea.

Setigera.

fpiralis.

Jpongiofa.

utrieularis.

veficata.

viridiviolacea.

zonata. Bi tenuior.
Batrachospermsm moniliforme.

viride.

Merienfia lumbricalis.

Rivularia angulisia.

dura.

elegans.

endiviefolia.

Linckia.

Ceramium denfum: junius. adult. a. B.

ploeamum. ๔. B.

falinum.

Scorpioides,

terminale.

tomentofum, $\alpha . \beta$.

vagum.

violaceum.

Fucus abrotanifolius.

acinarius, $\beta$.

bacciferus.

biferratus.

buccinalis. $\beta . \gamma . \delta$.

byssoides.

canaliculatus. $\alpha . \beta . \gamma$.

ceranoides. $\beta$. $\gamma$.

confervoides. $\beta . \gamma$.

corneus. $\gamma$.

cornigerus.

crinitus.

crifpus. B. $\%$.

criftatus.

cuneifolius.

diaphamus.

A 2 


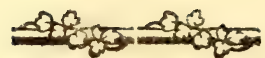

Fucus digitatus. B. $\gamma$.

fafcia.

filum. 10 2. 3.

flagelliformis.

flavus.

Fortteri.

fruticulofus.

hirfutus. $\beta$.

lichenoides.

longisfimns.

lumbricalis.

manmilofus.

membranifoliuc.

moniliformis.

muricatus.

mutcoides.

natans $\beta . \gamma$.

Opuntia.

pellucidus.

pencedanifolizs.

pinaftroides.

plagiophyllus.

plicatus.

punciatus.

ysurpurafcens.

radiciformis.

rotundus.

vufcifolins.

facharinus. $\beta . \gamma . \delta$.

falicifolius.

jedoides.

Serratifolius.

Serculatus.

Fucus filiquofus. B.

fpiciferus.

spinifex.

јрінолus.

spiralis, $\beta$.

Jpisjus.

subfuscus.

tamarififolius.

Thunbergii.

tomentofus.

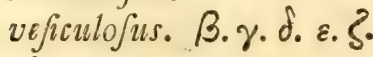

virgatus.

viridis.

Hippomane mancinella. $\beta$.

Jasminum hirfutum. $\beta$.

multiflorum. $\beta$.

Veronica Spicata. $\delta . \varepsilon$.

jpuria $\gamma$.

urticafolia. $\beta$.

Canna indica. $\delta$.

Phyllodes laeta. $\gamma . \delta$. vaginalis. $\beta . \gamma$.

Amomum paradifi.

Thalia geniculata. $\gamma$. $\delta$.

Epipaztis longifolia. $\varepsilon$.

Cranichis mejcofa. B.

Serapias lonchophylla. $\beta$.

Neoltia fpiralis. $\beta$.

Fraxinus pubefers. $\beta_{\text {. }}$

Salix

arenaria + . $\delta$.

caproea. ఫ

fol. a. $\beta$.

glanca of. $\gamma$. 


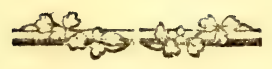

Salix haftata ㅇ $\gamma$, rojmarinifolia o. $\delta$. Serotina of $\beta$.

Ofyris alba. $\gamma$.

$$
\text { - viminalis. fol. }
$$

Valeriana montana. 2.

offinalis. B. $\gamma$.

pyrenaica. 2.

Camocladia ilicifolia.

Roiaia virticillaris.

Ixia folcata. B. $\gamma$.

Glodiolus fpicatiss. a. $\beta$.

Iris

graninize. $\varepsilon$.

temifolia, $\beta$.

Witfenia mawra. $\beta$.

Apium graveolens. 2.

petrofelinum. 4 .

Alfine media. $\delta$.

Crasfula cultrata. $\gamma$.

subulata. 2 .

umbellata. d.

verticillata. 2.

Folygonum mavitimum. $\gamma . \delta$. Laurus cinncomomeum. 10. II. Casfia horpetica fol. 2.3. Cafalpinia elata. 3 .

Prockia crencata.

Lightfootia crencta.

Mabea piriri.

$$
\text { pinata. }
$$

Hifngera

Mimoja glandulofa. longifolia. $\alpha . \beta$.

ovata.

rhombifolia. $\beta$.

virgata. 2.

Tigarca dentata. fol.

Boringionia fpeciofa. 2.

Cheiidonium glancum. 2 .

Capparis cynocephaliophora.

flexuoja. a. B.

filiquofe. 2.

triphylla $\beta$.

AEsa fpicata. B.

Calophyllum inophyllhm. $\beta$.

Cratava murmelas. fol. 2.

Talinum patens.

Plinia acida. 2.

Prunus canadenfis.

Jpinofa. 3 .

Ffidium cujavillus. fol. a. B.

Myrodendram baljamiferum.

Legnotis Casfipurea. 2.

Bunnetis paluftris.

Tilia americana. 2 .

pubefcens. fol. 2 .

Triumfetia cordata.

Cifius formolus.

ninfutus.

la laniferuis. B.

lavandulcefolius. $\gamma$.

laxius,

ledifulines. 2.

pilofis. $\gamma$.

Corchorus incifus. fol.

lomifolius. 
Roja

Rubus decunbens.

dirfectus.

hirfuins.

id ents. 3 .

odoratus 2.

rofofolius. B. $\gamma . \delta$.

Fragaria vefca. 4. 5 .

Potenitlla canloficens. 2.

hirta. 3.

miltifida. 2.

opaca. B.

vecta. 3 .

verna. 2.

Geum trilobum.

urbanum. $\gamma$.

Comarum paluftre. 2.

Kanunculus anplexicanlis. 2.

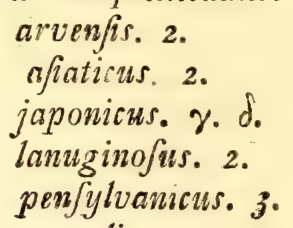

Adonis vernalis.

Anemone patens. $\delta$.
Grewia microcos

Jalvifolia. $\mathbf{8}$.

Banaza guianenfis. a. B

Aigemone mexicana. 2.

Clatia fiava.

venofa.

Blakea triplinervia. $\beta$.

Pla anus orientalis. 2.3. 4.

Nymphaca lotus. $\beta$.

lut $a .2$.

Nelumbium Speciofum. 3.3.4.5.6\%.

Ca pinus betulus. 3 . oftrya. 2

Cliffortia juniperina. $\hat{\jmath} \cdot 2$. trigona.

Liquidambar ftyracifluum.

Ioterium hybridum. 2.

fanguiforba. 2 .

Agrimonia repens.

Paonia officinalis. 2.

fibirica. 2.

Datif:a cannabina. $\hat{\text {. }}$

Begonia obliqua.

Delphinium ajacis. 6.

puniceum. 2.

ftaphifagria. 3.

Mefpilus phoenopyrum. 2.

Euphorbia Characias. 2. 3.

dubia. 2.

epithy moides. 2. 3 .

hysfopifolia.

oblitirrata 2. 3 .

Aquilegia grafudiflora. $\alpha . \beta$. 


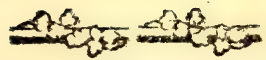

Aquilegia vutgaris. s. 6.

Nigtlla arvenfis. 2.

Spiraca arvenlis. 4.

palmiata 2.

forbifolia. 2 .

Mefembryanth. bracteatum. $\alpha . \beta$. folcatum.

Calla paluftris. 2.

Arum atrorubens.

bicolor.

colocafia. 2.

maculatum. 2. $3 \cdot 4$ muscivorum. 4.5.

Dracontium polyphyllum. 4.

Xylopia muricata. 2.

Piper adurcum. 2.

tohatum.

nigrum. B. $\gamma$.

pedicellatum.

umbellatum. 3 .

Sagittaria natans.

Clematis erecta. 5 .

grandis. 5 .

fimplex.

temuis.

qundulata.

Thalictrum majus. 3. 4.

fimplex. 2.

Zoftera marina 2. 3.

Lycopus exaltatus. $\beta$.

hirfutus.

paluftris.

Byftropogon canarieaje. $\beta_{\text {. }}$
Mentha aquatica. 2.

arvenfis.

tomento $a . \alpha . \beta$.

Sideritis incans. 2.

Phlumis biflora.

ceilendica. 2.

debilis.

elliptica.

fruticofa. 2.

hirfuta. 1.2 .

venti. 4.

Betonica gradifora.

hirfuta.

ftricta. 2 .

Lamium purpurenm. d.

Stachys lanata. 2.

Verbena fupina. $z$.

Marrubium peregrinum. $\beta$.

Scutellaria altisfima. 2 .

hys sopitolia. B.

japonica.

penfylvanisa.

Thymus ferpyllum. 7 .

virginicus.

$z y g y \cdot 4$.

Ocymum bafilicum. 2. 3. 4. 5. 6.

polyfachyon. 4.

Janctum. 2 .

teminfortom. 2.

Salvia ceratophylloides. 4.

foetida. 2 .

lyrata. $\%$.

fyriaca. 2 . 
Salvia verbenacea. 2. 3.

$$
\text { vifcolar 2. } 3 \text {. }
$$

Nepeta pectinata.

Cltonia lufiranica. 2.'

Colli fonia caratserzas. 2.

Dracophal canajicens. B. concrifum of $\beta$.

Trichoferna linseare.

Prenina ferrata $B$.

Melampyrum arvenfe. $\beta$.

$$
\text { criflatum. } 2 \text {. }
$$$$
\text { praterife. } 2 .
$$

Barlena prionitis 2.

Selago fruticosa 2."

$$
\text { rapunculoides. d. }
$$

- rotundifolia. 2.

Mamiea capergass. 2. 3 .

$$
\text { incisa. }
$$

- Sibthorpia glabra.

lobaia.

Lantana comara. 2.

Anicennia nitida. $\beta$.

Rucllia barbata. B.

cordata.

digitalis. $\beta$.

tupulina.

patula. 2.

Fuficia aggregata.

chinenfis. B.

japonica. B.

juligera. juricia litherpermifolie, procumbens. $\beta$. Spicata.

Bignomia pentaphylla. 4.

Digialis ferruginea.

Ca iola lucida.

Nature pratenfis.

Selantim orientale. 2.

Gesneria exferta. 2.

ferruginea.

Gerardia glutino $\int a . \alpha . \beta$. Anthirrinum junceum. $\beta$. linaria $\%$. minns. $\beta$. orontium. $\beta$.

Pedicularis refipinata.' 2. Gloxinia maculata. 2. Citharexylon ferratum. $z$. Vilex ternata. $\alpha . \beta$. Hyobanche fanguinea. 2 . Thunbergia javanica. $\gamma$. Iboris amara. 3 .

linifolia. $\beta$.

pimata. $\beta$.

Alysfume creticum. 2 halimifolinn 8 . faxatile. 3 .

Bifoutella apula. 4. auriculata.

Lepidium procumbens. 2.

fativnm. 2. Serratum. virginicum. 3. 4. 


\section{D. $D$. \\ MUSEUM NATURALIUM \\ ACADEMI $Æ$ UPSALIENSIS.

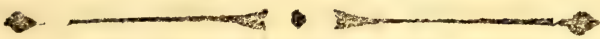 \\ C U J U S}

Appendic, XV.

IENIA EXP. FAC. MEDICAE UPSAL。

P R $Æ$ S I D E
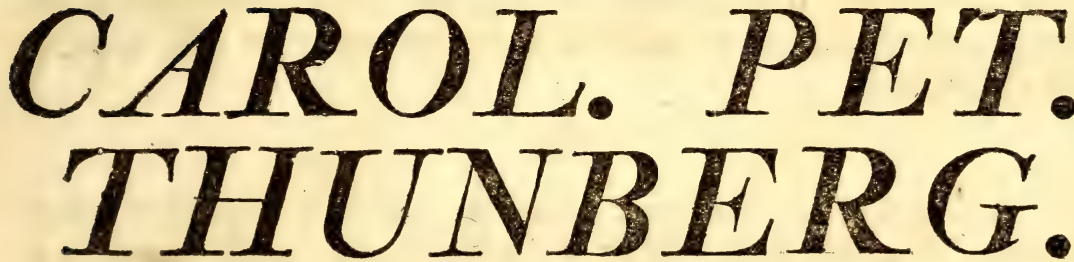

Equite Aurat. Reg. Ord. de Vasa,

Med. Doct. Prof. Med. ex Bot, Reg. et Ord. Acad. Casar. Petrotnc. et Nat. Curios. Reg. Scient. Lond. Holm. Societ. Scient. Ups. Philad, Patr. Holm, et Civ. Oecon. Fint. Berol. Nat. Scrut. Paris. Hafn. Moscou. et Halens. Hist. Nat. Lund. Harlem. Amsteld. Zeland. Nidrosiens. Ticinens. Ienens. Linn. Londin. Reg. Scient. Phys. et Phytourapi. Góttingens. Nanciens. Med, Paris. Emul. Monspel. Med. Matritens. Medic. et Nat. Studios. Edimburg. Membro nec non Acad. Scient. Paris. et Institut. Nation. Monspeliens. Agricuit. Paris. Medic.Londin.Scandinav. Florentin. et Batavin. Ind, Or. Corresponn.

PUBLICAE CENSURA COMMITTIT

$$
\text { JON JACOBI, }
$$

IN AUDIT. BOTANICO D. XXIV. MARTII MDCCCVIII. H. A. M. S. 


\section{TH E S E S.}

\section{I.}

Motum licet omnem plantis non denegaverit natura, nibilo tamen minus ab animalıbus eo inprimis differunt, quod feniu careant \& mota voluntario.

II.

Si quis negaret, plantarum fcrutationem aliquid phyfico hominum ufui præftare; tamen huic fcientiæ fuum ftaret pretium; nam illud fcrutinium femper eft homine dignisfimum.

\section{III.}

Varietatem colorum, quæe in corollis cernitur plantarum, ex effectibus lucis \& aëris, nec non varia ea: rum textura oriri, credimus.

\section{IV.}

Inftante difficultate annonæ, fi ufus Lichenis Iflandici cognitus esfet, utilitati quam maxime inferviret communi..

V.

Nemini faltim hucusque ullum in vegetabili regno invenire contigit medicamen, quod, noftro quidem im: mitiori cœlo, ad Siphilim radicitus tollendam valeat. 


\section{DONATION. THUNBERGIAN.}

APPEND. XV.

Vespertilio pipiftrallus. Canis familiaris.

B. Aquaticus.

$\gamma$. Avicularius.

ঠ. Fricator.

E. Nafo fisfo.

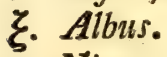

ท. Niger.

Lepus timidus $\gamma$. aftivus. Cuniculus $\beta$. cinereus. §. albus.

Ovis aries.

b. B. quadicornis. $\gamma$. quadricornis.

Capra hircus. + .

ঠ. monftrojis pedibus. Sus scrofa. \& caftrat. Equus caballus. oeland. Cavia porcellus. $\gamma . \delta$. Sorex araneus.

Phoca variegata. $\alpha . \beta$. Faleo tinnunculus. \&. Pfittacus galgulus. 1 . Oriolus xanthornus, \&. Corvus glandarius. $\beta$. Picus major. \&.
Cuculus canorus. $\$$. hepaticus.

Ardea minuta.

Tringa iflandica.

pugnax 8.4 .5 .6 .7 .8$. 9.10.11. 12.13.14.15.

Squatarola.

friata.

Charadrius apricarius $\sigma .7$. Scolopax rufticola. B. Anas anfer. \%. manjet.

fusca. 오. hismalis. ठ․ B. $\gamma$. marila. 8 . penelops \&. platyrynchos. querquedula. \$. $q$. Anatis caput roftris duobus. Colymbus criftatus. immer. parotis. a. B. subricollis. Mergus albellus. junior. Larus parafiticus. $\beta$. Alca torda: junior. Phafianus gallus: crispus. o. tetrapus. 


\section{) 41}

Tetrao bonafa. o.

coturnix. $\alpha$. $\beta$.

lagopus: maculat.

urogallus. ‥

Columba turtur. B.

Pavo criftatus $\hat{\delta}$.

Otis tarda: pullus.

Sturnus vulgaris: junior.

Ampelis garruhus: albus.

Alauda criftatella ?.

Loxia curviroftra. §. $\beta$.. .

\section{enucleator. $\phi$. $\beta$.}

Motacilla boartula.

curruca.

oenanthe. \&.

rubeculiu.

Jahoenobcenus.

tithys. \&.

$P$ arus biarmicus.

Emberiza nivalis. B. 8. fchomiclis. B.

Fringilla canarienfis. 4. 5o cannabina. के. $\beta$.

lapponica 2. 3. 4. linaria of $\beta$.

lulenfis. 2 . 3. 4. 5.

fpinus: nigra.

Teftudo caretta. $\alpha$.

$$
\text { B. }
$$

coltata.

fcabra. B. $\gamma_{0}$

Teftudinis cor:

Lagert agilis. $\eta$. ค. chamaleon. $\gamma . \delta$.

Lacerta cordylus. $\beta$.

gecko $\eta$.

iguana. $\gamma$ !

Lateralis. $\gamma . \delta$.

monitor. $\beta$.

orbicularis. $\beta$.

paluftris. $\varepsilon$. S. $\eta_{0} .9_{0}$

quadrilineata.

Draco volans. $\beta . \gamma$.

Siren lacertina. $\beta$.

Boa variegata $\alpha$. $\beta$.

Coluber Acontia.j

albus:

atropos.

aulicus.

bilateralis $\beta . \gamma$.

brunneus.

caspizs.

Haje.

lactens. $\alpha . \beta$.

Iemnifsatus. $\gamma$.

mexicamus.

natrix. $\gamma . \delta . \varepsilon_{0} \zeta . \eta$. श.

ordinatus.

ovivorus.

padera.

plicatilis. $\beta . \gamma$.

prefter. $\beta$.

regince. $\beta . \gamma$.

rhombeatus. $\beta, \gamma d \delta$

Scaber.

Ariatulus.

varius.

Amphisbana maculata. $\beta$. 


\section{0}

Anguis macutatus. $\beta$.

Ophidium imberbe.

Collionymus lyra.

Gobius jofo. B.

Silurus calichtus.

Cottus volins $\beta$.

Pleuronecies fiefur. $\beta$.

Chatodon macrolepidotus. $\beta$. vagabundus. $\beta$.

Sciana fulca.

$$
\text { taricata. }
$$

Cobitis fosfitis.

Trigla hirundo. B.

Ejox brafilienfis. B.

Mullus fasciatus.

Exocoetus exflitens.

Clupea Harengus $\beta$.

Cyprinus cephahus. $\beta$. griflagine. $\beta$.

Anarchicas lupus.

Petromyzon planeria

Squalus priftis.
Diodon hyftrix. $\gamma$.

Syngnatus acus $\beta$.

hippocampus. B.

Limax agreftis.

$$
\text { ater. }
$$

Scpia octopus. B.

$$
\text { officinaiis. } \beta \text {. }
$$

Aphrodita fquamata. $\beta$.

Pennatula aruldinacea.

Afcaris acus. 1)

blemnii. 2)

lttmbricalis. $\beta$.

nigrovenofa. 3)

Semiteres. 4)

(piralis. 5).

truncata.

veficularis, 6)

Cucullanus elegans. lacuftiris.

Strangylus armatus ơ. \$. 7). Ariatus.

Echinorynchus alcicornis. \&) attemuatus. 9)
1) ex Inteftinis Efocis Lucii.
2) ex Inteftinis Blenii vivipari.

3) e Pulmone Ranæ temporariæ. S. N. p. 3035, N:o 53. Afca. ris pulmonalis N:o 55. trachealis. N:o 58 dispnovs, N:o 59 infons.

4) Ex Inteft. Tringæ Vanelli.

5) Ex Alucone.

6) e Gallina Domeftica.

2) Ex Equi inteftino cœco.

8) Ex Alucone Ech. Percæ S, $N_{2}$

9) E Pleuronectro flefo. 


\section{) $6(\mathrm{C}$}

Echinorynchus cyprini. 10)

gracilis.

Fefucaria pileata. ventrico/a.

Gordius aquaticus. $\gamma$. argillaceus.

Amphiftoma fubclavatum. 11)

Cariophyllaus mutabilis. 12)

Faciola alata. 13)

armata. 14)

atomon.

cras $\left.\int a .15\right)$

crenata.

cirrhata.
Fafciola cylindracea. 16)

laureata. 17)

linearis. 18)

ocreata. 19)

Tania bacillaris.

calycina. 20)

clavipes. 21)

cyathiformis. 22)

expanfa. 23)

filum.

fragilis. 24)

lanceolata: $\beta$. nodofa.25)

longicollis. 26)

ofculata. 27)

I0) E Cyprino Erythrophtalmi.

II) Ex Inteft. Ranæ temporariæ. I. Ranæ. S. N. p. 3055 N:o 18.

12) Pifcium. S. N. e. Cyprino carasfia.

13) Fafciola Vulpis S. N.

14) E Muftela Putorio.

I5) Ex Erinaceo.

16) E Pulmone Ranæ temporarix.

17) Farionis \& Truttæ. S. N. 3058. N:0 33. 34.

18) E Phafiano Gallo.

I9) Fafc. latens. S. N.

20) Ex Inteft. Siluro glanidis.

2I) Ex Inteft. Murenæ anguillæ. T. Anguillæ. S. N.

22) Ex Inteft. Hirundinis rufticæ.

23) Ovina S. N. ex Agni Inteftinis tenuibus.

24) Ex Inteft. Clypeæ alofæ.

25) E Colymbo criftato.

26) E Vulpe. T. Frœlichii S. N.

27) T. Siluri S. N. \& Percæ \$. ibid. 


\section{6}

Tania paradoxa.

$$
\begin{aligned}
& \text { punctata. 28) } \\
& \text { rugofa. 29) } \\
& \text { ferpentiformis. } 30 \text { ) } \\
& \text { torquata. 31) } \\
& \text { torulo(a. 32) } \\
& \text { variabilis. 33) }
\end{aligned}
$$

Trichocephalus hominis. 34)

Uncinaria melis.

Ligula inteftinalis. 35)

Tricufpidaria nodulafa. $3^{6}$ )

Madrepora ananas.

areolata. $\alpha . \beta$.

fafcicularis. interftincta. $\alpha . \beta$. agaricits. faftigiata. $\beta . \gamma$. flexuofa. fungites. $\beta . \chi . \delta$. $\varepsilon$. $\zeta$.
Madrepara interfinetia. $\alpha . \beta$.

labyrinthiformis, mueandrites. $\alpha . \beta . \gamma$. muficalis. $\beta . \gamma$. oculata. B. $\gamma$. truncata.

Tubipora catemularis. $\alpha . \beta . \gamma$. Ifis hippuris. B. $\%$. nobilis. $\beta$. ochracea. B. $\gamma . \delta$. Millepora alcicornis. $\beta$. coriacea. $\beta$. polymorpha. $\beta . \gamma . \delta$. truncata.

Corallina opuntia. $\beta$. Cellepora Spongites. $\beta$. Gorgonia antipathes. $\beta, \gamma$. flabellum. $\beta . \gamma$.

28) T. Scorpii S. N. Ex Inteft. Pleurenectis maximi.

29). E Salmone Salar.

30) E Cornice, collo non articulato.

3I) Ex Anate domeftica.

32) Ex Int. Cyprini jefis.

33) Ex Int. Tringre Vanelli。

34) Ex Inteft. crasfis Mulierisn

35) E Colymbo criftato.

36) E Perca lluviatili. 



\section{D. \\ MUSEUM NATURALIUM ACADEMIE UPSALIENSIS.

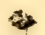

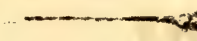 \\ Cu Jus \\ Appendic. XVI.}

VENIA EXP. FAC. MEDICE UPSAL.

\section{P R I I D E}
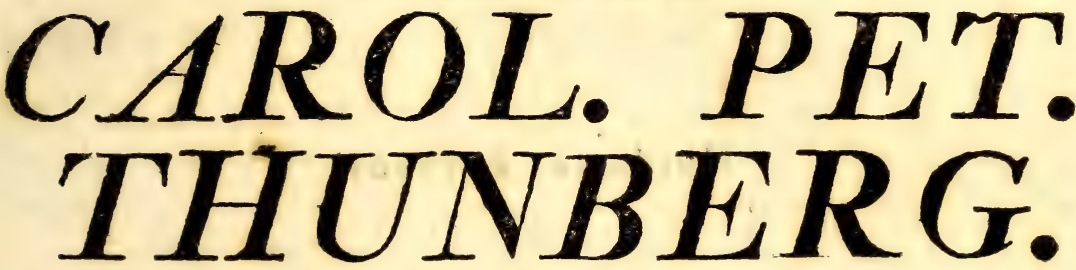

Epuite Aurat. Reg. Ord, de Vasa,

Med. Doct. Prof. Med. et Bot. Reg. et Ord. Acad. Cessar. Petropnd. ex Nat Curins, Reg. Scient. Lond. Holm. Socret. Scignt, Ups. Phir ad, Patr. Holm. ex Civ, Uecun. Fint. Berol. Nat. Scrut, Paris. Hafn. Moscou. er Halens. Hist. Nat. Lund. Harlem. Amsteld. Zeland. Nidrosiens. T icinene.

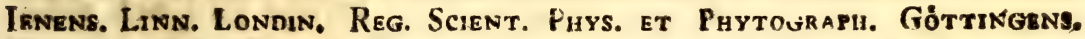
Nanciens. Men Paris. Emul. Monspel. Med. Matritens. Medic. ex Nat. Studios. Edimburg. Membro nec non Acad. Scient. Paris. et Instatet. Nation. Mongreliens. Agricuit. Paris. Medic.Londin.Scandinav,

Frorentin. et Batavin. Ind. Or. Correspond.

PUBLICA CENSURE COMMITTIT

O. A. ROBSAHM.

STIP. ROBSAKM, SUD. NER.

IN AUDIT. BOTANICO D. I. JUNII MDCCCVII.

F. A. M. S.

Upfalie, Typis Edmannienis. 


\title{
SLOTTSBYGMÅSTAREN ,
}

\author{
DIREKTO̊REN
}

HERR A BAHMNOBSAHM

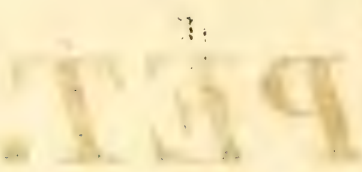

Huldaste Farbror!

Af Eder godhet stúddes hvar

Hvarje god och sko̊n

Frid o̊fver 


\section{DONATION. THUNBERGIAN.}

\section{AP.PEND. XVI。:}

Commelina midiflora. $\gamma$ : umbellata. undulata.

Corypha umbraculifera. 3 . Pontederia haftata. B. $\gamma$. vaginalis.

Leucojum autuminale. 2. Narcisfus orientalis. $3 .:$ tazetta. $\beta$.

Ariaryllis lutea. $\beta$.

$$
\text { regince. } 2 .
$$

Hamanthus punicens. 3 .

Fvitillaria imperialis. $\gamma$.

Tulipa Gefneriana. 21. 22. 23.24.25.

Scilla maritima 2.

Gethyllis cufpidata. flos.

Cyanella alba $\gamma$.

lutea. $\beta$.

Hyacinthus orientalis, $\varepsilon . \zeta . \eta, \vartheta$.

Lachenalia pallida. $\gamma$. Aloë perfoliata $\gamma$. Agave americana, $\gamma$. Alftroemeria pelegrina 2. Hemerocallis flawa. 2. 3. 4.
Dracena acuminata. elliptica. filiformis. hemichryfa. obliqua.

Funcus articulatus. $\delta$. bufoninus. $\delta$. capenfis. $\beta$. capitatus. $\gamma$. cephalotes. $\beta$. extenfus. $\beta$. luzuloides. $\beta$. maximus. parviflorus. $\beta$. spicatus. $\beta$. tenageia. $\beta$.

Prinos verticillatus. $\gamma$. Tectona grandis. 3. 4. Nandina domeftica. 2. Frankenia hirfuta. $\varepsilon$. Ehrharta calycina. a. B. geniculata. $\beta$. panicea.

Oryza mutica. a. $\beta$. 
Rumex aquaticus. 2.

nervofus.

palmatus. 2.

perficarioides. $\beta$.

fanguineus. $\beta$.

foutatus. 2.

tataricus. 2. 3. 4. undulatus. 2.

Melanthium turritum.

Trillium erectum.

Colchicum autumnate. 3 .

Alifma Plantago. ?.

Tamnus communis. $\delta$. $\beta$.

Smilax aspera. + .

ceilanica. \&.

china. त.

psendochina. 2. 3. 4. 5.

farfaparilla. \&: $\beta$.

Diofcorea bulbifera. 2. 3 . japonica. ?.

quinqueloba. 令. $\beta$.

Ariftolochia indica. 2.

$$
\text { odorata. } 2 \text {. }
$$

Cochlearia armoracia. 3.

$$
\text { glaftifolia. } 2 .
$$

Cakile myagroides. 2.

Cardamine pratenfis. 2.

Arabis filifolia.

heteroplinylla. 2.

thaliana. $\eta$.

Cheiranthus alysfoides.

incanus. 6. 7 .

littoreus. 2.
Cheiranthus Atritus. B.

Hefperis africana. 3 .

inodora. 2.

Sifymbrium indicum. 4.

irio. 2.

Loefelii. B. $\gamma . \delta$. tenuifolium.

Cupresfus thyoides. $\beta$. 7 uniperus fabina. 8 . Melochia borbonica. corchorifolia. 2. tomentofa. $\beta$.

Cisfampelos caapeba. 2. 3. 4. Ephedra monoftachya. $\beta$. Phyllanthus emblica. $\gamma$. ninuri $\gamma$.

Hermannia verticillata. $\beta$.

Geranium batrachioides.

chorophyllumin. ibericum. $\alpha$. $\beta$.

Pelargonium betulinum. $\beta$. denticulatum.

Oxalis repens. 2.

Sterculia javanica. 2. platanifolia. 2.

Acalypha javanica. ovata. a. B.

finuata.

Croton arganteum.

ceilanicum. fol.

chinense.

coriaceum.

glandulofim. $\beta_{\text {. }}$ 
Croton incanum. $\alpha . \beta$. latifolium. fol. mucronatum. fol. racemofum. fpiciflorum. $\beta$. tinctorium. $\gamma$. undatum. fol.

Pinus maritima. 1 . $\beta$. filveftris. $\gamma \cdot \delta$. frobus. \&. virginiana.

Ricinus trilobus. 1. 2.3. Gosfypium herbaceum. B. Sida acuta. B. althaifolia. $\beta$. crifpa. B. dioica. 2. 3. 4. javen/is. linifolia. $\beta$. pimpinellifolia. $\beta$. prooumbens. $\beta$. pufilla. repens. $\beta$. rhombifolia. 5 . ftellata. 2. tricuspidata. urens. 2. veronicareflia.

Pavonia fpinifex. 2. Hibijous paluftris. $\beta$. Malva ovata. furatten/is. 3 . polyftachya.
Malva finenfis.

Lavatera arborea. 4. olbia. $\beta$.

Alihae ficifolia. B. officinalis. 2. Melia azedarach. 2. 3. 4. Turras obovata. tomentofa. Malpighia ang"ffifolia. armeniaca. crasfifolia. B. glabra. $\mathbf{2}$. nitida. $\beta$.

Bannifteria bengalenfis. $\beta$. fuitgens. 4. 5 . javanica. 1. 2. Averrhoc carambola. 2. Aquilicia lavis. Zygophyllum fabago. $\beta$. Sesfilifotium. $\gamma$. Pasfiflora laurifotia. 2 . Serratifolia. $\beta$.

Polygala ciliaris. $\beta$. guianen/is. macrophylla. magna. $\beta$. pungens. Spinofa. B. viridef cens. $\beta$. Dahlbergia lancearia. 2. monetaria. 2. Amerimnon Brovnei. Erythrina picta. $\beta$. 
Fif idia erythrina.

Securidaca acuminata.

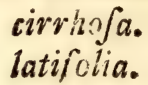

reflexa.

Scandens.

Galedupa multifisra. I. 2 .

pungens. 1.2.

jeandens.

Afpalathus affinis. B. flexuofa. 2 .

hifpida. B.

Rafnia angulata. $\beta$. $\gamma$. anguftifolia. $\beta$. filifolia. 2.3.

Crotalaria argentea.

bifaria.

biflora. $\beta$.

glabra.

juncea $\beta$.

linearis.

linifolia. 3.

nervaja.

proftrats.

pubefrens.

terminalis.

Vlex europaus 3.

Genifta canarien/is. $\beta . \gamma$. triangularis. $\beta$.

Ononis minutisfima. $\beta$. natrix. $\beta$. $\%$ pinguis. $\beta . \gamma$.

Anthyllis vulneraria. ๆ. ๆ. .
Phafeolus minge. $\beta$. radiatus. 2.

Orobus hitens. 2

Cicer arietivn?m. 2.

Vicia narboneryis. 2.

fativa. 4 .

Cytifus calycinus.

capitatus. 2. glutinofus. 2.

Scorpiurus muricata. $2^{\circ}$ vermiculata. 2. IIfcynomene diffuja. 2 . pallida. 2. fpinulofí. 2.

Dolichos enffformis. 2.

Hedyfarum coronarium. 2. Indigofer a Anil. 2.

enneaphylla. $\beta . \gamma$.

mauritunica. $\beta$.

proftrata.

p foraloides.

Aftragalus alopecuroides. $\gamma$

arenarius. 2.

crmpe?tris. 2.

glaux. 2.

phyfodes. 3 .

fulcatus. 2 .

virefcens. 2.

Ervum monanthos. 3.

Colutea frutefcens. 2.

Galega cinerea. 2.

jericea. $\beta$.

Arita. 
Galega villofa. 3.

Pforalea axillaris. $\beta$.

triflora $\beta$.

Trifolium agrarium. 2 .

flexunfum. $\beta$.

hybridım. 2. 3 .

officinale. $\beta$.

Lotus corniculatus. 2.

maritimus. 2.

Trigonella foenum. 3 .

Medicago laciniata. 2.

marina. 2.

Citrus decumanus. 4. medica. $\beta$.

Durio foetida. $\beta$.

Rhagadiolus edulis. 2.

Hieiacium incanum.

lyratum. $\beta$.

pahidofum. 4 .

fahaudum 8. 2.

fioiricum. 4.

Sprengerianum. 2.

amndulatun. 2.

Scorzonera hifpanica $\beta$.

purpurea $\beta$.

Lactuca javanica.

integrifolia.

Senegalenfis.

Rhotia integrifolia. $\gamma, \delta$.

Catananche lutea. 2.

Serratula coronata 3.

qutinquefolia.

Talja.
Carduus acanthoides. 2.

pycnocephahus. 4.

Cnicus helenioides. $\delta$.

Onopordon acanthium. 2. illyricum. 3.4 .

Carthamus lanatus. 3 .

mitis. 4.

Spilanthus acmella. 2. 3 . oleracea. 2. pSendoacmella. 2.

Bidens bipinnata. 3 . nodiflora. 2. piloja. 3 .

tripartita. 3 .

Mikania Scandens. $\gamma$. vohubilis.

Eupatorium ageratoides. 2.

diffufum. 2.

odoratum. 2.

finenfe. 4.

trifoliatum. $\beta$.

Ageratum conyzoides. $\gamma . \delta$.

Santolina chamcecyparisjus. ß. \%. d.

Tanacetum vilsare. $\gamma$.

Artımijia glacialis. 2, 3. 4 .

japonica. 2.

monoftachya.

parviflora.

Gnaphalium dioicum. 5 .

divaricatum. 2.

indicum. 2.

niveatm. 2.

orientale. $\beta$. 
Gnaphalium polyanthos. 2. Arrigofim 2.

Xeranıhemum inapertum. foloniferum. $\beta$.

Erigeron canadenle. S. uniforum $\beta$.

Conyza arborefcens. $\delta$. sinerea. 6 .

lacera. 2. 3. 4. virgata. $\beta . \gamma \cdot \delta . \varepsilon$.

Inula britannica. $\beta$.

Arnica doronicum.

montana. $\beta$.

Doronicum aufriacum. $\gamma$. bellidiaftrum. $\gamma$.

Senecio alpinus. B.

aquaticns. $\gamma . \delta$.

cruciatus. 2.

Squalidus, $\beta$.

Facobaa fulfa. 2 .

hieracifolia. 2.

After canus.

hysfopifolins. $\beta$.

macrophyllus: 2.

puniceus. 4. 5. 6.

frigofius. $\gamma$.

trailefcanti: 2 .

undulatus. $\gamma$.

Vaillantii. $\beta$.

Solidago gigantea. $\beta$.

minuta. 2. 3 .

fempervirens. 2.
Pectis humifusa. $\beta$. linifolia. $\beta$.

Helenium autumnale 3 .

Boltonia afteroides. 2.

Cotula bipinnata. 3 .

Chy yanthemum indicum. $\eta_{0}$.

Matricaria chamomilla. $\beta$.

Anthemis cota. B.

nobilis. $\beta$.

Achillea alpina. 3.

atrata. $\beta$.

criftata.

herbarota.

ptarmica. $\delta$.

Eclipta ereita. 3. proftrata. 3. 4.

Sigesbeckia occidentalis.

$V$ erbefina biflora. $\beta$.

calendulacea. $\beta$.

finticofa. $\beta$.

mutica. $\beta$.

nodiftora. 2 .

Ethulia ovata.

pinnata.

Virgilia

Ximenefia enfelioides.

Camuita perfoliata.

Stcvia linearis. 1. 2.

Serrata.

Galinfoga parviflora. triloba.

Lagafoa mollis, $\alpha_{0} \beta . \gamma$. 
Dyfoda vifco/a.

Buphthalmum aquaticum. 2. arborefcens. 2. Spinofum. 2.

Coreopfis auriculata. $\gamma . \delta$. Helianthus altisfimus. 2. dentatus.

Rudbeckia alata. $\alpha . \beta$. Centaurea afpera. $\beta$. $\gamma$. fcabiofa. .

Silphium afterifcus. 2. Calendula hybrida. S. pluvialis. 4 . ftellata. 3 .
Arcatis bellidifolia. calendulacea. 4. 5 . mudicaulis. 2. foechadifolia.

Ofteofpermum polygaloides. 3 . Othonna coronopifolia. 4. Ambrofia maritima. 3. 4. Spharanthus africanus. $\beta$. Echinops hifpidus. B. Spharocephalus. $\beta$. Micropus Jupinus. 2. Stoebe appera. 2. cinerea. 2. obliqua. virgata. 

D. D.

\section{MUSEUM NATURALIUM \\ ACADEMIE UPSALIENSIS.}

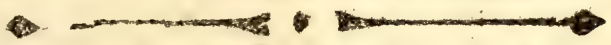

C U J U S

APPENDIC. XVII.

DENIA EXP. FAC. NEDICAE UPSAL.

PR $Æ S I D E$

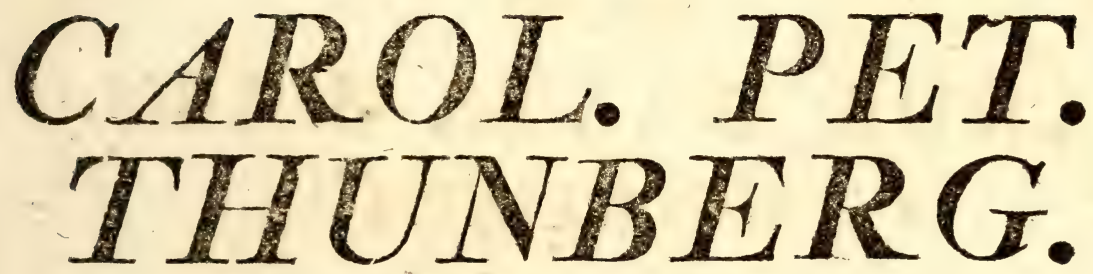

Equits Aurat. Reg. Ord. de Vasa,

Med. Doct. Prof. Med. ex Bot, Reg. ex Ord. Acad. Casar. Petropot. ex Nat Curios. Regr. Scient, Lond. Holm. Societ. Scient. Ups, Puilad, Patr. Holm. et Cin. Oecon. Fint. Berol, Nat. Scrut. Paris. Hafn, Mloscouner Halens. Hist. Nat ignd Harlem. Amsteld. Zeland. Nidrosiens. Ticinens.

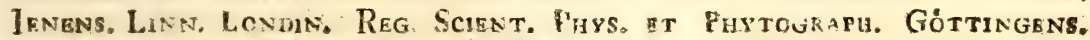
Nanciens. Mud Pakts Emul. Monspal. Med. Matritens. Medic. ex Nat. Siujros, timimag Membra nec non acad. Scient. Paris. et Insbitut. Nation. Manspeilens. Agricutt. Paril. Medic.Londin.Scandinav. Florenim. et batavin. Ind. Or. Corraspond.

PUBLICA CENSURE COMMITTIT ANDREAS OLAVUS HALL。 SMOLANDUS.

IN AUDIT. BOTANICO D. XXII. MART. MDCCCIX. H. A. M. S. 


\section{PASTORN OCH COMMINISTERN \\ VÅLÅREVO̊RDIGE OCH” HO̊GLÅRDE}

MAGISTER HERR.

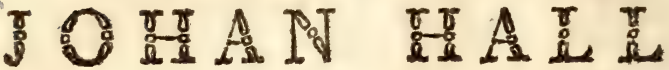

$\operatorname{SAMT}$

DESS FRU

\section{A P P P A FÓDD STRÖNY}

Mine Huldafte Foraldrar?

Om erkånfamheten 'jelo̊nar osmhetens upoffringar, och den ofőrikrácktł Aiten förtjenar dem; hoppas jag att kunna förlyckliga EDkA dagar. Det

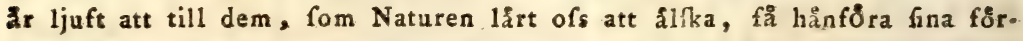
bindelfer, och om i en framtid jag kan gagna mina Likar och förmildra deras qual, år det till EDER, fom mitt hjerta fkall betala deras tackfågelfe.

Med vo̊rdnad fo̊rblifier,

Mine huldafte Fơråldrars 


\section{DONATION. THUNBERGIAN.}

APPEND. XVII.

Lopezia racemofa. B.

Syringa vulgaris. $\gamma . \delta$.

Veronica incana. $\beta$.

maritima. $\varepsilon$.

fpuria. $\delta . \varepsilon . \zeta$.

Cymbidium giganteum. $\beta$.

Diuris maculata. $\alpha$. $\beta$.

Salix anguftifolia. fol. $f u j c a$. fol.

Boerhavia ciliata. $\beta$. tomentofa.

Valeriana hexandra. $\beta$. officinalis. $\delta$.

Bryonia decumbens. elegans. laciniofa. B. $\gamma$. macrocarpa.

Cucrsmis acutangula. $\beta$ : aurantia, $\alpha . \beta$. colocynthis. $\beta . \gamma$, dudaim. $\beta$. hifpida. horrida. $\alpha . \beta$.

Trichojanthes amara. anguina. $\beta$. cordata. quinqueloba. triloba.

Momordica angulata.
Momordica cordata. disfecta. a. B. elliptica. hifpida. incija. quinqueloba. a. $\beta_{0}$ jpeciofa.

Iris fibirica. 5 .

fifyrinchium. $\gamma$. fqualens. $\beta$. tripetala. $\delta$. variegata. $\beta$.

Gladiolus Speciofus. triticeus. virefcens. Ixia coccinea. crocea. elliptica. monanthos. reflexa. Speciofa. fqualida. viridis.

Typha minor. $\beta$. Sparganium natans. $\beta$. Eriophorum gracile $\alpha . \beta$. polyftachyum. $\beta$. - Scheuchzeri.

Cyperus diftans. $\gamma$. 
Cyperus iria. B. $\gamma$. Atrigofus. $\beta$. $\gamma_{\text {. }}$

Scirpus ligularis. $\gamma_{0} \delta . \zeta . \eta_{0}$ miliasens. $\delta$.

Carex alpeftris.

Buxbanmii. $\beta$.

riparia. $\beta$.

Cenchrus Burmanni. $\beta . \gamma$. ciliaris. $\delta$.

orientalis.

Setacens.

Jetofus. $\beta$.

Coix gigantea. $\beta$.

Fuivena jeirpoides.

Saccharum Thunbergii. $\beta$.

Panicum agyptiacum. $\propto$. $\beta$.

Phleum annunu.

Milium vernum.

Agroftis hifpida.

linearis. $\alpha . \beta$.

pungens.

rupeftris.

Aira aquatica. $\beta$.

Poa anguftifolia. $\beta$.

bulbofa. $\gamma . \delta$.

capillaris. $\gamma$.

diftans, $\beta$.

difficha.

maritima.

pungens.

viccosa $\beta . \%$

Ifchamum congjugatum.

diflachyum.
Apluda tripolitana. $\beta$.

Dactylis hifpanica.

Cynofurus virgatus. $\varepsilon$. Alopicurus pratenfis. $\beta$. Fefluca arundinacea. $\beta$.

decumbens. $\beta$. elatior. $\beta$.

minuta.

Bromus confertus.

madritenfis. $\beta$. mollis. $\gamma$.

Secalinns $\gamma$

Arundo calcumagroftis. $\delta$.

Hordeum difichum $\beta$.

Triticum imbricatum.

turgidum. $\gamma$.

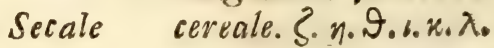

Avena elatior. $\beta$.

rigida.

Cativa. $\beta$.

Queria canadien/s. B.

Scabiofa africana. $\gamma, \delta$.

banatica.

caucafina.

srenata.

cretacea.

leucantha: $\beta$.

maritima. $\varepsilon . \zeta$.

rupeftris.

Speciofa. à. B.

Protea nitens.

patula. $\gamma$

verticillata. $\beta$. 


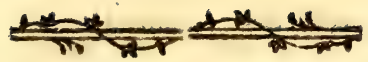

3

Hedyotis glabra.

hispida.

lavis.

lancea.

linearis. a’. $\beta$.

ovata

paniculata.

tenera.

Ernodea littoralis. $\beta$.

Spermacoce corymbofa.

ferruginea.

jamaicenfis.

Senegalenfis.

tenuior. $\beta$.

Oldenlandia biflora. $\beta, \gamma$.

nudiflora.

paniculata. $\beta$.

patula.

Jenegalenfis.

tenuifolia. $\delta$.

triflora. $\gamma$.

Galium maritimum. B.

tenuisfimum.

verum. $\varepsilon$.

Rubia lucida.

Callicarpa reticulata. tomentofa. $\gamma . \delta$.

Sangviforba officinalis, $\gamma$.

Plantago dentata.

Ammannia baccifera. $\beta$.

Elaggnus ceilanica.

Rivina brafilienfis. a. $\beta . \gamma . \delta$. octinara. 2 .
Camphorofma acuta.

Buffonia tennifolia. B. $\gamma$.

Vijcum obtufatum.

Potamogeton heterophyllum. $\beta . \gamma$.

lucens. $\gamma$.

perfoliatum. $\beta$.

Myrica papillofa. fol.

Morus alba. $\beta$.

nigra $\gamma$.

Tricera lavigata.

Dorftenia contrajerva. $\beta$.

Urtice ceilanica.

incana.

lavigata.

nivea. $\beta$.

pumila.

fcabra.

ferrulata. $\beta$.

Boehmeria crenata.

cuspidata.

hispida.

incequalis.

levis.

lancea.

ramiflora. $\beta$.

Scabra.

viliofa.

Fagara tragodes. $\gamma$.

Cornus obovata. fol.

Ludwigia hirfuta.

Pothos cordata.

Parietaria cretica.

\section{$x^{2}$}

hippida. 
Betula premila. B.

Heliotropium malabaricum.

Lycopfis pulla. $\gamma$.

Echium plantagineum. $\boldsymbol{\gamma}$. vulgare. $\delta$.

Tournefortia bicolor. $\beta$. volubilis. $\delta$.

Primula farinofa: $\gamma$. glutinofa. $\beta$. longiflora.

Cyclamen hederifolinum. $\beta$.

Phlox divarisata. $\beta . \gamma$. ovata.

Svaveotens. zindiulate.

Cortufa Gmelini $\beta$.

Convolvulus armatue. axillaris. biceps. capitatus. candatus. ceilanicus. cneorum. $\beta$. coarctatus. corymbofus. crenatns. $\beta$. cuspidatus. erojus. excifus. fulgens. gemelhis. 3. gibbus. \&. $\beta$. glomeratus.
Convolvulus gosfypifer. d. heterophyllhus. hir futus. hoinfericeus. javanicus. lanceus. latifolius. macranthus. ovatus. palmatus. pedunculatus. polygamus. purpureus. $\beta$. reniformis. rigidus. rotundatus. Sarmentofus. faxatilis. fcandens. frutatus. Senegalenfis. fibiricus. $\beta$. ftellatus. temuis. velutimus.

Ipomoea calycina.

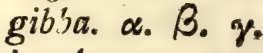
involucrata. quinqueloba. folanifolia. $\beta$. trifida. a. $\beta$.

Cantua Loitzia. Polemonium coesuleum, d. 


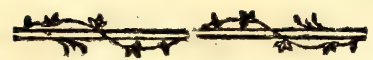

Campanula cethiopica. carpatica. $\beta, \gamma$. ciliata. glonerata. $\gamma . \delta$. iintegra. a. $\beta . \gamma$. laxa. linifolin. á.

B. Scheuchzeri. pulla. $\beta$.

rapunculoides. $\gamma$. rapunculus. $\beta$. fcabra.

fpeculum. B. jpicata.

Phyteuma hemifpharica. $\beta$. orbicularis. $\gamma . d$.

Tracheiium coeruleum. $\beta$. $\gamma$. Lobelia denfa. B.

fissa.

hifpida.

mudiflora.

racemefa.

farmentofa.

Aricta.

urens. B. $\gamma$.

Godenia villoja.

$$
\text { vifcofa. }
$$

Scavola Plnmieri. B. \&

Nauclea gemina.

grandis:

obtufata.

occidentalis. $\gamma$. tomentofa.
Pfychotria viotacea.

Lonicera diervilla. $\beta$. dioica.

Morinda volubilis. a. $\beta$. $\gamma$.

schoepfia americana.

Er ithalis fruticofa. $\beta$.

Musfanda frondofa. $\beta$.

glabra.

Mirabilis jalappa $\beta$.

Verbafcum foabrum.

taurinum.

Phyfalis angulata. $\delta$.

Solanum viculetisfimum. $\beta$. ceilanicum. cordatum. javanicum. indicum. $\beta$. laurifolium. moluccanum.

Sancitum. B. $\gamma$.

Capficum frutefrens, $\beta$.

Lycium barbarum. $\gamma_{0}$

Ardifia facbra.

Fasquinia ovata.

Cordia flavefcens.

febeftena. $\gamma$.

viburnoides. B.

Varronia auguffifolia. butlata. $\gamma$.

Laugeria hucida.

Bumelia cuneata.

Rhamnus catharticus. $\beta$.

aleoides. $\beta_{\text {. }}$. 
Ceanothus afiaticus. $\beta$. Celafirus buxifolius. $\zeta$. laurinus. $\beta$.

Diofma carnofa. coftata. rubra. $\delta$. Squarrofa.

Stavia incurva.

Mangifera indica. a: $\beta$. macrephylla.

Ribes alpinum. $B$. diacantha. $\beta$.

ftoridum.

reclinatum. $\beta$.

Viola elliptica.

frutefcens.

montana. $\gamma$. obliqua.

Impatiens cormuta. triflora. $\beta$.

$V$ itis arborea. $\beta$. $\gamma$. crenata. gibba.

Heliconia bihai. 4. pfittacorum. $\beta$. Achyranthis afpera. $\beta . \gamma$. echinata. $\beta$. expanfa. $\beta$. muricata. $\beta$. pubefcens. fcandens.

Celofia albida. saftrenfis. $\beta$.
Celofia repanda. a. B. virgata. a. $\beta$. Illecebrum Achyrantha. $\beta$. debile. a. $\beta$. decumbens. enfatum. ficoideum. $\beta$. laxum. lineare. paniculatum. B. \&. polygonoides. $\beta$. friatum. $\alpha . \beta$. triceps. $\propto$. $\beta$. vermiculatum. $\beta$. Irefine angufifolia. $\gamma$. Amaranthus candatus. $\delta$. melancholicus. $\% . \delta$. fanguineus. $\delta$.

Raurvolfia nitida. $\gamma$.

Gardenia aculeata.

armata. fol. $\beta$.

nervofa. pavetta. villofa. fol.

$V$ inea minor. $\beta$.

Echites edulise, 1. 2. elliptica.

ferruginea. a. $\beta$. obtufa. Scandens. à. $\beta$. fuberecta. tomentofa. $\beta$. vohubilis. 
Plumieria cufpidata.

$$
\text { obtuja. }
$$

Tabernamontana crasfa.

cuspidata. $\alpha$. $\beta$.

lanrifolia. $\beta$.

Periploea anguftifolia. efculenta. $\beta . \gamma$. macrophylla. ๔. $\beta$. obtufa.

Cinnanchum acuminatum. attenuatum. capillare. cordifolium. cuspidatum. diofcoroides. echinatum. extenfum. $\beta$. filiforme. 2. flavens. glabrum. macrophyllum. micranthum. maritimum. 2. ovatum. pallens. parviflorum. $\beta$. pedunculatum. pubefcens. varium.

Afclepias afimatica. crasfifolia. 2.2. gigantea. $\beta$. farmentofa.
Herniaria alpina. Anabajis oppofitifolia. Chenopodium album. dacumbens. dubium. $\beta$. haftatum. $\gamma$. murale. $\gamma$. pedunculatum. $\beta$. portulacoides. $\varepsilon$. fulcatum. 2. 2. 3 . vivide. $\beta$.

Beta maritima. vulgaris: $\delta$.

Salfola brachiata. tomiento $a$. tragus $\beta$. verrucofa.

Celtis iberica.

Tournefortii. Gentiana pannonica. $\beta$. Chironia lychnoides. $\gamma . \delta$. Panax attemunta. $\beta$. difcolor. fol. quinquefotia. fol.

Eryngium amethyfinum. $\beta . \gamma$. ciliatum. crinitum. dentatum. fruticofum. B. graminifolium. planum. $\gamma$. rugofum. $\alpha . \beta$. 


\section{T H E S ES.}

I.

riticen Hiftorix naturalis noftro ævo non utilem modo, fed admodum etiam necesfariam merito cenfemus.

\section{II.}

Miram in plantarum fponfaliis natura oeconomiam oculo attentiori-qui perfpiciat, cum Seneca deprehendet:- Omnia natnram adjuvare, ut naturce opera peragantur.

\section{III.}

In infectis defcribendis Tarfuum obfervatio, quam intulit perfpicax Geoffroi, quamque DeGeer et nuperrime Gyllenhal noftrates funt fecuti, non minimum fane affert commodum.

IV.

Quam Tarfuum obfervationem fi diligentius adhibuisfet cel. Fabricius, Syftema illius perfectius fuisfe contendimus.

\section{V.}

Enumerationem Naturalium Mufei cujusdam qui vili pendunt, funt cognitionis eorumdem expertes. 


\section{D. \\ MUSEUM NATURALIUM ACADEMI $Æ$ UPSALIENSIS.

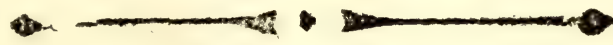 \\ CUJUS \\ APPENDIC. XVI!I. \\ VENIA EXP. FAC. NEDICAE UPSAL. \\ PR $Æ S I D E$
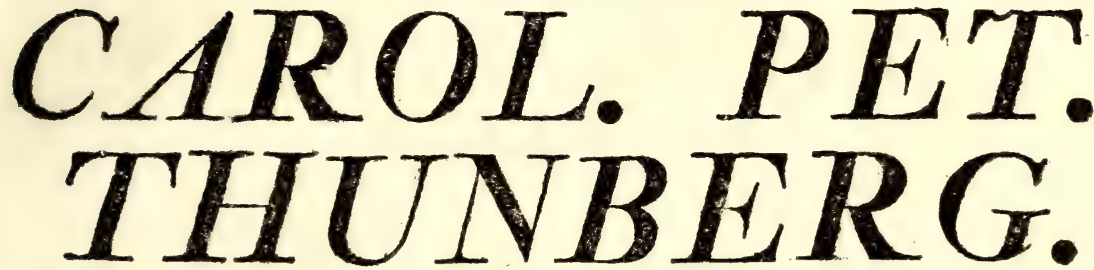

Equite Aurat. Reg. Ord, de Vasa,

Med. Doct. Prof. Med, et Bot. Reg. ex Ord. Acad. Casar. Petropor. ex Nat. Curios. Reg. Scient, Lond Holm. Societ. Scient, Ups. Phil.ad, Patr. Holm, ex Civ, Oecon, Find. Beror. Nat. Scrut. Paris. Hafn. Moscou. et Halens. Hist. Nat. [und. Hariem. Amsteld. Zeland. Nidrosiens. Ticinens. Irnens. Linn. Londin. Reg. Scient. Phys. et Phytograpit. Gótitingens. Nanciens. Men. Paris. Emol. Monsrel. Med. Matritens. Medic. et Nat. Studios. Edimburg. Membro nec non Acad. Scient. Paris. et Institut. Nation. Monspeliens. Agricur.t. Paris. Medic.Londin.Scandinan, Florentin. et Batavin. Ind, Or. Correspond.

PUBLICA CENSURA COMMITTIT

$$
\text { ERICUS NENSÉN. }
$$

BOTH NIEN S I S.

STIPEND. STIGL.

'IN AUDIT. BOTANICO D. X. MAJI. MDCCCIX.

H. A. M, S.

Up dalia . Typis Edmennianis. 
O̊FVER SJELEVADS OCH BJÓRNA FO̊RSAMLINGAR HO̊GÅREVO̊RDIGE OCH HÓGLÅRDE MAGISTER

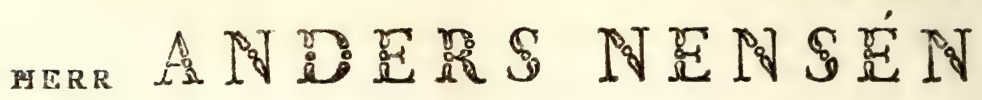
S A M T

H Oீ G ÅD L A F U

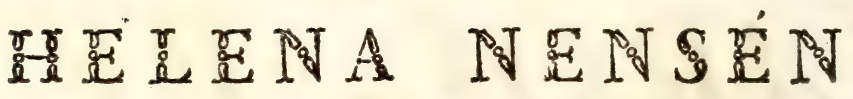 FóDD S.A.T. Tli $\mathbb{N}^{\top}$}

\section{HULDASTE FO̊RALDRAR!}

Kanflan af mina förbindelfer aterförde ånnu aldrig till mitt minne Edra dyrbara vàlgä:ningar, utan att hos mig tillika våcka en li ỉig ỏn. Ikan, at någon gång åga tillfålle gifva Eder et hỏgtidligare vädermåle af min vördnad och tackfamhet. Jag vore dock nog förmåten, om jag nu trodde mig fe fullkomligen upfyllas detta aflägsna hopp, och om jag ens förmodade, at denna ringa gård, eller nagot lofoffer kunde fullgơra de oåndliga fordringar, fom teknas af Desfa heliga namn. Det år likväl en tróft fỏr mitt hjerta, vid defs fitrid med min förmåga, da jag för Eder fär förklara, at den fednares brifter ej förmåt hindra det förras utbrått. Emottagen med ófverfeende godhet denna lilla gafua: den tilhör Er, och hvad ågde jag annars at gifva? O! måtte Förfynen förlånga Edra dagar! - För Eder fallhet har jag ej någon ho̊gre óntkan offrig.

E D E R

\section{Lydigfte Son}

ER. N E N S é N. 


\section{DONATION. THUNBERGIAN.}

\section{A P P E ND. XVIII.}

Sanicula marilandica. $\beta$.

Aftrantia major. B.

Hasfelquifia capillaris.

Selinum carvifolium. 3. 4. 5. Athamania alata.

Laferpiitum gallicum. 3 . Caucalis nodofa. B.

Heracleum alpinum. 3. 4. 5.

anguffifolium. 2.

fibiricum 2. 3. 4.

fphondylium. 2. 3. 4.

Ligufticum auftriacum. 2. 3.

Sium anguftifolium. 5 .

latifolium. 2.

nodiformm. 2.

Bubon galbanum. $\gamma$.

Cicuta maculata. 2.

Sonndix pinnatifida.

Sefeli Pallafii.

petrceum.

Smyrnium perfoliatum. 4.

Rhus procerum. 2.

thyphinum. B.

Viburn um incanum.

macrophyllum. $\delta$.

prunifolism. B.

Turnera elliptica.

Sesfilis.
Pharnaceum cefpitofun.

filifolium.

Juff uticofum.

Evolvulus crenatus. a. $\beta$.

linifulius. $\beta$.

Jericens.

Spinacia tetrandra. 0 . Acnida cannabina. .

Cannabis chinenfis. 战 3 .

Statice auriculifolia. $\gamma$.

opuntivides. peEtinata. 2. 2. 3 . reticulata. B. $\gamma$.

Linum gallicum. 2. frictum. $\beta$. villofum.

Xanthoxylon trifoliatum. 2 . Commelina lancea. nodofa. \&. $\beta$. paniculata.

radicans. tomentosa. trifida.

truncata. unifora.

Allium guttatum. odorum. B.

Ornithogalum fimbriatun. )$(2$

Orni- 
Ornithogalum fecundum. Anthericum ellipticam. japonicum. $\beta$. iatifolinm. $\beta$.

Lilium $c$ ndidnom, $\%$.

Tulipa gesneriana. 26.27.28.29. Aloe picta. 1. 2. Thrinax parvifora. 3.

Juneus glancus, 2. jpicatus $\gamma$.

Calamus mipcre. \& $\beta$.

Rumex hydrolapathum. $\beta$. tuberofis, $\beta$.

Smilax oblongata. $\beta$. taminoides. I. $\beta$. \&.

Diofcorea acuminata. elliptica.

ferruginea. japonica. 8. B. \&. ovata. tomentofa.

Rajania excija. Q. Ariffolochia acaulis.

$$
\text { longa. } \gamma \text {. }
$$

Ferreola buxifolia.

Oenother a fruticofa. $\beta$. Epilobium erofum. montanum. B. $\gamma_{0} \delta_{0}$. Combretum acuminatimn. Laxum. B.

Ornitrophie cominica. $B$. Amyris altisfinza for. anbrofiaca.
Amyris clanfona. heterophylla. fol.

Dodonce a latifolia. Schleicher a trijuga. \&.

Daphne alpina. 2. japonica. $\gamma$.

Pasferina anthylloides. $\beta$. canefcens. ericoides. $\propto$. B. Weinimannia glabra. $\beta$. Moèhringie mufcofa. $\beta$. Corylus maxima. 2. Acer opahis. B. Polygonum alpimum. $\beta$. arifolium. $\beta$. aviculare. $\delta$. barbatum. B. \%. $\delta . \varepsilon_{a}$ divaricatum. 4. glabrum. 2: glaudulojum. lapathifolium. maritimum. $\varepsilon$. minus. 2. perfoliatum. $\beta$.

Seriana trijuga.

Sapindus enuarginatils. foponaria. 2. Vaccinixm idxum. B. Casfia alcta 2. 3. 4 : bicapputaris: $\beta$. chine? is. liguftrina. B. metufa. 
Casfia tomentofa.

Tora. 4 .

Bauhinia aurita.

cirrhofa.

excifa.

grandis.

purpurea.

rubefcens.

Limonia monophylla. 3 .

Ruta fruticofa.

Bergera koenigii. $\beta$.

Ceratopetalum gummiferum. $\beta$.

Rhexia frigoja.

virginica.

Osbeckia glabra.

verticillata.

Melaftome alata.

aqvatica.

aspera.

difcolor.

grandiflora.

grosfularioides.

phyfiphora. 1. 2.

Splendens. 2.

tamonea. 2.

Rhododendron dauricum. 2.

Fusfieuia aubletii.

Anderfonia lanceolata.

Pyrola minor. 2.

rotundifolia. 3 .

Casearia elliptica. $\beta$.

Terminalia catappa. 2.

latifolia. 2.

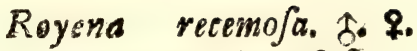

Trianthema humifusa. 2 .

Chryfofplenium alternifolium. 3.

Saxifraga aquatica.

cefpitofa. 2.

ciliata.

crenata.

decipiens. 2.3.

hirfuta. B.

hypnoides: $\gamma$.

juniperina.

recta.

tenella.

Saponaria glutinofa. officinalis. B. $\gamma$.

Gypfophila arenaria. viseofa.

Dianthus chinen/is. 2.3.

crinitus.

fimbriatus.

oxypetalus.

rigidus.

Silene chlorantha. 2.

fruticofa. B. $\gamma_{0}$

maritima. B. $\gamma$.

Spergulifolia.

Stellaria nemorum. 3 .

vifeida.

Arenaria cephalotes.

glomerata.

heteromalla.

securva. 
Carica papaja. $\bullet .2$. Sedum aizoon. $\beta$.

hifpanicum. 2. lineare. $\beta$.

Suriana maritima. 2.

Ceraftium dihotomum. 2. dioicum.

manticum. 2. rupeftre.

Spergula arventis. 2. 3 . nodofa. 1.2. procumbens. 2. jaginoides. 2.

Phytolacca octandra. 3 . Triumfetta hirfuta.

incana.

rotundata.

Semitriloba. 3. 4. trifida.

Euphorbia acuminata..

ciliata.

glabrata. 3.

heterophylla. B. $\gamma$. tivucalli. 3 .

Refeda undata. 3 .

Sempervivum tectornm. 2.

Cactus cocchenillifer. $\beta$.

Spirofisfimus.

Myrtus. moururi. $\beta$.

Pfidium aromaticum. gvineense.

Prunus padus. 2. rigida.
Cratagus oxyacantha. B. $\gamma$. Pyrus falicifolia. $\beta$.

Tetragonia frutisofa. $\beta$.

Dryas geoides. $\beta$.

Mabea pirivi $\beta$.

Capparis baducca. comofa.

cynophallophora. $\beta . \gamma$. fromdofa. 3 . grandis. 2. morifoni. 2.

Árgemone pyrenaisa. Bixa orellana. 3. Aconitum anthora. 2. Paonia hybrida.

Elsocarpus copalliferus. $\alpha$. $\beta$. dicera.

Corchorus plicatus.

Cliffortia trifida. 9 .

Begonia ciliata $1,2$.

crenata.

cufpidata. 1.2.

debilis.

pilifera.

Calla cethiopica. 3.

Stratiotes aloides. 2.

Ranunculus bulbofus. $\gamma$.

Anemone curonaria. 5.

Annona muricata. 5.

Trollius europcens. 4.

Compionia afplenifolia. 2. Eqvifetum arven $\int e . \delta$. $\varepsilon$.

Menifium reticulatum. 
Taninis blechroides.

Polypodium acroftioides. $\beta$.

calcarenm.

obliteratum. $\beta . \gamma$

Grammitis ferrulata.

Afplenium caudatum. $\beta$. radicans. $\alpha . \beta$.

phymatodes. $\varepsilon . \xi \eta$. Scolopendrium officinarum. $\gamma$. ftigmofun. $\beta$. Pteris aqvilina. $\gamma$.

Aspidium aculeatum. $\gamma$. $\delta$. $\varepsilon . z$. anthrifcifolium. axillare $\beta$.

biferratum. $\beta$. capenfe 2.

cicutarium. $\gamma$. cordifolium. criftatum. 2. exaltatum. $\beta$. femina. 2.3. tragile. 2. marginale. $\beta . \gamma$. mas 3. novceboracenfe. 2 . parafiticum. patens. B. $\gamma$. pennigerum. rhoeticum. 2. Cpinulofum. 1, 2, 3, 4. 5.Danaea cufpidata. trapezoides. trifoliatum. 7. 8.9. truncatuhim. umbrofum. 2. 3 . unitum. 2. 2. villofum. 2.

Splachnum froelichianum. spharicum. $\beta$. tenue. decurrens. $\gamma$. grandifolia. $\gamma$. involuta.

Vit taria elongata. lanceolata. Blechnum orientale. $\delta . \varepsilon$. spicant. $\gamma$. Adiantum denticulatum. trapeziforme. 3 .

Cheilanthes microphylla. Davallia aculeata. 3 . canarienfis. $\beta . \gamma . \delta$. elegans. $\beta$.

Dickfonia flaccida. $\beta$.

Cyathea glauca. $\beta_{0} \gamma$. Schizaa dichotoma. $\beta$. Mertenfia dichotoma. $\delta . \varepsilon$. z. Angiopteris erecta.

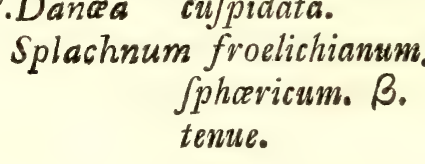




\section{THESES.}

I.

uod naturæ fcrutationi ferme omni facem pratulit recentius. Chemia Syftema, quamquam fit antiquiori longe preftantius, illud quidem perfectum tamen esfe atque omnibus fuis numeris abfolutum, vix ac ne vix quidem putabis, fi modum, quo fxpiu rerum caufas explicat, intentius confideraveris.

\section{II.}

Qvilitates enim, quas removere illud conari aquus aftimator nullus inficias iverit, occultas, pro certis adhuc indubiisque rationibus valere, videre nobis videmur.

\section{III.}

Ortas hinc cernimus de materialitate fimplicium, quas fert hoc fyftema, fubftantiarum, calorici, oxygenici \&c. adhuc fub judice lites.

\section{IV.}

Benevole autem judicandos esfe atque condonandos, quos infert animi humani fluctuatio, errores, quis neget? Eft filicet nulla, qux nifi fenfum pedetentimque perficiatur, difciplina.

\section{V.}

Sanandi vero fcientix, utpote qux experlentix præfertim nitifur fundamentis, hoc magis profuisfe credatur, quo melius ingenium ducuerit humanum naturx detegere myfteria.

VI.

Inter medicamenta, non parvi momenti funt nonnulla, quæ e regno minerali expromuntur. 


\section{MUSEUM NATURALIUM ACADEMI 2 UPSALIENSIS.}

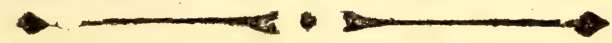

C U J U S

\section{Append. XIX.}

VENIA EXP. FAC. MED. UPSAL。

\section{P R E S I D E}

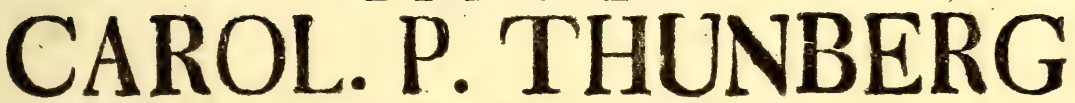

Equite Aurat. Reg. Ori). de Vasa,

Med. Doct. Prof. Med. et Bot. Reg. et Ord. Reg. Coll. Med. Memb. Hon. Acad. Ceesar. Petrofol, et Nat, Curins. Reg. Scient, Lond. Holm. Societ. Scient. Ups. Philad. Patr. Hoim. ext Civ, Oecon. Finl. Berol. Nat. Scrut, Paris. Hafn. Moscou. ex Halens. Hist. Nat, Gorenkens. Luni). Harlem. Amsteld. OEcon. et Inst. Litt. Zeland. Nidrosiens. Ticinens Ienens. Linn. Londin. Reg. Scient. Phys. et Phytograpi. Gót. tingens. Wet ter aw. Nanciens. Med. Paris. Emul, Monspel. Med. Matritens. Medic. ext Nat. Studios. Enmburg. et Petrop. Medico. Chir. Membro nec non Acad. Scient. Paris, et Institut. Nation. Monspeliens. Agricuit. Paris. Medic. Londin. Scandinav. Florentin. et Batavin, Ind, Or. Curresponn.

PUBLICO EXAMINI SUBMITTIT

GUSTAV. $\Im A C O B . O ̈ R T E N B L A D$

WERMELANDUS

IN AUDIT. BOTANICO D. III. DEC. MDCCCXII. H. A. M. S.

U P S A L I

excudebant Stenhammar et Palmblat

REG. ACAD. TYPOGR. 


\section{IN SACRAM REGIAM MAJESTATEM SPECTATE FIDEI VIRO,}

AD AULAM REGINA VID. CONCIONATORI, MAXIME REVERENDO MAGISTRO, DOMINO OLAVO ORTENBLAD, FRATRL

pignus hocce pietatis

FRATER 


\title{
DONATIONIS THUNBERGIAN $A$
}

\author{
A P P E N D I X XIX.
}

Chara batrachosperma.

funicularis.

globularis.

monosperma.

Mniarum pedunculatum.

Najas muricata.

Subulata.

Veionica acinifolia. $\xi$ arvengis.

multifida. $\varepsilon$. permularia. ponce. B.

Canna indica. $\delta$.

Orchis tonopsea. $\varepsilon$. planisiginea. $\beta$. viridis. $\varepsilon$.

Epidendrum ciliare.

Siylidium graminifoliann. Columellia clovata. Salix

argentea. crefia?

myrtilloides. \& $\beta$.

Valeriana tripteris. $\beta$.

Boerhovia difiufa. $\delta$.

Iris lutefecens.

Sparganium fimplex.

Rufcus androgynus. $\beta$.

Phoenix Elate. 4. 5. 6.

Carex bicolor.

diverfiffora.
Carex ferruginem.

firma.

hordeiformis.

Morhofei.

Oederi.

Spadicea.

Sylvatica.

teréiius cula.

Schoenus cephalotes.

finstus. B.

Scirpus ferruginents, $\gamma$,

Cyperus monofachyas. $\gamma$.

Juris amenfis. B. $\gamma . \delta$.

Fanicum poilygamum. $\beta . \gamma . \delta$.

refiactum.

repens. $\beta$.

Serratum. $\gamma$.

viride. $\xi$.

Andropogon aciculare.

bicorne.

infulare. B. $\gamma$.

Saccharum monandrum.

Cerefia elegans

Aira bothusica.

cespitofa. $\gamma . \delta$.

Spicata. $\beta$.

Poa alpina. $\gamma$.

anguftifolia. $\beta . \gamma$. chinenfis. $\gamma$. glauca. $\beta$. 
Pod tittorea.

Molineri.

torfacea.

Feftuca cefpitoja.

dura.

flavefcens.

uniglumis.

unioloides.

Agroftis bromoides, $\gamma$. derumbens. indica. $\beta$. minima. $\beta$. retroflexa. pfendoarenaria.

Dactylis littoralis. $\beta$. pafpaloides.

Holous borralis.

Cynofurus microcephalus. Bromus fylvaíicus.

Elymus monoftaehyos. Avena alpeftris. Rottboellia complanata. $\alpha . \beta$. Axyris ceratoides. $\gamma_{*}$ Excoecaria lucida. ?.

Scabiofa alba.

diandra. juaveolens.

Globularia mana. Rubia cordifolia. $\not$.

Galium maritiwam. premilum.

Afperula hirta. Potefia fpicatas
Callicarpa integrifolia.

Planlago arenaria. vaginata.

Hippophaë canadenfis. fol. Parietaria cretica. $\alpha$. $\beta$. Nigrina Serrata. Poterium polygamum. Betula laciniata $\varepsilon$. Myofot is finduatica. Cynoglosfium fylvaticum. Pulmonaria suffuticofa. Androsace chamojasme. Primula glacialis. grandiflora. nivalis. villosa.

Convolvulus paniculatus. Campanula lilifolia. $\gamma, \boldsymbol{1}, 2$. urticafolia. $V$ erbafcum pulveratum. Baobotrys nemroralis. Varronia monasperma. Schoepfia americana. Oxybaphus vifcofa. Hyoscyamus fenecionis. Cedrela odorata. $\beta$. Vivla pedata. $\gamma$. rhotomagenfis. tricolor. $\xi$.

Gẹntiana pannonica $\gamma$. pxeumonantha. $\gamma$. pulchella, $\beta$. 


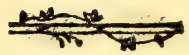

3

Chironia lychnoides. $\delta$.

Webera tetrandra.

Celofia criftata. $\xi$,

Sauvagefia adima.

Epacris daphnoides. odorata. reflexa.

Styphelia longiflora. Saljola arenaria. Afrantia minor. $\beta$, Bupleurum rigidum. B. $\gamma$. Sium nodifiorum. 3 . repers. $\gamma$.

Pevcedanum parifiense.

Selinum moniantin. Amms Boeberi. I. 2. Athamanta mongolica. $t .2$. Bubon macedonicum. $\beta \gamma$. Ulmus effufa. 1. 2. Sialice cordata 3. $\operatorname{diffu} / \mathrm{a}$. olvifotia. $\gamma$. d.

Armeria planiginea. Drofera media.

Chamerops palmetta. Pontederia azurea fol. Asphodelus clavatus. Anthericum osfifragum. 2. 3. 4. Saxifraga ajugafolia. B. Allium pedemontannm. rosenm. B. fibirictum. $\beta$.

Funcus articulatus. $\varepsilon$. $\xi$. Forfleri. monanthos.
Pyrola chlorantha.

Arbutus andrachne. $\gamma$.

Dianthus car yophyllus. 8. chloroleucus.

collinus.

orientalis.
Funcus pygmons.

fubverticillatus. $\alpha, \beta$.

Peplis alternifolia.

Smilax caduca. o.

Damafonium ftellatum.

Tetralheca glandulofa. fol.

juncea.

Pylygonum Bellardi.

Macrolobium hymanxooides.

Cubar paniculata. 1.2.

Perfonia falicaria.

Menziefia hifpida.

Paulinia divaricata.

Aieca olercicea. 12.

Quercus coccifera $\beta$. collina. 1.2 .

difcolor. $\beta$. lobulata. fol. sigra. B. marilandica. pubefcens. fol. robur. $\delta$. finuata. fol.

Mercurialis annua Q. B. $\gamma$.

Butomus umbellatus. $\beta$.

Osbeckia ceilanica. $\beta$.

Samyda dodecandra. Ruta intermedia. 


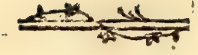

Silene bipartita.

conoider. B.

difficiur.

Lyahnis tecta.

piôa.

Arenaria hybrida.

Cutyledon orbiculata. 2.

Phytholacca abysfinica.

Tomex Sebifera $\beta . \gamma$.

Portulaca pilofa. 3 .

Euphorbia dendruides.

epithymoides. 4. myrfunites. B. $\%$

Cactus mammillaris. 2.

Myrtus commninis. ל.

Roja caroliniana.

cinerea.

corymbofa. $\alpha$. $\beta$.

gallica. $\beta$.

procimmbens.

rabrifolia.

Rubus, cefius. 2. faxatilis. 2.

Dalibarda ternata.

Geum potentilloides.

Cifius ilalicus.

Mimofa villoja.

Aconitum snontanams.

Poterium polygantm.

Thalictrum alatum.

Dracontium pert:k ium. 2.3. 4.5. Anemone cononaria 5. 6 . hepatica. $\delta$. trifolia. $\beta$.
Ranurculus aureus.

Adonis appennina. $\beta$.

Nipeia lanififila.

... pamonica. $\beta$.

Byfropogon princtatum.

Hysjopus orizntalis.

Lavandula glabra.

Miniha hybrida.

lavandulifolia.

Betonica birruta.

Sideritis taurica.

Stachys anguftifolia.

Thymus graveolens.

marfinallinus. pannonicus.

Ocymum bullatum.

Verbera hanata. 2. officinalis. 2 . orubica.

Ziziphore lafiantha: Serpillacea. tennior. $\beta$.

Pingvicula flave/cens. Favellana frapiflora. Srhizanihus pinzatus. Ballota foveolens. Scutellaria colunna. Ithlomis caribcea Bartifia alpina. $\gamma$.

Dracocephalum fibiricun: $\delta$. Euphrafia tricuspidata. 2. Buchnera foetida. Gerardia filiformis. 


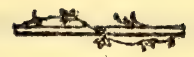

Chelone barbata.

Peloria hybrida. 2.

Pedicularis recutita. $\alpha . \beta$.

Ruellia tuberofa $\bar{\beta}$.

Conobaa aquatica.

7 ufticio bifida.

Mimulus guttatus.

Rhinanthus orientalis. $\beta$.

Melampyrum barbatum.

Gratiola lucida. $\beta$.

Antirrhinum bipurËatum,

linarioides.

pyrennicum.

ficuirum.

Scrophularia chryfanthemifolia. Stemodia aquatica.

orientalis. 2.

pygmiea.

Lantana acrileata, $\beta$.

Coruntia punctata.

Orobanche alba.

amethyftina.

epithymus.

purpurafcens:

Ruellia anagallis. 2.

blechnum. 2.

cordifolia.

Serrulata.

frepens. 2. curacaläna. $\alpha . \beta$.

ecbolium. $\beta$.

eniftachiana- 2.

formosa.

juligera $\beta$.

lithofpermifolia $\gamma . \delta$. paniculata. $\alpha$. $\beta$.

piEta. $\alpha$.

repens. 2.

thyrfiflora.

Refleria lutea. 2 .

Pentflemon campanulatum. 2 .

Barleria crifata. 2.

Volkamerid paniculata.

Clerodendrum fortunatum. $\beta$.

phlomoides. $\gamma$.

fpicatum.

Bunias afpera.

Syriaca.

Crambe ftrigofa.,

Draba nivalis.

repens. 


\section{T H E S E S.}

I.

Judicia de indole Plantarum ac viribus medicis Medico. rum diverfa e commutatione unius cum altera fæe derivanda cenfernus.

\section{II.}

Quod fi conftat, triftes exinde cafus perfape ortos esfe, haud equidem nimis nmquam admoneri poteft, quam fit Medico aque ac Pharmaceutico necesfaria integra omnino Botanices cognitio.

\section{III.}

Quantum autem vires atque qualitates Vegetantium ad quodcumque Clima, folum annique tempus varient, neminem fane fugit.

\section{IV.}

Topographiam ideo Plantarum plus jufto neglectam, quæ elaboretur, dignisfimam judicanuus:

$$
\text { V. }
$$

Unumquodque enim Clima folumque fuas fibi præcipue vindicant plantas; maximos hinc fructus Scrutatori Naturæ \& Oeconomo e Topographia rite examinata esfe percipiendos, uni quidem cuique gatefcit. - 


\section{MUSEUM NATUPALIUM ACADEMIIS UPSALIENSIS.

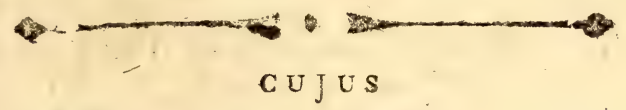 \\ Append. XX.}

VENIA EXP, FAC. MED, UPSAL.

\section{PR 压SIDE}

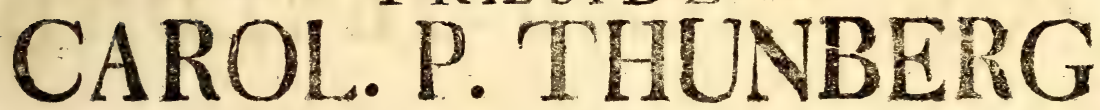

Egoite Auratit. Reg. Ord, de Vasa,

Med. Doct. Prof. Med. et Bot. Reg. et Ord, Reg. Coll. Med. Memb. Hon. Acad. Cefsar. Petropnl. ez Nat. Curios. Reg. Scient, Lond. Holm. Societ. Scientr. Urs. Pullad. Patr. Hor m. et Civ. Oecon. Finl. Berol. Nat. Scrut. Paris. Hafn. Moscou. et Halens. Hist, Nat. Gorenkens. Luni, Harlem. Amsteld. OEcon. et Inst. Litt. Zerand. Nidrasiens. Ticinens. Innens. Linn. Londin. Reg. Scient. Fiys, et Phytograpi. Gót. tingens. Wetter aw. Nanciens, Med. Paris. Emul. Monspel. Med. Matritens. Fífedic. et Nat. Studios. Enimeurg. ext Petrop. Medico. Chir. Membro nec non Acad. Scient. Paris. et institut. Nation. Monspeliens. Agricult. Paris. Medic. Londin. Scandinav. Florentin. ez Batavin, indo. Or. Currespond.

\section{PUBLICO EXAMINI SUBMITTIT}

\section{$N O A C H U S H E D R E \dot{E} N$}

A SACRIS VERMELANDUS.

IN AUDIT. BOTANICO D. III. DEC. MDDCCCXII. H. P. M. S.

\section{U'P S A I \&}

excudebant Stenhammar et Paladiad

REG, ACAD. TYPOGR. 


\section{- IN}

SACRAM REGIAM MAJESTATEM

SPECTATE FIDEI VIRO

CENTURIONI

DOMINO SPECTATISSIMO

รOํ.

J A C.

TORSLUND

Sacrum 


\section{DONATIONIS THUNBERGIANE}

\section{A P P E N D X XX.}

Lepidium graminifolium. 3 . veficarium, 2.

Cochlearia acaulis. Iberis Jempervirens. $\beta$. Alyssum clypeatum. $\gamma$. hirfatum.

Cardamine alpina. hirfuta. 4.

Arabis recta. regida. Arictio.

Ifat is tinctoria. 3 . Hesperis regida.

Sifymbrium amphibium, d. arenofum. pyrenaitum. B. tenuifolium. 2. Tillieri.

Eryfimum barbarca. 3. junкеum. officinale. 4. virgatum.

Heliophila coronopifolia. 1. 2. dissecta. (3. 4.

Sinapis laevigata. 2. 3 . orientalis. 2.3 .

Brassica napus. 2. oleracea, 11.12,13.14.15.

Cleome dodecandra. pentaphylla. z. 3 .
Cleome violacea. $\gamma$.

Cupresfus virens. Kitaibelia vitifolia. t. z. Pinus nigra. 9 . Phyllanthus grandifolins. $\gamma$. nigrefcens. $\gamma$. racemofus. $\beta$.

Funiperus excelfa. o, Waliheria microphylla. Melochia pyramidata. $\beta$. Hermannia incana. involucrata. lavandulifolia. a. $\beta$. Adelia neriifolia. Seriana lupulina. Pelargonium abrotanifolinum. $\beta$. acetofum, 2. betonicum. $\beta$. elongatum. 2 . exftipulatum. 2. graveolens. hispidum. 2. lobatum $\gamma$. IMyrrhifolium $\beta$. odoratum. 2. Steveni. trifoliatum. $\gamma$ trifte. $\gamma$ vifcofum. $\beta$. vitiolinn. 2 . 
Geranium divaricatum. $\beta$. macrorhizon. 2. pratense. 2 . purpurcum. pyrenaicum. $\beta$. rotundifolium. fonguineum. fibiricum. B tuberofun. $\gamma$.

Erodium chinm. 4. laciniatum. $\beta$.

Monfonia filia. petrceum. $\gamma$. Fatropha hughes. 2. pedata. fol. Sterculia incana. tol. Acalypha cordifolia. paftoris.

Croton angulatim. cuspidatum. $\beta$. rhomberm. Sebifernin. B,

Ricinus inermis. 2. mappa. 2.

Taxus elliptica. ठ. t. falcata fol.

Dombeija erythrocephala, ferriginea.

Carolincea princeps. Bombax eriantios. sida aczto. microfperma. palmata. 2. rotundifolia. tricufpidats. B.
Malachra capitata. Althace rosea. $\xi$. $\eta$. Lavathera thuringiaca. 2. Malope malacoides. $\beta$. Pavonia promorsa. Thespefia ovatc. triloba.

Myrodia turbinata. e. Laguncea lobata. $\delta$.

Gosfypium indicum. Polygala magua. $\gamma$. Bosfiaa haterophylla. $\alpha$. $\beta$. fcolopendrium Amerimnon Brownii. $\beta$. Pifciaia erythrina. $\beta$. Hypocalyptus capenfis. 1. 2. Spartium elevatum. 2. Ulex namus. Aspalathus galioides. incomta. villosa.

Amorpha funticofa. 3 . Ononis hirfuta. $\beta$. Anthylis tetraphylia. 2. Phafeolus helvolus.

lunatus. $z$. nanits. 2. Lupinus linifolius. Láthyrus angulatns. 3 . odoratus. 4.

Vicia fativa. s. Glycyrhiza glandiulifera. Indigofera Anit -3. difperma. 
Indizofer tinctoria. 2. s. Galega murpurea. 2. 3. rhombifolis. villofa 4 .

Orobus tuberofass. B.

- Afragalus canadenfis. 3 . canceficus. contortuplicatus. dealbatus. hamofus. 4. lanatus. physodes. 4. pilofus. 3. ponticus. fcorpioides. ftriatellus. Syriacus. $z$. tauricus. B. temuifolins. 3 . tragoides. 2. urctenfis. 2 . nitrigar. virgatus.

Trifolium campeftre. coeruleum. 2 ; elegans italicum. 3 . macrorhizon. maritinum. praterife. 5. 6. 7. repens. 2. 3 .

Lotus dorycniman. 2.2 . peregrinus. 2.

Trigonclla Senegalentis.
Medicago carftien/is. 1 . 2 . orbicularis. 2.

Genifla hifprinica. fericea.

Giycine violacec. Robinia mongolica. Speciofa. Hypericum alcyron. $\beta$. linearifolium. $\beta$. Richeri.

Tragopogan orientale. 2. Scorzonera antiviaca. laciniata. $\gamma_{0}$

Lactuca ceilnzica. Sonchus dichotomus. Plumierii. 2. tataricus. Leontodon palufore. Prenanthes alba. peniculata. Hieracium ambigunm. cydonifolium. echioides. elongatum. florentinun. foliofum. Halleri. lavigatam. porrifolinm. 2. 3. prencanthoides. faxatile. Schraderi. valdepilofun. xanthodion. 
4

Crepis nemancenfis. purpurea. retofa.

tectorum. 6 .

Hyoferis hedypnois. rhagadioloides. 2.

Artium lappa. 3 .

Vernonia prealta. 1.2 .

Laprana communis. 4.

Serratula arvenfis. ठ.

quinquefolia. $\beta$.

Carduus acanthoibes. 3 .

cyanoides. $\gamma$.

defloratus. 4.

medius.

rivularis. $\mathbf{z}, z$.

Cnicus niliginofus.

Cynara humilis. 2. 3 .

Carlina corymbofa. 2 .

Acarna cancella a. $\%$

Onopordon illyricum. 5. 6.

tanricum.

Carthamus lanatus. 2.' tanvicus.

Spilanthus atriplicifolia.

Bidens cermua. $\gamma$.

$$
\text { nodiflora. } 3 \text {. }
$$

Cacalia arbufcula. 2.

Eupatorium aromaticum. $2^{\circ}$

ivefolizum. 2.

Stchelina chamcepeuce 2

Cryfocoma graminifolia.

Calea lobata. 2.

Rothia rumeinata.
Cafulia axillaris. Artemifia Bocconi.

fafoiculata.

maritima. 4.

Salfoloides.

fantonica. 3.

vallefiaca.

vermiculata:

Gnaphalium arvense. 3 .

mòntanum. 2. 3 .

notatum.

pufillum.

Elichryfum lucidum,

Spinofum.

Conyza alopecuroides.

bifoliata. 2.

cinerea. 7.

lacera. 5. 6. 7.

odorata. 3.4. faxatilis. $\varepsilon . \xi$.

Erigeron acre. 4. finenfis, 2. 3. 4.

Tujfilago frigida. 4. paradoxa. 2.

facobaa ceilanica. fimenfis. 2. 3 .

Cineraria amelloides, $\gamma$. anrea. integrifolia. 2. paluftris. 2 ,

After paniculatus. 4. finenfis. $\delta . \varepsilon$. Inula glandilofa. 


\section{5}

Inula indica. 2. odora. 2 . vifcosa.

Doronicum auftriacum. $\gamma$. bellidiaftrum. $\gamma$.

Tagetes minuta.

Schkuhria abrotanoides. Zinnia hybrida. muitiflora. $\alpha . \beta$. verticillata. $\% \cdot \delta$.

Pyrethruim Sericeum. Cotula globifera. 2. grandis.

Anthemis monantha. peregrina. Achillace afplenifolia bijerrata. compacta. eupatoria. filicifolia. t. 2. impatiens. 2. liguftrina. 2 moschata. tanacetifolia.
Ecclipta latifolia. B.

Sigesbeckia orientalis. 2. triangutaris.

Rudbeckia oppofitifolia. Lagafca mollis .a. B. $\gamma$. Gorteria rigens. $\beta$. $\gamma$. Centaurea masrocephala ochroleuca. ovina. 3 , picris. pulcherrima. trichocephala.

Milleria qiinquefora. 2. Polyneria tetragonotheca. Arctotis - acaulis. 2, Dysoda vifcofa. Camutia perfoliata. Galling Joga parviflora. trilobata. Ambrofia trifida. $\gamma$. Buphthalmum cordifolium. Solidago lanceolata. Senecio eruccefolius. 3. 4. verbencefolius. -

Brasfica alba 1. 2. 3. 4. hvitkal. gongylodes. r. 2. Rabiskâl. laciniata. 1. 2. 3. Kruskâl. rubra. i. 2. Ródkàl. fabellica. 1. 2. 3. Blomkäl. jelenifta. Blakial. 


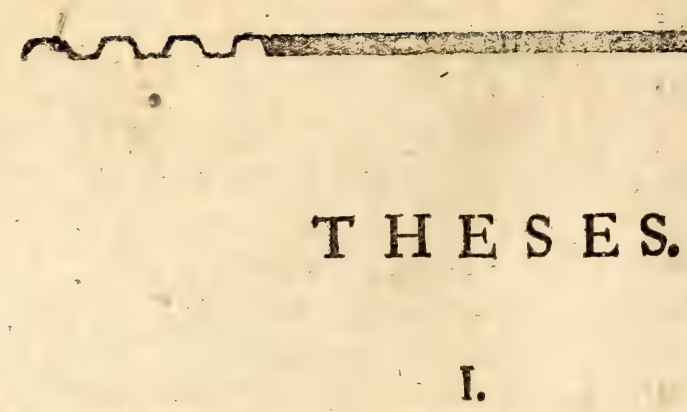

Secundum vita genus diverfum, diverfirodo variant in avibus non modo roftrum, lingua, ungues, pedes \& cauda, fed etjam interdux ipfum corpus.

II.

Calore artificiali ova volatilium excludi posfunt, que ac incubatione matris.

\section{III.}

Migratio avium obtine non modo in regionibus frigidisfimis, fed etian in jlis temperationibus, ubi hiems vix ulla.

\section{IV.}

Hujus migrationis causfæ funt plures, pro vario Climate diverfæe.

$$
\text { V. }
$$

Infecta panca quidem, nonnulla tamen præftantia nobis fubminiftrant remedia. - 


\section{MUSEUM NATURALIUM ACADEMIE UPSALIENSIS.}

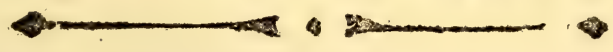

C U J U.S

Append. XXI.

VENIA EXP. FAC. MED. UPSAL.

\section{PR \& IIDE}

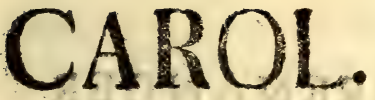

Equite

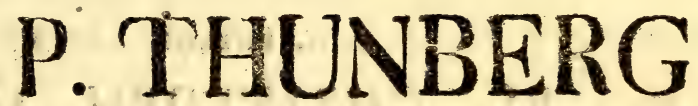

Aurat. Reg. Oris. de Vasa,

Mrd. Doct, Prof. Med. et Rot, Reg. et Ord. Reg. Coll. Sanit. Memb. Hon. Acad. Casar. Petrotol. ex Nate Curins. Reg. Scient. Lond. Holm. A gric. Societ. Scient, Ups. Philad, Patr. Holm, et Civ, Oecon. Fine. Berol. Nat. Scrut. Paris. Hafn. Moscou. et Halens. Hist. Nat, Gorenkens. Lund. Harlem. Amsteld. OEcon. et Inst. Litt. Zeland. Nidrosiens. Ticinens, Ienens. Linn. Londin. Reg. Scient. Phys. ext Phytograpit. Gót. tingens. Wetter. Nanciens. Med. Paris. Emul. Mongrei. Med, Ma. tritens. Madic. et Nat. Studios. Enimgurg. ext Petrop. MedicoChir. Memiro nec non Acad. Scient. Paris. et institut. Nation. Monspeliens. Agricuit. Paris. Mrdic. Londin. Scandinav. Florentin. ex Batavin.: Ind, Or. Currestond.

P ROPON I T

J. E. Wikström

VESTROGOTHUS.

IN AUDIT. BOTANICO D. XXVI. MAJI MDCCCXIII.

H. A. M. s.

\section{U P S A L I R}

excudebant Stenhammar ex Palmblad

REG. ACAD. TYPOGR. 
E. O. PHILOS, ADJUNCTO

MAG. EL. CHRISTOPH. GRENANDER

D. D.

Respondens. 
Inter Plantas Acotyledones, a Linnæo Cryptogamas dictas, infignem certe Lichenum cohors fibi vindicat locum. Cognitio tamen de hifce Algis per longam Sæculorum feriem fere nulla fuit. Sæculum præterlapfum ad Hiftoriam illorum illuftrandam refervatum erat, \& quod prefens eft, fine dubio hancce cognitionem infignius augebit, fanciet, \&, quantum fieri poteft, perficiet. Ante tempora Joh. Baubini vix defcriptiones unius vel alterius Auctoris, quæ nominentur, dignæ funt. Etenim illorum defcriptiones miferrimæ funt, \& perpaucos tantum Lichenes exhibent, Ipfius Joh. Bauhini cognitio hifce de plantis exigua fuisfe videtur, defcriptiones enim ejus imperfectæ funt ac vacillantes, \& figuræe ejus nequaquam meliores. Nec cognitio Cafp. Bauhini de Lichenibus major fuit. Morifonii defcriptiones hancce rem minime illuftrant, attamen figuræ ejus non nimis culpandæ. Rayus Lichenes melius novisfe videtur, multum tamen ad hanc fcientiam perficiendam non contulit. Defcriptiones Vaillantii utique levioris momenti funt: figuræ vero rem ipfam illuftrant. Michelius atque Dillenius autem de Botanicis quam maxime meruerunt. Ingenium illorum in fcriptis, quæ ediderunt, clarisfimis fatis elucet. Horum fcripta Lichenologis quain maxime necesfaria funt. 'Tandem vero in Botanicis fata perplurima cesfabant adverfa, \& poft nebulas Phobus. Venit Linnæus. Illuftris ille Vir primus fuit Auctor, qui Lichenis Genus in ordinem re vera fyftematicum dispofuit, fubdivifiones optimas in illo formavit, \& defcriptiones tandem dedit, quæe, quoad fimplicitatem, perfpicuitatem \& veritatem, non modo priorum, fed etiam recentiorum temporum perplurima opera fuperaverunt. 


\section{) 46}

De cæetero in omnibus fuis operibus de Lichenibus dedit obfervationes, æque de ingenio ejus præclari, ac derarisfimis ejus in obfervando donis, teftes. Abiit Linnæus, \& tabula fuos mutavit colores. Genus hocce notabilisfimas reformationes fubiit. Auctores, Lichenes plura in Genera diftingui oportere, putaverunt. Minime decidemus, utrum necesfarium fuerit, nec rte. Sed ad hanc tamen fententiam, quam ad illam, accedere magis inclinat animus. G. H. Weber varia Lichenum nova Gene. ra conftituit. Permultum ad cognitionem Lichenographicam infuper tribuisfe videtur. Schreber etiam plura nova Genera e Lichenibus diftinxit, variasque de hifce plantis maximi momenti dedit obfervationes, easque ejusdem omnino præftantiæ, ac ceteras omnes, quas in Hiftoria Naturali reliquit. Ehrhart fagacitate illa fingulari, quam omnes in illo Viro xeftimaverunt, unum \& alterum Ge. nus Lichenum ordinavit. Pulcherimmiæ ejus obfervationes Lichenologis inæftimabiles funt. Joh. Hedwig etiam unum \& alterum Genus diftinxit, \& Lichenum fecundationis \& fructificationis dedit Theoriam. Demonftravit, vel potius demonftrare tentavit, hafce plantas etiam fexus habere \& feminibus propagari. Jofeph. Gærtner autem docere tentavit, illas per gongylos, a fe dictos, propagari. G. F. Hoffman Lichenes ex profesfo tractavit, fed fcripta illius quoad dispofitionem principio univerfaliori carere videntur; Characteres ejus Generum va. cillant, determinatio fpecierum minus jufta eft, $\&$ in ipfo folo nativo illos ferutari, ut videtur, non multum curavit, id quod tamen Lichenologo magis fere, quam ulli alii Botanico eft necesfarium. Syftema illius nihilominus a popularibus ejus maxime celebratum fuic. Nunc vero id nemo fequitur. Ventenatii dispofitio Lichenum in totidem fere Genera, quot Linnæus habuit fubdivifiones, Botanicis non bene cognita fuisfe videtur. Forfan hancce 


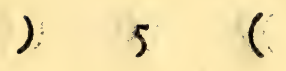

dispofitionem minoris etian momenti liabuerunt. St Hilaire, quantum novimus, unicus fuit Auctor, qui illam adoptavic. Perfoon varia Genera Lichenun conftituit, fungis affinia, idque maximo Botanico um applaufu; at dispofitio ejus generalis Lichenum eundem, ut videtur, non obtinuit. Quod Schrader de Lichenibus obfervavic fcripfitque Botanicis pergratum eft. Quis Botanicorum nefcit nerita Equitis Humboldt de harum, æque ac aliarum plantarum Geographia, \& quot conclufiones perutiles re. centisfimis annis ab hac cognitione deductæ fint? Tandem Syfterna Equitis Acharii omniam oculos in fe convertit. Quamquam Celebris Auctor in Act. Holm. 1794 declaravit, fubdivifiones intra Lichenis Genus æque, nifi magis, utiles esfe, ac plura Genera; attamen ab hac fententia in recentioribus feriptis longe recesfit, \& Methodus ejus Lichenum id primum demonftrat. Dispofitio ejus Organis, fic dictis Carpomorphis, nititur. Haud pauci dixerunt, Auctorem in hoc libro non bene diftinxiste inter gongylos, proprie fic dictos, \& eas partes, quæ jure quodam ut fructus æftimari debent. Terminos Artis in illo opere etiam præter necesfitatem multiplicasfe videtur. Lichenes in 23 Genera divifit, \& quam. quam varize objectiones maximi momenti contra hancce dispofitionem factre fint, tamen quoad magnam partem Botanicis hoc opus cum applaufu acceptum fuit, \& Viri Illuftrisfini, ut Swartz, Turner, Humboldt, ut videtur, etian Michaux, \& quoad partem Flœrke, Fr. Weber \& Mobr denominationes ejus adoptaverunt. In Lichenographia Auctoris univerfali Phalanges Lichenum multo meliores \& conftantiores funt, quam in Meth. Lich. \& terminos ad minorem numerum reduxit, fed Lichenes in 4i Genera divifit, hac vero dispofitio Botanicis minus grata fuit. Pauci certe, \& fere nulli Botanici præter Auctorem, fubtilisfimos \& minutisfimos illos characteres

Gene- 


\section{) 6 (}

Genericos invenire poterunt. Auctor quidem dicit, fundamento ejus femel jacto, hasce perquifitiones Characterum minime necestarias esfe, \& habitum naturalem ha. rum plantaruin tunc Generibus diftinguendis fufficere. At cui fini tunc character Genericus utilis? - In hoc opere Theoriam Hedwigii quam maxime refutat, \& fententiæ Gærtneri adfentit. In Synopfi Lichenum, naper Lundæ edita, Auctor plures, ut dicitur, emendationes priorum feriptorum dedit. Floerke varias Monographicas defcriptiones Lichenum conferipfit, feque ut magnum obfervatorem Naturæ præftitit. Scripta ejus pro Lichenographia fyftematica maximi nomenti certe futura funt. Adhuc tamen de Generica Lichenum dispofitione fibimet, ut videtur, non confentit. E Diariis Litterariis novimus quidem. Decandolle varia nova Genera Lichenum conftituisfe, fed Scripta illius Viri nobis nondum videre contigit. D:r Wahlenberg certe optime omnium Phalanges Lichenum conftituit \& firmavit. Varia Genera Achariana priora, vel, ut ipfe dicit, a fe primum conftituta, chasacteribus infigniter emendatis, adoptavit, \& fub nomine Lichenis habet Parmel. Urceol. Uesnas, Collen. \&c.; fubdivifiones ejus hoc fub Genere preftantisfimæe funt, nec minus characteres atque defcriptiones ejus exoptatisfiniæ. Infuper de Geographia illarum, æque ac aliarum plantarum in Septentrione noftro quam meritus eft hic Naturæ Scrutator?

Nunc Enumerationem exhibemus hiftoricam de fcriptis illorum Auctoruin, in quibus 1 defcriptiones 1. catalogi Lichenum, Svecire indigenorum, inveniuntur.

Prof. Frankenius primus fine dubio Botanicorum in Svecia, qui in Libro: Speculum Botanicum Upf. 1638 paucos Lichenes nominavit, partion hoc fub nomine, 


\section{) 7,6}

partim fub nomine Mufci, fine ullis defcriptionibus; adjectis tamen nominibus Svecanis.

Dr. Ol. Bromelius in Chloride Gothica Gothob. 1698 varios Lichenes, circa Gothoburgum nafcentes, partim hoc fub nomine, partim fub nomine $\mathrm{Mufci}$, adjectis nominibus $\mathrm{S}$ vecanis \& characteribus Cafp. Bauhini, enumerat.

Dr. Linder in Fl. Wiksb. Holm. 1716 etiam fub nomine Lichenis \& Mnlci varias Algas Lichenofas, circa Acidulas Wiksbergenfes a fe inventas, evumerat, adjectis nominibus Svecanis \& characteribus paucis vagis veterum Auctorum.

Th. Dr. Ol. Celfius Senior in Act. Upf. 1732 dedit Catal. Plantar. circa Upfaliam, \& in illo etiam Lichenes, a fe repertos, fub nomine Lichenis, Lichenoidis \& Mufci nominat, adjectis characteribus veterum Auctorum.

Linné in Fl. Lapp. \& Fl. Sv. Lichenes patriæ defcripfit, infuper in Sp. Plant. \& Mant. Plant. addidit plures Lichenes Sveciz; in Itiner. fuis varias de illis dedit oufervationes, in Disf. Herbat. Upf. loca habitationis plur. Lichenum nominavit, in Fl. Rybyenfi illas, circa Ryby obfervatas, \& in Stat. Plant, etiam variorum Lichenum ftationes defignavit.

Prof. Leche in Primit. Fl. Scanicæ, Lundæ 1744 duas tantum Lichenis fpecies, nt dicit, officinales, nominibus Pharmacevt., nominat, adjectis vero characteribus Linnæanis.

Prof. Rofenblad in Obf. Bot., Lundæ 1749 unum et alterum Licheriem, in Scania a fe repertos, nominat.

Præpofitus Samzelius in Nerikes Blomfterkrantz, Orebro 1760 perpaucos Lichenes, in Nericia a fe repertos, nominibus Linnæanis, enuinerat.

Prof. Retzius in Act Holm. 1769 \& Obr. Bot., Leipz. 1779 - 1791 varias nov. fp. Lichenum Sveciæ diftinxit, $\&$ in 


\section{). 8(}

\& in Prodr. Fl. Scand. ed. 1., Hohn, 1779 \& ed. 2., Leipz, 1795 Lichenes patriæ defcripfir.

Dr. Hoffeerg in Anvisn. till Wảxtrik. kånned. ed. r., Holm, $176 \%$ characteres Linnæi de Lichenibus Svecicis transicripfit.

Prof: von Linné fil. in Suppl. Plant. Brunsv., r $78 \mathrm{t}$ varios Lichenes, ut Svecanos, annotavit.

Eques Swartz in Meth. Mufc. Upf., 1781 \& Nov. Act. Upf. Vol. IV plures nov. fp. Lichenum Svecize diftinguit, in Act. Holm. 1789 de Lichene fuo frigido dẹdit obfervationes, \& denique in Sv. Bot. varios Lichenes patria delineavit \& delcripfit.

Piof. Ad. Afzelius in Disf. Obf. de Vegetab. Svecan. Upf. 1785 Act. Holm. 1788 obfervationes maximi momenti de Lichenibus Svecanis dedit.

Th. Dr. Osbeck in Fl. Halland. Act Reg. Soc. Scient. Gothob., 1788. 4 St., I, ichenes Hallandiæ enumeravit.

Ehrhart in Beyt?, zur Nat. kunde s B. Hanov. 1790 dedit Catal. Plantar. circa Upfaliam crefcentium, ubi etiam Lichenes, a fe obfervatos, enumerat, $\&$ in variis locis illius libri obfervationes de hifce Algis Sveciæ addit.

Eques Thunberg in Disf. Fl. Streng. Upf. $1791 \mathrm{Li}$ chenes circa Strengnefiam nafcentes adnotavit.

Prof. Liljeblad in Utk. till en Sv. Fl. ed, 1., Upr. 1792 Lichenes patrix vernacula lingua defcripfit.

Dr. Rutfroom in Disf. Spicil. Plantar. Cryptogam. Sveciæ, Aboæ 1794 varias nov. fp. Lichenum defcripfit. Eques Acharius in Act. Holm. 1794 dedit Fórfók till en fỏrbăttrad Lafvarnes indelning \& 1795 addidit Anmårkningar och forbåttringar vid Afhandlingen om Lafvarnes indelning, etiam 1. c. $1794 \frac{4}{8}, 95,96,1801$ perplu* rimas nov: fp. Lichenum Sveciae defcripfit \& delineavit, nlterius Lichenes patriz in Lichenogr. Svecix Prodr. Lincop. 1799, Meth. Lichen. Holm. 1803, Lichenogr. Uni- 


\section{6}

Univerf., Gotting. 1810 \& Sypops. Lichen., Lnndæ r813 defcripfit; infuper in Act. Holm. 1808, 9, 10 \& 11 dedit Fo̊rtekning pà de i Sverige văxande arter af Lafvarnes familj, hoc. in loco autem mixti cum Lichenibus Sveciæ etiam occurrunt Lichenes Nordlandiæe \& Finmarkiæ Norvegicæ.

Prof. Weber \& Mohr in Nat. Hiftor. Reife durch einen Theil Schwedens, Gôtting. 1804 loca habitationis variorum Lichenum adnotaverunt, \& infuper Recenfio. nem criticam Methodi Lichenuur Ach. dederunt.

Dr Wahlenberg in Fl. Gottl. Act. Holm, 1806 dedit Catal. Lichenum in Gottlandia nafcentium unacum defcriptionibus \& figuris aliquot fpecierum, in Act. Holm. 1810 \& Introduct. ad. Fl. Lapp., Berol. I8I2 de geographica Lichenum in Lapponia disftributione feripfit, \& in ipfa Fl. Lapp. Lichenes Lapponiæ defcripfit, adjectis variarum fpecierum iconibus.

Ufus Lichenum vel Oeconomicus eft vel Medicus, ad priorem vero quod attinet iterum dupplex eft; vel materiam exhibet alimentariam vel tinctoriam.

Ufus Oeconomicus, quoad materiam alimentariam.

Lichen Islandicus certe primus omnium Lichenum a longis retro annis ut nutrimentum cognitus fuit. De illo a temporibus Urbani $\mathrm{Hjårne}$ usque in dies noftros permultum fcriptum fuit. Primarii Auctores Svecani, qui hoc de Lichene fcripferunt, funt, qui fequuntur: Linné in Disf. Fl. Oecon., Upf, 1748 de eximio ejus ufu alimentari egit: qui optime hac de re fcripfit, eft Prof. Liljeblad in Patr. Sållfk. Journ. 1789; ulterius de ejus ufu fcripfit Anonymus 1. c. 1790. Med. Reg. Prim. Weftring in Act. Holm. 1794 huncce Lichenem ipfa ejus gelatina uberrima, ut dicit, valde nutrientem declaravit. 


\section{) 10(}

Analyfis hujus Lichenis dedit Prof. Berzelius in Ekon. Annal. 1808. - De ufu alimentari Lichenis Rangiferini optime fcripfit Præpofit. Hesfelius in Patr. Sâllfk. Journ. 1785. Infuper bene notum eft, omnem Oeconomiam Lapponum ab hocce Lichene dependere. - De cætero Linné in Disf. Plant. EEfcul. Patriæ varios Lichenes ut valde nutrientes indicavit. Prof. Acharius in Act. Holm. 1794 dixit, Lichenes foliaceos magnam nutriendi materiam continere, \& 1. c. 1796 de materiis conftitutivis Lichenum locutus eft. Prof. Retzius in Fl. Oecon. Lundæ 1806 valde fcienter modos varios Lichenes ad alimentum præparandi defcripfit. Hac de re etiam Med. Reg Prim.Weftring in Sv. Lafv. Fårghiftoria Holm. 18058 fcripfit. - Nunc bene novimus omnes Lichenes Umbilicatos, fruticulofos \& foliaceos valde nutrientes esfe mucilagine \& amylo, vel, fi magis placet, Amylo mucilaginofo, quod in illis continetur.

Ufus Oeconomicus quioad materiam tinctoriam.

Ufus Lichenum tinctorius apud noftrates diu incognitus fuir. A Rufticis quidem perpauci Lichenes in re tinctoria ufitati fuerunt, anteguam Linder $1728 \mathrm{~Sv}$. Fårg. konften edidit; poft illud autem tempus fenfim plures tinctorii Lichenes cogniti fuerunt. Varii Auctores experimenta tinctoria, Lichenibus Sveciæ facta, in Act. Holm. defcripferunt, ut Linné 174I, Kalm. 1744, Weftbeck 1754, Fifcherftróm 1761, Gadd 1767, \& in Patr. Sållk. Journ. Moller 1785 \& 87, Anonvinus quidam 1786 \& Holmberger 1790,91 \& 92 . Infuper Linné in Itiner. fuis, \& Disf. Fl. Oecon., Cui bono?, Plant. tinct. \& U. fus Mulc., atque Kalm. in It. Bahuf. plurium Lichenum ufus in re tinctoria explicaverunt. Hardt 1790 Libellum edidit de ufi tinctorio Lichenis tartarei. Tandem vero Med. Reg. Prim. Weftring omnibus hac in arte palmain præripuit. Cel, ille \& de Patria optime meritus Vir no- 


\section{) $\mathrm{II}($}

vam $\&$ certiorem viam ad hancce cognitionem aperuit, $\&$ cum ante 30 annos vix $20^{\circ}$ Lichenes tinctorios novimus, nunc Vir Laudatus nos docuit, plures quam 150 I.ichenum fpecies colores effundere præeftantisfimos. Perutilia fua experimenta primo in Act. Holm. 179r, 93 , 94, 95, 97, 98, 1801 \& 1804 defcripfit, \& ulterius in opere Sv. Lafv. Fårghiftoria multimodis aucta \& emendata edidit. Recenfio hujus operis exftat in Ekon. Annal. 1807. Prof. Retzius in Fl. fua Oecon. experimenta Weftringii ad compendium contulit. Fructum, qui fabricis adferri poteft, facile eft perfpicere. Pauci tamen adhuc ex Weftringii experimentis utilitatem ceperunt. Induftria enim communis apud noftrates adhuc deeft, \& forfan etiam diu defiderabitur.

Ufus Lichenum Medicus non tam infignis eft, quain Ufus Oeconomicus, Veteres Scriptores Rei Medicæ arbitrati tamen funt, magnam vim Lichenibus ineffe ad perplurimas calamitates Generis bumani depellendas, \& inter Noftrates Lect. Palmberg in libro: Sert. Fl. Svec. Strengn. 1681 diffufe \& fuperftitiofe locutus eft de utilitate variorum Lichenum Sveciæe in morbis, \& Ipfe von Linné in Disf: Plantæ Officinales, Purgantia indigena, Cui bono? \& Ufus Mufcorum magnam vim medicam Lichenibus adferipfit. At hæc utilitas medica Lichenum non omnino extra omnem dubitationis aleam pofita eft, excepto fere unico Lichene Island. quem in Morbis phthtificis, hecticis \& macie corporis univerfali fe cum fructu adhibuisfe complures putant Medici.

Dedimus quidem ante Catalogum Lichenum, qui in - herbario Mufæi Academici continentur; cum vero deinde multifariam fubierit mutationem \& Generis \& imprimis fpecifieæ denominationis Lichenographia, de novo jam communicare volu nus hunc Catalogum, fecundum recentiora Scientiz lumina, emendatum \& auctum. 


\section{DONATIONIS THUNBERGIANA.}

\section{APPENDIX XXI.}

Spilome levcofigma. melanaum:

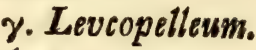

microdomum:

tumidulum.

vitiligo

xanthoftigma.

Arthonia radiata:

B. aftroidea

そ. anaftomofans

Swartziana

B. cinerafcens.

Solorine crocea.

Saccata.

Gyalecta Thunbergiana.

Lecidea Abietina.

alabaftrina

alba

B. rofella.

alboccerulescens:

amylacea.

atro-alba: $\alpha \dot{\alpha}$.

B. fimbriata.

atro.rufa

atro-virens: á.

B. geographica

aurantiaca.

cafio-rufa

sechumena

cinereo-f $n: f c a: \alpha^{\alpha}$.

B. jungermannice. citrinella.
Lecidea cocoës.

confluens.

coniops.

corticola.

Dick/onii.

B. b. Oederi.

Dilleniana.

dryina:

B. lilacina.

Ehrhartiana: $\alpha$.

B. polytrypa.

enterolevca.

B. grandiro/o:

epipolia.

epixantha:

B. Intea:

fumofa.

fufco lutea:

gos ypina.

icmadophila:

a. ericetorum.

immerfa.

B. arugino/a.

lapicida: $\alpha$.

d. monticola.

Lightfootii.

lurida.

luteo-alba.

luteola: á.

E. eryfibe.

margaritacea.

milcella. 


\section{) 136}

Lecided murina.

pallido-nigra.

parcola.

pantoftitia.

$\gamma$. Spilota.

parajema :

$\gamma$. athroa.

\&. punctata.

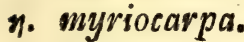

i. Saprophila.

petraa

rivulofa.

rupeftris.

rusfula.

$\gamma$. pyrithroma.

Sangvinaria.

Scalaris: $\alpha$.

B. myrmerina.

filacea.

Speirea. 1. 2.

tricophylla :

B. corallinoides.

tigillaris.

uliginofa.

Wahlenbergii.

varians.

vernatis.

veficularis.

viride/cens.

viridi-atra.

Gyrophora cylindrica

deufta

eroja

heteroidea:

a. glabra:
Gyrophoro

hirfuta.

B. polyphylle:

d. corrugata.

६. variegata.

hyperborea.

murina.

pellita.

pustulata.

Spadochroa

vellea. 1. 2.

Calicium cantharelhum.

capitellum.

chlorellum.

chryfocephalum.'

B. filare.

claviculare: $\propto$ ¿.

corynellum.

hyperellum.

fapiculare.

figonellum.

trachelinum.

trichiale.

B. femonenm.

tympanellum.

Glyphis cicatricofa.

labyrinthiformis.

Opegrapha diaphana: $\alpha^{2}$.

B. Spurcata

denigrata

enterolevea

epipafta

herpetica

macularis a.

y. faginea 


\section{) 146}

Opegrapha notha 1. 2.

parallela

profodea

rimalis

rubella á.

d. cenea

fenocarpa

vulgata

vulvella

$\gamma$. anatiera.

Graphis Afzelii

cerafi

inuifta

lineola.

pulverutenta $\alpha$.

r. flexuofa

Scripta

Serpentina

a. Sitterella

$\therefore$ J. Jpathea

\&. entypa

tortuofa.

Biatora turgida

Vorrucaria analepta

bysfacea

B. fictica

\%. minutisfima

cerafi

epidermidis

epigea

gemmata

mammillata

muralis $\alpha^{2}$.

B. concentrica
Verrucularia nitida

planorbis

pulla

punctiformis

rhyponta.

rubens

ftigmatella d.

B. micans

$\delta$. tremula

ع. atomaria

tetracerce

umbrina $\propto$.

B. nigrefcens

Endocarpon complicatum

cuplacum.

hepaticum

B. lacinulatum

leptophyllum

miniatum

finopicum

tephroides

Thusbergii

Weberi.

Trypethelium anomalum

favulofum.

maftoidenum

papillofum

Sprengelii

variolofum.

Porina lejoploca: $\alpha$.

B. hymanea

pertitsa

Thelotrema cavatum 


\section{)$\quad 156$}

Thelotrema difcoideum obturatum

typaneum

Pyrenula afpiftea

hematomma.

heteroclita: denigrata. maftoidea.

$V_{\text {ariolaria amara. }}$

aspergilla. communis.

¿. orbiculata.

B. faginea.

$\gamma$. alnea.

corallina:

B. oreina.

laitiea.

Sagedia depressa

verrucarioides.

Urceolaria Acharii.

bryophila.

cinerea.

gibbofa.

Hoffmanni:

B. contorta.

panyrga.

Schleicheri.

Lecanora alphoplaca:

B. inflata.

angulofa.

anomala:

B. ferrugino/a.

そ. cyrtella.

atra : $\alpha$.

$$
\begin{aligned}
& \gamma . \text { confragofa. } \\
& \xi \text { grymoja. }
\end{aligned}
$$

Lecanora badia. a.'

$\gamma$. fufcata.

brunnea.

candclaria.

cartilaginea.

cerina.

chrysoleuca.

citrina. 1.2 .

coaretata.

craspedia.

crassa.

decipiens.

elatina.

elegans.

epanora.

erythrella.

fallaria:

d. cyathoides.

fulgens: $\alpha$.

B. bracteata.

fusco-atra.

galactina.

glancocarpa.

glaucoma.

gramulofa.

grifea.

homatomma: $\alpha$.

B. coccinea.

Hageni:

$\gamma$. umbrina.

hypnorum.

lentigera.

lepidora. 


\section{) $16 \mathrm{C}$}

Lecanora hute/cens.

microptiylla.

multipunctata:

a, rivulo/a.

murorum.

orofthea.

parella : a.

B. pallefcens.

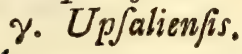

periclea:

B. pinicola.

rubra.

$\gamma$. exigua.

Sophodes.

Stonei.

Subfufca:

a. argentata:

ท. alloplaca.

J. atrynea.

fulphurea.

Swartzii.

tartarea: $\alpha$.

teftacea.

$\gamma$ frigida.

varia.

ventofa:

a. cruenta:

verrucofa:

B. agelaa:'

Villarfii.

$\gamma$. argena.

virella.

vittelina.

Roccelle fuciformis.
Roccella phycopfis.

tinctoria: a'.

B. hypomeca.

Evernia divaricata. prunaftri: $\alpha$. vulpina.

B. Aittocera.

Sticta crocata: $\alpha$.

B. gilva. damacornis. $\mathbf{I}, 2$. disfecta.

filicina. 1,2 . laciniata. pulmonaria: $\alpha$.

B. plevrocarpa, Scrobiculata. 2. 2. fylvatica. tomentofa. 2. 2.'

Parmelia aipolia.

$\gamma$. anthelina. alevrites. ambiqua. aquila. aureola. casfa. caperata. I. 2 junior. centrifuga. conoplea. confperfa. corrugata. I. 2. cyclofelis. diatrypa. encaufta: $\alpha$. B. candefacta. 


\section{) 176}

Parmelia Fahlunenfis. glomulifera. herbacea. hottentotta. lamuginofa. molliuscula. olivacea. omphalodes. pannofa.

parietina. perforate. $I, 2$. perlata: $\alpha$.

B. olivetorum. phyfodes. 1,2 . picita.

plumbea.

pulverulenta.

recurva.

rubiginofa., 2.2. faxatilis.

fortea. fellaris.

Aygia. tiliacer. vilothrix,

Borrera capenfis.

cryfophtalma. ciliaris. 2. 2. 3 . ephebea. flavicans. i. 2. furfuracea. 1,2 . levcomela. temella: á. B. leptalea.
Borrera villofa.

Cetraria cucullata. glauca: á.

B. fallax.

$\gamma$. junior.

islandica: $\alpha$.

B. thyreophora.

$\gamma$.

juniperina: $\alpha$. B. pinaftri. mivalis. 1. 2. 3. 4. Soepincola: $\alpha$.

Peltidea aphtofa. B. ulophylla. cainina:

a. Spuria.

$\beta$.

e. crispa. horizontalis: $\alpha$.

$\gamma$. lophyra. polydactyla. Scutata:

B. collina. venosa.

Nephrama parilis. polaris:

t. arctica.

2. antarctica. 3. lapponica. 4. americana. refupinata.

Dufourea flammea. madreporiformis. Cenomyce adunca. 


\section{) 18(}

Cenomyce aggregata. alcicornis. 1.2. allatropa:

- a. turbinata.

$\gamma$. fibula.

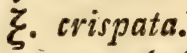

ท. corymbofa.

bacillaris.

d. Sparassa.

botrytes.

carinfa.

ceratophylle.

cervicornis.

coccifera.

coccocephala:

a. bellidiftora.

cornuta. $\boldsymbol{l} .2$.

damcecornis.

deformis. a.

delicata.

$\gamma$. digitata.

ecmocyna.

a. elongata.

B. roftrata.

$\gamma$. gracilis.

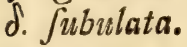

endivifolia.

epiphylla:

B. coefpiticia.

furcata: $\alpha$.

$\xi$. pungens.

ク. mivea.

d. Spinofa.
Cenomyce gonorega:

a. cenotra:

d. anomiea.

ع. virgata.

papillaria.

parecha: $\propto$.

B. cetrarioides:

pyxidata, $\alpha$.

radiata.

$\gamma$. fimbriata.

rangiferina. $\quad$ t. 2. 3 .

ع. ramo/a.

rubiformis.

Symphycarpa.

uncialis: $\alpha$.

d. obtufata.'

vermicularis. 2. 2 :

Boomyces fungoides.

placophyllus.

roseus.

rupeftris:

a. bysfoides.

Ifidium coccodes.

corallinum.

phymatodes.

Weftringii,

Stercocaulon botryofim.

cereolus.

nanum.

pafchale.

ramulofum. $x, 2$.

tabulare.

Spharophoron compressum, fragile. Alectoria crinalis. 


\section{) 196}

Aleçoria jubata:

$$
\text { d. prolixa. }
$$

jarmentola.

thraufta.

ulneoides.

Ramalina farinacea.

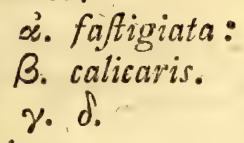

fraxinea. $z .2$.

linearis. 1. 2.

pollinaria.

polymorpha:

a. ligulata.

d. tinctoria.

Scoptilorum :

B. cuspidata.

Cornicularia aculeata. $\dot{x}$.

B. muricata.

bicolor.

divergens.

lanata.

ochrolevca.

pubefcens.

Spadicea.

triftis :

B. radiata.

Usnea barbata. I. 2 .

$\gamma$. articalata.

cornicularia.

florida. 1. 2. 3. 4. 5 .

jamaicenfis.

melaxantha.
Usnea plicata: a. B. d.

$\gamma \cdot \operatorname{como} \int \sigma_{\text {. }}$.

ह. glabrata.

trichoden. 1.2.

Collema Burgesfit.

diaphanum.

faficulare.

flaccidum.

fluviatile.

furvim.

hydrocharuin.

lacerum.

linofum.

marginellum:

melcenum: os.

B. marginale.

६. gyrofum.

muscicola.

myriococcum.

nigrefcens. $\alpha$. $\beta$.

palmatum.

pannofum.

plicatile.

pulpofum:

B. crispum.

$\gamma$. criftatum.

faturninum.

\&. gramulatum.

fotinum.

fubtile.

tenuisfomum.

tremelloides. 1. 2. 3.

tunceforme.
4. pichneum.

Le. 
Lepraria chlorina:

B. anvea incana: á.

20

Lepraria leiphama.

Rhizomorph fetiformis.

fubcorticalis. $t .2$.

B. latebrarum. 


\section{MUSEUM NATURALIUM ACADEMIE UPSALIENSIS. - $\infty$ \\ C U J U S \\ ApPEND. XXII.}

VENIA EXP. FAC. MED. UPSAL。
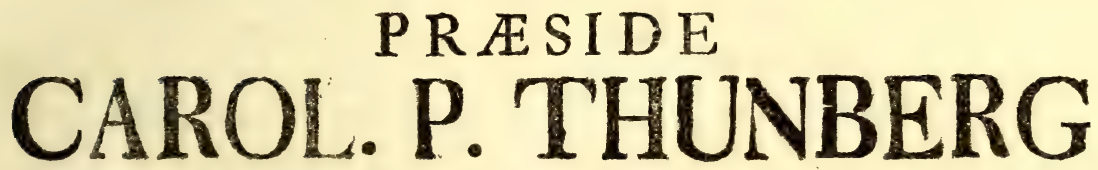

Equite aurat. Reg. Ord. de Vasa,

Med. Doct, Prof. Med. et Bot. Reg. ex Ord. Reg. Coll. Santt. Memb. Hon. Acad, Casar. Petrofol. ex Nat. Curios. Reg. Scient, lond. Holm. Agric. Med. et Evang. Societ. Scient. Ups. et Ofcon. Philad. Patr. Holm, et Civ, Oecon, Find. Beror. Nat. Scrut, Paris. Hafn. Moscou. et Haleng. Hist. Nat. Gorfinens. Lund, Harlem. Amsteid. Oecon. et Inst. Lit t. Zeland. Nidrosiens. Ticinens, Ienens, Linn. Londin. Reg. Scient. Phys. et Phytograpi. Gót. tingens. Wetter. Nanciens. Med. Paris. Emul. Monspel. Med. Matritens. Medic. et Nat. Studios. Enimbrg. et Petrop. Medico-Chir. Membro nec non Acad. Scient. Paris, et institut. Nation, Monspeliens. Agricuit. Paris. Medic. Londin. Scandinav. Florentin. et Batavin, Ind, Or. Corresrond.

PROPON I T

PETRUS CHRISTOPH. WESTRING AMANUENSIS ET STIPENDIARIUS STRANDBERGIANUS, OSTROGOTHUS.

IN AUDIT. BOTANICO D. XXIII. NOVEMB. MDCCCXIV. H. A. M. S.

U P S A L I

excudebant Stenhammar et Palmblad

REG. ACAD, TYPOGR. 


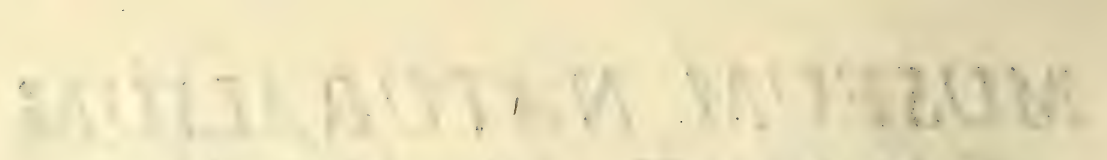

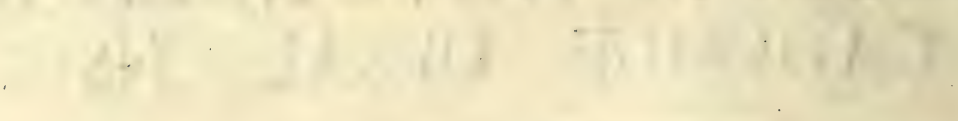

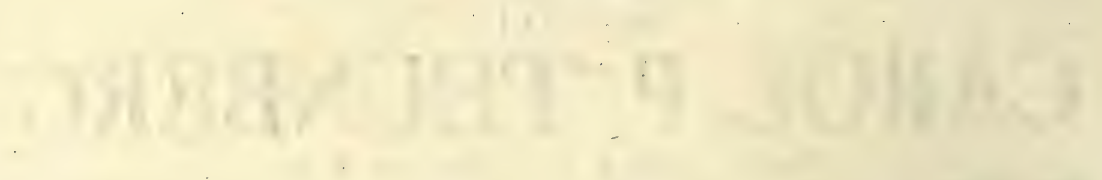




\section{DONATION. THUNBERG.}

\section{Appendix XXII.}

Arthonia elegans. polymorpha: : fubftellata. punetiformis: snalepta. galactina. radiata:

hypercha. tynnocarpa.

Gyalecta Waklenbergiana: truncigena. Opegrapha pedonta. fiderella: rufefcens. verrucarioides: hypolepta. marmorata.

vulvella: anachana.

Graphis fcripta: hebraica. macrocarpa. Verrucaria arcetina. carpinea. clopima. epipolcea. lignyota. papilloja. plumbea. fohraderi. tesfulata.

Endocarpon lacknoum. rufescens.
Pyrenula verrucoja.

Calicium cembrinum.

cladonifcum.

Lecidea ambigua.

artyta. candida. conglomerata. dolofa. fufcolutea: leucorhoca. inculcata. lapicida: illuta. latypea. lithophila: monticola.

timofa. lucida. luteoalba:

luteola:

pyracea. chloritica. muscorum. $a$. $\beta$ cupularis. papillosa. paradoxa. parafenia: cruftulata. ruguloja. petrea:

globulata. obfcurata. 
Lecidea rupeftris: irrubata. Lecanord epibryon: pachnoe. talcophila.

Variolaria tutejens.

vitiligo.

falfaria.

decusfata.

glaucóma:

Urcoolaria calcaria.

cinerea:

notaia.

polygonia.

suntabilis.

Collema cheileum:

bysfacenm.

elveloideum.

exasperativis.

lacerum:

catelenm.

puloinatum.

melcenum:

jacoborifoliums.

Lecanora albella:

Sordicefcens.

anomala:

cooperta.

atra:

tenebricofa.

argentea.

$\therefore$ caliginofa.

callopisma.

expanfi.

cerina:

gilva.

ftillicidiorum.

shorophana.

cireinata.

rimulofa.

grantilosa:

achroa."

inalpine.

aporetica.

intricata.

livida.

faxicols.

frraminece:

oreina.

fubcarnea.

varia:

ravida.

Parmelia caperata:

- vilophylla.

encanftia:

textilis.

muscigera:

lenta.

speciofa.

baceilaris:

apolepta.

macilenta.

Cenomyce cornuta:

exoncera.

chordalis.

coreophora.

merifta.

damcecornis :

phyllophora. 
Concmyce furcata: incrassata. lepidota.

Gonorega:

crefpitosa. fabirfa. trachylics.

papillaria: molariformis. pyxidata: exilis. pocillum.

fimplex. tuberculofa.

radiata:

holofchifta. actinota. rubiformis:

cefpiticia. Atrepefilis. vermicularis: taurica.

Ifidium lcevigatum. Alectoria jubata: capillaris.

Ramalina polymorpha:

Usnea barbata:

$$
\text { Areppilis. }
$$

$$
\begin{aligned}
& \text { dafopoga. } \\
& \text { plicata: } \\
& \text { implexa. }
\end{aligned}
$$

Cetraria fallax.

Marchantia fragrans. hirfuta. $\beta_{0}$
Marchantia polycephata. polymorpha $\delta$. $\beta$. quadrata.

Fungermannia adianthoides $\beta$. barbata a. minor. $\beta$.

bicornis. biroftrata. byssacea. connivens. dichotoma. diffusa. B. excifa. exfecta. filiformis. $\beta$. fragilis. gracilis. graveolens. implexa. incifa. inflata. $\beta$. lavigata. Leerfii. nigricans. paludofa. pinguis. platyphylla. $\beta$. pubefcens. Saxicola. Scalaris. Serpyllifolia. tomentella. trianguilaris. wnbrofa. 
Jfötes lacuifris. $\beta$. Sphagnum condenfatum. Phascum bryoides. curvi etum.

Splachnam gracile. helveticum.

Polytrichum holveticum. juniperinum $\beta$. pilofum.

Hypnum longiroftrum. penicilliforme. protenfum. 2.

falebrofum. Spiendens.

Arigofum.

Spharocarpons. uliginofum.

Conferva capillaris. $\beta$.'

linum.

mu/cortum.

rojea.

rubra. $\beta$.

Lemania fubtilis.

Fucus copillaceus.

cocinzeus.

coronopifolins.

luterus.

mamillofus. $\beta$.

muicronatus

palmatus. $\beta$.

priftoides.

jaccharinus. $\beta$

verticillatus.

vittatus. B.
Funaria hygrometrica. d. miihlenbergii.

Dicranum aquatioum.

elongatum.

longiroflrum.

pubefcens.

jubulatum.

undulatum.

Xanthodors.

Barbula apiculata.

gracilir.

paludofa.

Trichoftomum affine.

aquaticum.

cylindricum.

heteroftichon. $\beta . \gamma$.

nudim.

Serratum.

Tortula curvata.

mucronata. $\beta$.

Gymnoftomum aquaticum.

rupeftre.

Aelligerum.

Grimmia apocarpa. $\gamma . \delta$.

canefcens.

gracilis.

plagiopodia.

Weifra atrata.

gypfacea.

Mnium glaciale.

gracile.

pjeudotriqueirusi.

fellare.

Didymodon homomallum. 
Orthotrichum affine. cupulatum. diaphanum. octoblepharis. rupeftre.

Cynontodium cormutum. inclinatum.

Webera mutans.

Barthramia fontana.

longifeta,

Oederi.

Lefkea complanata. $\beta$. gracilefcens.

mollis.

Seligeri.

Neckera cladorhizans. crispa. $\beta$.

Bryum proliferum. punctatum.

Zierii.

Hypnum breviroftrum. cirrhofum. $\beta$. contextum.

curvatum. diverffolinm. dubium. falcatum. filicinam. $\beta$. fontanum.

Equifetum ramofum.

Lycopodium imbricatum.

Pteris nummularia.

Semipinnata. 2.
Polypodium aurenm.

Asplenium alternifoliun.

$$
\text { fontanum. }
$$

Lindfea iultrata.

Salvinia imbricata.

Marfilea dentata.

Adianthum capillus. 3 .

Cheilanthus odora.

Pterygonium Smithii.

Agaricus fipticus.

Merulius betulinus. B.

difcolor. 1. 2. 3. 4.

tremellofis.

Thaelephora coerulea.

cruenta.

ferruginea.

lavis.

Hydnum abietinam. decipiens.

Phallus impudicus.

Sclarotium coryli.

fraxini.

heraclei.

Cibraria purpurea.

Racodium cellare. rupeftre.

Namospora holofperma.

purpurea.

Spharia berberidis. bombarda. decidua. fibrofa.

fragiformis. 
Spharia fil/ca.

maxima.

nitida.

poronia.

prilchella.

punctiformis.

pyria.

Spermoides.

Spino/a.

Atigma.

typhina.

avda.

ulmi.

verruciformis.

Erineum vitis

Puccinia juniperi.

mucronata.

Xyloma acerinum.

hyfteroidies.

ilicis.

pezizoides.

populeum.

Onydena equina.

folicinum.

Domatium antennatum.

Uredo alchemillde.

alnea.

appendiculata.

candida.

caricis.

Cyani.

farinofa.

heliof copice.

idcei.
Uiedo miniata.

popuieum

Scutellata.

tusflaginis.

Pcziza aruginofa.

citrina.

coccinea

faficulata.

flavida.

poculum.

radiata.

ribefi.

ftercorea. tuberofa. urceolata.

Cyathas iruciluliformis. hirfutus. lentifer.

Clathrus ater. denuiatus.

Clavaria hypoxylon. $\beta$.

Lycoperdon minutrim.

Trichia rubiformis.

Lycogala miniata.

Leotia tremula.

Spumaria mueilago.

Stilbum nigrum.

Acidium berberidis.

cornutum.

crassum.

pini.

rumitis.

Sempervivi. tragopoginis. 
Amomum Spicatum.

Radermachia lakuska.

Chara translucens.

flexilis. $\beta$.

Fasminum arborefcens.

Scandens.

Philiyrea trachiata.

paniculata.

Veronica anagallis. $\beta$.

beccabunga. $\delta$.

marictima. $\xi$.

gentianoides.

Salix myrtilloides : $\gamma$.

Orchis fpeciofa. $\beta$.

$V$ aleriana olitoria. $\gamma$.

0 Syris poltata.

alba. $\gamma$. folia.

Ficus caricoides.

canglomerata.

cunea.

quercifolia.

repens.

Boerhavia Scandens. $\gamma$. Antholyza athiopica. $\gamma$. marginata. $\alpha . \beta$.

Spathacea.

Gladiolus bracteatus. $\beta$.

bullatus.

communis. $\beta$.

exfcapus $\beta$.

recurvus. $\beta$.

Sparrmanni, a. B.

fpiralis. $\alpha$.

trinervis.
Ixia ariftata. $\delta$. excifa $\beta$. humilis $\delta$.

Secunda $\beta$.

Iris compresja. $\beta_{.} \gamma$.

pavonia. B. $\gamma$.

tricuspis. 5 .

ventricofa?

Morca africana. $\gamma$. gladiata. $\beta$. undulata. $\beta$.

Eriophorum anguffifolium. triquetrum.

Digitaria Sanguinalis. $\delta_{0}$ linearis. $\delta$.

Bromus pendulimus. lanceolatus.

Triticum denffflorum. glaucum.

Lolium temulentum.

Coix aquatica.

Chloris paspaloides. $\gamma . \delta$. Avena bulbofa. $\beta$.

Stipa teneax. B.

Pappophorum fbiricum.

Eriocaulon decemangulare. $\gamma$.

Bryonia alba. $\beta$.

umbellata. $\beta$.

Cucurbita citrullus. $\beta$.

Carex arenaria $\beta . \gamma$. argentea $\beta$. atrata. $\beta$. brizoides. $\beta$. ciliata. 
Carex filiformis.

flacca, $\alpha, \beta, \gamma$.

foetida.

fus $c a$.

macrophylla.

microglochin. $\beta$.

montana. $\beta$.

nemorofa. $\alpha$. $\beta$.

ornithopoda.

tenuiflora. a. $\beta$.

veficaria. $\beta . \gamma$.

vulpina. $\gamma . \delta$.

Reftio Squamofus.

Fuirene involucrata:

Schoenus corymbofus. $\delta$.

Scirpus capillaris.

sefpitofus. $\beta$.

dipsafeus.

maritimus. $\gamma$.

romanus. $\gamma$.

Setaceus. $\gamma$.

Cyperus brizoides. $\beta$. efculentus. $\beta$.

Andropogon crinitum. $\gamma$. ivarancufa.

eniliaceum.

Ariftida tripilis. $\beta$.

Phalaris aspera. $\beta$.

Panicum coftatum.

flexнofum.

genicu'atum $\alpha_{0} \beta$.

miliaceum. $\gamma$.
Panicum farmentofum.

verticillatum. s. b. za

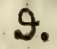

Agroftis compresfa.

miliacea. $\alpha, \beta$.

fetacea.

ftolonifera. d. minima. vulgaris. a. $\beta . \gamma . \delta$.

Poa hirta. $\beta$.

malabarica. 2.

Feftuca pauciflora. $\beta$. rubra. $\beta$.

Stilago diandra. t. 9.

Trophis afpera. \&. cylindrica. $\delta$.

Crucianella anomala. $\beta$.

Morus paniculata.

Urtica involucrata. tenacisfima.

Cufcuta capitata.

Sirium myrtifolism.

Bat is fpimofa. $\delta$.

Elacagnus conferta.

Ixora barbata.

parviflora.

undulata.

Bankfia ericifolia.

marginata.

Protea nitens.

pyramidalis.

Sericea.

Embothrium lanseum.

flaifolium. 2 .

mombellatum. 
Globularia vulgaris. $\delta$. Dipjacus pilosus. 2. 3. 4. Sylveftris, 1.2.

Scabioja caucafica. crenata. $\beta$. fpeciofa. $\gamma$. fuccifa. $\beta$. tartarica. $\beta$.

Pomax umbellata. $\beta$.

Spermacoce hirta. tenera.

Asperula cynanchica. $\gamma$. pentandra. 2.

Galium humifufum. $\beta$. Fusfienii. linifolium. tomentofum. $\beta$.

Callicarpa reticulata. Planiago coronopus. $\delta$. fubulata. vaginata. $\beta$. Scoparia dulcis. $\delta$. Fagara Euodia. $\beta$. Ptelea trifoliata. 2. 3 . Gonatocarpus micrantbus, $\beta$. Betula incana $\delta$. nigra. $\beta$. oblongata. Serrulata.

Ilex aquifolium. ज. 1 . canadenfis. fol.

Ruppia maritima. $\beta . \gamma, \delta$. Sagina erecta. $\beta$. Brucea fumatrana. Cerinthe aspera.
Samolus Valerandi. $\gamma . \delta$. Thefium Squarrofum. $\beta$. Diosma alba. $\beta$. siliata. $\delta$. pubefcens. virgata. $\beta$.

Brunia lanuginofa. $\beta$. Afclepias crifpa. $\gamma$. aftmatica. $\beta$. mucronata. tuberofa. B. filiformis. $\gamma$. fruticoja. $\delta$.

Cynanchum capense. $\beta$. bratteatum. Crasfula capitella. $\beta$. centauroides. $\beta . \gamma$. proftrata. $\beta$. retroflexa. $z$.

Rhus ellipticum a. $\beta$. levigatum. $\beta$. micranthus $\omega . \beta$. parviflorum. spicatum. $\alpha . \beta$. glaucum. 2 .

Pharnaceum incanum. $\beta$. mollugo. $\beta$. Lonicera bubalina. $\beta$. PhyJalis tomentofa. $\beta$. peruviana. $\beta$. Celaftrus linearis. 2. sutans. verticillata. obtufus. 
Celaftrus montante.

$V i t$ is qusadrangutaris, $\beta$.

dioica.

cordata.

cirrhofar $\beta$.

Chenopod sm glaucum. $\%$ gloucefcens. $\beta$. incifum.

lanceolatum.

virgatum.

Phylica capitata. 2. racemofa. $\beta$.

Rhamnus incanus. alaius. a. $\beta$. racemofus.

Zizyphus latifolius.

lucidus.

xylocarpus.

Gardenia campanulata.

dushetorum.

lucida.

laiffolia.

longispina.

randia. $\beta$.

Carisfa diffufa.

Ehretia Serruta.

Scavola taccada.

Xanthoxylon atatumi. P.

Rondelet id exferta.

paniculata.

fricita.

Convolvolus acutus. 1.2. reflexus.

Nerium tintiorium.
Nerium reticulatiuns.

Echites dishotoma.

lowis.

Coffea bengalenis.

Onosma echioides. $\beta$.

Echium calycinum.

Plumbago ceilanica. $\beta$.

europica. $\delta$.

rosea. $\beta$.

Heuchere americama.

Lyfimachia thyrfffora. B.

$$
\text { vetlgaris. } \gamma \text {. }
$$

Campanula cevvicaria. glomerata. latifolia. $\delta$. lilifolia $\gamma .{ }^{2} .2$. rotuindifolia. $\xi$.

Lobelia ceylanica. $\beta$.

Goodenia ovata. $\beta$.

Verbafcum compadtum.

Solanum bullatum.

nigrum. $k$.

$V$ arronia currasavica.

Roemeria inermis. $\beta$.

Violn eapenfiss.

cornuta. $\beta$.

pedata. $\delta$.

fricta. $\beta$.

Hirtella triandra. $\beta$.

Ribes crispum. $\beta$.

Celofia monforia.

paniculata. $\gamma . d$.

Illecebrum pubefrens.

Beta vulgaris. 3.4. 
Salfola arbufcula.

tragus. B.

Ulmus campeftris. 3 .

Gentiana campeftris. $\beta$. $\gamma$. ciliata. F.

Selinum montanum. 2. Cachrys alata.

Morifoni.
Sulcata.
taurica.

Oenanthe globulofa. 2. Scandix odorata. 3 . pecien. 2.

Pimpinella disfectu. 2. magna. 3. traquera.

Telephium imperati. 2. Corrigiola capenfis. Statice flexuoja. 2. tetragona.

Gifekia pharnacioides, 2.

Clutia collina.

Cordia latifolia.

Linum trigynum.

Hamanthus hydrophilus. aundulutatss,

Cyanella alba. d. finnuatus.
clla alba.
vineale. Allium vineale. Sanfeverinia athiopica, 2, Hypoxis decumbens. $\gamma$. Asparagus cordifolins. 2. erevilus. 2.3.
Ajparagus curilus.

Lachenalia pallida. $\delta$.

Funcus arcuatus. bothnicus.

Rumex perficarioides. $\boldsymbol{\gamma}$. aquaticus. 3.

Melanthium wurnęboa. $\eta$. 9. Mauhlia latifolia. tortilis.

Smilax fagittata. theezans. fol.

Loranthus bicolor.

Commelina mudicaulis. truncata. B. $\gamma$.

Musa paradifaca. 4.

Tilefia incarnata. Spiralis.

Ornithogalum caudatum. $\beta$. Tulipa Gesneriana. 30.

Dracana ferrea.

$$
\begin{aligned}
& \text { hirsuta. } \\
& \text { lancea. } \\
& \text { obliqua. } \beta \text {. }
\end{aligned}
$$

Anthericum unizaterale.

Scheushzeria paluftris. $\beta$.

Limeum cthiopicumn. $\beta$.

Efoulus paria. $\beta$.

Gvettarda crispiftora.

Sapindus fruticofus.

$$
\text { faponaria. fol. } 3 \text {. }
$$

Diospyros chloroxylon. cordifolia. glutinofa. montana. 3. 
Amyris heptaphylla.

\section{nana.}

punctata.

Combreium extenfum.

Ornithrophe Cobbes. $\gamma$.

Correa rubra.

Dodonac triquetra.

Erica caffra.

cerinthoides. $\beta . \gamma$.

comofa.

hirta.

imbricata, $\beta, \gamma, \delta . \varepsilon_{0}$ umbellata. $\beta$.

Pasferina glomerata. $\gamma$. involucrata. $\beta$.

\section{longifolia.}

Polygonum fagopyrum. 2.

Coccoloba excorticata. 2.3. Cardiospermum halicacabum. 2.

Laurus dulcis.

Areca oleracea. 2.

Quercus robur. 4. 5.

aesulus. $\gamma$.

nigra. $\beta . \gamma . \delta . \varepsilon . \xi$.

Casfia! auriculata. basilaris. 1, 2. sumatrana.

CaSalpinia cucullata.

Sepiaria.

paniculata.

Spinofa.

Perfonia Salicaria. Menziefia hispida.
Limonia pentagyna.

Bergera integerrima, 1.2.

Terminalia chebula.

Basfia latifolia.

Garcinia corva. \&. morella.

Iuglans cinerea. $1,2$. Eugenia ceilarica.

fruticosa. operculata.

Rosa involucrata. Rabus idaus. 4. 5. 6.

fonotus. 2.

faxatilis. 3 .

Eucalyptus obliqua. $\beta$.

Capparis ceilanica. heteroclita.

Mimosa concinna. latronun. lucida. xylocarpa. dulcis.

Grewia oppofitifolia. Sapida.

Lagerftrómia parvifora. grandiflora.

Uvaria cerafoides. speciofa.

Flacostitia cataphrafia. to 六 Ranunculus frigidus. Volkameria infortunata. odorata. ternata. Vitex incija. 
Bignonia undulata.

Glechoma ereita. Gmelina arborea. villosa.

Salvia bracteata. bengalentis.

Plectranthus fcutellarioides. Ajuga frutico $\int$.

Mentha Secunda.

Brasfica oleracea: alba. 5.

Eryfimum diffusum.

$$
\text { virgatum. 2. } 3 \text {. }
$$

Cheiranthus cuspidatus.

Cardamine graeca, 2.

Lepidium lyratum.

Myagrum dentatum.

Oxalis acetofella. 2.

bifida.

cuneifolia.

convexula.

lepida.

marginata.

macroftylis.

obtura. 1. 2.

polyphylla. $\gamma . \delta$.

rigidula.

temuifolia.

variabilis. $\beta$.

verficolor. 4 ,

Geranium laciniatum.

Hermannia alnifolia. 3 .
Butneria pilosa, $x, 2$.

Ricinus dioicus. $\delta$.

Swietenia mahagoni. 2.'

Erythroxylon monogynum.

Ekebergia indica. 1.2.

Hibifcus heterophyllus.

pariti.

Sida polyandra.

Leea ftaphylea.

-Phyllanthus Kirganelia. paiens.

Hedyjarum vijcidum.

Medicago maculata.

Trifolium effufum.

Lotus coimbricenfis.

hispidus.

Aftragalus leontiwus,

Vicia tenuifolia.

Phaseolus bipunctatus.'

dolichoides.

Polygala amara. $\gamma$.

Crotaleria paniculata.

pulcherrima.

virgata.

fulva.

Dalbergia angiennenfis.

paniculata.

fis $\int 00$.

Hopea octopetala. 2.

Hypericum tomentofum. $\beta_{0} \gamma$.

virginicum. 2. 


\section{Théres.}

I.

Perfectex in Algis fructificationis partes adhuc non demonfratx fuerunt.

\section{II.}

Textura Cellularis inter Organicæ texturæ characteres inprimis eminet. Hae vero algæ quædam, exque non pauex carere videntur.

\section{III.}

Leprofi a Linnæo nominati Lichenes eadem prafertim deftituuntur textura.

\section{IV.}

Confituti igitur hi videntur in confinio Regni Vegitabilis atque Mineralis.

\section{V.}

At in altiorem dignitatis gradum adfcendunt Lichenes Fo. liacei fic dicti, atque Úmbilicati.

\section{VI.}

Foliaceis Umbilicatisque his Filamentofi fic dicti Lichenes affines funt.

\section{VII.}

E Lichenibus leprofis pigmenta abundanti copia eliciuntur; e Foliaceis vero \& Umbilicatis, præter pigmenta fplendisfima alimenta etiam uberrima hominibus redundant.

\section{VIII.}

Adhuc non confat, an Algre veluti ceteræ plantx, aëri purificando inferviant. 


\section{MUSEUM NATURALIUM ACADEMIE UPSALIENSIS. cujus}

\section{APPEND. XXIII.}

VENIA EXP. FA, MED, UPSAL. P R E I D E

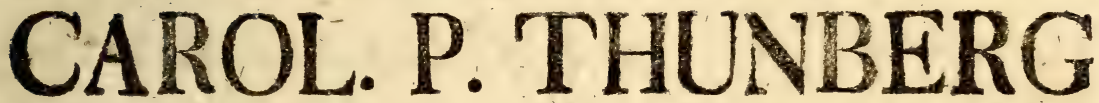

COMMENDATORE REG. ORD. DE WASA,

Med. Doct. Prof. Med. et Bot. Reg. et Ord. Reg. Coll. Sanit. Memb. Hon. Acad. Casar. Petropol. et Nat, Curios, Reg. Scient, lond. Holm. Agric. Med. et Evang. Societ. Scient, Ups. et Oecon. Philad. Ratr. Holm. et Civ, Oecort. Fine. Honot. Whrmel. Berol. Nat. Scrut, Paris. Hafn. Moscou, et Halens. Hist. Nat. Gorentens, Lund. Harlem. AmSteld. OECON. ET Inst. Litt.Zeland. Nidrosiens. TURICENS. InNens. Linn. Londin, Reg. Scient. Phys. ex Phytograph, Góttingens. München. Ertang. Wetter. Nanciens. Med. Parts. Emul. Monspet. Med. Matritens. Nedic. et Nat. Studios. Enimeurg. et Petrop. MedicoChir. Membro nec non Acad. Scientr. Paris. et Institutr. Nation. Mong. peliens. Agricutit. Paris. Medic. Londin Scandinav, Florentin. ez Batavin, Ind, Or. Correstond.

RESP.

\section{PETRUS ADAMUS STAF,}

STIP. STIEGLER. HOLMIENSIS.

IN AUDIT. BOTANICO D. XIII JUNII MDCCCXVI.

H. A. M. S.

UPSALI IE

Excudebant ZEIPEL ET PALMELA1\% 


\title{
KG VUNGENS
}

TROMAN, ÓFVER=KRIGSCOMMISSARIEN

\author{
HO̊GÅDLE
}

\section{HERR AND. PEHR ANDERSSON}

TACKSAMT OCH VO์RDNADSFULLT TILLÅGNADT

$$
\text { af }
$$

EDER

odmjukafte tjenare

PEHR A. STAF. 


\section{KON UNGENS}

*

$$
\text { TROTJENÁRE }
$$

TJENSTFO゚RRÅTTANDE O̊FVRRFÅLTLÅKAREN VID STOCK-

HOLMS GARNIZON, PROFESSORN VID MEDICO-

CHIRURGISKA INSTITUTET I STOCKHOLM, HO̊GÅDLE OCH VIDTBERO̊MDE DOCTORN

\section{HERR ER I C G A D E L I U S \\ VO̊RDNADSFULLT TILLÅGNADT}

af

EDER

¿dmjukafte Tjenare

PEHR A. STAF: 


\section{BOKHẢLLAREN}

I

RIKSENS STÅNDERS RIKSGÄLDS - CONTOIR

HERR J O H A N P E H R S T A SAMT DESS FRU

\section{FRU EVA MARGARETHA STAF FódD CARLSTEDT}

MINE HULDASTE FO̊RÅLDRAR

O̊MT OCH TACKSAMT TILLÅgNADT

af

EDER

lydigafte Son

PEHR ADAM. 


\section{DONATION. THUNBERG.}

\section{ApPENDiX XXIII.}

Lopezia hirrfuta.

Dahlia crinita. $\delta 2$.

Liguftrum vulgare. $\delta$.

$V$ eronica montana $\beta$.

Epipactis rubra $\beta$.

Difa excelfa. $\delta . \bar{\varepsilon}$.

Satyrium cucullatum. $\gamma$.

Pterygodium atratum. $\beta$.

Gunnera perpenfa. $\gamma$.

Valeriana dioica, f.

Fedia olitoria. $\delta$.

Morae umbellata. 2.

Ferreria pavenia.

Gladiolus laxus.

marginatus. $\varepsilon$.

Ixia veticulata

Stilago Bunius. $\delta$.

Bryonia s-loba $\beta$.

Thypha latifolia. $\gamma, 1,2$.

Carex limofa. $\gamma$.

Crocus ininimus

Cyperus compresfis. $\delta$. Jurinamenfis. $\varepsilon$.

Fuirena paniculata.

Schoenus lanceiss. $\alpha . \beta$.

Refio digitatus. $\beta$.

$$
\begin{aligned}
& \text { tetragomus. } \beta \text {. } \\
& \text { viminens. } \alpha . \beta \text {. }
\end{aligned}
$$

Panicum vulpinum.

Alopecurus utriculatus.
Agrofis interrupta. $\beta$.

pungens. $\beta$.

Phlewm nodofwsi. $\gamma$.

Andropogon bicome. $\gamma$.

Poa concinna.

$$
\text { crinitum. } \gamma \text {. }
$$

lapponica:

laxa. B.

marginata.

peruviana.

procumbens.

Sudetica.

Sparsina cynofuroides. B:

Bromus commustatus, $\alpha$. $\beta$.

mollis. $\delta$.

madritenfis, $\gamma$.

trivialis.

Avena carpatica

negleita

planicantis.

tenuis

Lolium pratenfe.

Triticum preftratum.

Scabioja africans $\varepsilon$. $\xi$.

atropurparea. 2.

mollis

Protea conifera. $\gamma \delta \delta$. heterophylla. $\beta$.

icuis.

levifanus. $\gamma$ ovata. 
Afperula cynanchiea. \&. Rubia lucida $\beta$.

Scoparia arborea. $\gamma$. Fagara capenfis. $\gamma . \delta$. Anihofpermum lanceolatum. $\beta$. $\gamma$, Potamogeton rufeccens

Myofot is Sparffifora

Echium papillofum.

$$
\begin{aligned}
& \text { fcabrum } \\
& \text { Arigojum } \\
& \text { verricofum } \\
& \text { violackum. } \beta . \gamma
\end{aligned}
$$

Menyanthes mymphoides. $\beta$. Anagallis arventis. $\varepsilon . \xi$. Convolvutus alt háoî́des. $\lambda$.

cordifolius. $\beta . \gamma$. crifpus

filiformis. a. $\beta$.

radicans

Sagittatus. 2.

finuatus. 2.

trilobus $\beta$.

Ipomoea folanifolia. $\gamma$.

Campanula lannginoja.

hifpidula. $\gamma$.

Phytheuma fpicata. $\beta$.

Lobelia hivjuta. $\gamma$.

$$
\text { pubefcens. } \gamma \text {. }
$$

Vangueria Jpinofa.

Lonicera canadenfis.

caprifolium. B.

Verbafcum pyramidatum.

rugulofum.
Solanum bracteatum.

humzile. melanocerafum miniatum nodiflorim.

Datura ferox. $\gamma$.

Nicotiana macriphylla. 2.2.

Cordia latifolia.

Olinia cymofa $\beta . \gamma$. Rhomnus circumscisfus. 2. Ciephaëlis alata. $\beta$.

Antidejma alexteria. fol. acida: 8. .

Celaftrus emarginatus.

excifus

flexiofus.

laurinus. $\gamma . \delta$.

linearis. 3 .

obtusus.

rigidus.

Phylica latifulia. $\alpha . \beta$. plumofa. $\beta$. Atipularis. $\beta$.

Diosma linearis $\beta$.

Amaranihus incomptus.

Viola arenaria.

Illecebrum Spinofum.

Thefium copitatum. $\gamma$.

Aricium. $\varepsilon$.

Cerbera odollum.

Aporynum filiforme. $\beta$.

Asclepias fufcata. 
Chenopodium angnfifolium. atriplicifolium.

Anabafis arbufcula.

Gompherena decumbens.

Bupleurum longifolinm. $\beta$.

Hydrocotyle linifolia. $\beta$.

Sanicula canadenjis. $\gamma$.

Selinum lineare.

Paftinaca opaca.

Rhus dentatum $\beta$.

dimidiatum.

erofum.

excifum. $\beta_{\text {. . }}$

lucidum. $\gamma$.

mucronatum. $\beta$. obliquum.

parviflorum.

panciforum. 3 .

rosmarinifolium $\gamma$.

Spicatum $\alpha . \beta$.

Crasfula cotyledonis. $\beta$. hirta.

Xanthoxylon alatum. 9.

Chutia alaternoides. $\varepsilon$.

Allium fragrams.

pallafiv.

Tulipa Chufana.

otulus.

Anthericum brevifolium $\beta$.

filiforme. $\gamma$.

planifolium. B. $\gamma$

Hyacinthus orientalis. ".

7 uncus bulbofus. $\delta$. $\varepsilon$.
Funcus cephalotes. $\delta$. capen/is. $\gamma_{\text {. }}$.

Rumex haftifolius.

Smilax copenfis. Q.

Ferraria buxifolia. 9 .

Epilobium dubium: $\gamma$. paluffre. $\gamma$. rofeum. $B$.

Combretum decandrums. $z$. Ornitrophe glabra. Diofpyros Ebemum. d. Erica planifolia. $\gamma$. Struthiola ovata. B. Populus angulata. fol: tremula, fol. $\gamma$. Polygonum Bellardi. $\beta$. Limonia pentagyna. Saxifraga Ajugafolia. $\gamma_{.}$. hieracifolia.

Sternbergi.

Casfia capenfis. 2. Saponaria officinalis. d: Royena lucida., 2* Stellaria glacua. Lychnis dioica. $\gamma$. Cucubalus vijcofus. 4. Dianthus nitidus. Cunonia capenfis. 2. Refeda lateola. 2. Euphorbia Humboldtii. literata. pieta. pubefcons B. 
Euphorbia heliofcopia. $\gamma_{.}$. Agrimonia eupatoria 2. Punica Granatum. $\beta$.

Cacius Ficus. 2.

Pfidium pyriferum. 2.

Eugenia jambolana.

Pyrus mahus: Sylveftis.

Rofa agrefis.

altaica.

Cafia.

fraxinifolia. .

laxa. a. $\beta$.

mollis. $\alpha$. $\beta . \gamma$.

ochrolenca. $\alpha$. $\beta$.

farmentacea. fol.

Senticofa; a. $\beta$.

Sepincola.

Sepium.

turbinella.

umbellifera.

venofa.

Rubus idaus. 7 .

Myjembryanthemum nmbillatum

(d. $\varepsilon . \xi$.

Aizoon paniculatum. 3 .

fecundum. 2.

Potentilla norvegica.

Cratagus monogyna. 4.

Rottlera alba.

tincteria. $\delta$. $\alpha . \beta$.

$$
q \alpha, \beta \text {. }
$$

Zamic pumila. $\delta$.

Adonis athiopica. 2 ,
Adonis mininta.

veficatorica. i:

Atragene capenifis 2.

tenuifolia. 2.

Anemone Regince:

Atsllata.

Thalidtrum teinusifolium. Ranunculus bulbofus. $\delta$. illyricus. 3.

Sagittaria Jagittifolia. 3 .

Hiper unbellatum. 4.

verticillatum.

Dryas ectopetala. B.

Nepeta colorata.

Thymus anguftifolius.

Ocymum thyrfiflorum, $\boldsymbol{z}$.

Maurandia jeandens. 2.

Crobanche racemofa.

purpurea. $\gamma$.

Barleria prionitis, $\gamma$.

Origanum pallens.

Verbena Aubletia. 2.

Phlomis Leonorus. 2.

Ballota ruderalis.

Salvia nepetifolia.

paniculata. 2.

Manulia tomentofa. 2.

Erinus africamus. $\delta$. $\varepsilon$. Antirrhinum bipartitnm."

Cymbalaria.

capense 2.

flavum.

linaria. \&. 
Antirrhinum finplex. Martynia longiflora.

Draba aizoon. mitricella.

Dentaria glandulofa. Arabis hifpida.

Heliophila iusana. 2.

$$
\begin{aligned}
& \text { pufilla. B. } \\
& \text { s-fida. } \varepsilon \text {. }
\end{aligned}
$$

Sifymbrium Arigofum. Sandoricum indicum. Klcinhovia hofpita. 3. Xanthium indicum. orientale.

Monfonia emarginata. $\beta$. Pelargonium auftrals. inodorum.

Sida tirboba. $\delta$. Malva microcarpa. Hibifcus athiopicus. $\delta$. fyriacus. $\delta$.

Taxus elongata \&.

Sterculia cordata.

- Croton Tiglium. 3. 4. variegatum 3 .

Polygala paniculata. 2.

Lebeckia Sepiaria. 4.

Borbonia lanceolata. 2. 3. villoja.

Liparia hirfuta. B.

Cytifus ciliatus. Vicia perpinianthe. villoja,
Phaca auftralis.

Galega grandiflora. 2. Trifolium hybridum. 4. ochrolaucum. $\beta$. pictum. fipulaceum. $\beta$. veficulofum.

Lotus Gebelia. tenuis. d. E. Meticago apiculata.

Echimus.

Hypericum athiopicum. B. hirfutum. B. quadrangulare. 2.

Cnicus unifloriss. Bidens grandiffora. Ofmites lancea. bipisnata. 4. Lactuca fonchifolia. 1. 2: Leoniodon lavigatum. Picris globusifera. Hier acium alpinum. $\sigma$. chondrilloides. cymofum. 2. glaucum.

montanum. neurorum $\xi$. paludofum. S. Pilosella. 3 . pramorfum. 1.2. fylvatisum.

Crepis agreftis. bamnaticfo. 
28

Crepis coronopifolia. pinnatifida.

Sprengeriana. 2. 3. 4. Stoebe cinerea. 3, Serratula pygmaa. quinquefolia. tincteria.

Lapsana grata.

Cynara glomerata. 2. Tarchonanthus camphoratus. $\beta$. Filago minima. Alhanafia indita. pinnata. 2.

Gnaphalium carpaticum cymofum: nudifolium. B. $\gamma$.

Senecio cermuss.

teretifolium. 5. 6.

\section{rosmarinifolius.}

Arnica cordata. 2.

Chryfanthemum pinnatifidum. 2. rotundifolium.

Anthemis fallax. Musfinia :nifora $\beta$. Rhoria lanseolata. $\beta$. monanthos. $\beta$. palmata. $\beta$.

Centaurea elongata. fulphutrea.

Gallinjoga triloba. Ofteojpermum Jpinofum. 3. Othanna abrotanifolia. 3 . coroncpifolia. 5 .

Calendula fcabra. $\dot{\beta}$.
Arctotis elegans. 2.

$$
\text { iniija. } \beta \text {. }
$$

fufca. 2.

Artemific coarctata.

laciniata.

Elichryfum rofeum.

Erigeron atticum.

Cineraria rapitata.

Pyreihrym pulvorulentam.

Achillac rojea.

$$
\text { Speciofa. }
$$

Inula Helenium. $B$. pulicaria. $\delta$.

Conyza balsamifera.

Eupatorium Ayapane.

Georgina Speciofa.

Equijetum variegatum.

finuroides.

Lycopodium feandens. 4. 5. Pteris fpinuloja. dimidiata. nemoralis. \&. B. $\gamma$. denticulata. $\beta$.

Polypodium adnafiens. alternifolium. elegans. phymatodes. ㄱ. ". $x$. puffulatum. $\gamma$. Afpidium anguftum. aureum. 9. . auriculatum. $\gamma$. attenuatum. 
Afpidium exfpanfum. heracleifolium. exaltatum. $g$. capenfe. 2o

incanum. molle. $t .2$. invifrism. $\gamma$.

montanusm. repandam. $\boldsymbol{I} .2$.

Lomaria feandens. Afplenium lineatum. Cheilanthus tenuifolia. 2 . Davalila elata. 2. falcata.

Lindfac guianenfis. Marattia fraxinifolia. Schizca criftata. mivicata.

Hymenophyltam axillare. Adianthum radiatum. $\beta$. Mertenfia furcata. ' $\beta$. $\gamma$. Canopteris flaccida, $\gamma$. Peltidea canina: lacinulofa. . fcitata: rufe/cerss. Cenomyce parecha: odintina. pyxidata: marginalis. Parmelia leptalea. firobiculata. teneila.

Lecanora Hageni: texticola. ocellata. protuberans.

Locidea decipiens: orphncea. fcrotina.
Lecided rivuloja. varians.

Gyrophor a anomea: cinerafcons. Limboria corrugata: $\propto$.

B. alnea.

$\gamma$. quercina.

fimbriata.

Erineum aureum.

Sparia argilacea.

bulista.

ochrarea.

quaternata.

tubaformis.

Collema triotophythum. urceoliferum.

Spiloma fphrarale. paradoxum.

Vaucheria cafpitofa. Hyfterium oblongum. Tmefipteris tannenfis, $1: 2$. Salix anguftelia. fol. cafia ₹. arenaria: dentata. $\delta$. machroftachys. $\delta$. obtuffolia. t. aurita: microphylla. ठ. \&. villof. ad. $\%$. capenfis. +... $\beta$. ambigua fol. coprea; anguftifol. \&. helix: brevif. 9. incubacea. angufifol. 3 . repens: microphylla, o. 


\section{T HE S E S}

TH. I.

Figura Floris in certior eft quam fruetus; proportio partium autem maxime diverfa, fed conftantisfima.

TH. II.

Fundamentum Botanices duplex eft, Difpofitio \& Denominatio.

TH, III.

Filum ariadneum Botanices eft Syftema, fine quo Chaos eft res herbaria.

\section{TH. IV.}

Secundum vita genus diverfum, diverfe variant in avibus non mo. do roftrum, collum, lingua, ungues, pedes \& cauda, fed etiam interduin ipfom corpas.

\section{TH. V.}

Calore artificiali ova Volatilium excludi posfunt, $x$ que ac incubatione matris.

TH. VI.

Migratio avium obtinet non modo in regionibus frigidisfimis fed etiam in illis temperatioribus, ubi hiems vix ulla.

TH. VII.

Hüjusque migrationis causfae funt plures, pro vario climate diverfo.

TH. III.

Infecta pauca quidem, non nulla tamen, præftantia nobis fubminiffrant remedia.

TH. IX.

$\mathrm{Si}$, ut credere fas eft, Exanthemata oriantur ex animalculis vivis, nobis certe non perfuadere posfumus, Variolas vaccinas eidem fpeciei faam debere originem, ac Variolæ ordinariz. 


\section{MUSEUM NATURALIUM}

ACADEMIÆ UPSALIENSIS.

-

$-$

cu.jus

Append. XXIV.

VENIA EXP. FAC. MED. UPSAL.

P R E I D E

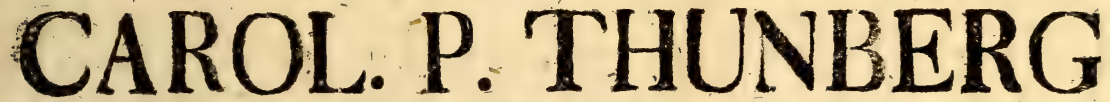

COMMENDATORE REG OHD. DE WASA,

Med. Doct. Prof. Med. et Bot. Keg. er Ord. Reg Coll. Santt. Memb. Hon. Acad. Casar. Petromt. ex Nat Curins. Rec. Scient. Lond. Holm. Agric. Med. et Evang. Societ. Oecon. upsat. Patr. Hoim, ext Civ. Oecon. Find, Honor. Wermel. Westman. et O̊rebr. Berol. Nat. Scrut. Paris. Hafn. Moscou. et Hatens. Hist. Nat, Goren k. Philap. Lund. Harlem. Amsteld. Oecon.et Inst. Litt. Zeland. Nidrosieng. turicens. Ifnens. Linn. Londin, Reg. Scient. Phys. et Phytograpis. fótmingens. Munach. Eklang. Wetter. Nanciens. Med. Paris Emulo Monspet. Med. Matritens. Medic. ex Nat. Studios. Emimbukg. et Petrop. Medtco Cht Membro nec non Acad. Scient. Paris. et Instiztot. Nation. Minnepliens. Agricuit. Paris. Medic. Londin Scandinav. Florentin. et Batavin. Ind. Or. Correstond.

\section{PROPONIT \\ $O L A V U S S \mathscr{F} S T R A N D$ \\ S M I A N D U S.}

N AUDIT. BOTANICO D: VIII JUN. MDCCCXVIIR

F. A, M. S. 


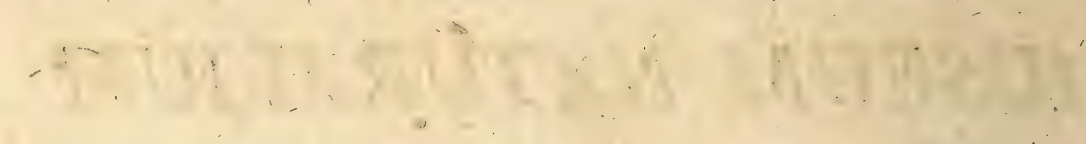




$$
\text { KONVNGENSTROIIAN, }
$$

OEVERSTE-LIEUIENANTEN OCH RIDUAWEN AF KONCL SW-ORD.

$$
H \stackrel{O G}{A} \mathrm{DL} E
$$

herR J O H A N F R. DE M A R E.

KONUNGENS: TROMAN,

BERGSRÅUET OCH RIDEA?EN AF KONGL WASA ORDEN

HO̊ G $\stackrel{\circ}{\mathrm{D} L \mathrm{E}}$

TERK J A C O B G U S T. DE MA R É.

KONUNGENS: TROTJENARE,

MAJOREN OCH RIDDAREN AF KONGL. SW, ORDEN

H! O̊ G Å D L E

HER C A R L DE M A E

Tillåten mig af EDRA för mitt hjerta höga och dyrbara namn låna en glans ât dessa blad, dâ de EDER, Vördade Morbröder, tillegnas. De äro et svagt och ringa offer, men förenadt med den renaste vördnad, hvarmed jag hax äran. framhärda:

\section{EDER}

odmjukaste tjenare - zOE SUÓSTAND. 
KRRKOHERTEO

O̊F VER

SPARRSÁTRA OCH BRED

HO̊GÅRVÓRDIG OCH HO゚GLÅRD

HER E L I A S W A L L I N DER

SAMT

ERU SO P H I A W A L L I N D E R

FöDD C O LL I N D E R

דód nadsfullt och tacksamt tillegnadt

a 


\section{DONATION. THUNBERGIAN.}

\section{APPENDIX XXIV.}

Simia Apella B. $\gamma . \delta$.

Capucina $\alpha . \beta$.

Yacchus $\beta$.

Midas.

Sphinx.

Sylvanus.

$V$ espertilio calceus $\alpha$. $\beta$.

noctulas.

ferotimus.

Myrmecophaga didactyla. $\beta$.

Bradypus tridactylus. $\beta$.

Da/ypus 9-cinotus. $\beta$.

Canis aurens.

familiaris. 9. เ. . $_{0}$ agyptius.

vulpes. B. cruciger $\gamma$.

Felis, capenfis.

catus. B. hifpanicus.

$\gamma$. grifeus.

chans $\delta$. + .

Leo. $\delta$. 9.

Leopardus.

Viverra felina.

foina.

grifea.

Ichneumon.

Putorius.

tigrina.

vulgaris. $\beta$. aftiva,

Zorilla.

Urfus Lotor.

mellivorus,
Didelphis cancrivora.

orientalis.

Oposfum.

Talpa europaca. $\beta$.

Lepus variabilis. $\gamma$. variegatus.

Sorex Araneus. $\beta$.

cunicularius.

muscardinus.

Cavia Aguti. B. braflienfis. Arctomys Marmota.

Mus amphibins. $\beta$.

barbarus. a. $\beta$.

nitela. $\beta$.

Sciurus aftuans.

glis. B.

Ariatus.

variegatus.

vulgaris. d. aftivus.

Hyftrix criftata. $\beta$.

Bos bubalus. $\delta$.

Ovis Aries. 4-cornis, Upland.

4-cornis, Gothl.

6 -cornis.

Capra Hircus 8. vulgaris.

turcicus.

Antilope Eleotragus. $\delta$.

Kevella.

maculata.

monticola. $:$

Oryx. $\delta$.

Strepficeros: junior. 
Cervus Alces. $\delta$.

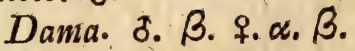

PSitiacus albifrons.

Equus Zebra: junior.

Caballus: mulus.

Sus Scrofa: Aper.

Chinentis.

Phoca variegata. $\gamma$.

$$
\text { pufilla. }
$$

Vultur leucocephalus. Falco ceruginofus. $\beta$.

Antillarum?

Buteo. ๙. $\beta$.

Chryfaëtos. columibarius. gallinarius.

Lithofalco. $\beta$. Melwiäthus. minutus.

Nijuis. B. $\gamma$. $\delta$.

Osfifragus. B.fulvus. Ramphaftos pifcivorus. pondicerianis.

tinnunculus. 8. B. q. Oriolus caftaneus.

Strix Aluco. $\delta$. vociferus.

Bubo. B.

Nyctaa. B. Scandiaca. nufa.

fridula.

Ulula.

Lanius madagafcarienfis. collavis. $\beta$.

Tyrannis. $\beta$.

Pfitacus ceftivus. $\beta$.
Ararauna.

capen/is.

cyanocephalus. crythrocephalus. luteus.

objcurils. fulphureus. Tuipara.

Corvus colvus.

Cornix. B alba. Pyrrhocorax.

Cuculus agyptius. iocevius. niger. fenegalenfis. Gracula criftata... nigriroftris. trifics. Tuianus. annulatus. domingenfis, Galbula. ?. Trochilus fimbriatus. latipennus. Mango. mellifugus. a. $\beta$. minimus. $\alpha$. B. cum nido. Certhia coerulea. erythrorynchos. 
Certhia furcata.

violacea.

Upupa Promerops. o. ?. Superba.

Pieus aurantins. ふ. ?. canaden is.

canipes. cinnanomeus. major. $\beta$. pileatus.

ividis. B.

Todus paradifacus. Alcedo bengalen/is.

criftata. B.

ifjida.

leucocephala. fenegalenfis.

torquata.

Merops viridis, a. $\beta$. Anas adunia.

agyptiaca. B.

Bernicla. ß3.

Cygnus.

fuligula. o.

fusca. B.

mofchata.

platyrynchos. $\delta$.

querquedula. ठ. 9.

Mergus fervator. ठ. $\beta$.

Pelecanus fufcus.

onocrotalus.

Larus fufcus. $\beta$.

glaucus. B. junior.
Larus marinus. B. junior.

Procellaria pelagica.

Aptenodytes patagonica.

Podiceps obfcurus.

Sterna minuta.

$$
\text { critatus. } \beta . \gamma \text {. }
$$

africana.

fibirica.

Cancroma Cochlearia.

Ardea cequinoctialis. 5. क.

caftanea.

comata.

malaccenfis.

$T_{\text {etrapteryx capenfis. }}$

Tantalus ruber.

Parra viridis. caijanenfis.

Tringa fufca. $\beta$ junior. alpina. B. junior. helvetica. of. pugnax. 万. 16. 9.

Scolopax agocephala. canefcens.

Glottis.

Charadrius Calidris.

Himantopus.

pileatus.

Rallus longiroftris. melanura. pifillus.

Palamedea cornuta.

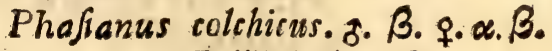
Gallus: domeftic. pullus: tetrapus. 
Pavo criftatus. 3.

Otis tetrax. ?. Tetrao Alchata.

Umbellus.

Urogallas. ठ B. eremita. ?. B. Perdix virginicus. Columba brafilien/is. ?. copen/s.
Emberiza citrinella. ?. Tanagra brafilia.

\section{Epifcopus.}

filens.

Fringilla canaria. $\xi . \eta$. গ. Amandava. ค. ठ. Carduelis. $\gamma$. domeftica. $\gamma$ Serinus. $\gamma$, arcuata.

จ. barbarica. gutturofa. $\beta$.

Onas.

Turtur. $\gamma$. finen/is.

Pipra brafilienfis. ?.

cinerea.

Manacus.

rupicola. ${ }_{\text {. }}$

Sturnus collaris.

ladovicianus.

Turdus arundinacus.

auftralis.

collaris, $\beta$.

rutus.

Tyranmus.

variegatus.

Alauda Senegalenfls.

trivialis.

Colius capenfis.

Loxia Cardinalis.

tafiata.

Pyrrhula: nigra.a. $\beta$.

Emberiza Cirlus.

violacea.

Queleas
Muficapa borbonica.

collaris.

grifola. $\beta$.

homorrhoulso.

lencomeia.

Andator alpinus.

Parus pendulinus. $\beta$.

Hirundo fuciphaga.

Apus. $\delta$.

Melba.

Motacilla atricapilla. ठ. $\beta_{0}$.

aftiva. 9. $\alpha$. $\beta$.

canactenfis. $\beta$.

Curruca. $\beta$.

cyanocephala.

dunnetoram.

fitis.

gibraltarienfis.

pileata.

rupicola. ㅇ.

fibilatrix.

$T$ eftudo discolor.

geometrica. $\gamma$. 


\section{4}

Teftudo mydas. $\varepsilon$ ficcata.

Apion flavipes.

$\xi$ ficcata.

pufilla.

roftrata, B. ferox. Scabia. d.

feripta.

tricarinata. $\beta$.

Lacerta ftincuss.

\section{Monitur. $\gamma$.}

Coluber ferruginofus.

$$
\text { Haje. a. B. }
$$

Angvis lineatus.

Ramphus bifafciatus:

Bruchus gvineenfis.

japonicus.

Lucanus Cervus. $\delta . \gamma$.

piceus. क.

Scarabaus punctatus.

Aphodius hamorrhoidalis.

iefericus.

lapponicus.

lutarius.

merdarins.

niger.

fullcicollis.

triftis.

Cetonia floricola.'

metallica. $\beta$.

Hoplia capucina.

Melulontho brunnea. $\beta$.

vividis.

Copris auftriaca. ㅎ. ?. Splevdens.

Coccinella abietis.

analis.

bifafciata.

lineola.

14. punctata. $\beta$.

5 - punctata. $\beta$.

Hydrophilus pygmaus.

Rynchites Baechus. $\beta$.

Curculio analis.

pulverulentus.

Hifter cadaverinus.

detritus.

12.-Ariatus. 
Hifter fimetarius. incequalis.

foybalariws. vicinus.

Elophorus brevis.

\section{minutus.}

Megatoma pellio. B. $\gamma . \delta$. Cryptophagus variabilis.

Catherétes Quenfelii.

Clerus apiarius. $\beta$.

Dafytes ater. formicarius. $\beta$.

Ips 4. puffulata.

Nitidula erythropa. oblonga. obfoleta.

Salpingus ater.

Boltrichus tridens.

Trogofina caraboides.

Blaps Jpinipes.

Akis punctata. $\beta$.

Mylabris Melanura.

Gyrinus minutus.

S aphylinus hirtus.

Chryfomela 10-punctata, 2.3.4.5. fait tuofa. $\beta$. lapponica, $\gamma$. pallida. $\beta$. viridula.

Haltica lurida.

Crioceris Lawefonice. tonzentofa.

Galeruca viburni.
Anobium hifpidum. nitidum.

Ptinus elegans.

$$
\text { Fiur. } \beta \text {. }
$$

Aleochara brevicollis.

Elater aneus. $\gamma$.

$$
\begin{aligned}
& \text { flavipes. } \\
& \text { pectinicornis. } \\
& \text { variabilis. }
\end{aligned}
$$

Nebria borealis. $\beta$.

Bembidium glabrum.

Harpalus albipes. catenulatus. nigrita. pubefcëns. $\alpha . \beta \gamma$

Brachinus crepitans. $\beta$.

Carabus ovatus. $\alpha$. $\beta$.

- Leptura maculicornis.

nigripes.

- pubefcens.

ftrigilata.

Cantharis affuis.

clypeata.

nigricans.

Calopus ferraticomis. $\beta$.

Saperda rufimana.

Callidium violaceum. $\beta$. Hyphydrus triftis. Dytifcus areticus. coftalis. $\beta$. dolabratus. limbatus. 古. 
Dytijcus maculatus. $\beta$.

Serricornis. $\delta$. $q$.

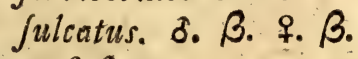

Pamphagus fufcus. maculatus.

Gryllus dimidiatus.

$$
\begin{aligned}
& \text { grosfus. } \beta . \\
& \text { lineola. } \\
& \text { marginalis. } \\
& \text { pedefris. } \beta .
\end{aligned}
$$

Locufta varia. $\alpha . \beta$,

Blatta gallica.

Cicada Jcanica. 9.

Cimex grifens. $\beta$.

Coraus miriformis.

$$
\begin{aligned}
& \text { Sylveftris. } \\
& \text { Thymi. }
\end{aligned}
$$

Miris albomarginatus.
ambiguus.
annulatus.
arbuftorum.
bilineatus.
ericetorum.
flavomaculatus.
magnicornis.
nigrita.
pinaftri. $\delta$.
rubicundus.
rubricatus.
rufifrons.
tanaceti.
tunicatus.
vagans.
variabilis.

Papilio Adonis. B. $\gamma$.

Antiopa B. p.

Argiolus. S.

Argus. $\int$.

Arion. B.

Fcarus. $p$. $\int$.

Idus. a. S. B. $\gamma$, p. J.

Bombyx trifolii.

Mnemofyne. $\delta$. f. \&. $p$.

Noctue bafilinea.

chryftis. $\gamma . \delta$

conigera.

oculuta. $\beta$.

Juffusa.

Phalana repandaria.

Agrion barbarum.

Puella. B. $\gamma$.

pinctata.

Uxor. vidua. 2.2 .

Phryganca evitata.

fuica.

grifes. B. $\gamma$.

marginata

rhombica. $\beta$.

Tipula crocata 3. ๙. $\beta$.

lunata љ. $\alpha . \beta$.

ornata. 9 .

prateniss. 9.

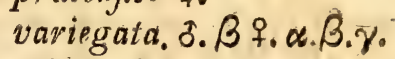

Trichocera hienialis.

Sciara hyalipennis. ?.

Mufca crasfipes.

Henops gibbofus, $\beta$. 


\section{THESES}

I.

Plantæ, quibus licet a natura omnis haud plane fit motrs dene. gatus, eo inprimis ab animalibus differunt, quod nullo letantur fenfu nec motu fpontaneo.

II.

Ufus quamvis \& beneficia haud forfitan paucarum, quæ cognitæ funt, herbarum non fatis perfpecta fint, nullam attamen per fe noxiam, nullam protfus inutilem contendimus.

\section{III,}

Qui foliis continetur infinite multis cumque pervolvitur, liber naturze ad Creatorem veneratione profequendum attentos adducit animad,verfores impellitque.

\section{IV.}

Exinde igitur pluresque praterea ob causfas in errore certe verfantur, qui hiftoria cognitionem naturalis non nifi medicis prodesfe contendunt.

$\mathrm{V}$.

Qui medicinam refpuendam omnino jubeant atque contemnendam; quia interdum erret, fine excidat, fpemque indigentium fallat, folem - mundo, quia caloribus nonnunquam maximis æeftuat \& perurit, fublatum volunt.:

\section{VI.}

Munere officioque, quxe Sacerdotis funt propria, omni, quibus posfit, cura, opera \& ftudio fungi debet, a quibus vero non diftrahitur, fi Medicinæe etiam fe tradat, eadem mentem inftruat nec non eam exerceat, qua quidem re parti fure aliorumque confuleret \& de Auditoribus bene mereretur. 


\section{MUSEUM NATURALIUM ACADEMI压 UPSALIENSIS.}

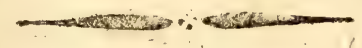

CUJUS

APpEND. XXV.

WENIA EXP. FAC. MED. UPSAL。

\section{$P R$ I S I E}

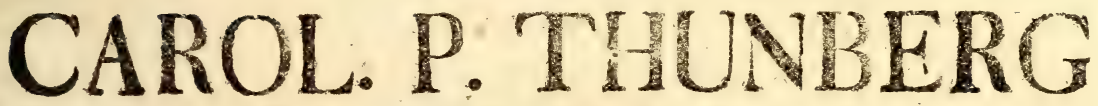

\section{COMMENDATORE RET: ORD. DE WASA,}

Med. Doct. Prof. Med. et Bot, Reg. et Qrd, Reg. Coll. Sanit. Memb. Hon. Acad, Ceesar. Petrome. et Nat Curtos. Reg. Sciqnt, lond. Hot m. Agric. Med. et Evang. Socret. Oecon. tipsal. Patr Horm. iet Civ. Oecon. Finl. Honor. Wermel. Westman. eórtebr. Beroto Nat. Scrut. Raris. Hafn. Moscou et Halens. Hist. Nat. Goren k. Pmilad. Lund, Harlem. Amsteld. Oecon et inst. Litt. Zeland. Nidrosiens. turichin Ienens. Linn. Londin. Reg. Scient, Pizs. et Phytcgrapi. Góto tingens. Munach, Erlang. Wetter. Nanciens. Med. Paris. Emulio Monspel. Med. Matritens. Medic. ex Nat, Stedios, Fimeurg. et Petrop. Medico Chi r. Membro nec non Acad. Scient. Paris et Insti. tur. Nation Monspeliens. Agricut.t. Parjs. Medic. Londin Scandinay. Florentin. et Batavini ind, Or. Correstond.
PROPONIT

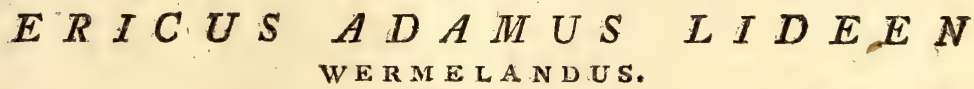

IN. AUDIT. BOTANICO D. X JUN. MDCCCXVII.

H. D. N. S.

U P S A L I E

IXPIS ZeIPEL ET PALMBLAD 
KONUNGEN

TKOTJENARE LIEUTENANTEN

$\mathrm{OCH}$

KQINGL., LIFURIBANTEN:

- HO̊GÄDLE HERR

D $A$ I I

S.A M : :

HO̊G ̊̊DLA FRU

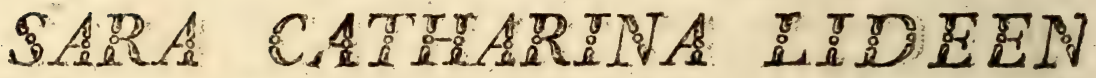
\&ÓDD W A

De: Huldaste: Föıälduà

Helgas: dessa blad sâsom elt vederıäle af barnslig; vördnad: och tillgifvenhet: 


\section{DOMINO CLARISSIMO}

PHILOSOPIE CANDIDATO

\section{O I A}

Beneficiorum memor, ex pia \& integra mente hasce pagellas sacravit 


\section{THE 5 S}

I.

Hiftoriæ Naturali \& fauftis, quos fecit, progresfibus merito id debet xtas noftra \& id etiam agnofcit, quod nempe non folum dispulf fint plurimæ ignorantiæ tenebræ, quibus vaftum illud Naturæ Regnum obductum erat, verum ctiam ex fcientia Medica, ex Oeconomia, quid? quod e vita hominum vulgari innumera opinionum commenta tandem exfulaverint.

H.

Quamvis vero Hiftoria Naturalis in Medicina ejus tit Momenti, ut partium illius nullam ignorare debeat Medicus folidus \& expertus, ftatuere tamen audemus, Botanicam ipfi omnium maxime necesfa. riam esfe.

\section{IIT.}

Eumdem, præ ceteris Hiftoriæ Naturalis partitibus eminentiorem locum, in Oeconomia, fcientix Botanicx adjudicamus.

IV.

Quo plura iuveniuntur nova genera plantarum, eo major difficultas oritur diftinctiones eorum rite determinandi。

\section{$\nabla$.}

Clasfis Pentandria \& quod ad numerum plantarum, \& quod ad ufum Medicum, præ reliquis maximam noftram attentionem merecur. 


\section{DONATION. THUNBERGLAN.}

\section{APPENDIX XXV.}

Culex ciliaris.

Myopa biccata. $\beta$.

Chryfops cecutiens. B.

Laphria flava. $\beta$.

Afilus Glaber.

Najas monofperma.

Chara crimita."

transhucens.

Mniarum fafciculatum.

Canna indica: d. hutecis

Epipatis paltens. B.

Diuris aiba.

Olax aphylla.

Salix fusca: q. B. argenter.

Circáa litetiana:

B. intermedia.

Notelea liguftrina. longifolia.

$\checkmark$ aleriana fupina. $\beta$.

Fedia coftata.

$$
\begin{aligned}
& \text { lafiocarpa. } \\
& \text { turgida. }
\end{aligned}
$$

Gladiolus communis, $\gamma$.

$$
\text { tricolor. }
$$

Ixia Bulbocodium. $\eta_{0}$

Crocus multifidus.

$$
\text { fpeciofus. }
$$

Morcea convolita:

Xyris operculata:

Carex arenaria. d. $\operatorname{extenfa.}$ i. 2 .

verna.

veficaria. $\delta . \varepsilon$

Scirpus rufus.

Zea Mays. D.

Phalaris arenaria.

Digitaria ciliaris.

Devauxia Billiardieri.

Polypogon divaricatum. a. B.

Alopecurus ruthenicus.

Agrofis' dulcis.

Setacea. 2:

Aira globofa.

Phleum asperum.

Boehmeri.

Melica uniflora. $\gamma$. Andropogon argenteum.

Milium dimidiatum.

Poa coefara.

gracilis.

megaftachyas.

- Serotina.

Eeftuca Adopecwrus. divaricata. glauca. tenuifolia.

Bromus gigantens. $\beta$. nanus. $\beta_{\text {. }}$ retufus.

Avena brevis. 
Avena calycina.

Triticum rigidum.

Protea hirfuta.

Bankfid articulata.

patula.

integrifolia.

paludofa.

jpinuloja.

Hakea acicularis.

arborefcens.

daclyloides.

gibboja.

glabra.

Petrophila pulchella.

Embothrium lineare.

longifolium.

polymorphaim.

flaifolium.

tinctorium.

Grevillea Banksii.

Baneri.

chryfodendrwm.

concinna.

Dryandri.

grodii.

juncea.

mucronata.

occidentalis.

polyftachya.

pungens.

refracta.

Sphacelata.

viftaria.
Perfoonia juniperina. latifolia.

Conospermum longifolinen. volubile. teretifolium.

Eriocaulia major. minor.

Brunonia auffralis.

Simfia tenuifolia.

Bellendera inontana.

Scabioja banatica. laxiftora.

Sylvatica. B. $\gamma . \delta$.

Knautia phumofa.

Valantia glabra. $\beta$.

fpuria.

Rubia peregrina.

Galium hierofolymitanum. B. hucidum.

provinciale.

- purpuresm. B.

Scaberrimum.

Sylvaticum. B.

vericillatum.

villo/un.

Dipfacus ferox.

Canfjeria fcandess.

Alchemilla Aphanes. B.

Simbuleta temuifolia.

Betula pubefcens.

Myrica Serrata.

Plantago arenaria. B. $\gamma$. lanceolata. $\xi . \eta_{*}$ 
Ilex balearica.

Myonima borbonica. $\beta$. Potamogeton denfum. $\beta_{\text {. }}$.

Vifcum latifolium.

Helioiropium bractea.

$$
\text { tenuifolizm. }
$$

Anchufa verficolor.

Cerinthe maculata:

Lithofpermum proftratum:-

Nyofoi is verficolor.

Tournefortia mutabilis.

Primula vifcoja.

Lyfimachia maculata:

Epacris grandiflora.

$$
\text { impresfa. }
$$

microphylla.

obtuiffolia.

paluidofa.

pulchella:

pungens.

Styphelic elliptica:

erisoides.

gnidium.

laurifolia.

microphylla:

oxycedrus.

Richei.

ftellata.

triflora.

tubiflora.

virgata.

viridiflora.
Lobelia dioica:

Scavola hijpida.

$$
\text { gibbofa. }
$$

microcarptio

revioluta.

Goodenia caléndulacea:

decurrens. geniculats. gracilis. grandiflora. repens. Atelligera. fricta.

Ehretic acuminata.

faligna.

Phyliea cryptandra.

Cefrum foetidum:

laurifolium.

Solanum fontanefium. punctigerum.

Zizyphus finenfis.

Viola lactea.

$$
\text { taberofa: }
$$

Capficum cerafiforme:

$V$ itis orientalis.

Claytonia cubenfis.

Gomphrana argentea.

Myrfine variabilis.

Ulmus Suber.

Eryngium helveticum.

Ferula nodiflora. 1. 20.

Sium bulbofum. 
Linum taurinum.

Drosera binata.

Statice anguiftifolia. Allium narcisffflerum. Anthericum bicolor.

Scilla umbillata. paniculatum.

Hyacinitus ciliatus. Blandifordia nobilis. Riumex maritimus. $\beta$. Alisma ranunculoides. $\gamma$

Gaura tripetala. Oenothera tetraptera. Polygonum incanum. Quercus pedunculata fol. prafina. fol. tausin.

Daviefia corymbofa. umbillata.

Crovsea faligna. Saxifraga groenlandica. irrigua. leucanthemifolia. pyramidalis.

Gypfophila Jerotina. Dianthas gallicus. Silene altaica.

bicolor. colorata. congefta. corossata. decumbens.
Silene dichotona.

geminiflora.

hirfuta.

hirta. imbricata. linifolia. livita. patula. petraca. pilosa. pungens. fodvides. tridentata. undulata. vefpertina. villofa.

Ceraftium holofteoides. Euphorbia aleppica. procera. rigida. Eugenia elliptica. Melalevea ericifolia. fquarrofa. Leptofpermum aromaticum. parvifolium. Rosa bracteata.

fquarrofum.

campeftris. $\boldsymbol{x} .2$. lucida. pyrifolia. pyriformis. psevdotomento/a. Swartziana. 1. 2. 
Rubus laciniatus.

Potcntilla inciinata.

Getum intermedium.

Papaver orientale. $\beta$.

Cifius pulverulentus. Anemone Halleri. umbellata.

Baucra rubioides.

Ranunculus alysfoides:

caucaficus. disfectus. hirfutus.

Hypericum humile. Teucrium orientale. Lavandula elegans. Sideritis taurica

Ballota Sepium. teucrifolia,

Marrubium aftracanicum.

Origanum majoranoides. Thymus villofis.

Clinopodium miwus.

Dracoctphalum guttatum.

Ocymum ceilanicum,

Plectranthus parviflorus. We, Aringia rubicefolia. Sälvic amara.

crasfffolia. lanigera. pyrenaica. Spielmanni.

Monarda rugofa.

$P$ inguicula graindiflora.
Pinguicula lnfitonica. Antirrhinum miercnthum. Bignonia paradoxa. Mariynia longiflora. Draba mollis Bifcutella raphanifolia. Cardamine matrophylla. fylvatica. Eryfimum pallens. Sijymbrium amplexicaule. Cheiranthus linifolius. longifolius. Arabis cancafica. Erodium alpinum. Geranium parviflornm. venofum. Acalypha acuta. ठ. alopecuroides. desumbens. obtula.

Sida reniflora. Lavatera unguiculata. Hibifcus rofeus. Polygala fasciculata.

Fumaria aurea. formoja. Bosfiaa heterophylla. fcolopendrium.

Platylobium formofum. Diivynia ericifolia.

Cytifus calycinus. Coronilla globojat 
Ornithopus ebralteatus. exftipulatus.

Melilothus dentatus.

Trifolium Molineri.

Lotws hifpidus.

fupinum.

Trigonella hybrida.

Medicago denticulata. glutinofa.

Vicia anginftifolia.

latyroides.

Hieracium eriopilorum. lanatum. Jylvaticum.

Crepis agreftis.

Carduus carlinoides. pauciflorus.

Cnicus echinocephalus.

Bidens dichotoma.

$$
\text { frondo } f a \text {. }
$$

pauciflora.

Chryfocama juncea. Aitemiifia inodora.

leuccanthemifolia, fol. palmata.
jalina.

Gnaphalium angufifolinum.

Senecio dentatus. graveolens.

Santolina ericosperma.

Soorzonera Brogiana.

Solidago elliptica. multiflora.
Solidago petiolaris. After parviflorus.

$$
\begin{aligned}
& \text { punciatus. } \\
& \text { Jalicifolius. } \\
& \text { fericeus. } \\
& \text { Jpurius. } \\
& \text { fibulatus. } \\
& \text { vinnineus. }
\end{aligned}
$$

Inula thapfoides.

Grindelia inuloides.

Stahelina alpina.

Chryfanihernum maritimum.

parthenifolinm.

Anthemis globofa.

Achilea diftans.

filipendulina.

flofculosa.

Helianthus lavis.

tubifloris.

Coreopfis nurea.

Babera glandulofa.

Tusfilago fragrans.

Zinnia violacea.

Centaurea nigrefcens.

Calendula ficula.

$$
\text { praten/is. }
$$

Pteris Plumieri.

Dickfonia pilofunfrula.

Vaucheria racemefa.

B'atrachojpernum vagum: temie.

Ceramium cirrhofum.

pulchellum.

filiculosum. 


\section{MUSEUM NATURALIUM ACADEMIE UPSALIENSIS.}

CUjUS

Append XXVI. ult.

VENIA EXP. FAC. MED. UPSAL.

P R E S D E

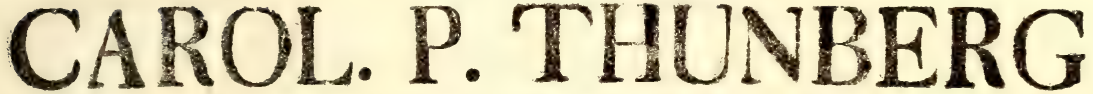

COMMENDATORE RES. ORD, DE WASA,

Med. Doct, Prof. Med. et Bot. Reg. et Ord. Reg. Coll. Sanit. Mema. Hon. Acad. Casar. Petropnt. et Nat. Curirs. Reg. Scisnt. lond. Holm. Agric. Med. ET Evang. Socret. OEcon. upsal. Patr. Hotm. et Civ. Oecon. Finl. Honor. Wermel. Westm. Calm. et o̊rebr. Berdr. Nat. Scrut, Paris. Hafn. Moscou. et Halens. Hist. Nat. Goren K. Puilad. Luni). Harlem. Amsteld, Oecon et Inst. Ltt turichin Ienens. Linn. Londin, Reg. Scient, Phys. et Phytcgrapi. Góttingens. Scient. Gothob. Monach. Erlang. Wetter. Nanciens. Med. Parts Emul. Monspel. Med. Matritens. Medic. ex Nat. Stu. dios. Fnimberg. ex Petrop. Medico Chir. Membro nec non Acad. Scient. Paris et Institut. Nation. Monspeliets. Agricult. Paris. Me. dic. Londin Scandinay, Florentin. et Batavin, Ind, Or. Currestond.

PROPONIT

OLAVUS WILHELMUS FLODSTEDT

VESTROGOTHUS.

IN AUDIT. BOTANICO D. X. MARTII MDCCCIX,

H. A, M. S.

U P S A I A

TyPis Zeipel et PaLmiLad. 
ENKEFRU

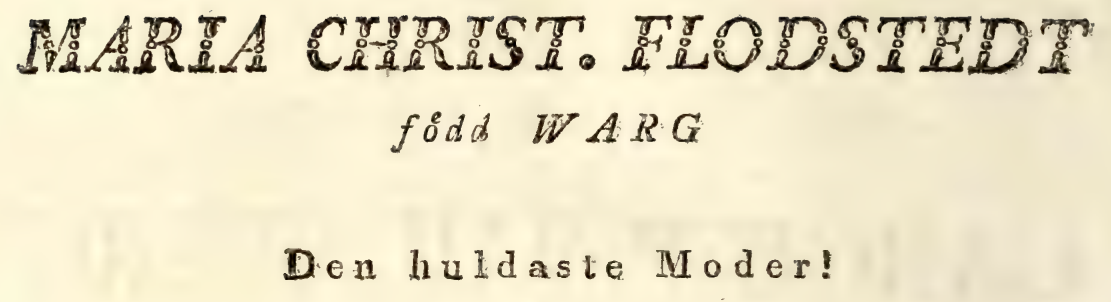

Helgas desse blad såsom ett vedermåle af barnslig vờd ad och tillgifvenhet

$24^{2}$.

en lydig son

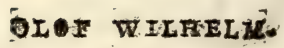




\section{DONATION. THUNBER GIAN.}

\section{APPENDIX XXVI, ult.}

Lopezia hirfuta. B.

Epidendrum guttatum. flor.

$$
\text { patens. }
$$

Epipactis latifolia. $\gamma$.

Dija chryjoftachya.

cylindrica. $\beta$.

Neottia pubefcens.

Satyrium coriifolium.

parviflorum.

Veronica perfica.

proftrata. $\gamma$.

Fedia carinata.

foutellata. $\gamma$.

dafycarpa.

Salix aquatica. fol.

$$
\text { cofia. \& } 9
$$

Bryonia macrocarpa. B. $\gamma$.

Momordica cylindrica.

Cucurbita pepo. a. 1, 2. B. $\gamma$.

Cucumis aurantia. $\alpha . \beta$.

Iris caucafica.

$$
\text { Melo. 2. } 3 \text {. }
$$

reticulata. fqualens. $\gamma$.

Crocus Pallafii.

$$
\text { reticulatus. }
$$

Eriophorum virginicum. Andropogon annulatum. barbatum. $\gamma$. fquarrof um.
Pollinia fulva.

Scirpus validus.

Cyperus confertus. B.

Polypogon Jubjpicatuw.

Milium coerulefcens. multiflorum.

Panicum gymnotrix.

$$
\text { nitidum. }
$$

Phleure Bellardi.

$$
\text { itenue. }
$$

Aira globoja.

Agroftis cinna. $\beta$.

folonifera. $\delta$.

Poa cegyptiaca.

cynofuroides. eragrofis. $\delta$.

maritima. B. $\gamma$. megaftachya. $\left.\propto, \beta . \gamma_{0} \delta . \varepsilon.\right\}$. mexicona. pilofa. B. $\gamma . \delta$. procumbens. $\beta$. recunda.

Melica minuta. $\beta$. ferotina. B. $\gamma$.

Dactylis glaucefcens. littoralis. B.

Holcus argentenis.

Briza elatior.

Feftuces digitata.

I

\section{elatior. $\%$.}

Sote: 
Feftuca heterophitho. B. hirfuta.

phleoides. B.

prateinfs. $\beta$.

Atricia.

Stipa ariftella. B.

Rottboellia incurvata. a.

Cynofurus durus. $\beta$.

Avena alopecurus.

Triticum patulum. pectinatum.

Bromus gynandrus.

maximus.
pubefcens.
rigidus.

Minuartia montanz. $\beta$.

Protea cinerea.

Bankfia latifolia.

Conofpermum ericafolium.

Corifpermum fpicatum.

Embothrium tinctorium. s:

Grevillea vemu/ta.

Scabiofa hybrida. trans $y$ lvanica. $\beta$.

Knautia phmoja. B.

Gyrocarpus Facquinii.

Hedyotis lancea. $\beta$.

spermacoce rubra.

$$
\text { Spino/a. }
$$

Galium boreale. $\gamma$.

infeftum.

litigiofum.

Valantia muricata.
Crucianella anguftifolia. 2:

Elxengnus ceilanica. 2.

Plantago Willenaii.

Cornus fericea. $\gamma$

Hypecoum pendulum. $\beta$.

Ifogonum Anemonifoliums.

Niorus alba $\gamma$.

Betula nigra. $\gamma$.

$$
\text { villofa. }
$$

Petamogeton demerfun: gramineum. 2.

Heliotropium inundatum. parviflorum. $\beta$.

Myofotis cordilophora. Lappula deflexa. a. $\beta$.

$$
\text { verficolor. }
$$

Symphytum. bullatum.

$$
\text { taurinum. }
$$

Onosma rupeftre. Anchufa ceilanica.

snilleri. 1.2.

Borago incana.

oehroleuca. x. $\beta$.

Cerinthe maculata. $\beta$.

Leucopfis veficaria. $\beta$.

Cynoglosfum virginicum:

Echium anguftifolium. italicum. B.

Tournefortia glabra. Androjace nana.

Hydrophyllum magellanicuns.

Lyfimachia verticillata. $\delta$.

Convolvulus disfectus. $\beta$. 
Convolvulus edutis. $\%$ unidulatus.

Ipomoea soccinea. $\beta$. difcolor.

repanda.

Campanule attenuata.

divergens. integra. $\delta$.

lancifolia.

sitida.

rapunaculoides. ?. unifiora. B.

Samolus incanus.

Phytevma fpicata, $\gamma$

Lonicers iberica.

orientalis.

perielymensm. $\beta$.

Oxybaphys vifcoja. $\beta$.

Nierembergia repens.

Verbafoum arcinus. B.

ferrugineum.

mugulo fum. 2. 3 .

Hyojoyamus pallidus. Atropa umbellata. a. $\beta$. Phyjalis barbadenfis. $\beta$.

Solanum buliatum. 2.

Humbolatii. $\beta$.

laciniatium.

mauritanicum:

nodiflorum.

pubigerum.

Strychnos cinnamomea.

Ehretia timifolia, 2.
Eaugeria lucida. 2.

Toddalia acnleata. $\alpha$. B.

Exocarpus cupresiformis.

Viola biflora. $\gamma$.

Impatiens nolitangere. $\gamma_{0}$

Billiardieria longifora.

Celofia japonica. $\alpha$. $\beta$.

Illecebrum gubefcens. $\beta$.

vermiculatum. $\gamma_{0}$

Rochefortia ovata. $\beta$. Achyranthes ficoidea.

Gomphrena decumbens. $\beta$. Afclepias amcena. $\alpha . \beta$. purpurafcens. $\beta$.

Amaranthus albus. $\gamma$. blitum $\gamma$. lividus. $\beta . \gamma . \delta$. proftrains. fangvineus. e. ?.

Chenopodium carthaginens $\mathrm{e}_{0}$ foetidnm. opulifolizms.

Salfola tragus. $\gamma$. Beta trigyna. I. 2:

Gentiana angulosa. gelida. pufilla.

Chironia tetragona. $\beta$.

Hamiltonia oleifera. 1. $\mathbf{s}_{0}^{4}$

Celtis occidentalis. $\delta$.

Gyrofpermum procumbens. Eryngium exaium.

Buplevrum terme. 3. 4. 
Bunium bulbocaftanum. 2. Caucalis marantha. 1. 2. Selinum auftriacum. Peucedantm ferulaceum. Ferula orientalis. Laferpitium chironium. lucidum.

Pimpinella rotundifolia. Heracleum liguftifolium. tourinum. 1. 2.

Liguficum candicans.

Crithmum maritimum. 1. 2. Angelica mongolica. 1. 2. Onanthe apifolia. Ethufa bunias. $\gamma$. cynapioides.

Scandix cerefolium. 2. fumarioides.

Cicuta virofa. 4. Rhus reticulatum.

tosicodondrum. s.

Statice cafpica.

Linum capenfe. 2.2. fafciculatum. marittimum. $\beta$. Squamulofum.

Bromelia acarna. Galanthus nivalis. $\gamma$. Narcisjus bulbocodium. G. tazetta. $\gamma . \delta$.

Hamanthus fnlcatus. 2. Allium baicalenfe. 2. Garinatum.
Allium mo/chatum. $\gamma$. Sativum. 3. Jubhirfutum. vineale. 2.

Pontederia cordata. $\beta$. Lilium bulbiferum. $\boldsymbol{\gamma}$. martagon. $\gamma$. Asphodelus prolifer. Anthericum ramofium. 3 . Ornichogalum umbellatum. $\gamma$. Hypoxis ovata. B. $\gamma$. $\mathcal{F}$ uncus acutus. $\gamma$. bothnicus. 2 . maximus. 2.

Berberis emarginata. Melanthium Hultgrenii. fol. Colchicum arenariswn flor. Rumex hybridus. paluftris.

Oenother a biemnis. $\beta$. purpurea. tetragona.

Boronia Serrulata. Cupania glabra. Pometic pinnata fol. ternata.

Mimufops kauki. B. Dodoncea triquetra. $\beta$. Amyris heptaphyll.. Populas monilifera. fol. Daphne Salicifolia. Yasjerina nivalis. Polygonum buxifolium. 
Quercus lobulata. B.

Daviefia denudata.

Pultenca flexilis. rubicefolia.

Arbutus thymifolia.

Hydrangaca arborefcens. 3.

Cunonia capenfis. 3 .

Saxifraga geum. palmata. trifurcata.

Gypfophila elegans.

Dianihus caricphyllus. 9. contcoficus. dintinus. fimbriatus. psevdoarmeria. petrous.

Silene piota $\beta$. Jquarrofns. faxatilis.

Stellaria longitolia. paluftris.

Arenaria heterophylla. $\beta$.

procera.

ramofisfma.

roff rata.

triflera.

Cerafium argenteam.

penfylvanicum.

femideca drium. $\beta$.

Sidum criflatum.

Syluaticums.

monregalense.
Sedum telephium. 2.

Euphorbia dendroides. 2. dentata. lucida. undulata.

Fuglans nigra. 3 . Sorbus hybrida. 2. Waldfteinia geoides. Mejembryanthemum linguafor. me.

Rubus cafius. 3 . corylifolius. 1.2.
occidentalis. 2.3.

Potentilla adfcendens. diffusa. inclinata. obscura. pedata. pilofa. ruthenica.

Spiraa alba. 3. Calycanthus pracox. 2. Capparis herbacea. Thoa urens.

Ciffus fumana. $\beta$. Calla cethiopica. 4. Ranunculus hirfutuss. B. Holleborus purpurajicns. Nymphaa mizunta. Alangium decapetalum. Gelonium Liforium. I 2. Rivibvidum. 1.2 . glabrim. 
Gelonium lanteolatum. Aquilegia bicolor. Arum maculatum s.

$$
\text { fpirale. }
$$

Teverium ehamopithys.

Nepeta graveolens.

$$
\begin{aligned}
& \text { longiflora. } \\
& \text { Mitfiniiz. } \\
& \text { reticulata. }
\end{aligned}
$$

Sideritis montana. 3 .

$$
\text { taurica. } 2 .
$$

Hyssopus orientalis.

Hyptis foparia.

Glechoma hirfuta.

Clinopodium agyptiacum.

Phlomis ferraginea

Thymus acinos. 3 .

$$
\begin{aligned}
& \text { melisfoides. } \\
& \text { villofus. }
\end{aligned}
$$

Ocymwm multiflorum. $\alpha . \beta$. urticafolium, a. $\beta$.

Stachys arabica.

Marrubium affine.

Salvis oblongata.

pratenfis. 4.

verbenacka. 4 .

$V$ erbena multifida.

$$
\text { nodiflora. } 50
$$

Antirrhinum cretaceum.

fragrans.

verficolor. $\beta$.

Scrophularia Balbifio.

Halleria lucida. 2.
Lantana ereîta.

Fufticia capenfis. 2.

Bunias cochlearioides.

Draba mollis.

Cochlearia coronopus. 2. danica. 2.

Iberis ciliata. maciocarpa. Alysfum draba. 1.2. edentulum. enaritimum. snicropetalum. Serpyllifolinm. vernale.

Bifcutella hispida. Eryfimum racox. Hefperis bituminofa. $t$. zo montana.

Sifymbrium aquaticum. contortum. $x_{0} 2$. gallicum. $x, 2$. haftatum. pannonicum.

Cardamine fylvatica. 1.2. Cheiranthus mollis. Arabis crifpata. hifpida. $\beta$. incana.

Brasfica napus. 1. 2. 3. 4. 5. oleracea: laciniata. 4.5. pracox.

Sinapis grifea. integrifolia. 
Ifatis tinctoria. 4.

Raphanus raphaniftrum. 2. tenellus. 3 .

Cleome ornithopodioides.

Finus cedrus. ㅇ

Croton polyandrum. variegatum. 3 .

Erodium alpinum. Acalypha glebrata.

Geranium colnnbinum. $\gamma$. Malva Alexuofa.

Trifolium albidum. 2. Trigonella efculentro. pinnatifida.

Lotus edulis. friata.

grecus.

Fumaria bianrita.

Polygala lutea. $\beta$.

Buica Juperba.

Genifta ovata.

Dilvynia ulicifolia.

Lathy us fpurius. 1. 2. Vicia faba. 2.

lathyroides. $\beta$.

purpurea.

villoja.

Galega Orientalis.

Scorzonera erio/perma. 2.

$$
\begin{aligned}
& \text { firicta. } \\
& \text { villofa: }
\end{aligned}
$$

Laciuca crifpa. intybacers.
Lactuca palmate.

Leontodon taraxacum. B.

Picris pyrenaica.

Hieracium cymo/um. 3 .

dentatum.

fruticofium.

Crepis agreftis, $\beta$.

taraxifolia. 1, z:

Seriola cretenjis.

Lapfana lyrata. a. t. 2. B. $\%$.

Cichorium divaricatum. 2.

Hedypnois mauritanica.

Serratula alata.

noveboracenfis.

tinitoria. 3.

Carduus argemone.

dentatus?

Carlina echinus:

Cephalophora glaucu.

Stevia paniculata.

$$
\text { pedata. }
$$

Stcehelina alpina. Artemifia alpina.

Gnaphalium cephnloideums.

$$
\text { erofum. } \beta \text {. }
$$

Elichryfum bracteatum.

Conyza thapfoides.

Tusfilago farfara, 3. 4.

Serecio cruciatus. 3.

$$
\text { ovatus. }
$$

Solidago novceboracenfis.

Grindelia imuloides, $\alpha . \beta$. 
Arnica marittima.

Pyrethrum macrophyllum. par vifiorum.

Anthemis caucajica. clavata. fallax. fu/cata. parthenioides.

Achillaa grandifora. pubefcens.
grandiflora.
myriophylla. seracea.

Balbifia elongata. Gymnoftyles integrifolia, Bidens chinenfis.

Servulata. tripartita. 4 .

Coreopfis retufa. Georgina rofea. Helianthus letiflorns. locvis.

tubaformis. B.

Centaurea Adami. axillaris.

macrocephala. 2. uigrefcens. 2. fonchifolia, $\beta$. trinervis.

Strumpía marittima. Lycopodium obfcurum. Osmunda claytoniana. Ackroftichum lineare.
Achrofichum forbifolium.

Polypodium auguftum.

Afpidium oppendiculatum. $\varepsilon$. montanum. 2.

Lomazia procera.

Afplenium anceps. afveftictum. pygmскит.

Spiloma cinnabarinum. paradoxum.

Graphis fulcata. Jpherale. Stieta limbata.

Collema cynalisfum.

Ceramium diaphanum.

Gymnoftomum japonicum.

Ulva terreftris.

Fueus balthicus.

Lemania fubtilis.

Rivularia damae.

Telephora rubiginoja.

Trichia botrytis.

Spharia quaternata. tuboeformis.

Hyfterium oblongun.

Ecidium ulmi.

Erineum aureum. populinum.

Puccinia phaseoli. Sclarotium mobile. Suffultum. 
Viverra fure.

Muftela hitra. $\beta$.

Cavia paca

Lepus timidus. $\alpha . \beta_{j} \gamma$ variabilis. $\delta$.

Sciurus glis.

Mus amphibius.

Marmota capen/is.

Areitomys citellus.

Falco ater.

$P$ fittacus aftivus. $\beta . \gamma$.
Trochilus imimus 3.

Palamedea cormuta.

Tetrapteryx capenfis.

Larus glancus: $\beta$. junior: ridibundus.

Colymbus Aellatus.

Phafianus gallus. to $B$.

Mufcicapa homorrhoufa. a. B.

Tetraodon oblongus.

Carabus granulatus. $c$. auronitens.

Monftroos Grankotte. $\alpha$. $\beta . \gamma$. Monftrós Gran gren,

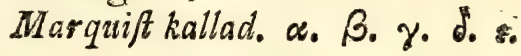

Naturx Thefauri, quos Cel. Præfes Regiæ A: cademix Upfalienfi, præterlapfis proxime XXXIII annis in publicum ufum benevole donavit, \& continuo auxit, individuis numerofis tam Animalium, quam Ve. getabilium conftare deprehenduntur, fcilicet

Mammalibus

Avibus 680.

Amphibiis 150.

Pifcibus 207 .

Infectis ultra soow.

Termibus 65.

Teftaceis ultra ins:
Ovibus Avium 70. ]

Nid. Avinm 8.

Craniis Anim. 20.

Seminib. Es fruti. plurimis.

Plantarum fol. 23,510.

quace in Herbario Species. sonfittunnt. 15,050\% 


\section{THE S E S.}

\section{I.}

Omnes res creatæ Divinx funt fapientix \& po. tentiæ teftes; nec Pietas in Deum, nec quanta huic - gratia debeatur, fine explicatione watura intelligi potert.

II.

Maximi igitur eft xutimandus, qui rerum naturx latebras dimover, nec exteriore eins confpeetu contentus, introlpicit \& in Divina fecreta defcendit.

III.

Animalia, herbe \& mineralia, quanquam alia ab aliis difcernere primo intuitu facile nobis videtur, regna tamen tria naturæ aretisfimis limitibus continentur, ut frpe difficile fit judicatu, ad quod naturære. gnum res nonnullas naturales rectisfime referamus.

\section{IV.}

Herbr, qux externa fua facie non inamænafunt \& ipfo adfpectu admirationem quandoque fui injicientes nos ad fe trahere videntur, ad nocendum fxpisfime maximas habent vires.

\section{V.}

Sicut clasfis pentandria medicorum maximam meretur attencionem, ita etiam triandria \& diadelphia a. griculx flurimas præbent utilitates. 

i 
